
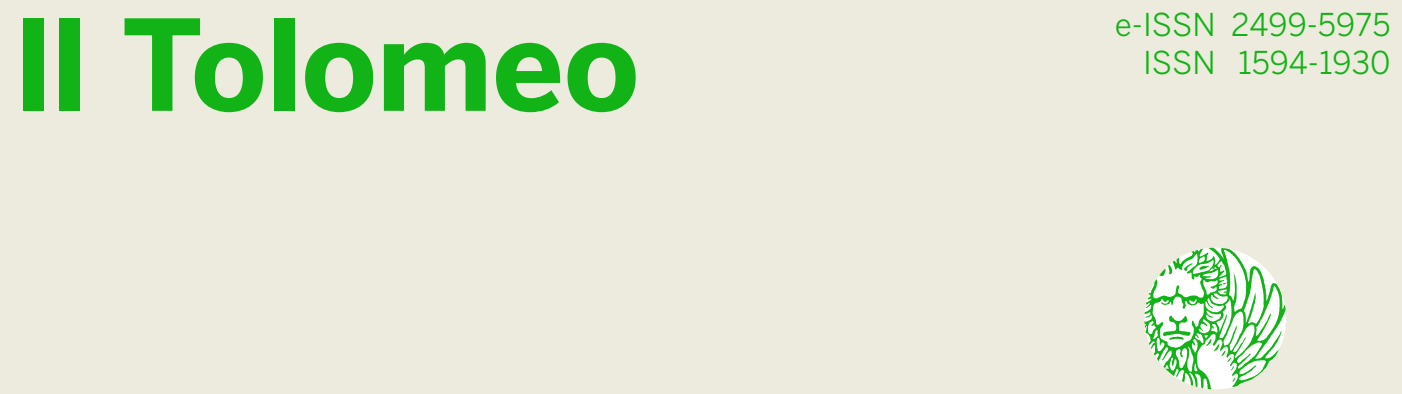

Vol. 20

Edizioni

Dicembre | December | Décembre 2018

Ca'Foscari
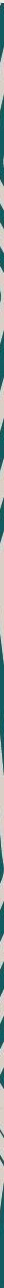
111 111111 N N N N N N N P 级

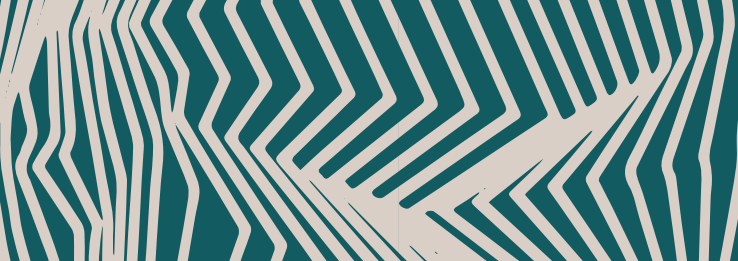





\section{Il Tolomeo \\ Rivista di studi postcoloniali \\ A Postcolonial Studies Journal Journal d'études postcoloniales}

Direttore | Director | Directeur

Alessandro Costantini

Edizioni Ca' Foscari - Digital Publishing

Università Ca' Foscari Venezia

Dorsoduro 3246

30123 Venezia

http://edizionicafoscari.unive.it/it/edizioni/riviste/il-tolomeo/ 


\title{
Il Tolomeo
}

\section{Rivista di studi postcoloniali | A Postcolonial Studies Journal | Journal d'études postcoloniales}

\author{
Rivista annuale | Annual journal | Revue annuelle
}

Direttore scientifico | General Editor | Directeur Alessandro Costantini (Università Ca' Foscari Venezia, Italia)

Consiglio di direzione | Board of Directors | Comité de direction Shaul Bassi (Università Ca' Foscari Venezia, Italia) Carmen Concilio (Università degli Studi di Torino, Italia) Marco Fazzini (Università Ca’ Foscari Venezia, Italia)

Comitato scientifico internazionale | International Advisory Board | Comité scientifique international Giulio Marra (fondatore | founder | fondateur /I Tolomeo, Università Ca' Foscari Venezia, Italia) Francesca Romana Paci (co-fondatore | co-founder | cofondateur /I Tolomeo, Università del Piemonte Orientale, Italia) Armando Pajalich (co-fondatore |co-founder|cofondateur II Tolomeo, Università Ca' Foscari Venezia, Italia) Elleke Boehmer (University of Oxford, UK) Andrea Cali (Università del Salento, Italia) Yves Chemla (Université Paris V, France) Graham Huggan (University of Leeds, UK) Jean Jonassaint (Syracuse University, USA) Józef Kwaterko (Uniwersytet Warszawski, Polska) Chris Mann (Rhodes University, South Africa) Marco Modenesi (Università di Milano, Italia) Radhika Mohanran (Cardiff University, UK) Sandra Ponzanesi (University College Utrecht, Nederland) Itala Vivan (Università degli Studi di Milano, Italia) Tony Voss (University of Kwa-Zulu Natal, Southafrica)

\begin{abstract}
Comitato di redazione | Editorial Board | Comité de rédaction Gerardo Acerenza (Università di Trento, Italia) Esterino Adami (Università degli Studi di Torino, Italia) Michela A. Calderaro (Università degli Studi di Trieste, Italia) Margherita Cannavacciuolo (Università Ca' Foscari Venezia, Italia) Marta Cariello (Università degli Studi della Campania «Luigi Vanvitelli», Italia) Carmen Concilio (Università degli Studi di Torino, Italia) Alessandro Costantini (Università Ca'Foscari Venezia, Italia) Irene De Angelis (Università degli Studi di Torino, Italia) Sara Del Rossi (Uniwersytet Warszawki, Polska) Alessandra di Maio (Università degli Studi di Palermo, Italia) Adriano Elia (Università degli Studi Roma Tre, Italia) Marco Fazzini (Università Ca' Foscari Venezia, Italia) Maria Paola Guarducci (Università degli Studi Roma Tre, Italia) Marie-Christine Jamet (Università Ca’ Foscari Venezia, Italia) David Newbold (Università Ca' Foscari Venezia, Italia) Luisa Pèrcopo (Cardiff University, UK) Biancamaria Rizzardi (Università di Pisa, Italia) Giuseppe Serpillo (Università degli Studi di Sassari, Italia) Silvia Riva (Università degli Studi di Milano, Italia) Eliana Vicari (Université Paris Nanterre, Nanterre Cedex) Alessia Vignoli (Instytut Romanistyki, Uniwersytet Warszawski, Polska) Ilaria Vitali (Università degli Studi di Macerata, Italia) Anna Zoppellari (Università degli Studi di Trieste, Italia)
\end{abstract}

\section{Segreteria di redazione | Editorial assistants | Secrétaires de rédaction}

Inglese | English | Anglais Lucio De Capitani (Università Ca' Foscari Venezia, Italia)

Francese |French|Français Fulvia Ardenghi (Università degli Studi di Trieste, Italia)

Direttore responsabile | Editorial Director | Directeur de publication Lorenzo Tomasin (Université de Lausanne, Suisse)

Direzione e redazione | Head office | Siège

Dipartimento di Studi Linguistici e Culturali Comparati, Università Ca' Foscari Venezia

Dorsoduro 1405, 30123Venezia| tolomeo@unive.it

Editore|Publisher|Éditeur Edizioni Ca' Foscari-Digital Publishing|Dorsoduro 3246, 30123 Venezia, Italia|ecf@unive.it

Stampa|Press|Impression Logo srl, via Marco Polo 8, 35010 Bogoricco (PD)

(C) 2018 Università Ca' Foscari Venezia

(C) 2018 Edizioni Ca' Foscari - Digital Publishing per la presente edizione

(ㅇ)(1)

Quest'opera è distribuita con Licenza Creative Commons Attribuzione 4.0 Internazionale

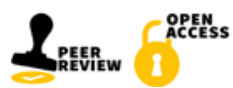

This work is licensed under a Creative Commons Attribution 4.0 International License 


\section{Sommario | Table of contents | Résumé}

TESTI CREATIVI | CREATIVE WORKS | CRÉATIONS

Les manuscrits trouvés sur eBay

La découverte de Robert Louis Vesque, écrivain algérien

pied-noir inédit d'avant l'exode

Alessandro Costantini

La main et le sein

Rita El Khayat

Rita El Khayat, une écrivaine de l'intime et du social

Anna Zoppellari

\section{Bioluminescence}

Jacqueline Bishop

Bioluminescenza

Traduzione di Federica Messulam

Jacqueline Bishop

The Julie Mango Tree

Jacqueline Bishop

Introduzione all'opera di Jacqueline Bishop

Michela A. Calderaro

ARTICOLI | ARTICLES | ARTICLES

Pudeur et splendeur de la prostitution chez Aimé Césaire et Zadi Zaourou

Représentations stylistiques du corps-tabou

Dorgelès Houessou 
De la dépossession à l'expérience de la possession dans Meursault, contre-enquête de Kamel Daoud Maha Badr

La fête et le spectacle carnavalesques dans Le Mât de cocagne (1979) et Hadriana dans tous mes rêves (1988) de René Depestre Irene Alvarez Domenech

"Un pays occupé est une terre sans vie »

Réflexions sur les subjectivités occupées et les espaces de résistance dans Kannjawou, roman de l'écrivain haïtien Lyonel Trouillot

Federica Cozzio

Stanley Péan et l'interculturalité en diaspora

Musique, zombis et marasa pour une haïtianité revisitée

Fabiana Fianco

Edward Elgar's Masque The Crown of India

Resonances of the Raj at the London Coliseum

Edvige Pucciarelli

James Joyce's 'Linguistic Musicality'

A Short Insight into Some Linguistic Musical Patterns in the First Chapter of $A$ Portrait of the Artist as a Young Man, and their Echoes in "Sirens"

Arianna Autieri

Thou Shalt not Kill! Or Notes on Caribbean Music as Literary Text on Being Human

Francio Guadeloupe, Ivette Romero

Caribbean Contemporary Poets and Artists Moored at the Venice Biennale

Margaret Spencer Matz

The Sieve of Translation

Reflections on the French Version of The Butcher Boy by Patrick McCabe 
INTERVISTE | INTERVIEWS | INTERVIEWS

"I Could Have Been Susanna From Thistlewood's Plantation Diary" Jamaican Poet Ann-Margaret Lim Discusses Her Second Poetry Collection Kingston Buttercup

Loretta Collins Klobah, Ari Hernández, Michelle Ramos Rodríguez

Doe Songs: Letting the Landscape Speak Through

the Truest Language, Poetry

Ann-Margaret Lim

Ngũgĩ wa Thiong'o: an Interview

Giulia Barison, Beatrice Carmello, Asmaa El Hansali, Dalia Pratali Maffei

RECENSIONI | REVIEWS | COMPTES RENDUS

Parisot, Yolaine (2018). Regards littéraires haïtiens. Cristallisations de la fiction-monde. Paris : Classiques Garnier, 385 pp. Coll. Bibliothèques francophones

Alessia Vignoli

Marty, Anne (2017). La littérature haïtienne dans la modernité. Paris : Éditions Kathala, 273 pp.

Fabiana Fianco

Martelly, Stéphane (2016). Les jeux du dissemblable : folie, marge et féminin en littérature haïtienne contemporaine. Montmagny : Éditions Nota bene, 379 pp.

Fabiana Fianco

Parisot, Yolaine (éd.) (2016). « Dany Laferrière : mythologies de l'écrivain, énergie du roman » (2016). Interculturel Francophonies, 30, novembre-décembre, $321 \mathrm{pp}$.

Alba Pessini

Charles, Jean-Claude (2018). Baskets. Coordonné par Alba Pessini. Montréal : Mémoire d'Encrier, 336 pp. Chronique

Emanuela Cacchioli

Sénac, Jean (2018). L'Enfant fruitier et Hadj Hamou, Abdelkader (2018). L'Offense 
Puccini, Paola ; Kirouac Massicotte, Isabelle (éds.) (2017). Bien-être en ville : espaces urbains, langues, culture et société. Bologna : I libri di Emil, 160 pp.

Sara Del Rossi

Puccini, Paola ; Kirouac Massicotte, Isabelle (éds.) (2016). Langue et pouvoir. Bologna : I libri di Emil, 128 pp.

Sara Del Rossi

Pfersmann, Andréas ; Porcher-Wiart, Titaua (éds.) (2017). "Francophonies océaniennes ». Interculturel Francophonies, 31, juinjuillet, 417 pp.

Anna Michieletto

Bernovsky, Victor (2017). « Francité, américanité et indianité dans le roman québécois contemporain ". Interculturel Francophonies, 32, novembre-décembre, $326 \mathrm{pp}$.

Veronica Brunetto

Biondi, Carminella (éd.) (2016). 1789: les colonies ont la parole. Anthologie, tt. 1-2. Paris : L'Harmattan, 498 pp. Autrement mêmes Elena Fermi

Modenesi, Marco (éd.) (2017). " Jouer avec les mots ". Ponts, Langues littératures civilisations des Pays francophones, 17, 245 pp.

Veronica Brunetto

Organisation internationale de la Francophonie [2006] (2013). Le Mouvement panafricaniste au vingtième siècle. Paris, 640 pp.

Giacomo Bottosso

Chambers, Iain; Curti, Lidia; Quadraro, Michaela (a cura di) (2014). Ritorni critici. La sfida degli studi culturali e postcoloniali. Milano: Meltemi, 245 pp.

La politica del ritorno: per una spettrologia dell'accademia Eleonora Meo

Kirsch, Adam (2016). The Global Novel: Writing the World in the 21st Century. New York: Columbia Global Reports, 105 pp.

Yuqian Cai

Concilio, Carmen; Festa, Maria (eds) (2016). Word and Image in Literature and the Visual Arts. Milano: Mimesis International, 397 pp. 
Collins Klobah, Loretta (2018). Ricantations. Leeds: Peepal Tree Press Ltd, 138 pp.

Michela A. Calderaro

NECROLOGI | OBITUARIES | NÉCROLOGIES

In Memoriam : Anne Marty (1947-2018)

Alessandro Costantini

In memoriam: Meena Alexander (1951-2018)

Shaul Bassi

BIO-BIBLIOGRAFIE | BIO-BIBLIOGRAPHIES | BIO-BIBLIOGRAPHIES 
La Direzione desidera esprimere un particolare ringraziamento a Francesca Romana Paci e Michela A. Calderaro per l'eccezionale ed essenziale contributo dato alla realizzazione di questo numero del Tolomeo.

The Editor would like to express special thanks to Francesca Romana Paci and Michela A. Calderaro for their exceptional and fundamental contribution to this issue of $/ /$ Tolomeo. 


\section{Testi Creativi | Creative Works | Créations}





\title{
Les manuscrits trouvés sur eBay La découverte de Robert Louis Vesque, écrivain algérien pied-noir inédit d'avant l'exode
}

\author{
Alessandro Costantini \\ (Università Ca' Foscari Venezia, Italia)
}

Sommaire 1 Prémisse. - 2 Historique de l'affaire Vesque'. - 3 L'auteur Robert Louis Vesque. - 4 La langue de ces textes : le pataouète. - 5 Les textes. - 6 Analyse contrastive de « La lettre au soldat » (§§ 1-7).

\section{Prémisse}

Mon but, ici, est celui de présenter et surtout d'offrir pour la première fois aux lecteurs, spécialistes et non, des textes d'un auteur francophone pratiquement inconnu du public, Robert Louis Vesque, disparu il y a plus de cinquante ans. Je présenterai en l'occurrence, en leur totalité, ${ }^{1}$ quelques textes courts qui présentent la particularité d'être écrits en pataouète, la langue populaire des Français d'Algérie.

Une analyse poussée de ces textes demanderait le concours de la philologie et de la linguistique ou de la dialectologie, qui ne sont pas de mise ici. Cependant, l'intérêt de ces textes est et reste certain ; ils constituent un témoignage et des documents de première main d'une langue (ou d'un dialecte, si l'on préfère) maintenant en voie de disparition : car c'est une langue ou un dialecte qui va disparaître fatalement, avec la disparition progressive de ses locuteurs naturels, les Pieds-Noirs, qui le parlaient normalement, tous les jours, chez eux en Algérie ou en Afrique du Nord, et de leurs descendants de première génération 'française'. Ceux-là souvent étaient nés là-bas (ainsi qu'on le dit en pataouète) et avaient été rapatriés avec leurs parents en 1962, au moment de l'indépendance de l'Algérie, non plus 'française', mais devenue une république indépendante habitée et gouvernée depuis par la composante majoritaire de sa population, jusqu'alors gardée dans une condition de subordination coloniale. La fin

1 J'inclus une page manuscrite (sans tapuscrit correspondant), « Précision », qui n'est que partiellement en pataouète et qui est assez difficile à déchiffrer ; ce n'est qu'un brouillon encore informe, mais c'était rangé avec les autres textes en pataouète, vraisemblablement par l'auteur, dont j'entends respecter la volonté ou le dessein en la publiant aussi. 
de cet état - historique et politique, mais aussi linguistique et culturel - des choses, constitue pour les uns, les victorieux restés sur place, une épopée ; pour les autres, les perdants, les vaincus poussés ou obligés à s'en aller, une tragédie, que beaucoup, de ce coté mais parfois ou souvent aussi de l'autre, ne souhaitaient pas.

Il reste que la langue pataouète, émigrée en France et arrachée de son habitat naturel et historique, n'a pu que perdre progressivement un bon nombre de ses locuteurs, de par leur décès, aussi bien que par l'assimilation dans la langue et la culture françaises de l'hexagone, pour ce qui concerne les jeunes générations issues des Pieds-Noirs.

Comment en suis-je arrivé à m'occuper de ces textes en pataouète, voire à les découvrir?

\section{Historique de l'affaire Vesque'}

Le 20 juillet 2014, sur eBay, j'ai pu acheter ce qu'on définissait comme « LOT œuvre littéraire Robert Louis Vesque (né 1895) Oran Algérie An 50 pied-noir ».

Important lot de travail littéraire (Romans et poésies) de Robert Louis VESQUE d'Oran né à Sidi Bel Abbès (Algérie) en 1895

Textes dactylographiés et parfois manuscrits sous jaquette et également des dessins originaux (en préparation d'édition) Oran années 50 Bon état général (voir photo)

Quelques titres : (Proses) La légende de l'étang maudit - Gai mémorial de Louis Parfait Bontour -

Les cimes tentaculaires - Pataouète - Le secretdes naufrageurs - etc... nombreuses poésies...à reclasser. ${ }^{2}$

Dans la courte liste de textes fournie par l'annonce, ${ }^{3}$ un mot a retenu tout de suite mon attention et suscité mon intérêt : Pataouète, le parler pied-noir (populaire) des Français d'Algérie. L'existence dans le lot en vente de textes censés être écrits en pataouète, a aguiché ma curiosité et mon intérêt pour cette trouvaille, puisque je cherchais et collectais depuis quelques temps les textes pataouètes publiés en vue de la constitution d'un corpus.

2 Interrogé à ce sujet, le libraire qui a mis en vente le 'lot Vesque' (manuscrits, tapuscrits, coupures de presse, lettres, photographies, films), a déclaré : «malheureusement je ne possède aucune information sur l'auteur, cet ensemble de documents se trouvait dans une bibliothèque particulièrement hétérogène (rare pour une bibliothèque généralement) et dont le propriétaire était décédé depuis longtemps » M.V. (courriel du 25-juillet-2014)

3 Il ne sera pas question de ces autres textes ici : ils seront traités et/ou publiés par la suite. 


\section{L'auteur Robert Louis Vesque}

Robert Louis Vesque est né à Sidi Bel Abbés en 1895 et mort à Oran en 1960. Marié à Oran en 1922 avec Anaïs Mathilde Fouque.

Le père de RLV était né en France (1851), fils de deux Français, tandis que sa mère était née en Algérie (1868), fille d'un Français et d'une Espagnole (mariés en Algérie, en 1854).

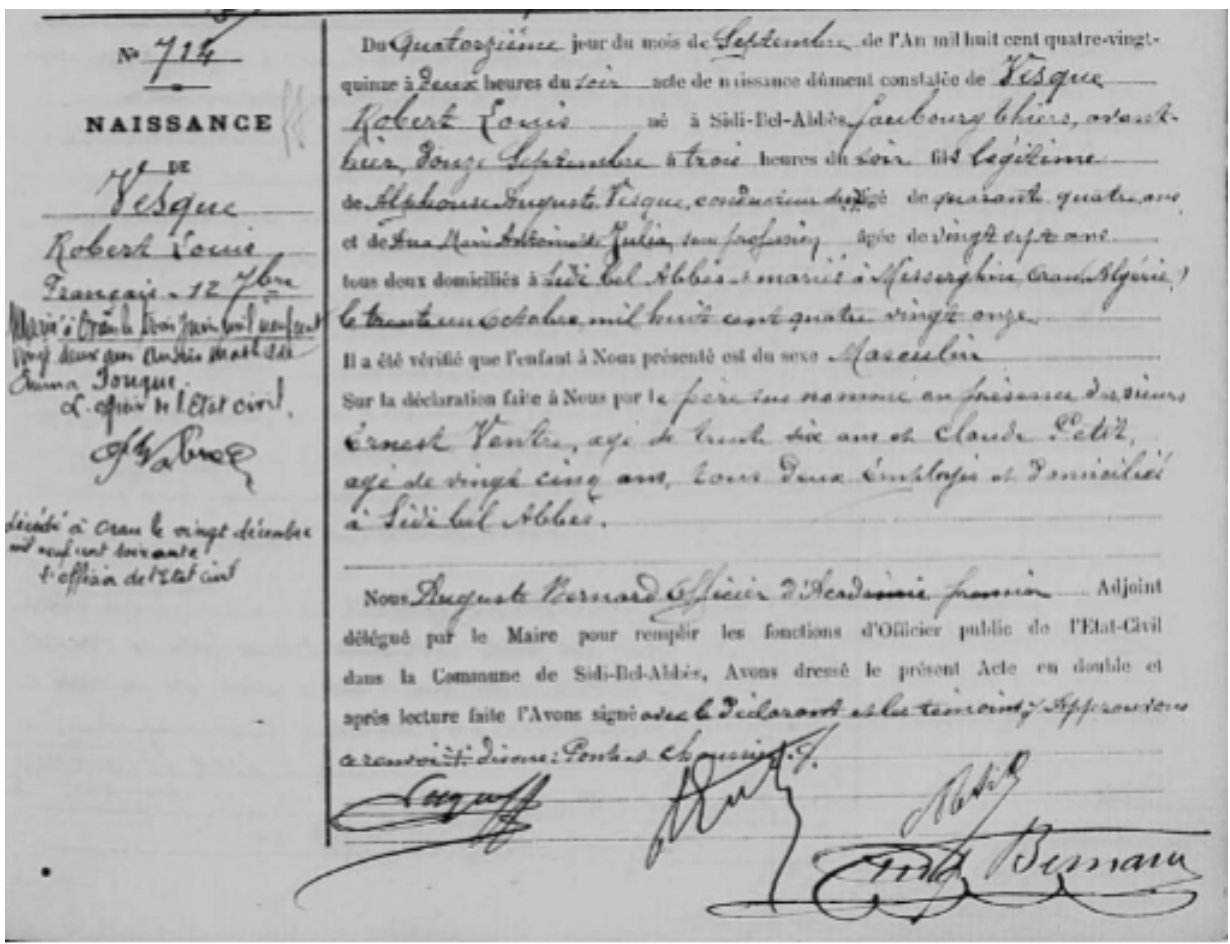

Figure 1. Robert Louis, acte de naissance, de mariage et de décès de Robert Louis Vesque

Robert Louis Vesque, Français d'Algérie ou Algérien Pied-Noir selon les différents points de vue (spatiaux aussi bien qu'idéologiques), apparemment n'a que très peu publié de son vivant ; simplement quelques textes courts dans la presse, surtout oranaise, ainsi qu'il le laisse entendre lui-même dans le document reproduit plus bas : «Auto - biographie sommaire ».

Pour l'instant, seulement quelques rares coupures de périodiques (incluses dans le 'lot Vesque') témoignent de la publication de quelques petits textes de sa main. Cependant il a, il avait écrit longtemps, ou toute sa vie durant peut-être : et il y a une œuvre de Robert Louis Vesque inédite et qui attend encore ses lecteurs. 
La valeur de ses écrits (romans, nouvelles, poèmes et autres textes) reste à établir, ${ }^{4}$ comme l'analyse en reste à faire : pourtant, l'ensemble est sûrement intéressant. Une œuvre non imposante, certes, mais non pas exiguë non plus, surgit du silence et de l'obscurité qui sont descendus pour recouvrir petit à petit, depuis des décennies, un peuple - les Français vivant dans l'Algérie dite coloniale et dite française aussi - en voie d'extinction physique peut-être, mais qui ne cesse pas moins de revendiquer son identité dans la déchirure du déracinement de sa terre natale. Une œuvre en quelques manière égarée et qui fait surface, cinquante après la mort de son auteur, tel un manuscrit dans une bouteille lancée à la mer ; qui fait surface dans la Mer, dans l'Océan de notre temps, c'est-à-dire sur internet, pour que quelqu'un puisse la recueillir et lire son message, ainsi que j'ai pu le faire.

\section{$4 \quad$ La langue de ces textes : le pataouète}

Il n'y a pas qu'un seul pataouète, contrairement à ce que l'on est amenés à penser de par le nombre grammaticalement singulier de ce substantif. La raison en est au fond assez simple : les individus venus peupler de manière coloniale surtout les territoires correspondant à ce que nous appelons aujourd'hui l'Algérie, étaient d'origines fort différentes. Il y avait les Français, certainement, venant de divers départements (entre-autres, plus nombreux les Corses, les Aquitains, les Provençaux, les Catalans, les Savoyards...). ${ }^{5}$ Par moments ou par endroits, les Français de 'souche' n'étaient pas toutefois les plus nombreux, du moins parmi les couches populaires de ce nouveau peuple, car il y avait des Européens de tous bords (Suisses, Luxembourgeois, Scandinaves, Roumains, Russes, Belges, Hollandais, Bulgares, Irlandais, Grecs, Polonais, Autrichiens, Serbes). Surtout il y avait des Méditerranéens du Sud : Espagnols (dont le pourcentage est le plus important), venant des îles Baléares, de Valence, du Levant espagnol ; Italiens venant de Campanie, Calabre, Toscane, Sicile, Émilie, Piémont, Lombardie, Vénétie et Sardaigne ; ensuite, troisième et dernier groupe important d'immigrés, les Maltais... Et enfin, installés sur les lieux depuis des siècles, les Juifs. ${ }^{6}$ Tout cela constituait la composante qu'on appelait non-indigène, en somme européenne, des habitants de l'Algérie française.

4 Dont les titres sont partiellement cités dans son « Auto - biographie sommaire » : cf. plus bas.

5 Pour des détails sur la composition départementale de la population de'souche' française : cf. Verdès-Leroux 2001, 194.

6 Sur les origines de la composante étrangère, ainsi que sur leurs proportions et sur sa distribution sur le territoire algérien ; cf. Verdès-Leroux 2001, 204-20 ; Hureau 2001, 269-71 ; Stora 1991, 27-8 ; Lanly 1962, 11-16. 
Le pataouète, à l'origine pour certains le parler des émigrants espagnols (les Pataouètes), a été le parler pied-noir d'Alger et de sa région, plus en général et par extension le parler populaire des Français d'Algérie, voire la langue pratiquée dans tout le Nord de l'Afrique du Nord. Ce parler, dans la conscience épilinguistique de ses parlants, est " composé de français, de catalan, de castillan, d'italien, de maltais et d'arabe » (Duclos et al. $1992,163){ }^{7}$

On dit aussi que le pataouète est un « français d'Afrique du Nord [qui] rappelle étrangement le 'latin provincial de Gaule' [...] et le latin vulgaire des Gaulois qui a pris sa place après les invasions barbares et la chute de l'Empire romain » (Lanly 1962, 5-6). Pour Lucien-Paul Fauque :

C'est le langage usuel du petit peuple des grandes villes d'Algérie, le ciment linguistique d'une société multi-ethnique algérienne en formation. Le mot pourrait dériver du vocable catalan 'patuet', patois. L'amalgame des cultures dont étaient porteurs les immigrants, les emprunts non négligeables à l'arabe dialectal, donna naissance au cours des générations à un français truffé de mots et d'expressions imagés avec accompagnement gestuel. L'apport linguistique provient du bassin méditerranéen avec, par ordre d'importance, l'italien (y compris le patois sicilien, napolitain, génois), l'espagnol (castillan, catalan), le provençal, le maltais, l'arabe. (Cité dans Anonyme 2000,4)

Aussi, « disons que le pataouète est un français gargantuesque » (Zakine $2015,9)$, bien que ses locuteurs «ne parlaient pas, dans leur grande majorité, et en permanence, comme Cagayous ou Roro. ${ }^{8}$ Pourtant certains termes, certaines tournures et surtout l'accent sont représentatifs du mode d'expression pied-noir » (Anonyme 2000,4).

Il n'a pas fait l'objet de descriptions et analyses linguistiques poussées, larges et détaillées, si ce n'est par Albert Lanly, dans son Le français d'Afrique du Nord. Étude linguistique, paru à Paris, chez les Presses Universitaires de France, en 1962, ${ }^{9}$ l'année même de l'abandon (le soi-disant 'rapatriement') de la part des Français d'Algérie d'un pays qui était désormais le leur aussi. Le moment de la consécration scientifique de ce parler par la publication d'une thèse d'État qui le décrit, coïncide avec l'exil de ce français africain,

7 Ou de mots juifs et portugais Moréno (1995, 199). Cf. en outre, sur la définition de 'pataouète' : Duclos 1992, 113-14 ; Vasquez 2004, 114.

8 Figures littéraires : Cagayous est le héros éponyme d'un cycle 'de gestes populaires piedsnoirs', publié par Musette à partir de la fin du XIXe s. ; Roro est le titre du dictionnaire pataouète de l'écrivain, journaliste, humoriste Roland Bacri (à l'instar de Littré, Larousse et Paul Robert, qui ont donné leur nom à leurs dictionnaires).

9 Très récemment, il y a eu aussi l'étude fouillée mais plus circonscrite de Ahmed Kaddour (2013), sur les hispanismes dans le Ouest de l'Algérie. 
avec la perte de ses racines, de son terroir et, fatalement, avec le début d'un long processus qui va porter, tôt ou tard, à sa disparition en tant que langue vivante parlée et pratiquée communément : ceux qui, au moment de quitter l'Algérie, étaient des jeunes parlants adultes, sont maintenant des personnes âgées, des octogénaires ou presque.

Quelle est, actuellement, la vitalité de cette langue ? En 1993, c'est-àdire trente-et-un an après l'exode, Gerard García $(1993,91)$ disait que le pataouète n'est pas mort avec le départ des Français d'Algérie : qu'il survit dans son vocabulaire, mais qu'il disparaît dans sa syntaxe qui prend une forme nouvelle sous la pression de la syntaxe arabophone. Le « pataouète est vivant, [mais...] il s'est différencié et il y a maintenant le nouveau pataouète algérien ».

On peut considérer que le pataouète c'était une langue qui serait comparée au bas latin mais cette langue n'a pas eu le temps d'évoluer. Elle évolue différemment actuellement mais [...] pas question de publier une grammaire pataouète, il est simplement question de traduire un dictionnaire pataouète-français. (García 1993, 92)

Par ailleurs, depuis longtemps, l'attention portée à l'Afrique du Nord de la part des scientifiques et des critiques littéraires est accaparée en mesure majeure moins par la langue que par la littérature des Pieds-Noirs, d'un côté $;^{10}$ de l'autre, inévitablement, c'est la langue des autres, des vainqueurs, qui occupe et occupera stablement le devant de la scène et fera l'objet principal des études et des analyses linguistiques des spécialistes, axées aujourd'hui surtout sur la langue et la littérature d'expression française maghrébines, donc des auteurs autochtones ('indigènes', disaiton autrefois) d'origine non européenne (arabe, berbère) et restés sur place. Quelques glossaires ou lexiques du pataouète, cependant, ont vu le jour aussi, surtout au cours des dernières décennies.

L'importance du pataouète et de ses manifestations culturelles, aussi bien que de ses études, est le propre d'un peuple neuf, quoiqu'à la vie brève : la lui reconnaître et accorder est une action historique et culturelle juste et nécessaire, qui n'a rien à voir avec une justification du colonialisme (cf. Dugas 2003, 143).

10 Voir les travaux de Paul Siblot, centrés principalement depuis une trentaine d'années sur l'analyse du français en Afrique du Nord ; travaux dont la perspective est, dans maints cas, moins intralinguistiques que sociolinguistique et culturelle. 


\section{Les textes}

Le lot de manuscrits et tapuscrits de RLV en ma possession, comporte un dossier intitulé 'Pataouète', comprenant cinq textes manuscrits et dactylographiés aussi :

1. «La lettre au soldat »

2. «Le rhume du tio Felipe »

3. «L'autre samedi... »

4. «La preuve a par neuf »

5. «El testament».

Un sixième texte - «Précision » - ne comporte que sa version manuscrite. ${ }^{11}$

Un seul de ces textes pataouètes, "La lettre au soldat », présente le manuscrit, le tapuscrit et une traduction française : est-ce bien la traduction du tapuscrit ? du manuscrit ? ou des deux à la fois et en même temps d'aucun des deux, étant plutôt une récréation originale?

$\mathrm{Au}$ suket de ce texte l'auteur déclare :

Cette lettre a été écrite dans un but d'initiation pataouétique. . Elle est suivie, en effet, de sa 'traduction' en langue française. Voilà pourquoi les phrases sont numérotées dans les deux textes de 1 à 16 environ.

Cette lettre déborde d'amour maternel

Je vais m'occuper ici de cette lettre - partiellement, pour les sept premiers paragraphes, à titre d'exemple -, pour donner un aperçu des problèmes, ainsi que de l'intérêt, linguistiques, culturels, esthétiques de ces textes et des textes en pataouète en général.

Dans le passage du manuscrit au tapuscrit, en général les variantes sont surtout de l'ordre de l'orthographe, de la phonétique, de la grammaire ou du style, avec quelques détails en plus ou en moins ; par contre, dans le passage du tapuscrit original en pataouète de « La lettre au soldat » à sa traduction française, les différences sont de l'ordre de la sémantique, de la sémiotique, de la culture et servent à montrer le décalage, la distance entre l'univers pied-noir et l'univers français hexagonal des Frankaouis.

11 Cf. ci-dessus, la note 1. 


\section{Analyse contrastive de « La lettre au soldat » (\$§ 1-7)}

1

Ms : t'écris

Tap. : Jé t'écri abec oun rayon tinta pourquoi j'a pas bésoin de lé tremper que ça mé fait tromper.

Tr. : Je t'écris avec un crayon fuschine car c'est plus commode.

2

Ms : y nous lé sommes plus depouis qué nous sommes guéris qué le médecin c'est oun tchambon.

Tap. : Mon fils, nous abons été maluchos et nous le sommes plus dépouis qué nous sommes guéris que le médecin c'est oun tchambon.

Tr. : Nous avons été malades et le médecin nous a guéris par hasard.

3

Ms : Depouis que tu t'es - bien aperçu - la

Tap. : Depoui que tou [t'] es parti pos on s'est [bien] aperçu que tou étais plus là.

Tr. : Nous ressentons vivement ton absence.

4

Ms : Mon fils y faut qué tu fasses - 1ère classe -

Tap. : Mon fils y faut qué tou soyes oun soldat dé prémière classe como ton padre qui sé lé ciré les bottines al capitaine

Tr. : Il faut que tu devienne soldat de Ière classe comme ton père qui était ordonnance.

5

Ms : con...concombres (tohé [?] jé té dirai pas !) qu'en français - y sé dit - Y ce jour -

Tap. : Ahier nous abons ramassé les petits con...concombres [...] qu'en français il sé dit les courts nichons. Y cé jour là yo né sé pas pourquoi ta mère il a fait qué penser à toi

Tr. : Hier nous avons récolté les cornichons. D'habitude c'est toi qui en étais chargé.

6

Ms : i sa marié - ton pauvre oncle - qué - $a$ dedans

Tap. : Ton cousin Pepe i s'a marié avec sa femme que tu la connais bien. C'est la tuerta qu'elle nous a tant fairt rigoler por l'enterrement dé ton pobre oncle Pascual que le Bon Dieu y le tienne à dedans ses bras. 
Tr. : Ton cousin a épousé cette femme importune et mal élevée qui n'a même pas respecté la tristesse d'un jour de deuil.

7

Ms : qué - la feste - y sont fait - mousique - Que mala suerte que tou étais pas la-de les bourricots - que tou serais arrivé lé premier.

vinga el baile - qui sé sont - les paniers - hasta quatro ores del matin - on sé - trouvé el lendemain al jardin poublic al desur les escaliers de la Mairie - que lé garde de nuit pour sûr il va se gagner

Tap. : Dommage que tou a pas vu la fête del village ! Premièrement ils ont fait des corridas, oun banquete y de la musique abec des variantes. Que si tou avais été là pour la course des bourricots tou seré arrivé le premier. Y lé soir à la replaceta vinga le bal, qui se sont remué lé panier de la crotte hasta quatre heures dou matin y qué les crottes on se les a trouvé devant de la Mairie y a desur les escaliers dé l'église ; que le gardien de nouit par force il va se gagner à la loterie.

Tr. : Que n'étais tu là pour la fête du village ! Il y a eu des réjouissances variées et comme tu es bon cavalier tu serais arrivé premier sur notre âne qui a déjà gagné plusieurs courses.

Le soir, il y a eu bal sur la place et les disciples de Tersichore ont tournoyé jusqu'au matin. Comme ils étaient nombreux, ils ont semé des immondices un peu partout et le gardien de nuit, trompé par l'obscurité a dû changer de souliers.

\section{Annexe - Détails biographiques et généalogiques}

Données de sources publiques

VESQUE Robert Louis :

- né à Sidi-Bel-Abbès le 12-09-1895 ;

- (annonce dans : Le Progrès de Bel-Abbès [ « puis » de Sidi-Bel-Abbès]. Journal de l'arrondissement de Sidi-Bel-Abbès, du 1895/09/22 puis dans : Le Messager de l'Ouest : journal de l'arrondissement de SidiBel-Abbès, du 1895-09-17 ;

- fils de Alphonse Auguste VESQUE, conducteur, âgé de 44 ans et de Marie Antoinette JULIA, sans profession, âgée de 27 ans.

- mariage annoncé dans : L'Avenir de Bel-Abbès. Journal agricole, commercial, industriel, politique et littéraire p... (1891-10-29), page 2 «Mariage - Nous apprenons avec plaisir le mariage de M. Vesque conducteur des Ponts et chaussées, qui a habité longtemps Bel-Abbès, et y a laissé les meilleurs souvenirs avec Mlle Julia, fille d'un des colons les plus estimés de Misserghin et qui a été maire de cette localité pendant 15 ans »(URL https://bit. ly/2PAhdh7). 
Renseignements de source privée (famille ; Pieds-Noirs rapatriés)

(deux courriels d'Évelyne Navarro Romero, du 4 mars 2018 : RLV est « un cousin issu de germain, à 5 generations)

VESQUE Robert Louis

- Il est né le 1895-09-12 à 14 h00 à Sidi Bel Abbes, faubourg négrier, Algérie ;

- Marié à Oran le 1922-06-06 avec Anais Mathilde FOUQUE ;

- Il est décédé à Oran le 1960-12-20.

Sa famille :

Le père de RLV (Alphonse Antoine Vesque ) était français, né en France (Eure, 1851) de parents français. Il s'était marié à Miserghin, Algérie, en 1891 avec Marie Antoine Julia, née à Miserghin (1868), fille d'un père français (n. en 1830 à Puyserguier, Béziers) et d'une mère espagnole (née en 1837 à Alicante), mariés en Algérie, en 1854.

Détails sur la famille

Son père :

- Alphonse Antoine VESQUE né le 14:01/1851 à Ecardenville la Campagne, Eure, France ;

- il était conducteur pour les Ponts Chaussées, il travaillait à la construction du barrage ; hydraulique de Cherfa, Saint Lucien, Miserghin, Algérie ;

- son père était le fils de Victor Désiré VESQUE, propriétaire terrien à Ecardenville la Campagne, Eure, France et de Marie Clotilde LUCAS ;

- marié à Miserghin, Algérie le 1891-10-31 avec Marie Antoine JULIA.

Sa mère :

- Marie Antoine JULIA née à Miserghin, Algérie le 1868-08-04 ;

- fille de Louis Pierre JULIA né le 1830-12-06 à Puyserguier, Béziers, France( de Louis JULIA et Rose FABRIER) marié le 28/01/1854 à Miseghin avec Maria NAVARRO née le 12/12/1837 à Novelda, Alicante, Espagne de Antonio NAVARRO et Maria Manuella Antonia BELTRA, originaires de Novelda, Alicante, Espagne.

Et c'est là où se rejoint ma branche (j'ai les actes de mariage et de naissance).

Je suis née en 1959, il est mort en 1960. Mes parents disaient qu'il était fragile des poumons, il avait participé à la guerre, on pensait qu'il y avait été gazé, comme d'autres hommes de la famille. Je $\mathrm{n}$ <ai pas d'autres éléments hélas, creuser peut être du côté du journal de 
l'époque « les Échos d’Oran »? [Évelyne Navarro Romero]

Courrier personnel reçu de Joseph Martinez (91 ans), le 7 mars 2018 :

Je pense que Mr Vesque Robert Louis avait deux frères.

Lucien né le 5-12-1899 à SbA [Sidi Bel Abbès]: Employé au chemin de fer. Armand né le17-4-1901 à SbA : Agent au service vicinal.

Et lui Robert Louis : Rédacteur de l'administration départementale. Voilà le résultat de mes recherches.

Joseph Martinez

Données ultérieures, dont la pertinence est possible, mais non vérifiée/ vérifiable

Sur un des deux frères possibles de l'auteur: Recueil des actes administratifs de la Délégation générale du Gouvernement en Algérie : Lois et décrets...du 09-09-1960

- Promotion de Vesque Armand, le 1958-02-04

- Affectation à : Ponts et Chaussées, $2^{\mathrm{e}}$ circonscription d'Oran (http://gallica.bnf.fr/ark:/12148/bpt6k9774914t/f30.image. $r=$ Vesque $\% 200$ ran? $r k=214593 ; 2$ )

- Un sous-lieutenant Vesque (pas de prénom), le 9 janvier 1915, s'est battu avec bravoure etc... S'agit-il de Robert Louis Vesque ou pas ? (https://bit.ly/2EpUbsg; https://bit.ly/2QIT6Sn)

\section{Bibliographie}

Dictionnaire, glossaires etc.

Bacri, Roland (1969). Le roro. Dictionnaire pataouète de la langue piednoir, étymologique, analogique,didactique, sémantique et tout. Paris : Denoël, $165 \mathrm{p}$.

Bacri, Roland (2000). Trésors des racines pataouètes. Paris : Éditions Belin, $223 \mathrm{p}$.

Duclos, Jeanne Massa ; CharlesAndré Monneret ; Jean Pleven, Yves (1992). Le pataouète. Dictionnaire de la langue populaire d'Algérie et d'Afrique du Nord. Calvisson : Éds. Jacques Gandini. 246 p.

Duclos, Jeanne (1992). Dictionnaire du français d'Algérie. Français colonial, pataouète, français des Pieds-Noirs. Paris : Éditions Bonneton. 160 p.

Kaddour, Ahmed (2013). Los hispanismos en el oeste de Argelia : cambios formales y deslizamientos semánticos. Saarbrücken : Publicia, ix, $548 \mathrm{p}$. Lanly, A. [André] [1962] (1970). Le français d'Afrique du Nord (Étude linguistique). Paris : Presses Universitaires de France, 367 p.; 2e éd., Paris : Bordas, 1970, 367 p.; réimpr., Monpellier : Mémoire de Notre 
Temps, s.d., 367 p.; 3e éd., Le français d'Afrique duNord : Qu'estce que le pataouète? Paris: Tchou Bibliothèque des Introuvables (coll. "L'Algérie d'Autrefois »), 2003, 367 p.

Mazella, Léon (1989). Le parler pied-noir. Mots et expressions de là-bas (lexique établi par). Paris : Rivages, 116 p.; avec une « Préface », 916 p. Moreno, Amédée (1995). Le parler d'Oran et d'Oranie. Memento-lexique avec anecdotes, histoires et souvenirs de là-bas. Calvisson : Éds. Jacques Gandini. 305 p.

Vazquez, Joseph (2004). Le DicOranais (La tchatche à l'oranaise). Nîmes : Éditions Lacour, $153 \mathrm{p}$.

Zakine, Hubert (2015). La langue de chez nous autres. Toulon : Les Presses du Midi, 90 p.

Bibliographie critique

Audisio Gabriel [1979] (1931). "Essai sur la langue de Cagayous ». Musette, Cagayous ses meilleures histoires. Paris: Gallimard ; rééd., Musette, Cagayous. Paris : R. Laffont, 1979, 11-30.

Audisio Gabriel (1979). «Lexique ». Musette, Cagayous. Paris : R. Laffont, 289-99.

Flores, Christian (1988). Le voleur d'huile. L'Espagne dans l'Oranie Française (18301962). s.l. [Montpellier] : s.é. (collection « Français d'ailleurs »), 197 p.

García, Gérard (1993). "Le français populaire d'Algérie ou 'pataouète' ». Comptes rendus trimestriels des séances de L'Académie des Sciences d'outre-mer. Tome LIII - 1 - 2, 81-92.

Hureau, Joëlle [2001] (2010). La mémoire des pieds-noirs de 1830 à nos jours. Paris : Perrin ; rééd., Paris : Perrin (coll. « Tempus »), 375 p.

Siblot, Paul (1985). « Mises en texte de la pluriglossie dans la littérature coloniale ». Cahiers de praxématique, 5 (Conflits diglossiques et production textuelle), 103-36.

Siblot, Paul (1987).« 'Cagayous antijuif'. Un discours colonial en proie à la racisation ». Mots, 15 (octobre), 59-75.

Stora, Benjamin (1991). Histoire de l'Algérie coloniale (1830-1954). Paris : Éditions de La Découverte, 124 p. ; nouv. éd. 2004.

Verdès-Leroux, Jeannine (2001), Les Français d'Algérie de 1830 à aujourd'hui. Une page d'histoire déchirée. Paris : Fayard, 492 p. 


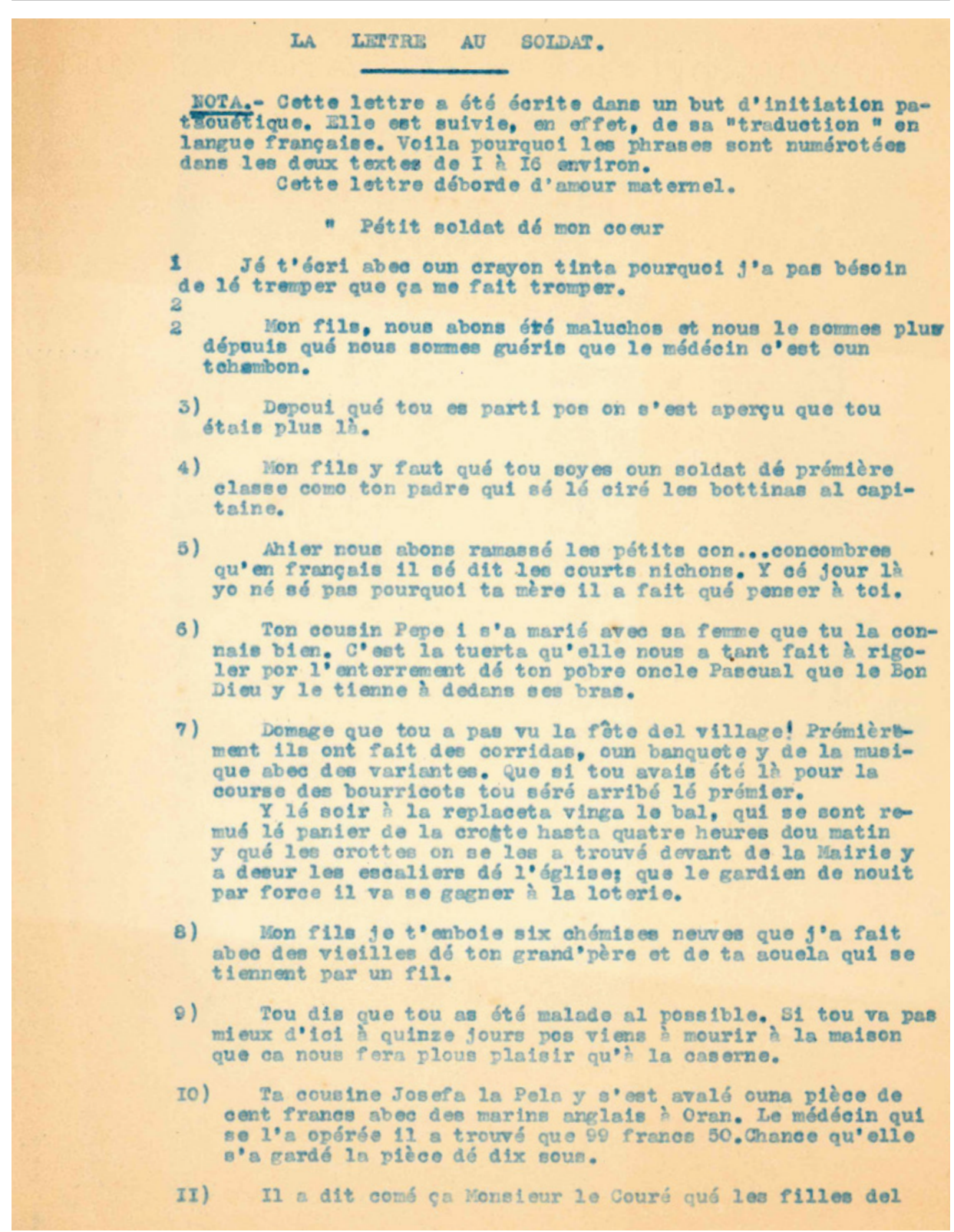

Figure A1. Lettre au soldat (tapuscrit) 


\section{( 2 )}

village e'est des pavas et des ofes blanches - qu'encore une j'ai pas vu- et qu'à la ville c'est des olseaux de nuit. Attention mon flis à ça qué tu manges y quand tou as fáim laisse toi de la carte.

I2) Quand tou sors lé solr, ne t'oublie pas la corbate y la capote que tu peux à choper oun rhoume cojonique;; coront que qu'il a dit ton parrain.

I3) La fiebre affreuse elle se mange les bichos à cornes que le plou malade natourellement $c^{\prime}$ est ton padre qu'il se 1 'est enganché de primera.

I4) Le temps il s'a mis al mauvais que la plouie 11 arrêté

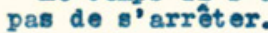

I5) Dans le journal 11 est mort le $111 \mathrm{~s}$ de Perico el Cartero. Le pobre défa qu'1l pouvalt pas marcher ni parler bolla qu'1l s'est oublié dé respirer. Cinq mo1s 11 avait. Sa mère Dolorè elle boudrait aller avec luí al Paradis mais elle peut pas pourquol elle a pas la elef. Ba y manquerait; elle et pas 1 es autres.

I6) Pos voila mon f1ls.Jé crois qué ta mère elle a dit des bêtisses mais to1 né $t$ 'on fals pas, attention $Y$ dit $t$ on

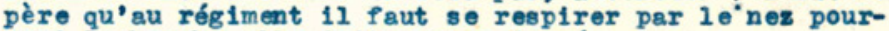
quoi la bouche elle dolt rester fermée.

Jé te mets à desur mon coeur abec ton padre, tes frères les primos y toute la famille.

Ta mère qui t'aime plous qué ton père 11 aimé l'anisette. MARIa la Pelua.

TRADUCTION.

( Ia traduction peut être inversée en tant que besoin .)

I) Je t'écris avec un erayon fuschine car c'est plus commode.

2) Nous avons été malades et le médecin nous a guéris par hasard."

3) Nous ressentons vivement ton absence.

4) Il faut que tu deviennes soldat de Ière elasse commet on père quil était ordonnance.

5) Hier nous avons récolté les cornichons. D'habitude e'eat toi qui en étais chargé.

6) Ton cousin a épousé cette femme importune et mal élevée qui n'a môme pas respecté la tristesse d'un jour de deuil.

7) Que n'étais tu là pour la fête du village f Il $\mathbf{y}$ a eu des réjouissances variées et comme tu es bon eaválier tu serals arrivé prèmier sur notre êne qui a défa gagné plusieurs courses. Le soir,il y a eu bal sur la place et les diseiples

Figure A2. Lettre au soldat (tapuscrit) 
de Terpsiohore ont tournoyé jusqu' au matin. Comme 11: étaient nombreux, 11s ont wemé des lmmondices un pou partout ot 1 gardien de nuit, trompé par l'obscurité a dû. changer de souliers.

8) Je t'envole six chemises talliées dans celles de tes grands perents. Les chemises $t$ 'iront mais les vieux ne vont pas bien.

9) Nous sormes $t$ rès inqui ets de te savo1r maladex et nous ne voudrions pas que tu meures loin de nous.

I0) Ta cousine au cours d'une fugue d̀ Oran a, par mégarde, avalé une pièce de monnaie. Ie chirurgien $y$ a vis bon ordre et 1 'opération a démontré que Joséphine avait conservé sa virginité.

II) M. Ie Curé $\mathrm{m}^{\circ} \mathrm{a}$ mise en garde contre les pilles de joie, cartées en général. İvite 1 es le plus possible quitte à passer pour un jeune homme quil s'amuse tout seul.

I2) Mets ta cravate et ta capote loraqu'il fait froid.

13) Ia fièvre sphteuse décime les bêtes c̀ cornes et ton père, qui les soigne, en est atteint.

I4) Ia récolte s'annonce mal à causê de la sécheresse.

I5) Le facteur a perdu un bébé de cinq mois. Ia mère est inconsolable.

I6) Ta mère 'exprime mal, mals elle te donne un dernier consell: pas de bêtises, surtout au Régiment où 11 ne faut jamaís répondre ̀े ses chefs.

I7) Nous t'embrassons tous de tout eour.

Figure A3. Lettre au soldat (tapuscrit) 


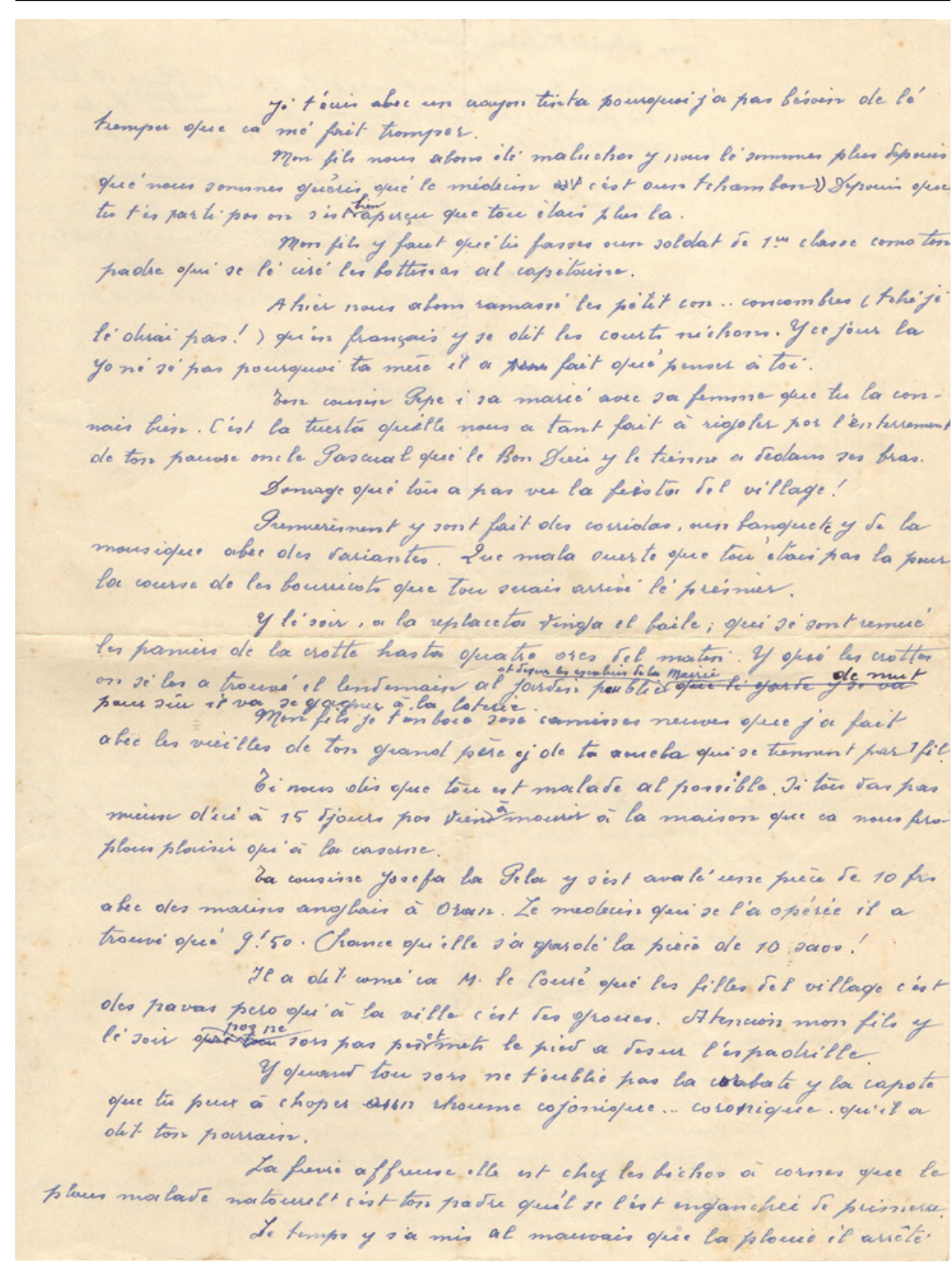

Figure A4. Lettre au soldat (manuscrit) 


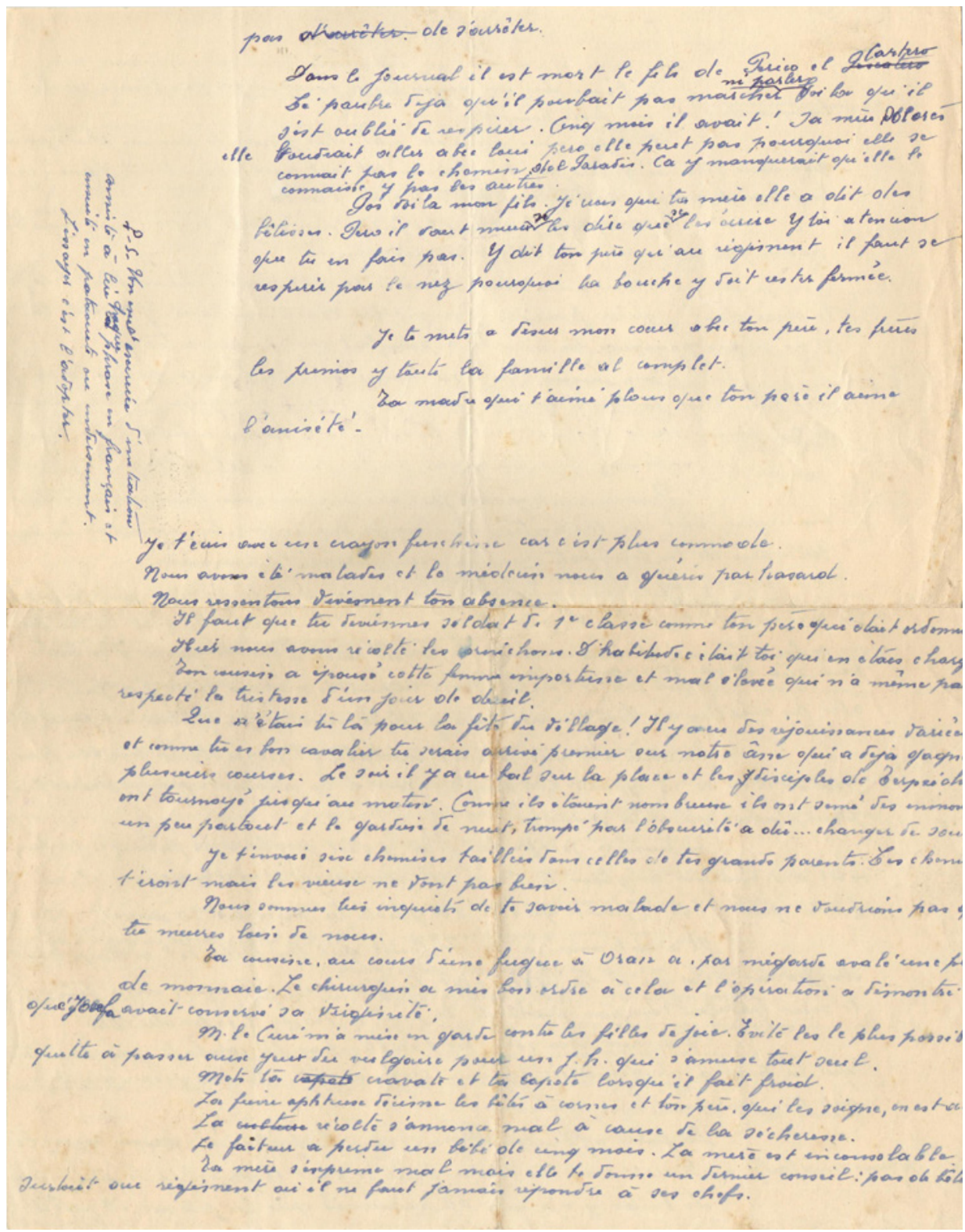

Figure A5. Lettre au soldat (manuscrit) 


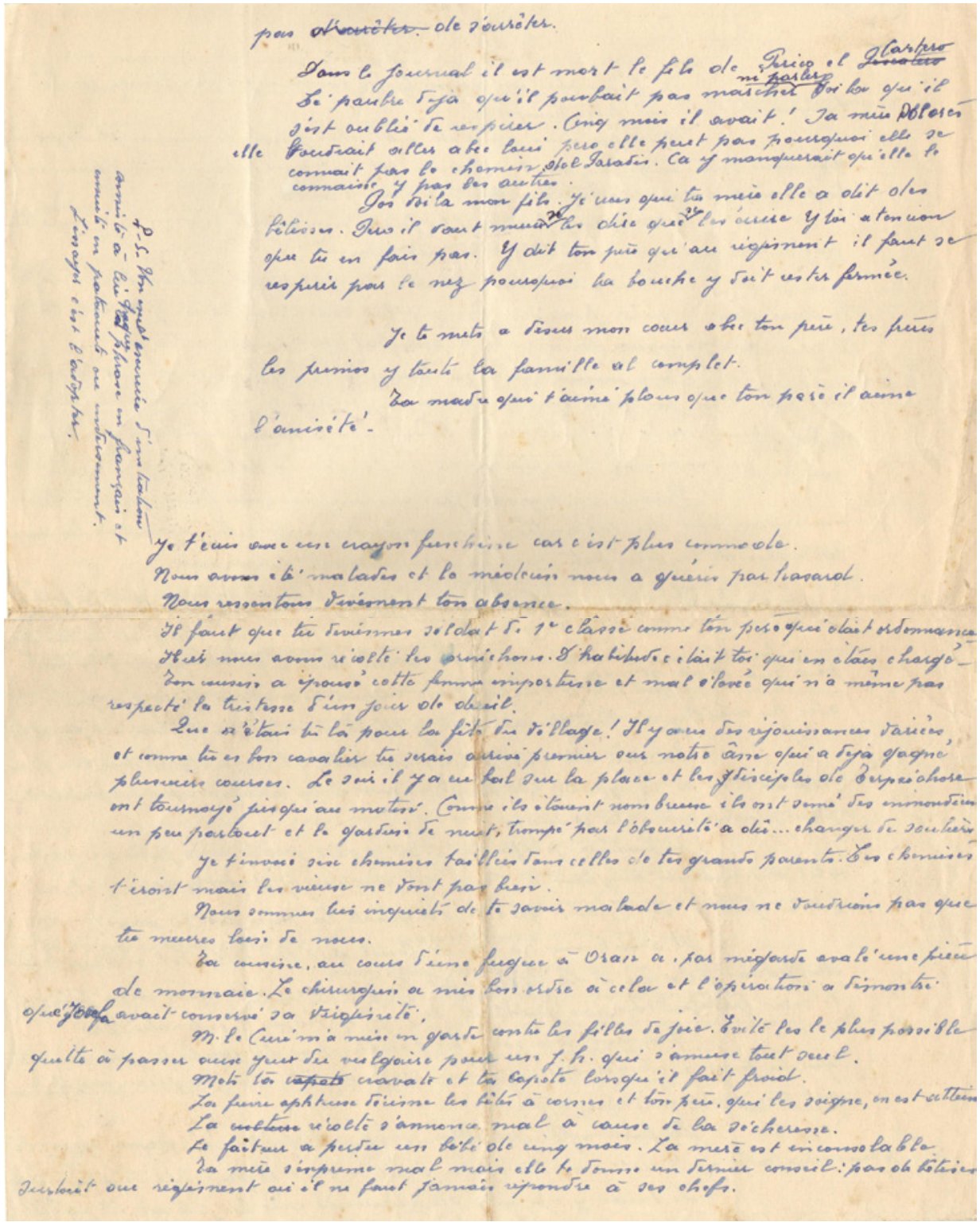

Figure A6. Lettre au soldat (manuscrit) 


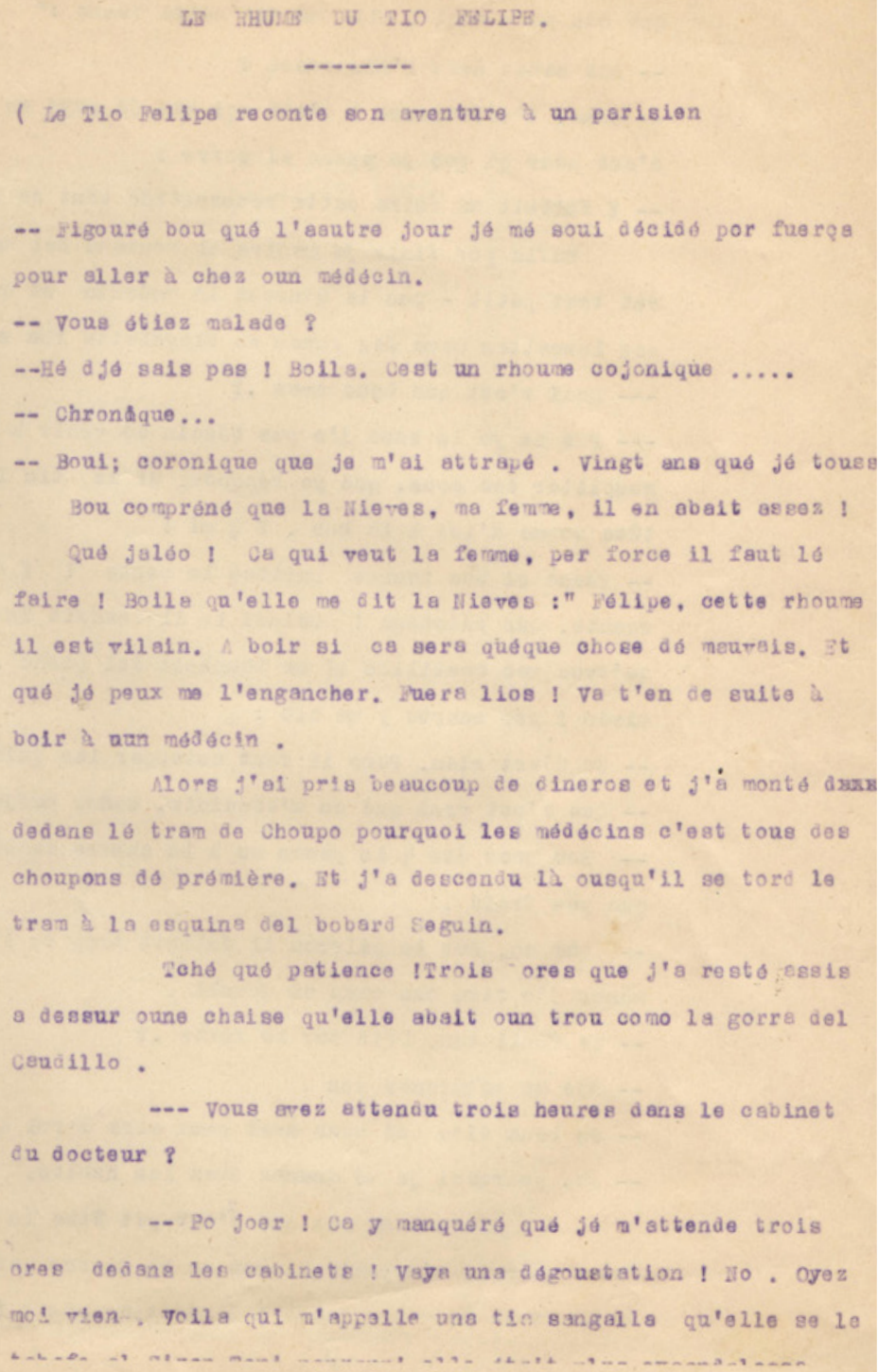

Figure B1. Le rhume du tío Felipe (tapuscrit) 


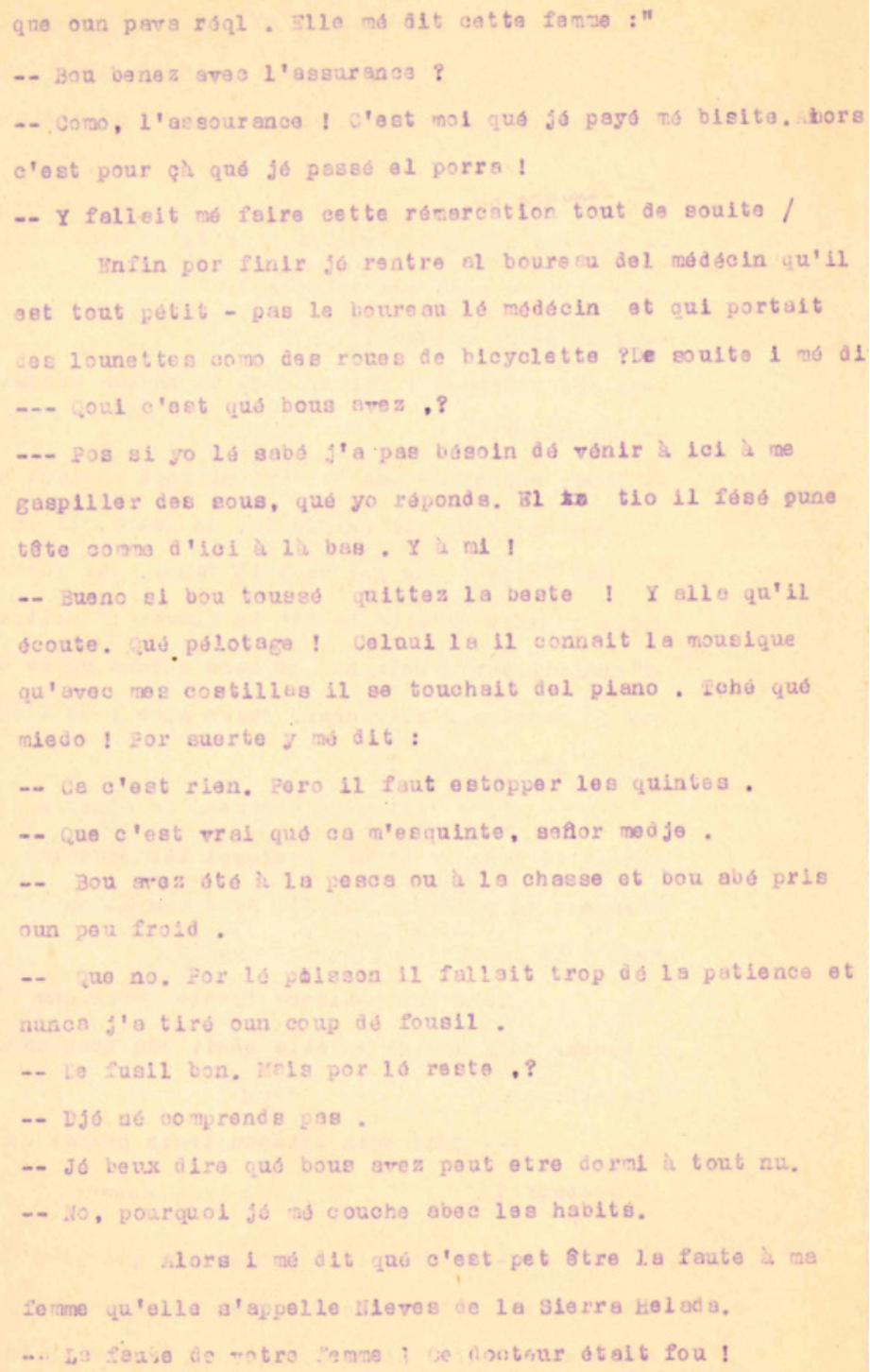

Figure B2. Le rhume du tío Felipe (tapuscrit) 


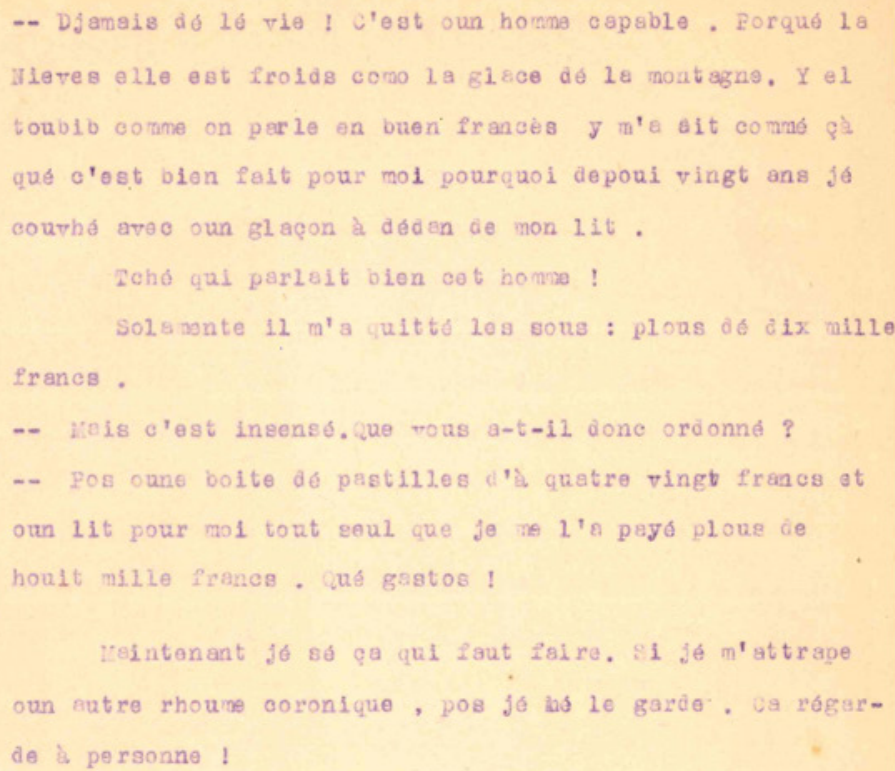

Figure B3. Le rhume du tío Felipe (tapuscrit) 
Le shern du bio Telipine

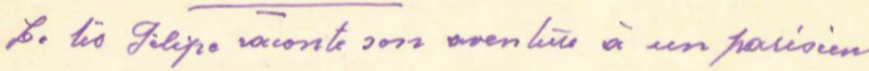

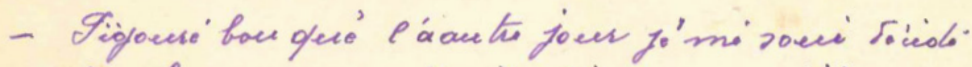

tras fuecra pous oulles à cho' seen méolócin

- Vous elly mabade.'

- Ho' djo'se'pras. Boila. Gist un rhoume corroniquer

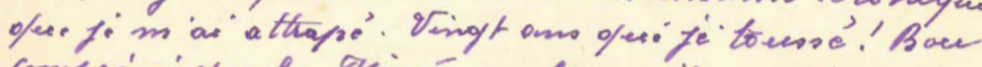

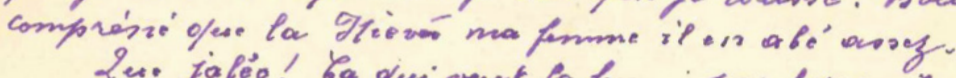

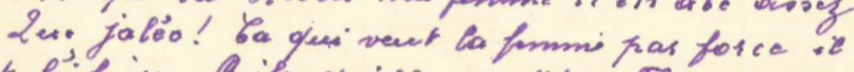

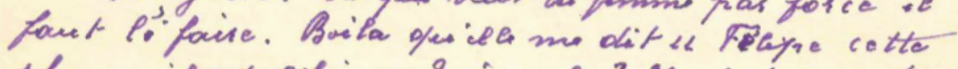

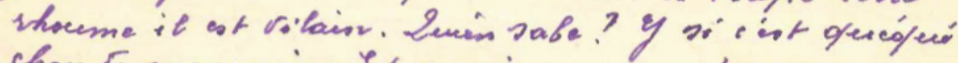

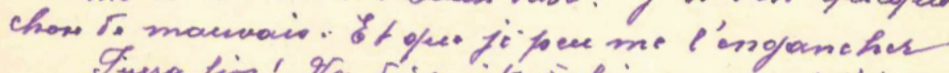

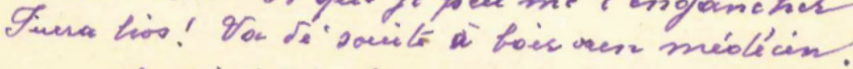

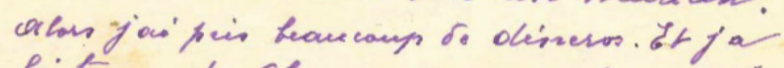

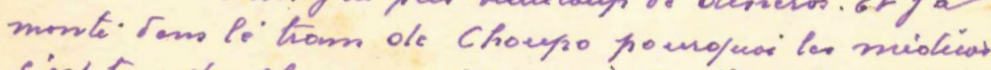

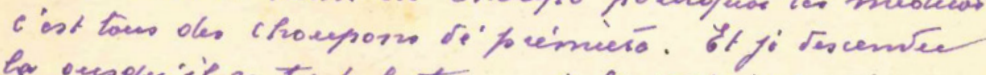

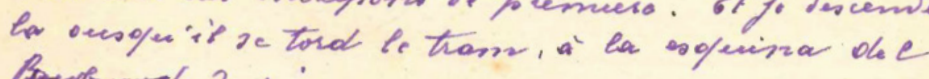
Troparar Jegien

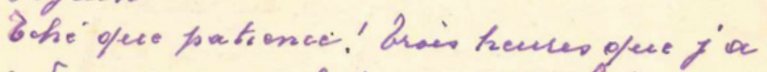

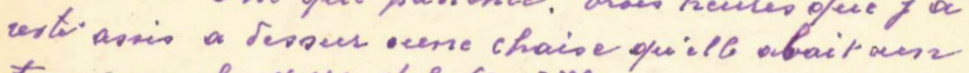
troe comme ha youra dol caursiles.

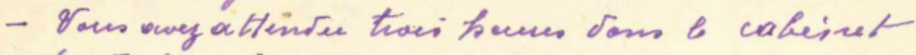
dee Footecer?

- To joer: Ca y monopuiro ofue foltter je mat.

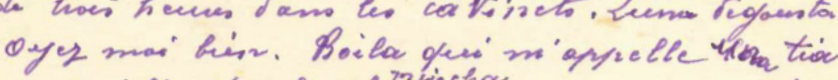

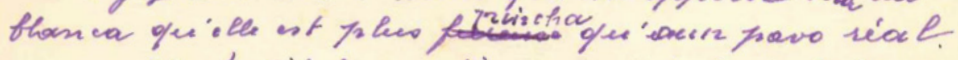

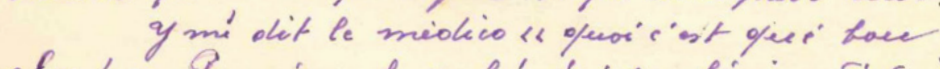
aby.' Pos si yo le sabe' jou' pas bisois ro' donir

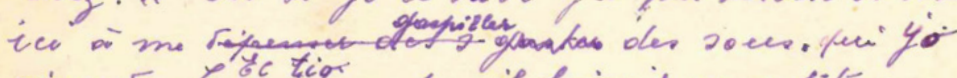

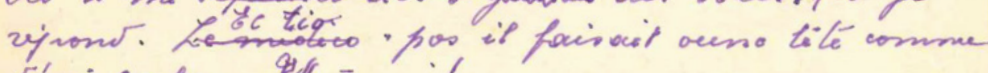
fivi cor bas. Elfoi mi!

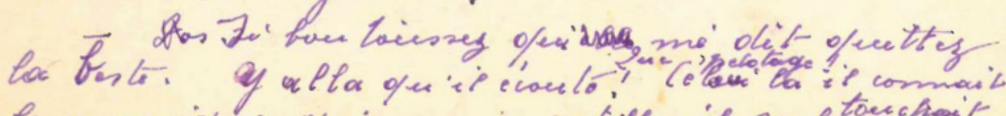

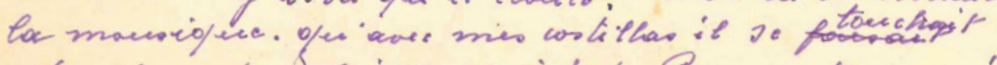
des priano!' Gcho opere miedo!' To I secesto y me'

Figure B4. Le rhume du tío Felipe (manuscrit) 


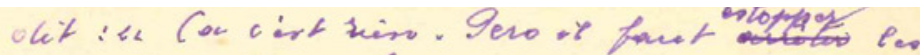

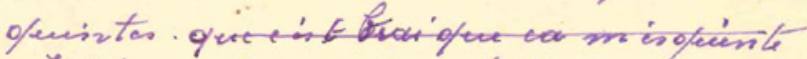

- Gublemment wor sopairlé,

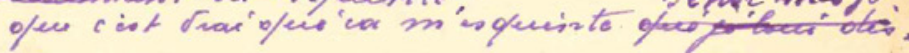

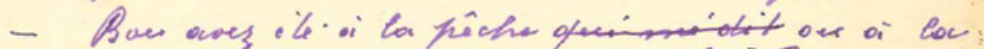
chasse. It fover avef pis rean freer frois.

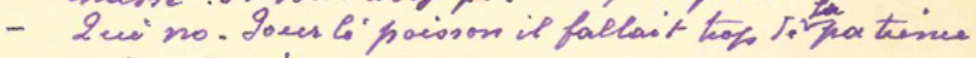

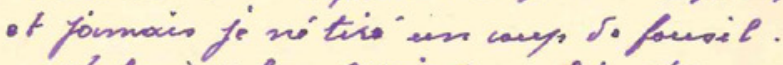

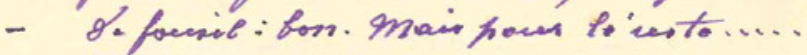

- Yo'me compuntron.' petôta

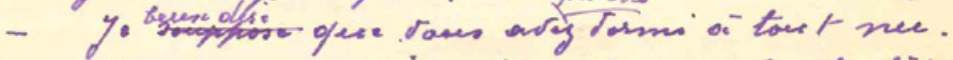

- No. poweroferas je me coesche wer les habils.

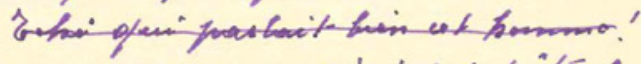

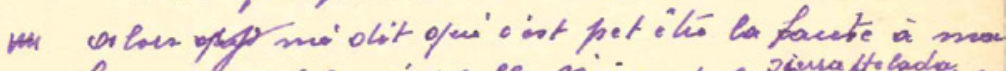

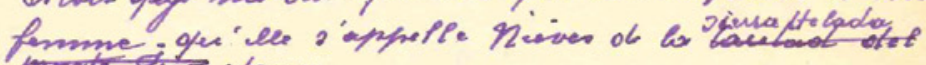
momo git . Le....

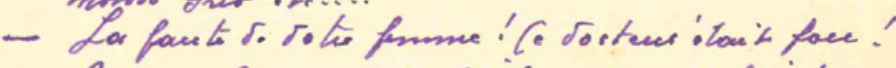

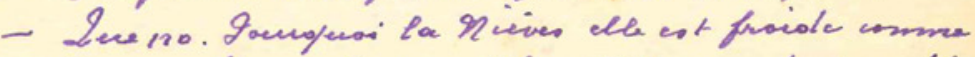

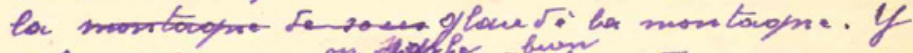

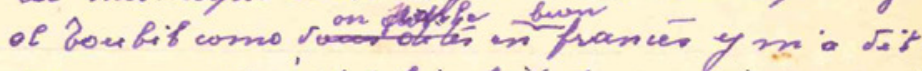

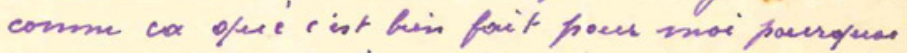

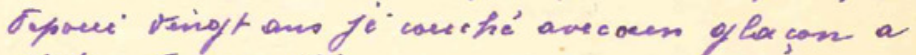
sleotan so mon lit.

$$
\text { bche oferi parboit beeri cet homme.' }
$$

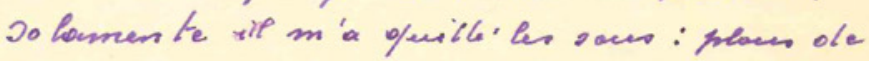
oki mile fromes.'

- Macis esteinense'. Lee soves at-ipostonne.

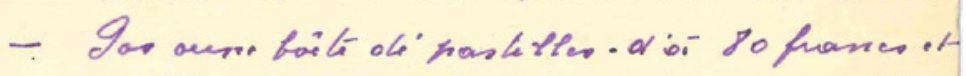

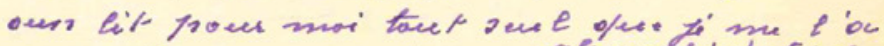

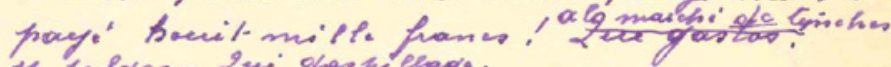

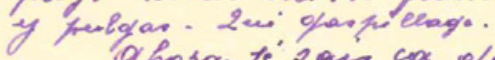

ahota fi' sain wa ofer 'y fourt fovie. De

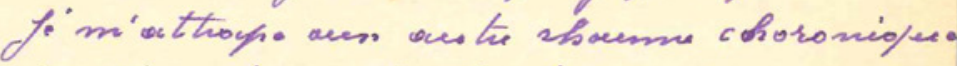

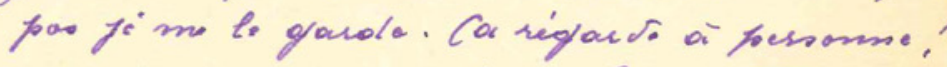
fromor Tere trattor!

Figure B5. Le rhume du tío Felipe (manuscrit) 

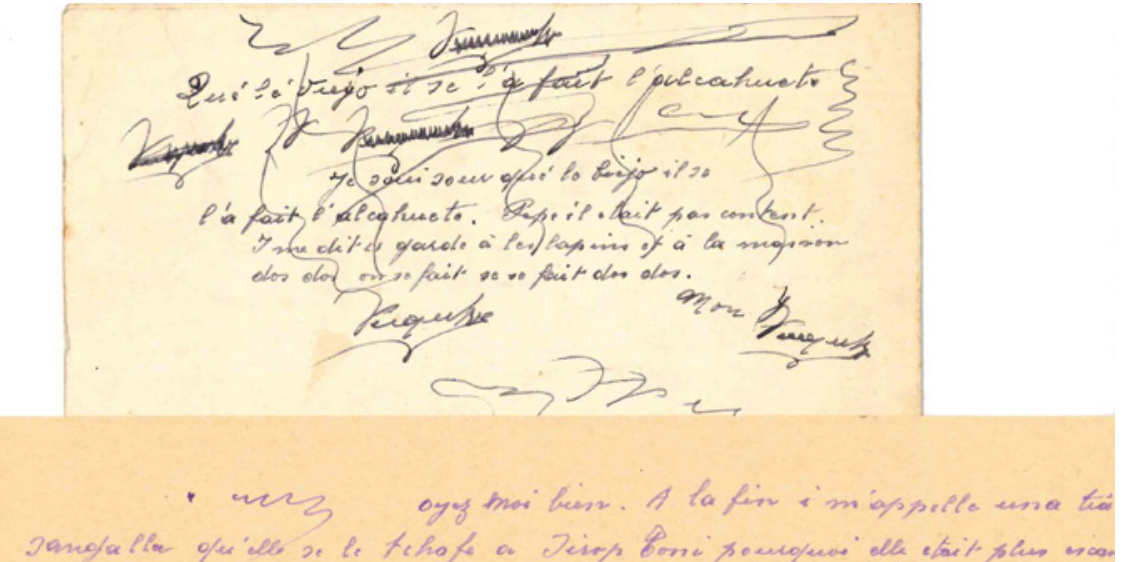

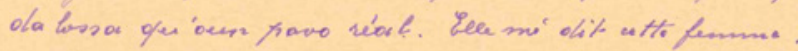

- bue bengy aber l'asso euance?

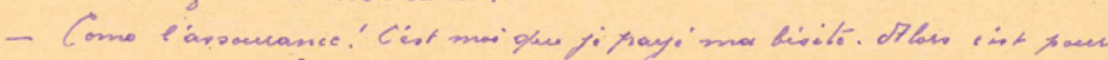

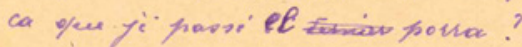

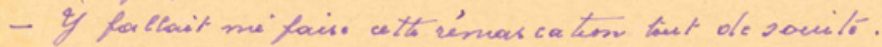

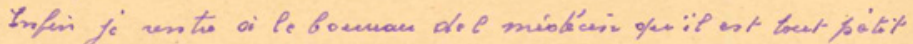

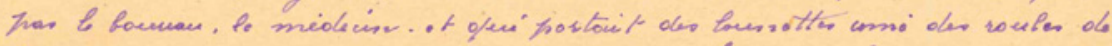

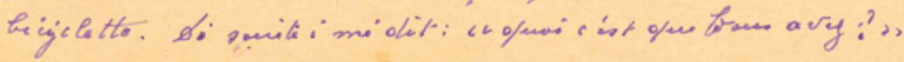

Figure B6. Le rhume du tío Felipe (manuscrit)

NOTES au manuscrit et au tapuscrit de « Le rhume de tío Felipe »

TAPUSCRIT, fig. B1, avant-dernière ligne :

« Voila qui m’appalle [sic] una tia sangalla qu'elle se le ... » Manque la dernière ligne semi-effacée

MANUSCRIT, p. 31

«Boila qui m'appelle una tia blanca qu'elle est plus [i ?]rvischa qu'oun pavo réal »

TAPUSCRIT, fig. B2, 1er ligne

« que oun pavo réal » 
TAPUSCRIT, fig. B2, bas de la page

il ne manque aucune ligne de tapée, en réalité.

MANUSCRIT, fig. B4. vers le bas de la page

Il manque un morceau du texte (présent par contre dans le tapuscrit, fig. B2, au début, de

« Elle mé dit cette femme [...] » à

«Qui c'est qué bous avez?»

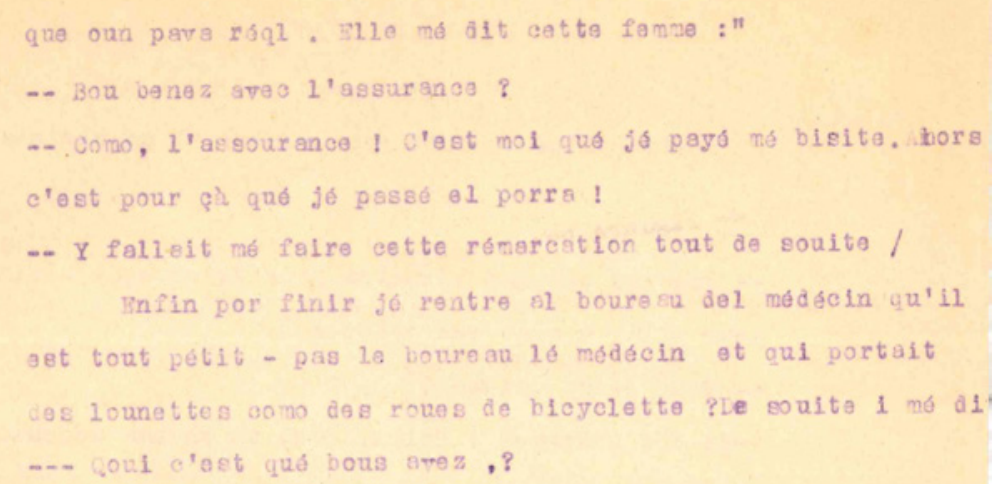

Cette partie du texte tapuscrit (fig. B2, ci-dessus) se trouve, à l'état de manuscrit, dans un feuillet détaché, reproduit ci-dessous :

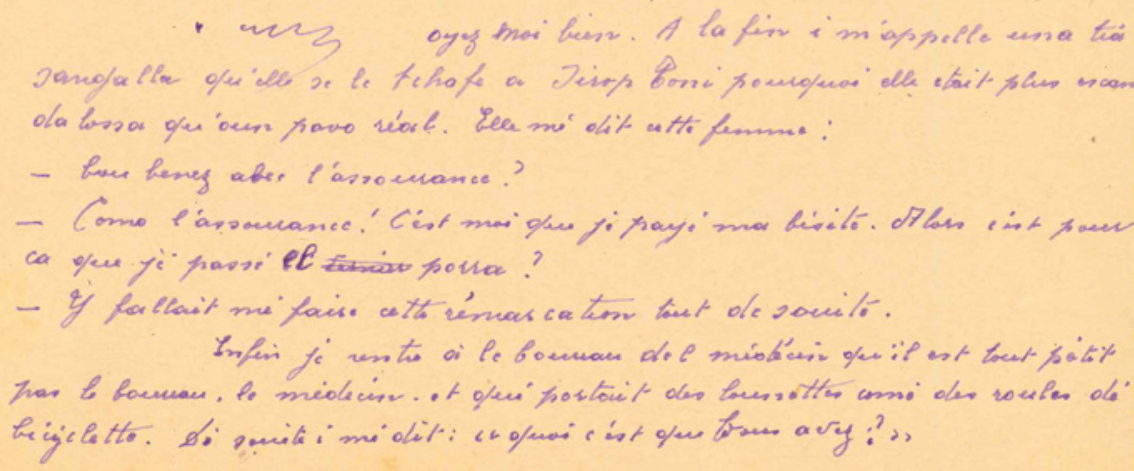




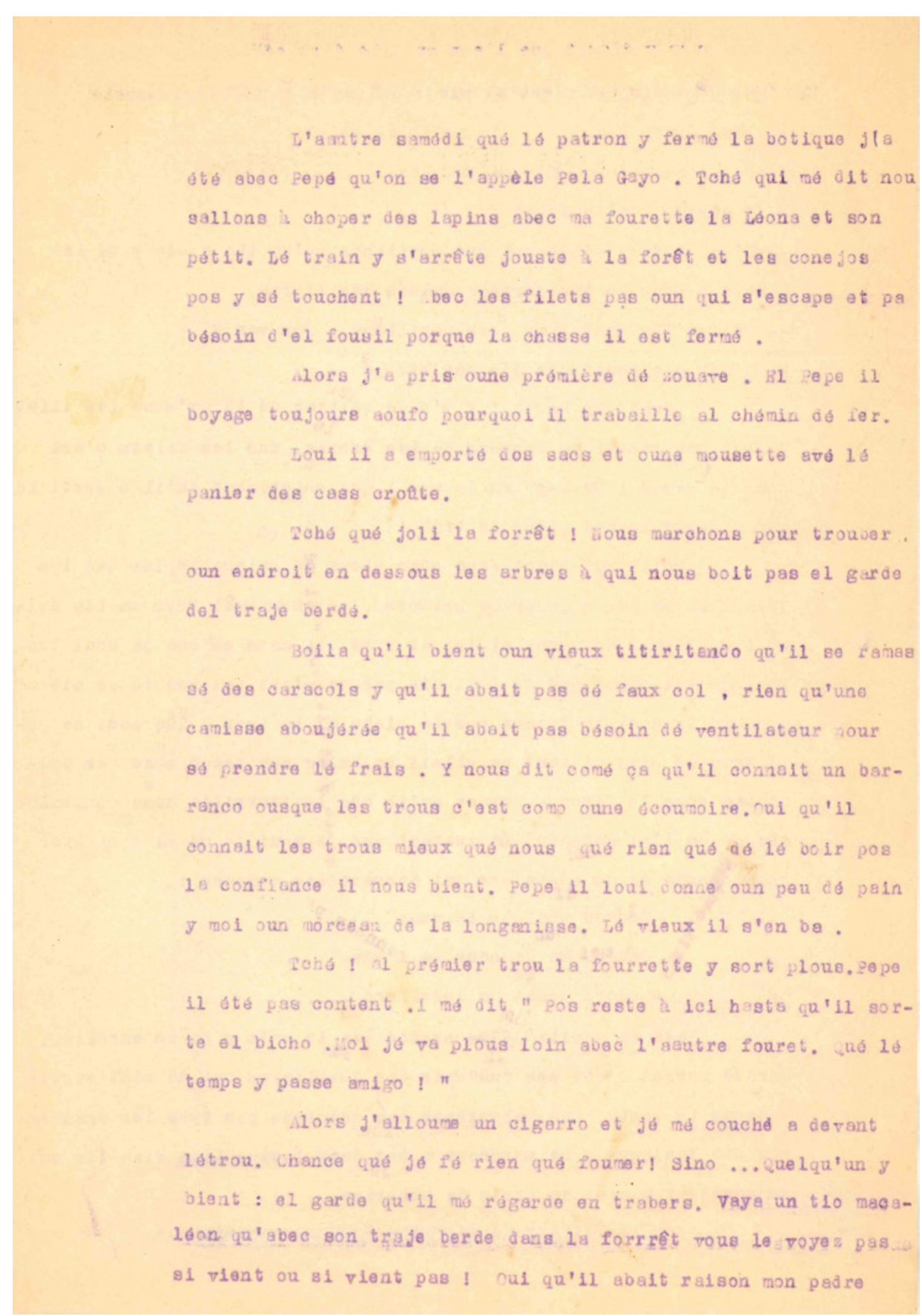

Figure C1. L'autre samedi (tapuscrit) 
qué toujours $y$ clesit que $1^{\prime} 0$ au qu'ello dort ê lé plous mauvais

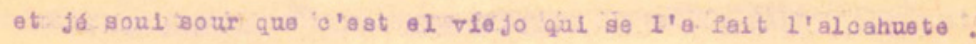
-- Qué fouté bou 1à, qui mé dit el garde.

-- pos rien; jé mé rápose oun peu.

-. Y porqal cette pilet?

-- C'est qug' je m'attrape des papillons qu'un tio esbio y mé 108 achète a pour gé lés écraser déáang des livres.

-- cul qu'ils sont gros ces papillone. Por ver ?

-. C'eat que encor oun j'a pas atrape...

Fohé qué bruto to $Y$ m'a fé quitter dé là qu'abec les lilet y faut pas rester on dessous de les arbres, qué les filets o'est pour la pesca I Le oggo en 18 mar I qué maintenant qu'il a parti le garde jé mé trouvo plong 16 trou !

Boila qui bient Fepe gbec plous de quinze lapias qué les quíze 11 mó leg a posés dé priméra . Qué chambon ! Vaya un tio felgo 1 Vite $y$ sé cache el as et $y$ mé dit comé q̧a que je goul ton to . Pos toi oussi qué jé loui dis porque e'est tol qué tu se mis dans lé trou et tu troube pas le bicho ni lé trou. Qué pour ce péti trou dé rien di tout on allait sé falre oun match abec des chlchotes sour la flgoure chance qu'il abé la fourrette uune campanlca que je me 1 'al entendu. De content qué jé, soui jé mé mi à chanter une chanson de me oùle por qui dorment les mấacos : Coucou cantaba 1 a rano Coucou en desaous de L'agus coucou passe un caballero .....

Pero de souite jé mé arrêté qué la vola y me sa enrollé.. enroué porqué c'est pas oun ms 1s dos caballeros qui mé sont sortis a desur la route. Les nelgulesos bou compréné pas ppos les agendarmès que toujours y se marchent à par deux I viva Dios rien 11s ont va pourquol $y$ gont on train de bobarder.

Dan í traía y mó dj, Pepe "t

Figure C2. L'autre samedi (tapuscrit) 


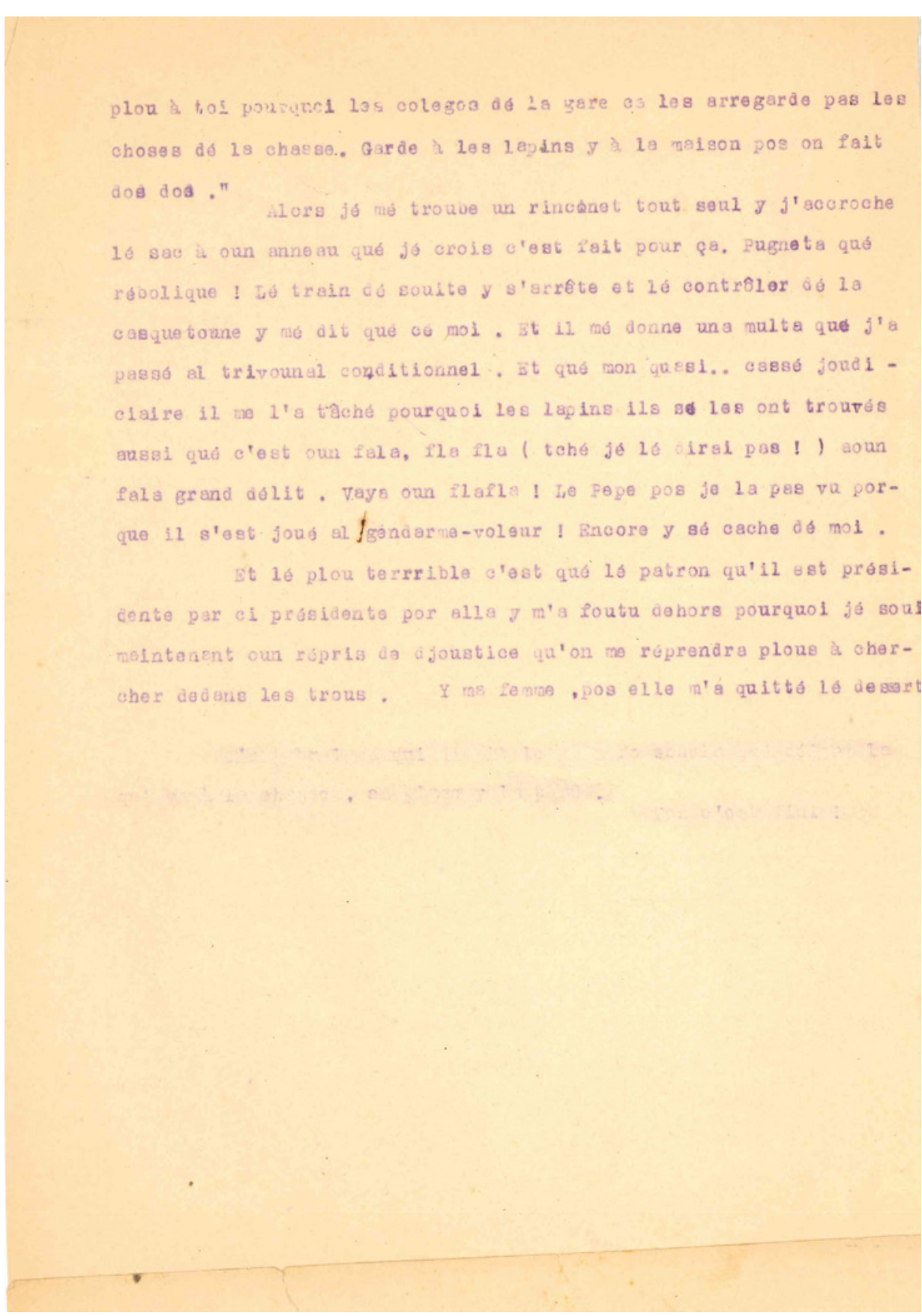

Figure C3. L'autre samedi (tapuscrit) 


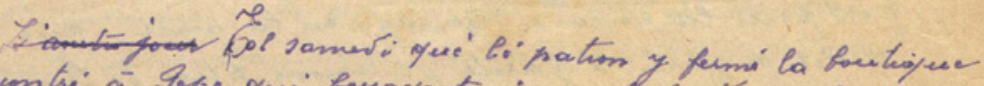

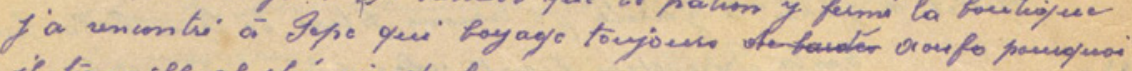
if liovaile al itimeni ole fer.

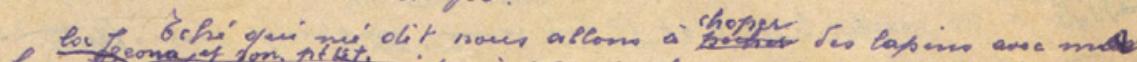

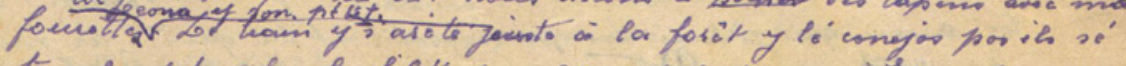

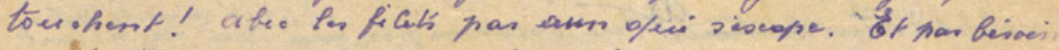

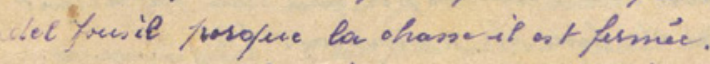

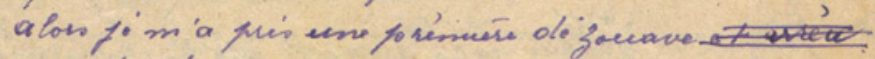

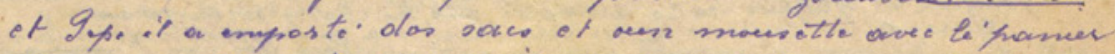

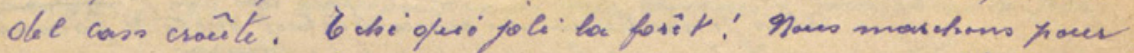

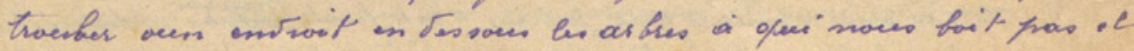
goude ole e costem vest.

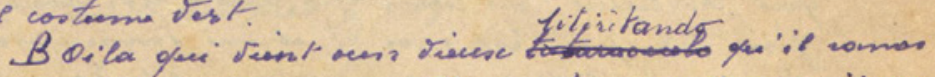

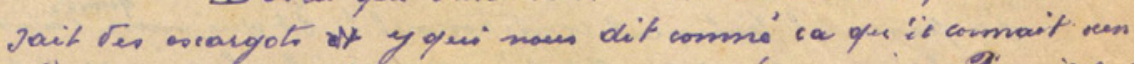

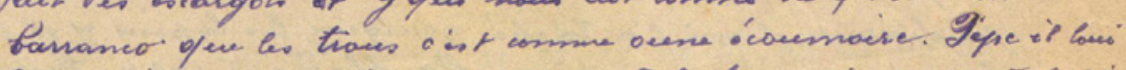

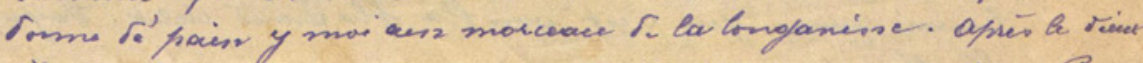

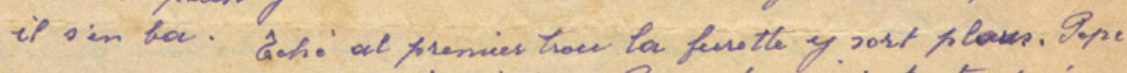

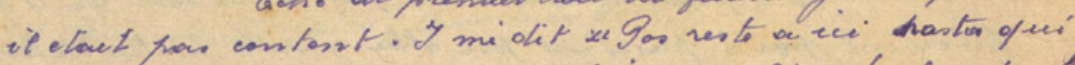

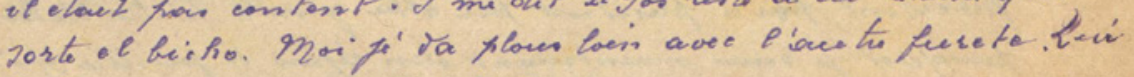
te.tempoy frarse omiolo $\therefore>$

ctlos $f$ ixlem la ciglasetto of fi me couchlie a

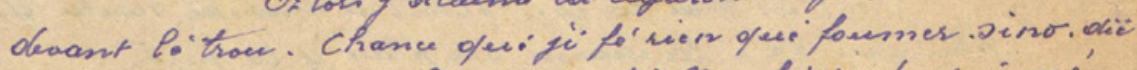

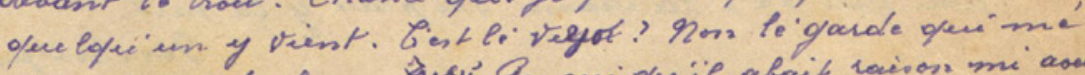

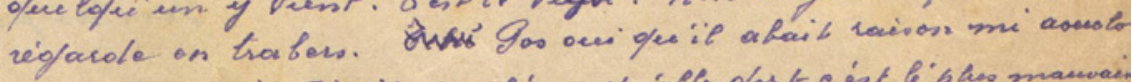

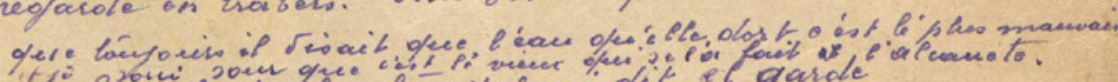
- Lue fortez boer la yber mo dit el garde. Dos rees : fo' mé vifoso oess toec.

- If posalevi cetto felet

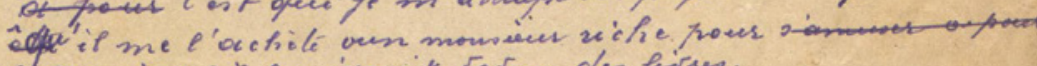

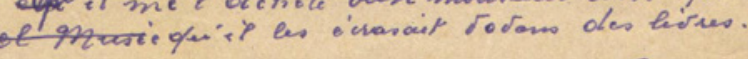

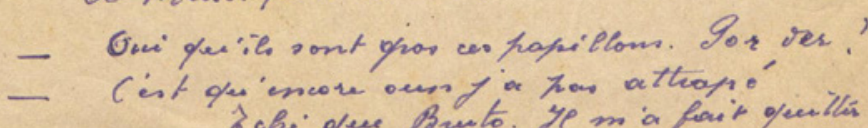

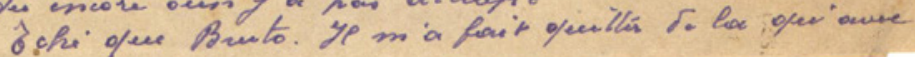

Figure C4. L’autre samedi (manuscrit) 


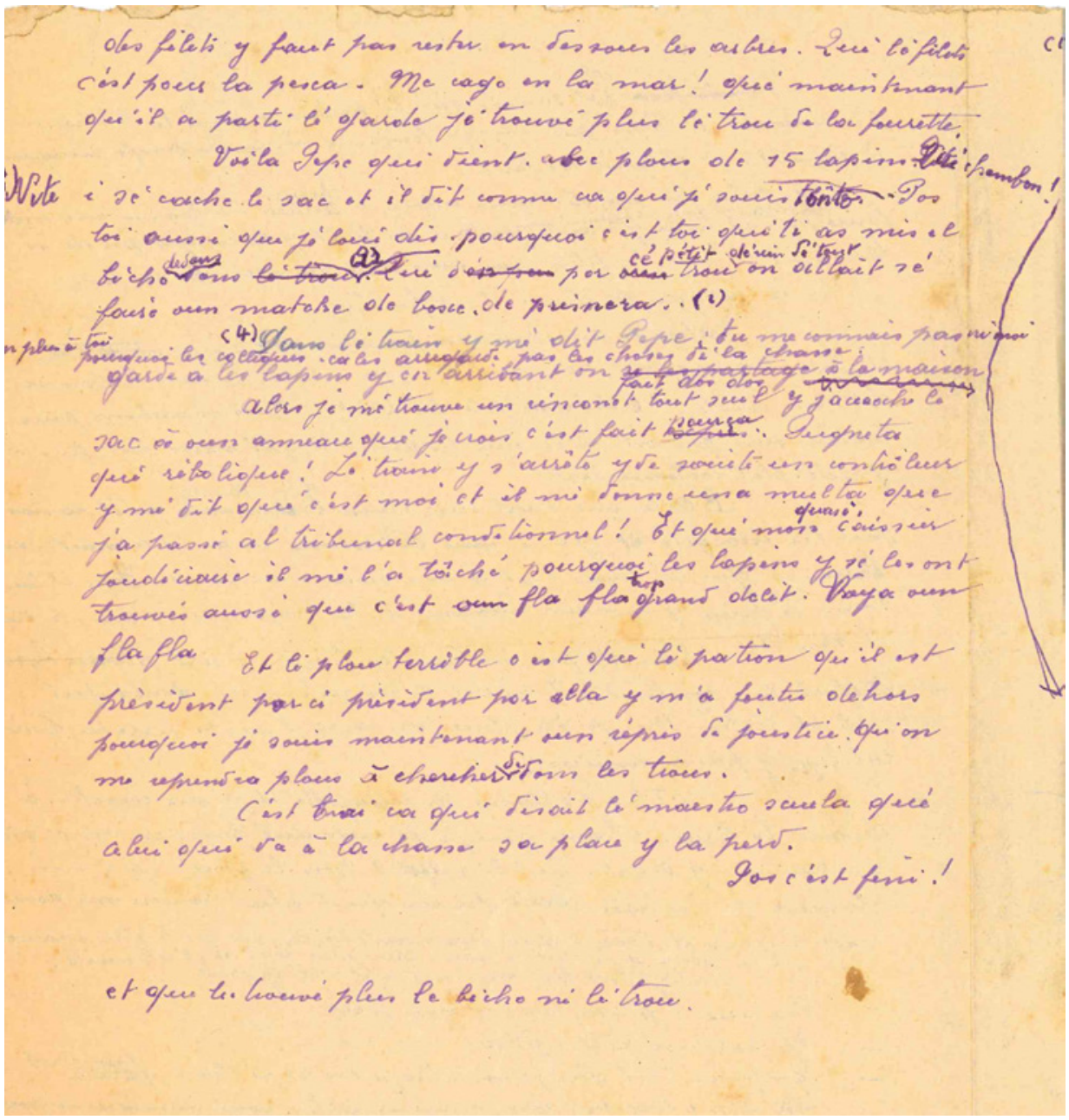

Figure C5. L'autre samedi (manuscrit) 
) Chance ofere ha fourtte is avait une ampromica opere fie

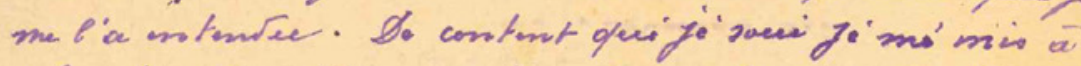

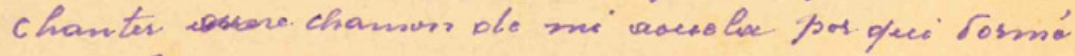
les mañacos:

Coucare cantaba la rana

Cacecace etz despous of liaglewa

Cancoer paso en cabatho.....

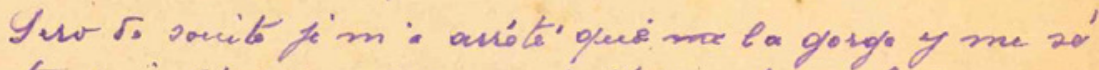

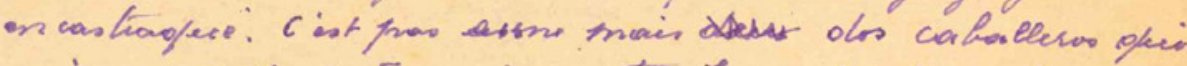

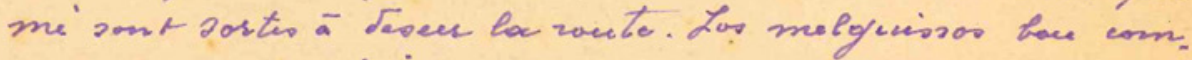

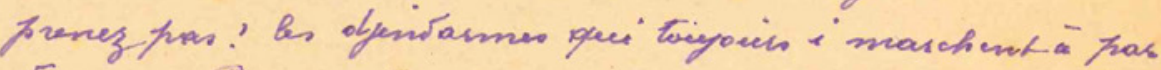
Fecese. Tos veis is ont vee trowgluev if sont en troin de tobardes. Lecil socespiso! (4)

ar ac ab ac ae ar ar at ay au ai ao ap va $v z$ ve $v r \cdot \nabla t \quad \nabla y \nabla u$

Bayn en tio palso.' Buer alb' Foir!. (3)

Buya un tio falso bou allé boir.

BI UN TIO FALSC BOU ALLE BCIR

Figure C6. L'autre samedi (manuscrit) 


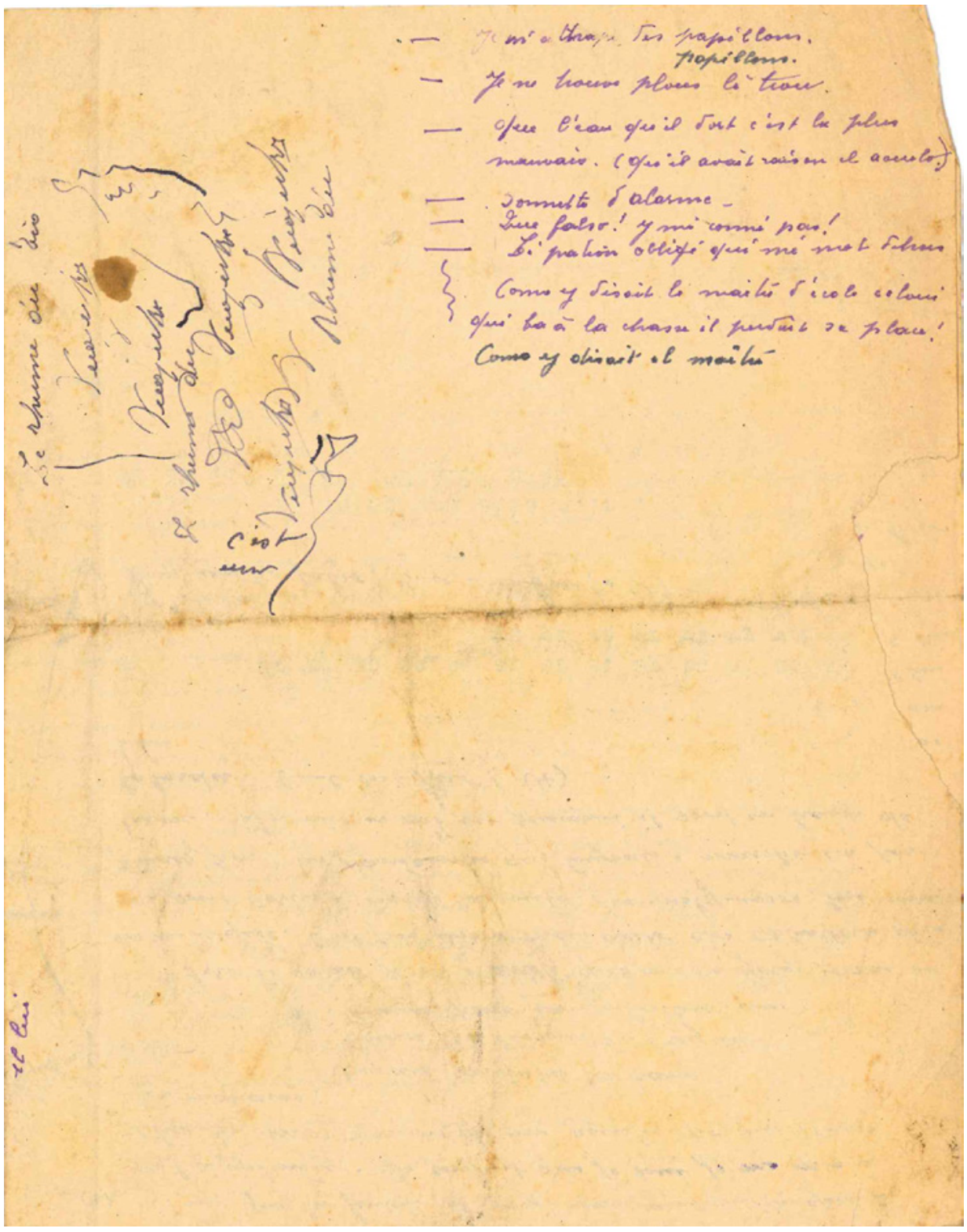

Figure C7. L'autre samedi (manuscrit) 


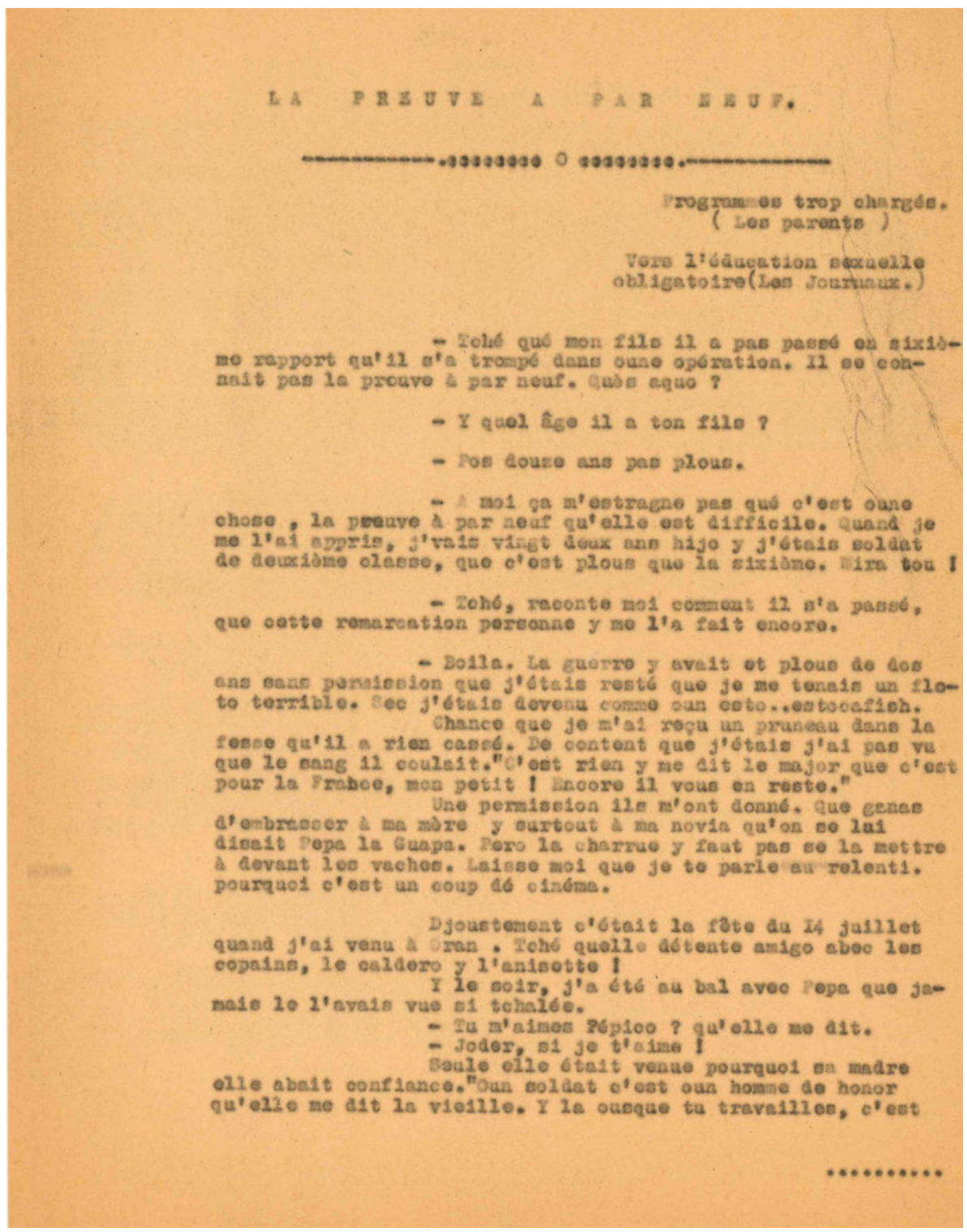

Figure D1. La preuve a par neuf (tapuscrit) 


\section{(2)}

tous dos carmain...gentlomen, yo que ed I

Fero dedene ie bel, 12 raladit cheud, tu conprende ? clotait come 16 four ga'd force de Irotter I'sil umotte pos $y$ e'all use. ga qu'12 a pased, andgo, je te le alrai pse quo e'est oun becret conildentiel.

fion ea'd mon padro je 2 ul a a1t tout.Y nos pedre que tu 10 conilala 12 eat plas p1120 que los oinges do la Tehourfa..de le chirfa. " J6 ve marquex gul mb dit" que 1 ul tout 12 ne narque a deeur son erdolse.

Aprذs, le guerre olle a Inl et f'a venu a Ia nalson libre oomo oun casam qui ne vauve de is eage

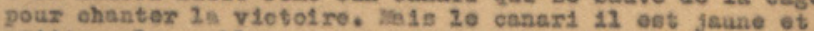
ogtte eouleur elle est pee bonae. De soulte, la fepe cile sta couche et la tla Antonia olle mo dit que mon 111011 e6 pòse plus de eing k120s. St lo viellie pemplinoses olle dit quo e'ost do ma reute of qu'11 leut d roparer lo pot eask carab: It mol je ale que ous; come nol tu aurale fast, pas

ATe 1 quelle serregate. Ion padro 11 s prie son ardoleee ot sea bêton de olivier neuvege et de soulto on a $6 t 6$ a cher in Popa. Un escandale qulil a falt mon peares plue fort que plete $11 \mathrm{a}$ ex 16 ; venu on permiesion, male Joeguin que e'est un narin, avent 2 aj 11 a venu. $Y$ ga eaesi, je me $i^{\prime}$ a marque $t^{n}$

- I apris ? qu'elle e falt la Popat voue avez pas la preave que votre $t$ ile 11 a sien falt."

- Que a1 I Hafntanant mâne je vas vouo

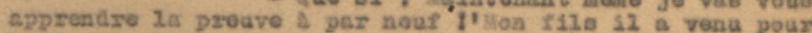

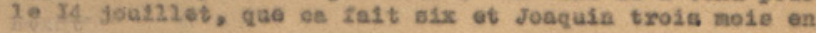
sveak, que os falt neuf. Pour mon 111s, la opdration elxe est pes jouste pero elle eat jouste pour josecusn. Je veux pes cue mon $111 \mathrm{~s} 11$ se rélte ga quian autre 11 a send. Y a preseat, \& 1a maseon $I^{n}$

- I bolla cowanent mon padro $11 \mathrm{~m}^{2} \mathrm{a}$ sauve 1e mise. Tohico que 2 ardoloe je 2 's mis decene us carre

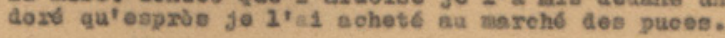

- Pos tu as raison. lisintenant les bouroes, y les conoours y les cxameng $y$ sont trop alffielles. $Y$ se 2. ohsuffent trop la tie a les gosees. Tohb i je compreade pas qu's des mahacos de douze ans on se leur comande la prouve \& pas neus i"

Figure D2. La preuve a par neuf (tapuscrit) 


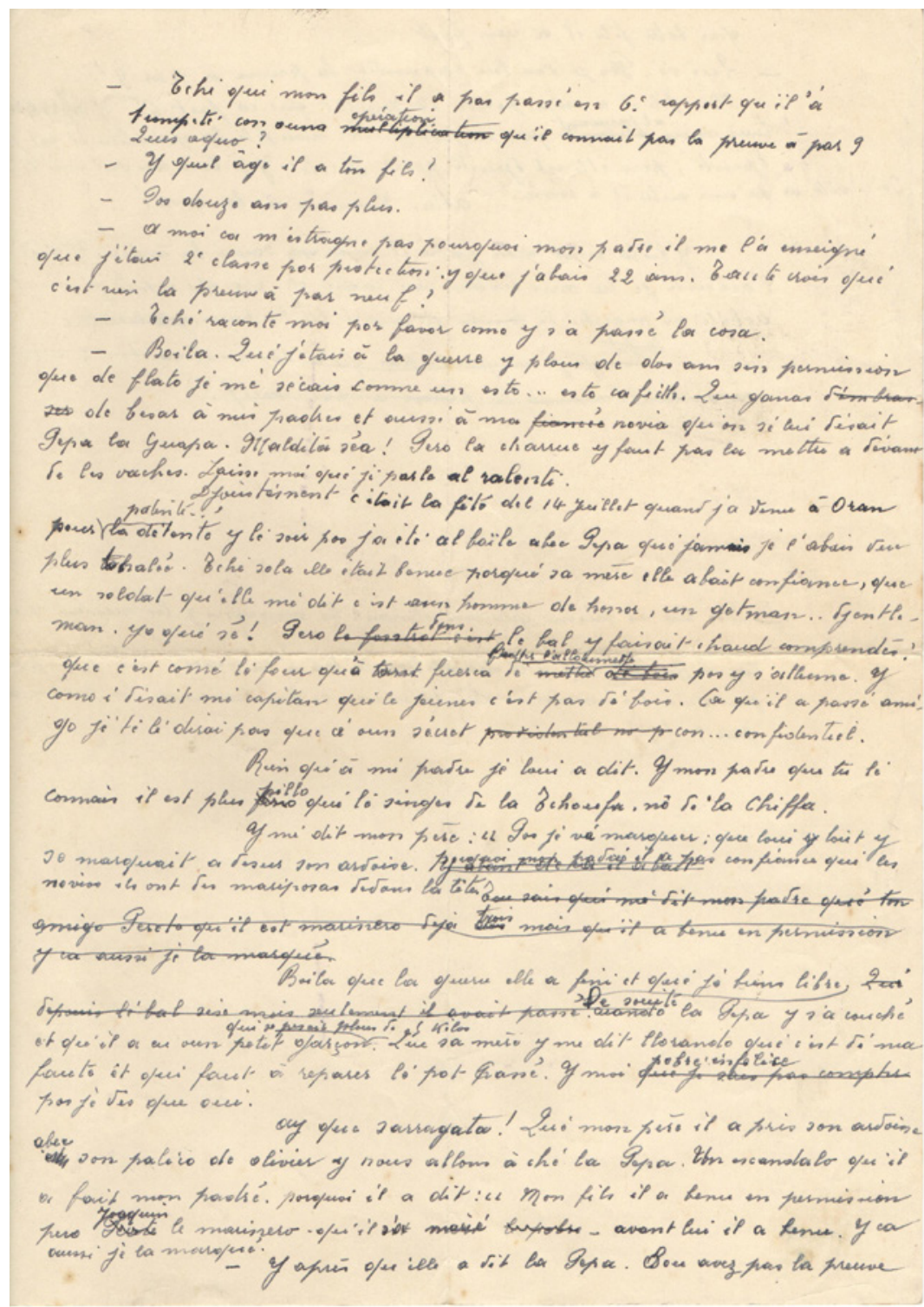

Figure D3. La preuve a par neuf (manuscrit) 


$$
\begin{aligned}
& \text { ofee totu filh it an win froct }
\end{aligned}
$$

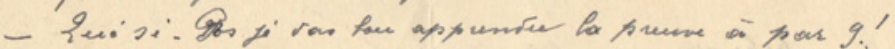

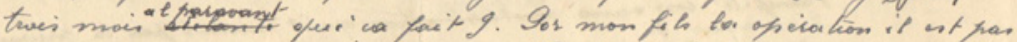

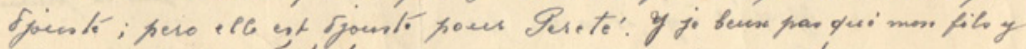
Jo'vicolts ca qui our autu il a Démé.' alla. Vamonos á la cava.'

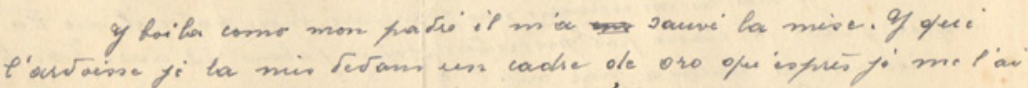

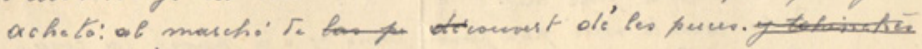
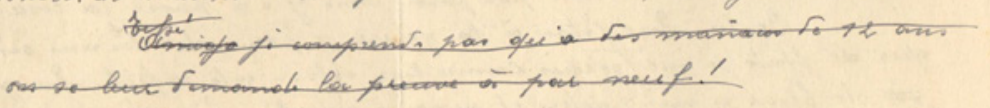

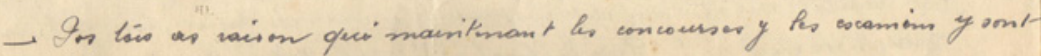

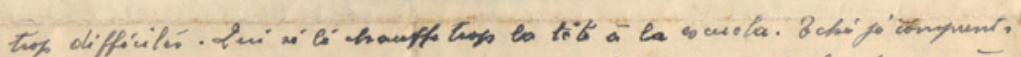

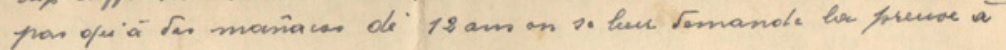
proug!.

Figure D4. La preuve a par neuf (manuscrit) 


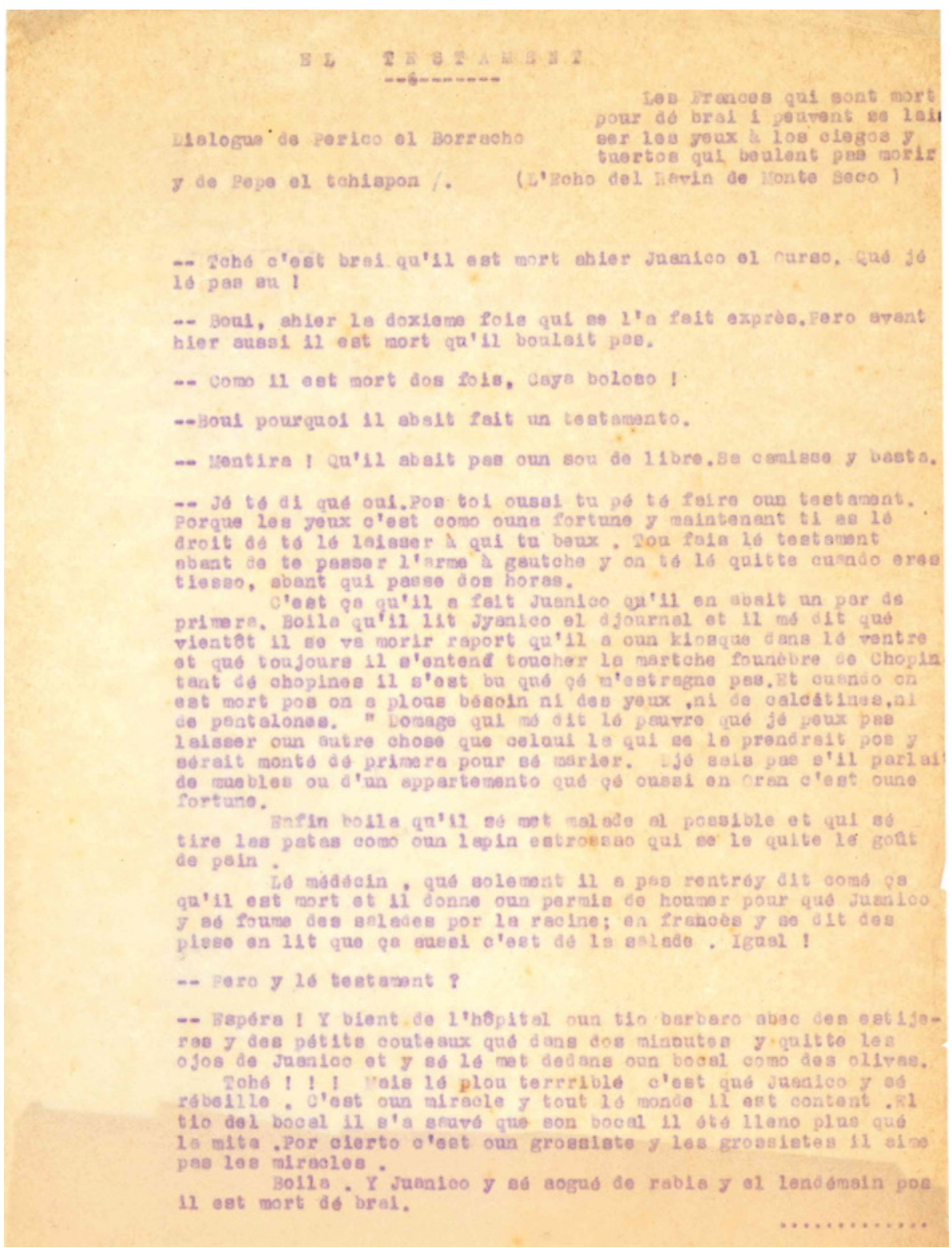

Figure E1. El testament (tapuscrit) 


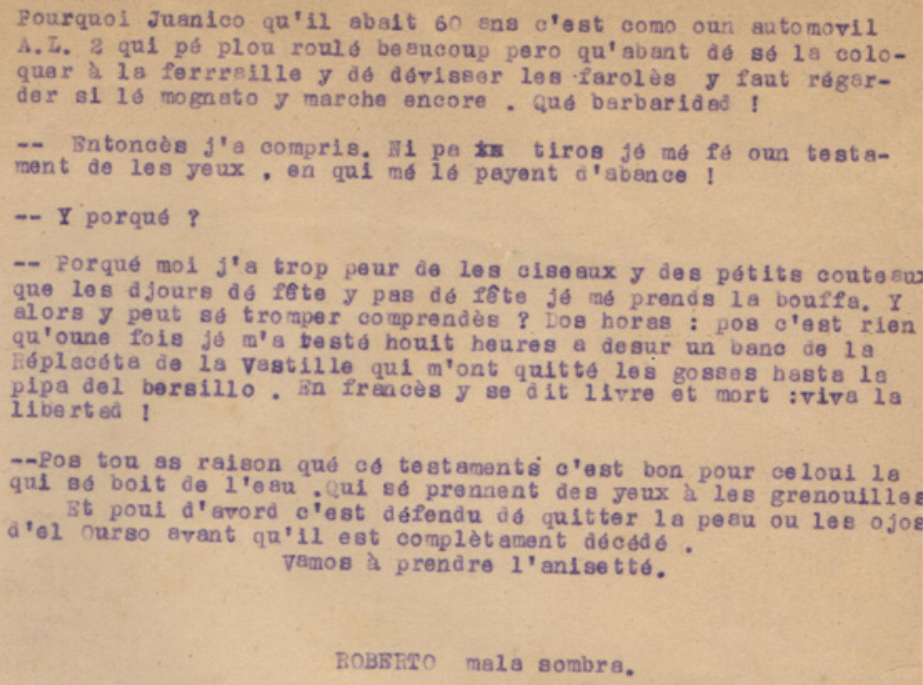

Figure E2. El testament (tapuscrit) 


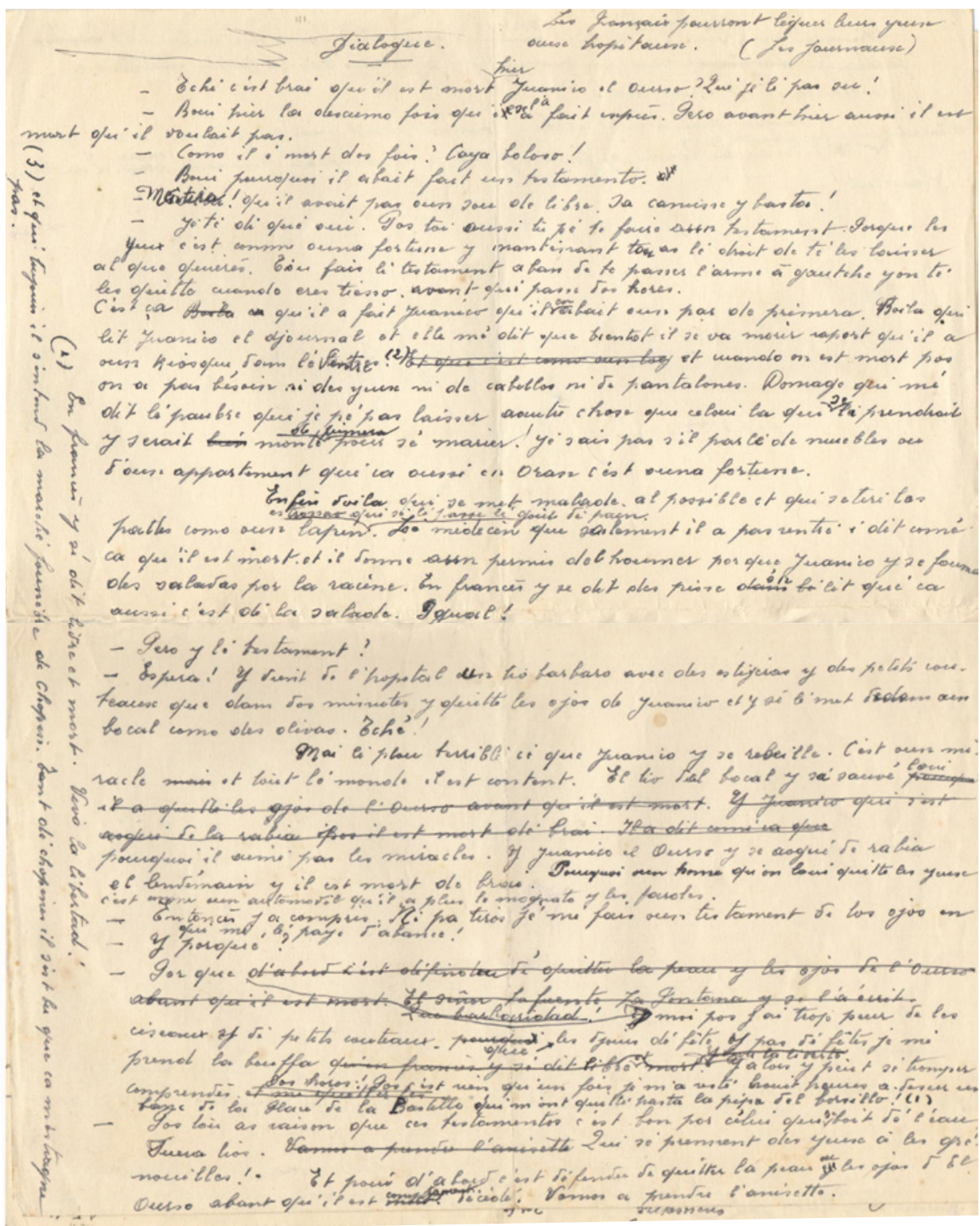

Figure E3. El testament (manuscrit) 


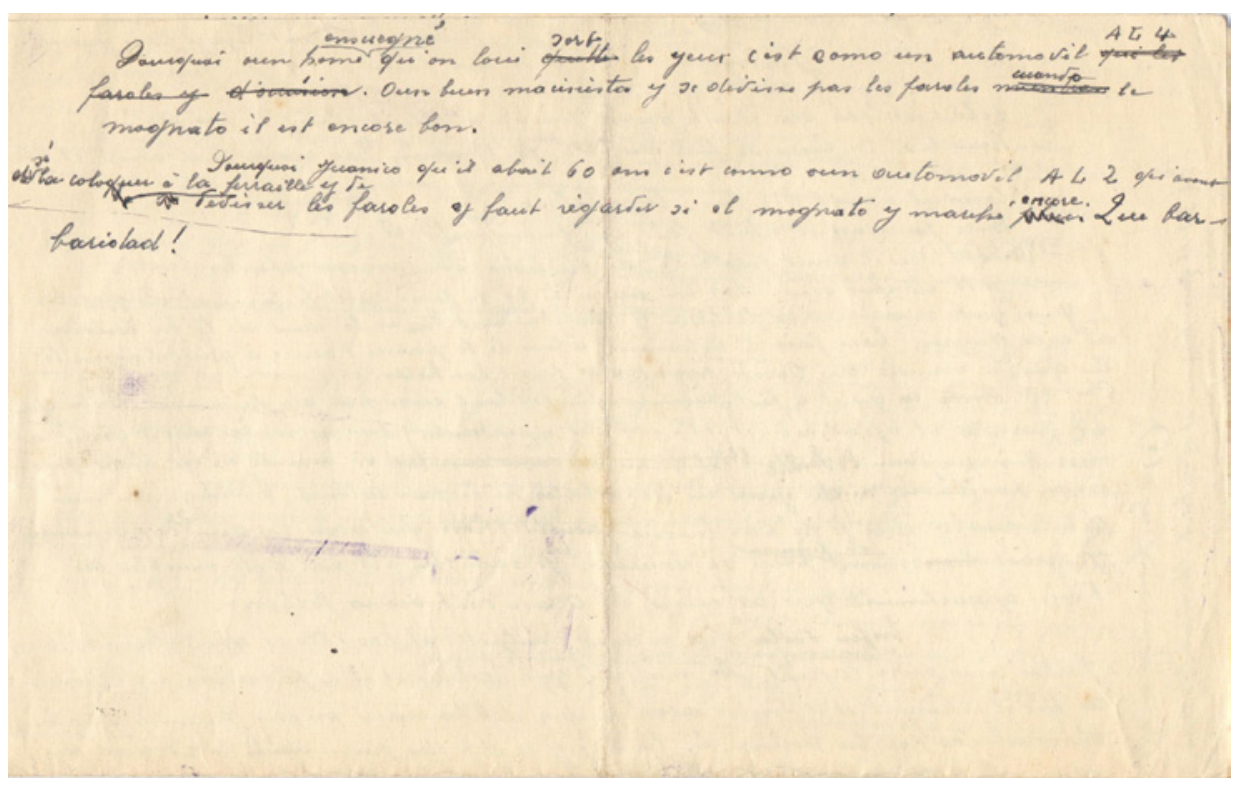

Figure E4. El testament (manuscrit) 
II Tolomeo, 20, 2018, 15252

e-ISSN 2499-5975

ISSN 1594-1930

Dementior

Sreiciear

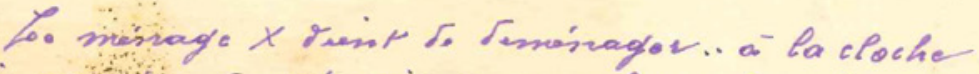

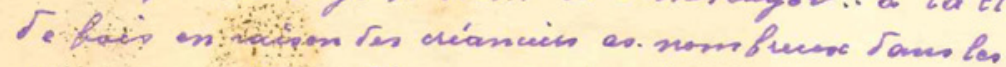

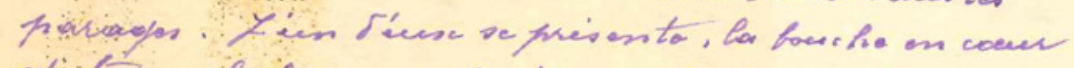

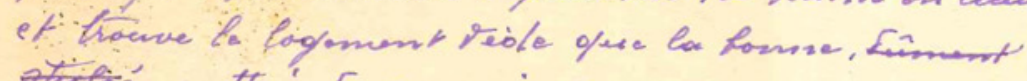
nottové re son micer.

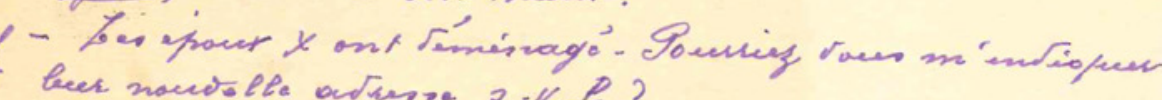

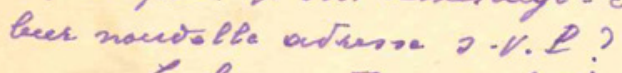

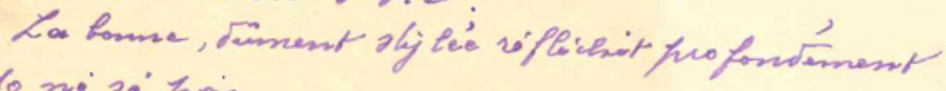

- go no so fais.

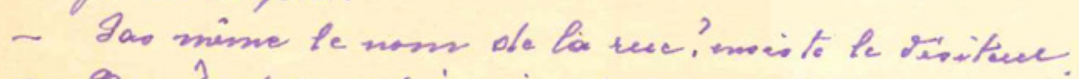

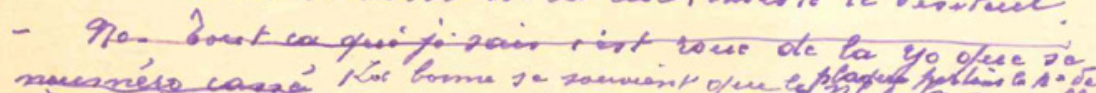

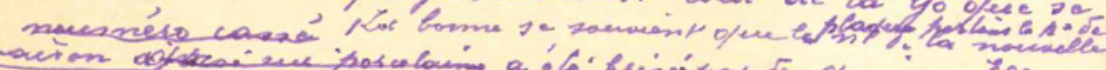

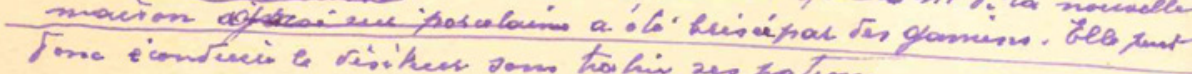
Zo

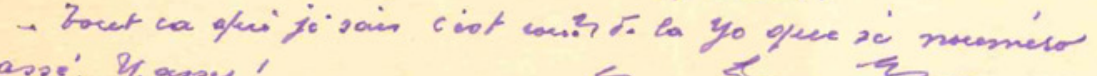
casoe: If aroy!

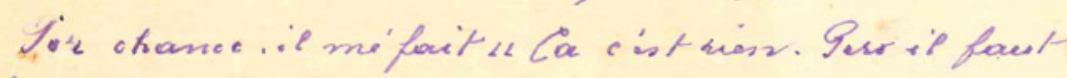
Dlojpies bes ofueintas.

- 2uro int Trai ofer ca minqueinto saños medjo.' 2.0. ist Thai que ca minguente penos medije

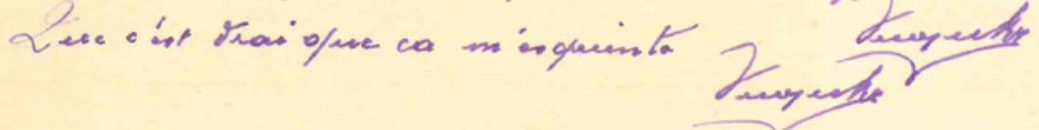

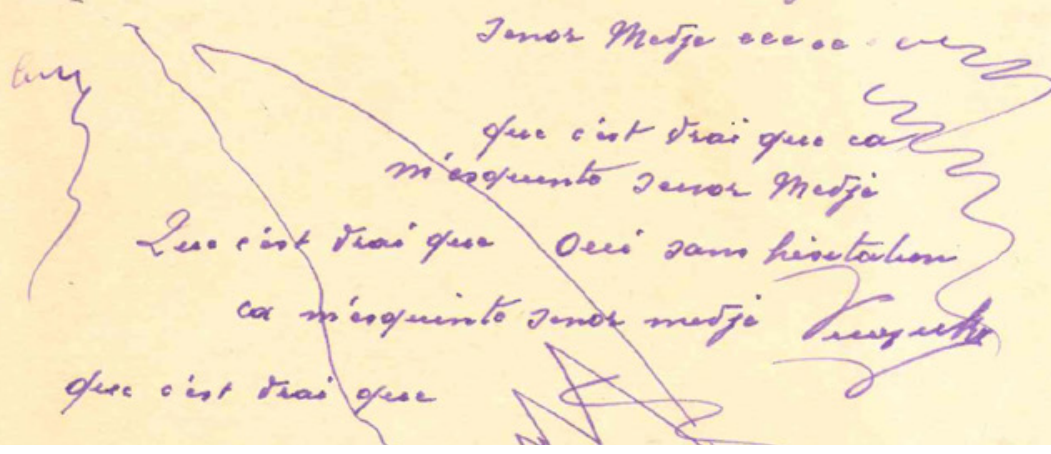

Figure F. Précision

Costantini. Les manuscrits trouvés sur e.Bay

51 



\section{La main et le sein}

\author{
Rita El Khayat \\ (Author)
}

J'ai mis mon sein dans ma main et mon œil dans la tombe.

Mon œil regardait Mon sein débordant de lait Dans ma main vide.

Une gazelle en but une goutte Un Bonze caressa mes doigts Le lait coula

Comme une cascade au printemps

Mon œil buvait

Au milieu des vers

La blancheur liquide

Sourdant de ma main...

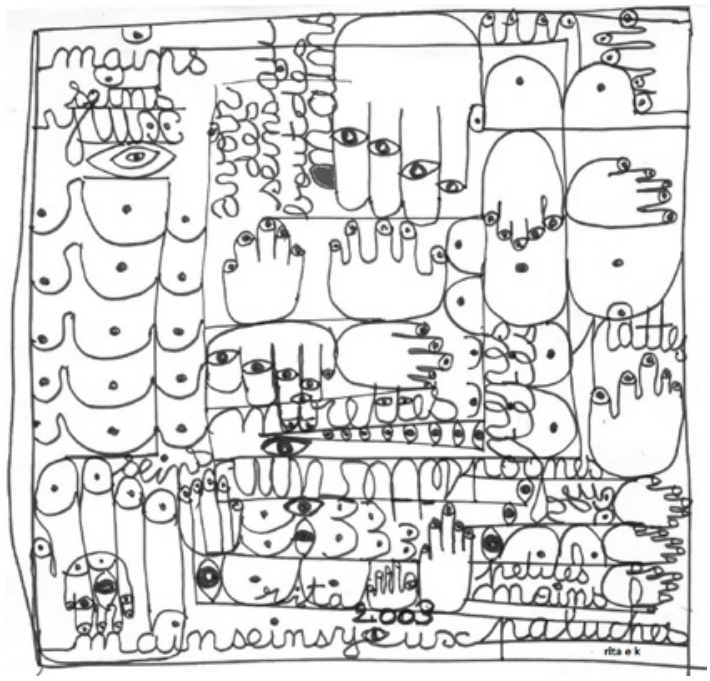

Figure 1. Peinture en noir et blanc, encre, sur papier glacé ; réalisée par l'Auteur

DOI 10.30687/Tol/2499-5975/2018/20/002

Submission 2018-07-06

(C) 2018 | @) Creative Commons Attribution 4.0 International Public License 


\section{Hand and Breast}

I put my breast

In my hand

And my Eye

In the grave.

My eye was looking

My breast overflowing with milk

In my empty hand.

A gazelle tossed off a drop

A Bonze fondled my fingers

Milk run

Like waterfall in spring.

My Eye was drinking

Among worms

The liquid whiteness

From my hand springing...

(Saturday 18th January 2003, Casablanca)

\section{La Mano e il Seno}

Ho messo il mio seno

Nella mia mano

e il mio Occhio

Nella tomba.

Il mio occhio osservava

Il mio seno straripante di latte

Nella mia mano vuota.

Una gazzella ne trangugiò una goccia

Un Bonzo accarezzò le mie dita

Il latte scorreva

Come una cascata in primavera.

Il mio Occhio beveva

Tra i vermi

Il liquido bianco candido

che sgorgava dalla mia mano...

(Sabato, 18 gennaio 2003, Casablanca)

(traduzione italiana di Antonella Perlino) 


\title{
Rita El Khayat, une écrivaine de l'intime et du social
}

\author{
Anna Zoppellari \\ (Università degli Studi di Trieste, Italia)
}

Née à Rabat en 1944, Rita El Khayat est écrivaine, psychiatre et anthropologue. ${ }^{1}$ Elle a étudié la médecine à Rabat et la psychiatrie à Casablanca. Partie pour Paris, elle y continue ses études de psychiatrie et se spécialise en Médecine du travail et Ergonomie de la médecine de l'espace. Au cours de son séjour à Paris, elle étudie l'arabe classique à l'Institut national des langues et civilisations orientales. C'est au cours de cette période que commence son activité littéraire : depuis lors, elle a écrit plus de trente livres parmi lesquels Le Maghreb des Femmes (2001), La Liaison (1995), Le Désenfantement (2002), Correspondance ouverte (avec Abdelkébir Khatibi) (2005). En tant que psychiatre, elle a été l'élève de Georges Devereux, l'un des fondateurs de l'ethnopsychiatrie. En 2008, Rita el Khayat a été proposée pour le Prix Nobel de la Paix et a reçu la nationalité italienne des mains de l'alors président Giorgio Napolitano ; elle a enseigné dans plusieurs universités italiennes, à Milan et à Chieti entre autres.

Sa formation scientifique éclectique influence ses intérêts culturels qui embrassent des perspectives différentes. Poète, romancière, essayiste, directrice de maisons d'édition, animatrice culturelle, El Khayat peut être considérée comme une voyageuse entre les civilisations et les cultures. D'un point de vue esthétique, dans son œuvre, nous avons affaire à une créolisation de langages qui s'associe à la tendance à considérer l'action créatrice comme le lieu d'une liberté totale et d'une vitalité qu'il est impossible de contenir et ou d'enfermer dans des limites. En répondant à Abdelkébir Khatibi sur ce sujet, l'écrivaine se compare à " une chèvre, animal impossible à tenir et qui s'égaille partout, y compris sur les arbres, les arganiers du Sud, impossible à contenir » (El Khayat, Khatibi 2004, 19). Cette nécessité de ne pas « avoir de limites, de contenant, de règles fixées, au moins dans le domaine de l'Écriture » (19) fait que l'œuvre de Rita El Khayat est caractérisée par une dynamique intellectuelle qui lui permet

1 Nous remercions Rita El Khayat pour nous avoir donné la permission de publier "La main et le sein" dans l'original anglais, dans son autotraduction française et en traduction italienne.

DOI 10.30687/Tol/2499-5975/2018/20/003

Submission 2018-11-25

(c) 2018 | (1) Creative Commons Attribution 4.0 International Public License 
de passer continûment du social au personnel. D’un point de vue thématique, cette liberté d'expression porte l'écrivaine à aborder des questions particulièrement aiguës telles que le suicide, la folie, la condition féminine et le travail féminin dans le monde arabe, la sexualité, la migration, en passant toujours du plan de l'individu à celui de la collectivité. Ce lien entre le social et le personnel est particulièrement perceptible dans $L a$ Liaison et dans Le Désenfantement.

La Liaison est un roman érotique, sentimental et d'amour écrit en Espagne en 1985 et publié à Paris et au Maroc une dizaine d'années plus tard. La volonté d'écrire un roman érotique (le premier qu'ait écrit une femme arabe) s'inscrit justement dans cette double voie à la fois intime et sociale : d'un côté, El Khayat envisage d'aller au-delà de sa pudeur habituelle pour expérimenter la possibilité d’une libération personnelle par l'écriture ; de l'autre, l'écrivaine entre de plein pied dans une question, l'écriture de l'intime, qui est particulièrement problématique dans le monde arabe et dans l'écriture féminine. Mettre en scène le moi, c'est une tentative de mettre en scène l'image d'un individu-femme en transformation, d'une société changeante, dans laquelle, ainsi que le signalait Déjeux $(1994,61)$ «l'analyse intime du soi est rare », mais où l'émergence du concept d'individualité devient de plus en plus importante. Il ne s'agit pas en fait de parler de son moi intime, mais de s'appuyer sur un sentiment de vide total, éprouvé à un certain moment de sa vie, pour s’insérer dans un parcours de découverte et d'observation introspective.

Le Désenfantement est en revanche un texte ouvertement autobiographique, que l'écrivaine compose à la suite de la perte de sa fille Aïni. Dans ce «jet d'écriture » (Ahnouch 2005, 105), la fonction thérapeutique se lie à la constatation que toute parole est inadéquate et ne peut exprimer réellement la douleur. C'est ainsi que la douleur, en tant que telle, devient le moteur de l'écriture. Mise en face d'une douleur totale et totalisante, l'écrivaine éprouve un sentiment d'impossibilité. La parole ne parvient pas à atteindre l'angoisse éprouvée. Mais cette douleur totalisante et la conscience d'une insuffisance du mot à dire le sentiment éprouvé sont comme revendiquées et constituent paradoxalement le mobile qui permet à l'écrivaine de renverser le stéréotype d'une inaptitude spécialement féminine. El Khayat renverse le sentiment de frustration et trouve dans la désolation, cette « douleur plus forte que celle de Lamartine et Hugo réunies » (Ahnouch 2005, 35), le seul barrage possible à la perte individuelle et le point de départ pour l'écriture.

Correspondance ouverte, volume qui rassemble la correspondance échangée avec Abdelkébir Khatibi, constitue un texte ultérieur et très important dans l'élaboration d'une poétique de la liberté. Ce texte devient bientôt un dialogue entre le féminin et le masculin, une possibilité de s'interroger sur le rapport entre les sexes, mais aussi d'établir une ligne de démarcation entre les écritures, entre des conditions différentes. Les 
lettres sont donc un échange d'idées sur l'amour, la poésie, la philosophie, l'art, la politique, la vie, l'au-delà. Le fantasme de la mort de sa fille persiste et les lettres posent à nouveau le motif du pouvoir thérapeutique de la littérature : " 'Cher' ami... il faudra juste que l'on écoute de nouveaux les poètes » (El Khayat, Khatibi 2004,5) écrit El Khayat, comme pour exorciser le silence et la solitude, comme pour suggérer la possibilité de retrouver « une trace aussi tenue soit-elle, chemin de soie et de vers arabes, legs sublime par-delà les siècles en écoutant Imru’al-Qays évoquer les femmes » (5).

Le choix de la langue d'écriture s'insère lui-aussi à l'intérieur d'un parcours de définition de son image d'écrivaine. En s'engageant sur un chemin connu, El Khayat écrit en français, langue qu'elle considère comme maternelle ou, pour être plus précis, liée à la figure du père. C'est avec son père, mort très jeune, que la jeune Rita parlait en français, surtout pendant ses derniers jours. Le français est donc une langue aimée, qui s'inscrit sur le fil d'un lien profond, mais reste la langue de l'ancien colonisateur. Une langue dont la diffusion au Maroc suit un parcours complexe : langue imposée pendant le protectorat, mais dont l'utilisation s'est amplifiée avec l'indépendance et doit être analysée à l'intérieur d'une situation sociolinguistique complexe, où le français vit à côté de l'arabe classique, langue officielle, et d'autres langues ou dialectes (dialectes arabes et berbères), qui sont les langues que parlent couramment les habitants du pays. C'est dans ce contexte que le français continue à avoir un statut double, langue seconde et langue étrangère, et c'est dans ce contexte qu'El Khayat, née pendant le protectorat, mais devenue écrivaine quand le Maroc était un État indépendant depuis plus d'une vingtaine d'années, considère le français comme sa langue première. Le français est donc une langue aimée, mais c'est une langue qui porte en elle un poids de souffrance historique et personnelle, pour laquelle l'écrivaine ressent un lien fort, fait de prédilection mais aussi d'éloignement.

Pour toutes ces raisons, on comprendra le choix de proposer, dans cette revue, un texte écrit à l'origine en anglais et que l'écrivaine a traduit en français elle-même. Écrire dans une autre langue, différente du français, mais différente aussi de la langue officielle du pays, s'explique par une série de motivations profondes qui permettent à l'écrivaine de mettre une distance entre son être social et son être intime : la langue aimée est aussi à l'origine de souffrances personnelles et collectives. Mais ce choix est aussi une façon de se mettre en contact avec un milieu différent, pour se mettre à l'épreuve sous un autre vêtement. Cette pratique fait penser, d'ailleurs, au Dreams of Trespass de Fatema Mernissi, roman biographique publié en 1994 en anglais et traduit en français en 1996 avec le titre Rêves de femmes.

Le poème que nous publions, « La main et le sein », propose le thème du corps féminin comme mémoire d'un lien primordial. Des images vitales (la 
main, le sein, la gazelle) se confrontent à des images de mort (le vide, la tombe, les vers). Le corps féminin est évoqué par quelques parties désagrégées ; il s'agit d'un corps maternel, d'une main d'où jaillit « un liquide blanc et candide », d'un " sein débordant de lait » et d'un corps-animal qui évoque l'image filiale de la gazelle buvant une goutte du lait. Le lait coule « comme une cascade au printemps ». À ces signes de vie et de plénitude s'opposent les traces d'une perte, d'un vide impossible à combler. La main ne parvient pas à contenir le lait et reste vide. L'œil regarde à partir d'une tombe et « boit au milieu des vers ». À la fraîcheur du sein s'oppose la pourriture des vermisseaux qui entourent l'œil. Par un effet de dédoublement, le corps voit et est regardé, il est mort et vivant à la fois, sans que cette opposition puisse trouver une synthèse, ni l'image d'une pacification intérieure.

\section{Bibliographie}

Ahnouch, Fatima (2005). « La Parabole de la brisure dans Le Désenfantement de Rita El Khayat ». Gontard, Marc (éd.), Le récit féminin au Maroc. Rennes : Presses universitaires de Rennes, 101-08.

Déjeux, Jean (1994). La littérature féminine de langue française au Maghreb. Paris : Khartala.

El Khayat, Rita ; A. Khatibi, Abdelkébir (2004). Correspondance ouverte. Rabat : Marsam. 


\title{
Bioluminescence
}

\author{
Jacqueline Bishop
}

I have followed you to this place of pink and lilac crepe myrtles, Spanish moss, and green and gold beads bleeding from trees along St. Charles Avenue. The man beside me on the tram is heading towards the Creole Tomato Festival, but me, I am heading towards you, Mr. John James Audubon, whose name is now like bioluminescence lighting the way all over this town. It is mid-June and hotter than I expected. What must July or August be like, I wonder? Truthfully, Mr. Audubon, I am tired of following you around. Or is it the other way around, you are the one following me around? For surely you were there with me in London, last March, you remember, I introduced you to my grandfather?

All three of us, you, me and my grandfather, we took a walk together around Piccadilly and we stopped and had fish and chips in some dive place that my grandfather recommended --he who knows this City better than the country where he was born. It was difficult, all three of us trying to talk to one another. My grandfather wanted me to know that he was never Jamaican, always British --- He came to this country before there was any such thing as a black green and gold Jamaican flag. I smile, for despite his harsh denials I can hear Jamaica coating his words. I can hear her too in his laughter.

I can see her too, this gal Jamaica, biding her time and just looking at him, as all good Jamaican girls are wont to do. I see this gal Jamaica, this gal from Nonsuch district really, chocolate brown skin with hair down to her back, on a bicycle that my grandmother told me about once, a red postman's bicycle a young and earnest lover rode miles and miles to pick her up on, in Port Antonio Bay where she was going to school to learn sewing. A bicycle my grandfather now claims to know nothing about. That long and exhausting ride through all those mountains. But tell the truth, can't you see it too dear reader? Can't you see the two young lovers on an old rickety postman's bicycle and my grandmother's slender frame fitting nicely between the bicycle's handles --my grandmother laughter, years and years later, carrying in the wind, one of the few times she ever talked about my mother's father --how was a young woman supposed to resist a man like that? You tell me my granddaughter? He had ridden that old jalopy thing across all those mountains? You were an old man then, Mr. Audubon, last March in London. I had pulled up out from the grave.

DOI 10.30687/Tol/2499-5975/2018/20/004 
All you wanted to do was to relive your glory days

in a time and a place where everyone knew your name.

You wanted to walk these streets and reminisce

about bringing your painted birds here,

how they were immediately applauded.

This was before you grew old and lost your mind

and reverted to the Kreyol and French songs of childhood.

This was before no one knew what you were saying.

It was here in London that people could see what you had given up

years and years of your life to creating. Europeans

have so much better taste than those people across the Atlantic.

Never mind the wife and children you had left back in America.

Never mind the wife and children who for years you had abandoned.

Never mind your cracked and broken English.

You are and were a Frenchman first and foremost.

For his part, my mother's father was having none of it.

He was tired, he said, of hearing pompous

white men yell their story. In any case, he was anxious,

my mother's father was, to set the record straight

as to why he left my mother and her brothers to fend-for-themselves

as children on the island of Jamaica.

Why he chose one woman over another.

He wanted me to know my great grandmother Celeste threatened lawsuit

against him and his mother for child support and to strip herself naked

before them in Port Antonio Bay --- such indecent people!

And what was I doing? I was doing what I am always doing ---

I was in search of my grandmother and a place called Nonesuch

that was simultaneously hidden away in the folds

of the dark blue mountains of Jamaica, and to be found right outside London

in majestic splendor. When things got tense between my grandfather and I,

especially when he brought up anything having to do with that-woman-Emma, it was you Mr.

Audubon and your love of birds

that saved the day, all the conflicting stories that we tell ourselves

and each other, these grand narratives of migration. 


\title{
Bioluminescenza \\ Traduzione di Federica Messulam
}

\author{
Jacqueline Bishop
}

Ti ho seguito fino a questo luogo di mirto crespo rosa e lilla, e muschio spagnolo e perle verdi e oro che sgorgano dagli alberi lungo St. Charles Avenue. Il tizio accanto a me sul tram sta dirigendosi al Creole Tomato Festival, mentre io, io sto venendo incontro a te, Mr. John James Audubon, un nome che ora come bioluminescenza illumina le strade di tutta la città. Siamo a metà giugno ed è più caldo di quanto pensassi. Come saranno luglio e agosto, mi chiedo?

A dire la verità, Mr. Audubon, sono stanca di seguirti.

$\mathrm{O}$ è il contrario, e sei tu che mi insegui?

Perché di certo tu eri con me laggiù a Londra, lo scorso marzo, ricordi che ti presentai mio nonno?

Noi tre, tu, io e il nonno, passeggiammo insieme per Piccadilly e ci fermammo a mangiare fish and chips in una bettola che il nonno consigliava --lui che conosce questa città meglio del paese in cui è nato. È stato complicato, noi tre che cercavamo di parlarci. Mio nonno desiderava io sapessi che non è mai stato giamaicano, ma inglese da sempre --- $\grave{E}$ venuto in questo paese prima che esistesse qualcosa come una bandiera giamaicana verde e oro. Sorrido, perché nonostante i suoi bruschi dinieghi sento che la Giamaica permea le sue parole. La sento anche nelle sue risate. Riesco persino a vederla, una ragazzina della Giamaica, che resta in attesa e lo guarda soltanto, come tutte le brave ragazze giamaicane sono solite fare. Posso vedere la ragazza della Giamaica, questa ragazza del distretto di Nonsuch, la pelle scura come cioccolato e i capelli sciolti sulla schiena, su una bicicletta di cui mi parlò mia nonna tempo fa, una bicicletta rossa da postino che un giovane sinceramente innamorato cavalcò per miglia e miglia per andare a prenderla a Port Antonio Bay dove lei frequentava una scuola per imparare a cucire.

Una bicicletta di cui mio nonno oggi nega di sapere qualcosa.

Quella lunga ed estenuante corsa tra le montagne.

Ma dimmi la verità, non riesci a vederli anche tu, caro lettore?

Riesci anche tu a vedere due giovani innamorati su una vecchia e malconcia

bicicletta da postino e il corpicino esile di mia nonna che si infila a pennello nello spazio del manubrio --la risata di mia nonna, anni ed anni dopo, porta con il vento una delle rare volte in cui ha parlato del padre di mia madre --come avrebbe potuto una giovane donna resistere ad un uomo cosi? Dimmelo tu nipote mia? Lui che aveva cavalcato un catorcio simile attraverso tutte quelle montagne? 
Eri un uomo ormai anziano, Mr. Audubon, lo scorso marzo a Londra.

$\mathrm{Ti}$ avevo trascinato fuori dalla tomba.

Tutto ciò che desideravi fare era rivivere i tuoi giorni di gloria

in un tempo e in un luogo in cui tutti conoscevano il tuo nome.

Volevi camminare per quelle strade e rievocare

quando portasti i dipinti dei tuoi uccelli qui,

e di come furono immediatamente applauditi.

Questo era accaduto prima che tu invecchiassi e perdessi la testa

e ritornassi alle canzoni creole e francesi della tua infanzia.

Questo era accaduto prima che nessuno riuscisse a comprendere ciò che dicevi.

Fu qui a Londra che la gente si rese conto di ciò che avevi sacrificato

per anni ed anni per le tue creazioni. Gli Europei

hanno gusti più raffinati rispetto a quelli che stanno al di là dell'Atlantico.

Non importa se hai lasciato moglie e figli in America.

Non importa se hai abbandonato moglie e figli per anni.

Non importa se il tuo inglese è zoppicante e scorretto

Tu sei stato e sei ancora innanzitutto un francese.

A suo dire, nulla di tutto questo importava al padre di mia madre.

Lui diceva di essere stanco di starsene ad ascoltare

tronfi uomini bianchi che strillavano le loro storie. Comunque, era davvero ansioso,

il padre di mia madre, di mettere in chiaro

la ragione per cui aveva lasciato mia madre e i suoi fratelli a cavarsela da soli

ancora bambini nell'isola di Giamaica.

Sul perché aveva scelto un'altra donna.

Voleva che sapessi che mia nonna Celeste minacciò un'azione legale

contro di lui e sua madre per il sostentamento dei figli e si spogliò nuda

davanti a loro a Port Antonio Bay - che gente indecente!

E io cosa stavo facendo? Stavo facendo quello che faccio di continuo ---

cercavo mia nonna e un luogo chiamato Nonesuch

che si nasconde tra le pieghe delle montagne

color azzurro cupo della Giamaica, ma che si può trovare nel contempo anche subito fuori Londra

nel suo maestoso splendore. Quando l'aria si è fatta tesa tra me e mio nonno,

soprattutto quando ha iniziato a tirar fuori discorsi che avevano a che fare con quella tale Emma, fosti

tu Mr. Audubon e il tuo amore per gli uccelli

a salvare la giornata, tutte le storie contraddittorie che raccontiamo a noi stessi

e agli altri, fantastiche narrazioni sulla migrazione. 


\title{
The Julie Mango Tree
}

\author{
Jacqueline Bishop
}

Small things give way to large things and large things give way to even larger things. This was how the man explained to himself what he was doing in the First Circuit Court in Down Town Kingston over a mango tree. Not just any mango tree, mind you, he told anyone who bothered to ask, but a Julie mango tree. His favorite type of mangoes. He, who had lived a peaceful nondescript life for most of his life, now had to see his face plastered all over the Jamaican newspapers as some kind of special deviant in this country full of deviants. He'd had to listen to all kinds of cheap on-air radio and television program analyses made of him, as if the people running these radio and television shows and those calling in to these shows did not all know why he had done what he had done. As if he had not been forced to do what he had done. As if they were in his position they would not do the same damn thing. People called him a sociopath. Others said that his actions pointed to how deeply indeed the island was in trouble, because the crimes committed in a society were but a reflection on the state of the society. On and on it went. Very few dissenting voices, saying that he had been provoked. That his actions were understandable. That this was something any last one of them would do. The man looked down at himself. This was the first time in his fifty-five years that he had ever gotten himself into trouble. He who had never even gotten so much as a traffic ticket before. He did not even eat meat or meat products of any kind, in this country where meat was god. Barely made any impact on the environment. The few people who knew him described him as a saint-like character. One who years before had a wife, who left him when he left his job, left corporate Jamaica to become an artist and refused to take on any other kind of regular full time employment. The wife, they said, had stuck around for a while, but when it became clear he had no intention of going back to work, she packed her bags and walked straight through the front door. This was not what she had signed up for. He has been driving the same car for some thirty years now, and could not sell the car now even if he wanted to, it was so old. His one saving grace was the house in Harbour View he purchased all those years ago when he had a good job in corporate Jamaica and all the fruit trees that he had planted in his yard.

DOI 10.30687/Tol/2499-5975/2018/20/006

Submission 2018-07-28

(c) 2018 | () Creative Commons Attribution 4.0 International Public License 
"Jamaica was a very different place then," he would eventually tell the lawyer assigned to his case, "in those long-ago days us Jamaicans seem to have more respect for ourselves and for people's property."

The house in Harbour View. It was on a slope that faced the often tranquil blue Caribbean waters. He remembered the days when the beaches were free and open to the public and he and his wife would saunter over on a Sunday evening and walk along the beach, him skimming stones along the water's edge. Sometimes, his wife would finish cooking early and they would have dinner on the beach. Other times, she would tell him to go, and he would take up his paint brushes and you would find him on the beach painting. He had so many views of that beach, before it all changed. Paintings of fishermen pulling in their dingyes after being at sea all night, and women coming to buy the fish to sell in Coronation market down town. He had thin clear seines of web spread out in pristine white sand under the dappled light of almond trees. He even had paintings of Air Jamaica planes flying low coming in for the landing with the orange and yellow humming birds on the tail of the planes. Air Jamaica no longer existed. He used to know that beach like the back of his hand. He used to know this Palisadoes strip of landing before it all changed, before Jamaica all changed, like the back of his hand. He had painting after painting to prove it.

Sometimes he would go along the Palisadoes Road that led out to the airport and to Port Royal. God, that road used to be so beautiful with its white sandy beaches and coconut trees. This was before the government ugly it up and spoil it up by removing all the natural vegetation and putting in a flat ugly highway and those awful bill board signs saying "welcome to Jamaica, your new island home." He wondered who the hell they were welcoming with bill boards like that? He had lovely paintings of the old Palisadoes before it was "improved" upon, and he now wondered if he was the only artist on the island who had such paintings. That might be important, he found himself thinking distantly as he sat in the court room hearing all the charges being read against him. That he would be the only artist on the island with what that part of Jamaica used to be like before it changed. Before it had been "improved" upon. And old beautiful Port Royal, he had some paintings of there too.

Of all the places, maybe only Port Royal maintained any semblance of its former self. Most Jamaicans didn't have access to most of the beaches on the island anymore, never mind that beaches were supposed to be crown land. A fence would go up around the property where the beach was and a big "Trespassers Keep Out" sign would appear on the fence. There would be a club or some such thing before long on the property, and the trespassers were now common workaday Jamaicans who could not meet the fees of going into those establishments. Anything for the almighty dollar, he found himself saying one day, when he was first denied entry to the beach 
in Harbour View where he had gone since he was a boy. Anything to keep down --- keep out --- poor black Jamaican people.

He must have said something like this to the security guard at the high fence of the beach, because the security guard laughed and said,

"The new owners of this establishment my friend, is someone looking just like you and me. The rules have changed. People looking like you and me, they the ones now keeping out people looking just like you and me. They the ones now mashing up this country."

The security guard then looked away, before he lighted up a cigarette. He did not know why he lingered, but he did and then the security guard spoke again.

"I know you. You don't know me, but I know you. I used to do security work over at the supermarket in the square and would see you come there all the time with your wife. I don't feel good about this, about keeping you out, but this job paying me more money than that security job over at the supermarket used to pay me, and I need the money. But no, I don't feel good keeping out people who been 'bout here forever. But I have to do what I have to do. You have a problem, you take it up with management."

After that, and especially after he left his job in advertising, his home with the still glorious view of the harbor became his refuge. They hadn't put up some horrible hotel yet to block his view of the harbor. He turned his attention from painting as much broad landscape work to doing more portraits of people, and still-lives of the fruits and flowers in his yard. He ended up actually, with what he considered a series of truly lovely portraits of children from Harbour View, many of whom were grown men and women now, and who, from time to time, came back to purchase their portraits as children. The things he'd had to do to get those children to sit still. He'd had to bribe them with the fruits in his yard, and he'd had to do the portraits under a tree out in the yard so everyone could see what he was doing, because by then his wife had left and there were all sorts of rumors going around about him. To quiet those rumors he sat the children, especially the little girls out in the yard, and set up his easel and painted. As he worked, there would always be a little group of people who either stopped by to see what he was doing, or gathered around to see what he was painting. They would ooh and ah at every brush stroke, and to be frank about it, he found it all quite irritating, but there was nothing really to be done. He got some lovely portraits out of endlessly fidgeting children. Some sitting under his panganat trees with their yellowish-green fruit and bright orange flowers. The leaves of the tree casting endless shadows across the 
children's innocent upturned faces. Children posed against bright red or yellow hibiscus flowers. Sometimes he heard the things whispered about him as he worked.

"He is a vegetarian you know," he heard one child saying to another while he was intently drawing a flower.

"What is that?" The other child wanted to know.

"Someone who does not eat any meat."

"No meat at all?" The child asked, astonished.

"No, no meat at all?"

"Not even oxtail or chicken?" The child asked, still a little confused.

"You stupid or what?" The other child wanted to know, "I said no meat didn't I?"

"Not even ham at Christmas time?"

"No, not even ham at Christmas time."

The child was quiet for a while, before she continued. "That just sounds strange to me. A person not eating any meat at all. I hate it when my mother cooks callaloo or cabbage even."

"Even if the cabbage have bully beef?"

"That make it a little bit better, but I just can't imagine not eating any meat at all."

He made the mistake of getting into the children's conversation and explaining to them that he was not vegetarian but really vegan.

Four even more confused eyes looked back at him wanting to know what the hell that was?

"Well," he explained, relishing his role as teacher, "this is when you not only stop eating meat but you also stop eating meat products as well. So, no eggs nor cheese for me."

He was to regret telling the children this, because they of course told other children and before long it got to the ears of adults and parents. Finally, they all seemed to understand something about the tall skinny man who every Friday morning drove the old dilapidated car that he still had to Coronation Market in downtown Kingston to buy all sorts of nuts and beans and fruits and vegetables that he blended together and ate.

"Tell you the truth if I was his wife, I would leave him too." A woman named Deloris was heard to say passing his house. "Man just looking for all sorts of ways not to spend any money! Man don't even want to spend money on himself and definitely don't want to spend money on no woman nor any children! Give up him good-good job to sit home 
every-day and call himself artist! That is for rich people living up in hills not for no man that drive a car looking like that!"

Another day and another woman was heard loudly saying, "Man don't even have a television in him house is how I understand it, listening day and night to some old rickety radio. And look at that house. Nice house like that. Years now it could do with a nice coat of paint! Talking 'bout him is artist and vagan whatever that is. Not even vegetarian but some extra-ness called vagan. I wonder what that is? And I wonder how many paintings him sell! No sir, I wouldn't do it either if I was his wife. I would leave him too. Is not like him putting up him things to sell in the craft market or something!".

The woman was right about that. He did not sell that many of his paintings, for each one of Patrick's paintings became his babies. Something he could not bear to part with. Or something he had the greatest difficulty parting with. Not that there were people beating down his door for his paintings. Patrick had the weirdest ideas about art. On the one hand, he believed that he should give up everything for his art, which was exactly what he had done. He gave up his life in advertising and he gave up his wife and god bless the few friends that he still had.

But on the other hand, he did not believe that he should do anything to bring attention to his art. People should find him, he seemed to think, start talking about him. People should immediately see his genius and pursue him for his work. In the meantime, he seemed to be prepared to be the starving artist. After a while, even his lawyer would start to wonder, as he came to know him, if this was not some role that he was playing. Something he had read about in a book. That one should suffer to create good art? Patrick seemed to be playing that role to the fullest.

The one thing the publicity did do though was to bring Patrick to the attention to the art establishment on the island and when they came calling there was no denying the mastery in his paintings. His still-lives, it was decided, were particularly remarkable. Over the years, Patrick had worked and reworked the West Indian cherry, lime tree, ackee, sweet sop, East Indian mangoes, and especially Julie Mangoes into perfection. Bunches of guineps, custard apples, dark purple Othaheite apples, June plums, moved effortlessly on his canvas, and oh the flowers! He had a huge Lignum Vitae tree and a Flamboyant tree he painted over and over again to the point where they seemed not so much to even be on the canvas anymore but were fully realized creations in their own right in and on the world of the canvas.

In his backyard, Patrick had planted a patch of escellion, thyme, onion, hot and sweet peppers, and carrots and turnips, and before they made it into a meal, they made it onto his canvas. His work was simply stunning. 
Then there were the landscape paintings. Patrick had been right all along. They not only captured aspects of the island that no longer existed, but they captured growing tensions on the island as to who had access to certain parts of the island, the beaches in particular, for in his anger, Patrick had started painting from the outside all the new clubs and establishments set up on the seaside in Harbour View, the no-trespassing signs posted on the chain link fences of these establishments, and the security guards in their smart new uniforms standing guard at the entrance posts of these establishments and patrolling these places. He even started painting the ridiculous billboards welcoming people to the island on their way in from the airport along the Palisadoes and the new airlines that had taken the place of Air Jamaica --- American Airlines, Air Canada, and Jet Blue carriers --- as they flew in to land on the island.

"Why you never showed anyone all of this before?" His lawyer wanted to know, when he became aware of Patrick's paintings.

"I did my work for me, not for me and company" was the reply.

But Patrick was secretly delighted at all the acclaim finally coming to his work. He was being compared to Cezanne! Was being called Jamaica's very own Cezanne and there was not another artist he loved more than he loved Cezanne! When the police was trampling through his house after the killing, pushing things aside, and throwing down books and canvases, he made sure to safeguard as many of his canvases as he could and all the books and pamphlets he had collected over the years on Cezanne.

"We might have to sell some of your works to pay your fees," the lawyer tested.

Patrick did not answer.

"Anyway Patrick, go ahead and tell me your side of the story. Tell me what happened."

They were in the lawyer's office and Patrick did not know where to begin. He kept looking around him. The lawyer had a small office in downtown Kingston. Maybe this man too was feeling the pinch, Patrick thought, looking at the small cramped hot place. Maybe he would understand what he was talking about. How change often happened imperceptibly. Maybe this man would understand that it's not like you could say things was one way one day but totally different the next day. Instead, what he started to notice, what everyone started to notice, about a dozen or so years back, was just a lack of respect for people and people's property. The lawyer nodded his head when Patrick started talking. How, it may be that the ordinary Jamaican was feeling squeezed out of the things of their society, but instead of taking it out on the people doing the squeezing, they started taking it out on each other. 
The lawyer looked on closely, again nodding his head knowingly at what Patrick was saying.

The Jamaicans being squeezed needed to take out what they were feeling on the people putting up the "no trespassing" signs on Jamaican beaches and keeping ordinary Jamaicans out, on the politicians and people in power allowing them to do this, Patrick was saying and the lawyer was rapidly writing it all down on yellow legal pads, but instead they started taking it out on each other. What did he mean exactly by this, the lawyer wanted to know, and Patrick explained further. The people in Harbour View for example started to realize that they could not enjoy the fruits of their labor. No, he was not speaking in riddles and parables, he assured his lawyer, he had a point that he was getting to. Whereas before, you could have a fruit tree in your yard that you plant and you water and you bring to fruit and enjoy the fruit of your labors, literally, this was no longer the case, people were casing the place and picking off all the fruits from off the trees and leaving not even one fruit from your own tree for you to eat. They pick the ripe fruits and they shake the green ones off the trees to get to the turn fruits. What was worst, they climb on your own house-top to get the fruits, damaging your own house-tops to do their picking. Gangs of roving boys and grown-ass men doing this. They wait until you gone to work or to a doctor's appointment or somewhere and then they all over your yard as if they own the damn place. They even found his little garden in the back and made all his vegetables their own.

One day he said to one of the fleeing men, grown, big as him, "Why the rass you doing this man? You have no conscience at all! You can't even leave one or two of the ackees on the tree for me!"

The man stopped, leaned nonchalantly against a large white pail and said something about feeding his family. But somehow, he knew the man was lying, that this was just a quick and easy way for the man to get some money.

"So, this is how you teach your family that you feed them? That you take care of them? Is by thieving people property? What kind of example you setting?"

The man laughed a snickering little laugh which really got under Patrick's skin. "What I teaching my family is that God make the ackee tree and God make me and so I have a right to the ackees that God make grow on the tree," the man said, before he sauntered off up the road.

Patrick could not believe how upset what the man said made him. He could not believe the insouciance of the man. A few other people gathered on the street shook their head in dismay and disbelief at what the man said. People were beginning to cut down the fruit trees in their yards to keep the brazen thieves out. Other people picked the fruits themselves and left them 
at the front of their gate, to stop the thieves walking on their house-tops and damaging their roofs. One woman complained long and loud on the radio how she came upon a lady thief cutting her prize-winning heliconias and red gingers from out of her yard. When she confronted the lady thief about it, the woman insisted the flowers were to decorate her church altar! As if that somehow absolved her of what she was doing! The hysterical woman kept screaming on the radio call-in show. On Facebook people were posting videos of thieves sauntering into their yards and making off with fruits, vegetables and even prize-winning flowers as you please. The people of Harbour View decided not to buy fruits from fruit sellers in the area, because these people had the nerve to thief your fruits and try to sell them back to you! He would have to cut down his precious ackee tree, is what Patrick was thinking, because ackee bore all year round, and people thieved nothing more than they thieved ackees. It was afterall part of the national dish of the island. Where were these people when he had to sweep up the ackee leaves in his yard? When he had to pick up rotten yellow fruit that splattered to the ground? He loved to cook red green and gold rice with his ackees and now he was going to have to cut down his cherished ackee tree to keep the scoundrels out of his yard.

When he got rid of his ackee tree though, that was when he started to notice just how many people had gotten rid of the fruit trees out of their yards. What a shame, he kept thinking. What a god damn shame, that you could not even enjoy the fruits that you had growing in your own yard. Fences kept getting higher and higher, and topped by barbed wire and broken bottles, and there were flood lights everywhere at night. Not that any of that seemed to be helping. People were becoming prisoners in their own homes. Harbour View wasn't even an inner-city neighborhood, Patrick found himself saying to himself. It was solidly middle class. But that didn't seem to matter anymore. From what he was hearing on the radio, scoundrels were ransacking the interior of the island as well. Jesus Christ, Patrick found himself thinking, people will thief and sell just about anything. People will thief and sell their own damn mother. There was no respect for people or their property at all on the island. People were under siege in their own damn country. When had all of this happened? When had Jamaica become this kind of place?

Then one of the newscasters brought a group of thieves onto his show. Wanting them to explain why they did what they did. Why they thieved people's fruits and vegetables and animals and flowers. The thieves gave the usual explanations of poverty and hunger and not being able to find a job. The newscaster said he was glad to hear them say this, because he had a friend that had a farm in St. Catherine and he was looking for strong young men like them to hire. Good enough pay and they would have a place to stay when they worked with his friend if they wanted, but St. Catherine right next door to Kingston so they could go to work every day and come 
home every evening if they wanted to. Patrick leaned closer to the radio to hear what the thieves would say. The thieves were upset. They did not see themselves as thieves at all and resented people calling them that. They wanted to make that crystal clear. Thieves broke into people's houses and cars, or held up people at gun or knife point and they did not do that. They even asked for permission to enter the yards that they entered if someone was at home. But what about the job offer the newsman pressed them? Patrick leaned closer still towards the radio. He believed he could hear the men shuffling uncomfortably. Then there was dead silence. Someone? Anyone? The newscaster wanted to know. There was more uncomfortable silence. Still no one answered. After the young men left the station, the newscaster continued. "Poverty and hunger are very real things. But I humbly suggest to you, that that is not the real reason why you have so much widespread thieving going on, on the island."

Patrick could not believe how angry the news segment made him. He did not know why he even bothered to listen to it. He was even more pissed off the next morning when he went outside and saw that someone had picked off all his tomatoes and uprooted all his turnips and carrots. The onions and scallions and peppers were gone too. That was the day when he started stockpiling rocks underneath the Julie mango tree for the next thieving scoundrel that would come bothering his Julie Mango Tree. The one he had planted and grown at the front of his house from a sapling. Of course, he did not know that one of the rocks would connect with one of the thieves and he and his long stick and big white bucket who come tumbling down through the limbs of mango tree and end up tangled up in and electrocuted by the electrical wires at the front of the house. Of-course he did not see the people in Harbour View and all over the island who would be calling for his head and saying what kind of thing that was, him killing a man over a few Julie mangoes. Of-course he did not see himself sitting miserably in the First Circuit Court in downtown Kingston. Of-course Patrick did not see himself being charged for murder. 



\title{
Introduzione all'opera di Jacqueline Bishop
}

\author{
Michela A. Calderaro \\ (Università degli Studi di Trieste, Italia)
}

Jacqueline Bishop, professore associato presso New York University, è nata a Kingston, Giamaica, dove ha trascorso l'infanzia e l'adolescenza circondata da una famiglia allargata, zie, zii, cugini, sorelle, nonni e bisnonni. La figura che più influenzerà la sua vita e le sue scelte artistiche resta però la nonna.

Dopo aver terminato la scuola superiore, si è trasferita negli Stati Uniti, a New York, dove già viveva la madre, per completare gli studi presso il Dipartimento di Creative Writing di New York University.

Sempre a New York, e spinta dall'interesse per il legame tra le arti visive e la parola scritta, ha fondato Calabash: A Journal of Caribbean Arts and Letters. La rivista, che nei dieci anni di pubblicazione si è imposta come una delle più influenti riviste letterarie e di arti visive, comprendeva nell'Advisory Board, scrittori come George Lamming, Kamau Brathwaite, Wilson Harris e Paule Marshall.

Il primo numero verrà pubblicato nel settembre 2000, con in copertina un dipinto dell'albero di calabash, di Tony Monsanto, un artista di Curaçao. L'immagine di questo albero, che ha un posto particolare nella mente e nei cuori dei Caraibi, ritorna nella poesia d'apertura (di Bishop) a forma di foglia, per guidare i lettori attraverso un viaggio di parole e immagini.

Jacqueline Bishop è al tempo stesso una poetessa, una scrittrice, una fotografa, una pittrice, una cineasta e una produttrice.

Ha scritto, diretto e prodotto When In Doubt, Check It Out: A Promotion Of Men's Health Awareness; ha scritto, diretto e prodotto Voices In Prevention: The Sexual Assault, Prevention, Education \& Support Office presso la New York University.

A Venezia per completare un Master in Arte (programma gestito dalla New York University), ha creato e prodotto Two Visions from Venice: The Art of Maria Morganti \& Federica Marangoni. Two Female Venetian Artists; ha fondato la società cinematografica indipendente Dyaspora Films e in seguito ha ideato e ottenuto finanziamenti per I Came Here by a Dream: The Jamaican Intuitive Artists, un documentario su artisti giamaicani.

$\mathrm{Da}$ anni è responsabile del supplemento letterario domenicale del $\mathrm{Ja}$ maica Observer, «Bookends», con articoli ed interviste.

Ha pubblicato due raccolte di poesie, Fauna (2006) e Snapshots from Istanbul (2009); un romanzo, The River's Song (2007); un libro d'arte, Writ- 
ers Who Paint, Painters Who Write (2007); una raccolta di storie orali di donne giamaicane che vivono a New York, My Mother Who Is Me: Lifestories from Jamaican Women in New York (2006); alcune serie di fotografie, tra cui Childhood Memories; una serie di poesie e disegni, The Tempest Poems (2014) che offre al pubblico una resa perfetta del legame tra le arti visive e la parola scritta, per il 9th Annual Liberal Studies Colloquium, Shakespeare's Globe, per celebrare il $450^{\circ}$ anniversario della nascita di Shakespeare; una raccolta di storie, saggi, interviste, The Gymnast and Other Positions (2015).

La sua ultima produzione artistica, By the Rivers of Babylon, una mostra di quadri in cui esplora la relazione tra lo spazio e il tempo nei Caraibi, ha riscosso un grande successo di pubblico e di critica. La mostra, inaugurata a New York nel mese di marzo 2018, verrà ripresentata presto in Europa. 


\section{Articoli | Articles | Articles}





\title{
Pudeur et splendeur de la prostitution chez Aimé Césaire et Zadi Zaourou Représentations stylistiques du corps-tabou
}

\author{
Dorgelès Houessou \\ (Université Alassane Ouattara, Bouaké, Côte d'Ivoire)
}

\begin{abstract}
In the Cahier d'un retour au pays natal, Césaire depicts prostitution in its abject and reprehensible dimension. Many stylistic processes, such as the reference to the biblical account of the fall, thus define a servile and shameful practice. The reason is that the poet subtly encodes the vice of the sex trade that he makes the equivalent of cultural assimilation. In contrast, Zadi Zaourou Bernard, Ivorian poet and disciple of Césaire, generally depicted prostitution under the aegis of admiration. Elegy for those bodies offered to the libidinal satisfaction of whoever is able to pay the price, some poems from his collection titled $\hat{A}$ Califourchon sur le dos d'un nuage raise and sublimate the prostitute.
\end{abstract}

Sommaire 1 Introduction. -2 La topique stylématique de la figuration macrostructurale de la prostitution. - 2.1 L'allusion. - 2.2 L'ironie. - 2.3 L'humour. - 2.4 La périphrase. - 2.5 L'hyperbole. - 3 La topique stylématique de la figuration microstructurale de la prostitution. - 3.1 La métaphore. - 3.2 La métonymie. - 3.3 La répétition. - 3.4 L'épitrochasme. - 4 Conclusion.

Keywords Stylistics. Styleme. Rhetoric figure. Poetry. Figure of the prostitute.

\section{Introduction}

Selon Brochier « la prostitution peut être définie comme le fait d'établir avec d'autres personnes des relations dont l'aboutissement logique est un acte sexuel, avec pour but d'obtenir à court terme une rémunération » $(2005,79)$. Ainsi envisagée, cette pratique a vu naître à la fois un processus d'héroïsation, un culte célébrant la liberté sexuelle de la femme, symbole de son émancipation envers et contre la domination masculine (Borrillo 2002) et un autre processus de désacralisation, un blâme de cette victime de l'impératif phallocratique, de celle qui en subissant la marchandisation de son corps, se rend coupable de vices de tout genre (Tabet 2005). L'ouvrage de Tabet intitulé La grande arnaque : sexualité des femmes et échange économico-sexuel est déjà évocateur du principe selon lequel la domination masculine (Bourdieu 1990, 25) organise à son seul profit les modalités économiques inéluctables de la sexualité féminine. 
Il ressort de ces représentations autant que de maints imaginaires discursifs que la prostitution est saisie par glissement métonymique comme symbole de la sexualité féminine. Au nombre desdits imaginaires figure, sans doute au premier plan, celui qui à en croire le récit mythique du péché originel, en a fait le plus vieux métier du monde. D'où le postulat de Pheterson pour qui, il ne suffit que d'« ôter de l'échange économico-sexuel le stigmate de « putain » et la prostitution s'évapore » (Pheterson 2001, $10)$. Son analyse du phénomène de la prostitution envisage :

la position stratégique des femmes portant cette marque, qu'il s'agisse de travailleuses du sexe rémunérées, ou de femmes arbitrairement accusées de prostitution, ou de femmes stigmatisées comme putains en raison du statut que leur donnent leur travail, leur couleur, leur classe, leur sexualité, les sévices qu'elles ont subis, leur ethnie, leur situation matrimoniale, ou simplement leur genre. (Pheterson 2001, 10-11)

On verra dans cette étude comment la topique stylématique de la figure aussi bien macrostructurale que microstructurale selon la typologie de Molinié (2011) organise les représentations de la prostitution chez Césaire et Zaourou à partir du Cahier d'un retour au pays natal de l'un et de $\dot{A}$ Califourchon sur le dos d'un nuage de l'autre. ${ }^{1}$ On s'y intéressera, au-delà des déterminations esthético-conceptuelles, au processus de la fabrication de la figure de la prostituée et de la symbolique qui en découle.

\section{La topique stylématique de la figuration macrostructurale de la prostitution}

Molinié définit la figure comme le processus de signifiance à travers lequel s'opère une rupture entre l'information et le système expressif utilisé. "La figure est donc-dit-il-une différence, une quantité langagière différentielle entre le contenu informatif et les moyens lexicaux et syntaxiques mis en œuvre » $(2011,83)$. En tant que telle, la figure est définie par Molinié à la fois comme un indice stylématique, c'est-àdire un constituant formel du style littéraire, et une topique (un lieu) de manifestation de la littérarité. Dans la taxinomie qu'il opère au sujet des figures, il distingue les figures macro structurales et les figures microstructurales. Ces dernières sont d'un point de vue définitoire, identifiables et interprétables au sein du microcontexte, obligatoirement

1 Pour l'ensemble du présent document les références des deux œuvres du corpus seront les suivantes : Aimé, Césaire [Bordas 1947] (1983). Cahier d'un retour au pays natal. Paris : Présence Africaine ; Bottey, Zadi Zaourou (2009). À Califourchon sur le dos d'un nuage. Abidjan : L'Harmattan. 
identifiables pour l'acceptabilité sémantique de l'énoncé et enfin attachées à des éléments formels précis dont elles dépendent. Ce sonten gros les figures de répétition, les figures dépendant de l'arrangement syntaxique ou figure de construction et les tropes. A contrario, les figures macrostructurales auront pour caractéristiques de n'être ni identifiables, ni interprétables qu'à partir du macrocontexte énonciatif, de ne pas obligatoirement être identifiées comme figure pour l'acceptabilité sémantique de l'énoncé et enfin de ne pas être isolables sur des éléments formels précis. Il s'agit notamment des figures de l'allocution, de la personnification, des figures de la caractérisation quantitative, des figures d'opposition, des figures de l'amplification, et enfin, de l'hypotypose.

\subsection{L'allusion}

Cette figure macrostructurale « consiste en ce que, dans un segment de discours s'étendant en général sur plusieurs phrases, un terme a un sens à l'égard d'un autre terme de la phrase, et un sens différent par rapport à la situation d'énonciation ou à l'univers de culture » (Molinié 1992, 44). On la déclinera ici à travers les univers de culture mythologique et historique.

\subsubsection{L'allusion mythologique}

Soient ces extraits du poème «Swilia » de Zadi Zaourou :

Qui donc prétendait

Mais qui...

Qu'elle n'est pas de l'Eden un bocage secret,

La pleine lune de ton visage boréal

Ah ! Swilia !

(Zaourou 2009, 68)

Je te proclame vieille cuvée des réserves de Dieu

Brise câline des rivages marins

Embrun qui tressaille et soupire entre ciel et mer

Orage orage cyclone aux quatre rives de l'âme

Quand te tient la transe des désirs que tu inspires

Comment te nommer, femme,

Ô ! Vaste plaine à éclore mille vergers

Graine bourgeon fleur succulent fruit de pleine saison

Emerge donc féline et radieuse 
Swilia mon SIDA !

Et qu'à mon signal

Chaque étoile s'incline et te salue au passage du gué !

(70)

L'évocation du mythe du jardin d'Éden comme source première de l'éveil au désir du féminin permet au poète d'introduire une vision de la femme aux mille amants à travers la métaphore « Ô ! Vaste plaine à éclore mille vergers » qui constitue une allusion à la prostitution. Mais l'énonciateur joue aussi sur la référence doxique de la prostitution comme métier le plus vieux au monde pour en célébrer la première figure : Ėve. On perçoit ensuite, alors qu'un ensemble de descripteurs mélioratifs vantait la superbe de cette figure totémique à laquelle il assimile sa bien aimée, que cette dernière est aussi représentée sous les traits d'une femme fatale. Il s'agit là d'un cas d'inférence au sens où l'entend Kerbrat-Orecchioni c'est-à-dire comme une forme d'implicite qui concerne « toute proposition implicite que l'on peut extraire d'un énoncé, et déduire de son contenu littéral en combinant des informations de statut variable (internes et externes)» (Kerbrat-Orecchioni 1986, 24). Ainsi, l'énoncé « Swilia mon SIDA ! » revêt un caractère amphibologique. Il traduit premièrement l'implicite doxique que cette femme aux mœurs légères ne pouvant qu'être séropositive du fait de la multiplicité de ses partenaires, lui aura transmis ce mal vénérien. Mais cet énoncé allusif de la prostitution renvoie aussi à l'idée métaphorique que le poète est porteur de la marque de sa partenaire comme il serait porteur du SIDA c'est-à-dire jusque dans les tréfonds de ses leucocytes. Elle le dévorerait de l'intérieur d'une passion incurable au point où, ayant eu le privilège des délices de son corps, le poète sonne le tocsin pour que tout homme ( " chaque étoile ») digne de ses plaisirs la découvre en ses jardins secrets (« te salue au passage du gué »). Cette image de la femme décrite comme ayant volé au paradis le feu du plaisir pour le faire connaître des hommes constitue aussi une allusion prométhéenne que l'on peut retrouver dans ces extraits :

C'était au temps zéro

[...] Aïessa brillait de mille carats

Flamme sacrée consumant l'iris

Corps de jaspe et de rubis

Sexe d'étoile pubère !

(Zaourou 2009, 20)

Flamme sonore sur les lèvres de minuit

Ivresse ivresse

Délire du temps quand s'éloignent tes feux

Que pourchassent d'autres feux 
Puis d'autres encore qui parachèvent, féerique,

Le fier embrasement de la nuit

Minuit !

(26-7)

La représentation sexuelle de l'étoile joue ainsi sur la double topique de la visibilité/brillance et de la désirabilité tout comme le symbolisme du feu en tant que métaphore du désir érotique. L'énonciateur amant évoque ainsi le douloureux (délire) éloignement des désirs de son amante nommée Aïessa qui s'en va, pourchassée par une multitude d'amants ardents (d'autres, puis d'autres encore) jusqu'à féconder la vie, car la nuit pour le tradi-oraliste que fut le poète, symbolise la fécondité. La femme aux multiples amants est donc représentée comme une hérö̈ne prométhéenne offrant aux hommes la félicité des sens dérobés aux dieux.

On retrouve dans le Cahier des allusions à la prostitution qui empruntent le biais de l'évocation mythologique. C'est le cas dans cet extrait où l'évocation de la Bible tient lieu d'emploi topique sous la plume de Césaire qui en fait un instrument d'aliénation mentale et culturelle :

Et voici ceux qui ne se consolent point de n'être pas faits à la ressemblance de Dieu mais de diable, ceux qui considèrent que l'on est nègre comme commis de seconde classe : en attendant mieux et avec possibilité de monter plus haut; ceux qui battent la chamade devant soimême, ceux qui vivent dans un cul de basse fosse de soi-même ; ceux qui se drapent de pseudomorphose fière ; ceux qui disent à l'Europe : « Voyez, je sais comme vous faire des courbettes, comme vous présenter mes hommages, en somme, je ne suis pas différent de vous ; ne faites pas attention à ma peau noire : c'est le soleil qui m’a brûlé ». (Césaire [1947] 1983, 58-9)

L'extrait dépeint le Noir assimilé qui, reniant toute dignité, vend jusqu'à son identité à l'ogresse blanche qui dévore son histoire, sa mythologie et sa culture. Sous les traits métaphoriques de la prostituée, le nègre assimilé est décrit avec sarcasme comme prisonnier d' « un cul de basse fosse de soi-même ». L'emploi du mot « cul » en régime de vulgarité connote «le stigmate de la putain » dont parle Pheterson. Ces Noirs indignes aux yeux de l'encodeur se vendent comme des prostituées. Cette hypothèse de lecture transparaît aussi dans la phrase " c'est le soleil qui m'a brûlé » qui extraite du Cantique des cantiques rapporte les propos d'une femme noire aux mœurs légères :

5 Je suis noire, mais je suis belle, filles de Jérusalem, Comme les tentes de Kédar, comme les pavillons de Salomon. 
6 Ne prenez pas garde à mon teint noir : C'est le soleil qui m'a brûlée. Les fils de ma mère se sont irrités contre moi, Ils m'ont faite gardienne des vignes. Ma vigne, à moi, je ne l'ai pas gardée.

7 Dis-moi, ô toi que mon cœur aime, Où tu fais paître tes brebis, Où tu les fais reposer à midi ; Car pourquoi serais-je comme une égarée Près des troupeaux de tes compagnons? (Cantique des Cantiques 1, 5-7)

Lallusion permet ici au poète de mettre en parallèle le personnage biblique de cette femme noire incapable de garder sa vigne, c'est-à-dire sa virginité, sa pudeur sexuelle, et ces Noirs qui abdiquant toute fierté, se complaisent en courbettes et obséquiosités face au Blanc et au mépris des valeurs et de la dignité humaines, quémandant comme elle l'amour, le droit à l'identité d'homme.

\subsubsection{L'allusion historique}

L'allusion à la prostitution emprunte aussi parfois les traits de la référence à l'histoire sous l'inspiration des poètes. Ainsi peut-on lire dans le Cahier : « Et il y a le maquereau nègre, l'askari nègre, et tous les zèbres se secouent à leur manière pour faire tomber leurs zébrures en une rosée de lait frais » (59). Dans ce passage, le lexème « maquereau » désigne en effet l' « Homme qui débauche et prostitue les femmes et qui reçoit d'elles l'argent qu'elles tirent de la prostitution ${ }^{2}{ }^{2}$ Si les dictionnaires en font ainsi le synonyme de proxénète, c'est en raison de ce que le maquereau comme poisson avait selon une croyance populaire attestée surtout à partir du XIXème siècle pour rôle de rapprocher les harengs mâles des harengs femelles, qu'il accompagne dans leurs migrations. Quant au terme «askari », il constitue un sème diachronique et un indicateur de lecture renvoyant historiquement à la période de l'empire colonial où il désignait le corps des troupes indigènes des empires coloniaux européens. Cette allusion à l'histoire de la colonisation rencontre celle de la prostitution telle que l'entend Césaire ici. Le terme existe chez Zadi Zaourou dans une graphie différente mais avec la même acception lorsqu'il affirme notamment :

La femme que j'aimais n'a même plus d'âme

Quand aux Saturnales s'annonça le temps des criquets

Le bal des chacals et des vipères futures

Et que l'Antéchrist accoucha de millions

2 Cf. http://www.cnrtl.fr/definition/MAQUEREAU (2018-11-27). 
d'ascaris et de reptiles fauves

(Zaourou 2009, 55)

L'ascari ou askari est donc décrit à la fois en nombre pléthorique voire hyperbolique et comme le fruit d'une union trompeuse puisque l'engendre l'Antéchrist qui, selon le livre de l'Apocalypse, est l'ennemi du Christ qui viendra prêcher une religion hostile à la sienne un peu avant la fin du monde. Or cette pratique même est encore assimilée à la prostitution dans de nombreux passages bibliques. ${ }^{3}$ La représentation de la prostituée dans ces deux extraits est donc axiologiquement dysphorique. C'est de l'indignité capitale que de marchander son sexe que Césaire accuse les Noirs qui ont collaboré avec le Blanc aux fins d'asservir le continent africain avec pour seul paiement de la verroterie et de la pacotille. Zadi Zaourou pour sa part fait des Africains indignes de la confiance de leurs frères, non seulement les équivalents d'animaux à sang froid (《 vipères », « reptiles »), symboles de froideur, d'égoïsme et d'indifférence, mais encore des charognards (chacals) dont la culture populaire fait des êtres malfaisants, et enfin des avortons diaboliques. Le recours à l'allusion historique s'enrichit ici d'une symbolique remarquable. D'une part, la lexicalisation des appellatifs 《 reptile », « serpent » et « vipère » dénote la trahison et la félonie car « on traitera de reptile une personne qui démontre un caractère bas, rampant, obséquieux et servile ; est-elle sournoise, méchante dans son agir et acerbe dans ses paroles, on la qualifiera de 'serpent' ou de 'vipère' » (Girard 1991, 827). D'autre part, ces mêmes appellatifs connotent la lubricité voire la prostitution dans un tel contexte d'écriture poétique quand on sait que chez les freudiens « le serpent est perçu comme l'un des symboles les plus constants et invariables de l'organe sexuel mâle. L'analogie de forme et de fonction saute passablement aux yeux » (Girard 1991, 827). Peut-être est ce là encore un rappel du mythe édénique du serpent tentateur.

3 Cf Mi 1:7 « Toutes ses images taillées seront brisées, Tous ses salaires impurs seront brûlés au feu, Et je ravagerai toutes ses idoles : Recueillies avec le salaire de la prostitution, Elles deviendront un salaire de prostitutions »; ou encore le Ex 34:15-16 «Garde-toi de faire alliance avec les habitants du pays, de peur que, se prostituant à leurs dieux et leur offrant des sacrifices, ils ne t'invitent, et que tu ne manges de leurs victimes ; 16 de peur que tu ne prennes de leurs filles pour tes fils, et que leurs filles, se prostituant à leurs dieux, n'entraînent tes fils à se prostituer à leurs dieux ». 


\subsection{L'ironie}

Georges Molinié indique dans son Dictionnaire de rhétorique que « l'ironie et une figure de type macrostructural qui joue sur la caractérisation intensive de l'énoncé : comme chacun sait, on dit le contraire de ce que l'on veut faire entendre » $(1992,180)$. Ce jeu d'inversion du sens doit avoir une finalité humoristique. Il précise notamment qu' « il importe de bien voir le caractère macrostructural de l'ironie : un discours ironique se développe parfois sur un ensemble de phrases parmi lesquelles il est difficile d'isoler formellement de terme spécifiquement porteur de l'ironie » $(1992,180)$. Le diasyrme et l'astéisme en sont les deux modalités fondamentales.

\subsubsection{Le diasyrme}

Le diasyrme qui consiste en un faux éloge est une figure macrostructurale qui laisse entendre que, étant donné un énoncé laudatif, « chaque expression emphatique et méliorative est à comprendre à l'envers, au sens d'une attaque particulièrement mordante » (Molinié 1992, 116). Il en est ainsi dans ce passage du Cahier :

C'était un très bon nègre,

la misère lui avait blessé poitrine et dos et on avait fourré dans sa pauvre cervelle qu'une fatalité pesait sur lui qu'on ne prend pas au collet ; qu'il n'avait pas puissance sur son propre destin ; qu'un Seigneur méchant avait de toute éternité écrit des lois d'interdiction en sa nature pelvienne. (Césaire 1983, 59-60)

Si le contexte permet de dire que cette envolée laudative est insincèrement encodée, le recours au parallèle sexuel et par ricochet au dévergondage lié à la prostitution résulte de l'emploi de l'expression « nature pelvienne ». La critique a longtemps perçu l'adjectif « pelvienne » pour les connotations sexuelles qui en découlent par glissement métonymique. L’hypothèse est juste sans doute. Mais la concaténation du lexème « nature »y implique surtout l'idée de fatalité. Ce serait donc nécessairement, et sous les ordres péremptoires de celui que l'oxymoron désigne « Seigneur méchant », que les Noirs seraient des êtres lubriques et incapables d'autres conditions sinon la misère et la servitude. 


\subsubsection{L’astéisme}

Selon cette figure de rhétorique consistant en un éloge implicite du positif par le négatif, « un discours apparemment dépréciatif (de blâme) ou négatif s'interprète, pour des raisons largement macrocontextuelles, en discours d'éloge, de faveur ou d'orientation nettement positive » (Molinié 1992, 63). Il en est ainsi chez le poète Césaire qui s'écrie :

Mais ah ! la rauque contrebande

de mon rire

Ah ! Mon trésor de salpêtre !

Parce que nous vous haïssons vous et votre raison, nous nous réclamons de la démence précoce de la folie flambante du cannibalisme tenace. (Césaire 1983, 27)

Ce passage évoque encore le crime de flibustier dont l'Europe s'est rendu coupable. Mais fidèle à sa logique métaphorique 'flibuste = prostitution des Noirs coupables de collaboration', Césaire emploie donc le possessif «mon » associé au rire lubrique des contrebandes à cette fin. Le basculement pronominal au «nous » inclut cette fois-ci les Noirs qui n'ont pas pris part à l'assaut contre le continent, ceux pour qui l'astéisme prend ici tout son sens. Car le poète, raisonnant par l'absurde, dit réclamer la démence et les bacchanales cannibalistiques dont les Noirs sont accusés par les esclavagistes soucieux de justifier leurs actions. Ce procédé met en relief la volonté de l'énonciateur de vanter a contrario les vrais mérites de ces derniers à savoir leur dignité et leur humanité.

\subsection{L'humour}

L'humour est une forme d'esprit railleuse soulignant, par le langage, le caractère comique d'une situation donnée. Césaire l'emploie souvent pour montrer le ridicule de la discrimination fondée sur la couleur de la peau comme dans cet extrait :

Et ce pays cria pendant des siècles que nous sommes des bêtes brutes ; que les pulsations de l'humanité s'arrêtent aux portes de la négrerie ; que nous sommes un fumier ambulant hideusement prometteur de cannes tendres et de coton soyeux et l'on nous marquait au fer rouge et nous dormions dans nos excréments et l'on nous vendait sur les places et l'aune de drap anglais et la viande salée d'Irlande coûtaient moins cher que nous, et ce pays était calme, tranquille, disant que l'esprit de Dieu était dans ses actes. (Césaire 1983, 38-9) 
Ce passage reprend la même topique de la prostitution comme marchandisation du corps à travers l'emploi des syntagmes nominal « un fumier [...] de cannes tendres et de coton soyeux » et verbal « et l'aune de drap anglais et la viande salée d'Irlande coûtaient moins cher que nous ». La domination masculine est dépossession du corps de la femme. De même la marchandisation du corps des esclaves est-elle perçue par le poète comme de l'esclavage proxénétique dont il paraît presque drôle que la morale religieuse de ceux qui se dirent « civilisateurs » ne put, sinon l'empêcher, au moins en juguler les excès.

\subsection{La périphrase}

Cette figure consiste à nommer une réalité donnée par un groupe de mots dont l'encodeur est juge de la fiabilité et de la logique. Ainsi, lorsque Césaire affirme : « Beauté je t'appelle pétition de la pierre » (27), il construit une sorte de périphrase in praesentia, en explicitant d'un point de vue intra-syntagmatique la périphrase à travers l'élément référent "Beauté » et l'élément désignateur " pétition de la pierre ». Mais la beauté qui mériterait la lapidation ne serait-elle pas une femme infidèle ? Une femme fatale ? Une femme indigne ? Les préjugés moraux quant à la perception axiologique négative de la femme non attachée à un homme sourdent encore dans ce vers césairien allusif à la tradition orientale du châtiment de la femme infidèle par lapidation. C'est que l'Afrique, en vendant ses fils, s'est révélée infidèle à ses valeurs de solidarité et de spiritualisme (par opposition au matérialisme) et a subi le contrecoup du viol de ses terres comme pareil châtiment.

Le poète ivoirien emploie aussi couramment la périphrase dans un effet de poétisation de la débauche sexuelle comme dans cet extrait :

Ce frisson de désir

Bise ou brise... caresse

Ombre d'éther sur ton corps qui se souvient

La valse des libellules en rut

Ô ! kaléidoscope féerique

Et le chant du passant : trois chocs à la porte du mont !

Regarde

Le temps s'émeut

Et ses fébriles adieux ramènent le passant d'un soir de lune

(Zaourou 2009, 61)

L'énonciateur choisit de nommer par l'expression « La valse des libellules en rut » le défilé avide et concupiscent des amants de la dénommée Swilia. De même le syntagme « le passant d'un soir de lune » désignera l'amant 
occasionnel de cette dernière, celui qui en cache toujours un autre, chaque soir, l'un après l'autre. Le singulier associé au terme a donc valeur de généralité exprimant une quantification de l'infini tant sont innombrables les amants en question. Enfin, « la porte du mont » désignera, certes par périphrase mais aussi par le truchement de la réticence euphémique et synecdochique de l'expression « mont de vénus », l'organe sexuel féminin comme objet fondamental de désirabilité.

\subsection{L'hyperbole}

L'hyperbole est une figure qui consiste en l'exagération d'un fait ou d'une réalité. Zadi Zaourou en fait un usage quasi systématique surtout dans le poème intitulé « La chute ». Ainsi dans cet extrait où le poète associe la multitude des amants à une meute :

Une meute de mécréants a franchi sa porte sculptée

Aïessa exultant cœur et sexe au vent

(Zaourou 2009, 54)

Ou encore dans cet autre extrait où persiste l'idée de multitude pour désigner le nombre infini des amants de la bien-aimée du poète :

La femme que j'aimais n'a plus de lèvres

Mille pieuvres en rut les ont anémiées

(53)

Ici l'hyperbole du nombre (mille) se dédouble d'un glissement de l'idée de multiplicité liée au sème de tentacule poly-brachial qui définit la pieuvre et que le poète fait l'égal des amants scélérats de l'Afrique personnifiée en le personnage d'Aïessa.

La femme que j'aimais n'a plus de seins

Toutes les mains du monde s'y sont essayées

(53)

Cet extrait achève ce poème qui est tout entier construit sur une représentation hyperbolique de la prostitution. Avec le syntagme «toute les mains du monde », le poète accentue la multiplicité des individus, métaphoriques des grandes nations dont les seuls avis font autorité pour l'ensemble des pays du monde, et qui sont coupables aux yeux de l'énonciateur d'abuser de l'Afrique sous le prétexte fallacieux d'échanges économiques équitables. 


\section{La topique stylématique de la figuration microstructurale de la prostitution}

\subsection{La métaphore}

La métaphore est le trope par excellence qui consiste à établir une comparaison entre un thème et un phore sans terme explicite de comparaison. Elle permet en poésie d'induire des glissements, transferts et modifications sémantiques qui tout en préservant la qualité ontologique de chacun de ses constituants, enrichit le langage non seulement esthétiquement mais aussi sémantiquement. On le voit par exemple dans cet extrait du recueil du poète ivoirien :

Ils ont violé l'inviolable et accompli l'impensé

Et tu ruisselais de plaisir femme cœur et sexe au vent

Que n'as-tu préservé la vallée du crime Aïessa...

Oui le sanctuaire

Pour te consacrer à jamais médium aux sept mystères ?

Que n'es-tu demeurée aux banquets des Immortels?

(Zaourou 2009, 54-5)

La jeune amante du poète est décrite comme une femme perverse qui bien qu'ayant été violée, en a pris du plaisir en s'offrant à tout venant « sexe au vent». Pour le poète qui regrette cet incident, le sexe de sa bien-aimée est désormais et métaphoriquement «la vallée du crime » alors qu'il fut jadis représenté sous le phore du «sanctuaire ». Mais la réelle transposition sémantique opérée par le truchement de la métaphore concerne le parallèle marial entre la vierge aux sept douleurs et ce " médium aux sept mystères » dont parle l'énonciateur. En effet, selon l'historiographie chrétienne, Marie affichant une grossesse alors qu'elle était fiancée à Joseph sans qu'il n'y ait eu entre eux le moindre contact susceptible de provoquer une fécondation, passait ainsi pour une femme aux mœurs légères. Joseph, le fiancé jugé cocu, était alors bien tenté de se passer de l'épouser jusqu'à ce que durant l'Annonciation (Mt 1:16-25), l'ange l'en dissuade définitivement. La suite de l'histoire étant qu'elle eut, par la suite, plusieurs enfants de Joseph paraît aussi incompatible avec sa dénomination de «vierge éternelle » (Duquesne, Houziaux 2006). Cette image de la mère de Jésus, anathème aux yeux de l'Église, est pourtant revendiquée historiquement au point où l'expression « Marie-couchetoi-là » désigne une prostituée ou à tout le moins une femme qui offre sans difficulté ses faveurs à de nombreux partenaires. La métaphore joue ici sur ce double encodage et désigne en réalité, par le truchement des ambiguïtés d'Aïessa, une Afrique coupable de prostitution, d'infidélité à 
ses valeurs et à son identité mais qui est, comme Marie dans la tradition catholique, promise à la félicité des « banquets des Immortels ».

Et Césaire de traduire en ces vers la construction de l'image du nègre prostitué par la métaphore de la sexualisation de l'esclavage :

autour des rocking-chairs méditant la volupté des rigoises

je tourne, inapaisée pouliche

Ou bien tout simplement comme on nous aime !

Obscènes gaiement, très doudous de jazz sur leur excès d'ennui.

Je sais le tracking, le Lindy-hop et les claquettes.

Pour les bonnes bouches la sourdine de nos plaintes enrobées de

ouaoua

\section{(Césaire 1983, 35-6)}

Le « rocking-chair » est une chaise longue dotée d’un mécanisme de balancement. Le glissement qu'il en résulte envers le va et vient constitutif de la copulation se justifie non seulement par des emplois dénotatifs mais aussi connotatifs. D’un point de vue dénotatif, la « volupté » renvoie aux douceurs de la sensualité. L'adjectif « obscènes » met en lumière l'encodage vulgaire de la sexualité tandis que l'hypocoristique « doudou » constitue une appellation tendre entre partenaires érotiques et évoque un exotisme outré dans un but de séduction. ${ }^{4}$ De même il transparaît dans cet extrait un ensemble de phores à connotation érotique : les « rigoises » qui sont des fouets sont associés au phallus par l'évocation paronomastique de la rigidité ; la « pouliche » est une jeune jument souvent associée à la désirabilité, à la pétillance et à la pétulance d'une jeune femme, ou d'une femme pauvre ; quant au Jazz, il est perçu dans Le nouveau dictionnaire $d u$ Jazz comme encodage connotatif de l'acte sexuel. On sait par exemple que « les prostituées de la NouvelleOrléans sont appelées jazz-belles, en référence à la Jézabelle de la Bible... En général, il y a association de jass (ou jazz) à la danse, à la vitalité, à l'acte sexuel » (Carles, Clergeat 2011, 651). De même pour Pierrepont citant Duke Ellington, « Le mot [jazz] n'a jamais perdu la connotation qui lui venait des bordels de La Nouvelle-Orléans » $(2002,23)$.

Comme on le voit, ce passage révèle d'importants indices quant à la sexualisation de l'esclavage. Le syntagme «Ou bien tout simplement comme on nous aime !» traduit dans cette représentation de la prostitution la présence de l'esclavagiste abuseur sous l'indéfini « on ». Sous la houlette du sarcasme, le poète indique que celui-ci aime surtout à posséder des esclaves par le rudoiement de la cravache qui, à défaut de se révolter, chantent, dansent et s'amusent de leur condition d'hommes dépossédés de leurs corps pour le plaisir et le bénéfice d'autrui.

4 Cf. http://michel.balmont.free.fr/pedago/cesaire.html (2018-11-27). 
Puis je me tournais vers des paradis pour lui et les siens perdus, plus calme que la face d'une femme qui ment, et là, [...] j'entendais monter de l'autre côté du désastre, un fleuve de tourterelles et de trèfles de la savane que je porte toujours dans mes profondeurs à hauteur inverse du vingtième étage des maisons les plus insolentes et par précaution contre la force putréfiante des ambiances crépusculaires, arpentée nuit et jour d'un sacré soleil vénérien. (Césaire 1983, 7)

Le poète décrit dans ce passage un nègre en évoquant ses "paradis » perdus et par expansion la perdition même de ce dernier et des siens. Cette transposition phorique du passé glorieux de l'Afrique s'accommode de l'évocation du mythe biblique de la chute à travers la description ironique (calme) de cette Ėve stéréotypée du mensonge originel par glissement copulatoire avec le serpent dont les Écritures disent qu'il est le «père du mensonge » (Jn 8:44). S'il est juste d'interpréter le dernier syntagme verbal - « arpentée nuit et jour d'un sacré soleil vénérien » - en disant que « ce segment est un complément de phrase et non une simple expansion de mot » (Kouassi 2006, 60), l'ambivalence herméneutique qui se déploie comme caractéristique de la poétique césairienne permet aussi de voir ce segment comme complément du nom «femme », désignant ainsi par là le syntagme nominal « soleil vénérien » comme l'appendice phallique du colonialisme arpentant continuellement (nuit et jour) le sexe de la femme incarnant l'ensemble des Noirs subissant un viol collectif dans leur chair et dans leur âme. Pour Césaire, la pratique de ce viol qui sonne comme un acte de prostitution impliquant le proxénétisme de certains Noirs et des négriers, constitue une pratique incessante qui se soldera par l'apogée conséquentielle de ce mal « vénérien » que le poète désignera aussi « abâtardissement ».

\subsection{La métonymie}

Figure microstructurale de substitution, la métonymie autorise des glissements qui sont, entre autres, de l'ordre de l'effet pour la cause et de la partie pour le tout et inversement. Dans cet extrait du recueil de Zadi Zaourou, il la mobilise selon ces deux modalités précisément :

La femme que j'aimais n'a plus de mains ni de langue

Car il n'est corps plus rugueux que la pierre des champs

Que n'ait caressé la femme que j'aimais

Râpées jusqu'aux phalanges les paumes en coussinets de velours (Zaourou 2009, 53-4)

Les « mains » et la « langue » évoquées sont certes des parties renvoyant métonymiquement à l'ensemble du corps mais elles sont riches d'un imagi- 
naire que le lecteur décodera sans mal en faisant des indices représentatifs du travail et de la parole. De même l'emploi du verbe « caresser » semble la cause de la déliquescence et de la déhiscence pathologique du corps de la femme-Afrique ainsi décrit, alors qu'il n'en est que la conséquence. Conséquence figurativement encodée de cet amour à relents économiques du continent noir séduit par un étincelant mimétisme du modèle économico-social occidental, le rêve de développement «caressé »par les pays africains s'avère le résultat d'un miroitement frauduleux au bout duquel ceux-ci sont dévorés par l’ogre mondialisant.

\subsection{La répétition}

\subsubsection{La répétition générique}

La répétition est dotée d'un effet stylistique de rare portée du fait qu'elle joue aussi bien sur les sonorités que sur les rapports sémantiques de l'identique et du différent et donne lieu à une grande variété de procédés. Au sens strict, c'est-à-dire en son acception basique qui veut qu'elle consiste en la répétition d'un mot ou d'un syntagme, Césaire en fait un usage quasi compulsif comme dans cet extrait :

Et une honte, cette rue Paille, un appendice dégoûtant comme les parties honteuses du bourg qui étend à gauche et à droite, tout au long de la route coloniale, la houle grise de ses toits d'essentes. Ici il n'y a que des toits de paille que l'embrun a brunis et que le vent épile. Tout le monde la méprise la rue Paille. C'est là que la jeunesse du bourg se débauche. C'est là surtout que la mer déverse ses immondices, ses chats morts et ses chiens crevés. Car la rue débouche sur la plage, et la plage ne suffit pas à la rage écumante de la mer. (Césaire 1983, 19 ; emphases ajoutées)

Le lexème « honte » est repris dans le dérivatif « honteuses », le lexème «bourg » et les syntagmes nominaux « rue Paille », « la mer », « la plage », et le possessif «ses » sont repris par dédoublement à mesure que se déploie l'extrait, mais à quel prix ? Le premier syntagme « rue Paille » désigne la rue la plus pauvre de la Martinique au moment où Césaire écrit le Cahier. De ce fait, elle est construite de case coiffée par des toits de pailles d'où elle tire probablement son nom. Mais comment imaginer que Césaire ignore la paronomase insidieuse que le syntagme forme avec le lexème « ripaille » qui traduit dans une expression telle « faire ripaille » le fait de mener une vie de plaisir ? Pour ne pas être dupe, le lecteur devra dévoiler le ton sarcastique qui en sourd puisque la population du bourg affamée n'a d'autre choix, pour les jeunes du moins, 
que de s'adonner à la «débauche » de la prostitution pour ne pas se laisser mourir de famine. Ici la prostitution que le poète dit « honteuse » relève d'un emploi dénotatif. S'ensuit une représentation connotative de celle-ci car le poète, en dressant le portrait de cet endroit de la rue Paille, la définit comme représentant « les parties honteuses du bourg ». Il en résulte une personnification métonymique des " parties honteuses du bourg » pour les parties intimes (objet du péché) recouvertes après la chute des premiers hommes. Cet encodage tient lieu d'indicateur de lecture et justifie sémantiquement les assauts répétés et continuels de la mer comme constitutifs de la métaphore du ressac pour le viol des flibustiers. On le remarque dans l'extrait suivant notamment :

$\mathrm{Au}$ bout du petit matin, ce plus essentiel pays restitué à ma gourmandise, non de diffuse tendresse, mais la tourmentée concentration sensuelle du gras téton des mornes avec l'accidentel palmier comme son germe durci, la jouissance saccadée des torrents et depuis Trinité jusqu'à Grand-Rivière, la grand'lèche hystérique de la mer. (Césaire 1983, 14)

La répétition sert ainsi à construire une double sémantique de la prostitution c'est-à-dire dénotative et symbolique. Elle se déploie plus spécifiquement cependant à travers l'anaphore et la polysyndète.

\subsubsection{L'anaphore}

L'anaphore est la reprise identique d'un même terme en début de phrase, de segment ou de vers. Soit l'extrait suivant du poète ivoirien :

L’amour a chaussé ses béquilles

L'amour s'est éborgné

L'amour est bossu qui tente

Impénitent repris des justices divines

De noyer ses soifs multiples dans l'onde si pure que tu crées

(Zaourou 2009, 62)

La triple répétition initiale du syntagme « L'amour » est associée à une gradation descendante si l'on en croit les modalités verbales et sémantiques de l'action grammaticale. Le premier vers présente un sujet actif et un objet extérieur à celui-ci, les béquilles représentées comme un accessoire du corps personnifié de l'amour. Le second vers présente par pronominalisation un sujet actif et un objet passif constitutif du sujet, c'est-à-dire l'œil de l'amour personnifié crevé dans un accès d'autodestruction. Le troisième vers enfin montre l'amour non plus en tant que sujet mais en tant qu'objet passif, subissant l'infirmité de la protubérance dorsale. On perçoit 
donc la régression statutaire de l'amour comme un indice de son indignité croissante ; celle qui justifie au vers suivant le courroux divin du fait de la multiplicité des partenaires de la femme infidèle (ses soifs multiples).

Avec le chantre de la négritude, l'anaphore est d'un usage tout aussi riche sur le plan de la connotation de la débauche :

terre grand sexe levé vers le soleil

terre grand délire de la mentule de Dieu

terre sauvage montée des resserres de la mer avec dans la bouche une touffe de cécropies [...] Il me suffirait d'une gorgée de ton lait jiculi pour qu'en toi je découvre toujours à même distance de mirage - mille fois plus natale et dorée d'un soleil que n'entame nul prisme - la terre où tout est libre et fraternel, ma terre. (Césaire 1983, 21-2)

L'anaphore sert ici de prétexte à une accumulation expansive au phore de «terre » (Kouabenan-Kossonou 2017, 212). Alors que le poète présentait le bourg sous les traits du sexe féminin, ce passage évoque une masculinisation de la Martinique, sa terre, à travers le genre évocateur des syntagmes « grand sexe » et « grand délire » qui lui servent d'expansions. Mais alors que les deux premiers vers en font l'équivalent de la virilité de Dieu, le troisième présente le référent du lexème « terre » sous des traits féminins à en croire l'accord de l'épithète "montée ». Il en résulte un questionnement lexicographique dont la résolution aboutit à l'acception suivante du verbe " monter » fournie par le CNRTL : « Monter avec (un homme, un client) ou, absol., monter. Se rendre dans une chambre pour y faire l'amour, généralement dans les conditions de la prostitution $» .^{5} \mathrm{On}$ peut en déduire que la mer dans ce contexte mélioratif $n^{\prime}$ est pas suspendue (montée) au dessus de la Martinique mais qu'elle polarise les sèmes d'un accouplement à connotation économique car la resserre est un grenier, un garde manger dont la disposition copulatoire en rajoute à la dimension rémunératoire de l'union revendiquée par l'énonciateur entre la mer et sa terre. On observe ainsi une inversion du schème de la représentation de la prostitution telle qu'elle se déploie dans le Cahier. Une sorte de symbolisation nouvelle à en croire Tricaud pour qui « hors du système de référence défini par cette parole [institutionnelle], l'impureté cesse d'être. Et cette parole institutionnelle donatrice d'un sens symbolique, peut se charger à son tour d'une signification symbolique » (Tricaud 1977, 177). C'est le cas dans cet extrait du Cahier car le phore marin ne représente plus l'indigne flibustier s'enrichissant de la misère des peuples noirs mais un partenaire dont la présence est balsamique. D'ailleurs la suite de cet

5 Cf. http://www.cnrtl.fr/definition/monter (2018-11-27). 
extrait notifiant la « gorgée » du « lait jiculi » de la terre-mentule n'évoquet-elle pas paronomastiquement l'ejaculari étymologique de l'éjaculat?

\subsubsection{La polysyndète}

La polysyndète est une figure microstructurale de construction. « Elle consiste en l'usage systématiquement abondant d'outils de liaison, explicitement marquée, entre les groupes, notamment en ce qui concerne les coordinations »(Molinié 1992, 275). On en retrouve ci-dessous un extrait illustratif :

Une détresse cette plage elle aussi, avec son tas d'ordures pourrissant, ses croupes furtives qui se soulagent, et le sable est noir, funèbre, on n'a jamais vu un sable si noir, et l'écume glisse dessus en glapissant, et la mer la frappe à grands coups de boxe, ou plutôt la mer est un gros chien qui lèche et mord la plage aux jarrets, et à force de la mordre elle finira par la dévorer, bien sûr, la plage et la rue Paille avec. (Césaire 1983, 19-20)

Cet extrait compte six occurrences de la coordination copulative « et ». Il est construit par empilement de segments phrastiques d'axiologie dévalorisante. Le portrait dépeint, auquel la répétition de ce coordonnant donne un rythme plus vif, décrit la violence du ressac sur une plage insalubre comme représentation du viol dont nous avons déjà fait cas plus haut et comme en témoigne la chute de l'extrait à travers le syntagme « rue paille » précédemment analysé. Le passage suivant exploite la même représentation sémantique avec quatre occurrences du copulatif « et »:

Et je cherche pour mon pays non des cœurs de datte, mais des cœurs d'homme qui c'est pour entrer aux villes d'argent par la grand'porte trapézoïdale, qu'ils battent le sang viril, et mes yeux balayent mes kilomètres carrés de terre paternelle et je dénombre les plaies avec une sorte d'allégresse et je les entasse l'une sur l'autre comme rares espèces, et mon compte s'allonge toujours d'imprévus monnayages de la bassesse. (58)

Ici la répétition du coordonnant suggère l'idée arithmétique d'addition, voire de capitalisation dont un lexique financier (argent, entasse, compte, monnayage) attestera la portée. Le jugement axiologique dévalorisant du poète pour le travail du sexe transparaît dans les expressions « battent le sang viril » et «monnayages de la bassesse » qui constituent des indices d'échanges érotiques numéraires en rapport avec la représentation figurative principale de la prostitution dans le Cahier. Mais une autre représentation de la prostitution s'y déploie, à travers cet extrait notamment : 
Alleluia

Kyrie eleison... leison... leison,

Christe eleison... leison... leison.

Et ne sont pas seulement les bouches qui chantent, mais les mains, mais les pieds, mais les fesses, mais les sexes, et la créature toute entière qui se liquéfie en sons, voix et rythme. Arrivée au sommet de son ascension, la joie crève comme un nuage. Les chants ne s'arrêtent pas, mais ils roulent maintenant inquiets et lourds par les vallées de la peur, les tunnels de l'angoisse et les feux de l'enfer. (16)

Dans ces versets, la double reprise de la copulative « et », de même que la quadruple occurrence de l'adversative «mais », crée un effet de chevauchement d'où sourd l'idée d'un entêtement des personnages évoqués dans une adoration abrutissante. Les Noirs dont il est ici question, sont décrits adorant le Christ au détriment de leurs divinités authentiques. L'emploi d'un lexique à connotation érotique («fesses », « sexes ») en contexte d'adoration divine, renvoie à une acception biblique de la prostitution envisageant l'orientation vers d'autres dieux comme acte de débauche. Cela se perçoit particulièrement à travers le livre d'Osée en son douzième verset du quatrième chapitre «Mon peuple consulte son bois, Et c'est son bâton qui lui parle ; Car l'esprit de prostitution égare, Et ils se prostituent loin de leur Dieu ». La référence aux écritures bibliques configure le propos de Césaire comme un sarcasme car il emprunte au christianisme une acception de la prostitution qui consiste en l'adoration de dieux étrangers en vue de condamner l'adoption par les Noirs d'une religion importée et de surcroit introduite par l'esclavagiste même. On comprend dès lors pourquoi les chants d'adoration décrits par le poète finissent par virer au cauchemar de l'« angoisse » infernale.

\subsection{L'épitrochasme}

Selon Molinié, l'épitrochasme est un procédé figural de l'ordre du microstructural. «Il consiste en une suite de mots brefs, coordonnés par juxtaposition, formant une accumulation d'autant plus saisissante que chaque mot est en soi fortement significatif. L'effet de série peut faire ressentir comme brefs des termes qui, dans un autre environnement matériel, ne produirait nullement la même réception » (Molinié 1992, 141). Cet extrait du Cahier l'illustre :

Au bout du petit matin, l'échouage hétéroclite, les puanteurs exacerbées de la corruption, les sodomies monstrueuses de l'hostie et du victimaire, les coltis infranchissables du préjugé et de la sottise, les prostitutions, les hypocrisies, les lubricités, les trahisons, les mensonges, les faux, les 
concussions... l'essoufflement des lâchetés insuffisantes, l'enthousiasme sans ahan aux poussis surnuméraires, les avidités, les hystéries, les perversions, les arlequinades de la misère, les estropiements, les prurits, les urticaires, les hamacs tièdes de la dégénérescence (Césaire 1983, 12)

Le poète met ici en œuvre une très longue énumération associant des syntagmes et des mots brefs qui procèdent de cette figure au sens propre. Une vingtaine de syntagmes se décline par le truchement de virgules coordinatives et amplifie à l'excès le rythme de cette phase. L'énumération qui se dédouble d'un effet d'accumulation suppose un tel écœurement de l'énonciateur que la suspensive médiane semble ouvrir à une infinité de possibles interprétatifs en vue d'accroître l'intensité de la misère décrite. On remarquera d'ailleurs la concaténation de la suspensive au lexème « concussions...». Ce dernier étant lui-même prosonomasique du motthème "prostitutions », l'énonciateur les rapproche sémantiquement et confirme, par le fait de leur coprésence phrastique, la construction d'une représentation polysémique de la prostitution. Par ricochet, l'usage du pluriel, associé autant au lexème "prostitutions » qu'à l'ensemble des amoralités décrites, induit une pluralité des modalités et acceptions du phénomène de la prostitution que le poète fait ici synonyme de tous les vices nommés. Ce passage est donc construit comme une « accumulation de substantifs en phrases nominales prédicatives » (Kouassi 2006, 101) censée décrire le contact entre les négriers et les côtes africaines depuis « les échouages » des premiers jusqu'à la " dégénérescence » des Noirs qui en a été conséquente. Ainsi, ce passage se donne à lire comme une succession d'actes ayant effet de condensé narratif au sujet de l'esclavage et de la colonisation qui en a découlé sous la représentation générale de la prostitution.

\section{Conclusion}

« La prostitution dérange, inquiète, choque ou fascine, (mais) ne laisse jamais indifférent »(Gil 2003, 112). Cette assertion est davantage vraie en littérature où la poétisation de la prostitution couve une symbolique que de nombreux mythes et imaginaires sociaux encodent. Au cœur de cette représentation archétypale des sexes, la question du pouvoir et de la domination masculine couve. Césaire en déduit par exemple le parallèle de l'esclavage et de la colonisation comme viol déguisé, car les populations noires sont souvent et l'ont été déjà jadis, complices de la pénétration des Blancs sur les terres africaines ; il s'agit bien du topos de l'image du dirigeant politique nègre proxénète offrant son pays à la libido économique insatiable du colon. D'ailleurs pour Césaire la prostitution des Noirs n'est que la conséquence de l'amoralité blanche ayant conquis par 
contagion la pudeur nègre. Avec Zadi Zaourou, la prostituée n'est point toujours négativement perçue. C'est l'amante aimée, sacrifiant son corps par passion, pour que les hommes s'élèvent à la dimension symbolique de Dieu à travers la performance phallique. Mais c'est aussi la muse du poète qui, en commerçant avec lui, l'introduit dans les dimensions éthérées de l'immatériel et de l'inaccessible. C'est enfin la poésie, qui s'offre à quiconque sait lui payer le prix de sa liberté et de sa raison pour être soulagé de ses blessures existentielles. Cette étude a souhaité mettre en évidence, par une analyse formelle des procédés figuratifs convoqués, les modalités expressives qui concourent à poser la prostitution en tant que référent discursif portant, au-delà de la tensivité phorique marine et mythologique, un univers doxique où le mystère religieux et la métaphysique du monde sensible sont solidaires de la construction du sens social. De ce fait aussi bien dans le texte de Césaire que dans celui de Zaourou, s'entrecroisent les modalités d'un dire nivelé en strates axiologiques pour repenser la domination, d'une part en ce qui concerne les rapports entre hommes et femmes, et d'autre part pour ce qui est de l'ontologie même des rapports interpersonnels.

\section{Bibliographie}

\section{Corpus}

Césaire, Aimé [Bordas 1947] (1983). Cahier d'un retour au pays natal. Paris : Présence Africaine.

Zaourou, Zadi Bottey (2009). À Califourchon sur le dos d'un nuage. Abidjan : L'Harmattan.

\section{Ouvrages cités}

Bozon, Michel (2002). Sociologie de la sexualité. Paris : Nathan. Bonhomme, Marc (1998). Les figures-clés du discours. Paris : Seuil. Borrillo, Daniel (2002). « La liberté de se prostituer » [online]. Libération, 5 juillet. URL https://www.liberation.fr/tribune/2002/07/05/la-liberte-de-se-prostituer_409197 (2018-11-27).

Bourdieu, Pierre (1990). "La domination masculine ». Actes de la recherche en sciences sociales. Masculin/féminin, 84(2), 2-31.

Brochier, Christophe (2005). « Le travail des prostituées à Rio de Janeiro ». Revue Française de Sociologie, 46(1), 75-113.

Carles, Philippe ; Clergeat, André ; Jean-Louis Comolli (éds.) (2011). Le nouveau dictionnaire du Jazz. Paris : Robert Laffont.

Duquesne, Jacques ; Houziaux, Alain (2006). La Vierge Marie : histoire et ambiguité d'un culte. Paris : Éditions de l'Atelier. 
Fromilhague, Catherine; Sancier-Château, Anne (1999). Analyses stylistiques, Formes et genres. Paris : Dunod.

Gil, Françoise (2003). « De la prostitution... ». Gradhiva, 33, 111-17.

Girard Marc (1991). Les symboles dans la Bible : Essai de théologie biblique enracinée dans l'expérience humaine universelle, vol. 1. Montréal : Les Éditions Fides.

Kerbrat-Orecchioni, Catherine (1986). L'Implicite. Paris : Armand Colin. Kouabenan-Kossonou François (2017). Stylistique et poétique, pour une lecture impliquée de la poésie africaine. Paris : L'Harmattan.

Kouassi, Germain (2006). La Poésie de Césaire par la langue et le style : l'exemple du "Cahier d'un retrour au pays natal". Paris : Publibook.

Molinié, Georges (1992). Dictionnaire de rhétorique. Paris : Librairie Générale Française.

Molinié, Georges [1986] (2011). Éléments de stylistique française. Paris : PUF.

Pheterson Gail (2001) Le prisme de la prostitution. Trad. de l'anglais par Nicole-Claude Mathieu. Paris : L'Harmattan.

Pierrepont, Alexandre (2002). Le champ jazzistique. Marseille : Éditions Parenthèses.

Tabet, Paola (2005). La grande arnaque : sexualité des femmes et échange économico-sexuel. Paris : L'Harmattan.

Tricaud, François (1977). L'accusation. Recherche sur les figures de l'agression éthique. Paris : Dalloz. 


\title{
De la dépossession à l'expérience de la possession dans Meursault, contre-enquête de Kamel Daoud
}

\author{
Maha Badr \\ (Université Libanaise, Liban)
}

\begin{abstract}
There is a clear correlation between the text of L'Étranger of Camus and Meursault, contreenquête of Kamel Daoud. Daoud, living in a previously colonised minority context, manipulates Camus' memorised coded speech to make himself heard. Is it a confrontation or a duplication of speech? The writing attempts to find points of instability and mobility likely to ruin the 'temple' of a monumental literary building. Emphasis will be placed on this gesture of appropriation and the polyphony of the text sited between recovery and transgression. This play of intertextuality creates a discursiveness that destabilizes the reader. The reading follows a retroactive investigation that goes beyond an experience of negativity by traversing a path between myth and fantasy that visualises a peaceful relationship between East and West.
\end{abstract}

Sommaire 1 Intertextualité : entre reprise et transgression. - 2 Entre identité et identification. - 3 De la négation. - 4 Écrire dans la langue de l'autre.

Keywords Algeria. Francophone literature. Intertextuality. Trace. Identity. History. Mixed languages.

\section{Intertextualité : entre reprise et transgression}

À la réception du titre Meursault, contre-enquête de Kamel Daoud, le lecteur averti se trouve confronté à un rapport de filiation, de continuité, de rebondissement et de réappropriation d'un 'produit littéraire' qu'est L'Étranger de Camus. Ancré dans le territoire algérien, le récit de Daoud tente le défi de donner suite à celui de Camus en redonnant un nom à l'Arabe anonyme tué par un français au bord d'une plage. Il revisite le texte matrice : des scènes se répondent comme celles du crime et de l'arrestation; le destin de Haroun et de Meursault s'entrecroisent dans un jeu de miroir. Mais si les échos se répercutent d'un livre à un autre, ils se présentent avec des changements comme pour explorer d'autres angles de l'œuvre camusienne. C'est ainsi que le vecteur de la focalisation change : si Camus adopte, dans son roman le point de vue d'un français qui tue un Arabe au sein de la période coloniale, Daoud, lui, en faisant référence à la période postcoloniale, entreprend le point de vue de l'algérien Haroun - frère de l'Arabe abattu par Meursault - qui finit par assassiner un français. Et si le narrateur-personnage, dans Meursault, contre-en- 
quête, ancre son récit en terre algérienne, il rattache son histoire aux contextes sociologique, religieux et politique actuels pour penser l'Algérie d'aujourd'hui et y poser la condition de l'existence. C'est ainsi que Daoud réécrit L'Étranger qui est, a priori, un texte connu ou mémorisé ; il le transforme en espace brouillé taxé d'interrogations et d'ambiguïtés propres à rendre compte des incertitudes et des inquiétudes d'une époque hantée par l'imminence de la guerre, de l'injustice et de la violence. Ainsi face au texte de Camus, l'écriture se voit prise dans deux voies contradictoires : c'est tantôt l'adhésion, tantôt une distanciation qui suppose transformation ou réécriture de l'histoire «dans la même langue, mais de gauche à droite », non pour atteindre « la justice des tribunaux, mais celle des équilibres » (16). Au sein de Meursault, contre-enquête (Daoud 2014), le texte de Camus se voit éclaté ou disséminé. En effet, des segments camusiens mis en italique ${ }^{1}$ ou entre guillemets ${ }^{2}$ se trouvent juxtaposés, insérés ou collés au texte de Daoud transgressant parfois la linéarité narrative d'une 'nouvelle' fiction. Mais il s'agit surtout de les pasticher ou de les commenter pour faire coexister deux voix différentes. Dans cette perspective, le narrateur dans une relation métatextuelle fait référence à l'œuvre de Camus ; 3 il y a un rapport d'évaluation ou de critique comme si l'écriture ne voulait pas rester immobilisée au sein du périmètre camusien tant au niveau de l'action, de l'histoire, des personnages, de la philosophie qu'à celui du style : des variantes ${ }^{4}$ se laissent y percevoir.

Daoud, vivant dans un contexte minoritaire auparavant colonisé, manipule le discours mémorisé, codé de Camus pour se faire entendre. S’agitil d'une confrontation ou d'un dédoublement du discours ? La mémoire littéraire se trouve-t-elle ainsi honorée ou outragée ? Il ne s'agit pas, dans ce présent article, de débrouiller cet écheveau intertextuel, mais tenter d'interroger cette œuvre qui est prompte à entrecroiser les cultures, les langues pour en déconstruire les présupposés idéologiques, religieux et parfois institutionnels. Dans une interview, Daoud semble en donner la réponse :

1 "C'est sûr, il y aura beaucoup de spectateurs le jour de mon exécution et ils m'accueilleront avec des cris de haine » (152; emphase dans l'original).

2 Exemple : « Donc l'histoire de ce meurtre ne commence pas avec la fameuse phrase, 'Aujourd'hui, maman est morte' » (20) ; « J'arrivai enfin à la dernière phrase du livre : '[...] il me restait à souhaiter qu'il y ait beaucoup de spectateurs le jour de mon exécution et qu'ils m'accueillent avec des cris de haine' » (141).

3 «C'est l'un des livres les plus lus au monde » (74); «A sa sortie, l'assassin écrit un livre qui devient célèbre où il raconte comment il a tenu tête à son Dieu, à un prêtre et à l'absurde »(75). Le narrateur manifeste parfois une critique acerbe vis à vis de L'Étranger le qualifiant par exemple d'une « terrible arnaque » (74) ou d'un « mensonge cousu du fil blanc » (63).

4 Il suffit de comparer quelques épisodes comme l'incipit, l'arrestation, la demande en mariage pour se rendre compte de cette 'transformation' ou cette transposition entre les deux textes. 
HD Pour un jeune écrivain tel que vous, se mettre dans la peau d'un Camus, à contre-courant, est-ce une récupération de l'auteur vers la littérature algérienne ? Une réappropriation d'un monde perdu ?

Kamel Daoud Je ne me mets pas dans la peau de Camus. Le texte de Camus, je m'en sers. L'essentiel pour moi est de poser mes propres questions. Je mets en scène ma propre vision du salut et du sens. Camus est là, c'est une belle esthétique. Il est incontournable dans la généalogie de l'écrivain algérien, mais je ne suis pas un camusien. Je n'aime pas cette tendance à construire un temple autour de Camus. Lorsque j'ai commencé à écrire " Meursault, contre-enquête », mon éditeur m'avait proposé d'écrire sur Camus. J'ai refusé. Je veux garder cette lecture moyenne, fausse, approximative qu'on a sur Camus. Je ne veux pas faire le procès de Camus. Il est mort, mais moi, je suis vivant, et c'est donc à moi de trouver du sens avant de mourir. Je n'ai pas son génie, mais je ne veux pas vivre son texte avec infériorité. J'adore cette citation ancienne : "Nous sommes des nains juchés sur les épaules de géants ». J'ai ma façon d'écrire, je la défends et je l'impose. (Seklaoui, Déchir 2014)

Comment ne pas percevoir dans ces propos un nouveau dispositif légendaire s'appropriant les faits pour «troubler », ${ }^{5}$ faire "trembler » un préjugé, une œuvre ou un héritage ? L'écriture ne tenterait-elle pas de trouver des points d'instabilité et de mouvance susceptibles de ruiner « le temple » d'une édification monumentale littéraire ? S'il avoue qu'il refuse d'habiter la pensée ou de se mettre dans la peau de Camus, Daoud semble aussi s'en prendre à la « figure » religieuse de l'écrivain et maintenir sa propre vision des faits. Il tente de laisser faire entendre la « voix » de Camus par une contre voix qui ne cesse de dire sa perception propre.

Les livres et la langue de ton héros me donnèrent progressivement la possibilité de nommer autrement les choses et d'ordonner le monde avec mes propres mots. (47)

En effet, des intertextes camusiens subsistent : le texte est ancré à Oran, cadre spatial de La Peste dans un mode narratif qui n'est pas sans rappeler celui de La Chute. Mais en instillant le désordre dans l'image mythique de L'Etranger, l'écriture laisse subsister la confrontation rassurante d'un lecteur face à un objet intellectuel. De ce fait, l'écriture ne cible pas l'impression ou l'admiration auprès du récepteur; elle n'entreprend pas un mouvement giratoire centré uniquement sur l'imitation servile en forme

5 'Troubler' serait pris dans le sens que nous donne le Littré : « Action par laquelle on inquiète quelqu'un dans la jouissance de sa propriété ». 
d'hommage à L'Étranger de Camus. Dépouillé de son caractère sacré voire « classique », le livre ne serait pas « gardé bien sagement « « comme un soleil dans une boîte » (135). Le narrateur semble puiser dans l'œuvre de Camus ce qui est nécessaire au plaisir de l'écriture et à la satisfaction de la possession ou de l'appropriation. Le pastiche, sorte d'écriture oblique, serait déployé, à cet égard, comme une sorte d'appropriation de la langue et du texte pour raconter une propre version des faits et représenter son monde en affirmant une identité individuelle qui déstabilise une mémoire collective littéraire. L'œuvre de Camus serait pensée comme mythe ou structure sur laquelle repose celle de Daoud.

Le meurtrier est devenu célèbre et son histoire est trop bien écrite pour que j'aie l'idée de l'imiter. (12)

Mais l'entreprise d'écriture se limite-t-elle à l'imitation ou à une forme d'hommage ? Dans Meursault, contre-enquête pullulent les inversions et les antithèses par rapport au texte de Camus. Il suffit de citer l'incipit et la clausule pour se rendre compte de cet acte de défiguration ou de distorsion, du texte initial dans une pratique d'actualisation.

Aujourd'hui M'ma est morte / Aujourd'hui, M'ma est encore vivante. (Camus 1972, 9 ; Daoud 2014, 11)

Il me restait à souhaiter qu'il y ait beaucoup de spectateurs le jour de mon exécution et qu'ils m'accueillent avec des cris de haine / Je voudrais, moi aussi, qu'ils soient nombreux, mes spectateurs, et que leur haine soit sauvage. (Daoud 2014, 153 ; Camus 1972, 188)

Intercalée entre deux virgules, l'expression « moi aussi », pourrait constituer un dialogue avec le texte source ; ce serait un moyen d'imposer une voix, d'affirmer une singularité qui s'exprime par le biais d'un « je » à l'encontre de la formulation impersonnelle du personnage de Camus. Le texte de Daoud décèle, à cet égard, l'existence d'une tension vers les limites du connu, du familier, faisant ressortir, parallèlement dans un détournement, une forme d'altérité. Lécriture se situe, d'une manière subtile, dans une dialectique/un dialogue entre le même et l'autre, entre la différence et la répétition. L'appropriation serait considérée comme un processus d'usage et de recyclage qui réactualise, qui re-sémantise, redonne du temps à ce qui est suranné ou à ce qui est taxé de 'classique'. Dans ce processus de 'renversement', Daoud semble confirmer la théorie d'Umberto Eco sur l'œuvre ouverte, œuvre qui ne serait achevée que dans son épuisement absolu. Et Valéry de confirmer cette idée du travail toujours en progrès de la production littéraire : « L'œuvre est pour moi l'objet possible d'un travail indéfini », dit-il. 
Le lecteur se voit ainsi mené au point d'intersection de la lecture et de l'interprétation. Il serait devant « un texte double, deux mains, deux regards, deux écoutes, ensembles à la fois et séparément » (Derrida 1972). Il aurait une attitude réflexive et critique vis-à-vis d'un texte qui, intégrant un avant-texte (intertexte/transtexte/le texte des autres), joue sur les limites de la lisibilité.

S'agit-il pour Daoud d'interpréter les avant-textes en introduisant des gloses?

Les textes s'écartent ou semblent se répondre plutôt que se répéter. Entre « ressembler » et « créer » (Poulet 1967, 20), sous le halo transparent de la fiction, le texte de Daoud dessine le mouvement d'un projet d'écriture qui est «critique en acte » (Genette 1982) ou « une variété de lecture active » (Proust 1992,108) qui est une prise de conscience d'autrui et de compréhension.

Réécrire n'est pas seulement changer un texte en un autre, c'est les échanger, comme il se dit de la correspondance. Echange de textes, échanges entre deux sujets d'écriture, réécrire suggère le schéma dialogique d'une socialité. L'autre du texte, c'est aussi le texte de l'autre. (Domino 1987, 3)

Des énoncés ou fragments mémorisés de L'Etranger sont insérés (disséminés) dans un nouveau contexte allié au temps présent pour composer une "vocalisation individuelle qui devient la trame même du sujet » (Macé 2015, 4). Ce geste d'appropriation met en œuvre une polyphonie énonciative et se montre surtout comme une « réinterprétation de l'appareil de l'énonciation littéraire » (Macé 2015, 4). Surtout, dans cet acte de réécriture qui nous « apprend quelque chose du phénomène littéraire » (Domino 1986, 10), le texte dépasse la hantise d'une fonction autotélique. ${ }^{6}$ L'appel à l'intertextualité (non seulement le texte de Camus, le Coran, Michel Tournier, etc.) agit contre la 'tyrannie' d'un sens ${ }^{7}$ ou d'une vision unique traduite, d'une manière métaphorique, par l'image du lieu circonscrit ou fermé du bar dans lequel débute le récit de Daoud. L'Etranger devenu mythe joue la fonction d'un intertexte, d'un corps « étrange » jamais inerte. L'enjeu de cette écriture qui glose ou agit sur le texte littéraire serait de célébrer la rencontre entre livre et « lecteur professionnel »,

6 S'agit-il pour Daoud d'opter pour une littérature restreinte, dans le sens où le texte n'explique pas sa genèse dans une approche structuraliste

7 Ceci peut rejoindre un concept barthesien hérité de Nietzche pour qui « donner du sens est une force ». Cette enquête qui, à l'instar du sens, devient une question de vie et de mort.

8 Le terme est celui de Bourdieu : « la relation entre le lecteur et l'œuvre, entre le critique et l'œuvre, n'est pas une relation fascinée. Elle se dissocie d'ailleurs en de multiples niveaux de 
dans une ouverture à l'altérité. Ce jeu d'intertextualité, entre reprise et transgression, entreprend une langue qui devient une direction et fait appel à une discursivité pour délivrer une force communicative traduite d'ailleurs, d'une manière pragmatique, par l'appel direct à un «tu » et l'emploi des questions rhétoriques qui déstabilisent le lecteur et le poussent à interpréter et créer du sens. Le discours est adressé à celui qui est réduit à une impasse et ne sait pas répondre. L'autre (le lecteur) est impliqué et apparait comme le but d'un discours qui cherche à inquiéter, à entretenir le doute et à installer l'angoisse. Dans la jouissance du texte, le lecteur est gagné par l'inquiétude ; il se montre perplexe quant à son rôle : est-il vraiment inclus dans ce roman qui semble, au demeurant, un monologue ? Va-t-il prendre celui-ci en possession ? Ou bien, dans un ton ironique et peut-être sarcastique, Daoud, tel un Raymond Queneau, dépossède-t-il son récepteur de son rôle et le prive-t-il du plaisir de construire un roman «à sa façon »?

Mon histoire te convient-elle ? C'est tout ce que je peux t'offrir. C'est ma parole, à prendre ou à laisser.

[...]

Deux inconnus avec deux histoires sur une plage sans fin. Laquelle est la plus vraie ? Une question intime. A toi de trancher. (153)

Si le statut du destinataire apparaît hypothétique, il s'avère cependant nécessaire au « spectacle $»^{9}$ du texte. L'écriture ne semble pas avouer son besoin de l'autre et de ses réactions pour subsister, justifier son entreprise et garder «trace », chercher une identité et surtout redonner un nom.

\section{Entre identité et identification}

Si l'anonymat ôte à l'arabe son identité, il l'affirme, d'une manière paradoxale, pour supposer, non seulement une valeur éthique, mais aussi construire la dimension du langage. Dans cette perspective, le langage et le sens seraient pensés à partir de cet impersonnel qui devient l'origine de l'itinéraire narratif de Daoud. Il s'agit d'interroger cette dimension impersonnelle ou cette illéité dont parlait Emmanuel Lévinas et qui met en

réception : celui du lecteur mais aussi du critique. Le lecteur a ses attentes, au nom desquelles il appréciera l'œuvre. Le critique est à la fois déterminant (il a droit à la parole) et relatif (sa parole comme celle de l'œuvre, ne sera pas entendue si elle n'est pas attendue » (Riondet, Odile (2003). « L'auteur, le livre et le lecteur dans les travaux de Pierre Bourdieu ». BBF, 48(2), 87. URL http://bbf. enssib. fr/consulter/15-riondet. pdf).

9 « Je voudrais, moi aussi, qu'ils soient nombreux, mes spectateurs, et que leur haine soit sauvage »(153). 
cause la notion même d'identité. Le « il » ou le « lui » avec qui on désigne Moussa habitent le dedans ou l'identité fictionnelle du « je », sujet narratif, afin d'entretenir une fonction ou une fiction. L'énigme de la recherche devient une fabulation, une histoire.

Je suis le frère de Moussa ou le frère de personne. Juste un mythomane que tu as rencontré pour remplir tes cahiers... (153)

Lidentité d’un «je » se sait désormais fictive ; l'expression restrictive « Juste un mythomane » situe l'énoncé entre la fiction, l’hypothétique, le doute et l'altérité insaisissable d'un autre en mettant en doute l'identité de tout « moi ». Cette permanence inquiétante de l'écriture ouvre la possibilité d'un vide, d'un rien ou d'une fêlure de sens; et le mouvement de l'enquête serait controversé ou inabouti : il serait une « contre-enquête », une résistance au mouvement de la recherche de la vérité qui, dans un projet herméneutique, veut déranger voire « troubler » un ordre préexistant.

Moi, je connais ce livre par cœur, je peux te le réciter en entier comme le Coran. Cette histoire, c'est un cadavre qui l'a écrite, pas un écrivain. On le sait à sa façon de souffrir du soleil et de l'éblouissement des couleurs et de n'avoir un avis sur rien sinon le soleil, la mer et les pierres d'autrefois. (14-15)

La fiction s'inscrit dans un projet d'une façon de dire qui va à l'encontre d'une idéologie fragile ou d'une vérité absolue. «Hors des livres qui racontent, point de salut, que des bulles de savon qui éclatent » (34), est dit-il dans le récit. Comme moyen de secours ou de salut, la fiction serait une promesse d'une révélation au moment où, imaginer, critiquer voire penser sont vus dans une optique de révolte contre le joug religieux. Se déclarer comme «mythomane » souligne la tendance plus ou moins consciente à la fabulation et le retour à un aspect nécessaire du mythe qui permet à l'homme de donner un sens à la vie. Elle permet une maîtrise du monde et un éloge de la fabrication du sens. L'histoire «n'est pas le 'le réel' », elle est « du réel ce que l’on peut dire, et moyen d'agir sur ce réel » (Domino 1987, 9). La fiction semble permettre à l'être de prendre sa place au monde ; et le mort, par sa réincarnation, reprend une identité, un nom et un linceul.

Sans m'en rendre compte, et des années avant que j'apprenne à lire, je refusais l'absurdité de sa mort et j'avais besoin d'une histoire pour lui donner un linceul. (31)

La fabulation et le pouvoir des mots semblent creuser un lien entre l'écriture et la vie pour défendre la possibilité et le droit au roman. Elle s'incarne 
surtout par la répétition de l'épisode du crime et de la disparition de Moussa qui, non seulement se perpétue d'un chapitre à un autre, mais présente aussi des variantes; les bribes de journaux « religieusement » cachés « dans la poitrine » (130) de la mère et la thèse préparée par Meriem suggèrent la potentialité d'éclatement des différents points de vue narratifs et analytiques. Ce qui met en doute le sens univoque de l'histoire et rejoint l'aphorisme de Cioran cité en exergue. Lépisode du crime revient comme un leitmotiv et le sens de l'histoire se voit, par la suite, produit par le mouvement de l'écriture entre reprise et répétition. Cette perpétuation ne prend véritablement son sens que parce qu'elle est elle-même répétée dans l'acte criminel du narrateur qui, hanté voire obsédé par l'écho du passé, tue un français. La fabulation serait ainsi le moteur de l'histoire et non sa fuite.

Tu devines donc tout le génie qu'il a fallu pour transformer un fait divers de deux paragraphes en une tragédie décrivant la scène et la fameuse plage, grain par grain. (130)

Le mensonge et l'amplification tolérant une extension multidimensionnelle et protéiforme de l'épisode criminel assurent l'évolution et surtout la cohérence progressive du texte. La « reconstitution imaginaire du crime » (131) se décline comme un élargissement d’ordre épique. "J'ai gonflé leur volume jusqu'à en faire un cosmos » (131). Se dessine ainsi une esthétique du dépassement et non de la restriction; le récit acquiert, de ce fait, un caractère modulable, une flexibilité qui est l'expression d'une adaptation au mouvement de l'écriture en train de se faire ou d'une écriture mouvante qui ne peut se contraindre à un carcan fixé par « le génie d'un mathématicien penché sur une feuille morte » (137). La résonance polyphonique contribue à produire cet effet d'ouverture ou de suspension d'une écriture en « quête ». En ce sens, le texte acquiert un pouvoir de signification supérieur à celui d'une enquête policière. La multiplicité des récits et leur dissémination rendent possible l'élaboration d'un sens mythique de l'événement. Ce côté mythique ou fictionnel qui aura une fonction mobilisatrice dans la multiplications des niveaux pour la production du sens éloigne le texte d'un réalisme sec. Bien qu'elle donne l'impression d'arrêter la linéarité du texte, la répétition permet paradoxalement sa progression dans une dimension double ; les mémoires spatiales et temporelles du lecteur seront interpellées. A part le retour aux mythes (Thésée, Caïn, Sisyphe...) qui se trouvent dispersés dans l'épaisseur fondatrice du texte, le lecteur, grâce à une lecture active, fera foisonner la trace d'une rencontre entre le texte et l'Histoire.

A Hadjout, le paysage est le même qu'à l'époque où ton héros a accompagné le cercueil de sa prétendue mère. Rien ne semble avoir changé [...] Moi, nostalgique de l’Algérie française ? Non ! Tu n’as rien compris. (41) 
C'est justement le lecteur qui tourne en rond. Daoud semble induire le lecteur en erreur : déjà le titre inspire l'engagement du narrateur vers une voie d'investigation policière dans le but apparent de faire ressortir l'Arabe de l'anonymat et de l'ère de soupçon (selon les dires de Nathalie Sarraute) et de lui redonner une épaisseur existentielle à l'encontre de L'Etranger. Ainsi en insérant des gloses interprétatives sur le texte de Camus, Daoud semble montrer la responsabilité des écrivains qui, à l'instar des hommes politiques, agissent sur les récepteurs et véhiculent l'image d'un oriental réduit à l'anonymat, pétrifié par le regard autoritaire de l'Occident. Cette image appauvrie et négative qui caractérise la relation entre l'Orient et l'Occident est d'ailleurs démontrée par un Edward Saïd (Saïd 2005) ou confirmée d'une manière lapidaire par un Farjallah Haïk (Haïk 1946) par exemple. Dans le roman de Daoud, il y a tentative de dépasser, à la manière de Saïd, l'image restreinte ou stéréotypée qu'un occidental a de l'orient :

Arabe, je ne me suis jamais senti arabe, tu sais, c'est comme la négritude qui n'existe que par le regard du blanc. (152)

Cette attitude qui se veut subversive voire libératrice pourrait se muer en une attitude " réparatrice », conciliatrice entre ces deux mondes. La dichotomie raciale entre les arabes et les roumis semble être de mise. Le roman de Daoud se lit ainsi dans l'optique de récupération de cette sphère fondamentale de la subjectivité identitaire liée à la mémoire comme lieu de conflit réciproque $:^{10}$

Moussa est un arabe que l'on peut remplacer par mille autres de son espèce ; les français dévisageaient les quelques Arabes présents. (110)

Moussa perd toute particularité ; le nom propre (« Moussa »), marque sémiotique de la désignation, glisse dans une situation d'indétermination (un Arabe) et acquiert une valeur généralisante qui semble être liée à une mémoire acquise ou à une représentation mentale construite à partir des discours. En d'autres termes, cette déclinaison du nom propre à un nom commun semble réduire le personnage au trait qui en constitue (d'une manière apparente) l'essence et qui provient d'un lourd fardeau de la mé-

10 Souvent dans le roman, les Français sont désignés par « les Roumis » qui prennent à cet égard un sens péjoratif ; Majid El Houssi, dans une étude linguistique explique, dans une remontée à l'espace-temps, la signification du mot Roumi : « Dans sa (celle de l'Arabe) bouche, le mot roumi [...] a pris une nuance péjorative, tout en étendant son domaine pour englober tous les chrétiens quelle que soit leur origine. De plus, le mot a été identifié avec la religion de l'envahisseur de sorte que roumi $=$ chrétien européen $=$ envahisseur sont devenus synonymes dans l'esprit de l'Arabe algérien » (cité par Majid El Houssi, «Le mot roumi ». Imbroscio, Carmelina ; Minerva, Nadia ; Oppici, Patrizia (éds.), Des îles en archipel... Flottements autour du thème insulaire en hommage à Carminella Biondi. Peter Lang, 300. 
moire rattachée principalement à la figure de l'arabe dominé, anonyme et facilement substituable. Cet acte de dépossession, épreuve d'un manque, fantôme du passé réel et matérialisation illusoire de celui-ci, touche non seulement le nom, mais surtout une identité. Prenant conscience, comme l'énonce Derrida, qu' « une identité n'est jamais donnée, vécue ou atteinte, non, seul s'endure le processus interminable, indéfiniment fantasmatique, de l'identification » (Derrida 1996, 53), le narrateur entame ainsi un itinéraire obligatoire ou une enquête qui peut mener à la renaissance et au désir de se reconstruire et de s'affirmer dans le présent à l'épreuve du tourbillon de l'histoire et la mémoire du monde ; au moins, Haroun semble atteindre un objectif : attiser la colère qui l'habite en posant un problème de conscience. Il relève Moussa de l'oubli et l'inclut dans l'existence. ${ }^{11}$ Dans un mouvement 'inversé' qui suit la trace et retourne même à la naissance, l'écriture tente de combler un vide en restituant une identification ou un droit à l'identité lié au nom :

C'est important de donner un nom à un mort, autant qu'à un nouveau-né. C'est important, oui. (32)

A l'instar de Kateb Yacine et de Yasmina Khadra, Daoud semble vouloir à Camus l'écriture de L'Étranger. L'arabe tué reste inconnu et son corps baigne dans l'anonymat sur la plage ; il se trouve dépossédé de son nom propre qui peut construire son identité voire son existence. Il n'est désigné que, comme le souligne bien Daoud dans son incipit, par ce côté éphémère, accidentel, poussiéreux qui git dans « l'ère du soupçon ». Vingt-cinq occurrences au singulier ou pluriel du mot Arabe sont disséminées de part et d'autre dans l'œuvre camusienne. Ce côté dérisoire ou insignifiant, le lecteur le retrouve aussi dans La Peste où les Arabes sont littéralement effacés de la ville d'Oran. Et c'est justement pour n'avoir retrouvé ni leur corps ni leur incarnation dans les romans, que les Algériens s'y sont sentis comme des occupants forains acculés aux confins d'une écriture qui les nie radicalement. À Yasmina Khadra qui déclarait :

J'ai tout simplement voulu lui dire que l'Algérie, ce n'est pas ce type qu'on abat sur une plage parce qu'il fait chaud. J'ai voulu montrer que l'Algérien est une histoire, une épopée, une bravoure, une vaillance, une intelligence, une générosité. (Diffalah 2010)

11 «Moussa, Moussa, Moussa... j’aime parfois répéter ce prénom pour qu'il ne disparaisse pas dans les alphabets. J'insiste sur ça et je veux que tu l'écrives en gros. Un homme vient d'avoir un prénom un demi-siècle après sa mort et sa naissance » (23). 
Daoud pourrait répondre en écho :

Et encore ! Quand je repasse cette histoire dans ma tête, je suis en colère - du moins à chaque fois que j'ai assez de force pour l'être. (13)

À l'instar de Nedjma de Yasmina Khadra qui entame la quête du corps de l'arabe classé parmi «des figurants, des fantomatiques», le narrateur entreprend la mission de suggérer l'image d'un corps éprouvé qui pourrait assurer une épaisseur existentielle et constituer un simulacre relationnel servant à la sensibilisation.

C'est le français qui y joue la mort et disserte sur la façon dont il a perdu sa mère, puis comment il a perdu son corps sous le soleil, puis comment il a perdu le corps d'une amante, puis comment il est parti à l'église pour constater que son Dieu avait déserté le corps de l'homme, puis comment il a veillé le cadavre de sa mère et le sien, etc. (13-14)

Par sa dissertation sur son corps, l'assassin gagne l'empathie du lecteur au profit de l'arabe qui demeure un étranger, un « lui » avec qui le lecteur n'a pas de rapport. «Le corps de Moussa n'a jamais été retrouvé »12 (43). L'image du corps de Moussa est quasiment omise de L'Étranger. Il n'y a pas de représentation du corps éprouvé qui, comme forme de reconnaissance du sujet, suppose la rencontre de l'autre et de sa douleur, ou du pathos en soi. Tout se passe comme si l'écriture camusienne abolissait cette dynamique de l'empathie qui pourrait animer un sens de responsabilité obsessionnelle ou de culpabilité exposant le « je » à l'altérité, indispensable à la conscience de soi, selon une conception hégélienne. Par l'écriture, Daoud essaie de ressusciter un disparu. Tout se passe comme si la recherche du corps de Moussa était un appel à la prise de conscience de l'identité algérienne. L'écriture se veut ainsi la gardienne d'une trace qui non seulement présente l'intention de réparer la mort ou de résister à la disparition mais de raconter une histoire. Elle entreprend un dialogue avec un «tu », dans une démarche herméneutique qui permet la rencontre d'autrui et la suggestion d'une liaison affective entre sujet représenté et lecteur. Retrouver le corps serait comme lieu de médiation utilisant le fond commun reconnu de la souffrance humaine. En d'autres termes, il y aurait l'instauration d'une intersubjectivité et d'une 'empathie'. L'arabe devient un signe à montrer, une trace à suivre ou une énigme à résoudre comme dans une enquête policière. Et la trace, en se référant à Derrida,

12 Idée répétée aussi au début du chapitre IV : «Je te l’ai dit, le corps de Moussa ne fut jamais retrouvé » $(51)$. 
Husserl ou à Lévinas, serait marquée par le rapport à l'autre. ${ }^{13}$ Le lecteur, en tant qu'instance invitée, allégorie du regard d'autrui, peut interroger le statut de cet « arabe » mort en le transformant en sujet ayant un nom, une identité. C'est ce qui pourrait expliquer aussi, dans le roman, l'apparition de Meriem qui, pour préparer une thèse sur le sujet intitulé « L'Autre », entame une recherche de la famille de la victime, de cette trace qui « nous place dans une relation latérale, inconvertible en rectitude (ce qui est inconcevable dans l'ordre du dévoilement et de l'être) et qui répond à un passé irréversible » (Lévinas 1967, 198). Le passé symbolisé par la trace se place dans l'ordre d'une distance, synonyme de la non-identification, de l'inconnu ou de l'irrécupérable. Et c'est justement cette irréversible absence qui marque l'aboutissement d'un destin et le processus d'une œuvre « inachevée » mobilisant le négatif ou le « rien »dans la création des significations et la permanence de la mémoire :

Rien que de la routine éblouissante. Sa vie ? S'il n'avait pas tué et écrit, personne ne serait souvenu de lui, est-il dit. (73)

\section{De la négation}

Dans le texte de Daoud, remarquable est le foisonnement de la négation pour montrer le risque de la déperdition de l'histoire et de l'origine, marquer l'absence de la trace et de toute inscription scripturale du déroulé ; le manquement du passé à la conscience est récurrent. La vie de Haroun, dès son enfance, se voit, en fait, marquée par le vide et la négation ; c'est ainsi qu'il parle de son père :

J'ai appris à écrire ce nom comme on écrit une adresse, sur mes cahiers d'écolier. Un nom de famille et rien d'autre. Aucune trace de lui, [...] M'ma a toujours refusé de me décrire ses traits, son caractère, de lui donner un corps ou de me raconter le moindre souvenir. Et je n'ai pas eu d'oncles paternels ou de tribu pour jouer à en redessiner les contours. Rien. (76)

Le narrateur, lui-même, semble être tendu entre un monde dont il voulait provoquer la perte et une intériorité, produit de ce monde méprisé à savoir le côté maternel qui l'obsède et le dépouille de son identité en ne voyant en lui qu'une réincarnation du défunt, son frère. Tout se passe comme si le narrateur se livrait à un mode de constitution du sujet qui échappe

13 «Qui, l'autre ? Tu te demandes, hein. Il y a toujours un autre, mon vieux. En amour, en amitié, ou même dans un train, un autre, assis en face de vous et qui vous fixe, ou vous tourne le dos et creuse les perspectives de votre solitude » (83). 
au jeu infaillible de l'absence ou du manque. Sous l'influence de Cioran, cité en exergue au début du roman, la négation agit comme un moteur de l'écriture ; elle est associée à l'emploi presque obsessionnel, dans le texte de Daoud, du lexème « rien » qui ne semble témoigner d'aucun choix, ni d'aucune détermination. Cependant, ce «rien » ne serait pas le signe du vide ou du chaos mais le vecteur d'une attente - déçue - qui bascule le lecteur et l'écriture dans le doute voire le scepticisme. Il y aurait une corrélation entre l'étonnement et l'angoisse qui font chanceler et dériver. On n'affirme rien, on installe le doute et la dépossession d'une forme de vérité. Le roman pose des questions et des contestations dans une expérience des limites.

Tu n'y trouveras rien. J'ai déjà essayé, l'ami. Je te l'ai annoncé d'emblée, cette histoire se passe quelque part dans une tête, la mienne et la tienne et celle des gens qui te ressemblent. Dans une sorte d'au-delà. (67)

L'écriture n'explique pas, elle revient sur sa trace, elle peut rester « intransitive », comme le disait Roland Barthes. Elle semble rester à la frontière du non-dit. Le texte présente, souvent, une brutale affirmation du « rien », sans présent ni absent, dans une forme elliptique ou laconique :

A six reprises... Oui, j'y suis allé six fois, sur cette plage. Mais je n'ai jamais rien retrouvé, ni douilles ni traces de pas, ni témoins, ni sang séché sur le rocher. Rien. Pendant des années. (66)

Et un peu plus loin,

Tout s'est passé sans nous. Il n'y a pas de trace de notre deuil et de ce qu'il advint de nous par la suite. Rien de rien, l'ami ! (74)

Répété, le « rien » serait, à la manière de Maurice Blanchot (Blanchot 2002), une pensée dominée par l'impossible restauration d'un passé et la préparation d'un avenir. Daoud entraîne le lecteur dans un jeu fascinant face à ce qui n'est pas encore le néant ; la lecture se laisse abîmer dans un horizon d'attente, dans un intervalle qui ne relève « rien » de présent ou d'absent et qui oscille entre possession et dépossession. C'est que le vécu de l'attente demeure, dans le récit, un temps suspendu, arrêté qui ne voit pas la réalisation de son objet à savoir la libération et le salut loin de l'oppression religieuse, politique (et même maternelle). Le narrateur se montre, ainsi, conduit à tuer un français pour « rien » dans un absurde obsolète qui rappelle celui de L'Etranger de Camus ; la boucle est bouclée et la quête agit « contre » son intentionnalité. Le « rien » serait l'aboutissement de l'attente, dans un mouvement réflexif; il peut aussi supposer une allégorie de l'attitude des Algériens ou des Arabes qui, après 
une période d'attente, «tournent en rond », semblent ne pas agir contre l'emprise du manque ou de la dépossession.

Je voulais juste te dire qu'à l'époque, nous, les Arabes, donnions l'impression d'attendre, pas de tourner en rond comme aujourd'hui. (41)

L'écriture serait une métaphore de ce moment de latence ou d'attente lors de la colonisation et le fait de piétiner dans la régression après l'indépendance. Ainsi naît une corrélation entre la forme littéraire et la thématique de la recherche et de l'attente comme actualisation d'un passé et d'une mémoire. Le narrateur se refuse à boucher le « trou » du rien, en lui substituant des histoires fallacieuses. Si le « rien » entraine l'angoisse sur laquelle la conscience et la raison n'ont rien à dire, il provoque une tension sans cesse reconduite, une fièvre qui cause le besoin d'écrire dans une fuite salutaire ou un "don de spiritualité ». ${ }^{14}$ Dans un hors temps, il suppose une vision sans cesse en mouvement et une mobilité dans la pensée. "Que faire d'autre, dis-moi, avec mon histoire, sinon la rejouer à l'infini ? » (147), est-il dit dans le roman. Le passé, loin d'être résorbé, se donne à voir sous l'espèce du virtuel, "dans une sorte d'au-delà », qui suppose à la fois détachement et sentiment de rejet violent à l'encontre du monde et de soi. Ceci pourrait suffire à montrer le caractère relatif des représentations, loin de l'universalité d'un discours qui tente de se transformer en dogme figé ou cliché équivalent à une forme de dictature ${ }^{15}$ selon Daoud. Ainsi, la trace recherchée, loin de se résumer à un présent vivant, expose à un événement énigmatique ; elle permet une possibilité illimitée de renvoi dans un mouvement ou une sorte de renversement ${ }^{16}$ qui fait même retourner au point de départ, peut-être vers un passé immémorial. Dans cette perspective, il ne serait pas anodin de retrouver des allusions mythologiques comme celle de Moïse et de Jacob ou de citer la légende de Caïn au sein du roman, dans un retour aux origines : « D'une certaine manière, ton Caïn a tué mon frère pour... rien ! » (67) pour dire le recommencement, la répétition jusqu'à l'épuisement, « la permanence de l'histoire » dans la langue de l'autre.

14 Et le critique de continuer : « Ecrire pour sauver quelque chose ou quelqu'un, telle est la leçon des errants qui peuplent l'univers fictionnel, [...] telle est la parabole du nouveau roman de Daoud, Zabor ou les psaumes » (Belhabib 2017).

15 «Une dictature, c'est d'abord l'empire du cliché » dit Daoud (Lapaque 2017).

16 «Cette histoire devrait donc être réécrite, dans la même langue, mais de droite à gauche » (16). 


\section{4 Écrire dans la langue de l'autre}

J'ai donc appris cette langue, en partie, pour raconter cette histoire à la place de mon frère qui était l'ami du soleil. La langue française me fascinait comme une énigme au-delà de laquelle résidait la solution aux dissonances de mon monde. (129)

Adopter la langue d'apprentissage - tel le français - est évidemment l'un des gestes les plus tranchants qui puissent s'accomplir ; évitant « une concordance avec [sa] langue » (126), elle serait une entreprise à double tranchant : d'une part, elle rejoint la perspective d'un locuteur faisant le pari d'une libération possible de l'emprise étroite voire étouffante du complexe œdipien ou maternel qui l'a dépossédé de son corps et qui a pu neutraliser en lui «l'incandescence, le désir, la rêverie, l'attente, l'affolement des sens » (139). D'autre part, en apprenant et en écrivant la langue française, le narrateur essaie, dans un acte de confrontation ou de dédoublement du discours, de reprendre la parole à /de l'autre. La langue française représente pour Daoud et le narrateur ${ }^{17}$ la langue du corps, de l'imaginaire, de l'interdit, de la dissidence face à l'arabe qui 'représentait l'autorité' et qui, relié au sacré du religieux, enferme et peut empêcher un échange entre les civilisations, les croyances et les cultures. L'écriture semble cibler un territoire où loisibles sont les faits d'imaginer, de critiquer et de penser pour donner un nouveau sens au monde. Cependant, bien qu'il souligne le double poncif de l'élégance et de la clarté de « cette langue parfaite qui donne à l'air des angles de diamant » (12), le narrateur n'opte pas impérativement pour un usage académique du français. Marquée par son oralité, l'écriture brise l'ordre syntaxique et paradigmatique de la langue; désormais, celle-ci n'est pas un refuge à laquelle on devrait déférence. Elle serait un bien à conquérir ; dans cette perspective, il faut cesser de la tenir comme objet mais être au sein « du grand territoire de la langue française » (Barthes 1967, 142), selon des termes barthésiens. En ce sens, il n'y a pas ici coupure entre deux langues chronologiquement déterminées, mais giration elliptique autour du manque, du vide et même du nom qui se dérobent à l'expression; il s'agit de la recherche d'une identité linguistique et littéraire dans un acte de réappropriation, de possession de la langue, mais sans effraction ou violence :

Pour moi, la langue française est beaucoup plus un bien vacant, un bien sans maître. Je me la suis approprié ni par la violence, ni par la guerre. J'ai un rapport pacifié au français. (Mechaï 2014)

17 On pourrait soulever un pacte entre Daoud et Haroun, le narrateur mais aussi une ambiguïté entre Meursault et Camus. 
Confie Daoud dans un entretien accordé au Point. Ces paroles résonnent en écho dans la bouche du narrateur :

Le meurtrier est devenu célèbre et son histoire est trop bien écrite pour que j'aie l'idée de l'imiter. C'était sa langue à lui. C'est pourquoi je vais faire ce qu'on a fait dans ce pays après son indépendance prendre une à une les pierres des anciennes maisons des colons et en faire une maison à moi, une langue à moi. Les mots du meurtrier et ses expressions son mon « bien vacant » (12)

La langue se plie à la recherche d'une individualité. C'est ainsi que Daoud greffe la langue maternelle sur la langue française. Les emprunts, par exemple, émaillent le texte : zoudj, chahid, Ouled el-assesse, Ouled-elbled, gaouri, ouled el-houmma, El-Hadj, etc. Par un acte réfléchi et une conscience délibérée, Daoud insère des mots, des expressions de sa langue maternelle, " une langue minorisée », ${ }^{18}$ en quête d'une reconnaissance au sein d'une 'culture' dominante. La langue maternelle, sorte d'héritage conservé, féconde le récit écrit en 'français' pour chercher un compromis entre langue et identité. Cette écriture culturelle hybride nous permet d'assister à une véritable appropriation de la langue et de se trouver dans la langue de l'autre. Elle devient un lieu-dit, un terrain où se pense et se construit l'avenir à partir de l'origine. Dès lors appropriée, la langue servirait à réclamer un enracinement, à l'affirmation d'une identité face à une dépossession, à une désappropriation et au non-sens. Il s'agit, peutêtre, d'une sorte d'indépendance ou de libération mentale du préjugé d'une langue (celle du colonisateur) qui, un « jour vous possède », " donn[ait] des noms à ce qu'il s'approprie » (23) et " prend l'habitude de saisir les choses à votre place » (17) ; une langue qui altère le sens de la propriété et rend l'oranais « un étranger [qui] ne possède rien » (126). Le rapport pacifié à la langue française permet de relativiser ces déterminismes culturels et idéologiques hérités. Le français n'est plus la langue du pouvoir opprimant, celui du colonisateur ; au contraire, il permet de dire la révolte, le dévoilement et la recherche de l'identité. Dans cette langue de l'autre, serait diffusée, dans un acte créatif, une vision de l'intérieur conforme à la réalité : l'Arabe ne saurait vivre dans une infériorité ou dans l'anonymat, réduit souvent à l'image violence, du terrorisme et de l'extrémisme religieux. La fin du roman marque, à ce sujet, une déclinaison du nom français Meursault en «El-Merssoul » (l'envoyé) soulignant un métissage linguistique et une symbolique identitaire. Ainsi, loin de rester enfermée dans sa communauté linguistique et de favoriser une binarité,

18 Cf. Deleuze, Gilles ; Guattari, Felix (2005). Qu'est-ce la philosophie ?. Paris : Éditions de Minuit. 
l'écriture se veut un lieu de reconnaissance de la pluralité, un territoire «des équilibres » (16; emphase dans l'original), à l'encontre des « choses improbables et des histoires de lutte à bras-le-corps entre Moussa, géant invisible et le gaouri, le roumi, le Français obèse, voleur de sueur et de terre » (26; du réel ce que l'on peut dire, et moyen d'agir sur ce réel).

Meursault, contre-enquête demeure un roman de la reconnaissance et une tentative de libération. Hormis la violence culturelle coloniale qui est écrite d'une manière fragmentaire et parfois d'une manière suggestive, l'écriture semble entreprendre une opposition entre le temps historique d'une modernité révolutionnaire 'laïque' et celui de la prosternation religieuse en pensant les barrières langagières.

\section{Bibliographie}

Barthes, Roland (1978). Leçon. Paris : Seuil.

Barthes, Roland (1967). Système de la mode. Paris : Seuil.

Belhabib, Assia (2017). " Le mal de mère. Le sanctuaire de restitution dans Meursault contre-enquête de Kamel Daoud ». Babel Littératures plurielles, 3, 119-27. URL https://journals.openedition.org/babel/4985 (2018-11-28).

Blanchot, Maurice (2002). L'attente, l'oubli. Paris : Gallimard.

Daoud, Kamel (2014). Meursault, contre-enquête. Paris : Actes Sud.

Derrida, Jacques (1972). Marges de la philosophie. Paris : Minuit.

Derrida, Jacques (1996). Le Monolinguisme de l'autre. Paris : Galilée.

Diffalah, Sarah (2010). Camus, L'algérien ou l'étranger ? URL http://www. nouvelobs.com (2018-11-28).

Domino, Maurice (1987). «La réécriture du texte littéraire : Mythes et Réécriture ». Semen. Revue de sémio-linguistique des textes et discours, 3. URL http://journals.openedition.org/semen/5383 (2018-11-28).

El Houssi, Majid (2008). « Le mot roumi ». Des îles en archipel, Flottements autour du thème insulaire en hommage à Carminella Biondi. Bern : Peter lang, 281-306.

Genette, Gérard (1982). Palimpsestes. Paris : Seuil.

Haïk, Farjallah (1946), Dieu est libanais. Beyrouth : Nouvelles éditions orientales.

Khadra, Yasmina (2010). "L'Arabe ne comptait pas à mes yeux » (online). Le matin d'Algérie. URL www.lematindz.net (2018-11-28).

Lapaque, Sebastien (2017). « Kamel Daoud : Il n'y a pas de dictature sans consentement ». Revue des deux mondes. URL WWw. revuedesdeuxmondes.fr (2018-11-28).

Lévinas, Emmanuel (1967). "La trace de l'autre ». En découvrant l'existence avec Husserl et Heidegger (1949). Paris : Vrin, 261-82. 
Macé, Marielle (2015). "Questions de lecture, entre expérience et appropriations ». Fabula-LhT, 14. URL http://www.fabula.org/lht/14/ mace.html (2018-11-28).

Mechaï, Hassina (2014). "Kamel Daoud, sur les traces de Camus ». Le Point Afrique. URL http://afrique.lepoint.fr/culture/kamel-daoudsur-les-traces-de-camus-28-09-2014-1867354_2256.php (2018-11-28).

Poulet, Georges (1967). Les chemins actuels de la critique. Paris : Plon.

Proust, Marcel (1992). Pastiches et Mélanges. Paris : Gallimard.

Saïd, Edward (2005). L'Orientalisme. Paris : Seuil.

Seklaoui, Hafida ; Déchir Smaïl (2014). «Kamel Daoud : Je revendique Camus comme auteur algérien ». L'Humanité. URL https://www. humanite.fr/ (2018-06-20). 


\title{
La fête et le spectacle carnavalesques dans Le Mât de cocagne (1979) et Hadriana dans tous mes rêves (1988) de René Depestre
}

\author{
Irene Alvarez Domenech \\ (Université Sorbonne Nouvelle - Paris 3, France ; Università Ca' Foscari \\ Venezia, Italia)
}

\begin{abstract}
The aim of this article is to bring together two of René Despestre's novels, Le Mât de cocagne (1979) and Hadriana dans tous mes rêves (1988), and place them within the tradition of the carnivalesque as it was discussed by Mikhail Bakhtin in L'œuvre de François Rabelais et la culture populaire au Moyen Âge et sous la Renaissance. By taking into account this wider perspective, I intend to show that the presence of a diegetic spectacle reinforces the subversive value of the two works by including a moment of utopian relief that invites both the characters and the readers to engage in a fight for freedom.
\end{abstract}

Sommaire 1 Introduction. - 2 Épisodes diégétiques de fête et le spectacle de la vie à Haïti. - 3 Le merveilleux carnavalesque. - 4 L'élan vital de la fête. - 5 La fête comme initiatrice d'une révolte collective. -6 Conclusion.

Keywords Depestre. Haitian literature. Carnival. Subversion. Bakhtin.

\section{Introduction}

Le carnaval et la fête sont deux termes qui semblent être chers à René Depestre qui les emploie souvent pour décrire sa conception de l'écriture et de l'histoire et de la culture haïtiennes. Ces leitmotivs traversent aussi de manière particulièrement signifiante deux de ses œuvres : Le Mât de cocagne (1979) (désormais désigné comme Le Mât) et Hadriana dans tous mes rêves (1988) (désormais désigné comme Hadriana). Dans ces œuvres sont exposés, sous couvert du thème de la fête et du spectacle, du mélange, d'une écriture comique et d'une imagination débordante, les sévices subis par le peuple haïtien pendant la dictature des Duvaliers (19571986). En effet, la mise en place d'une esthétique et d'une thématique 'carnavalesques' permet la révélation et la critique de la situation politique et sociale du pays. Conscients qu'il s'agit d'un sujet amplement discuté dans la critique, nous souhaitons tout de même nous attarder sur ce thème capital et récurrent chez Depestre dans ses deux romans les plus célèbres. 
Nous analyserons ces caractéristiques en prenant aussi comme point de référence l'ouvrage de Mikhail Bakhtine L'œuvre de François Rabelais et la culture populaire au Moyen Âge et sous la Renaissance. Nous chercherons à étudier comment les idées depestriennes sur le carnaval pourraient s'entrelacer avec les théories bakhtiniennes pour mettre en relief la valeur contestataire du corpus. Nous soutiendrons que la fête représente dans ces « romans de la dictature $»^{1}$ tant un système politique et un héritage historique grotesques qu'un moment de révolte symbolique et cruciale. Le carnaval dans Hadriana et le concours du mât de cocagne dans Le Mât « donn[ent] un aspect du monde, de l'homme et des rapports humains totalement différent, délibérément non officiel, extérieur à l’Église et à l'État » (Bakhtine 1970, 13). La fête et le spectacle carnavalesques incarnent un tournant dans la diégèse car ils représentent le moment propice au renversement des valeurs préétablies. Le spectacle donne naissance, par la restitution de l'élan vital et du sentiment de communauté, à la possibilité d'une révolte populaire face à la domination opprimante du pouvoir en place.

\section{2 Épisodes diégétiques de fête et le spectacle de la vie à Haïti}

Tout d'abord, la fête, moment concret dans la diégèse de nos deux romans, se caractérise par une démarche volontaire de mise en scène et par une ampleur sans pareil. Dans Hadriana, un débat suit la mort de la jeune femme pour déterminer quelle cérémonie, catholique ou vaudou, doit honorer son décès. Au début de la partie 7 du deuxième chapitre, les questions sont retranscrites au discours direct ainsi que les dialogues entre les différents personnages pour faire apparaître avec plus de saillance les difficultés de la prise de décision et, par ce geste, mettre l'accent sur le choix final du carnaval. Celui-ci se distingue par son exorbitance dénotée par des expressions telles qu' « un carnaval sans précédent » (Depestre 1988, 37 ), « nuit de ripaille et de folie que tout le monde attendait » (41), « une nuit de bombance et de folie » (52), « un carnaval de cinq cent diables » (159), ou encore « carnaval chauffé à blanc » (162). L'excès est une particularité aussi du concours du mât de cocagne. Tous les moyens sont mis en place par le Grand État Zacharien pour garantir un divertissement pour le peuple à l'occasion de la « Fête d'Électrification des âmes » (Depestre $1979,49-50)$. La diffusion sera inédite - « Ce sera la première fois que des monteurs de mât suiffé apparaîtront sur un écran de télévision » (38) - et il y aura même, selon les mots de Barbotog, une «principale attraction » (50): la présence d'Henri Postel.

1 Formule empruntée dans son application au roman haïtien à Alessandro Costantini (2016). 
Or, le carnavalesque dépasse les limites des moments précis de fête et de spectacle. Il obéit, à vrai dire, à un principe global, tragique et burlesque à la fois, qui règne sur l'histoire, la politique et les mœurs haïtiennes. René Depestre appellera plus tard dans sa carrière cette conception oxymorique du vécu la « carnavalisation de l'Histoire » (Depestre 1998, 2011). ${ }^{2}$ Le carnaval dans Hadriana présente l'histoire d'Haïti en version concentrée. Le narrateur propose une description minutieuse des costumes des Jacméliens parmi lesquels nous pouvons observer des Indiens caraïbes, des Frères de la côte, des barons et des marquises de la cour de Louis XIV, des officiers de la Grande Armée de Napoléon, Toussaint Louverture, Alexandre Pétion, l'empereur des Haïtiens Jacques I, Simon Bolivar, les troupes du président Wilson, et même Joseph Staline. Lécrivain s'amuse à dépeindre les périodes de l'histoire de son pays natal en allant des tribus natives jusqu'à l'occupation américaine sous la forme de masques hétéroclites. Il introduit en outre, de manière subreptice, l'ombre de la dictature duvaliériste. Dans la foule de personnes costumées, il y a « des rôles moins spectaculaires de porcs, d'orangs-outangs, d'oiseaux de proie, de taureaux, [...] de tontons-macoutes et de léopards » (Depestre 1988, 62). L'inclusion anachronique des tontons-macoutes - le temps de la diégèse est 1938 - au milieu de cette accumulation de costumes animaliers rend les corps paramilitaires duvaliéristes grotesques et brutaux et signale qu'ils ne sont pas à leur place dans le pays, indépendamment du moment historique de leur apparition. Avec ce tableau caricatural Depestre insiste sur les combats continuels des Haïtiens pour obtenir et garantir leur liberté sous les différents régimes et occupations. Cette même mouvance historique carnavalesque est prolongée dans les «Prolégomènes à un essai sans lendemain » (125-33) de Patrick où le jeune homme explore les stratégies de justification de la colonisation à travers l'image du masque. Léquivalence entre les costumes du carnaval et l'histoire est reprise ainsi :

Tout s'était passé comme si les maîtres entreprenants de la colonisation avaient eu besoin, sur le mode 'sorcellaire', de mettre des masques à la fois à leur champ d'action et aux protagonistes des traversées triangulaires qui mobilisaient les hommes des trois continents (Europe, Afrique, Amérique) [...].

2 D'après le romancier, Christophe Colomb aurait apporté avec lui lors de son arrivée aux Amériques la culture européenne du carnaval qui a imprégné les mœurs de l'île (Depestre 2011, 135). Il explique que dans son enfance il a découvert dans « les livres d'histoire d'Haïti que le Mardi gras n'est pas seulement une fête qui tombe chaque année au mois de janvierfévrier, mais qu'on trouve ses déguisements et ses masques à tous les épisodes du tragique passé des Haïtiens » (Depestre 1998, 149). Depestre ajoute qu'« après leur fantastique libération du système colonial, les Haïtiens passaient le meilleur de leur temps à tourner en dérision l'État de droit, les règles de la démocratie, et les institutions de la société civile inventées par les anciens maîtres blancs...» (149). 
Haïti [...] serait entrée dans l'histoire moderne affublée d'un jeu de masques (blanc, noir, indien, mulâtre, etc.), c'est-à-dire sous une fausse identité. (129-30)

Le fait que le romancier reprenne cette même image dans une interview plus de vingt ans après la parution d'Hadriana ${ }^{3}$ laisse sous-entendre une connivence entre les théories exprimées par le personnage et les pensées de son auteur qui adhère encore, malgré le passage du temps, à la justesse de son image.

Dans Le Mât, la vie sous le pouvoir dictatorial de Zoocrate Zacharie est décrite comme une représentation ridicule. Cette idée est énoncée explicitement à plusieurs reprises par Henri Postel : "Rideau, maître Horace, sur le répugnant spectacle ! Je pars pour toujours » (Depestre 1979, 16), "Le mardi gras était permanent : tous les masques étaient à l'effigie du Grand Électrificateur » (23), «Au fait, pourquoi un mât de cocagne, ces jours-ci ? Il y a dans la ville un cirque ouvert toute l'année, avec des numéros où se distinguent toute sorte d'animaux de proie » (24). La métaphore filée souligne l'uniformisation et la cruauté de la vie sous la dictature où les personnages-spectateurs-comédiens doivent cacher leur véritable identité pour survivre. De plus, comme certaines de ces occurrences le sous-entendent, cette carnavalisation institutionnalisée semble découler directement de l'identité des personnages au pouvoir. Ceux-ci « remplisse[nt]», selon la formule de Malika Hadj-Naceur, « l'espace fictionnel l'investissant d'une présence grotesque et bouffonne » (2016, 226). Ainsi, après la mort de Zoocrate Zacharie d'une crise cardiaque, « le Pouvoir est apparemment entre les mains de l'Ange Zacharie. L'ONEDA porte mini-jupes et robes de chez Chris Bior » (Depestre 1979, 177). Le spectacle de la dictature se clôt dans le roman avec une dernière blague grotesque : notons la prise de distance d'Hadriana narratrice par rapport à son énoncé et la référence détournée à la marque de luxe Christian Dior. La profusion des noms du Président Zacharie participe du même phénomène carnavalesque. L'accumulation comique d'appellations périphrastiques, grandiloquentes et hyperboliques où règne l'emploi excessif de la majuscule - voir par exemple le discours d'ouverture du concours prononcé par Barbotog (Depestre 1979, 72-3) - résulte de « l'obsession maladive d'exister en chef suprême » (Hadj-Naceur 2016, 226). En effet, «[d]ans le roman haïtien le Dictateur est seulement, et surtout, un Nom » (Costantini 2016, 172). Il est important de souligner, d'ailleurs, le sous-texte historique de ces surnoms dans Le Mât : en plus

3 « Elle [« la carnavalisation de l'histoire »] a commencé très tôt, dès l'époque coloniale, comme une tendance à poser des masques sur les réalités [...]. Les membres des différentes ethnies des Afriques ont été ensuite déguisés en Noirs et Nègres du fabuleux mardi-gras colonial !» (Depestre 2011, 135). 
des inventions de Depestre «à des fins burlesques », une partie de ces dénominations « avaient effectivement été donné[e]s à Papa Doc par ses adulateurs » (Hoffmann 1995, 330).

Le caractère ridicule de ces institutions gouvernementales pourrait, nous semble-t-il, être l'héritier de la culture carnavalesque décrite par Bakhtine : «Nul festin ne se déroulait sans qu'interviennent les éléments d'une organisation comique comme, par exemple, l'élection pour la durée du festin de reines et de rois 'pour rire'» $(1970,13)$. Cependant, le lecteur ne peut réagir qu'avec un rire légèrement grinçant lorsqu'il comprend que la fin de la farce n'est jamais annoncée et que le ridicule et le comique de l'absurde sont la seule manière de représenter la vraie absurdité de la vie sous la dictature. L'histoire haïtienne ressemble donc à une troublante mascarade où les épisodes historiques graves sont dépeints sous le prisme du carnavalesque qui cache une volonté de sédition railleuse. Nous croyons que les propos que tient Hadj-Naceur sur La Vie et demie de Sony Labou Tansi pourraient s'appliquer aussi au Mât : «l'univers de mascarade aux allures rabelaisiennes [caractérise] l'État-farce, l'État-spectacle, présenté comme une place publique quasi-foraine donc festive, lieu de toutes les libertés, non sérieuse » $(2016,236){ }^{4}$

\section{Le merveilleux carnavalesque}

Bien plus, la description des figures et des stratégies de la colonisation et de la dictature en des termes carnavalesques provoque un premier renversement de leur caractère normatif et officiel en leur ôtant toute crédibilité. D'autre part, s'oppose à ce carnaval ridicule étatique, telles deux forces cosmiques confrontées, la véritable fête, symbole de jouissance, de rébellion et de vie. ${ }^{5}$ Le carnavalesque infiltre d'abord les narrations et provoque une disparition de la réalité matérielle douloureuse : l'apparition du spectacle et de la fête brouille les frontières entre réel et fantaisie. Sa présence donne lieu à un « second monde et une seconde vie » (Bakhtine $1970,13)$, à une représentation de « la vie même présentée sous les traits particuliers du jeu » (15) et de l'imagination. Depestre se sert du conte de

4 Nous voulons tout de même exprimer une réticence envers son expression « lieu de toutes les libertés » parce que, dans notre corpus, les stratégies de privation des libertés et les modes de représentation et de maintien du pouvoir sont l'essence même du ridicule carnavalesque qui définit la dictature.

5 Nous pourrions rapprocher ceci d'une remarque de Marina Ondo (2010) sur la langue créole : "Surtout, la langue créole se nourrit d'un 'Pleurer-Rire' constant, résurgent dans toutes les manifestations de la parole. À n'en point douter, le proverbe haïtien [sic]: 'ridiri, ou ké pléré lanti' (tu peux rire après avoir pleuré) indique comment aborder avec un humour ironique les remous sociaux et politiques pour mieux les affronter.» 
fées pour faire régner cette atmosphère de fantaisie dans les deux œuvres. Il est, d'une part, question de récits imaginaires dans Hadriana. Un effet de mise en abîme apparaît dans la volonté des Jacméliens d'incorporer le personnage éponyme à leurs récits : «les Jacméliens [...] l'intégrèrent [Hadriana] le soir même, au répertoire des fables du pays, dans une fantastique histoire » (Depestre 1988, 51); « des Jacméliens à l'imagination nécrophile ont incorporé leur fille à un conte de fées » (92). Dans les deux cas, nous avons affaire à un type de récit traditionnel ancré dans l'imaginaire collectif. Il s'agit, de plus, d'un phénomène de surcadrage où le récit fictif nous est décrit comme tel. Le récit du Mât se présente lui-même, de manière analogue, comme une narration fictive. Le narrateur-auteur indique à ses lecteurs que «Le Grand pays zacharien dont il est parlé est, de toute évidence, une contrée imaginaire qui ressemble, en plus fou, au pays de cocagne » (Depestre 1979, 9) et, que par conséquent, il « décline fermement la responsabilité, au nom de ce que des esprits éclatants de rigueur et de tendresse ont appelé les 'droits imprescriptibles de l'imagination'» (9). La supposée véracité de ces déclarations est confirmée par les premiers mots de l'incipit qui sont une reprise de l'expression traditionnelle du début des contes : «Il était une fois » (11). Depestre semble déployer une ironie mordante reposant sur la métatextualité. Il joue avec la présence du mode comique qui traverse l'ensemble des romans et avec les attentes du lecteur, qui soupçonne fortement que les œuvres soient inspirées d'événements réels de l'histoire haïtienne.

En plus de cette hybridation diégétique entre le réel et le fictif, Depestre met en place un mélange entre le réel et le merveilleux. Le merveilleux n'est pas que fictif, il s'appelle, en effet, "réel merveilleux ». ${ }^{6}$ Des expressions qui jouent sur la dérivation de cette formule ponctuent Hadriana. Patrick Altamont affirme « guett[er] l'incident qui mettrait [s]on imagination sur quelque piste du surréalisme quotidien » (Depestre 1988, 17) et, plus

6 Cette mouvance est en fait redevable de ce que la littérature latino-américaine appelle " realismo mágico » et de sa version haïtienne analysée par Jacques Stephen Alexis ([1956] 2002) dans « Du réalisme merveilleux des Haïtiens ». Alejo Carpentier ([1949] 2012) relie précisément le « real maravilloso » à Haïti dans le prologue de son roman El reino de este mundo. Lors d'un séjour à Haïti en 1943, il remarque l'omniprésence du réel merveilleux dans les terres qu'il visite : " al hallarme en contacto cotidiano con lo real maravilloso » (en me trouvant en contact quotidien avec le « real maravilloso ») (9), « A cada paso hallaba lo real maravilloso » (À chaque pas je trouvais le « real maravilloso ») (9). Il déclare avoir compris à ce moment que le réel merveilleux faisait partie de l'héritage de l'Amérique entière : « lo real maravilloso no era privilegio único de Haití, sino patrimonio de la América entera » [le « real maravilloso » n'était pas le privilège d'Haïti uniquement mais le patrimoine de l'Amérique entière] (9). La traduction en français est de nous. Depestre se réclame explicitement de cette esthétique américaine en déclarant que "S'il y a un élément de merveilleux dans [s]es modestes travaux, c'est que cet état est d'abord une composante de la réalité du pays. [...] Il y a une sorte de surréalisme populaire qui rend plus apparents qu'ailleurs les côtés les plus insolites de la vie en société » (Depestre 1998, 143). 
tard, il précise qu' « à la veillée et aux funérailles d'Hadriana, en hommage émouvant à sa beauté, le réel merveilleux haïtien était entré en éruption » (92). Le personnage éponyme se réfère aussi au réel merveilleux mais comme un besoin vital, alimentaire, qu'elle ressentait dans son enfance : « fringale de merveilleux quotidien » (178). Tout comme le carnaval qui est selon Bakhtine la « seconde vie du peuple, basée sur le principe du rire. C'est sa vie de fête » $(1970,16)$, le réel merveilleux insuffle une nouvelle vie dans l'existence des personnages et des villes décrites dans les deux romans. En effet, Depestre soutient que

[1]a notion de réel merveilleux est la négation poétique et romanesque des circonstances historiques de la conquête coloniale. C'est l'antithèse artistique de la realpolitik qui aura été à l'œuvre aux Amériques à partir de l'aventure de Christophe Colomb. C'est son inverse, que j'appelle aussi la realutopie des humiliés et des offensés. (Depestre 1998, 112)

Le réel merveilleux équivaudrait alors à une sorte de réparation des dommages causés par l'histoire qui passerait par la revendication et la récupération d'une vision du monde purement haïtienne. Par exemple, la disparition des catégories temporelles au début d'Hadriana lorsque la voiture de Germaine Villaret-Joyeuse se dirige vers la côte n'est pas perçue comme une source d'angoisse mais comme un moment de fête et de fantaisie faisant partie du quotidien :

Dans leur panique les chrétiens-vivants emportèrent le temps avec eux : il pouvait indifféremment être la demie de trois heures de l'après-midi ou du matin, en l'an 1938 ou bien 38 av. J.-C. Cinq mètres plus loin, à bout de souffle, je crus rattraper le jour d'octobre et le $\mathrm{XX}^{\mathrm{e}}$ siècle dans la grand-rue qui allait droit s'embrancher sur l'avenue La Gosseline. (Depestre 1988, 21)

Pour mettre en valeur le syncrétisme entre passé et présent, le narrateur dispose sur le même plan le temporel et le spatial et produit ainsi un effet comique. La perturbation produite par la juxtaposition non articulée des différentes périodes historiques, patchwork temporel semblable à la tenue d'Arlequin, est imprégnée par la jovialité intrinsèque au carnaval. 


\section{L'élan vital de la fête}

L'emploi du calque "chrétiens-vivants ${ }^{\boldsymbol{7}}$ dans cet extrait est aussi significatif parce que nos deux romans mettent en scène une rencontre entre la vie et la mort. La première prend le dessus sur sa rivale et la transforme en un moment de célébration et de fête. Cette joie est proprement carnavalesque parce que, comme l'indique Bakhtine, dans le carnaval « la mort est suivie de la résurrection de l'an neuf, de la nouvelle du nouveau printemps » (1970, 199). Tant la mort d'Henri Postel que celle d'Hadriana sont accompagnées de fêtes à leur demande. Dans Le Mât, Postel s'adresse ainsi à Zaza : " Emportez l'ex-Henri dans nos montagnes. Chantez, dansez, vivez sa mort avec les tambours des jours d'allégresse » (Depestre 1979, 124). Plus tard, dans sa lettre, Élisa rapporte que « Tandis qu'Henri était étendu sur une natte, on a chanté, dansé, vécu son départ avec les congos et les tendres mouvements de nos corps, au rythme des tambours » (174). Les deux rythmes ternaires constitués d'impératifs et de participes passés respectivement, l'oxymore «vivez ma mort » reprise dans les paroles d'Élisa et soulignée par l'emploi de l'italique mettent en évidence un élan vital voulant dépasser les limites de la mort. Pareillement, selon la mère du narrateur d'Hadriana, le personnage éponyme a manifesté que « Si [elle] mourrai[t] jeune, [elle] aimerai[t] que [s]a mort soit vécue, par tous ceux qui [1'] auraient aimée, avec les tambours et les masques des jours de carnaval !» (Depestre 1988, 65). Dans ce cas, le rapprochement avec le carnaval est explicite, mais le fait que Depestre répète les mêmes images, l'oxymore «mort [...] vécue » et les « tambours », semble indiquer que son premier roman se plaçait aussi sous le signe de la fête perturbatrice de l'ordre canonique de la société et de la vie humaine.

Cette célébration de la mort est d'autant plus importante qu'elle accompagne un mouvement de résurrection des morts-vivants, c'est-à-dire des zombies. Dans Le Mât, Henri Postel est victime de la « zombification par soi-même » (Depestre 1979, 12) mais il essaie de se déjouer de cette étiquette de zombie en la détournant à travers le concours du mât de cocagne. Au début du roman, le protagoniste montre son refus d'accepter cet attribut en accrochant une affiche devant sa boutique - l'Arche de Noé - où il est écrit « Fermé pour cause de dézombification » (18). Or, plus tard, lors de l'inscription au concours, il affirme : « Je suis un zombie-grim-

7 «Chrétien-vivant » est un calque en français sur le terme créole kretyen vivan. Selon Jean Targète et Raphaël G. Urciolo, il s'agit d'un «[h]uman being, person (as in many Romance languages and dialects », où 'chrétien' signifie tout simplement et justement 'être humain, personne'). Kretyen vivan Living soul » $(1993,106)$. L'origine de ce terme dériverait de la nécessité de distinguer dans la culture haïtienne les zombies aussi appelés morts vivants - mò vivan en créole (Valdman, Iskrova 2007, 489) -, des êtres humains vraiment vivants et en possession de leur âme. 
peur » (48). Nous sommes d'accord avec Alessandro Costantini $(2016,25)$ pour dire que grimper le mât de cocagne signifie pour Postel que

[1]a Dictature ne lui laisse - et ce malgré elle - que cet unique mot d'ordre : une espèce de je grimpe donc je suis comme unique message humain et politique encore possible pour celui qui est écrasé et quasiment annulé.

Dans Hadriana, le phénomène du zombie est lié au réel merveilleux haïtien. La zombification de la jeune femme emporte avec elle la vitalité qui était propre à Jacmel : « La filiation naturelle entre le réel et le merveilleux a été interrompue par la disparition d'Hadriana Siloé » (122). Elle devient ainsi la personnification de sa terre natale et sa métamorphose en zombie se transforme, comme pour Postel, en symbole du passage au régime dictatorial. Néanmoins elle revient dans le monde des vivants quelques années plus tard. Elle resurgit de manière comiquement métatextuelle dans l'amphithéâtre où Patrick, à présent devenu professeur d'université à Kingston, est en train de donner un cours sur le réel merveilleux. Sa réapparition révèle d'un détournement du mythe original du zombie. En effet, Depestre

a dû ainsi commettre un écart épistémologique et faire d'Hadriana, non plus une Haïtienne ni même une femme noire, mais une femme blanche d'Europe qui, dépourvue de toute croyance ou d'idée préconçue, serait plus susceptible de se défendre contre un mal - la zombification - qui

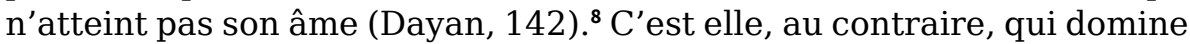
ce mal, l'observe du dedans, l'analyse et trouve les moyens d'en triompher. (Salien 2000, 88)

Le dépassement de l'état de zombie et de la mort équivaut à une rébellion utopique contre les injustices sociales et politiques. L'affirmation de la vie qui passe par une acceptation de l'hétérogène et du merveilleux devient une attitude assurément subversive.

\section{La fête comme initiatrice d'une révolte collective}

Le spectacle et la fête produisent donc un foisonnement qui renverse les valeurs préétablies du temps, de la vie et de la mort, du sérieux et du comique, et de la représentation des événements historiques. Le car-

8 Quoted in Salien. Cf. Dayan, Joan (1993). «France reads Haiti : René Depestre’s Hadriana dans tous mes rêves ». Yale French Studies. Ed. Lionnet and Scharfman. New Haven : Yale Uiversity Press, 154-75. 
navalesque offre un espace d'affranchissement, une opportunité idéale pour la révolte, un instant où les libertés confisquées par la dictature redeviennent possibles. Lors de ces moments s'opère un renversement symbolique du pouvoir rendu possible par l' « abolition provisoire de tous les rapports hiérarchiques, privilèges, règles et tabous » (Bakhtine 1970, 18) du carnavalesque. Dans Hadriana, ce moment est représenté par le choix du «papa-Mardi gras » (Depestre 1988, 81). À la fin du carnaval, les jumelles Philisbourg apportent un mannequin représentant Balthazar Granchiré, le coupable des malheurs de Jacmel. Dans Le Mât, la victoire d'Henri Postel sur le mât de cocagne équivaut à une double victoire sur le pouvoir dictatorial. Non seulement l'ex-sénateur réussit à dépasser ses adversaires dans l'épreuve physique mais il vainc aussi le président Zacharie personnellement puisque le chef était devenu un seul être avec le mât après une cérémonie vaudou. Ces instants de liberté reconquise transparaissent, d'autre part, dans le langage des spectateurs. Selon Bakhtine, la « perception carnavalesque du monde » a besoin d'un langage particulier capable d'exprimer des «formes d'expression dynamiques changeantes (protéennes), fluctuantes et mouvantes » $(1970,19)$. Ainsi, toujours dans Le Mât, au fur et à mesure que le concours se déroule, l'attitude des spectateurs devient de plus en plus osée jusqu'au point où « Tout le monde riait et hurlait des moqueries au baron à plat [Nildevert]. Merdoie ne pouvait se faire entendre »(Depestre 1979, 162). L'instant de liberté que le spectacle offre est tellement important que le peuple, dont la connivence dans la jovialité railleuse est mise en relief par la formule «Tout le monde », réussit à écraser le pouvoir des fonctionnaires de la dictature.

La fête induit alors un changement de mentalité qui mène à la prise de conscience et à l'acquisition d'une attitude séditieuse et dessine même une lueur d'espoir. «[A]ffranchissant de tout utilitarisme, de tout but pratique, [elle] donne le moyen d'entrer temporairement dans un univers utopique » (Bakhtine 1970, 276). Le carnaval et le concours du mât de cocagne sont des moments éphémères mais décisifs dans le parcours des personnages. À partir de ce tournant, les personnages échappent à la privation de liberté de la dictature en faisant le choix de la lutte ou de l'exil. Les deux romans se closent avec une image positive : Elisa s'est engagée dans la révolte après la mort de Postel et une histoire d'amour naît entre Patrick et Hadriana en Jamaïque. L'exploit de Postel devient, en outre, dans l'oraison funèbre d'Élisa, l'augure de l'arrivée de temps plus prospères : « Tu seras un berceau joyeux pour tout ce qui naîtra de bien et de beau sur nos terres. [...] Ta mort soutiendra la lumière des tiens, parce que de ton vivant tu as su élargir leur droit à l'espoir et à la liberté » (Depestre 1979, 175). La juxtaposition des pronoms personnels et possessifs se rapportant à la deuxième personne du singulier qui se réfèrent à Postel et de la première personne du pluriel « nos terres » fait ressortir la transition entre l'acte individuel du protagoniste et l'impact qu'il a sur la collectivité. 
Le renversement symbolique du pouvoir est en effet lié à la renaissance du sentiment de communauté permise par la fête. Bakhtine remarque que « Les spectateurs n'assistent pas au carnaval, ils le vivent tous, parce que, de par son idée même, il est fait pour l'ensemble du peuple » (1970, 15 ; emphases dans l'original). L'évolution de la situation sociale du protagoniste du Mât est assez révélatrice à cet égard. Le président Zacharie ordonne comme partie du processus d'auto-zombification « qu'il n'y ait plus jamais à ses [d'Henri Postel] côtés rien de vivant ni de chaud : ni enfants, ni parents, ni amis, ni partisans, ni le moindre animal domestique !» (Depestre 1979, 13). L'accumulation de particules négatives souligne la sévérité d'une mesure qui n'est pas respectée dans la suite du roman. Postel est assisté dans son entreprise séditieuse par maître Horace, sor Cisa, Élisa et d'autres habitants de Tête-Bœuf. Voire plus, au moment de sa mort « la place était si effervescente que peu de gens entendirent le coup de fusil qu'un franc-tireur posté sur le toit de la Tribune tira contre l'éclat de rire d'Henri Postel » (168). Le climax de l'action capitale de Postel coïncide de manière assez remarquable avec une manifestation externe du comique carnavalesque, « de ce rire de fête, dirigé contre toute supériorité » (Bakhtine 1970, 21). De même, dans Hadriana, malgré les premières dissidences des catholiques, toute la ville de Jacmel finit par participer au carnaval. Le narrateur indique que «Plus personne ne pouvait continuer à prier à genoux. Tout le monde fut violemment happé par l'envie de chanter, danser, crier, d'éclater à la face sacrée de la mort » (Depestre 1988, 76). Les deux expressions totalisantes «Plus personne » et «Tout le monde » révèlent par leur position au début des phrases l'ampleur du mouvement d'inclusion qui se produit dans ces moments de communion festive et séditieuse.

Le foisonnement et le mouvement inclusif du carnaval se transmettent aussi aux structures narratives. Depestre joue avec la polyphonie, la transcription de la langue orale et le croisement de différents points de vue. Les deux romans se fondent sur une succession de voix narratives, comme si les différents personnages endossaient à tour de rôle le masque du narrateur, pour mieux retranscrire le grouillement de la fête débordante de vie. Dans Hadriana, la même histoire est racontée deux fois. La première, vue sous l'angle de Patrick Altamont en position de témoin, est suivie de la version d'Hadriana, emprisonnée dans son corps en état catatonique, qui retrace les évènements de cette journée dont elle était la protagoniste. À ceci, nous devons ajouter entre autres l'article de La Gazette du Sud-Ouest au début du deuxième mouvement (Depestre 1988, 37-9), l'interview imaginaire de Patrick et de l'auteur de la Lettre de Jacmel (120-4) et l'essai de Patrick sur le zombie (125-33). Le Mât repose similairement sur une mosaïque de textes différents : la narration générale à la troisième personne d'un narrateur d'origine haïtienne, la lettre d'Élisa Valéry à la fin du roman (Depestre 1979, 170-8), ou encore les extraits de journal comme 
l'éditorial du Courrier de Sam Daumac (62-3), ou l'article de Claude-Marc Nidang, un envoyé d'un quotidien de Paris (81-4). Cette fragmentation déstabilisante va à l'encontre de l'emploi traditionnel du narrateur-auteur omniscient et d'une vision individualiste où une seule instance détiendrait la vérité. En accordant la parole à divers personnages et sous des formes variées, Depestre fait de ses romans des symboles de la liberté de parole, des ouvrages collectifs qui refusent tout point de vue hégémonique.

Finalement, la communauté que rétablissent les fêtes de nos romans ne se borne pas à un cadre local. Les deux œuvres de Depestre veulent atteindre l'universel en traitant des sujets en apparence spécifiquement haïtiens. Cette caractéristique se place dans la lignée de Bakhtine qui affirme que «[l]e carnaval revêt un caractère universel, il est un état particulier du monde entier : sa renaissance et sa rénovation auxquelles chaque individu participe » $(1970,15)$. Cette volonté est explicite dans Hadriana où le personnage éponyme évoque «[s]on village qui, au milieu de la mascarade générale, se prenait pour la scène comique du monde » (Depestre 1988, 62). La transition entre le local et l'universel se fait en maintenant la référence au carnavalesque et à son pouvoir englobant. Cette transfiguration est, en plus, mise en valeur par l'insertion de cette phrase à la fin de la première partie du troisième chapitre. Ce mouvement de globalisation se traduit aussi en syncrétisme entre culture locale haïtienne et culture européenne, à l'image de l'hybridité des usages religieux des vaudouisants. ${ }^{9}$ Ainsi, les nombreuses évocations du vaudou - voir la cérémonie de sor Cisa pour revitaliser Henri Postel et celle de l'union entre le mât de cocagne et le président Zacharie, les deux décrites en détail - sont mises en parallèle avec des références bibliques. Postel travaille dans un magasin appelé " l'arche de Noé » et il est représenté sous des traits christiques : il incarne la figure du martyr qui se sacrifie pour le bien de son pays en montant « en compagnie des mauvais larrons de la ville » (Depestre 1979, 41) sur un mât de cocagne qui ressemble fort à la croix du calvaire. Depestre choisit ainsi volontairement de se placer dans un cadre multiculturel et hybride, de s'adresser à un lectorat international et de lancer un appel global à la lutte pour les libertés.

\section{Conclusion}

La fête et le spectacle apparaissent donc comme une invitation globale au vitalisme, à la lutte pour les libertés et à la régénération. Ils deviennent « la forme que revêtait la seconde vie du peuple qui pénétrait temporellement

9 Parmi ceux qui assistent aux cérémonies vaudou, une partie seulement a reçu l'initiation (les hounsi). 
dans le royaume utopique de l'universalité, de la liberté, de l'égalité et de l'abondance » (Bakhtine 1970, 17). Le foisonnement, l'excès et la folie du carnaval et du concours du mât de cocagne bouleversent l'univers diégétique, emportent avec eux l'unicité de la narration et brouillent les distinctions entre le réel et le merveilleux, la vie et la mort et le passé et le présent. La vague de vitalité que ces moments représentent contraste avec la paralysie de la vie haïtienne comparée à un zombie et à un carnaval où les participants sont obligés de porter des masques grotesques. Sous couvert d'une scène de réjouissance éphémère, la fête incarne un instant de répit où les libertés sont reconquises et où les personnages, grâce à un sentiment de communauté retrouvé, renversent symboliquement le pouvoir en place. Depestre exploite ainsi le potentiel subversif du carnavalesque, tel qu'il a été décrit par Mikhail Bakhtine, en se réclamant d'une tradition européenne qu'il se réapproprie pour l'adapter à la culture haïtienne.

\section{Bibliographie}

Alexis, Jacques Stephen [1956]) (2002). « Du réalisme merveilleux des Haïtiens ». Présence africaine, 1, 91-112.

Bakhtine, Mikhaïl (1970). L'ouvre de François Rabelais et la culture populaire au Moyen Age et sous la Renaissance. Traduit du russe par André Robel. Paris : Gallimard.

Belleroche, Jean Élie (2011). The Nature of the Marvellous in René Depestre's "Hadriana dans tous mes rêves". Austin : University of Texas, Digital Repository.

Blanchaud, Corinne (2013). "René Depestre, l'homme-banian ou les tribulations du 'Tout en un' ». Brodziak, Sylvie (éd.), Haïti : enjeux d'écriture. Saint-Denis : Presses Universitaires de Vincennes, 53-73.

Carpentier, Alejo [1949] (2012). «Prólogo ». El reino de este mundo. Barcelona : Seix Barral, 5-12.

Colletta, Antonella (2001). " La Vie et demie de Sony Labou Tansi et Le Mât de cocagne de René Depestre : la plénitude de l'homme désagrégé ». Blachère, Jean-Claude (éd.), Sony Labou Tansi. Le sens du désordre. Montpellier : Presses de l'imprimerie de l'université Paul Valéry, 23-53. Costantini, Alessandro (2016). « Formes et figures de la dictature dans le roman francophone et hispanophone de la Caraïbe ». Interfrancophonies, 7, 157-80. Nouvelles formes de l'engagement dans les littératures francophones.

Couffon, Claude (1986). René Depestre. Paris : Éditions Seghers.

Delpêche, Bernard (2005). Magouilles d'une esthétique. René Depestre et le vodou. Paris : Éditions Caractères.

Depestre, René (1979). Le Mât de cocagne. Paris : Gallimard.

Depestre, René (1988). Hadriana dans tous mes rêves. Paris : Gallimard. 
Depestre, René (1998). Le métier à métisser. Paris : Éditions Stock.

Depestre, René (2006). «Retour à l'île de la Tortue ». Beniamino, Michel ; Thauvin-Chapot, Arielle (éds.), Mémoires et cultures. Haïti, 1804-2004. Actes du colloque international de Limoges 30 septembre - 1er octobre 2004. Avant-dire de René Depestre. Limoges : Presses universitaires de Limoges, 15-9.

Depestre, René (2013). «Dans les décombres du carnaval ». Cultures \& Conflits, 84. URL http://conflits.revues.org/18265 (2018-11-28).

Fauchier, Joël (2002). Le 'réel merveilleux' chez Alejo Carpentier, René Depestre et Gabriel Garcia Marquez. Thèse présentée en octobre 2002. Université de la Réunion. URL https://tel .archives-ouvertes.fr/tel00464189 (2018-11-28).

Hadj-Naceur, Malika (2016). La dérision comme stratégie d'écriture. L'exemple des littératures africaines et antillaises de langue française. Paris : Éditions Karthala.

Hoffmann, Léon-François (1982). Le roman haïtien. Idéologie et structure. Sherbrooke : Les Éditions Naaman.

Hoffmann, Leon-Francois (1995). Litterature d'Haiti. URL http://classiques.uqac.ca/contemporains/hoffmann_leon_francois/litterature_dHaiti/litterature_dHaiti.html (2018-11-28).

Hoffmann, Leon-Francois (1998). «Haiti ». Corzani, Jack ; Hoffmann, Léon-François ; Piccione, Marie-Lyne (éds.), Les Amériques. Vol. 2 Littératures francophones. Paris : Editions Belin, 7-85.

Joqueviel-Bourjea, Marie ; Bonhomme, Béatrice (éds.) (2015). René Depestre. Le Soleil devant. Paris : Hermann Éditeurs.

Kundera, Milan (1993). « Rabelais et les Misomuses ». Entretien avec Guy Scarpetta. La Règle du Jeu, 9. URL https://laregledujeu. org/2014/08/25/17684/milan-kundera-rabelais-et-les-misomuses/ (2018-11-28).

Ondo, Marina (2010). «Hadriana dans tous mes rêves de René Depestre ou la littérature haïtienne francophone entre réalisme social et croyances mystiques ». La Revue des ressources. URL http://www.larevuedesressources .org/hadriana-dans-tous-mes-reves-de-rene-depestreou-la-litterature-haitienne-francophone, 1650.html (2017-05-26).

Salien, Jean-Marie (2000). « Croyances populaires haïtiennes dans Hadriana dans tous mes rêves de René Depestre ». The French Review, 74, 1, Octobre 2000, 82-93.

Targète, Jean ; Urciolo, Raphaël G. (1993). Haitian Creole-English Dictionary. Kensington : Dunwoody Press.

Valdman, Albert ; Iskrova, Iskra (2007). Haitian Creole-English Bilingual Dictionary. Bloomington : Indiana University Creole Institute. 


\title{
«Un pays occupé est une terre sans vie » Réflexions sur les subjectivités occupées et les espaces de résistance dans Kannjawou, roman de l'écrivain haïtien Lyonel Trouillot
}

\author{
Federica Cozzio \\ (Università Ca’ Foscari Venezia, Italia)
}

\begin{abstract}
The article proposes a reflection on how the intertwined themes of power, subjectivity and agency are articulated in Lyonel Trouillot's 2016 novel Kannjawou. In its narrative space, the hermeneutics of collective reality is produced by an individual memory engaged in questioning the relations of power that affect subjectivity and action of both the occupied Haitians and the foreigners occupiers. A close reading of the novel seeks to unpack its ethnographic thickness, its capacity to built layers of understanding around the stakes of a disadvantaged microcosm in contemporary Port-au-Prince.
\end{abstract}

Sommaire 1 Introduction. - 1.1 Pouvoir et subjectivité. - 2 Le journal d'un jeune de la rue de l'Enterrement. - 3. D'une terre sans vie et des corps occupés. - 3.1 Le corps heureux de l'occupant. - 3.2 Quelle résistance?

Keywords Haitian literature. Ethnography. Power and subjectivity. Lyonel Trouillot. Kannjawou.

\section{Introduction}

L'anthropologie et la littérature s'interpellent depuis longtemps, et les perspectives adoptées pour questionner leurs intersections, alliances et irréductibles spécificités sont nombreuses. Cet article se propose d'expliciter comment le roman Kannjawou de Lyonel Trouillot sollicite une analyse conduite avec les outils interprétatifs de l'anthropologie. Ce texte, en interrogeant certains aspects du rapport entre individu et pouvoir, adresse des problèmes théoriques existants au cœur de la discipline. Bien entendu, ces problèmes n'animent pas exclusivement le débat anthropologique, mais sont partagés par bien d'autres disciplines (par exemple, la sociologie, la philosophie, l'histoire). C'est la capacité du roman de les dégager à travers la description d'expériences particulières et subjectives, et de les situer dans la voix, la vision du monde et les expériences des subjectivités qui peuplent le microcosme décrit qui interpelle une lecture anthropologique. L'analyse du roman, donc, ne se propose pas de prendre en compte sa 
dimension esthétique ou d'en faire une critique littéraire, mais se lance dans une recherche différente. Dans le roman de Trouillot le moi narratif, en se référant à une réalité sociale spécifique, située dans l'Haïti contemporaine, s'engage dans la description de l'expérience du milieu social qui l'habite et des relations de pouvoir que le construisent. Je soutiens que, dans l'espace narratif ainsi créé, se construit sur un mode implicite une théorie engagée autour des concepts de subjectivité, agency et résistance. C'est à l'explicitation de cette théorie que le présent article se prête, à travers une lecture attentive du roman. Cela demande une méthodologie, qu'il faut repérer préliminairement à travers deux opérations : définir comment le statut de fiction du roman n'exclut pas la légitimité d'une lecture ethnographique ; expliciter en quoi cette lecture consiste et, finalement, spécifier comment elle puisse enrichir le savoir anthropologique, et ce grâce à la fonction heuristique et cognitive des romans.

L'anthropologie est considérée comme la plus littéraire des sciences sociales. Comme textes finis, les ethnographies bien écrites et les romans fondés sur l'observation sociale semblent se superposer dans un continuum de formes narratives (Narayan 1999, 136 ; Symons, Maggio 2014, 2). Le débat anthropologique, notamment pendant les années quatre-vingt, a accentué cette promiscuité, nébulisant la spécificité de l'anthropologie et menaçant de dissiper son savoir dans la fiction (ce qui l'a poussée vers une crise de la représentation). Et ce, en se focalisant en particulier sur la question de l'écriture ethnographique (Clifford, Marcus 1986). Il s'agissait d'une démarche auto-réflexive et auto-critique, motivée largement par la nécessité de se libérer de certaines excès, moralement et politiquement compromettants, de l'objectivisme. Cependant, s'il est indéniable qu'une dimension d'interprétation créative est inhérente à toutes les représentations de la vie sociale, effacer les confins entre les deux champs est surement moins productif que tenter de les définir en leur reconnaissant une certaine élasticité. Du moins, si l'on a pour but d'explorer les parfois insaisissables zones d'intersection qui subsistent dans cette relation (Łebkowska 2012, 36).

Il est évident que les logiques de représentation mobilisées par les deux champs sont hétérogènes, si l'on considère que l'ethnographie appartient au champ du véridictionnel, et la littérature au champ du fictionnel. L'anthropologie est une discipline à vocation empirique. Soit dans sa dimension plus strictement descriptive, que dans celle plus réflexive elle doit toujours répondre a des contraintes épistémologiques, méthodologiques et morales qui en assurent pertinence, référentialité et, finalement, validité scientifique (Olivier de Sardan 1995, 2003). Ces conditions de production du savoir ne peuvent aucunement être effacées par les choix stylistiques que tel ou tel autre anthropologue adopte, même s'il manifeste une inclination littéraire dans ses descriptions. Parler d'anthropologie et littérature signifie établir une « relation entre deux modes de restitution du réel qui ne présentent ni les mêmes usages sociaux ni les mêmes fonctions » (Le- 
dent 2015, 378). De plus, les ethnographies ne sont jamais désamarrées de l'expérience de l'enquête de terrain, basées sur la permanence prolongée de l'anthropologue dans le(s) milieu(s) enquêté(s) et sur la fameuse " observation participante ». Il est nécessaire tout de même de rappeler qu'il n'existe pas une « boîte à outils » définissant cette pratique, mais une « politique du terrain » (Olivier de Sardan 1995) soumise à une vigilance méthodologique qui garantit la «plausibilité » du texte ethnographique. Le souci de validité des données est au centre du travail et malgré toutes les contraintes de l'écriture, il existe une sorte de

'pacte ethnographique', qui atteste pour le lecteur que l'anthropologue n'a pas inventé les discours dont il rend compte et qu'il n'a pas rêvé les descriptions qu'il propose. Cet 'effet de réalité', dû à la mobilisation sélective de données produites lors du terrain, n'est pas qu'un procédé rhétorique. Il témoigne aussi de l'ambition empirique de l'anthropologie. (Olivier de Sardan 1995, 88)

Au contraire, le travail littéraire " même quand il s'appuie, ce qui est plus souvent le cas qu'on ne le croit, sur une documentation fournie, des observations répétées ou de véritables enquêtes » (Lahire 2010, 490) n'a pas le même souci de référentialité, explicitation et conceptualisation que le travail anthropologique. Malgré cela, le débat à l'intérieur de l'anthropologie a exploré la possibilité de «lire anthropologiquement» des textes hétérogènes, produits aux dehors des exigences de validité du savoir de la discipline (Comaroff 1992 ; Łebkowska 2012 ; Ledent 2015); et pourtant les traitant comme s'ils pouvaient contenir des données ethnographiques, ${ }^{1}$ capables de stimuler des interprétations ayant une valeur cognitive et herméneutique pour la compréhension anthropologique. Pendant les dernières décennies on a assisté au développement d'analyses concernant les traits anthropologiques en littérature. Les mondes littéraires - construit sur les confins entre cultures, avec des personnages porteurs de différents points de vue - sont les cibles de ces recherches (Łebkowska 2012, 36). Clifford Geertz a attribué une fonction cognitive aux œuvres romanesques dans un article intitulé « A Strange Romance. Anthropology and Literature » (Geertz 2003, 31). L'anthropologue Sherry Ortner a suggéré que certaines romanciers américains, comme Philip Roth, sont tout à fait des « ethnographes » dont les commentaires sur la société sont aussi importants que leurs contributions esthétiques (Ortner 2006, 35). Les romanciers ont une connaissance implicite, intuitive et théorique du monde social, grâce à l'expérience qu'ils

1 Les Comaroff, par exemple, dans Ethnography and the Historical Imagination (1992), ne cherchent pas à définir l'ethnographie dans le sens d'enquête de terrain (fieldwork), mais tentent d'explorer la possibilité d'une lecture ethnographique des fontes historiques, partiellement, comme si ces dernières s'étaient produites à travers une recherche sur le terrain. 
en font. Ils sont capables de produire de la connaissance sur un contexte anthropologique spécifique et sur les enjeux qui les hantent. ${ }^{2}$ Et ce, à travers la densité des descriptions, ${ }^{3}$ la restitution des sentiments, des expériences, des relations, des horizons de sens, des conceptions du soi, du temps, de l'espace, du pouvoir, et ainsi de suite. Comme les bons ethnographes, ils ont la faculté d'humaniser les luttes des personnes et donnent la possibilité d'explorer les façons dans lesquelles les sujets vivent leurs réalités, leurs conflits, leurs contradictions et leurs rapports avec les autres (Mellino 2005, 27). Donner corps, même si sur un plan fictionnel, aux enjeux d'un contexte réel est dans le même temps organiser un discours théorique implicite sur la réalité sociale ; discours qui a la faculté d'être explicité à travers une analyse informée par le débat de la théorie sociale. ${ }^{4}$ Ce travail d'explicitation peut avoir une fonction cognitive et heuristique pour l'anthropologue (Łebkowska 2012 ; Ledent 2015). On peut considérer le roman comme un apport descriptif et analytique à l'anthropologie, un apport qui dépasse la seule connaissance de l'écrivain. Il est donc possible pour l'anthropologie d'utiliser les œuvres romanesques en confrontant leurs récits respectifs du monde social en vue d'interroger, stimuler, illustrer, exemplifier la connaissance anthropologique. Et ce, en décelant, dans une lecture a posteriori, les commentaires sur la société dont Ortner parlait. L'idée est bien d'insister sur la capacité du roman à activer la faculté de penser. La lecture anthropologique des récits romanesques devient une exploration de problèmes théoriques existants ou émergents (Ledent 2015, 383) qu'il faut, au cas par cas, selon la spécificité du texte littéraire, définir et expliciter.

Kannjawou, de l'écrivain haïtien Lyonel Trouillot, représente les expériences de subjectivités qui habitent la Port-au-Prince contemporaine,

2 L'approche ici adoptée restreint le champ d'application d'une lecture anthropologique en mobilisant un roman qui repose sur l'« illusion référentielle » propre au réalisme (Barthes 1982). Si on veux s'appuyer à la valeur heuristique de la description d'un contexte il faut tenir compte de « la bonne connexion [qu'ils établissent] entre figuration interne et référence externe, entre le travail de l'imagination et la réalité sociale que celle-ci affronte » (Ledent 2015, 383).

3 J'évoque ici le concept de « description dense » (thick description), essentiellement comme il a été formulé par Clifford Geertz $(1998,2003)$. Cette démarche permet une herméneutique complexe et approfondie, produite grâce à un travail interprétatif joué sur une multiplicité de niveaux, de l'imprégnation culturelle incorporée à l'analyse théorique.

4 «En reconnaissant la fonction cognitive du roman pour l' [anthropologie], on reconnaît dans un même temps sa valeur heuristique, c'est-à-dire sa capacité à s'immiscer, par les problèmes potentiels qu'il pose, dans un débat théorique, immixtion qui demeure a posteriori. » (Ledent 2015 : 380). Je renvoie ici à l'article "Peut-on parler d'une sociologie implicite du roman ? » (Ledent 2015). Je considère les réflexions présentées dans cet article pleinement valides pour l'anthropologie, car l'approche implicitement considérée est celle de la sociologie qualitative. Je donne pour acquis la proximité des deux champs : on a proposé en effet le terme " socio-anthropologie » pour unir sous un même terme la sociologie dite parfois qualitative et l'anthropologie (Olivier de Sardan 2003, 14). 
ville façonnée par un contexte géopolitique complexe. Le roman est pivoté sur une mémoire individuelle, engagé dans la description dense d'un territoire occupé depuis 2005 par des délégations onusiennes et des organisations non gouvernementales. La littérature, «toujours en décalage, ou en situation d'exception', par rapport aux mots d'ordre géopolitiques » opère une « mise en connexion des espaces », en répondant à « la pression interculturelle exercée par un monde globalisé » (Garnier, Zoberman 2006, 11). Le roman met en effet en connexion les espaces, dans plusieurs sens et sur différents niveaux : les espaces physiques, ceux habités par les Haïtiens pauvres et ceux réservés aux forces d'occupation ou aux privilégiés ; les espaces émotifs, idéels et politiques qui reflètent la dialectique insaisissable et pourtant envahissante entre macro-structures de pouvoir et l'agir des individus ; les espaces de choix, ceux qui en ont à volonté et pourtant suivent des binaires constitués et ceux qui n'en n'ont pas et se lancent dans l'imagination d'un monde bouleversé, dépourvu de relations de pouvoir et habité par la solidarité.

\subsection{Pouvoir et subjectivité}

En décrivant et enquêtant sur la vie des habitants de la rue de l'Enterrement, l'agir des « corps » incarnant l' « occupation » et les contraintes et les motivations qui façonnent leurs relations, le narrateur de Kannjawou interroge un sujet cher à l'anthropologie, c'est à dire la relation entre individu et structures de pouvoir. C'est un motif de réflexion classique de la théorie sociale. Cependant, afin d'encadrer la façon dans laquelle le roman questionne le thème, je m'appuierai sur un cadre théorique qui a touché disciplines différentes et qui a eu un impact épistémologique et analytique fort sur la pensée anthropologique des dernières décennies, c'est-à-dire la théorie de la pratique. Je me référerai aux travaux de recherche de Sherry Ortner, qui se placent dans ce cadre large. ${ }^{5}$ Elle en met à jour la structure théorique avec des contributions provenant de différents secteurs de la théorie sociale (Ortner 2006). C'est pourquoi, en me nourrissant des réflexions dégagées par Ortner dans « Anthropology and Social Theory : Culture, Power, and the Acting Subject » (2006), j'ai organisé une réflexion sur le roman de Trouillot autour des concepts de subjectivité, agentivité

5 Réduite à son squelette, l'idée fondamentale de la théorie de la pratique postule que la culture, dans un sens très large (qui inclut les grandes structures sociales, économiques, politiques et les formes spécifiques des relations de pouvoir qui y sont imbriquées), construit les êtres humains comme acteurs sociaux particuliers ; mais simultanément les acteurs sociaux, à travers leur vie quotidienne, leurs pratiques variables, leurs actions sur le terrain (on-theground), reproduisent ou transforment - normalement les deux en même temps - la culture qui les a produits. L'idée, ainsi simplifiée, semble évidente, mais a beaucoup d'implications dans la méthodologie de l'analyse sociale (Ortner 2006, 129). 
et résistance. Je m'interrogerai sur la question suivante : dans l'espace de représentation du roman, comment les relations de pouvoir sont-elles conçues et quels effets ont-elles dans la structuration des subjectivités et de leurs espaces d'action?

La théorie de la pratique reprend certains éléments du concept de culture, élaboré par l'anthropologie culturelle américaine, mais donne une pertinence analytique particulière aux relations de pouvoir. Selon Ortner, les subjectivités ne sont pas culturellement façonnées seulement en tant qu'appartenant à des groupes culturels particuliers mais en tant qu'imbriquées dans des régimes de pouvoir spécifiques et historiques qui saturent la vie des individus et leurs expérience du temps, de l'espace et du soi (Ortner 1984, 14). La subjectivité est entendue comme l'ensemble des modes de perception, affection, pensée, désir et peur qui animent les sujets agents. Ce concept est situé à la base de l'« agentivité » (agency), qu'on peut entendre comme la faculté des personnes d'agir dans et sur le monde, en même temps que le monde agit incessamment sur eux. Celle-ci n'est pas le résultat d'une intentionnalité libre, mais prend la forme spécifique de désirs et de motivations à l'intérieur d'une matrice subjective. Pour lire la complexité et la signification politique de l'action des acteurs sociaux, surtout dans de forts contextes d'inégalités sociales, il est nécessaire de considérer dans le même temps tant la subjectivité que les formations culturelles et sociales qui forment, organisent et provoquent ces modes d'affection, de pensée, etc. (Ortner 2006, 107).

Dans les paragraphes suivants, je mettrai en exergue les subjectivités façonnées par le pouvoir dans le roman, et je présenterai les espaces d'action et de résistance des dominés.

\section{Le journal d'un jeune de la rue de l'Enterrement}

Le roman est construit tel un journal écrit par un jeune habitant de la rue de l'Enterrement. Cette rue se termine dans le vieux cimetière du centreville de Port-au-Prince. Encouragé par man Jeanne, doyenne de la rue, le narrateur trace une chronique de la vie sous l'Occupation molle (Trouillot $2016,171)$ du gouvernement humanitaire et militaire imposé à Haïti par la communauté internationale. Il décrit les petits destins des morts et des vivants qui peuplent son microcosme, ainsi que les structures de pouvoir incarnées dans les lieux, les corps et les discours.

Différentes générations se côtoient dans ce quartier. Tout d'abord, celle qui a connu la première occupation américaine (1915-1934). Ensuite, celle qui a vécu sous la dictature des Duvalier (1957-1986). Et enfin, celle qui a grandi sous la période que le narrateur définit seconde occupation (2004-présent). Ces générations ont en commun la lutte quotidienne pour la survie. Au centre de la narration se trouve la « bande des cinq ». C'est 
un groupe d'amis d'enfance - désormais jeunes adultes - qui se désagrège au fil des années. Cette bande est composée du narrateur lui-même, de son frère Popol, Wodné et de deux sœurs, prénommées Sophonie et Joelle. Tout comme les autres membres de la bande, le narrateur fréquente la faculté de sciences humaines de l'Université d'État d'Haïti. Par contre, Sophonie a quitté les études après la licence. Durant son temps libre, le narrateur questionne les itinéraires de vie des gens avec le petit professeur, ex-militant qui enseigne à l'Université. Ils interrogent leur contexte de vie en puisant l'inspiration dans la lecture des romans. Les deux en effet aiment dédier leur temps à la lecture. Les personnages fictionnels des œuvres romanesques stimulent leur enquête des motivations, des désirs, des émotions qui guident les actions des habitants des quartiers défavorisés et des étrangers qui occupent leur pays. A travers cette enquête, les effets des relations de pouvoir sont questionnés. L'alliance entre « anthropologie en tant que discipline et littérature en tant que forme de connaissance » (Fabre dans Danetti, Lo Feudo 2013, 8) est ainsi évoquée à travers une démarche métalittéraire, qui réfléchit implicitement sur les usages possibles de la littérature dans la compréhension de la réalité sociale.

Bien qu'il soit issu d'un milieu social plus aisé, le petit professeur est arrivé à la rue de l'Enterrement pour soutenir le centre culturel ouvert par la bande des cinq. Et ce, dans le but d'offrir un lieu d'agrégation et la possibilité d'une éducation aux enfants de la rue. Dans les itinéraires urbains tracés par le narrateur lors de ses promenades, un nœud est le bar Kannjawou, qui se situe au dehors de son microcosme défavorisé. Le fait que Sophonie y travaille comme serveuse offre au narrateur et à son frère Popol - petit ami de la fille - l'occasion d'y aller chaque mercredi, à la soirée dansante. Ils y observent à distance les clients étrangers qui travaillent pour le 'bras civil de l'Occupation'.

\section{D'une terre sans vie et des corps occupés}

À partir de la période de la dictature de Duvalier, l'espace textuel de la littérature haïtienne est pénétré par une « esthétique de la dégradation » (Lucas 2004). Dans le système symbolique qui la caractérise, basé sur le sens de ruine et du délabrement, les personnages sont souvent menacés par un processus de zombification (56). L'image du zombie, enracinée dans l'histoire culturelle d'Haïti, est porteuse d'un un grand potentiel pour la production de sens. Dans le contexte de Kannjawou il faut l'entendre comme l'état provoqué par la forme envahissante et violente du pouvoir, qui affecte mentalement et physiquement les sujets, en les démembrant, les privant d'esprit vital et neutralisant leur capacité à se révolter.

À partir des premières pages du roman, les dimensions émotives dominantes se réunissent autour du thème de l'impuissance (Trouillot 
2016, 21) et d'états d'esprit connectés : " sentiment d'abandon », « colère », « défaite » $(21,22)$, qui sapent la faculté d'action des sujets. Par exemple, le débarquement des troupes de l'ONU en 2004 est accueilli dans l'immobilité. À l'exception de quelque voix de protestations, c'était « comme si les gens s'étaient couchés », abandonnés à l'idée " qu'il ne restait plus rien à préserver. Ni rêves. Ni dignité » (21). C'est le début de ce que man Jeanne, témoin de la première Occupation des marines (1915-1934) a baptisé seconde Occupation. Le narrateur déclare que dans son journal «ne se passe rien », parce que " un pays occupé est une terre sans vie, [...] une terre sans ciel et sans ligne d'horizon » (29) et que tout ce qu'il pourrait livrer " ne serait que l'expression du désespoir ou le combat pour la survie ». Le thème de la zombification se manifeste sur les visages tristes que l'aube surprend aux fenêtres, sur le corps des femmes qui sortent balayer l'entrée de leurs maisons « avec des gestes mécaniques » en s'échangeant « des saluts aussi mécaniques que leurs gestes » (30). Ces états d'esprit s'entrecroisent dans un sens du temps calqué sur le présent, lié aux nécessités de la survie quotidienne : "Survivre peut être un travail à plein temps qui consomme toute [...] énergie. Quand tu ne sais comment tu vas finir le jour, il n'y a dans ta vie ni hier ni demain, ni rêve ni mémoire » (13). Le pouvoir, tissé dans toutes les expériences de temps, espace, interaction, participe activement à l'usure des liens affectifs.

Aujourd'hui, il n'y a plus de bande des cinq. [...] Peut-être n'y a-t-il rien de pire que d'atteindre l'âge adulte dans une ville occupée. [...] Nous avons perdu ce bien commun, toujours virtuel, qui s'appelle l'avenir. Nous sommes dans un présent dont nous ne sommes pas les maîtres. Chaque uniforme, chaque démarche administrative que nous devons entreprendre, chaque bulletin de nouvelles, tout nous rappelle à notre condition de subalternes. (45)

Un autre aspect touché par l'esthétique de la dégradation est le paysage - tant à la campagne que dans la capitale - qui se dégrade d'une façon étroitement liée à la décomposition du tissu social. Le paysage urbain, traversé et habité par le narrateur et les autres personnages, est lié à leur compréhension émotive, cognitive et politique de la réalité, et façonne l'intégralité de leurs subjectivités. Les lieux dans le roman sont souvent le reste d'un passé précipité dans un état de ruine. C'est le cas de l'architecture du Grand Cimetière :

les fleurs qui bordaient autrefois les allées avaient séché. Le bleu roi avait pâli et l'on voyait sous la peinture la couleur triste de la pierre. [De la dernière demeure] ne restait qu'un réduit aux dehors délabrés, exilé dans un marécage. (39) 
Le grand cimetière est un lieu qui porte l'inscription d'un temps révolu, lorsqu'il accueillait des gens riches et importants, mais aussi les dynamiques successives de l'augmentation de la pression démographique, de l'exode rural et de la bidonvilisation de la capitale. Les riches, avec la croissance exponentielle des vivants dans l'espace urbain et, par conséquent, des morts dans un cimetière désormais débordant, "sont allés mourir ailleurs » (18), explicitant la « guerre de mouvement » (18) qui amène la classe sociale dominante à se distancier de la masse pauvre. L'espace urbain matérialise les profondes inégalités sociales : il est clairement polarisé entre les espaces des gens riches et ceux des gens pauvres, lieux accessibles et lieux inaccessibles. En habitant et en traversant le paysage, en faisant quotidiennement l'expérience de la physionomie inégale de l'espace, les personnages comprennent corporellement la structure de la société, l'action du pouvoir, leur position de marginalité. Le paysage est dressé par une dimension intime faite de mémoire, d'affect, d'émotion. Par exemple, la nostalgie des promenades sur les collines est animée de sentiments positifs. C'est une époque où ces amis étaient unis ; mais c'est aussi une période qui coïncide avec le souvenir de la prise de conscience précoce de leur condition d'exclus : "Nous avions la ville à nos pieds. Deux villes. Celle que nous connaissons. Et celle que nous ne pouvions qu'imaginer » (40).

\subsection{Le corps heureux de l'occupant}

Le narrateur livre des commentaires sur la logique du pouvoir en confrontant l'occupation molle du temps présent (171) aux moments historiques révolus. Les centres de pouvoir, disloqués globalement, agissent dans le pays via discours, pratiques et interventions qui se fondent sur les concepts de coopération, développement, stabilisation, aide humanitaire. Une forme de « gouvernementalité » (Foucault 2004, 111, 112) que Didier Fassin a défini « raison humanitaire » (Fassin 2010). La violence de l'histoire habite une économie morale que le narrateur lit en termes de mystification : la structure humanitaire perpétue la dépendance ; la démocratie formelle des élections libres et de la liberté de parole cache le contrôle exercé par la communauté internationale, en oblitérant les dynamiques qui reproduisent les écrasantes inégalités sociales. La « ruse de l'occupant » (21) a produit une situation dans laquelle, comme le petit Professeur le dit « tout est mou et se donne pour autre chose » tandis que dans le passé « les choses étaient peut-être plus nettes. Moins molles. L'Occupation. La Résistance » (160).

Le fait clair et évident est que les occupants ne partagent pas les espaces de vie des Haïtiens : "On peut deviner leur présence derrière les vitres fumées des voitures de luxe et des véhicules officiels » (16). 
Mais il y a un lieu qui permet au narrateur de les observer, le Kannjawou. Chaque mercredi son frère et lui passent le temps dans un coin à l'écart de ce bar et, ignorés par les clients, ils s'amusent à les baptiser à partir d' « un trait ou un rituel » qui les caractérise - avec des surnoms comme Petite brune, Grande blonde, Le petit chose, La hyène et Les trois mousquetaires (73). C'est surtout leur appartenance au même clan qui est soulignée. Le narrateur décrit les étrangers se réunissant au bar pour consommer un divertissement frénétique et excessif, comme « un monstre compact » à plusieurs têtes et à plusieurs jambes : experts, consultants, coopérants, technocrates, personnes jeunes, éduquées et liées entre elles non par une réelle connaissance réciproque mais plutôt par leur style de vie, par les dispositions et les habitus (Bourdieu 1980) de leurs professions :

N'ayant rien partagé d'intime à part le fait de vivre bien au royaume des pauvres [...] nous les entendons parfois se demander : ne nous sommesnous pas rencontrés à Dhaka ou à Kigali ? Mais oui, moi j'étais avec tel organisme. [...] Eux-mêmes, entre eux ne sont pas des personnes, mais des fonctions. Ce qui les lie, c'est les coutumes du clan. (Trouillot 2016, 75)

Les membres de ce clan ont adopté et naturalisé les règles, les idéologies et les pratiques de la position structurelle qu'ils occupent dans un champ social globalisé. Le narrateur en dépeignant leurs techniques du corps, leurs habitudes, leurs interactions et leurs attitudes mentales, fait émerger le discours qui fonde leurs habitus. Ce discours nous montre que le pays est réduit à sa seule condition de sous-développé sans tenir compte de l'aspect historique et présuppose que le corps haïtien (pauvre) soit marginal et ambivalent : objet d'aide et de violence en même temps, corps à penser à travers les lentilles de la « raison humanitaire » ou bien objectivés et destitués d'humanité (nombreuses sont les références aux épisodes de violence sur des jeunes Haïtiens, impliquant le personnel des forces d'occupation). Qu'ils soient Haïtiens, Bangladais ou Africains - peu importe - ces corps sont cristallisés dans le besoin et ne sont pas vus dans leurs complexité subjective, culturelle, historique. Au contraire :

Au Kannjawou on voit des corps qui ne doutent pas. Comme une pensée unique. Heureux les corps des occupants. Les intellos ont beau parler de structures et de faits globaux, l'Occupation, c'est d'abord des corps. [...] Le monde n'est que corps. Rapport d'un corps à l'autre. À lui-même. À l'espace. (Trouillot 2016, 133, 138)

Le corps de l'occupant est protégé et libre. Protégé comme celui du coopérant qui, sorti ivre du Kannjawou, a renversé un Haïtien avec sa voiture de service et a été prestement mis sur un avion par son organisation dans le but d'enterrer l'accident (Trouillot 2016, 139). 
Le corps de l'occupant est libre car il peut aller et venir, changer de pays, traverser des confins : "Ils détruiront le monde pour payer leurs voyages » (Trouillot 2016, 143). La structure du pouvoir se fonde sur la mobilité des experts et sur le principe de rotation, rendant problématique la connaissance approfondie des contextes locaux. Dans le roman, il y a une seule rencontre entre les occupants et les occupés. Celle-ci devient possible parce que la souffrance a momentanément dépouillé une habituée du bar, une fille française surnommée " petite brune », des règles et des conduites liées à sa position, permettant une rencontre sur le plan humain avec les jeunes de la rue de l'Enterrement. Une nuit elle se retrouve ivre et en proie au désespoir amoureux « dans la mauvaise moitié de la ville, celle dans laquelle les documents qu'elle avait signés pour obtenir son poste lui interdisaient de circuler » (Trouillot 2016 104). Devenue corps vulnérable au dehors des espaces protégés de son clan, elle est aidée par Sophonie, Popol et le narrateur. Elle est hébergée par man Jeanne. Au début, elle est effrayée et méfiante des mains et des visages noirs qui vont à son secours. Progressivement, elle prend confiance et accepte l'aide qu'on lui offre. La petite brune montre qu'elle n'a pas de compréhension critique de sa position dans les structures de pouvoir. Lorsque man Jean l'interroge sur sa capacité à distinguer le vrai du faux, elle lui répond qu'elle

N'était quand même pas venue ici pour commettre des crimes ! [...] elle ne comprenait pas. Il y avait un poste, une offre d'emploi. Une compétence. Un boulot. Avec l'accord des autorités locales. Ça lui suffisait. [...] C'était une façon de découvrir. D’aider. Enfin, elle croyait. (Trouillot 2016, 105)

Ici est représenté la difficulté, voire la presque impossibilité, de rencontres avec les étrangers. Ces dernières, dans l'espace de représentation du roman, incorporent les structures de pouvoir et leur agir, qui, apparemment voué à l'aide, n'est en fait qu'une adhésion aux politiques qui dominent le pays. Leurs horizons symboliques et leur pratiques en sont saturés et jouent à reproduire le système. Tout autre espace d'action demeure au dehors du pensable, à moins que, comme le cas de la petite brune, la douleur et la frustration ne le poussent, involontairement, à la recherche d'alliances humaines, de soutien et de solidarité temporaire.

\subsection{Quelle résistance?}

On a vue quel scénario émotif prédomine dans la vie des Haïtiens. L'absence d'espoir et le sentiment d'être occupés sur le niveau des conditions matérielles de vie (l'occupation perpétue la misère) et psychologique (l'absence d'espoir, le cul-de-sac émotif). Mais quel genre d'espace d'action est 
possible pour les « subalternes » (Trouillot 2016, 45)? Quel est leur pouvoir d'intervention sur la réalité ? Il faut considérer la notion d'agentivité.

La notion d'agentivité est prise dans deux champs de signification, deux « visages » qui s'entrelacent dans une relation façonnée comme le ruban de Möbius (Ortner 2006, 139). D'un côté le concept se réfère à la vie activement jouée par les sujets et orientée vers des fins culturellement constituées, impliquant pratiques routinières et actions internationalisées. ${ }^{6}$ De l'autre côté, l'agentivité concerne plus spécifiquement le pouvoir, l'agir à l'intérieur des relations sociales marquées par l'inégalité, l'asymétrie et la force. Même si la résistance est l'une des tant de formes d'agentivité, elle a été souvent assimilée à l'idée d'agentivité tout-court. Dans un sens très général, il est question de contexte. Il est évident que l'effort de soutenir une vie culturellement significative dans des situations de domination à large échelle (par exemple l'esclavage, le colonialisme, le racisme) se confond avec la manière dont les sujets résistent à la nature envahissante du pouvoir. La résistance inclut un éventail qui va des révoltes générales jusqu'à l'acceptation ambivalente et complexe des catégories du dominant. Ce qui donne un sens nouveau à une pratique. À l'intérieur des relations de pouvoir, l'agentivité dégage pour les dominés un ensemble d'émotions, raisons, actions et motivations complexes et contradictoires, qui articulent des positions différentes. Qu'il soit question de poursuivre des projets culturellement significatifs aux marges du pouvoir ou de poursuivre une résistance collective organisée, presque toujours et presque nécessairement, il y a des relations internes de pouvoir, au niveau local des communautés, à considérer.

La résistance n'est pas tout simplement une réaction compacte des sujets dominés et le roman l'explore de l'intérieur, avec ses complexités, ses contradictions et ses conflits. Dans le roman une tension se manifeste entre les projets collectifs, fondés sur la tentative difficile d'élaborer une cause commune. Les projets individuels semblent inexorablement se dessouder de la dimension collective. Les cinq amis de la bande ont tous eu accès à l'instruction universitaire, ayant ainsi une position relativement privilégiée par rapport à la majorité des jeunes Haïtiens : " N'en déplaise à Wodné qui aime voir en nous les damnés de la terre, nous sommes déjà allés dans des lieux où d'autres n'iront jamais » (Trouillot 2016, 54-5). Ils ont une compréhension critique de leur propre condition et depuis l'enfance ils ont été animés par le désir de s'opposer activement à l'occupation en vue d'un kannjawou final. Mot lui-même détourné par un occupant qui s'est approprié le pouvoir et les joies, ce mot créole signifie

6 L'intentionnalité indique toutes les manières dont l'action est cognitivement et émotionnellement orientée vers quelque objectif, mais elle n'implique jamais un agent sans entraves et libre dans un vacuum social : l'acteur est toujours imbriqué dans une multiplicité de relations sociales et, pour cela, n'a jamais un plein contrôle sur les résultats de ses actes. 
originairement 'fête, partage'. La signification traditionnelle du mot se trouve chez Anselme, le père de Sophonie et de Joelle, corps faible et malade, qui « avec ses jambes de peau et d'os » (Trouillot 2016, 47) est contraint à rester au lit. Avant de mourir, Anselme rêve de retourner sur la terre qui lui a été soustraite tant d'années auparavant dans la région de l'Archaïe, en le forçant à migrer dans la capitale. Le rêve du retour coïncide avec le rêve d'un dernier kannjawou organisé en son honneur. Une grande fête dans laquelle tous les voisins ainsi que les autorités locales du vaudou seraient invités. Ils y partageraient la nourriture et le clairin et danseraient sur les rythmes de la musique rara. ${ }^{7}$ Anselme ne réalise pas que tout cela n'est plus possible parce que la campagne d'où il vient « est une tombe et personne n'a envie d'être enterré vivant » (Trouillot 2016,47). Si cette pratique sociale coopérative a disparu à cause de l'état de dégradation du tissu social à la campagne, le mot qui la définit se charge pour la bande des cinq et pour le petit professeur d'une signification plus large, quasi mystique. À leurs yeux le mot incarne un élan vers le bouleversement du pouvoir, vers la fin de l'occupation et l'égalité de tous les êtres humains. Mais l'élan se dissout à cause de l'occupation. La possibilité d'émancipation de la population semble s'ouvrir seulement sur un plan individuel et individualiste. Pour des jeunes cultivés mais pauvres, l'échappatoire est la bourse d'étude vers l'étranger : «ce sera bientôt chacun pour soi dans la course à la bourse » (Trouillot 2016, 49). Le narrateur remarque que les séjours à l'étranger font revenir des gens parlant un autre accent et succombant aux discours désintégrateurs qui représentent Haïti de l'extérieur. Il cite par exemple un professeur de l'Université qui « est né à trente ans lors de son premier voyage à l'étranger. Sa bourse a accouché de lui » (Trouillot 2016, 32). À l'étranger il a appris que la condition de sous-développement du pays est due à une carence chronique de pensée scientifique qui en caractérise la culture. Un exemple parfait de violence symbolique (Bourdieu 1994, 188), où les catégories des dominants sont adoptées et incorporées par les dominés. Le fait que «dans un pays occupé, il y a de fortes chances que ceux qui travaillent soient des subalternes de l'occupant » (Trouillot 2016, 91), contribue à défaire la possibilité de la construction d'un soi collectif et crée de la division.

Le roman décrit pourtant des petits actes de résistances quotidiennes (man Jeanne jette du pissat de chatte sur la tête des gens trahissant les règles de base d'humanité ou collaborant avec l'occupant) ou des pratiques de coopération et solidarité au niveau communautaire. Les subjectivités gardent un espace de contestation et un sens de dignité en travaillant à

7 Clairin : eau-de-vie produite en Haïti à partir de la canne à sucre et de façon artisanale. Musique rara : forme musicale traditionnelle jouée avec des trompettes en bambou, tambours, maracas et d'autres instruments à percussion. 
l'intérieur de l'espace contrôlé par le dominant (de Certeau 2001, 60-2), en soutenant des projets culturellement constitués aux marges du pouvoir (Ortner 2006, 147). À ce propos, on peut mentionner le centre culturel, résultat du désir de la bande de créer un lieu d'agrégation et d'éducation pour les enfants du quartier, très souvent orphelins (comme le narrateur et son frère Popol) ou enfants d'un seul parent (comme Sophonie et Joelle). Cet endroit est en même temps un laboratoire de résistance - ce n'est pas un hasard que l'un des seuls éléments décoratifs ici présent soit une photographie de Charlemagne Péralte, héros de la résistance durant la première Occupation. ${ }^{8}$ Mais la résistance ouvre aussi la voie à des rivalités et des conflits. Les fortes tensions qui s'ouvrent parmi les cinq amis, ainsi que les raisons qui conduisent à l'expulsion du petit professeur du centre culturel, gravitent en particulier autour de l'attitude de Wodné. Il est le chef d'un groupe radical d'étudiants militants et chef autoproclamé du centre culturel. Wodné a une façon de conduire la lutte qui rappelle dangereusement au narrateur la logique de division de l'occupant. Wodné est animé par la peur et la haine et suit la logique de l'exclusion. Son groupe est autoréférentiel et fermé. Ses actions et ses choix définissent les confins d'un petit royaume où une seule ligne de conduite et de pensée est approuvée. Cela et les situations narratives qui en suivent - y compris la soumission imposée par Wodné à sa copine Joelle, qui accepte de lui être subordonnée et en même temps rêve la fuite à l'étranger - provoquent chez le narrateur une profonde amertume, sentiment qui amène à l'interruption temporaire du journal.

Le premier des deux chapitres s'arrête sur la perte de confiance du narrateur dans l'utilité des mots. Tandis que dans le second chapitre - qui marque la reprise du journal après quelque mois d'interruption - la parole lui redevient nécessaire. Tout d'abord pour chercher à donner sens à un événement dramatique, c'est-à-dire la mort du professeur. Le suicide de ce dernier lie des motivations intimes (il était amoureux de Joelle) à une usure générale de son horizon d'espoir pour le pays. Le narrateur est lié au professeur par une affinité profonde qui dépasse leurs différents milieux sociaux et sa mort le pousse à défier sa propre nature contemplative et l'amène à prendre une position active dans la société. Son écriture reflète ce choix. Il veut devenir militant. Pour cela, il prend contact avec un vieux militant, Monsieur Laventure, puni de plusieurs années de prison pour son action politique, et avec qui le petit professeur avait milité dans sa jeunesse. La cohérence de Monsieur Laventure et la clarté pragmatique de ses luttes pour les paysans semblent au narrateur un moyen d'agir plus efficace que les mots.

8 Chef de la résistance durant la première occupation, tué par les marines des États-Unis. Ils ont ensuite photographié son corps martyrisé et ont lancé par avion des reproductions de cette image, afin de dissuader les Haïtiens de la révolte. 
D'un côté, quelque chose de partagé par man Jeanne, le professeur et Sophonie : l'idée que chaque interaction humaine doit être fondée sur l'amour et le respect de l'autre. De l'autre côté, le rêve du kannjawou, lequel, à la fin du roman, prend la forme d'un conte créé par le narrateur pour les enfants du centre culturel. Ce conte incarne l'idéal mystique et utopique de bouleversement du réel : la fin de toute occupation, de toute domination, l'instauration de l'égalité, l'abolition des confins et des injustices. Lucas remarque que dans la littérature haïtienne contemporaine la forte présence d'une esthétique de la dégradation se tisse à une forte et radicale dénonciation des maladies sociales, et la possibilité du changement prend une valeur mystique (Lucas, Mitsch 2004, 54). Cela est vrai pour le roman de Trouillot, où le kannjawou final rêvé par le narrateur devient un lieu inexpugnable, parce que c'est un non-lieu, une utopie (de Certeau 2001, 48).

\section{Bibliographie}

Barthes, Roland (1982). "L'effet de réel ». Genette, Gérard (éd.), Littérature et vérité. Paris : Seuil, 81-90.

Bourdieu, Pierre (1994). Raisons pratiques. Paris : Seuil.

De Certeau, Michel (2001). L'invenzione del quotidiano. Roma : Edizioni Lavoro.

Disegni, Silvia ; Lo Feudo, Michela (2013). «Littérature et anthropologie ». Recherches \& Travaux, 82(1), 3-28.

Clifford, James; Marcus, George E. (1986). Writing Cultures. Berkeley : University of California Press.

Comaroff, Jean and John (1992). Ethnography and the Historical Imagination. Boulder ; San Francisco ; Oxford : Westview Press.

Fassin, Didier (2010). La raison humanitaire : une histoire morale du temps présent. Paris : LeSeuil/Gallimard.

Foucault, Michel (2004). Sécurité, territoire, population. Paris : Seuil.

Garnier, Xavier ; Zoberman, Pierre (2006). Qu'est-ce que l'espace littéraire ?. Vincennes : Presses Universitaires.

Geertz, Clifford (1998). Interpretazione di culture. Bologna : il Mulino.

Geertz, Clifford (2003). " A Strange Romance : Anthropology and Literature ». Profession, 9, 28-36.

Ingold, Tim (1993). "The Temporality of the Landscape ». World Archaeology, 25(2), 152-74.

Lahire, Bernard (éd.) (2011). Ce qu'ils vivent, ce qu'ils écrivent. Mises en scène littéraire du social et expériences socialisatrices des écrivains. Paris : Éditions des archives contemporaines.

Łebkowska, Anna (2012). "Anthropology of Literature and Literary Anthropology ». Teksty Drugie, special issue, 2, 19-29. 
Ledent, David (2015) « Peut-on parler d'une sociologie implicite du roman ? », Revue d'anthropologie des connaissances, 9(3), 371-86.

Lucas, Rafael ; Mitsch, Ruthmarie (2004). «The Aesthetics of Degradation in Haitian Literature ». Research in African Literatures, 35(2), 54-74.

Mellino, Miguel (2005). La critica postcoloniale. Decolonizzazione, capitalismo e cosmopolitismo nei Postcolonial Studies. Milano : Booklet.

Narayan, Kirin (1999). "Anthropology and Fiction : Where Is the Border ? ». Anthropology and Humanism, 24(2), 134-47.

Olivier de Sardan, Jean-Pierre (1995). « La politique du terrain. Sur la production des données en anthropologie ». Enquête, 1, 71-109.

Olivier de Sardan, Jean-Pierre (1998). «Émique ». L'Homme, 38(147), 15166. Alliance, rites et mythes.

Olivier de Sardan, Jean-Pierre (2003). « Observation et description en socio-anthropologie ». Blundo Giorgio ; Olivier de Sardan, Jean-Pierre (éds.), Pratiques de la description. Paris : EHESS, 13-39. Enquête.

Ortner, Sherry Beth (1984). "Theory in Anthropology since the Sixties ». Comparative Studies in Society and History, 26(1), 126-66.

Ortner, Sherry Beth (2006). Anthropology and Social Theory: Culture, Power, and the Acting Subject. Durham : Duke University Press.

Symons, Jessica ; Maggio, Rodolfo (2014). "'Based on a True Story': Ethnography's Impact as a Narrative Form ». Journal of Comparative Research in Anthropology and Sociology, 5(2), 1-6. 


\title{
Stanley Péan et l'interculturalité en diaspora Musique, zombis et marasa pour une haïtianité revisitée
}

\author{
Fabiana Fianco \\ (Università Ca’ Foscari Venezia, Italia)
}

\begin{abstract}
In spite of being viewed as a young writer until the '90s, Stanley Péan is now known as one of the most distinctive and established voices in the Haitian-Canadian literary scene. The pivotal moment in his career happened in 1996, when Zombi Blues was published. This novel displays a cultural space in which Haitian traditions and Canadian modernity converge and allow intercultural exchange to take place. Drawing from this perspective, the following article aims to analyse how Péan creates a fictional universe through the blending of cultural elements. Using the collection of myths and beliefs that permeate the Haitian and African cultural panorama as a reference point, we will investigate the ways in which Péan adapted and transposed these traditions to the Haitian diasporic context. Particular attention will be given to the use of jazz and African American music, as well as to the reinterpretation of mythological creatures such as the zombie and the marasa twins. Hence, the article tries to show how Péan's cultural crossroad contributes to the foundation of a new literary interpretation of Haitianity.
\end{abstract}

Sommaire 1 Introduction. - 2 Zombi Blues: un jeu de funambule entre jazz, blues et tam-tam. 2.1 Une narrative musicale : quand la musique parle ‘culture'. - 3 Un zombi à moitié, ou le trompe-l'œil haïtien. - 3.1 L'irruption du fantastique métissé. - 4 Marasa : la rencontre des altérités. - 5 Conclusion.

Keywords Stanley Péan. Interculturalism. Haïtianité. Diaspora. Music and semiotics. Fantastic. Marasa and zombie.

\section{Introduction}

Port-au-Prince, années '60. Dans le climat de terreur politique diffusé par la dictature duvaliériste, une « Négresse fluette » (Péan 1996, 24) erre dans les rues de la capitale haïtienne en pleine nuit. Dans un état de confusion et de désespoir, elle s'introduit furtivement dans l'habitation d'un diplomate canadien, Ben Reynolds, et de sa femme, Corinne. Grièvement blessée, elle tient dans ses bras un bébé emmailloté dans une serviette et implore la Canadienne de ne pas prévenir les tontons macoutes qui la poursuivent. Elle meurt peu après, laissant au couple de visiteurs le petit bébé à l'étrange lueur écarlate dans les yeux. C'est ainsi que Stanley Péan, écrivain québécois d'origine haïtienne, nous plonge dans l'univers merveilleux et énigmatique 
de Zombi Blues. Publié en 1996, c'est le deuxième roman pour adultes signé par un auteur qui n'a pas directement connu les atrocités de la dictature des Duvalier, ni la violence effrénée des tontons macoutes. Forcé à déménager au Québec en 1966 quand il n'avait que quelques mois, il a pourtant hérité de sa famille un patrimoine culturel lui permettant d'intégrer à son univers nord-américain les us et les coutumes du pays natal haïtien.

Dès les débuts de sa carrière, Péan a ainsi réussi à faire de sa littérature le carrefour idéal entre Haïti et le Québec, en jouant quant au premier sur ses « réminiscences enfantines » (Olivier 1998, 8) et sur le pouvoir de la fiction Aussi, dans ses premières productions pour la jeunesse il construit un imaginaire haïtien nourri par « le regard et la mémoire d'autrui » et riche de «souvenirs empruntés et de plagiat éhonté » (8). En effet, contrairement à d'autres écrivains d'origine haïtienne en diaspora, Péan n'a visité son île natale que sporadiquement et par intermittence : pendant des brèves vacances d'été à l'âge de 9 ans ; en 1997 lors du tournage du documentaire Francoeur : Exit pour Nomades; en 1999 pour prendre part à un symposium multidisciplinaire avec des compatriotes venus du monde entier. ${ }^{1}$ En raison d'une situation politique fortement instable et des fréquentes catastrophes naturelles qui ont récemment frappé Haïti, son dernier voyage date de 2014. Le manque d'un contact continu avec son pays natal explique en partie le sentiment de déracinement que l'auteur évoquait dans La Plage des songes en 1988 : «Tu t'absentes de ta patrie trop longtemps, la métamorphose s'opère, l'exil fait de toi un intrus, un étranger partout même chez toi, la distance et les années creusent un gouffre-indifférence » (cité dans Hoffmann 1995, 305).

Malgré la conscience de ne pas complètement appartenir à une réalité enfantine très changée depuis son départ, Haïti reste un élément constant de la production romanesque péanienne. Tant son style que les thèmes abordés dans ses œuvres témoignent d'une identification symbolique et affective avec le peuple haïtien et restituent avec force les traumatismes qui en ont marqué la mémoire collective. D’ailleurs, ce qui fait l'unicité de Péan est surtout sa capacité à osciller entre deux cultures qui lui appartiennent au même degré, l'une haïtienne et l'autre québécoise. Ce dualisme identitaire l'a rendu sensible et perméable à une écriture interculturelle, qui échappe ainsi à tout classement esthétique se voulant rigide ou définitif. Dany Laferrière est parmi ceux qui ont saisi en premiers la singularité du jeune écrivain :

C'est l'écrivain du world beat. Il peut travailler le matin sur un sujet haïtien pour une maison d'édition haïtienne, tout en corrigeant l'après-midi ce

1 Péan, Stanley (2014). Carnet de Voyage en Haïti par Stanley Péan [en ligne]. URL http:// WWW.clownssansfrontieres.ca/carnet-de-voyage-en-haiti-par-stanley-pean-fier-portevoix/ (2018-11-30). 
roman à l'intention des jeunes Québécois [...] et terminer le soir un recueil de nouvelles de science-fiction pour des lecteurs qui passent le weekend sur la planète Mars. Tout cela a tellement décontenancé la critique haïtienne qu'elle ne sait vraiment pas où classer Péan. (Laferrière 2000, 62)

Cette indétermination littéraire est directement liée au pluriculturalisme qui caractérise son identité. À ce propos, Péan affirme : "Dans la vie de tous les jours, je ne suis pas obnubilé par la nécessité de me définir »(Olivier 1998, 8). Et c'est précisément cette porosité définitionnelle qui traverse Zombi Blues, nous permettant d'explorer la manière avec laquelle un auteur à mi-chemin entre deux mondes et ouvert aux nouvelles vagues artistiques américano-québécoises réussit à tout condenser en 310 pages. Nous commencerons par interroger l'usage que Péan fait de la musique comme espace de construction d'une identité interculturelle dans la perspective de la sémiotique narratologique et musicale. Ensuite, nous nous focaliserons sur la reconfiguration fantastique de la figure mythique du zombi haïtien. Finalement, nous analyserons les jumeaux marasa, les dieux du vaudou, comme éléments fonctionnels à l'achèvement de l'univers 'métissé' du roman.

\section{Zombi blues : un jeu de funambule entre jazz, blues et tam-tam}

D’après les références bibliographiques de la base des données de la MLA, il y a une dizaine d'années les études académiques concernant Stanley Péan et son esthétique romanesque pouvaient se compter sur les doigts d'une main. ${ }^{2} C^{\prime}$ est seulement depuis peu que la critique a su apprécier une écriture qui bouleverse le panorama des romans haïtiano-québécois. ${ }^{3} \mathrm{Si}$, à une première lecture, Zombi Blues paraît présenter plusieurs points de convergence avec les romans de Phelps, Ollivier, Étienne ou Laferrière, il est néanmoins indéniable qu'il y a aussi des éléments déstabilisateurs par rapport à ces grands prédécesseurs. En effet, nous observons que d'un côté, Péan a recours au réalisme merveilleux de la même manière que ses collègues, c'est-à-dire comme solution à l'impossibilité de reproduire fidèlement tous les changements qui ont affecté la mère patrie pendant leur

2 Cf. Ransom 2009, 79. Il faut néanmoins préciser que notre recherche sur Stanley Péan n'a produit à l'heure actuelle que 8 résultats, dont 4 sont des articles écrits par l'auteur luimême. Cf. Modern Language Association. URL https://www.mla.org (2018-11-30).

3 Nous signalons pourtant que les premières publications portant sur Stanley Péan et sur ses œuvres ont commencé à paraître à partir des années 1990. Nous renvoyons à ce propos aux références bibliographiques établies par Thomas C. Spear sur Île-en-île. Cf. Spear, Thomas C. (2016). «Stanley Péan ». Île-en-île. URL http://ile-en-ile.org/pean/ (2018-11-30). 
absence (Hoffmann 1995, 311). De l'autre, il donne à ce réalisme quelque peu figé une fraîcheur culturelle incontestable et par le recours constant à la musique qui envahit tout le tissu romanesque.

Fervent mélomane depuis sa petite enfance, Péan reconnaît effectivement à la musique une place privilégiée dans son écriture. Plutôt que concevoir la littérature et la musique comme séparées l'une de l'autre, il les envisage selon une perspective esthétique d'intégration. Aussi, il « rédige au son de la musique » et « pour l'équilibre, pour la musique de la phrase (sa musicalité, son caractère mélodieux), il relit son texte à voix haute » (Noël-Gaudreault 2003, 110). Du reste, Péan a cultivé sa sensibilité musicale grâce à une discothèque familiale « hyper éclectique » (Péan 2014) qui lui a offert une culture musicale parmi les plus diversifiées et complètes : à côté de la musique populaire de danse haïtienne et africaine, en passant par la chanson française de Brel, Ferré et Gréco, il a découvert le rock'n'roll, le pop et, immanquablement, le jazz. C'est ce dernier qui occupe une place de premier plan dans son univers artistique et qui s'est révélé une véritable charnière entre musique et littérature. Son penchant pour le jazz, dont il est d'ailleurs spécialiste, ${ }^{4}$ émerge clairement dans plusieurs de ces textes littéraires : Blues, en rouge sur blanc (1993), Zombi Blues (1996) et Le Temps s'enfuit (1999). Le premier n'étant qu'une brève nouvelle où le jazzman apparaît comme simple figurant, c'est avec Zombi Blues que l'auteur accomplit le mélange longtemps souhaité entre le jazz et le romanesque.

Mais pourquoi le jazz ? D’abord, parce que Péan établit un rapport de complémentarité entre l'homme de lettres et l'homme de musique quand il affirme : «Comme écrivain, j’aime penser qu'on peut être aussi libre qu'un jazzman », en précisant que dans les deux cas « on part d'une base commune et on essaie de raconter une nouvelle histoire » (Péan 2014). Ainsi, par l'affinité reconnue entre ces deux arts, il redéfinit le champ d'expérimentation de l'écrivain dans une optique interdisciplinaire qui rend les frontières culturelles plus floues que jamais. Du reste, cette perméabilité entre musique et littérature renforce ultérieurement son inclination et son amour pour le jazz. Né de la rencontre entre la musique classique européenne et les musiques africaines, le jazz permet à ce « borisvianesque écrivain-jazzman amateur » (Hamel 2007, 43) d'explorer plusieurs cultures en même temps. Il s'agit en effet d'un genre qui dépasse la seule dimension musicale et qui permet ainsi à un auteur comme Péan d'exploiter le jazz comme « un pont entre des aires culturelles » (44). Autrement dit, parler de jazz :

4 Voir à ce propos Jazzman, l’anthologie du jazz rédigée par Stanley Péan et parue en 2006 aux éditions Mémoires d'encrier. 
C'est aussi parler de sexe, de politique et d'Histoire, de la fonction sociale du musicien et de l'écrivain, [...] des interactions entre cultures populaire et savante. (43)

Par ailleurs, Péan «prend un plaisir manifeste à se jouer des hiérarchies culturelles et des frontières identitaires » (44), peut-être plus que d'autres écrivains de la diaspora haïtienne. Bien que le merveilleux soit sans aucun doute leur commun dénominateur, contrairement à Péan ils ont eu l'avantage d'avoir vécu en Haïti et de mieux la connaître. Pour cette raison, Péan inaugure une écriture diasporique plus influencée que les autres par les apports culturels provenant de l'extérieur, notamment des États-Unis. Le jazz, si bien répandu chez les Haïtiens, avait d'abord évolué dans les bordels et les cabarets de Storyville à New Orleans, pour être ensuite introduit en Haïti lors de l'occupation des marines en 1915 (Barson 2017, IV). Il s'agit donc d'un élément que les Haïtiens se sont appropriés, mais qui reste en même temps étranger à une haïtianité traditionnelle liée plutôt aux musiques populaires (cf. Dauphin 1983). Dans cette optique, il est intéressant d'étudier comment Zombi Blues exalte le métissage culturel si cher à son auteur (Noël-Gaudreault 2003, 110) par le biais de l'élément musical.

\subsection{Une narrative musicale : quand la musique parle 'culture'}

Zombi Blues est entièrement envahi par le jazz, à commencer par le paratexte. Tant le titre que les définitions qui l'accompagnent précisent qu'il sera surtout question de blues, un « morceau de jazz lent et mélancolique » (Péan 1996, 11). En clôture du roman, le glossaire final est suivi d'une liste de compositeurs et de titres jazz qui permettent aux moins expérimentés d'attribuer un sens au bizarre titrage des chapitres, comme Melancholia, Hallucinations ou Equinox. Ces derniers sont en effet un clin d'œil à des œuvres plus ou moins célèbres du répertoire jazz de l'auteur (316) dont tout le récit est savamment parsemé. ${ }^{5}$ Bien que la présence de musiciens américains soit prédominante, l'interculturalité musicale de cette liste n'échappe pas au musicologue avisé : Gismonti et Jobim (Brésil), Seress Rezsó (Hongrie), Zawinul (Autriche) et Piazzolla (Argentine) contribuent ainsi à un véritable mélange culturel sur une toile de fond narrative haïtiano-québécoise. Quant à la structure interne, elle s'articule en cinq parties précédées d'une ou deux citations de poètes, écrivains ou

5 Il faut pourtant préciser que certains titres comme Desafinado et Pensativa se réfèrent plutôt à la bossa nova, un genre musical né au Brésil autour des années 1950. La bossa nova se rapproche notamment de la samba, mais aussi du cool jazz dans les formes musicales de «fusion». 
musiciens concernant le domaine musical : on y trouve Langston Hughes, Toni Morrison, Gustav Mahler, Miles Davis et un extrait d'une complainte haïtienne traditionnelle. Seules font exception celles d'Aimé Césaire et de Countee Cullen, se référant plutôt à un discours racial et colonial. Les mots de Morrison et de Hughes placés tout au début sont particulièrement intéressants, puisqu'ils renouent la musique blues à des états d'âme spécifiques : angoisse, souffrance, sagesse, colère et humour (12). Le rapport qui s'établit entre sentiments et musicalité est symptomatique de la valeur émotive, cognitive et sémantique que Péan confie aux différentes musiques évoquées tout au long de la narration.

Aussi, il est possible d'interpréter les apports culturels du jazz, du blues et du tam-tam de Zombi Blues dans la perspective de la narratologie musicale pour laquelle la musique deviendrait un canal de transmission de sens et de signification au même niveau que les mots, créant une symbiose entre langage verbal et production musicale. C'est pourquoi notre analyse part du présupposé que la musique, comme le langage verbal, est constituée d'un code doté de capacité expressive et communicative. En possédant une dimension syntaxique et temporelle, la musique produit des « énoncés instrumentaux » (Nattiez 1990, 72-4) inscrits dans un récit littéraire où l'évocation des sons peut donner vie à une narration ou y contribuer. ${ }^{6}$

Il vaut ainsi la peine de s'interroger davantage sur les titres qui introduisent les chapitres du roman. En effet, au fur et à mesure que la lecture avance, on saisit l'ingénieux mécanisme sémiotique et narratif mis en place par Péan : loin d'être le fruit d'un simple effort stylistique, chaque titre musical marque une nouvelle étape dans l'évolution narrative du récit. Par conséquent, ces titres deviennent des porteurs de sens actifs dans l'univers fictionnel de Zombi Blues, des véritables signes qui transmettent un contenu ou un message précis. Qu'il parle de jazz, de blues ou de musique africaine, Péan exploite donc cette substance sonore pour donner vie à une sorte de « récit musical » (Toolan 2011,5). Ce dernier finit par se superposer harmonieusement au plan narratif verbal et même par l'anticiper. Parmi d'autres, le premier chapitre, « Misterioso », est un exemple assez parlant. Il s'agit en effet d'un titre jazz à valeur diégétique non seulement pour le référent du signe verbal, c'est-à-dire quelque chose d'insaisissable et de vague, mais surtout pour son aspect proprement matériel et sonore.

6 Pour des approfondissements sur la relation entre musique et sémiotique, voir : Barthes, Roland (1972). « Le grain dans la voix ». Musique enjeu, 9, 74-96 ; Barthes, Roland (1982). «La musique, la voix, la langue ». L'Obvie et l'obtus. Paris : Seuil, 246-52 ; Benveniste, Émile (1969). "Sémiologie de la langue ». Semiotica, I(1), 1-12 ; Tarasti, Eero (1987). « Le Rôle du temps dans le discours musical ». Sémiotique en jeu : À partir et autour de l'œuvre d'A.J. Greimas. Paris : Éditions Hadès-Benjamin, 105-29 ; Tarasti, Eero (1994). Sémiotique musicale. Limoges : Presses Universitaires de Limoges; Tarasti, Eero (2010). I segni della musica. Che cosa ci dicono i suoni. Roma : Le Sfere, 3-23, 79-103. 
En écoutant le morceau de Theolonious Monk, on comprend tout de suite l'association entre ce titre jazz et le contenu du chapitre : le sentiment de dépaysement, d'incompréhension et de mystère évoqué par l'entrée en scène d'une mère haïtienne en fuite avec son enfant est transposé en musique par un morceau éveillant les mêmes sensations. Cela montre qu'avec Zombi Blues « les sons sont classés dans des champs sémantiques différents selon le timbre » (Basile, Galloni 2013, 12), en créant un continuum entre mots et musique.

Cette prémisse nous permet de postuler que la narratologie musicale telle qu'exploitée par Péan joue un rôle fondamental dans l'inauguration d'une haïtianité interculturelle. Sans compter que nous avons affaire à une forme de narrativisation de la musique proprement « sémiotique », c'està-dire celle qui est influencée par la culture de l'auditeur (Marty 2011, 6). Le premier auditeur n'étant autre que l'auteur, il construit la valeur sémantique de la musique sur son interaction avec le discours culturel du roman. Par conséquent, en admettant que l'aspect musical a une signification principalement culturelle, il serait difficile de saisir la complexité du personnage principal, Gabriel D'ArqueAngel, sans en tenir compte. À ce propos, les notes du blog personnel de Péan nous donnent la possibilité de partager le processus créatif qui a amené à la naissance de Gabriel, un véritable produit de la rencontre entre littérature et musique :

$\mathrm{Au}$ tout début, longtemps avant d'entreprendre l'écriture de $Z o m b i$ Blues, j'ai eu en tête une image obsédante, empruntée à une nouvelle de mon mentor Harlan Ellison : celle d'un trompettiste de jazz, qui se tient debout devant une tombe fraîchement comblée, la nuit, et joue un blues triste à fendre l'âme, [...] l'image était restée dans ma mémoire et s'était amalgamée à d'autres histoires de jazzmen signées par quelques-uns de mes écrivains-fétiche (notamment « The Truth » et « Memory of a Muted Trumpet » dans Gentleman Junkie d'Ellison, « The Black Country » de Charles Beaumont, et j'en passe). [...]. Le trompettiste de ma scène nocturne, ce serait donc lui, Gabriel D'ArqueAngel, cet émule contemporain de Miles Davis, inspiré à la fois de Wallace Roney (le véritable dauphin de Miles) et du personnage de Bleek Williams (interprété par Denzel Washington dans Mo' Better Blues de Spike Lee). (Péan 2009)

Ajoutons à ce portrait hétéroclite que, malgré sa naturalisation québécoise après l'abandon d'Haïti, Gabriel est profondément tourmenté par des troubles identitaires qui le rendent un « musicien apatride » (Péan 2009). Néanmoins, c'est l'élément musical qui lui confère progressivement une identité culturelle métisse, au croisement entre tradition africano-haïtienne et héritage nord américain.

La première composante sonore à fonction culturelle qui saute aux yeux du lecteur est sans aucun doute l'africaine. Le son du tam-tam est en ef- 
fet présent dès la fuite de Gabriel et de sa mère à Port-au-Prince, où les battements du cœur « épousent le kata scandé par les tambours au loin » (Péan 1996, 20). Une fois à Montréal - malgré la prédominance d'une culture musicale québécoise plutôt américanisée - la diaspora n'efface pas les sonorités haïtiennes, qui deviennent un véritable trait identitaire. Laura s'en rend compte lors de la sortie de l'album de Gabriel :

Elle examine le boîtier, intriguée par le nom de scène et les titres émaillés de références africaines [...]. Une drôle de sensation l'étreint, comme si, pour la première fois, elle prenait conscience que son frère est noir. (37)

L'image que Laura projette sur son frère est celle d'une musique classée comme représentative de ses origines, un symbole de son haïtianité, ce qui prouve qu'un seul aspect d'une structure sonore peut dégager plusieurs caractères d'une culture donnée (Basile, Galloni 2013, 7). Toutefois, le contexte diasporique fait que les sonorités haïtiennes sont modifiées par « la formation classique que lui a fait suivre, dès son plus jeune âge, son père adoptif » (Péan 1996, 137), de sorte que Gabriel joue du piano aussi bien que de sa trompette. Le jazz est ainsi la troisième composante du panorama culturel et identitaire du musicien, qu'il a perfectionné pendant les années passées à jouer aux États-Unis. Par rapport aux autres, il s'agit pourtant de l'élément clé qui inclut rythme africain et classique, en amenant la journaliste qui interviewe Gabriel à le questionner sur « le rapport entre métissage culturel et jazz » (137).

Aussi, la musique permet un mélange culturel là où l'haïtianité traditionnelle n'est qu'une base perméable aux variantes externes. L'ensemble qui en résulte s'accorde si bien à l'identité de Gabriel, qu'il arrive même à faire coïncider signe verbal et signe musical : « En guise de réponse [...], D'ArqueAngel pointe son biniou vers le plafond pour un chorus électrisant » (94). Dans ce cas, la mélodie de l'instrument a la même fonction que la parole humaine, en devenant « une représentation métonymique du personnage » (Toolan 2011, 7). Les thèmes et les sons musicaux deviennent ainsi des actants narratifs qui déterminent la perception culturelle de Gabriel. Il exprime par exemple sa tristesse par le piano :

Dans son crâne résonnent encore les sons irréels de la veille. [...], il décide d'utiliser ces dissonances comme matière première pour une nouvelle composition. [...]. Il abandonne son déjeuner, va au piano. [...]. Une mélodie triste bleue s'élabore en douceur. (Péan 1996, 114)

Il exprime aussi l'amour par le swahili de Michael Jackson :

D'une voix monocorde, Gabriel déclame les vers qui font office de préface à Liberian Girl, cette ode sirupeuse empruntée au répertoire de 
Michael Jackson : [...]. D'où lui vient cette conviction ridicule que cette déclaration s'adresse à elle ? Laura refuse d'admettre l'évidence. (64)

Et la rage par le son de la trompette :

Les mains de Gaby ont trouvé ses poings qui martelaient le sol, leurs doigts s'entrecroisent, il lui tord les phalanges. À la faveur d'une montée de la trompette de Miles, il la pénètre d'un violent coup de reins. (198)

Nous voyons bien que Péan contribue à créer une haïtianité en diaspora tout à fait inattendue et originale, dans une narration qui est « le reflet du monde où nous vivons » aujourd'hui (Olivier 1998, 9).

\section{Un zombi à moitié, ou le trompe-l'œil haïtien}

L'usage péanien de la musique est d'autant plus intéressant si l'on tient compte de la nature de Gabriel. Vers la fin du roman, nous découvrons que le jeune musicien a été victime des expérimentations de Barthélemy Minville, impitoyable bourreau du duvaliérisme. Afin de mettre en place « un bataillon de surhommes assujettis à sa volonté », il lui a injecté un sérum capable de transformer des enfants haïtiens en bas âge « en des véritables fauves » (Péan 1996, 227). Ambiance dictatoriale, poison mutagène et titre du roman amènent ainsi le lecteur à considérer Gabriel comme un zombi tombé en proie à la dictature, mais dont la zombification n'a pas été correctement achevée. ${ }^{7}$ Telle est du moins la lecture que la plupart des études sur Zombi Blues proposent et que nous résumons par ce qui suit :

Zombi Blues présente plusieurs personnalités traditionnelles du vaudou et du folklore haïtien en réponse à l'expérience traumatique de certaines abominations. [...] Celui du zombi est un motif récurrent dans l'interprétation magicoreligieuse du pouvoir politique. (Cévaër 2009, 11) ${ }^{8}$

Et pourtant, le roman ne fait jamais mention du mot « zombi » pour définir la nature de Gabriel, malgré l'évidente bestialité meurtrière qui s'empare de lui (Péan 1996, 290). Minville le considère comme une « arme absolue » (278), alors que l'investigateur Apollon le définit comme un « baka » (301), un monstre malfaisant du folklore haïtien. Certes, il est indéniable que le

7 Cf. Alfred Métraux (1958). Le Vaudou haitien. Paris : Gallimard, 71-140 ; 216-36 ; 249-52.

8 «Zombi Blues presents most of the traditional characters of vodou folklore as a response to the traumatic experience of certain abominations. [...] The theme of the zombie appears as a recurrent motif in the traditional (magico-religious) interpretation of political power » (traduit par l'Auteure). 
roman renvoie volontairement à une haïtianité désormais classique aux années 1990, celle qui joue sur la rencontre entre la politique dictatoriale haïtienne et l'imaginaire vaudouesque du zombi. Elle restitue ainsi la figure du dictateur - Duvalier en l'occurrence - en zombificateur « accusé de s'être emparé de l'âme du peuple haïtien, de l'avoir réduit à l'état de mort-vivant désespéré, incapable de réagir » (Hoffmann 1995, 344). En même temps, les allusions à ce genre de fictionnalisation n'empêchent pas l'auteur de brouiller avec astuce les pistes traditionnelles.

C'est en effet Péan qui manifeste une prise de distance intentionnelle par rapport à la tradition des romans dictatoriaux qui le précède. ${ }^{9}$ Dans son blog, il raconte avoir commencé un roman au même titre que Zombi Blues dont l'intrigue s'inscrivait dans la lignée des œuvres de Depestre et de Jacques Stephen Alexis. Comme eux, il voulait écrire une histoire de «zombi dézombifié » (Péan 2009). Néanmoins, il affirme n’avoir jamais mené à terme ce roman et s'être ensuite consacré au livre publié en 1996. Ce changement est important lorsqu'on se penche sur les modèles culturels et littéraires suivis par Péan pour la rédaction de Zombi Blues. S'il est évident que d'un côté il a intégré le patrimoine culturel haïtien, de l'autre il déclare l'avoir profondément innové :

On comprendra donc qu'en dépit des nombreuses références au système de croyances du vodou, à la mythologie populaire et au folklore haïtien, il n'y a au fond pas de zombi, au sens propre du terme, dans ce roman. (Péan 2009)

Loin d'être présente de manière complète et traditionnelle, la figure du zombi semble être marquée par d'autres influences que celles du folklore haïtien. La remarque de Péan nous demande ainsi de reconsidérer cer-

9 Voir à ce propos les principaux romans haïtiens sur la dictature : Chauvet, Marie (1968). Amour, Colère et Folie. Paris : Gallimard ; Clitandre, Pierre (1979). Cathédrale du mois d'août. Port-au-Prince : Île en île ; Fardin; Colimon, Marie-Thérèse (1974). Fils de misère. Port-au-Prince : Éditions Caraïbes; Depestre, René (1979). Le mât de cocagne. Paris : Gallimard ; Dorsinville, Gérard (1980). Mourir pour Haït (ou les croisées d'Esther). Paris : L'Harmattan ; Étienne, Gérard (1974). Le Nègre crucificié. Ottawa : Éditions Francophones et Nouvelle Optique et (1979) Un Ambassadeur macoute à Montréal. Montréal : Nouvelle Optique ; Ollivier, Émile (1983). Mère-Solitude. Paris : Albin Michel ; Phelps, Anthony (1972). Moins l'infini. Paris : Les Éditeurs Français Réunis et (1976) Mémoire en colinmaillard. Montréal : Les Éditions Nouvelle Optique. Quant à la littérature critique sur le sujet, voir : Borst, Julia (2009). " Violence et mémoire dans le roman haïtien contemporain ». Les Caraïbes : convergences et affinités, 10. URL http://www.publifarum. farum.it/ezine articles.php?art_id=103; Costantini, Alessandro (2002). Fantasmi narrativi e sovversione linguistica nel romanzo haitiano moderno e contemporaneo. Milano : Cisalpino ; Férdinand, Joseph (1983). «Le nouvel énoncé politique du récit haïtien ». Collectif Paroles, 22, 15-19; 23, 21-5 ; 24, 24-32 ; Hoffmann, Léon-François (1995). Littérature d'Haïti. Vanves : EDICEF/ AUPELP, 326-33 ; Jonaissant, Jean (1986). Le pouvoir des mots, les maux du pouvoir. Montréal : Arcantère/PUM ; Rama, Angel (1976). Los dictadores latinoamericanos. Mexique : FCE. 
taines lectures du roman proposées jusqu'à maintenant, du moins celles fondées sur la présence d'un zombi typiquement haïtien qui, tout compte fait, n'en est pas un.

Il est aussi vrai que Péan semble consciemment laisser le lecteur dans un état d'indécision et de désarroi, en parsemant la narration d'indices tout au plus ambiguës sur Gabriel. Ainsi, certaines caractéristiques peuvent effectivement dérouter le lecteur, en l'induisant à classifier le jeune musicien comme un zombi véritable. D’après les nombreuses recherches qui ont été conduites sur le mythe du zombi, nous savons que le mythe haïtien est enraciné dans la culture africaine, mais dans celle qui a été réadaptée au contexte sociopolitique de la colonisation caribéenne (Laroche 1975, 480). Malgré des traits qui distinguent profondément les deux traditions, Laroche précise qu'elles partagent l'idée centrale selon laquelle la mort et l'esclavage du zombi ne seraient pas naturels, mais artificiellement provoqués par une instance humaine à l'insu de la victime (482). Dans cette optique, le cas de Gabriel est l'emblème d'une soumission involontaire, en ce qu'elle est causée par Bébé Doc, sans être le résultat d'un acte délibéré de servitude et de soumission. Ainsi, sa transformation physique et spirituelle est d'autant plus frappante qu'elle a été pratiquée sans qu'il en ait conscience, et dont seul une faible réminiscence enfantine persiste :

Depuis son enfance, il souffre d'un étrange mal, une froideur constante qui le dévore de l'intérieur, comme si on lui avait arraché une part de son âme. (Péan 1996, 40)

À la sensation d'être dominé par une force extérieure s'ajoute l'émergence d'une série d'effets collatéraux qui renvoient à la figure mythique du zombi. Métraux affirme que ce dernier se reconnaît à son air absent et à ses yeux « éteints, presque vitreux » $(1958,251)$. En effet, l'un des traits distinctifs de Gabriel est une paire de lunettes dont il ne se sépare jamais. Sa demi-sœur Laura constate qu'il « paraît si distant derrière ses lunettes fumées » (Péan 1996, 35), cachant habilement ses émotions ainsi que la lueur écarlate de ses yeux. S'ils ne sont pas tout à fait vitreux, il est vrai qu'ils sont « les seuls à briller de la sorte »(119). En plus, comme le zombi est doté de « propriétés surhumaines » (Ackermann, Gauthier 1991, 469), Gabriel fait preuve d'une « force physique et agilité décuplées » le rendant presque invulnérable (Péan 1996, 278). Finalement, comme tout zombi qui se respecte, le trompettiste est enchaîné à un maître qui le réclame à travers des «flashes visuels et sonores »(111).

Néanmoins, ce portrait de zombi presque canonique ne saurait pas, au final, suffire à satisfaire une haïtianité ad hoc. Le mythe africain aussi bien que l'haïtien décrivent le zombi comme une personne « dont le décès a été dûment constaté » et « que l'on retrouve quelques années plus tard chez un boko (un méchant sorcier dans le vaudou) dans un état voisin de l'idiotie » 
(Métraux 1958, 249 ; emphase dans l'original). Rien de plus loin de ce qui est arrivé à Gabriel. Puisque pour les Haïtiens le zombi se caractérise par « l'inexistence d'une véritable distance entre la vie et la mort » (Laroche 1975, 483), il faut préciser que Gabriel ne passe préalablement pas par la mort. Péan opère ainsi un détour par rapport à l'imaginaire classique du zombi. Contrairement aux étapes rituelles qui feraient d'un mort un zombi, Gabriel n'a pas été physiquement tué pour être ensuite ressuscité par un houngan. Il n'a en effet jamais été autre qu'un être vivant en chair et en os, et au lieu d'avoir été un mort transformé en vivant, il est un vivant chez lequel on a installé un germe meurtrier. Aussi plus qu'être ce qui est traditionnellement appelé un 'mort-vivant', Gabriel est-il plutôt ce que nous pourrions définir un 'vivant-mort', car en dépit des scènes de mort qui hantent sa mémoire, il est un être humain et vivant par-dessus tous les effets.

Impossible à ce point de ne pas lui associer Henri Postel depestrien du Mât de cocagne, un véritable mort avant l'heure. Et pourtant, la distance entre eux ne saurait être plus grande. Avec Depestre, il est indubitable que Postel est un zombi puni par la dictature de Zoochrate Zacharie pour ses élans révolutionnaires. En revanche, Gabriel est un personnage autonomisé par rapport à l'histoire parallèle de Minville dans la mesure où il ne se pose jamais des questions concernant la situation politique d'Haïti. Par conséquent, son état ne dépend pas de ses convictions politiques comme celui de la plupart des personnages romanesques haïtiens à partir des années $1970 .{ }^{10} \mathrm{Gabriel}$ adhère avant tout à l'imaginaire plutôt moderne du jeune homme tourmenté qui fuit les fantômes d'une enfance de transplanté. Péan suit en ce sens les tendances narratives du roman québécois de la deuxième moitié du XXe siècle, dont les héros « fuient le destin ou eux-

10 Les romans les plus représentatifs d'une zombification dictatoriale rendue sous forme narrative sont Le mât de cocagne (1979) par René Depestre (Paris : Gallimard et Dézafi, 1975) par Frankétienne (Paris : Vents d'ailleurs). La même symbolique est repérable dans les œuvres suivantes, où le peuple haïtien est souvent décrit comme un ensemble de zombis : Étienne, Gérard (1974). Le Nègre crucifié. Ottawa : Éditions Francophones et Nouvelle Optique ; Laferrière, Dany (2000). Le Cri des oiseaux fous. Paris : Éditions Zulma ; Ollivier, Émile (1986). La discorde aux cent voix. Paris : Albin Michel ; Phelps, Anthony (1973). Moins l'infini. Paris : Les Éditeurs Français Réunis; Trouillot, Lyonel (2000). Thérèse en mille morceaux. Paris : Actes Sud. Pour la littérature critique à ce sujet, voir : Bonnefoy, Claude (1979). « René Depestre, le « zombi de la liberté ». Les Nouvelles littéraires, 2(703) ; Coates, Carroll F. (éds.) (2006). « Vodou in Haitian Literature ». Vodou in Haitian Life and Culture : Invisible Powers. New York : Palgrave Macmillan, 181-98. URL http://miltonthed.weebly. com/uploads/1/4/1/6/14162844/vodou_in_haitian_life_and_culture_invisible_powers. pdf ; Hoffmann, Léon-François (1995). Littérature d'Haïti. Vanves : EDICEF/AUPELP, 311-16, 328-33, 343-6 ; Kwaterko, Józef (2002). « Les fictions identitaires des romanciers haïtiens du Québec ». Revue de littérature comparée, 2(302), 212-29 ; Lucas, Rafael (2002). " L'esthétique de la dégradation dans la littérature haïtienne ». Revue de littérature comparée, 302, 191211 ; Ransom, Amy J. (2009). « Ce Zombi égaré est-il un Haïtien ou un Québécois ? Le vaudou chez les écrivains haïtiano-québécois ». Canadian Literature, 203, 64-83. 
mêmes » pour accomplir « un acte de prise de possession » (Éthier-Blais 1971, 161-2). Ainsi, la dépendance de Gabriel à l'alcool et son affection incestueuse pour Laura pourraient être les conséquences d'une adoption tant précoce que violente en pays étranger. Au croisement entre plusieurs cultures, sa nature à lui serait moins le résultat d'une zombification au sens haïtien et traditionnel du terme qu'une forme de quête identitaire, ayant du mal à se reconnaître dans sa famille québécoise et à se réconcilier avec son héritage haïtien.

\subsection{L'irruption du fantastique métissé}

Si Ransom affirme que le zombi est devenu l'emblème des maux haïtiens et que des références à la zombification « semblent incontournables dans un texte littéraire qui veut afficher son haïtienneté » $(2009,66)$, pour Zombi Blues nous proposons de maintenir l'idée d'une haïtianité affichée, mais celle qui est revisitée par des influences culturelles et littéraires autres qu'haïtiennes. Outre la musique, c'est donc la figure du zombi qui enrichit le métissage culturel du roman. Voilà nous arrivés au paradoxe péanien : dans Zombi Blues le zombi n'apparaît guère, et pourtant un zombi y est bien présent. Il s'agit d'un zombi lié au genre fantastique qui s'affirme au Québec à partir des années '60 et qui est très proche du roman d'horreur américain, où la peur naît « d'une exploitation originale des thèmes classiques des morts vivants, du vampirisme, des fantômes et des lieux mystérieux » (Émond 1983, 31). D’ailleurs, Péan se déclare ouvertement l'héritier de Poe et des écrivains de l'école californienne du fantastique d'après-guerre (Olivier 1998, 9), en se révélant ainsi un grand expérimentateur quant à la richesse esthétique offerte en diaspora. Aussi, Zombi Blues est moins inscrit dans la lignée diasporique d'un Ollivier, d'un Depestre ou d'un Laferrière. Il est plus orienté vers le mélange entre tradition haïtienne et courants américano-québécois :

Évidemment, au-delà de ces questionnements existentiels qui soustendent l'intrigue, mon souci a été de faire de Zombi Blues un thriller captivant, voire haletant, qui flirte autant avec le fantastique et la science-fiction que les bandes dessinées de superhéros qui ont bercé mon enfance. (Péan 2009)

Péan exploite donc une histoire lui permettant de faire un clin d'œil au lecteur quant à l'allégorie de l'Haïtien aliéné, en donnant pourtant priorité à un usage du zombi plus fantastique qu'haïtien.

Dès lors, le zombi est fictionnellement réinventé et habilement transformé dans un personnage topique de l'écriture noire. C'est la raison pour laquelle chez Péan «l'élément québécois [...] ressort encore plus » que 
chez ses collègues de la diaspora et le vaudou est exploité comme « catalyseur du fantastique » (Ransom 2009, 65-70). Il est en effet question de fantastique et non pas de merveilleux haïtien, car dans le deuxième cas il n'y aurait aucune distinction entre naturel et surnaturel. Autrement dit, Gabriel et les autres personnages du roman traiteraient la tentative de zombification ainsi que l'arrivée de Caliban, le monstrueux jumeau du musicien, comme des évènements tout à fait ordinaires. En revanche, il est évident que chaque actant impliqué est choqué et incrédule face à ce qu'il ne peut pas expliquer selon les coordonnées de son univers rationnel (Ransom 2013, 157-8).

D'après Ransom - qui a eu le mérite de valoriser l'écriture péanienne à la lumière du fantastique - Péan est parmi les rares auteurs qui ont su jouer avec un " fantastique pur », c'est-à-dire qui fait constamment douter le lecteur quant à la véridicité des éléments de l'histoire (156). En même temps, Zombi Blues correspond à ce qu'elle appelle un " texte hybride », capable de mettre en confrontation l'Haïtien avec le Franco-Québécois (151). Aussi, l'œuvre déploie un fantastique métissé, « un usage des codes du fantastique occidental pour des fins spécifiques aux écrivains migrants » (147-8).

Gabriel devient ainsi un sujet extraordinairement transculturel : il émet des échos haïtiens qui sont fonctionnels pour la création d'un personnage fantastique dans un univers teinté d'horreur à la Edgar Allan Poe. N'oublions pas non plus que c'est Péan même qui reconnait une filiation entre son personnage et ceux d'Ellison, écrivain américain de fantasy et de science-fiction. Gabriel est le point de contact entre plusieurs contextes culturels grâce à ses contours estompés et au manque d'une essence précise ou définitive. Tout compte fait, Zombi Blues est sans aucun doute « le thriller haïtien le mieux construit » (Saint-Éloi cité in Mininni 2002, 132), mais il faudrait mieux s'interroger sur ce que l'on entend par 'haïtien'.

\section{$4 \quad$ Marasa : la rencontre des altérités}

Dans ce monde fantastique, il ne pouvait manquer une part de mystère. Le motif des marasa, les jumeaux divins du folklore haïtien, ${ }^{11}$ donne au récit la juste pointe d'horreur qui manquait jusque-là. Aux yeux de Gabriel, son jumeau Caliban est un automate inhumain « dont l'unique fonction est d'assassiner les gens » (Péan 1996, 297). Il est un djab, être maléfique dont la transformation en « monstre surnaturel » (246) a été achevée et

11 Les jumeaux marasa sont « des véritables protecteurs que l'on vénère depuis des générations et que personne ne pourrait 'rejeter' sous peine de s'exposer à toutes sortes de malheurs » (Marcelin 1947, 68). Ils figurent à côté des loas du panthéon haïtien et des morts, tout en étant plus puissants $(1950,138)$. 
qui incarne l'ennemi juré de Gabriel. La symbolique des noms est assez parlante : nous avons d'un côté le prototype du Caliban shakespearien, une bête hybride et sauvage assoiffé de mort et de sang ; de l'autre, D'ArqueAngel, peut-être un clin d'œil à l'archange Gabriel, connu comme porteur d'harmonie et révélateur de rêves prophétiques similaires aux visions que Caliban envoie à son frère. Deux forces opposées mais affines qui semblent ne coexister que dans une tentative d'anéantissement mutuel entre survie et dégradation meurtrière (Marcelin 1950, 128-9).

Dans les cérémonies vaudou, les marasa sont en effet invoqués après Legba, le maître des carrefours et de la barrière mystique qui sépare les hommes des esprits (Métraux 1958, 89). Les jumeaux divins seraient ainsi à mi-chemin entre le monde des morts et celui des vivants, au point que leur culte est souvent associé à celui des morts (Marcelin 1950, 138). Toutefois, dans Zombi Blues il nous semble qu'il n'est pas tant question de perméabilité entre ces deux mondes, ni d'une véritable exploration du panthéon haïtien. En nous éloignant de la vision traditionnelle et religieuse des jumeaux marasa, nous suggérons d'interpréter ce couple et leur vie « double » et « polarisée » (Rigaud 1953, 415) comme une rencontre entre des altérités culturelles. Dans cette perspective, le lien entre Gabriel et Caliban est l'apothéose d'un métissage suggéré tout au long du récit bien avant le dénouement final. En effet, il y a dans le roman plus qu'un seul binôme fraternel, ce qui permet à Péan d'adapter ce topos haïtien à un univers diasporique multiculturel et pluriel. Les deux autres couples sont formés par Gabriel et Daniel et par Gabriel et Laura. Même dans ces cas un lien fraternel est présent et il est également conflictuel. Dans le premier, le conflit est dû au fait que Gabriel pense avoir usurpé la place du fils légitime des Reynolds (Péan 1996, 33). Ce malaise l'amène à la conviction de ne pas être à la bonne place à Montréal et déclenche le premier dépassement des frontières vers les États-Unis. Il devient ainsi un personnage déplacé, confronté à l'altérité québécoise qu'il refuse ou qu'il n'arrive pas à assimiler. Il en est de même avec Laura, où l'attraction sexuelle incestueuse pourrait vraisemblablement se traduire par une tentative extrême de métissage (Mininni 2002, 141), tant métaphorique que physique. Et Laura et Daniel placent Gabriel dans une dualité qui le pousse à la conciliation de deux pôles apparemment incompatibles où ils représenteraient le dualisme (franco-anglais) canadien.

Quel est alors l'autre pôle ? Il serait du côté de Caliban, avec qui la question identitaire se fait plus compliquée. Il symbolise une identité haïtienne dans toutes ses nuances et avec toute son histoire de douleur, violence, sécularité et richesse. Gabriel voit en lui ce qu'il aurait pu être et qu'il n'a pas été au moment où le roman devient une véritable « fiction identitaire » aux nuances culturelles (Kwaterko 2002, 215). Caliban est le différent dans l'égalité - vu qu'ils partagent la même origine - mais qui est éliminé au nom d'une autodéfinition nécessaire. Les trois binômes font donc partie 
de son inconscient culturel et il ne lui reste qu'à bâtir son haïtianité à lui à partir de l'identité américano-québécoise et de l'héritage haïtien qui s'offrent à lui. Aussi, Gabriel renaît de la négociation entre ces différentes cultures qu'il assume à sa manière à la fin du roman, en se défaisant du clivage « identité-différence » (223) et inaugurant ainsi une

vocation transaméricaine de l'écriture, dans laquelle l'expérience haïtienne et québécoise sont toutes les deux présentes, où l'expérience transculturelle, interculturelle et intraculturelle ne s'annulent pas, mais se côtoient et s'interrogent. [...] non pas au sens d'un pluralisme de surface, mais par rapport à des liens et des liaisons tissées avec l'espace caribéen, interaméricain et mondial. (227)

\section{Conclusion}

En conclusion, nous pouvons affirmer que Stanley Péan a tissé un roman aussi complexe qu'intriguant. Il fonde une nouvelle vision de l'haïtianité par le mélange d'une pluralité d'apports culturels et identitaires que le contexte diasporique lui a offerte. Musique, zombi et marasa vont ainsi au-delà des significations symboliques typiquement attribuées aux romans de l'exil. Loin d'être inscrits dans un univers culturel et narratif attendu, ces éléments contribuent à une nouvelle écriture romanesque de l'haïtianité dans une perspective interculturelle. Péan joue savamment sur l'ambiguïté en regard de la réception de son œuvre, dans la mesure où il reprend des aspects culturels québécois, haïtiens et afro-américains littérairement familiers mais reconfigurés dans une nouvelle configuration narrative. Il élargit en effet la perspective du roman diasporique au-delà d'une simple dualité. Pour cette raison et à la lumière de ce qu'on vient de montrer, il nous semble plus pertinent de parler de 'métaspora', ${ }^{12}$ un concept qui traduit mieux une haïtianité aux facettes plurielles et interactives, ouverte à des nouveaux imaginaires qui dépassent l'horizon québécois et haïtien (Kwaterko 2002, 228). Aussi, Zombi Blues est un véritable jeu entre apparence haïtienne et identité interculturelle, qui confond le lecteur autant qu'il le fascine.

12 Celui de la 'métaspora' est un concept développé par Joël Des Rosiers, écrivain et psychiatre québécois d'origine haïtienne. Il avance la notion pour la première fois aux années '90 dans l'essai Théories caraïbes. Poétique du déracinement (1996) pour l'approfondir ensuite dans Métaspora : essai sur les patries intimes (2013). 


\section{Bibliographie}

Ackermann, Hans-Wolfgang ; Gauthier, Jeaninne (1991). "The Ways and Nature of the Zombi ». The Journal of American Folklore, 104(414), 466-94. Barson, Benjamin (2017). "Return to My Native Land". Vodou Jazz in PostOccupation Haiti. Master's Thesis in Cultural Studies, University of Pittsburgh. URL http://d-scholarship.pitt.edu/32063/1/BarsonMA_ ETD Final\%205-18.pdf (2018-3-15)

Basile Marco, Galloni Gloria (2013). « Sul significato in musica. I limiti epistemologici di un'analisi semiotica musicale ». Testo \& Senso, 14, 2-18.

Beauquis, Corinne (2004). Quatre écrivains haïtiens du Québec : Gérard Étienne, Émile Ollivier, Dany Laferrière, Stanley Péan. Alter-rature ou ubiquïté réussie ? Ottawa : Library and Archives Canada.

Cévaër, Françoise (2009). " Haitian crime fiction : Re-interpreting Haitian History through Vodou ». The International Journal of Humanities, 7(9), 11-18.

Dauphin, Claude (1983). La chanson haïtienne folklorique et classique. Montréal : Société de Recherche et de Diffusion de la Musique haïtienne.

Depestre, René (1979). Le mât de cocagne. Paris : Gallimard.

Des Rosiers, Joël (1996). Théories Caraïbes. Poétiques du déracinement. Montréal : Tryptique.

Des Rosiers, Joël (2013). Métaspora : essai sur les patries intimes. Montréal : Tryptique.

Émond, Maurice (1983). «Le fantastique au Québéc : le XXe siècle ». Québec français, 50, 26-31.

Éthier-Blais, Jean (1971). «Le roman québécois ». Naamaan Antoine, Painchaud Louis (éd.), Le roman contemporain d'expression française. Sherbrooke : CELEF, 156-64.

Hamel, Yan (2007). « Autobiojazzographie/Jazzman. Chroniques et anecdotes autour d'une passion, de Stanley Péan ». Spirale, 214, 43-5.

Hoffmann, Léon-François (1995). Littérature d'Haïti. Vanves : Edicef/Aupelf. URL http://classiques.uqac.ca/contemporains/hoffmann_leon francois/litterature dHaiti/litterature dHaiti.html (2018-11-28). Hurbon, Laënnec (1993). Les mystères du vaudou. Paris : Gallimard.

Kwaterko, Józef (2002). « Les fictions identitaires des romanciers haïtiens du Québec ». Revue de littérature comparée, 2(302), 212-29.

Laferrière, Dany (2000). J'écris comme je vis. Genouilleux : La Passe du vent.

Laroche, Maximilien (1975). « Mythe africain et mythe antillais : le personnage du zombi ». Revue Canadienne des Études Africaines, 9(3), 479-91.

Laroche, Maximilien (1989). La Découverte de l'Amérique par les Américains. Sainte-Foy : GRELCA.

Lucas, Rafael (2002). "L'esthétique de la dégradation dans la littérature haïtienne ». Revue de littérature comparée, 302, 191-211. 
Marcelin, Émile (1947). " Les grands dieux du vodou haitien ». Journal de la Société des Américanistes, 36, 51-135.

Marcelin, Milo (1950). Mythologie Vodou (Rite Arada) II. Pétionville : Éditions Canapé-Vert.

Mars, Louis (1945). « The Story of the Zombi in Haiti ». Man, 45, 38-40.

Marty, Nicolas (2011). "Vers une narratologie naturelle de la musique ». Cahiers de narratologie, 21, 1-14. URL http://narratologie. revues. org/6476 (2018-11-28).

Métraux, Alfred (1958). Le Vaudou haïtien. Paris : Gallimard.

Mininni, Maria Isabella (2002). Ombre di realtà e ladri di anime. Torino : Tirrenia Stampatori.

Modenesi, Marco (2014). «'On se dirait en Haïti' : Stanley Péan, écrivain québécois à statut particulier ». Plaisance, 32, 101-12.

Nattiez, Jean-Jacques (1990). «Peut-on parler de narrativité en musique ? ». Canadian University Music Review, 10(2), 68-91.

Noël-Gaudreault, Monique (2003). «Comment Stanley Péan a écrit certains de ses livres ». Québec français, 130, 110-11.

Olivier, Nathalie (1998). "Stanley Péan : l'œuvre au noir ». Lettres québécoises, 90, 8-10.

Olmos Fernàndez Margarite, Paravisini-Gebert Lizabeth (2011). Creole Religions of the Caribbean : An Introduction from Vodou and Santería to Obeah and Espiritismo. New York : NYU Press, 116-55.

Péan, Stanley (1996). Zombi Blues. Montréal : J’ai Lu.

Péan, Stanley (1998). La plage des songes et d'autres récits d'exil. Montréal : Bibliothèque québécoise.

Péan, Stanley (2006). Jazzman. Montréal : Mémoire d'encrier.

Péan, Stanley (2009). «Zombi, quel ténébreux chant entonnes-tu ? ». Clair/Obscur, 9. URL http://wWw.stanleypean.com/textes-divers/ zombi-quel-tenebreux-chant-entonnes-tu/ (2018-11-28).

Péan, Stanley (2014). « Histoires de jazz avec Stanley Péan ». La Fabrique culturelle.tv. URL https://www.lafabriqueculturelle.tv/capsules/211/stanley-pean-histoire-de-jazz (2018-11-28).

Ransom, Amy J. (2009). « Ce Zombi égaré est-il un Haïtien ou un Québécois ? Le vaudou chez les écrivains haïtiano-québécois ». Canadian Literature, 203, 64-83.

Ransom, Amy J. (2013). « La gamme du fantastique : l'éclatement des genres et l'écriture migrante haïtiano-québécoise (1979-2001) ». @nalyses, 8(2), 146-74.

Rigaud, Milo (1953). La tradition voudoo et le vaudoo haitien. Paris : Éditions Niclaus.

Toolan, Michael (2011). « La narrativité musicale ». Cahiers de narratologie, 21, 1-11. URL http://narratologie.revues.org/6489 (2018-11-28). 


\title{
Edward Elgar's Masque The Crown of India Resonances of the Raj at the London Coliseum
}

\author{
Edvige Pucciarelli \\ (Università degli Studi di Bergamo, Italia)
}

\begin{abstract}
The music for The Crown of India was written by Edward Elgar in 1912 to accompany an 'Imperial Masque' with a libretto by Henry Hamilton. The impresario Sir Oswald Stoll had commissioned Elgar to compose the Masque music for the lavish celebration of the coronation of King George $V$ as Emperor of India as part of a larger entertainment in the Coliseum Theatre in St. Martin's Lane. The Masque was part of an ample music-hall programme, involving shows as different as mime, pantomime and music. Elgar's 'Imperial Masque' was meant to be an assertion of the British Empire, bringing to the London stage the crucial political happenings behind all the pageantry of the Delhi Durbar for the crowning of George $V$ as Emperor of India in December 1911. This event had marked the climax of the only royal tour of India undertaken by a reigning King-Emperor and had caused much public excitement in England. The Durbar ceremony itself was an adaptation of a court ritual of the Mogul Empire, an event where the ruling princes used to meet to discuss politics and legislative changes. To listen to works such as Elgar's The Crown of India (Opus 66), it is necessary to acknowledge that at the beginning of the 20th century the British nation believed in the Empire and in its concept.
\end{abstract}

Summary 1 Henry Hamilton's libretto: Nation and Empire in The Crown of India. - 2 Edward Elgar's The Crown of India, Opus 66, 1912 as the Representation of Places of the British Empire. 3 Representations of the Raj on the London Coliseum Stage.

Keywords Elgar. Hamilton. Masque. Imperialism. Said.

\section{Henry Hamilton's libretto: Nation and Empire in The Crown of India}

The impresario Stoll wrote to Elgar early in January 1912 offering him to compose the music for an 'Imperial Masque' to be performed at the Coliseum in celebration of the newly-crowned Emperor George V. The cultural, political and royal issues were the chief motivation of Stoll's commission, ${ }^{1}$ events strictly related to the Royal visit to India at the end of 1911. The Delhi Coronation Durbar 1911 was organised by the British as a means of

1 Sir Oswald Stoll owned theatre chains in Britain and he is reputed to have transformed the music-hall entertainments into a more socially acceptable entertainment of multi-class appeal drawing more audience to the theatres. The London Coliseum opened in 1904, becoming an attraction for the middle class. See Barker 1957, 72. 
demonstrating British power over the colony of India as well as a means of rendering manifest the close relationship that existed between the Raj and much of the Indian elite.

However, by the time of the royal visit in 1911, the first signs of deterioration in the relationship with local Indians at an institutional level were beginning to show, creating difficulties in maintaining the British hold over India and more specifically, over the totality of the Indian subcontinent. That these emerging problems in the ruling of and in India were not apparent to the British public knowledge is demonstrated by the fact that Britons were fascinated with the royal visit to India of November 1911. This very fact encouraged Stoll to commission a piece of music informed with imperialistic values as a means of celebrating the royal imperial coronation event, trying to portray and represent Britain's view of what was thought India to be from the perspective of the United Kingdom theatre and national audience.

Elgar was presented with a challenging proposal from the outset of the composition by the imperial sentiments, expressed in rather bombastic terms and by the structure devised by the librettist Henry Hamilton, a playwright of little distinction but known to the general public who appreciated his overt ways of presenting the imperial powers and achievements, also expressed in philanthropic terms, of Britain. ${ }^{2}$

The propagandist imperialist aspects of the 1911 Durbar, attended by the first time by the King-Emperor of Great Britain in Delhi, on the long-colonised Indian subcontinent, were carefully and very precisely transferred into the masque's libretto by Henry Hamilton (1912) in a straight-forward way. $^{3}$ So that the home audience, who had read eagerly in the newspapers about the ceremony that took place in the exotic distant 'other' space of the Empire, could easily understand and identify itself with the ideology and rhetoric Hamilton inserted in his libretto. Although such an audience could not relate to the ceremony's description in a real sense, of course, the public attending the performances at the London Coliseum could respond to an imaginary exotic far-fetched, far-flung unreal recreation of it. Hamilton's text is a visionary encounter, a successful hybridisation, between

2 Henry Hamilton, the writer of the libretto, also wrote a series of melodramas in the later years of the nineteenth century. At the Theatre Royal, Drury Lane, he became one half of 'the Gilbert and Sullivan of melodrama' with his writing partner Cecil Raleigh. See MacQueenPope 1945, 292. It is significant that Henry Hamilton, the librettist chosen by Stoll for The Crown of India, does not appear in The New Grove Music and Musicians Dictionary edited by Stanley Sadie and John Tyrrel in 2000. See also "Grove Music Online" in Oxford Music Online, http://www. oxfordmusiconline. com/grovemusic/ (2018-12-04). We have already anticipated the scant information on Elgar's music.

3 The only article devoted to the study of Hamilton libretto's for The Crown of India is that by Pellegrino 2010, 13-36. Pellegrino's article focuses on the cuts made by Elgar himself in Hamilton's text. 
a simplified and effective version of both Indian history and mythology with the British history and tradition. It is an epic fantasy echoing and, at times, resembling the description of romance and Arthurian chivalric literature. The themes in the passage of the text referred to Saint George as 'The Spirit of Chivalry', the personification of Britain embodying knightly canons. St George, who makes his entry accompanied by Tradition and Romance, introduced in the stage directions as personifications. This literary commonly-shared background was also known to the working-classes, who enjoyed the shows, which helped feed this ideological and political image of the Empire to the average general public of late Edwardian Britain. The matter of romance-chivalry literature with its topographical and cartographical vision, although itself an imaginative invention, or rather re-reinvention of exotic places that were part of traditional British imagery, was well adapted to describing and representing the subservient role attributed to the 'uncivilised' colony of India, with its simultaneously real and 'unreal' territory.

The libretto is an allegory of imperial British rule: the twelve most important cities of India personifying Indian ancient history and traditions, both cultural and political, as well as incarnating the economic and commercial values that were essential to the wealth of the empire and through which the cities were known to the general audience in Britain. The beginning of the libretto is dominated by feminine images of the geographical and cultural Indian references - actors impersonated the Cities and India, staging the urban topography of India as Agra, Benares (Varanasi), Mysore, Haidarabad, Lucknow, Bombay, Madras, Lahore, Allahabad and Gwalior along with the Mogul Emperors - sharply contrasting with the inserted British male figures (real, mythical and mythological): John Company, the colloquial name for the East India Company; St. George representing England, the King-Emperor George V. Only at the end of the second tableau, the Queen-Empress Mary is mentioned, accompanying the King as they both enter the Durbar in Delhi.

Moreover, the text was able to further the concepts of paternalistic philanthropic views, which the general public strongly believed in: views portraying the British rule, and functioning as a sort of evangelical, philanthropic civilising agent, defining the role the Empire had assumed over all its conquered territories. This political stance, shared by the government elite and the general public in Great Britain were everyday attitudes of the colonisers themselves in the colonised territories, commonplace beliefs for the colonised peoples of the Empire as well and were the colonial discourses of the time, which Edward Said in Culture and Imperialism and in Orientalism explains, criticises and exemplifies. Such were the ideological imperialist bases conveyed by Hamilton in his libretto.

The libretto of The Crown of India consists of two tableaux: the first, named The Cities of Ind, opens with the controversial announcement that 
the capital of India would move from Calcutta to Delhi; the second, is called Ave Imperator and concerns King George V's visit to India; it is a re-enactment of the Durbar ceremony itself. These two tableaux serve the dramatic function offering us a historical background and assessment of the situation in India. It seems that the subcontinent is presented as a kingdom of peace and prosperity due to the intervention of Great Britain in establishing the Pax Britannica. 'Britannia'/England is portrayed in terms of the Good Shepherd who is capable of ensuring domestic tranquillity.

In the first tableau, Mother India greets and welcomes her twelve most renowned Cities: this underlines the importance of the destinies of the personified cities of India, as recorded by historical and mythological accounts: and the insistence on a historical and mythological framework is a structural literary device used by Hamilton to write a new history of the British nation and of the Empire, all inscribed in the European chivalric tradition.

The libretto also posits dichotomies with the ideological, political values and attributes of Great Britain and those of India, thus the text plays out the primary binary opposition between England and India. As a matter of fact, quite typically of colonial discourse, India is referred to in the libretto as Mother India, an old trope identifying the land as feminine, a 'feminine other', enforcing the British rule over a colonial territory also by means of a trite gendered representation and display of imperial male power. The rule of the King-Emperor is celebrated and everyone involved seems to be happy in kneeling before him in a colourful and exotic manner intended, as it was also intended by the organisers of the Durbar that took place in Delhi, to fascinate and draw the general public to the theatrical rendering of the official ceremony performed at the Coliseum.

\section{Edward Elgar's The Crown of India, Opus 66, 1912 as the Representation of Places of the British Empire}

It is important to stress that The Crown of India for Elgar was a real breakthrough in his career since the commission not only gave him the kind of commercial success he had been looking, and striving, for but also confirmed him as the royal composer of the Empire. ${ }^{4}$ His involvement with royal occasions (most notably Queen Victoria's 1897 Jubilee and the coronations of Edward VII and George V - not forgetting his The Spirit

4 After the success of 1912 two-weeks' performances at the Coliseum, The Crown of India was never performed on stage again in its entirety. Sadly, the original complete orchestral score was lost in the '60s. In 2007, the Elgar Society commissioned an orchestration of the piano score from Anthony Paine. This version was recorded by the BBC Philarmonic and Sir Andrew Davis in 2007 (Chandos). 
of England (L. Binyon) in 1914, Empire March in 1924, The Pageant of Empire (A. Noyes) in 1924 - culminated in his appointment as Master of the King's Music in 1924. His participation in The Crown of India was another way of showing his loyalty to the Crown, and of demonstrating the national and imperialistic beliefs and values he so revered (Hughes 1989, 41-68; Kennedy 1990, 107-17).

Elgar could not but be fascinated by the inheritance from the seventeenth-century court masque. That masque was a multi-faceted, multilayered, complex genre, an aesthetic expression of the early modern Stuart court, offering its various political ideologies as theatrical event. The genre was basically an elaborate costumed dance party, with dramatic conceits (derived from classical myth) and prose romances conceived to praise and honour the monarch. This courtly panegyric contained many different expressive forms: the masque-text, music (Walls 1996), elaborate painted scenery, stage machinery, songs, dancing and, at the end, the final revels, when the aristocratic masquers joined and danced with the spectators (cf. Butler 1994, 91-115).

Careful analysis of the multiple cultural and socio-political contexts that went into the masque's structure shows it deals with national issues and was a very powerful tool of propaganda for the Crown's policies (Orgel 1965, 62; Orgel, Strong 1973). In the masque, were embedded not only local-political concerns regarding Whitehall's closed circle of the participants in royal power discourses (also in virtue of their physical proximity to the royals). The masque also embodied the ongoing views regarding national political affairs as well as England's contemporary relations with Europe.

Such contents and purposes of the Stuart masque were ideologically shared by Elgar in his approach to a 20th-century Masque. Elgar seems to have understood that the socio-economic and political context of The Crown of India supports the contention of Raymond Williams that:

from at least the mid-nineteenth century, and with important instances earlier, there was this larger context [the relationship between England and the colonies, whose effects on the English imagination 'have gone deeper than can easily be traced'] within which every idea and every image was consciously and unconsciously affected. (Williams 1973, 165)

Williams has a telling grasp of the historical situation that can be found in Elgar's masque. As Williams points out, it is difficult to ascertain the strength and depth of Empire mythology in the British consciousness during the late Victorian and Edwardian times. The relationship between England and its colonies is embodied and represented in the work by Hamilton and Elgar and in how their artistic creations help the public's attitudes towards Empire. 
Edward Elgar's incidental music for the 'Imperial Masque' for The Crown of India consists of seven orchestral numbers, two songs, and six pieces of melodrama. The general idea expressed in the libretto, and in Elgar's music itself, is that Indian music and culture was barbaric and inferior when compared with the European tradition, including English tradition (Cowgill, Rushton 2006). As Sharon Hamilton, has noted:

According to Victorian thought, European music like European society, worked precisely because it was ordered and systematic, it was everything which 'native' music - and by extension 'native society' - was not'. The Crown of India is full of indications that Elgar used Orientalist tropes when composing: the Masque constructs a binary opposition of East and West: India represented by pseudo-Oriental chromatic melody and harmony while Britain is illustrated by ceremonious diatonic invention. (Hamilton S. 1998, 53)

At this point, we must try to gain a true insight into Elgar's own beliefs and ideologies (Kennedy 1987, 252) since Elgar's life and works are also an expression of an ideology of Empire commonly acknowledged and shared in this period. We must bear in mind that his beliefs were built upon a political vision entirely in accord with the dominant imperialist doctrines of his time. Moreover, with his music for The Crown of India, Elgar was making a conscious effort, willing to confirm to the general audience, themselves enmeshed in the imperialist credo, as 'nation' and 'the people', that his own personal ideology was completely identical with the national vision of Empire. In fact, he was trying to give musical voice and artistic expression to what they believed in. Through a short composition meant to articulate structurally and culturally, Elgar wants to represent, through long-established musical tropes, a summa of these political doctrines both national and imperial.

Elgar expresses his imperialistic feelings and views in his music for the masque by rendering the binary opposition of East and West through the juxtaposition of an 'orientalised style' of composing to represent India and an overtly 'ceremonial pomp' music to represent St George-England and the King-Emperor George V (Gardiner, Crump 1986, 164-90). Elgar's music for the masque, with its dialectic of musical styles, really possesses the same simplicity of idea and ideology of Colonialism as 'mission' (Kipling's 'white man's burden' is not far off). ${ }^{5}$

5 The term 'imperialism' requires some in-depth definition since its meaning has altered over time. As anticipated earlier, in the nineteenth century, imperialism possessed a clearly defined, policy-driven agenda to expand the Empire and maintain control over its constituent parts. Here, the term is used in accordance with Said's definition to mean the "practice, the theory, and the attitudes of a dominating metropolitan centre ruling a distant territory". In Edward Said's (1993, 3), the term becomes ideologically rather than politically defined in 
When viewed as part of the contemporary ideological matrix, Elgar's treatment of the Orientalist subject of The Crown of India reveals sympathy with the dominant imperialist tropes of the period. Musically, the East is portrayed as weak and indecisive, the West as bold and powerful, assertive in major mode against the chromaticism in the minor tonality of the Orient.

This polarity is nowhere more evident than in the music of the two marches - the "March of the Mogul Emperors" and "The Crown of India March". The "March of the Mogul Emperors" appears in the First Tableau, as Delhi summons the ancient Emperors to testify to her greatness. The stage directions indicate the spectacle of the procession as the Emperors enter. To match this striking scene, Elgar created an animato, with relentless drive and changing rhythm. Perhaps he was playing a musical joke on the Emperors with the use of this tempo, to which it is impossible to march properly so that it forces an uneven, clumsy stride. One might suggest even that Elgar composed the march to force the Mogul Emperors to demonstrate their inferiority in 'race', education and manners. Thus, their lack of choreutic skills were put on show to suggest their political incompetence as compared to the British imperial political and cultural values.

Musicologist Corissa Gould asserts that the march is a 'musical expression of the common Orientalist tropes' (Gould 2007,15) so much so that Elgar, to stress the opposition, devised and composed a noble, sombre orderly march for the 'civilised West' to show British supremacy through the music. The prevalent Orientalist trope of the time proposed by the composer was one of the central justifications in reaffirming the rights of Western domination. With this trope, the unchanging ancient old barbaric, unmannered and unpolished East is denied any modernity or progress. It is seen as without grace or artistic development throughout the millennia; it follows that without the West's intervention it would have remained a land uncivilised without grace or power. ${ }^{6}$ Thus Rudyard Kipling, an almost exact contemporary of Elgar and an imperialist poetic voice, wrote that if the British were to leave India, the country would dissolve into "one big cockpit of conflicting princelets" within six months. ${ }^{7}$ It was this perceived aspect of the, uncivilised, uncouth nature of the Indian people that Elgar aimed to portray in his incidental music - the Indian Moguls despite their best attempts to march cannot quite manage the dignified imperial stride in respect of the civilised British King-Emperor.

The music critic Nalini Ghuman expresses this very view in her assessment of the Elgar's masque stating that:

Said's theoretical framework. Our aim is to use Said's insights and also integrate his views with a more political discourse when possible.

6 See Hughes 1989, 41-68; Kennedy 1990, 107-17 and Moore 1985, 242; Anderson 2002, 311.

7 Quoted in Kennedy 1987, 252. See also Percy 1955, 355. 
The masque is a fascinating work of imperialism: historically illuminating and often musically rich, it is nevertheless a profoundly embarrassing piece - a significant contribution to the orientalized India of the English imagination. We might hear it, in some ways, as the realization of British imperialism's cumulative process: the control and subjugation of India combined with a sustained fascination for all of its intricacies. ${ }^{8}$

In the "March of the Mogul Emperors", the music becomes incessant in rhythm and takes on a rather ferocious quality that propels the movement forwards. The incessant rhythms suggest the volatility of the Indians, with the syncopated motive of the first theme becoming an unrelenting ostinato behind the more lyrical second theme and towards the end of the movement, a new ostinato figure begins which was the leading-note but avoids resolution and this gives the orchestral texture a disquieting and insistent dissonance, as minor chords close the movement.

Corissa Gould affirms that:

Elgar apparently sympathetic with the imperialist ideology inherent in the libretto is demonstrated by more than his written comments on the work; he also endorses it on the musical level. In his score, he renders the binary opposition of East and West through juxtaposition of a quasiOriental style of writing to represent India with ceremonial pomp to represent St. George and the King. (Gould 2007, 154)

"The Crown of India March", composed for the imperial procession of functionaries, soldiers, courtiers, and of course the Emperor and Empress, appears to be in stark contrast to the preceding dissonant march. It contains the stately pomp one might expect Elgar to produce in response to such a stately imperial parade also remembering that the composer had already written an 'Imperial march' and a 'Coronation Ode'. The sliding chromaticism of the previous march disappears and the music is dominated by the major mode throughout. The difference in tonality makes a strong contrast to the minor mode of the 'oriental' march (Gould 2007, 156). It seems to me both Ghuman and Gould fail to recognise that Elgar's "The Crown of India March" is deeply influenced by the chromaticism and tonality of Rimsky-Korsakov's "The Song of India" (1861), which Rimsky-Korsakov later developed in a complete opera entitled Sadko (1891). Elgar, in his composition, derives the Oriental sound-scape and stately pomp directly from the great Russian composer and uses it to set the mode and tone for the celebration of the British Empire.

8 Ghuman 2007, 249-85 (278). Ghuman's and Gould's are the only two scholarly contributions devoted to Elgar's masque existing in the literature on the composer. 
Once this opposition is asserted, Elgar proceeds with it to confirm the imperialist attitudes which have been exemplified by the contrasting marches: "The Crown of India March", which had been diatonic for British officials against "The March of Mogul Emperors", an apparent parody of Indian culture with a 3/2 - a time inappropriate for a march, crude orchestration and sudden modulations and at the end a trumpet ostinato with a shocking dissonance. The military suggestions in the text are also overtly expressed in Elgar's music: the fanfare theme of the opening is taken up during the song and will serve also as the start of "The Crown of India March". One must stress that, whereas the song accompaniment contains these military suggestions, Elgar seems not very attentive to the word setting. Elgar also includes here a rendition of the chorus of the song "The Rule of England", offered as an interlude, thus emphasising repeatedly the 'honour and righteousness' of English rule in overt terms. Moreover, it is important to declare here that this repetition does not feature in the libretto at this point and it was, one supposes, Elgar's own idea to add it as an explicit reaffirmation of the sentiments he wished to portray in the music and convey to the audience of the Coliseum in London. One must underline, in addition, that the binary opposition of musical styles is incessantly maintained throughout Elgar's music for the masque: India's music is constantly in the minor mode, Britain's is always in the major; India's is largely chromatic in contrast to Britain's diatonicism; India's rhythmic volatility contrasts with the regular rhythms of the West. Having established this musical style and mode, Elgar is able to use these musical signifiers to reiterate the ideologies inherent in the libretto of Hamilton, even though the composer does not pay much attention to the lyrics.

The music for the final scene of the composition reaffirms Britain dominant role in its rendition of a eulogy for imperial power: the theme is in the minor tonality, always directly associated with India throughout the masque. The India theme in the minor chord is placed against the theme of "The Crown of India March" in the relative major and so yet again, musically Britain overpowers the East and India is made to submit to her British rulers -once again faithful to the sentiment of the libretto. The general effect that Elgar wants to obtain with the marches is to represent aspiration and hope brought to India by British rule - in fact, this incidental music is a testimony to Elgarian imperialism and it expresses a political ideology he fully supported. The Crown of India marches are magnificent display pieces, apt for their time, and still of some worth, if they can be listened to without nostalgia for an imperial past remembering that Elgar's march style can cause embarrassment if read and understood from our contemporary post-colonial critical theories. ${ }^{9}$

9 For further discussions on Elgar's marches, see McVeagh in Riley 2009. 
On this topic, musicologist Jeffrey Richards states that:

Elgar's vision of Empire [...] is a vision of justice, peace, freedom, and equality, of the pax Britannica and of the fulfillment by Britain of its trusteeship mission, to see the countries in its charge brought safely and in due course to independence - a far from ignoble dream. $(2002,51)$

The critic Lawrence James maintains that, through the tonal qualities mirroring the power balance inherent in the masque's text, Elgar provides a musical depiction of the imperial doctrine of the time, portraying the "foibles of Eastern people, their iniquities, their mindless autocracies, and their general inadequacy in the face of an easy Western superiority" (MacKenzie 1984, 54). The available evidence leaves no doubt as to Elgar's imperial ideals and beliefs; the discomfort surrounding this work, with imperialist content, is entirely the result of later postcolonial generations attempting to relieve the guilt of what was revealed to be a morally questionable regime, motivated primarily by greed. The scholars do not stress enough that the production and reception of imperial works was not subject to the sense of immorality felt by our contemporary world. Exposed to a huge propagandist effort in the years between the Boer War and the First World War, an 'imperial consciousness' was manufactured in the minds of the Edwardian public, extolling the virtues of Empire, both for coloniser and colonised, creating a sense of righteousness and pride that few thought to question (James 2001, 327). It was not until after the First World War that this vision began to disintegrate.

By recapturing the innocent view of imperialism held by the majority of his contemporaries, a reading of Elgar's The Crown of India as a social text of the early twentieth century reveals and confirms many aspects of the mechanisms of British imperialist culture at that time. Elgar's work becomes a constituent of the ideological web which "supported and perhaps even impelled" imperialism, and includes the "notions that certain territories and people require and beseech domination, as well as forms of knowledge affiliated with domination" (Said 1998, 8). On a more local level, The Crown of India also opens a window on Elgar's ideology allowing one to see behind the constructed image of the composer and providing a starting point for a more honest and historically accurate reassessment of Elgar's life, devoid of the effects of the embarrassment and discomfort provoked by England's imperialist past.

It must be stressed that Elgar's Orientalism was an imaginative recreation of the idea of the Orient that was commonplace in Great Britain and in Europe at this time. The general public was acquainted with harmonies and rhythms and its typical music to represent the East. In contrast to the British Imperial music style and mode, oriental music was interpreted entirely within a Western musical context with a dialectical mode meant 
to underline the differences between the two contrasting and opposing worlds, values and 'races' both in the East and in the West. As such, Elgar echoes the libretto's story of British dominance, observing India from a British viewpoint. In fact, the perspective given is only British and India is not allowed to speak for herself. For all its chromaticism and ostinati (by then old musical signifiers of Western musical depictions of the exotic), the "March of the Mogul Emperors" is essentially a march in the form of Western art music, albeit, as we noticed, with an unusual tempo. The obsolete tropes used by Elgar's oriental signifiers were not lost on contemporary commentators, some critics observing that with the use of such tropes, his music lost part of its characteristic individuality.

However, the majority of audiences at the Coliseum would have been familiar with the conventions of Orientalism in music and their significance. The exotic was a highly popular subject in music-hall, theatre, songs, and salon pieces; musical comedies reinforcing standard Indian stereotypes were commonplace in the theatre tradition at the end of the nineteenth century. By employing standardised techniques, music language and tropes, Elgar was communicating with the audience in terms that would have both appealed to them and been understood as representing the East. In addition, he was adhering to the propaganda made also by the press that the music was deliberately intended to be popular and for the secular stage (Head 2000, 164). It seems for Elgar, then, Orientalism was simply a tool for communication and representation of commonly shared ideological values through music tropes that had become clichés. He felt no need to experiment further with the sense of 'otherness' and never appears to have considered that it might be used to further his own musical language or convey India in its own terms. Instead, he inserts in his musical suite the oriental tropes already accepted and fashionable and the resulting music is ultimately, music of the Raj - of the Englishman abroad - composed in a setting no more exotic, as Lawrence James wrote in 1997, than Elgar's Hampstead home (James 1997, 4; see also Gardiner, Crump 1986, 389). In the imperial counterpoint between centre and periphery, the critic John MacKenzie wrote in 1995 that Elgar's work provided overwhelmingly dominant melodies (MacKenzie 1984, 54; see also Said 1998, xv).

The relationship between Imperialism, Orientalism and the East-West power balance has become familiar also to contemporary music critics from the writings of Edward Said. In his book Culture and Imperialism (1994), Said emphasises that all Western discourse on the Orient, including art and music, is dominated by the East-West power display and balance - most notably he devoted a section to Verdi's Aida, set in Egypt, and there Said shows how the power of Western dominance is constructed, enacted and performed from a Western perspective. It is a pity that Said does not investigate other cultural musical phenomena. 
To Said, Orientalism, defined as Western discourse on 'the Orient', is a regime of power inextricably linked to Imperialism and he seeks to demonstrate the centrality of Western culture in the rationalisation, justification and transmission of imperialist values, stating that "culture is a sort of theatre where various political and ideological causes engage each other" (MacKenzie 1995, xiii-xix).

In doing so, Said warns us to remain critical of the Imperialist discourses developed by the West to defend its political and cultural supremacy. Even though, in contrast to Said's position, John MacKenzie suggests that Elgar's Orientalism can have functions other than the political one; the critic states, for instance, that composers at the beginning of the twentieth century began to experiment with oriental ideas as a way of extending their musical language, a venture distinct from imperialist ideas (Head 2000, 25). In the case of music, it has been suggested by Head that "sometimes, a musicologist works at a level of musical detail that can make Said's thesis of a binary opposition of Self and Other seem too broad to account for what matters most: the music itself" (25).

Though of course accepting the opinion of Head, we cannot but agree with Said's critical insights offered on Verdi for our current analysis of The Crown of India, and, making Said's affirmations our own, we might say that all regimes depicted in music should be approached with reference to 'the imperial process of which they are manifestly an unconcealed a part' (Said 1998, xiv). In this critical perspective, the Imperial Masque is not only influenced by the East-West power relationships of imperialist regimes' ideologies, it is constructed to display the dominant power of the British-rule ideology and render it manifest as an inherent civilising factor as understood by British subjects at home and abroad - an attitude we may derive from the 'Courtly Music Panegyric', a form inherited directly from the Stuart period through the Jonsonian Masque.

\section{Representations of the Raj on the London Coliseum Stage}

The performances of Elgar's Masque at the London Coliseum helped 'feed' the ideological and political image of this imperial space to the average British home-public - with the masque's topographical and cartographical re-reinvention of the exotic places of the colony of India then represented as simultaneously real and 'unreal' territory on the stage. The constitution of two contradictory spaces, the real and the mythical, makes such spaces at once both a perfect microcosmic analogy of the libretto's wider universe and in the theatre offers privileged locations where such spatial disjunctions can occur. In fact, The Crown of India's opening scene also presents an encounter on stage with foreign female outsiders coming from a different nation and continent and, hence, from a different culture and 
'race', thus momentarily on the threshold of two apparently incompatible worlds and spaces. In its explicit juxtaposition of binary oppositions of cultures, 'races' and geographical territories and boundaries, The Crown of India embodies and enacts a 'landscape' of the British Nation and Empire through a representation of different sites. This visionary welcoming encounter with outsiders in The Crown of India also reflects the political notion of the imperial dominance of the British Isles over the foreigner travellers coming from the colonial East. And yet again, the broader implication, displayed throughout the masque's performance, is that of the British control over the outer distant colonial world.

Moreover, the meeting on-stage predicts the inclusion of the female personifications of the Indian Cities-Daughters of the Empire and of Mother Ind before the audience at the London Coliseum Theatre. Through the act of summoning and naming the Indian Cities, Mother Ind lists their origins, their histories, individual worth and purpose, locating them spatially and geographically at the core of the Empire on the stage of the London Theatre. This first meeting in the Coliseum represents an extended moment in which both performers and onlookers dwell, visually and physically, on the threshold of assimilation and inclusion on either side of the proscenium, creating a structurally hierarchical, ideological coherence for both the British audience and the represented sub-continental colony as a microcosm of the Empire.

The audience thus witness the incorporation and assimilation of the outsiders on-stage. This is the moment in which the Empire's cohesion expands to incorporate the new members. But before full assimilation can take place, 'Britannia' must experience itself as an ethnic hybrid, a Nation and an Empire made coherent by its ability to expand and incorporate at the same time.

By recalling India's imperial forbears, Mother Ind expands the encounter happening across the proscenium, allowing both the onlookers and the Indian Cities impersonated by the singers to identify themselves as active agents of the Empire, all playing different roles in a process of a dramatized meeting for assimilation. In fact, the description rendered by Mother Ind offers a fitting analogy and a perfect metaphorical symmetry between the British and Indian nations and their histories, with their intertwining ancient histories of national and imperial rule, thus creating a mythical and historical framework for British imperial rule. All the while, each group is a focal point for the other and the masque invites them to dwell on their respective forms of sameness and difference. Thus, the masque magnifies their spatial and social coherence by extending their coming-together in the dramatic action. The stage offers the onlookers a romantic vision of the world outside the British Nation and frames the foreign travellers who are about to enter the threshold of two spaces, calling attention to their outsider position and status, while reaffirming the 
Empire's own integrity. The masque stresses the ability of the British Raj to assimilate the East into the West in a grandiose epic narration in which the histories of the nations, of the colonised and the coloniser, as well as that of the Empire, are strictly intertwined. In this perspective, The Crown of India asserts the Coliseum Theatre as a geopolitical and topographical centre of the British Raj, also in the felt need for a welcoming ceremony to accept outsiders into the 'West', a kingdom where the 'East' must be rightfully welcomed, contained and dominated by negotiating the incorporation of its out-sidedness.

Such transactions enabled the masque to construct the identity of the two parties involved and to merge them, by affirming the coherence of the group through performance. These spatial interactions mark these spaces as different from the spaces surrounding them. In the most literal sense, these spaces exist as part of the Empire's 'landscaped' sites at the Coliseum. These 'other spaces' coexist and are asserted by the innumerable thresholds entered and exited by the characters-singers on stage in achieving coherence and unity inside the hall. This unity is only created by reconfiguring the Coliseum as an 'other space' in which its occupants can perceive and see themselves as a self-reflexive commentary on the British Raj. Thus the masque staged implies an imperial coherence between singers-performers and public looking at themselves across the proscenium as if they were both reflected in a looking-glass.

The mirroring acts as symbol for the seemingly contradictory facets of the dramatic action by coalescing different spaces and we find combined the opposing nations and 'races' present in the hall during the masque's performance. Thus, the masque enacts the participants' inclusion within British society at the very centre of the Empire, while simultaneously mirroring the imperialist ideology embodied by the audience in the Coliseum Theatre through the spectators' visual access to the scene's illusions performed on stage. These overlapping and interpenetrating spatial dynamics were also active within the Coliseum's physical space. In a way, the hall functioned as a containing, private-event space in which participants were a uniform collective group of British loyal subjects -united by virtue of their inclusion in the event and reminded in several visual and spatial ways of their coherence. This process not only symbolises but also constitutes an exterior perspective from which two different contrasting realities are made to enact and coexist.

There are also geographical references in the libretto, along with detailed topographical indications and descriptions linked to the mapped colonised Indian nation, reminding the audience of British colonial territories. Remembering that all maps are politically-charged representations of space, this textual material emphasises the unreality of the space they enclose - since the act of mapping brings together a unified, all-embracing vision of different spaces, with their political, colonial, national and impe- 
rial coefficients. This unification happens in the Coliseum where with the physical inclusion on stage of the Indian Cities, 'Britannia' creates a new cartographic 'reality' that allows one to conceive the Coliseum as comprising the multitude of incompatible worlds described and enacted by the masque. The audience perceives the hall as a protected realm of display of power in a fictive geographical 'other' space of 'Britannia'. Inside the Coliseum, the theatre is capable of coalescing these different and incompatible 'other spaces', and onlookers are encouraged to conceive of that space as a framework for their own supposed coherence.

Thus, the Crown of India as a whole constitutes an imperial world, with its two contradictory Nations-Empires now merged into the British Raj ostensibly occupying exactly the same space. The British Empire, rather than being exclusively located in the libretto's impossible cosmology is a space defined as being both in particular exact places that make up the landscape described in the epic fictional world of the libretto and in the 'landscaped' performing space of the London Coliseum stage and Theatre at the same time. It is a spatial divide, with its geometrical and topographical anomaly, which represents the intrusion of a different world into the onlookers' sense of reality, leading to a different mode of perception and consciousness of this 'other space'. The space enacted on the stage is flawed by numerous inconsistencies and impossibilities because the topographical referents are in a quite different place and are always wandering into an 'other space' continually suggested in the repeated performance when they are slid into another dimension - at a geographical, historical and political level. Throughout the narrative the characters personifying places metamorphose into the real British place and space of the Coliseum, where they become visually assimilated to the British Nation and Empire. The performance on the stage functions as a reflection of the libretto's central theme. The masque itself works as a mirror characterised by projecting the material worlds existing apart from each other into a unified and unifying geopolitical transcendent prototype of the British Empire. The ordered world of 'Britannia', as Nations-Empires merged into the British Raj, is contained in the Coliseum as cartographic representation, a map conflating multiple nations ostensibly occupying the exact same space and creating also a geographical integrity in the 'landscaped' hall. The theatre-stage, itself imaginatively conceived as a map of the Empire, becomes a site where different 'races' and cultures are merged in incommensurable and impossible spaces, thus reinforcing a political unifying vision of 'Britannia'.

By suggesting a strange configuration of space in the mapped colony, the personifications of India can be in two places at once. The illusory quality of the map reflects the mingled realities of the Colony and the Empire. The map functions as a space where the imperialist ideology and policies are symbolically enacted and thus the action in the masque assumes mean- 
ings as reference to realities outside its own limits. Through the map, the masque, by reasserting colonial dominance on the exotic territory of the Indian subcontinent, acquires a full political meaning as a social enactment of power ordering social and political coherence. The staging of this cartographical vision renders the London Coliseum a precise site of courtly imperial inclusion and cohesion and it inserts the British Raj into this cohesion. Thus the London Coliseum Theatre becomes the microcosm of the British Empire.

\section{Bibliography}

Adams, Byron (ed.) (2007). Edward Elgar and His World (The Bard Music Festival). Princeton: Princeton University Press.

Anderson, Robert (2002). Elgar and Chivalry. Rickmansworth: Elgar Society Edition.

Barker, Felix (1957). 'The House that Stoll Built': The Story of the Coliseum Theatre. London: Allen.

Butler, Martin (1994). "Ben Jonson and the Limits of Courtly Panegyric”. Sharpe, Kevin; Lake, Peter (eds), Culture and Politics in Early Stuart England. Basingstoke: MacMillan, 91-115.

Clayton, Martin; Zon, Bennett (eds) (2007). Music and Orientalism in the British Empire. Farnham: Ashgate Publishing.

Colls, Robert; Dodd, Philip (eds) (1986). Englishness: Politics and Culture 1880-1920. London: Routledge.

Cowgill, Rachel; Rushton, Julian (2006). Europe, Empire, and Spectacle in Nineteenth-Century British Music. London: Routledge.

Gardiner, John; Crump, Jeremy (1986). “The Identity of English Music: The Reception of Elgar 1898-1935". Colls, Robert; Dodd, Philip (eds), Englishness: Politics and Culture 1880-1920. London: Bloomsbury Academic, 164-90.

Ghuman, Nalini (2007). "Elgar and the British Raj: Can the Mughals March?”. Adams, Byron (ed.), Edward Elgar and His World (The Bard Music Festival). Princeton: Princeton University Press, 249-85.

Gould, Corissa (2007). "An Inoffensive Thing: Edward Elgar, The Crown of India and Empire". Clayton, Zon 2007, 146-60.

"Grove Music Online”. Oxford Music Online. URL http://www.oxfordmusiconline.com/ (2018-12-05).

Hamilton, Henry (1912). The Crown of India: an Imperial Masque. London: Enoch.

Hamilton, Sharon (1998). "Musicology as Propaganda in Victorian Theory and Practice”. Mosaic: A Journal of Interdisciplinary Study of Literature, 31, 35-56. 
Head, Matthew (2000). Orientalism, Masquerade and Mozart's Turkish Music. London: Routledge.

Hughes, Meirion (1989). “The Duc D'Elgar: Making a Composer Gentleman". Norris, Christopher (ed.), Music and the Politics of Culture. London: St. Martin Press, 41-68.

Kennedy, Michael (1990). "Elgar the Edwardian”. Monk, Raymond (ed.), Elgar Studies. Aldershot: Scholar Press, 107-17.

Kennedy, Michael (1987). Portrait of Elgar. Oxford: Oxford University Press.

Lawrence, James (1997). Raj: The Making and Unmaking of British India. London: Routledge.

Lawrence, James [1994] (2001). The Rise and Fall of the British Empire. London: Routledge.

Lycett, Andrew (2000). Rudyard Kipling. London: Weidenfeld and Nicolson.

MacKenzie, J.M. (1984). Propaganda and Empire: the Manipulation of British Public Opinion, 1880-1960. Manchester: Manchester University Press.

MacKenzie, John M. (1995). Orientalism: History, Theory and the Arts. Manchester: Manchester University Press.

MacQueen-Pope, W.J. (1945). Theatre Royal, Drury Lane. London: W.H. Allen.

Monk, Raymond (ed.) (1990). Elgar Studies. Aldershot: Scholar Press.

Moore, J.N. (1985). Edward Elgar: Letters of a Lifetime. Oxford: Oxford University Press.

Norris, Christopher (ed.) (1989). Music and the Politics of Culture. London: St. Martin Press.

Orgel, Stephen (1965). The Jonsonian Masque. Cambridge (MA): Harvard University Press.

Orgel, Stephen; Strong, Roy (1973). Inigo Jones: the Theatre of the Stuart Court, 2 vols. Berkeley: Berkeley University Press.

Pellegrino, Joe (2010). "Mughals, Music, and "The Crown of India” Masque: Reassessing Elgar and the Raj". South Asian Review, 31(1), 13-36.

Young, Percy M. (1955). Elgar, O.M.: a Study of a Musician. London: Collins. Richards, Jeffrey (2002). Imperialism and Music: Britain 1876-1953. Manchester; New Yor: Manchester University Press.

Riley, Matthew (2009). Edward Elgar and the Nostalgic Imagination. New York: Cambridge University Press.

Sadie, Stanley; Tyrrel, John (eds) (2000). The New Grove Music and Musicians Dictionary. Oxford: Oxford University Press.

Said, Edward (1998). Culture and Imperialism. London: Vintage.

Sharpe, Kevin; Lake,Peter (eds) (1994). Culture and Politics in Early Stuart England. Basingstoke: MacMillan. 
Williams, Raymond (1973). The Country and the City. New York: Oxford University Press.

Walls, Peter (1996). Music in the English Courtly Masque 1604-1640. Oxford: Clarendon Press. 


\title{
James Joyce's 'Linguistic Musicality' A Short Insight into Some Linguistic Musical Patterns in the First Chapter of $A$ Portrait of the Artist as a Young Man, and their Echoes in "Sirens"
}

\author{
Arianna Autieri \\ (Warwick University, UK)
}

\begin{abstract}
The central aim of this paper is to show the similarities of some stylistic features of $A$ Portrait of the Artist as a Young Man with musical code. A second purpose is to verify how these musical features are echoed in "Sirens". In order to initially describe the common properties of language and music and to define how their acoustic and rhythmic similarities are relevant in written texts, the paper will draw on the theories of the Science of Rhythm - a non-academic discipline that influenced many modernist writings, and also studied the common rhythmic features of music and language. After detailing a musical method for the analysis of the linguistic texture of written prose, I focus on the first chapter of $A$ Portrait. Hence, I identify the musical characteristics of the novel's style through a comparison between some Joycean scholars' theories on music in A Portrait and the principles of the Science of Rhythm. Finally, a few examples of the musical language in "Sirens" will provide a benchmark for a comparison with $A$ Portrait.
\end{abstract}

Summary 1 Introduction. - 2 Lanier and the Science of Rhythm. - 3'Linguistic musicality' in the First Chapter of $A$ Portrait of the Artist as a Young Man. - 4.1 Echoes of $A$ Portrait in the Music of "Sirens": "Verbal Music". - 4.2 Echoes of A Portrait in the Music of "Sirens": staccato and martellato. - 5 Conclusions

Keywords Joyce. Style. Music. Linguistic musicality. Science of Rhythm.

\section{Introduction}

The aim of this paper is to propose a rhythmic and acoustic perspective on James Joyce's prose, with particular reference to some peculiar stylistic features of the first chapter of A Portrait of the Artist as a Young Man and their echoes in "Sirens". My main focus here is Joyce's 'linguistic musicality'. Although music has been vastly dealt with in Joycean Studies (eg. Bowen 1995, 1974; Martin 1991 and, more recently, Witen 2018), Joyce's style has seldom been analysed in musical terms, especially as far as the first works of the author are concerned. In this paper, I propose a small contribution to this field, suggesting a comparison between the language of $A$ Portrait and musical code. My theory draws on some findings of the 
Science of Rhythm - a non-academic discipline which developed at the end of the 19th century with the specific purpose of studying the composition of rhythm, and which also described some common rhythmic properties of music and language. The connection between Joyce's works and the Science of Rhythm has been firstly considered by William Martin in Joyce and the Science of Rhythm (2012). As Martin proves (2012, 4-13), the Science of Rhythm served to legitimise the stylistic experiments of modernists writers such as Pound, Yeats, and Joyce. ${ }^{1}$ Joyce read Herbert Spencer's First Principles (1862) while studying at the University College of Dublin; Martin claims that the chapter "The Rhythm of Motion" was particularly influential in Joyce's aesthetics, because the word "rhythm" started being used consistently in his theoretical writings ever since. According to Martin, Joyce also knew about Lanier's theory on the musicality of poetic language through his engagement with Arthur Symons's treatise on symbolism. Moreover, the principles of Helmholtz's studies on the properties of sound, upon which the scientific framework of Lanier's theory depends, are referred to by Bloom throughout the chapter "Sirens" (Martin 2012, 4-13 and 145; Plock 2009). ${ }^{2}$

Before proceeding, I would like to clarify what I mean by 'linguistic musicality' and to define how this concept is relevant to my analysis of Joyce's texts. The term 'musicality' has often been used with a metaphorical meaning in relation to literary texts, and it is usually associated with a general lyrical tone in novels or poetry. As Theodor W. Adorno's affirms $(1993,113)$, real similarities between the micro-level and the macro-level of verbal code and musical code can be identified. Music and language are initially composed by concrete sounds (notes and words) that are then organised in an ordered form to create a structural meaning (words are organised in sentences, and sentences in paragraphs, while notes are organised in melodic and rhythmical themes that then constitute the musical phrases and sentences). My hypothesis here is that, thanks to the author's musical ear, poetic language could assume several qualities of the musical compositions, both in its sound patterns and its rhythmical organisation: 'linguistic musicality' is hence used in this essay to refer to the acoustic and rhythmical qualities of poetic language that bear some resemblance to music.

1 Martin affirms: "For the purpose of studying the development of modernist poetry, free verse, and prose, the interaction between the fields of experimental psychology and prosody is of particular interest to the literary critic, as the theories of rhythm developed during this time not only worked to motivate the creation of new poetic forms, but also served to legitimate the stylistic experiments of modernists such as Pound, Yeats, and Joyce" $(2012,2)$.

2 As an example, Helmholtz's theory of harmonics is addressed in "Richie cocked his lips apout. A low incipient note sweet banshee murmured: all. A thrush. A throstle. His breath, birdsweet, good teeth he's proud of, fluted with plaintive woe. Is lost. Rich sound. Two notes in one there" $(U, 11: 630-3)$, where Bloom describes "the simultaneous sounding of Harmonics" (Martin 2012, 148) - "two notes in one". 
In Joyce's texts, the issue of musicality is particularly delicate. It is well known that Joyce was himself a musician. According to his brother Stanislaus Joyce, when writing Dubliners, he was "a musical singing... voice (a tenor), a good undeveloped talent in music" (Joyce S., 1958, XIV). Music is particularly relevant in Joyce's literary aesthetics, and several references to songs and operas have been found in his works. As Timothy Martin affirms in Joyce in Context (2009):

Over the years Joyce scholars - most notably Ruth Bauerle, Zack Bowen, Matthew Hodgart and Mabel Worthington - have unearthed a huge number of allusions that reflect Joyce's lifelong experience of opera, the music hall and pantomime, liturgical music and popular song. (Martin 2009, 278)

According to Martin, music is not only present in the allusions to real song in Joyce's work, but in several different forms, relating "to its texture, its structure and its ethos or general character" (278). Regarding language, Martin affirms: "certainly, there is a musicality reflected in the tonal and rhythmic qualities of Joyce's language, in his sound as opposed to his sense" (279). As far as musical language is concerned, not only can a generic musicality be identified in the style and in the rhythmic elements of the texts, but in "Sirens" the writer has set out to explicitly imitate music. This fact has sometimes led to the idea that if readers wish to understand how Joyce used music to shape his language, "Sirens" is the only text to be considered. However, as several scholars have pointed out, Joyce's own experience as a musician had immediate consequences for all his writings, from Dubliners onwards. For example, in his article "Music in Dubliners" (1992), Robert Haas claims:

Clearly Joyce in Dubliners was already beginning to use language beyond its customary limits; if music could help achieve his expressive purposes, it is reasonable to expect that he might use it too. (Haas 1992, 23)

Similarly, in his essay "The Distant Music of the Spheres", Thomas Jackson Rice (1999, 216-19) argues that "The Dead" is characterised by the presence of a "verbal music" that derives from some sonic expedients as alliterations and internal rhymes, and peculiar punctuation forms. In this sense, musicality could be identified mostly in the last pages of "The Dead", which are separated from the rest of the story through a blank space. Take, as an example, the following lines, which are the last lines of Dubliners: 
His soul swooned slowLY/ as he heard the snow falLING faintLY/ through the universe/and faintLY falLING/, like the descENT/ of their last END/ upon all the living and the dEAD. $(D: 224)^{3}$

Musicality here is mainly achieved thanks to the use of alliterations, "soul swooned slowly" or "falling faintly", repeated consonants, $s, w, f$, internal rhymes and assonances "descENT/END/dEAD", "slowLY/falLING/faintLY", and chiasmic figures "falLING faintLY", "faintLY falLING". ${ }^{4}$

Similarly, discussing A Portrait of the Artist as a Young Man, Jack Weaver claims that the text is characterised by the presence of a musical melos. This melos is defined through Minahan's words for Words like a Bell (1992), as:

the quality of sound and rhythm... created by... the conscious or intuitive arrangement of various consonant and vowel groupings... and degrees of stressed syllables. (Cited in Weaver 1998, 27)

According to Weaver, this musical melos mostly characterises the first chapter of A Portrait, and assumes different forms, which will be analysed in the sections below.

For the purposes of this paper, the theory of "primary and secondary rhythms" developed by Sidney Lanier in the field of the Science of Rhythm might be particularly significant, since it allows a comparison to be drawn between the real acoustic properties of language and of music, and provides a framework for a musical analysis of Joyce's language. While in Joyce and the Science of Rhythm (2012) William Martin investigates "the impact of rhythmic science on Joyce's critical and creative writings" (Martin 2012, 27), suggesting a simultaneous three levels analysis of the texts focused on "the study of (1) discourse, (2) influence, and (3) stylistics" (27), my purpose here is to apply Lanier's rhythmical principles, as presented in The Science of English Verse, to a mere stylistic analysis of A Portrait in order identify the concrete similarities between language and music in Joyce's prose.

3 In the example and in the whole essay, bold is used to indicate a remarkable vowel and consonant texture; capital letters indicate rhymes, assonances and consonances; and slashes indicate intonation units.

4 As we will later see in this essay, many of these expedients cause a concrete musicality, and bear upon the parameter of timbre. 


\section{Lanier and the Science of Rhythm}

Lanier's The Science of English Verse (1880) proposes a new contribution "towards a complete theory of the technic of English verse" (Lanier 1880, xv) through a musical analysis of the expedients used in poetry. In his study, the similarities between poetical language and music are identified through a comparison of the common acoustic qualities of their sonic material. Even though Lanier's work is mainly focused on the description of poetry, the rhythmical principles individuated could be considered relevant also in prose, as Lanier himself affirms: "It would not serve to discriminate verse and prose. Prose has its rhythms, its tunes, and its tone-colors, like verse" (Lanier 1880, 57).

In order to identify the musical features of poetry, Lanier investigates how the perception of read aloud language is characterised by rhythmic principles similar to those perceived in music. According to Lanier (50), since words are sounds produced by a "reed-instrument which can alter the shape of its tube (the buccal cavity) at pleasure", their sonic material could be compared to the sonic material of music:

'Words' means simply one kind of musical sounds, and 'musical sounds' means simply another kind. (Lanier 1880, 50)

The hypothesis of Lanier's theory $(1880,22)$ is that the acoustic perception also takes place during silent reading:

The science of verse, then, observes and classifies all the phenomena of rhythm, of tune, and of tone-colour, so far as they can be exhibited to the ear directly by spoken words, or to the ear, through the eye, by written or printed signs of spoken words. $(1880,58)$

Several later studies on 'auditory imagery' have confirmed Lanier's hypothesis, explaining how the duration, pitch and timbre perceived when reading silently maintain their acoustic qualities, thanks to the activation of the auditory cortex while decoding verbal meanings (e.g. Hubbard 2010; Perrone-Bertolotti et al. 2012).

To formulate his theory, Lanier initially draws on the concept of "compound rhythm" by Herbert Spencer:

Rhythm is very generally not simple but compound. There are usually at work various forces, causing undulations differing in rapidity; and hence it continually happens that besides the primary rhythms there are secondary rhythms, produced by the periodic coincidence and antagonism of the primary ones. Double, triple, and even quadruple rhythms, are thus generated. (Spencer [1862] 2009, 316) 
According to Lanier, in poetry as well as in music, rhythm is given by an initial "primary rhythm" which is subsequently organised by other "secondary rhythms". As Martin points out $(2012,124)$, there are several passages in A Portrait that prove Joyce's familiarity this theory. As an example, Stephen appears to distinguish between primary and secondary rhythms when listening to the sound of the train track during the journey to Cork with his father. Stephen in fact composes a prayer which

ended in a trail of foolish words which he made to fit to the insistent rhythm of the train; and silently, at intervals of four seconds, the telegraph poles held the galloping notes of the music between punctual bars. $(P, 73)$

According to Martin $(2012,124-5)$ the intuition of a primary rhythmic form precedes the composition of the poem, and "the choice of diction (and its order) is predetermined by the perception of the metrical pattern".

The theory of composed rhythm is particularly useful in order to understand the similarities between rhythmical patterns of music and poetry. Lanier argues that a primary rhythm is perceived whenever a sequence of sounds and silences is present. In order to understand this sequence of sounds, the mind needs to organise them in patterns; the organisation is carried out with reference to the four parameters of sound, duration, intensity, pitch and timbre (or colour):

(1) How long a sound lasts (duration); (2) How loud a sound is (intensity); (3) How shrill that is, how high, as to bass or treble a sound is (pitch); (4) Of what sounds a given sound is composed [...] (tone-color). ${ }^{5}$ (Lanier 1880, 24)

The result of this organisation is a perceived secondary rhythm of the same sonic material.

In order to illustrate his theory, Lanier proposes the following examples, which are meant to show the primary rhythm and its organisation in secondary rhythms, and which are particularly useful in understanding how Lanier's theory could be applied to a stylistic analysis of Joyce's texts. In the picture below, primary rhythm is composed of a sequence of eight sounds, each followed by a silence:

5 As far as tone-colour (or timbre) is concerned, it is important to underline that a peculiar timbre is perceived thanks to the harmonics emitted when a sound is played, both in music and in language. 


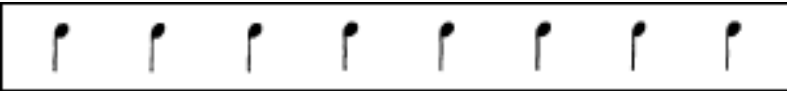

Figure 1. Lanier $1880,41^{6}$

The initial sequence can be grouped according to the duration of each sound or the duration of the silences,

the ear [...] may again coordinate the same sounds with secondary reference to their duration in order to divide them into groups of two or more units; each group being distinguished by some variation in the duration of either of its sounds, or in the duration of the silences between them (Lanier 1880, 43)

which musically could be written as: ${ }^{7}$

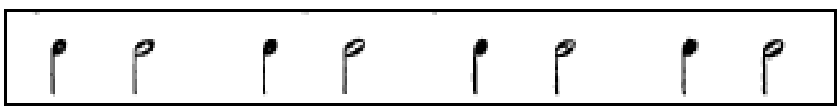

Figure 2. Lanier 1880, 43

The sequence can be grouped also according to the pitch of the sounds,

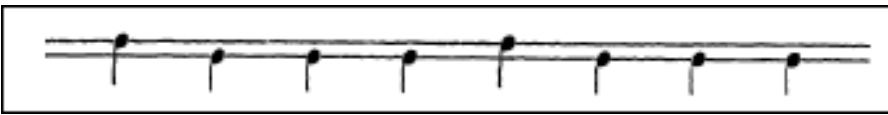

Figure 3. Lanier 1880, 45

their colour (timbre),

The illustration as to the eye readily suggests that the eight notes might be grouped for the sense of sight by making every second, third, etc., note of a certain color, say red, while the other note or notes of each group were blue. (Lanier 1880, 46)

6 The example refers to the musical notation in a mere graphical sense.

7 We should note that this example was used by Lanier to explain how intensity works in secondary rhythm using two different blacks (one more intense than the other). However, as the musicians among the readers can possibly notice, the example proposed suits better the first definition of duration. I will provide an example designed following Lanier's description of intensity later on in this essay. 
which we can graphically represent as

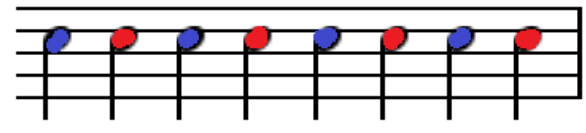

Figure 4. Graphic representation of the rhythmic organisation of eight sounds according to their colour

In real acoustic perception, the sounds can also be grouped according to their intensity (i.e. their volume),

Suppose, for example, the first be printed in ink of an intenser black than the second, and this variation be consistently carried on through the eight, (Lanier 1880, 43)

which we can illustrate as:

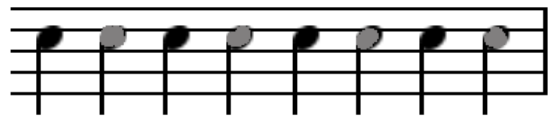

Figure 5. Graphic representation of the rhythmic organisation of eight sounds according to their intensity

As far as intensity is concerned, Lanier explains that in written texts this parameter does not play the same distinguishing role that it plays in real hearing. The minor role of intensity in the perception of written sounds is confirmed also by Hubbard's studies on auditory imagery (Hubbard 2010, 304-5).

In order to provide a further explanation of his theory, Lanier also proposes an acoustic example, and describes how the hearers perceive the regular sequence of the sounds of the clock (primary rhythm) as if they were divided in groups of two (secondary rhythm):

[we perceive] a difference in pitch, as if the clock, instead of saying, "Tick-tick, tick-tick, tick-tick," and so on, should say, "Tick-tack, ticktack, tick-tack," and so on [...] but also a difference in emphasis, stress, or accent (that is, in intensity), as if the clock said "Tick-táck, tick-táck," and so on. (Lanier 1880, 63-4) 
As Martin points out, this example was significant also in Joyce's aesthetics:

It is clear that Joyce associated the perception of the primary rhythm with the ticking of a clock, for the third section of the novel depicts a "Fire Sermon" in which Father Arnall constructs a rhetorical conceit that links the opposition between heaven and hell to the alternation between the 'tick' and the 'tock' of a great clock. (Martin 2012, 126)

Following Lanier's theory, we can state that a musical composer can influence the perceived rhythm of the composition by melodic, durational and timbrical expedients that organise the primary rhythm (a sequence of notes and silences) - hence determining a pre-organised secondary rhythm. By recurring to long and short notes, to the repetition of phrases (musical sentences), as well as by dividing the composition in sections, the composer influences the rhythmic duration of the musical text. Similarly, melodic patterns of notes influence the secondary rhythm according to the parameter of pitch, and a different set of musical instruments, or effects on the same instrument, influence the rhythm according to the parameter of timbre. Finally, recurring to musical dynamics (forte, piano, crescendo), the composer influences the rhythm according to the parameter of intensity.

Although in the rhythmic organisation of language the meaning is also to be considered, the writer can use several poetic devices to organise the verbal material of poetry and prose. According to Lanier, primary rhythm in the English language is given by "verse sounds" $(1880,97)$, small sonic units that are represented by words and silences. Metrical expedients and the organisation of the poem in verses and stanzas (in poetry), or, we can infer, the organisation of the prose text in phrases, sentences, and paragraphs (achieved also thanks to punctuation), as well as the recurrence of words and sentences, influence the secondary rhythm according to the parameter of duration. Intonation units ${ }^{8}$ and prosodic elements ${ }^{9}$ are signalled through the punctuation, which can be used in peculiar ways to modify the pitch of the text. The intonation or pitch of the text is also modified through emphatic groups - such as an unusual word order, or peculiar word plays which condition the stresses of the sentences. Finally, because each vowel and consonant have their peculiar timbre, devices such as alliterations, rhymes, assonances and consonances, or a remarkable vowel

8 Spoken language is characterised by prosodic units, called "intonation units", with one or more picks of intonation. Each of them is characterised by a peculiar melodic movement in its final part, and they are separated by pauses (Lanier 1880; Chafe 1988; Knowles 1987).

9 As reported in Anatomy and Physiology for Speech, Language and Hearing (2010), "Pitch and intensity play a significant role in the suprasegmental aspects of communication" (Seikel et al., 2010, 254). 
and consonant texture have a bearing on the timbre of the text. This latter statement might need some more explanation. In fact, although it is commonly thought that vowel and consonants have different pitches, ${ }^{10}$ because the pronunciation of linguistic sounds depends on the physical shape of the vocal cavity, phonemes are characterised by different timbres and not different pitches. On this topic, Lanier writes:

Every vowel-sound, every consonant, every combination of letters in a syllable, every shade of pronunciation, is simply a difference of tonecolor made by the almost instantaneous changes which the muscles of the mouth and throat can effect in the shape of the buccal cavity. (Lanier 1880,52 )

The connection between phonemes and timbre has been confirmed in later studies; for instance, in Pitch and Timbre: Definition, Meaning and Use (1997), Houtsma writes:

The timbre, although not commonly named this way in speech literature, is different for each phoneme and depends physically on the shape of the glottal air flow pulse and the instantaneous shape and length of the vocal tract (throat, oral and nasal cavities). (Houtsma 1997, 110)

Drawing on Lanier's theory, I will now show how it could be useful to define musicality in the first chapter of A Portrait of the Artist as a Young Man and to compare it with "Sirens".

\section{3 'Linguistic Musicality' in the First Chapter of A Portrait of the Artist as a Young Man}

Although references to music and rhythmic patterns could be found in the whole Portrait, as Jack Weaver points out, it is in the first chapter of the novel that musical language is particularly significant $(1998,27)$. In the first pages of $A$ Portrait of the Artist as a Young Man, the acoustic dimension of the language and its poetical qualities are used to show Stephen's artistic potentialities. Weaver notes this as follows:

Stephen's focus upon the sounds and meanings of words combines music and rhetoric and suggests he has a future in the arts. (1998, 29)

10 For instance, in his study, William Martin refers to vowels and consonants as if they were different in term of pitch: 'the 'bright' timbre of the Sirens' voices can be reflected by the choice of vowel sounds with a high pitch (i/ee)" $(2012,152)$. 
Through what Kershner defines "pseudo-stream-of-consciousness" (Kershner 1986, 885) and the free indirect discourse, Stephen's awareness of the properties of the language is mirrored in a narrative style in which sounds play a significant role and can thus be considered 'musical'. In order to describe the musicality in the first chapter of $A$ Portrait, I will initially make reference to the definition of melos given by Weaver in Joyce's Music and Noise (1998). Weaver describes three forms of melos in A Portrait: the lullabies and the 'baby talk' of little Stephen, Stephen's concern with the meaning and the sound of words, and, finally, theme and variation forms - id est repetitions of sentences, 'augmented' or 'diminished' with more words or less words when repeated. In order to make some comparisons with "Sirens", I will focus on two modalities of melos: namely Stephen's concern with words, and the theme and variation forms.

Stephen is interested in the relationship between the meaning and the signifier of words. Therefore, he reflects on words which imitate real sounds, such as "suck" ( $P: 9)$, on words with different meanings, such as "belt" ( $P: 7)$, on the meaning of his name or on the relationship between the word "God" and the meaning of "God" in different languages. Stephen's attention to words often results in their repetition throughout the whole chapter. As we have seen considering Lanier's theory, these periodical repetitions mainly influence the secondary rhythm according to the parameter of duration. However, Stephen's interest for the sonic material of the words sometimes results also in the use of timbrical expedients such as a peculiar consonants and vowels texture, alliterations, imitative phonemes and internal rhymes.

If we consider the following passage from A Portrait,

1 It made a roar like a train at night. And when he closed the flaps/ the roar was shut off/ like a train going into a tunnel./ That night at Dalkey the train had roared like that/ and thEN, /whEN it went into the tunnel,/ the roar stopped. He closed his eyes/ and the train went on,/ roaring/ and thEN stopping;/ roaring agAIN,/ stopping. It was nice to hear it roar/ and stop/ and thEN roar out of the tunnel agAIN/ and thEN stop, $(P: 11)$

we can notice that the most used rhythmic expedient is the repetition of words - "roar", "stop", "train", "tunnel", "then", "again", "going in/out". Timbrical expedients are also used: there are internal rhymes - like "agAIN /thEN", "nOISe/ bOYS" - and phonemes that imitate real sounds - such as the occlusive $t$ and $p$ in "stop", which could imitate the dampened sound of the train in the gallery, or the liquid $r$ that allows the sound to expand as the noise of a train out of the tunnel or of the refectory. In this example, punctuation, which influences both the duration and the intonation of the text, is used to distinguish the sentences in such a peculiar way that the 
reader can perceive how the rhythmic motion of the phrases mirrors the motion of the train.

Similarly, in the following passage,

2 First came the vacation/ and thEN the nEXT tERM/ and thEN vacation agAIN/ and thEN agAIN another tERM/ and thEN agAIN the vacation. It was like a train going in and out of tunnels/ and that was like the nOISE/ of the bOYS/ eating in the refectory/ when you opened and closed the flaps of the ears./ Term,/ vacation;/ tunnel,/ out;/ noise,/ stop./ How far away it was! $(P: 14),{ }^{11}$

the rhythmical recurrence of the words "then", "again", "term", "vacation" is the most used musical device. The passage is musically similar to example 1. The alternation between "term" and "vacation", in particular, resembles the alternation between the verbs "roar" and "stop", and the same rhythmical words "then" and "again" are also used. Timbre effects - rhymes, "thEN/agAIN" (or "nOISE/boYS, as in the preceding example), and assonances, "nEXT/tERM" - are present too. Intonation units, although not signalled by punctuation, similarly reflect a recurring movement, like the one of the train. However, a new musical device is introduced: in "Term, vacation; tunnel, out; noise, stop" we can notice an unusually excessive presence of commas and semicolons, which determines the perception of short intonation units.

Martin also refers to this last example in his analysis of Joyce's writings. Although Martin draws on the rhythmic principles of the Science of Rhythm too, his analysis mainly focuses on the poetic significance of the rhythmical patterns identified in the text. In fact, he suggests that when presenting the rhythmic experiences of Stephen, Joyce "reveals the 'tension' that motivates the biographical development of the hero", since Stephen's personality is "'stretched' between music of the maternal sphere (the metrical rhythm) and the intellectual image of the paternal sphere (the structural rhythm)" (Martin 2012, 30). The maternal and paternal spheres are physically separated, then, as the former is connected to home and the latter to the boarding school at Clongowes Wood. In Martin's view, the train journey from Bray to Clongowes Wood provides Stephen with an experience of continuous movement. Although Stephen is at school in the above example, "the memory of the train journey allows him to simulate the alternation between home and school, creating a continuous rhythm that dramatizes the structural rhythm as the opposition between two poetic symbols" (119). In example 2, Martin therefore identifies "three rhythms with different periods of recurrence": he argues that "term" and

11 Repeated words and phrases are underlined. 
"vacation" are two spatially and temporally separated periods, and "noise" and "silence" ("open" and "closed ears") represent a continuous rhythm controlled by the movements of the body. For Martin, since the alternating noise and silence of the boys in the refectory is similar to that of the train, the "continuous rhythm allows one form of experience to be transformed into another" (119), resulting in a symbolic correspondence between "term" and "noise", and "vacation" and "silence". As we have seen, the rhythmical periods identified by Martin play a significant role in the 'linguistic musicality' of the text. In fact, these words also influence the rhythmical duration of example 2 , together with other words already used in example 1, like "again" and "then", and their alternation is significant also as far as the intonation of the text is concerned. When the two examples are analysed together, the connections between "term" and "noise", "vacation" and "silence" could also be considered as the result of a musical linking between the two passages. In example 1 the noise and silence of the refectory is represented by the words "roar" and "stop". These words are then changed in example 2 - the first with "term" and the second with "vacation" - in order to reproduce a similar rhythmical effect. The association between noise and "term" and silence and "vacation" is thus also inferred due to the position of the musical patterns in the rhythmic structure of the sentences.

Returning to Weaver's musical definitions, the other form of melos considered here is theme and variations. According to Weaver, this form of melos consists in larger rhythmic patterns (more than a phrase) that recur throughout the first chapter of $A$ Portrait with some minor variations, as the following examples show:

3.1 That was mean of Wells to shoulder him into the square ditch/ because he would not swOP/ his little snuffbOX/ for Wells's seasoned hacking chestnUT,/ the conqueror of forty./ How cold and slimy the water had been!/ A fellow had once seen a big rAt jUMP/into the scUm. (P: 8)

3.2 It was Wells who had shouldered him into the square ditch the day before/ because he would not swOP/ his little snuffbOX/ for Wells's seasoned hacking chestnUT,/ the conqueror of forty./ It was a mean thing to do;/ all the fellows said it was. And how cold and slimy the water had been! And a fellow had once seen a big rAt jUMP/ plOP/ into the scUM. The cold slime of the ditch covered his whole body. $(P: 11)$

The effect of theme and variations is achieved here through phrases and sentences that are repeated in a variated form - augmented or diminished with more or less words. Similarly, in music, an original theme is developed in different variations, usually thanks to some changes in the duration of the rhythmical patterns, or the addition or subtraction of some 
notes; the theme could also become less and less recognisable each time it is repeated. In this case, the first (and in this case only) variation of the original theme, as in music, is similar to the original theme. Only a few words are added, "it was a mean thing to do; all the fellows said it was" or "and" and "plop", and "that was mean of Wells" is changed in "it was wells who". Theme and variations thus influence the secondary rhythm of the text according to the parameter of duration, since sentences and phrases repetitions recur in time, resulting in a rhythmical period. The recurrence of the passage about Wells has a strong rhythmical effect that could be perceived by the reader as the main musical expedient. As in the examples above, however, other linguistic expedients influence the rhythm of the sentences. Among the timbre devices that Joyce employs, there are alliterations, "was Wells who", internal assonances, such as "swOP" "snuffbOX" ("ChestnUT"), and phono-symbolic consonants (s $1 \mathrm{~m}$ in "slime").

Word repetitions and themes and variations are significant also from a melodic point of view. As Richard Kershner affirms in The Artist as Text: Dialogism and Incremental Repetition in Joyce's Portrait $(1986,885)$, repetitions in the first chapter of A Portrait are usually typical of Stephen's schoolmates' jargon, of the adults' language, or represent mysterious signifiers. Words and sentences are repeated in the main narrative mainly because of their sound, or because Stephen is trying to master their meaning. Quoting is a typical feature of Stephen's mind, hence also Stephen's own words are sometimes subject to a similar repetition process, and especially when they result in "poetical compositions" - as in the above examples (3.1-3.2) mentioned by Weaver and also by Kershner (1986, 886). In order to assess the significance of these recurring words and sentences repetitions, Kershner draws on Bakhtin's theory of "intonational quotation marks". According to Bakhtin $(1981,50)$, "indirect discourse [...] the representation of another's word, another's language" is reported in intonational quotation marks. Intonational quotations are a form of "intentional hybrid" (Bakhtin 1981, 76). Although grammatically belonging to only one speaker, hybrid sentences are composed by two voices - "two utterances, two speech manners, two styles, two 'languages'” (Bakhtin 1981, 305). Bakhtin's definition is useful to understand how repetitions could be musically influential in determining the pitch of the text. Normal melodic movements in the sentences of texts may be in fact modified by the presence of different voices in the narrator's discourse, because these repetitions are characterised by a different intonation with respect to the main narrative. An example of how intonational quotation marks determine the intonation of Joyce's text is provided by the following lines:

4 Was that a sin for Father Arnall /to be in a wax/ or was he allowed

/to get into a wax/ when the boys were idle because that made them 
study better or was he only letting on/ to be in a wax? (P: 40) (Kershner $1986,884)^{12}$

In this passage, the lack of punctuation makes it difficult for the reader to understand the intonation units of the text and to mentally pronounce the sentence. However, the rhythmical recurrence of another voice in the text assists the reader in distinguishing the curves of intonation: "to be/ get in a wax" is mentally pronounced with a different voice and pitch with respect to the main narrative, because it is connected to the jargon of Father Arnall; it divides the prosody of the sentence in melodic units, as the slashes in the passage show.

\section{Echoes of $A$ Portrait in the Music of "Sirens": "Verbal Music"}

As I have shown, a 'linguistic musicality' is identifiable in the first chapter of $A$ Portrait, although musicality is not explicitly declared as the writing technique of the book. Instead, as Joyce himself affirms in a conversation with George Borach in June 1919 and later that year in a letter to Harriet Weaver (Ellmann 1982, 459-62), the imitation of music is precisely the stylistic skopos of "Sirens". According to Bowen $(1995,28)$ linguistic musicality in "Sirens" is achieved in two ways: through a musical language (poetical devices, such as alliteration, rhymes and onomatopoeia) and through the verbal imitation of actual musical techniques, such as staccato or sostenuto. Although several critics have described the musical techniques imitated in the chapter, generic musical language has been less considered. Among the scholars who have attempted to describe how Joyce exploits the properties of the language in a musical way in the 11 th episode of Ulysses, Andreas Fischer (1999, 256-7) identifies a generic linguistic musicality in the episode, described as "verbal music". It is interesting to note that several aspects of the highly musical linguistic texture of the examples provided by Fischer and reported below bear some similarities with the musical melos identified in A Portrait.

1.1 Bald Pat at a sign drew nigh. A pen and ink. He went. A pad. He went. A pad to blot. He heard, deaf Pat. (U, 11: 822-3)

1.2 Bald deaf Pat brought quite flat pad ink. Pat set with ink pen quite flat pad. Pat took plate dish knife fork. Pat went. $(U, 11: 847-8)$

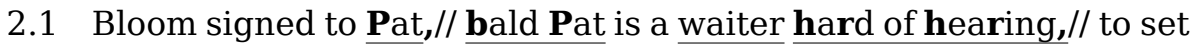

12 Kershner uses italics to imdicate the intonation quotation marks. 
ajAR/ the doOR of the bAR. The doOR of the bAR.// So.// That will do.// Pat,// waiter,// waited,// waiting to hear,// for he was hard of hear/ by the doOR. $(U, 11: 669-72)$

2.2 Pat is a waiter hard of his hearing.// Pat is a waiter whO wAIts whIle yOU wait.// Hee hee hee hee.// He wAIts whIle yOU wait.// Hee hee.// A waiter is he.// Hee hee hee hee.// He wAIts whIle yOU wait.// WhIle yOU wAIt/ if yOU wAIt/ he will wAIt whIle yOU wait.// Hee hee hee hee. Hoh.// WAIt whIle yOU wait. $(U, 11: 915-19)$

Firstly, in each sentence it is possible to identify the first of the forms of melos analysed earlier: a peculiar concern with words. The narrator's interest in the meaning and the sound of the words results in a highly musical discourse, in which many of the rhythmical expedients signalled by Lanier can be identified. Primary rhythm is mainly characterised by monosyllables, while the many repetitions of words and of phrases create a secondary rhythm, - "Pat", "waiter", "went", "pad" "hard" "hear" "the door of the bar", "while you wait" - variated repetitions of words - "waiter, waited, waiting" - and of phrases and sentences - "He waits while you wait", "He waits while you wait", "While you wait if you wait he will wait while you wait", "Wait while you wait", "Hee hee hee hee". These patterns recur periodically in different paragraphs influencing the rhythmic duration of the text. There are also several timbre expedients, as in A Portrait: alliterations - "wait while you wait/ went", "hard of his hearing" -, an internal rhyme - "ajar", "bAR" -, a wordplay between "he" and the laughter "hee hee". Some vowel transformations are also used: the timbre effect of "wait/ while/ wait/ will" is similar to that of the famous phrase "pick, pack, pock, puck" ( $P$ : 35) from A Portrait, which imitates the sound produced by the cricket bats. Consonant repetitions play a remarkable timbre role: an example is the repetition of the stops $p, b, t, d$ e $k$ in "Bald deaf Pat brought quite flat pad ink/ Pat set with ink pen quite flat pad", which significantly influence the colour of the passage. Interestingly, as we will later see, the repetition of stop consonants is also linked to the musical technique of martellato.

Secondly, these examples from "Sirens" are also partly linked together like the sentences about "Wells" in A Portrait $(P: 8 ; P: 11)$, meaning that they could be associated with the other form of melos described by Weaver - theme and variations. In this case, however, the themes are much more rephrased when variated than Well's sentences are. In the two first examples, for instance, the "pen ink and pad" are firstly asked and then taken to Bloom. In 1.2 the cluster of words is initially repeated, though in a different order, "pad ink [...] ink pen quite flat pad", and then substituted by "plate dish knife fork". Similarly, the phrases "Bald Pat" is variated in "Bald deaf Pad" and the sentence "He went" is changed in "Pat went". 
In the two second instances, the variated phrases, "hard of hearing" and "waiter, waited, waiting" from 2.1 become "hard of hear" (in the same passage) and "hard of his hearing" (in 2.2), and "waiter whO wAIts whIle yOU wait", which is repeated and changed several times in 2.2.

If, as I have shown in example 2.2, a different voice or a different thought seems to repeatedly interrupt the narrative, "hee hee hee hee" - as intonation quotation marks interrupted the narrative of $A$ Portrait - then the examples themselves represent a different voice inside the narrative of "Sirens", fitting in with the structure of the fugue that Joyce aims to recreate in the chapter. Similarly, if 'quoting' is a typical procedure of Stephen's mind and quotations of previous thoughts influence the intonation and the duration of the first chapter of $A$ Portrait, this happens even more with Bloom's mind, whose creations generate rhythmical patterns throughout the whole of Ulysses, and with the narrating voice. In this sense, the examples of "verbal music" identified by Fischer could be considered part of a recurrent theme in "Sirens" - namely the theme of the deaf waiter Pat. Although the examples provided by Fischer are among the most musical, we can notice that many of the passages in which Pat is described are in fact characterised by a "verbal music", and they are related to each other in a musical way.

Some further examples include:

3.1 To the door of the bAR/ and diningroom came bald $\underline{\mathbf{P A T}}$,/ came

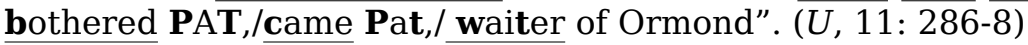

3.2 Pat paid for diner's popcorked bottle:/ and over tumbler,/ tray and popcorked bottle ere he went he whispered,/ bald and bothered,/ with miss Douce. $(U, 11: 317-19)$

\subsection{Wait,/ wait./ Pat,/ waiter,/ waited. (U, 11: 393)}

3.4 Bald Pat, bothered waiter, waited for drink orders. (U, 11: 444)

3.5 Here, Pat, return. Come. He cAme, he cAme, he did not stAY. (U, 11: 994)

3.6 Deaf, bothered. But perhaps he has wife and family waiting,/ waiting Patty come home./ Hee hee hee hee./ Deaf wait while they wait. $\overline{(U, 11: 1113-15)}$

As we can notice, the two form of melos described in A Portrait are similarly important in these examples. There are recurring sounding words associated to "Pat" - "bothered", "bald", "deaf", "wait" in all its possible forms, and "came" -, which are alternatively present when Pat is mentioned. 
Among timbre effects, there are alliterations, "Pat paid", "wait while", the repeated consonants $p, b, t$ (martellato consonants) and internal rhymes, "CAME/cAME/stAY". As in the examples analysed above, word clusters are also repeated - "he came", "popcorked bottle" - and variated - "came bald Pat,/ came bothered Pat, /came Pat". A frequent phrase in the lines that Fischer analyses - "waits while you wait" - is variated here in "wait while THEY wait". An instance of the laughter used in the examples above is also present: "waiting Patty come home./ Hee hee hee hee./ Deaf wait while they wait". Considering all the possible associations of words, Pat's theme recurs in its variations throughout almost the entire episode.

Interestingly, some other examples of what Fischer defines as "verbal music" can be found in the sentences related to the appearance of the blind piano tuner, towards the end of the episode. The blind man's arrival is introduced by the sound of his cane "Tap", which interrupts the main narrative more and more often since verse 933, until the young man makes his appearance in the streets and in the Ormond. Since the appearance of the piano tuner's musical theme $(U, 11: 1190)$, Pat's one disappears. The sentences describing the blind man - represented by the sound "tap", which can be read back to front as "Pat" - could be considered a final variation of deaf Pat's music, in which the theme of not seeing is opposed to the theme of not hearing. The blind man's gestures are described by a musical narrating voice as:

\subsection{Tap blind walked tapping by the tap the curbstone tapping,/ tap} by tap. $(U, 11: 1190)$

4.2 Tap. Tap. A stripling,/ blind,/ with a tapping CAne CAme taptaptapping [by Daly's window where a mermaid hair all streaming (but he couldn't see) blew whiffs of a mermaid (blind couldn't), mermaid,/ coolest whiff of all] $(U, 11: 1234-6)$

4.3 Tip./ An unseeing stripling stood in the door. /He saw not bronze. He saw not gold. Nor Ben nor Bob nor Tom nor Si nor George nor tanks nor Richie nor Pat./ Hee hee hee hee./ He $\overline{\operatorname{did}}$ not see. $(U, 11 \overline{: 12} 81-3)$

We can notice that the first form of melos, attention to words, is mostly heard in examples 4.1 and in the first line of example 4.2 , where rhythmical patterns are given by timbre devices, like the insistence on the stops $t, p, b$ - the same used in Pat's theme - "CAne/CAme", and, mostly, the continual repetition of varied forms of the word "tap". In example 4.3, the repeated conjunction "nor" influences the parameter of duration in the secondary rhythm of the text, and some timbre devices are also present - such as the alliteration "un-seeing stripling stood", and the repeated consonant $h$. As far as the theme and variations form of melos is concerned, it can be 
seen that in example 4.2 the first line is a variated occurrence of example 4.1. The second part, instead, is a variation of some lines from the beginning of the episode:

Two sheets cream vellum paper [...] in Daly's Henry Flower bought [...]. Wise Bloom eyed on the door a poster, a swaying mermaid smoking mid nice waves. Smoke mermaids, coolest whiff of all. $(U, 11: 295-301)$

Similarly, the laughter from Pat's theme - "hee hee hee hee" - returns in example 4.3. Finally, some words and phrases introduced in example 4.2 - "stripling", "he could not see" - become the source of a different music in example 4.3: "An unseeing stripling stood", "/He saw not bronze./ He saw not gold/ He did not see."

A final comment on the connections of the generic linguistic musicality in "Sirens" concerns some specific musical punctuation devices used in the 11 th episode of Ulysses. Discussing this topic, Fischer $(1999,225)$ underlines that Joyce uses punctuation in two rhythmical ways in "Sirens": through underpunctuation, the lack of punctuation, and through overpunctuation, the excessive use of punctuation marks. Both these expedients are not meant to signal units of meaning, as punctuation usually does in written texts. Rather, they re-organise the sentences only according to their form in order to create rhythmical effects: in this sense they have a bearing upon the mere signifier, as the rhythmical expedients used in music do. Both over and under punctuation were already used in A Portrait - for instance in the lines "Term, vacation; tunnel, out; noise, $\operatorname{stop}^{\prime}(P: 14)$, and in the example about Father Arnall ( $\left.P: 40\right)$, discussed above - and create a similar musical effect in the text. The following two examples show how overpunctuation and underpunctuation are used in Ulysses in a musical way:

5 Miss Douce halfstood to see her skin askance in the barmirror gildedlettered where hock and claret glasses shimmered and in their midst a shell $(U, 11: 118-20)$

\section{Will?/ You?/ I./ Want./ You./ To. (U, 11: 1096) (Fischer 1999, 255)}

In example 6 the melodic curves of a naturally punctuated "Will you?/ I want you to/" are interrupted by an excessive use of full stops and question marks, "Will?/ You?/ I./ Want./ You./ To". The melody reproduced in the line is hence independent from its meaning, because the units of sense are segmented: there are short curves of intonations that are limited to single words, while the whole sentence is not characterized by its peculiar intonation patterns. Similarly, the opposite absence of punctuation marks to indicate the sense units in example 5 makes it necessary for the reader 
either to mentally pronounce the sentence without pauses and melodic differences, or to look for other intonation expedients in the text to understand the patterns of sense. As with "to be/get in a wax" in A Portrait, in this case some other expedients may also help the reader identify the intonation units. For instance, the wordplay "skin askance", the compound words "barmirror" and "gilderlettered", or the rhyme "gilderletterED/ shimmerED", as well as the emphatic position of "in their midst" in the sentence may be considered an aid for understanding the melodic distinctions, because they could be pronounced with a different speed and voice with respect to the main narrative. With recourse to these, the reader could mentally pronounce the example as:

Miss Douce halfstood to see her skin askance/ in the barmirror gildedletterED/ where hock and claret glasses shimmerED/ and/ in their midst/ a shell (U, 11: 118-120) ${ }^{13}$

\subsection{Echoes of $A$ Portrait in the Music of "Sirens": staccato and martellato}

In this last section, I consider some musical techniques that Joycean scholars have identified in "Sirens". In particular, I will focus on the linguistic imitation of staccato and martellato, as they could be linked to the musical expedients identified in A Portrait. In music, staccato is a form of articulation through which notes of shortened duration are separated from the notes that may follow by silence. In "Sirens" Joyce achieves a staccato effect though the use of overpunctuation, and by shortening words. Unlike in music itself, in which staccato influences only the duration of the notes, in language, the prosody of the phrases is also affected, because, as seen above, the melodic curves of overpunctuated sentences are not the same of those of sentences punctuated according to their meaning. Bowen identifies staccato in the following example $(1995,28)$ of "Sirens":

1 Miss Douce, Miss Lydia, did not believe: Miss Kennedy, Mina, did not believe: George Lidwell, no: Miss Dou did not: the first, the first: gent with the tank: believe, no, no: did not, Miss Kenn: Lidlydiawell: the tank. $(U, 11: 818-20)$

The words "Miss Douce", "Gentleman", "Miss Kennedy" and "tankard", and the phrase "did not believe" are abbreviated in "Miss Dou" "Gent", "Miss Kenn", "tank" and "did not", and the musical pauses are recreated through the use of the asyndeton. In these lines, the curves of intonation 
are short, and they end after each comma or colon. The example used above to define overpunctuation - "Will?/ You?/ I./ Want./ You./ To" (U, 11: 1096) - is also considered by critics (e.g. Gilbert 1963, 223) as an occurrence of staccato, even though in this case words are not shortened, and staccato is achieved only through punctuation. As seen before, this example is also similar to the one analysed in the previous sections - "Term, vacation; tunnel, out; noise, stop" ( $P: 14)$. Although the use of excessive punctuation marks in A Portrait are possibly a consequence of Stephen's experimentations with language, the telegraphic style resulting from this attempt could also be seen as a first effort to reach what Declan Kiberd $(2000,968)$ defines "staccato style", a narrative technique which will be used not only as a musical expedient in the 11th episode, but also as a narrating device in the whole Ulysses, and which is peculiar to Bloom's interior monologue.

The second musical technique considered here, martellato, is a music timbre device used mostly in bow instruments and obtained by holding the bow against the string with pressure, then releasing it explosively to produce a sharp, biting attack. It is sometimes required together with staccato. In "Sirens", Joyce appears to rely on the modality of articulation of the stops consonants, in which sound is explosively released after the blockage of the vocal tract is removed, to imitate martellato. An example is:

One rapped on a door, one tapped with a knock, did he knock Paul de Kock, with a loud proud knocker, with a cock carracarracarra cock. Cockcock. $(U, 11: 986-8)$

In these lines, the most used consonants are $c / k$ - "knock" "Kock" "cock" "carracarra" - although also other stops are used (mostly $p$ and $d$ ). Martellato is imitated also in the examples of "verbal music" in "Sirens" considered above, as part both of Pat's and the piano tuner's themes, in which $p, b$ and $t$ were frequently used. In all the examples, the stops imitate the timbre of the sharp attack in bow instruments, bringing the martellato technique in "Sirens" to bear mostly upon the parameter of timbre, exactly as it happens in music. As seen above, in A Portrait vowels or consonants timbre were sometimes used to imitate sounds - which are often real sounds (e.g. when Stephen compares the noise of the train to the noise in the refectory, or when he considers the sound of the word "suck" [P:9]). The properties of the language and the capacity of the language to imitate real sounds seem to be initially used to show the poetical discoveries of young Stephen, and then fully developed when Joyce tries to imitate musical sounds and techniques in the 11th episode of Ulysses. 


\section{Conclusions}

As briefly shown, the stylistic expedients used in the prose of the first chapter of $A$ Portrait can have analogous correspondents in the rhythmical and acoustical devices used in musical compositions; the 'linguistic musicality' that several scholars have claimed to be present in Joyce's texts - what Rice and Fischer term "verbal music" (Rice 1999; Fischer 1999) or "melos" as per Waver (Weaver 1998) - could thus be considered more than just a metaphor. Verbal rhyme patterns (consonant repetitions, words and phrases patterns, and even the recurrence of sentences) characterise both the micro and the macro-level of Joyce's literary discourse, just as rhythmical patterns and melodic clusters recur in the micro and macro level of musical compositions. Considering the parameters of sounds, which have in the organisation of the "secondary rhythm" (Lanier 1880) both music and language, some comparisons between their role in Joyce's A Portrait and in music could be attempted.

Given the importance of repeated patterns of words and phrases in the text, the sound parameter of duration seems to play a remarkable role in establishing the 'secondary rhythm' of several passages of Stephen's poetical pseudo-stream-consciousness. Similarly, in music, duration is among the most relevant sound parameters since the time length of notes is the most recognisable feature of musical themes, and recurring rhythmic patterns are important in the structure of all musical compositions. Timbre devices (such as alliteration and internal rhymes) are among the second most used musical expedients in Joyce's text. Unlike in music itself - where timbre is often a mere colouristic expedient (e.g. the sound of a violin proves sweeter than the sound of a trumpet and hence can be preferred for certain motifs), while the substance of the composition is given by melodies with different pitches - in A Portrait timbre plays a more significant role than pitch does. A few melodic expedients are also present in the text. Although less used in A Portrait than in "Sirens", the different pitches of intonation units help the reader in identifying the voices quoted in the narrative - through what Bakhtin terms "intonational quotation marks" (Bakhtin 1981, 50) - and understanding some of the character's thought associations. Concerning the different role of intonation in music and in Joyce's texts, it is interesting to note that while in A Portrait the use of intonational quotations marks is not widespread, the quotation mechanism will become more and more complex in the interior monologue of the specifically musical episode of Ulysses, because self-quotations and repetitions of others' words and expressions are typical of Bloom's thinking processes and of his mental associations, and also characterise the narrator's voice. This could show a more musically-balanced use of the parameters of sound in "Sirens", in which pitch seems to be at least as relevant as timbre. 
Another proof of the musicality of the style in the first chapter of $A$ Portrait is the presence of the same musical devices in "Sirens", which is explicitly written to imitate music. Although the features of the musicality of "Sirens" that have been considered here are only a few examples of the several musical characteristics of the episode, their connection with A Portrait shows that Joyce's musical experiments with language started much earlier than during his writing of the 11th episode of Ulysses. Stephen's attention to words in A Portrait and the melos that derives from it are echoed and expanded in the wordplays of "Sirens"; similarly, the mechanism of theme and variations identified by Weaver in the first text is repeated and developed in Ulysses. Furthermore, as stated above, intonation patterns that are sometimes used in A Portrait to represent different voices become an important stylistic feature of the narrative of "Sirens". Finally, the peculiar punctuation devices found in short passages of $A$ Portrait - over and underpunctuation - are also frequently used in the 11th episode, determining both a musical language and the musical technique of staccato.

To conclude, the examples provided in this paper, as well as others still to be found in A Portrait and "Sirens", can possibly show how the simple rhythmical devices used by young Stephen in the pseudo-stream of consciousness may be considered the starting point of a more complex musical style, as simple musical themes and basic musical techniques could be expanded to create a sonata, a fugue or even a symphony. In this sense, "Sirens" could also be understood as a full development of the musical and poetic potentialities of the young artist shown in the first chapter of A Portrait. 


\section{Bibliography}

Adorno, Theodor W. (1993). "Music, Language, and Composition". Essays on Music. Edited by Richard Leppert. Berkeley: University of California Press.

Arnold, Denis (ed.) (1983), The New Oxford Companion to Music. Oxford: Oxford University Press.

Attridge, Derek (1982). The Rhythms of English Poetry. London: Longman. Attridge, Derek (2004). Joyce Effects on Language, Theory, and History. Cambridge: Cambridge University Press.

Bakhtin, Mikhail M. (1981). The Dialogic Imagination: Four Essays. Edited by Caryl Emerson and Michael Holquist. Austin: University of Texas Press.

Berio, Luciano (2013). Scritti sulla Musica. Milano: Einaudi.

Berruto, Gaetano (2006). Corso elementare di linguistica generale. Novara: Utet Università.

Bowen, Zack (1974). Musical Allusions in the Works of James Joyce: Early Poetry Through "Ulysses". Albany: State University of New York Press. Bowen, Zack (1995). Bloom's Old Sweet Song: Essays on Joyce and Music. Gainesville: University Press of Florida.

Chafe, Wallace (1988). "Punctuation and the Prosody of Written Language". Written Communication, 5(4), 395-426.

Ellmann, Richard (1982). James Joyce. New York: Oxford University Press.

Fischer, Andreas (1999). "Strange Words, Strange Music: The Verbal Music of the "Sirens" Episode in Joyce's Ulysses". Knowles 1999, 245-62.

Gilbert, Stuart (1963). James Joyce's Ulysses. Harmondsworth, Mitcham: Penguin Books.

Green, Jon D. (2002). “The Sounds of Silence in 'Sirens': Joyce's Verbal Music of the Mind". James Joyce Quarterly, 29(3), 489-508.

Haas, Robert (1992). "Music in Dubliners". Colby Quarterly, 28(1), 19-33. Houtsma, A.J.M. (1997). "Pitch and Timbre: Definition, Meaning and Use". Journal of New Music Research, 26 (2), 1997, 104-15.

Hubbard, Timothy L. (2010). "Auditory Imagery: Empirical Findings". Psychological Bulletin, 136(2), 302-29.

Joyce, Stanislaus (1958). My Brother's Keeper: James Joyce's Earlier Years. London: Faber and Faber.

Kershner, R.B. Jr (1986). "The Artist as Text: Dialogism and Incremental Repetition in Joyce's Portrait". ELH, 53, 881-94.

Kiberd, Declan (2000). "Notes". Ulysses. Annotated Student Edition. London: Penguin Classics, 941-1195.

Knowles, Gerald (1987). Patterns of Spoken English. An Introduction to English Phonetics. London: Longman.

Knowles, Sebastian (ed.) (1999). Bronze by Gold: The Music of Joyce. New York: Galand Publishing Inc. 
Lanier, Sidney (1880). The Science of English Verse. New York: Charles Scribner's Sons.

Macy, L. (ed.). Grove Music Online. URL http://www.grovemusic.com (12 - 12 - 2017).

Martin, Timothy (2009). "Music". McCourt, John (ed.), James Joyce in Context. Cambridge: Cambridge University Press, 275-84.

Martin, William (2012). Joyce and the Science of Rhythm. New York: Palgrave Macmillan.

Napoli, Ettore, Polignano, Antonio (2006). Dizionario dei termini musicali. Milano: Mondadori.

Perrone-Bertolotti, Marcela et al. (2012). "How Silent Is Silent Reading? Intracerebral Evidence for Top-Down Activation of Temporal Voice Areas during Reading". The Journal of Neuroscience, 5, 17554-62.

Plock, Vike M. (2009). “Good Vibrations: 'Sirens,' Soundscapes, And Physiology". James Joyce Quarterly, 46(3-4), 481-96.

Power, Arthur (1974). Conversations with James Joyce. Chicago: University of Chicago Press.

Rabaté, Jean-Michel (1986). "The Silence of the Sirens". Beja, Morris et al. (eds.), James Joyce in The Centennial Symposium. Urbana: University of Illinois Press.

Rice, Thomas Jackson (1999). "The Distant Music of the Spheres”. Knowles 1999, 213-27.

Seikel, J. Anthony et al. (2010). Anatomy and Physiology for Speech, Language and Hearing. Delmar: Cengage learning.

Spencer, Herbert [1862] (2009). First Principles. Cambridge: Cambridge University Press.

Weaver, Jack (1998). Joyce's Music and Noise: Theme and Variation in His Writings. Gainesville: University Press of Florida.

Witen, Michelle L. (2018). James Joyce and Absolute Music. New York: Bloomsbury Academic.

\section{List of abbreviations}

D: Joyce, James (1996). Dubliners. Text Criticism and Notes. Edited by Robert Scholes and A. Litz. London: Viking critical Library, Penguin Group. Reference to page number.

P: Joyce, James (2008). A Portrait of the Artist as a Young Man. Edited by Jeri Johnson. Oxford: Oxford University Press. Reference to page number.

U: Joyce, James (1986). Ulysses. Edited by Walter H. Gabler. New York: Random House. Reference to chapter and verse number. 



\title{
Thou Shalt not Kill! Or Notes on Caribbean Music as Literary Text on Being Human
}

\author{
Francio Guadeloupe \\ (University of St. Martin, Philipsburg, USA; \\ University of Amsterdam, Netherlands) \\ Ivette Romero \\ (Marist College, Poughkeepsie, USA)
}

\begin{abstract}
One of the important things that we who share in the human condition do when we write, read, interpret, and discuss written works, is relate to death; the death of the unruliness of Life. We are referring here to that counter, that unforeseen, that chaos, that deconstructive constant, that je ne sais quoi that perpetually undoes all the certainties and structures and truths we hold dear in our attempt to master and colonize our existence. Let us call this the aesthetic-real understanding of death that, at its best, is ethical in character. But - and this is crux of our argument - Caribbean literature, as it is also expressed in the musical production from the region, reminds us that this general aesthetic-real of relating to death is inextricably bound up with the specific historical-real of non-Europeans, and those who Europeans deemed lesser creatures, being murdered by overwork, guns, disease or poverty as a result of western greed and anti-human humanism. Again, to repeat, in this essay, we explore this and its summoning of an alternative conception of being human via the literature housed in Caribbean music: those written, sung, performed, and sometimes, danceable texts.
\end{abstract}

Summary 1 Introduction. -2 The Aesthetic-Real and Historical-Real in the Caribbean. -3 Caribbean Literature and the Question of the Human. - 4 Human Identity in Caribbean Music.

Keywords Aesthetic-real. Historical-real. Caribbean music. Caribbean songs. Caribbean literature. Embodied reading.

\section{Introduction}

In this essay, or rather brief set of notes, we explore an alternative understanding of what it means to be human inspired by the literature housed in Caribbean music. We wilfully conflate the two art forms literature and music, as for us, the beauty of every art is that it can manifest another art; be another art. Some may read this as a hip provocation in the spirit of Bob Dylan winning the Nobel Prize for Literature. We prefer to see it in the vein of Kendrick Lamar winning the Pulitzer Prize for music - as an invitation to other ways of seeing/reading/understanding. We who know 
that some of the first books that we read with our whole bodies were the albums of Bob Marley, Rubén Blades, Ismael Rivera, and Willie Colon are not surprised that this Caribbean wisdom is becoming more common. For us, the literatures - those written and sung discourses - of Caribbean musicians are just as important to us as the books in which Kamau Brathwaite, Maryse Condé, Edwidge Danticat, Junot Díaz, Robert Antoni, Lasana Sekou, and the late Aimé Césaire, Derek Walcott, Antonio Benítez Rojo, Stuart Hall, and Édouard Glissant house their prose and poetry. Nevertheless, we are not speaking of music-as-literature, underlining the centrality of the language of popular culture, or examining the relationship between literature, orality and music. These aspects have been explored amply through the decades, and we are indebted to many Caribbean thinkers (Carolyn Cooper, Kwame Dawes, Mervyn Morris, Gordon Rohlehr, Emilio Jorge Rodríguez, Hanétha Vété-Congolo, along with the aforementioned Brathwaite and Benítez-Rojo, among others) whose works have enriched and expanded our conceptions of literature in its myriad manifestations as it interplays with other modes of cultural production. Instead, we entertain a comprehensive consideration of Caribbean music/songs, in which an embodied reading - a reading that involves lyrics, music, performance, and active (often collective) participation - becomes a life-affirming act. At times, we may refer to a particular 'message' or formal elements of the songs we chose, but we do not see the latter as a collection of discursive forms independent from the material emplacement and interpretative engagement of the body - our bodies. We begin, however, by clearing the ground in a more general sense.

Academic common sense of the orthodox kind would consider that to appreciate Caribbean literatures we need first to know what literature is. We have to delve into the commonalities and differences between poetry, short stories, drama, plays, and novels in their most basic form, and then zoom in on their regional variations. To us, this is not a useful exercise, as there is no such thing as literature, only specific literatures.

If we subtract the adjectives - e.g. African, African American, Caribbean, Chinese, Indian, Western European, Women's, etc. - from the noun literature, we are left with little to talk about. Yet we cannot abstain from entertaining the question - 'what is literature?' - in some way. We cannot endorse that Plato is dead. We have to contend that it is only by keeping the idea of literature alive, engaging in a wilful abstraction, that we can acknowledge Caribbean literatures, and have them speak to one another and to the manifold forms of cultural expression on our planet. Therefore, the right question for us is not what literature is, but rather what literature does. One of the important things that we (who share in the human condition) do when we write, read, interpret, and discuss written works is relate to death; the death of the unruliness of Life. We are referring here to that counter, that unforeseen, that chaos, that deconstructive constant, that je 
ne sais quoi that perpetually undoes all the certainties and structures and truths we hold dear in our attempt to master and colonize our existence.

Let us call this the aesthetic-real understanding of death that, at its best, is ethical in character. But - and this is crux of our argument in this essay - Caribbean literature as it is also expressed in the musical productions from the region, remind us that this general aesthetic-real of relating to death is inextricably bound up with the specific historical-real of non-Europeans, and those who Europeans deemed lesser creatures, being murdered by overwork, guns, disease or poverty as a result of western greed and anti-human humanism. Again, to repeat, in this essay, we plan to explore this and its summoning of an alternative conception of being human via the literature housed in Caribbean music: those written, sung, performed, and often, danceable texts.

\section{The Aesthetic-Real and Historical-Real in the Caribbean}

For obvious historical reasons that the spirits that inhabit the bottom of the Atlantic and Indian ponds and the official and unofficial burial grounds of plantation America will not allow us to forget, Caribbean literary artists at their best dance both with the aesthetic-real and historical-real realities of death. Moreover, they do so referring to what we today have come to call a 'multicultural setting'. The world lives here. Thus, the ethical understandings of what it means to be human that they put forth matters to all who share in the human condition.

If we wish to demolish colonial racisms while recognizing their continuing (detrimental) force, it is best to state that this archipelago of ours is inhabited by the ancestors of the band who left Africa to populate the planet and who eventually misrecognised each other in the Caribbean, engaging in the most horrendous forms of dehumanization. The words Middle Passage, Asian indenture, Amerindian genocide, the Irish plight, Jewish persecution, white European supremacy, patriarchy, Christian imperialism, capitalism and colonialism cannot truly ever express the carnage that took place here. Wrongs and the miracles of human emancipation that somehow emerged from our man-made inferno are too great for words, and yet we must use words too.

We use the vocabularies we inherited, mindful of the proposition that colonial racisms are our common socialized ways of thinking and acting, whereby most take for granted an imperially fabricated world of bounded continents, sub-continents and regions, peopled (supposedly) by civilizations, with their incommutable somatic norm images and world views. This dead logic of destruction and carnage must always be handled with care (for we must unspeak it as we speak, and unwrite it as we write about it, to be heard and to contribute to a more just planetary conversation). In 
this respect, we call into question the 'powers' of reason that have been immersed in this process of socialization and stress the importance of nonrational forces implicit in our corporeal participation in these histories as well as our acts of resistance to them. Therefore, our acts of speaking and unspeaking, writing and unwriting must be informed by the written/sung discourses as well as our emotional and physical enactment; let us call it our 'dancing' of/to the vocabularies. After all, as Stuart Hall asserts, "identities are formed at the unstable point where the 'unspeakable' stories of subjectivity meet the narratives of a history, of a culture" (Hall 1996, 115).

Our use of the 'we' pronoun is both an invitation and an entreaty to remember our elemental humanity. By that term, we mean the undeniable real of our being anchored to specific bodies. In somewhat cryptic terms, one can state that we are bodies, have bodies, and do bodies in a body politic. Everybody, which is every body and every face, is specific; yet, we resemble each other enough to mate, relate, cohabit and, of course, fight and subjugate; yet, nevertheless, we recognize each other and provisionally call ourselves a species: humans.

In his documentary, Residente, Puerto Rican singer-songwriter René Pérez Joglar (better known as Residente, from the duo Calle 13) says:

Music is full of blood, and that blood is also nomadic because it runs through the veins of the world, that blood that feeds on meat and covers itself with animal skin. They say music cures, that it takes away our pain, that it saves lives and helps us remember those moments we are about to forget. Music carries with her everything she has lived in the past and with every millisecond, she tells us something new. We are everything that sounds, and our contribution is infinite, like the sound of silence. (Residente 2017)

In his ruminations, he captures the undeniable real of our being anchored to bodies of flesh, bone, and blood, while linking us, as a species - humans of the globe (with blood that "runs through the veins of the world") - indissolubly to music, to sound ("We are everything that sounds"). Our human condition is tied to blood - the blood of menstruation, birth, wounds, battles, and death - as well as sound - in every one of our modes of expression and communication. But it is always blood-in-movement: flowing, running through veins, channels, fissures and furrows.

Note that Residente set into motion his exploration of the body (his body, his DNA) in the body politic through a global voyage in search of his roots, documented through film. His traveling cinematic project was inspired by the results of a DNA test, and was both a journey to become acquainted with all the regions of the world reflected in his genetic mapping and a quest for musical inspiration. A constant thread running through the documentary is blood: the blood of hunting for survival, cooking, war, and 
music-as-blood connecting us all, the blood that links us and leads us to recognize ourselves in the face of the other, in sum, the blood of various spheres and modes of perception, including the aesthetic-real and the historical-real.

We are aware that the term human has a bloody history. Its hegemonic interpretation cannot be separated from the last 500 years of colonialism, imperialism and capitalism. We are also deeply aware of the deep anthropocentrism embedded in our ways of seeing, reading, thinking, speaking, and being; of our dream or desire to position ourselves as the central subject of knowledge and to feel separate from and superior to nature. For now, we will focus on cultural production/interpretation and our understanding of what it is to be human. However, since any hegemony, when carefully scrutinized, points to contesting voices and conceptions, other genealogies of what it means to be human can be explored.

\section{Caribbean literature and the question of the Human}

We will make an attempt to present a Caribbean-inflected alternative version of what it means to be human that contests the racist ideas associated with what black activists term "the Man" and with what in poststructuralist talk is called "Man." We are encouraged and humbled by the fact that renowned social theorists of the likes of Katrine McKittrick, Achille Mbembe, Patricia Saunders, and Gary Wilders, among others, have begun doing this work by exploring the rich inclusive humanist thought of Caribbean literary artists such as Nicolás Guillen, NourbeSe Phillip, Maryse Condé, Aimé Césaire, and Sylvia Wynter. We will not take that route, but instead turn to known and lesser known cultural producers of the wider Caribbean as our sources - artists such as Jacob Desvarieux (Guadeloupe); Ismael Rivera and René Pérez Joglar, aka Residente (Puerto Rico); Peter Tosh and Bob Marley (Jamaica); Edwin Yearwood (Barbados), Sundar Popo, Ras Shorty I, Calypso Rose and the Mighty Shadow (Trinidad and Tobago) and from our rock, King Vers, Shakiya, and Shadowman (St. Martin/St. Maarten).

Again, you might question why we choose to focus only on musicians, singers and composers, instead of/also on novelists or poets. Our point is more radical. We appreciate Caribbean songs (including lyrics and music) as unfinished discursive fragments that only truly take on meaning when read by a body that dances and sings along with other bodies. In engaging in that particular form of "reading," a reasoning emerges. This constitutes a rehearsal of a liberating way of being where all bodies recognize the higher synthesis of which they are both expressions and co-creators.

For us all, the arts - music, dance, literature, architecture, theatre, sculpture, painting, cinema, and other visual arts - are present in each other and in conversation with each other. Literary pieces have a rhythm 
to them that entices an internal dance as we paint images in our minds, therewith talking back to things we read or heard elsewhere, and sculpting - with our remade bodily aesthetics and politics - a place with others. What we produce, subsequently and often almost simultaneously, finds its way in social media, the surveys of cultural industry magnates, and sometimes in our own creative pieces or scholarly articles, which in turn might influence artists. What we are referring to, in other words, is the blurring, without a thorough negation, of the distinctions between the categories of receptive and disruptive audiences, active artists, the culture industry, and reflective scholars. Everyone and every art is implicated.

And, of course, there are performers that cultivate the blurring between artistic categories, and make it problematic to state unequivocally that we are speaking of musicians instead of writers. For Rita Indiana (Dominican Republic), who went from writing novels and short stories to writing songs to sing on stage and back to writing 'literature', it is difficult to separate the arts. Music and lyrics are interwoven in her written production. Referring to her decade-long singing career in an interview with Dominique Lemoine Ulloa, Indiana says, "My project as a singer was a kind of performative fiction. A lived novel". About her music, the writer/singer explains, "The process of composing and producing is very similar to writing. I love this part and that's why I'm still producing music for movies". What she defines as the main difference between writing (literary) texts and singing/performing (musical) texts is the bodily effort required, in her words, "hay que trabajar con el cuerpo, sudar" [one has to work with the body, one has to sweat] (Lemoine Ulloa 2015). Indiana is also poignantly mindful of the legacies of slavery, our shared histories of colonization and domination. She underlines the affect that music and literature conjure up as an effect of our historical groundings: "I have a special fascination for music and literature from the southern United States. Blues, jazz, work songs. Richard Wright, Faulkner, Mark Twain, Carson McCullers. This art is a product, like mine, of the plantation culture, of the slave trade, and it is easy for me to identify with it". ${ }^{1}$

If the struggle for freedom - freedom from and freedom to - means anything in the Caribbean, it is a happening in which the downpressed and those who downpress are equally involved. Sometimes, individuals that we structurally position as victimized and victimizers resemble each other in their vices and virtues, a resemblance that does not erase, however, their individuality, specific socio-economic locations, and privileges. The arts in the Caribbean are an avenue through which this manifold truth can be discerned.

Moreover, in the Caribbean, the arts are symptomatic of super-creolization in the sense that the various traditions and contemporary cultural

1 English translations are made by the Author. 
heritage of the planet are alive within them - they are Old and New World, Old and New School, black, non-black (i.e. usually on the receiving and issuing end of power), and future and past oriented, without being fully one or the other. The supposed original cultural source of the arts is mortally stabbed during Caribbean artistic performances, while being simultaneously resuscitated to keep the transaction going.

What better examples of how these manifold truths and simultaneous (inter)actions are expressed than through our feasts and festivals: Carnival, Carnaval, Mas, Crop Over, Junkanoo, and Fiestas de Santiago Apostol? Here, the tensions between the downpressed and downpressors, Old and New Worlds, black, non-black, and everything in-between are joyfully, playfully, musically played out, contrasted, blended, in a syncretic play - through these rituals, games, and processions our language, rhythms, and bodies keep the transactions going while concurrently questioning them as we move through equally syncretic spaces. We repeat the word 'play' because, in Caribbean music, especially in this type of event, dance and humor come together as ways of warding off Death, and fear of Death, from our everyday existence. Our various types of masking/masquerading to the beat of the drums are ways of 'playing'. The enactments, performances, rehearsals of life allow us to disguise and temporarily reinvent ourselves, moving in and out of diverse, and sometimes contradictory, roles. At the same time, we stage enactments of agency and sovereignty to the beat of songs that sometimes become anthems - think Leave Me Alone, ${ }^{2}$ performed by Calypso Rose, with Machel Montano and Manu Chao.

The soca tune Leave Me Alone became a feminist rallying cry for women festival-goers during Trinidad's 2017 Carnival. Written and composed by Calypso Rose (née McCartha Linda Sandy-Lewis, AKA McCartha Lewis) and Manu Chao in 2016, the song represents a stand against misogyny and gendered violence. Many, especially women, adopted it as a reaction to the murder of a professional musician from Japan, Asami Nagakiya, who had played at several Carnivals with a Trinidadian steel pan orchestra. She was found strangled in Queen's Park Savannah the morning after Carnival ended. According to Michael E. Miller (The Washington Post), after her death, the mayor of Port of Spain, Raymond Tim Kee, blamed the musician's death on the lack of dignity and the "vulgarity and lewdness" of women's behavior at the yearly event. Trinidadians were enraged at the way the mayor blamed the victim by claiming that the musician's skimpy outfit had led to her death. Among the reactions to his words, several organizations - led by feminist activists such as Attillah Springer, Angelique V. Nixon, and others - sprung up to work against gendered violence. In Martine Powers's article, “'Leave Me Alone': Trinidad's women find a rally-

2 Cf. https://www.youtube.com/watch?v=KQ0otZhMXTk (2018-11-30). 
ing cry for this year's Carnival", Springer explains what the song meant to women at that moment: "It's like a rallying cry for women who just want to be able to have the option of enjoying their Carnival - Carnival being that space of freedom. And then you have to deal with people who are trying to control how much freedom you feel". At the end of this quote, you may add: Springer put into words what women were demanding through this song - the right to participate fully in the Carnival celebrations, to wear any outfits they desire, to play music, to march, and to dance without repercussions, threats and danger to their bodies. If Carnival is a space where strict categories are suspended, where people of all social classes, ethnicities, and beliefs are free to celebrate together, then it should be no different for gender. Springer, and other feminists, were saying that regardless of what women wear or how they 'wine' (dance) during Carnival, their actions are not an invitation to be sexually assaulted, abused, punished, or attacked in any way. In the song, Calypso Rose sings about a woman trying to dance in the streets and participate fully in the Carnival celebration, rejecting advances or any type of control by men, simply demanding that they leave her alone and let her enjoy the music:

Boy doh touch me like you goin crazy | Let go me hand, lemme jump up in de band I I don't want nobody to come and stop me | Leave me, let me free up, myself let me jump up. (Rose 2016)

In the song's corresponding video, a woman sneaks out of the house to take part in the Carnival festivities. Not finding her in bed when he awakens, her partner searches through crowds of people dancing in the street. When he finds her, he reacts angrily, but after some coaxing, he decides to stop fighting and join in the collective dance. In contrast to the circumstances that made the song 'go viral' and become so important for women claiming agency and demanding safety, the video maintains a playful, humorous storyline, focusing on the potential to come together in a communal celebratory gesture.

\section{Human identity in Caribbean music}

Now to end, let us turn to the cultural producers we mentioned before. Their work presented us with literature on being human via the medium of music. We read their 'books' with our entire body. We can only be sketchy here, expressing what these works summoned in us in our coproduction while singing along, studying the lyrics, watching the music videos, discussing the artworks with others, and dancing. Do not expect biographical details on these artists but an account of the lessons of what it means to be human that we drew from their works. 
Many Caribbean artists enact myths of relation to the Old World settings of their ancestors. Growing up in a Caribbean that was considered and still considers itself Dutch, we nevertheless learnt to acknowledge Mama Africa $^{3}$ through Peter Tosh as others learnt to appreciate Mama India via Sundar Popo's hits like Nana and Nani. ${ }^{4}$ These are examples of Pan Africanism and Pan-Indianism in the Caribbean arts whereby the loss and the hardships faced by the descendants of Africans and Asians are continuously highlighted. Nevertheless, there is another way. Instead of focusing on what the wicked did - think, for instance, of Bob Marley's Redemption Song $^{5}$ - some legendary artists actually us to privilege the pleasure of belonging to the African Diaspora. Ismael Rivera comes to mind, as does Jacob Desvarieux of the Zouk band Kassav'.

In Rivera's interpretation of Las caras lindas (de mi gente negra) ${ }^{6}$ [The Beautiful Faces (of My Black People)], a guagancó composed by Catalino 'Tite' Curet Alonso, he sings about "his black people” as a "molasses parade" that carries everything - beauty, sweetness, sadness, pain, laughter, truth, and love:

Las caras lindas de mi gente negra/son un desfile de melaza en flor/ que cuando pasa frente a mí se alegra/de su negrura, todo el corazón. Las caras lindas de mi raza prieta/tienen de llanto, de pena y dolor/son las verdades, que la vida reta/ pero que llevan dentro mucho amor. [...] Melaza que ríe, melaza que ríe, jajajaja/ay, que canta y que llora/y en cada beso [es] bien conmovedora.

[The beautiful faces of my black people | are a parade of molasses in bloom | which, when passing before me, my entire heart is overjoyed by its blackness. | The beautiful faces of my black race | which carry weeping, sadness and pain | are the truths, that life challenges | but they hold so much love inside. [...] Molasses that laughs, molasses that laughs, hahahaha | Ay, that sings and cries | and in each kiss [is] very moving.] (Rivera 1978) (Transl. by the Author)

In one rendition of this song, Rivera - in the improvisational segment that is expected in Afro-Latin American genres like guagancó, salsa, and son montuno, among others - adds a line about the black faces beyond Puerto Rico. The original song mentions Panama, another country where the com-

3 Cf. https://www.youtube. com/watch?v=buVm-D3eChM (2018-11-30).

4 Cf. https://www.youtube.com/watch?v=73FVMjfXFGE (2018-11-30).

5 Cf. https://www.youtube.com/watch?v=0FGgbT_VasI (2018-11-30).

6 Cf. https://www.youtube.com/watch?v=t9gsjztx3f8 (2018-11-30). 
poser (Curet Alonso) has fallen in love with “a beautiful black face”. In his improvisation, Rivera calls out to Llorens Torres - an urban, public housing sector in the San Juan, Puerto Rico. From here, he moves on to the beauty of the beautiful black faces of people of all Latin America - a shift from the particular to the plural. We also see this in songs by Pérez Joglar, such as $L a$ Perla (featuring Rubén Blades) and Los de atrás vienen conmigo (Those in back are coming with me). In the first song, the slum known as La Perla becomes a focal point (as emblem of fierce Puerto Ricanness) from which other similar communities in Latin America and around the world are interpellated, establishing a broader community and creating a conversation: "Esa risa en La Perla la escuché en el Chorrillo | y de Tepito hasta Callao y donde se acallan chiquillos" (That laughter of La Perla, I heard it in El Chorrillo | and from Tepito to Callao and where children are kept quiet). Thus, he links all those who live on the margins: from La Perla (in San Juan, Puerto Rico) to El Chorrillo (in Panama City, Panama), Tepito (in Mexico City, Mexico), and Callao (in Lima, Peru). Similarly, in Los de atrás vienen conmigo, the singer calls for all of Latin America to march along (for the drumming is distinctly a marching/procession tune) with the "underdogs" (los de atrás). Here, Pérez Joglar mentions all the impoverished urban areas of Puerto Rico, calling out to those living in similar conditions in Panama, Peru, Mexico, Argentina, Chile, Colombia, Paraguay, Uruguay, Brazil, and Venezuela - all linked by hardships and the shadow of Death, all linked by a recognition of blackness, and all marching to a cumbia villera or bloco afro rhythm.

Now we will focus more comprehensively on Jacob Desvarieux. The mid-tempo beat in Desvarieux's hit African Music allows us, in the springs of our existence, to sway from left to right as he refers to the numerous repertoires of the music of Africa and its Diaspora in a way that makes us feel happy to be part of that community of practice. As scholars, we are of course always mindful, as we swing to Desvarieux, that Death brought the trans-Atlantic community that many others and we herald into existence. But we enjoy the dance nevertheless. As this is a community of practice and not an everlasting phenomenon immutable to change, the exchange between the two symbolized worlds in Desvarieux's music - Africa and the African Diaspora in the New World - has to constantly take place. Every time we download the song via YouTube these days, that bond comes alive. It is not about a pure Africa meeting the, say, impure African Caribbean Diaspora, but about two historically specific, yet related, creolizations transacting. We learned to appreciate this more fully via Desvarieux's collaboration with the Angolan Creole music artist Nelo Carvalho, in Mamã Falou, with the latter singing in Portuguese-Angolan while Desvarieux sings in Guadeloupean/Martinican Creole.

In the accompanying music video to Mamã Falou, a new factor that is usually associated with Europe enters the equation: the Catholic Church. What to make of this? As we watched the video several times, and dis- 
cussed the matter, we found ourselves acknowledging the ambivalent role the Church played in the lives of Africans and their descendants in the Caribbean. Desvarieux and Carvalho's video prompts a study of the ways in which, despite racial prejudice and institutional discrimination, Catholic canonical law, as opposed to civil law, was the venue for the victimized to assert their personhood in legal terms (as members of fraternities, sisterhoods, husbands and wives, etc.) when they were deemed nothing but slaves and indentured servants - as lesser humans undeserving of equal concern and supposedly unfit to govern themselves or decide what they considered the good life. The ambivalence with which we were invited through Mamã Falou to approach Catholicism of course also holds for Protestantism and its evangelical offshoots. We have come to appreciate Christianity in the Caribbean as a particular working out of justice through creolization: the traditions of the subjugated and subjugators melding and blending while somewhat retaining their specificities under inhuman conditions. Visually, through this video, we are reminded of how and where two (and echoes of more) historically specific, yet related, creolizations transact. We see our two musicians on the stage, which is set in front of the altar. In some scenes, we see the figure of Christ on the cross in a central position but we also glimpse a brown Madonna to the right. Given the syncretism displayed in the dancing, the repetition of the chorus, the modes of dress, the dugout canoe, the landscape, and objects in the church, the phrase "Eo verbo se fez carne", shown on a light blue banner, becomes much more than a biblical quote. Does the red candle (prominent at the beginning of the video) only represent the transubstantiated blood and body of Christ or does it signify much more? If the church is venue for the victimized to assert their personhood, they also assert the plurality of related beliefs. The red candle may just as well represent the flesh (blood and body) in other ways, just as it may represent love, passion, courage, fertility, vitality depending on the participant in this space.

It is in the music that came to life in these houses of worship that insightful existential truths can be found. We learnt from Caribbean music never to confuse the necessity of humanism with its ultimate sufficiency. Man makes his and her world, yes, but we do not make the world. As Edwin Yearwood of the Barbadian Soca band Krosfyah exclaims, we must believe in Something Greater than ourselves.

Man still tries to separate | His own brothers with class and creed | Upper class, middle class and lower class | With the lower following lead | Separation shows like a sore thumb, aye | And it spreads out rapid and fast | Man on dying bed need a donor's blood | Does he request a certain class? (no no) | But now who am I just to wonderer | Passing judgement and kicking fuss | Because death is a crucial part of life | When man returns to nothing but dust | Now I wipe my tears and I start to 
fear | Smiling off the class and creed game | 'Cause when rich and poor convert back to dust | So I must believe, That there's something greater than me. | To keep up the fight I just got to see, That there's something greater than me | Take your head out the clouds and look till you see, That there's something greater than me | Jail me wrongfully and I'll still be free, Cause there's something greater than me | Jah Jah Jah Jah Calling | Jah Jah Jah Jah (Calling)...back to dust we shall all go, for all man is equal. (Yearwood 2005)

In the Mamã Falou video, "Eo verbo se fez carne" reminds us of all the ways in which survival, celebration, and affirmation of life are expressed in this song: a mother lovingly pours holy water on her child's head, girls dance. The affirmations of our being in the world are expressed through work - shown in the people transporting goods on the dugout canoe - and through play - boys play football, girls splash water on one another, our two performers "air-guitar" the rhythm using the oars in their shared canoe, and then we see them dancing with the community. And yet, in its textual reference, the phrase reminds us of "something greater than ourselves".

When we do not show that reverence and, to use Ras Shorty I's title of his classic, Push the Creator Out, the result is anomie and a perpetuation of the worst of colonialism. This is how Ras Shorty I skilfully interprets the behavior of renegade youngsters in crime torn Trinidad and Tobago. Reminiscent of the heydays of formal colonialism, many youth violently treat those who inhabit the twin islands as expendable means to their ends. Yet, Ras Shorty I hearkens his generation remember that they are not without blame. In refusing to acknowledge 'something greater', and therefore, by implicitly privileging a crass colonially derived way of being, they have fostered the current malady:

How my country overnight | get you angry and uptight | I searched out the answer | And this is what I discovered | All you push the Creator out | you didn't want His name in your mouth | so you take Him out of the school curriculum | No knowledge of God for your children | Look around you | All you have is teenage bandits | Their minds have nothing in it | But Os and As and CXCs [Here, Ras Shorty I is referring to students who may be bright in mathematics and economics, but lack morals and spiritual wisdom.] | But no knowledge of the Almighty. Just because you pushed the Creator out $\mid$...You're living the fruits of your action | So tell me | Weh [why are] you bawling for | don't bawl | we all you crying for | don't cry | ... It's you who pushed the creator out | Every generation that comes | is nothing more | than a reproduction of the ones gone before | all you double standards | and your false value | come back and catch you off guard | and confusing you | what you seeing is yourself | manifesting nothing else | check the children performance 
and then look deep in your conscience | you gone see | All you push the Creator out.

[...] Don't condemn the children for their inequity | Because it is all you made them and shape their mentality | What you did in secret they doing openly | Don't run away from it | it's your own cruelty | A mango can't bear a fig | a donkey can't make a pig | you sow seeds of darkness | Now you reaping wickedness. Just because you push the Creator out. (Shorty I 2000)

Listening and dancing to that highly influential song of Ras Shorty I, we find ourselves acknowledging Death in our societies without falling into despair or nihilism. We realize in all profundity that nothing goes harmoniously when we do not seek His face in the face of every other we encounter (to whom we must utter the primal mystical Rastafari greeting "Give thanks", despite what they do, for seeing, being, and becoming with us in concord with 'Cosmic Love'). ${ }^{7}$

This quest to recognize his Face in every other face led us to connect Push the Creator Out to the Mighty Shadow's latest tune Doh Mix Me $U p .^{8}$ Shadow's song was a warm awakening that though we may revel in the African and African Diasporic community of practice summoned by Jacob Desvarieux, we must never lose our singular sense of moi in the belief in a collective Us, as different from a They. Taking a substantive approach, we come to realize that we can never be black without being specifically ourselves, meaning, encumbered in our particular black way of being connected to context and personal histories. And paradoxically, in a non-disturbing way, we also appreciate ourselves as an unencumbered self that can and must don identity labels - black, brown, woman, man, hetero, professional, etc. - in order to be, but can never to be reconciled with any of these. In The Repeating Island, Antonio Benítez Rojo speaks of "supersyncretism" and how our being/acting "in a certain kind of way", through polyrhythm, improvisation, and other modes of expression, can conjure away Death (although his words are "apocalypse" and "violence"). In his introduction, he explains how we, "in a certain kind of way" - which we may read as "in our particular black way" - may come together, while differentiating ourselves from one another, expressing our desire to sublimate apocalypse and violence:

7 Cosmic Love - what some call the Creator, the Unmoved Mover, the Most Moved Mover, God, Brahman, etc. - can be conceived as a he, she, or it, but is none of the above. Rather awkwardly put she/he/it is best understood as an impossible possibility that the human mind in a variety of cultures and ways of life has imagined, imaged, and intuited, as that which enables the emergence and disappearance of phenomena.

8 Cf. https://wWw.youtube. com/watch?v=G9Uqwc5psPY (2018-12-01). 
The peoples of the sea, or better, the Peoples of the Sea proliferate incessantly while differentiating themselves from one another, traveling together toward the infinite. [...] If I were to put this in two words, they would be: performance and rhythm. And nonetheless, I would have to add something more: the notion that we have called "a certain kind of way," something remote that reproduces itself and that carries the desire to sublimate apocalypse and violence; something obscure that comes from the performance and that one makes his own in a very special way; concretely, it takes away the space that separates the onlooker from the participant. (Benítez-Rojo 1996, 16)

To echo his words, through performance and rhythm, "in our particular black way", we may preserve both our singular and collective sense, and be both onlooker and participant, encumbered and unencumbered.

In this state of being, specifically encumbered and simultaneously unencumbered, we have been able to wholeheartedly sign on to the radical equality promoted by Shakiya, the young Sint Maarten Soca artist from our rock, in her anthems such as None Better than the Rest. We join her in her quest on and off stage for all to recognize that the social and factual deaths caused by poverty, political indifference, and general indecency in our society and the world, are unacceptable.

But, at those moments of missionary zeal, another beat luckily interrupts our concentration. We sense the very real danger of a possible form of camp-thinking emerging, namely, that of a morally correct minority (Shakiya, us, and those we regard politically) versus an immoral majority. It is then that we come to truly appreciate why most of Caribbean music is apolitical, if we think politics as always involving non-contradictory and static appreciations of Us versus Them.

We recognize why artists from our rock (Sint Maarten/St. Martin) like King Vers - following in the footsteps of the country's legendary Soca star, Shadowman - is the Lord of bacchanal. In Vers's music he summons us, as his more widely known Caribbean counterparts Machel Montano, Destra, Peter Ram, Skinny Fabulous, and Tall Pree do, to acknowledge that all we can do, if we do not want to fall into camp-thinking, and yet infuse our ethics of singularity into political moves, is to continuously exorcize our indecent tendencies to think in terms of clear-cut friends and enemies, by collectively Getting on Bad, as King Vers heartens us to do in his 2015 Carnival jammer. Like Rivera does in Las caras lindas (de mi gente negra), the Getting on Bad video also reaches beyond one island, presenting the communal bacchanal in St. Maarten, New York, Jamaica, and Barba- 
dos - waves of bodies of all shapes, colors, and ages collectively wining. ${ }^{9}$ None can be said to be stealing our coveted enjoyment and transgression of that enjoyment as we routinely release ourselves from stiff regimes of colonially derived respectability and societally sanctioned forms of rudeness! We are simultaneously advised and enticed (to use another of King Vers's hits, his collaboration with the Aruban band Tsunami) to give each other Pressure in the way that dances with Death: meaning to perform acts of radical truth telling about the inextricable bond of the philosophicalreal and historical-real manifestations of death in the Caribbean and the world after the colonial nightmare without anyone having to literally die.

Thou shalt not kill!

\section{Bibliography}

Allen-Agostini, Lisa (2006). "Wining Words: a Trinidad Carnival Wining Dictionary". Caribbean Beat Magazine, 77. URL https://www. caribbeanbeat.com/issue-77/wining-words\#axzz5WW6wihDq (2018-11-30).

Benítez-Rojo, Antonio (1997). The Repeating Island: The Caribbean and the Postmodern Perspective. Translated by James E. Maraniss. Durham: Duke University Press, 16.

Desvarieux, Jacob (1999). “African Music”. Euphrasine's Blues. France: Sonodisc.

Hall, Stuart (1996). "Minimal Selves”. Baker Jr., Houston A.; Best, Stephen; Lindeborg, Ruth H. (eds), Black British Cultural Studies: A Reader. Chicago: University of Chicago Press, 115.

Krosfyah (2005). “Something Greater.” Featuring Edwin Yearwood. Next to You. VP Records.

Lemoine Ulloa, Dominique (2015). "La dominicana Rita Indiana: Islas diabólicas y ruidosas" [online]. Arcadia, 17. URL https://www.revistaarcadia.com/impresa/musica/articulo/rita-indiana-musica-escritora/41885 (2018-09-05).

Marley, Bob (1980). “Redemption Song.” Uprising. Tuff Gong/Island Records.

Miller, Michael E. (2016). “Outrage in Trinidad after mayor blames Carnival killing on dancer's 'lewd behavior'”. The Washington Post. https:// WWW.washingtonpost. com/news/morning-mix/wp/2016/02/12/outrage-

9 As Lisa Allen-Agostini explains in "Wining words: a Trinidad Carnival wining dictionar", "To wine, if you don't know, is to move your hips and waist in a 'winding' motion, hence the name. The dance is peculiar to calypso, although someone with real skill and dedication could wine to any kind of music". 
in-trinidad-after-mayor-blames-carnival-killing-on-dancers-lewdbehavior/?utm term=.bb6075d2bff8 (2018-11-10).

Pérez Joglar, René (2008a). “La Perla.” Los de atrás vienen conmigo. Sony Music Latin.

Pérez Joglar, René (2008b). “Los de atrás vienen conmigo.” Los de atrás vienen conmigo. Sony Music Latin.

Popo, Sundar (1979). "Nana and Nani." Come Dance with the Champ. Trinidad and Tobago: Windsor Records.

Powers, Martine. “'Leave Me Alone': Trinidad's women find a rallying cry for this year's Carnival”. The Washington Post. URL https://wapo. st/2QVR4hy (2018-09-05).

Residente (2017). Dir. René Pérez Joglar. Documentary. Produced by Paraíso Pictures. 90'. Distributed by Sony House Entertainment.

Rivera, Ismael (1978). "Las caras lindas (de mi gente negra).” Composed by Catalino 'Tite' Curet Alonso. Esto sí es lo mío. Tico Records.

Rose, Calypso (2016). “Leave Me Alone." Featuring Manu Chau and Machel Montano. Far from Home. Stonetree Music, Because Music.

Shorty I, Ras and the Love Circle (2000). "Push the Creator Out.” Jamoo Victory. Charlie's Records/VP Records.

Tosh, Peter (1983). Mama Africa. Emi Records. 


\title{
Caribbean Contemporary Poets and Artists Moored at the Venice Biennale
}

\author{
Margaret Spencer Matz \\ (New York Institute of Technology, New York School of Interior Design, \\ Pratt Institute, Saturday Program of The Cooper Union - Schools of \\ Architecture and Design)
}

\begin{abstract}
The Teatrino del Mondo of Aldo Rossi, a performance space set on a barge that could be reached by boat (1979-80), became an iconic metaphor for the first Venetian Biennale of Architecture. At the Venetian Biennale of Architecture 2018, this metaphorical representation was engaged by Caribbean contemporary poets Lasana M. Sekou and Charles Matz II and visual artists Cozbi Sanchez Cabrera and Jean-Ulrick Désert. In exploring ties that link the vessels of Caribbean contemporary literature and art to Venice, witness is the audacity of their opera, created while confronting intensifying natural disasters such as earthquakes and hurricanes, and recalling horrifying unnatural conditions of Slavery and war. The artists participated in collateral events in which poetry or visual art intersected marvellously with Venetian idioms.
\end{abstract}

Keywords Caribbean. Venice Architecture. Poetry. Art. Biennale 2018.

The Teatrino del Mondo, designed by the distinguished architect Aldo Rossi in 1979-1980, a temporary performance space set on a barge and reached by boat - a new island added to the archipelago of extant Venetian islands - became an iconic metaphor for the early Venetian Biennale of Architecture. The Teatrino still represents the Biennale metaphorically, and recently this metaphorical representation was engaged with Caribbean contemporary poets and visual artists participating as embodied vessels who intrepidly arrived at this new Venetian utopia. An unsusceptible temporary world tourist presence at this utopia sharply contrasts with a vulnerable permanent citizen inhabitation within the city of Venice; most inhabitation is marginalized at the periphery of the lagoon instead. This momentary juxtaposition of fiction to actuality, belying the existential threat posed by mass-tourism to Venice, is startling to those who become aware of its imbalance.

In exploring the invisible ties that link the vessels of Caribbean contemporary art to the island of Venice, to related aspects of Venetian culture, one discovers their existence and persistence. An insular landscape generates a visceral rapport, between humankind and the sea, and prompts experiences, of trade and travel, which constitute the essential affinities 


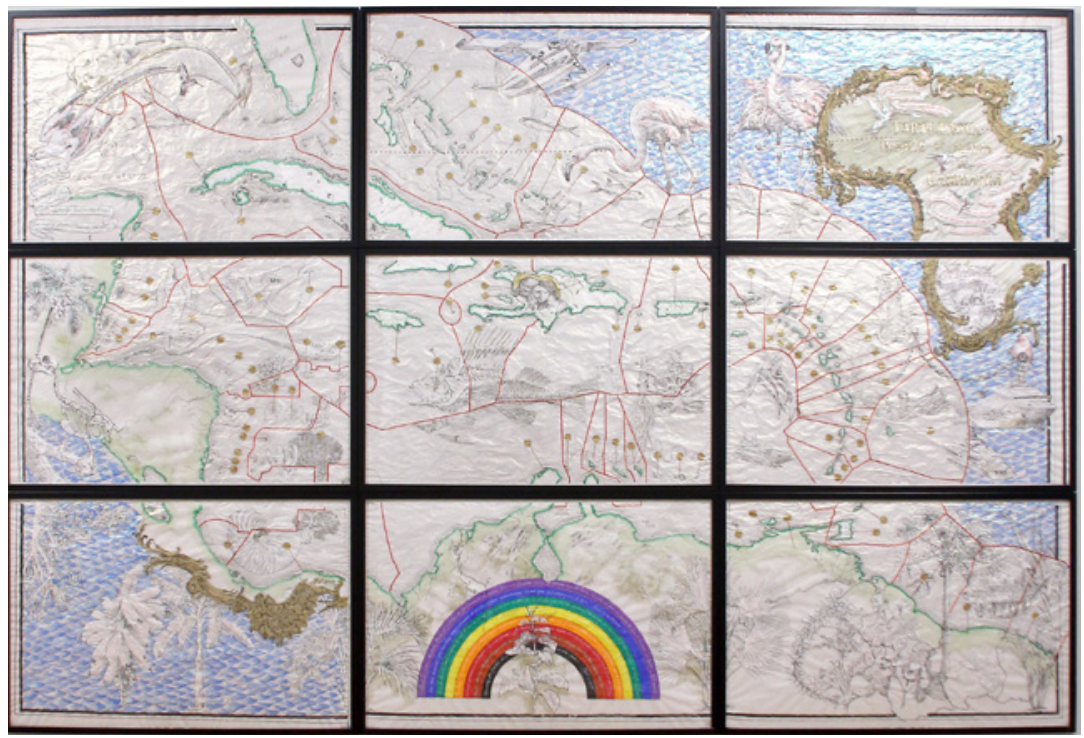

Figure 1. Jean-Ulrick Désert, The Waters of Kiskeya $(274.32 \times 182.88 \mathrm{~cm}$, composed of 9 panels each $91.44 \mathrm{~cm}$ by $60.96 \mathrm{~cm}$ ), acrylic and ink-embellished xerography on vellum, 2017

between these cultures. The adoption and adaptation of Venetian idioms by these artists in their works most poignantly expresses the unseen ties. Witness the audacity of the artists' works created as these individuals confronted intensifying natural disasters, like earthquakes and hurricanes, ushered in by human artifice; the audacity of their opus engendered while recalling horrifying unnatural conditions of slavery and war.

The performance art and poetry of contemporary poets, such as Lasana Sekou and Charles Matz II, and graphic and new media art of artists, such as Cozbi Sanchez Cabrera and Jean-Ulrick Désert, express identity and resilience, values which address challenges of epic proportions currently faced by both the Caribbean and Venetian communities. These artists were invited by Venetian citizens to participate in events surrounding the Venetian Biennale of Architecture 2018 and Biennale of Art 2019 because their work resonates with Venetian culture and with its present vulnerability. Some of the authors had previously been summoned to present in Venice in the context of other cultural forums: symposia such as Incroci di Civiltà at Ca' Foscari, presentations at the Casa Carlo Goldoni, residencies at the Emily Harvey Foundation and collaborations at the Centro Tedesco di Studi Veneziani. Returning to Veni Etiam, these artists arrived with poetry or art compositions that intersected marvellously with Venetian idioms. 


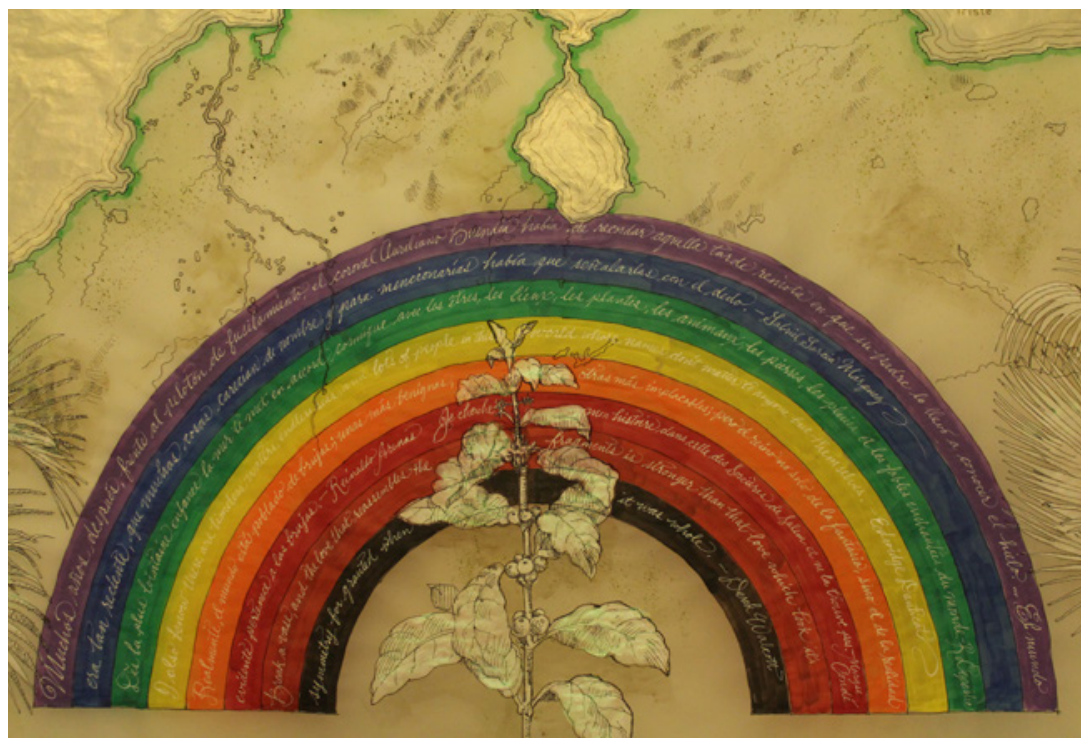

Figure 2. Jean-Ulrick Désert, The Waters of Kiskeya (detail of panel 8 of 9), acrylic and inkembellished xerography on vellum, 2017

The works of these artists, specific to the Dimension: Two Venetian Scole exhibit, a collaborative event that will be described later, are examined here as well as in the context of earlier works by the same persons. The literary and visual genres of the exhibit's contributors are compared to one another to explore their consonances. A transient paradigm, reappropriating identity through cultural belonging, is a connection among these pairings of author/artist. This correspondence bridges cultural and generational difference and informs the chronological arrangement of the works in this essay.

The Atlantic World epic Columbus, the Moor (2015) by poet Charles Matz II, and the artist Jean-Ulrick Désert's The Waters of Kiskeya/Quisequeya (figs. 1-2) are paired here to demonstrate affinities between their artistic endeavours. The collage of contemporary images/words applied upon a vellum/text of a historical narrative is one correlation between these two works - the art of Désert and the poetry in the book by Matz. A conception of a comprehensive multi-dialectical and multilingual region, the Caribbean with references to Middle-Atlantic passage/Middle Passage, is another. The assumption by the authors of another identity and/ or idiom(s) with which to convey artistic significance is one more.

The poet Lasana Sekou's Book of The Dead (2016) is paired with artist Cozbi Sanchez Cabrera's illustrations (figs. 3-4) reaching for synergies 

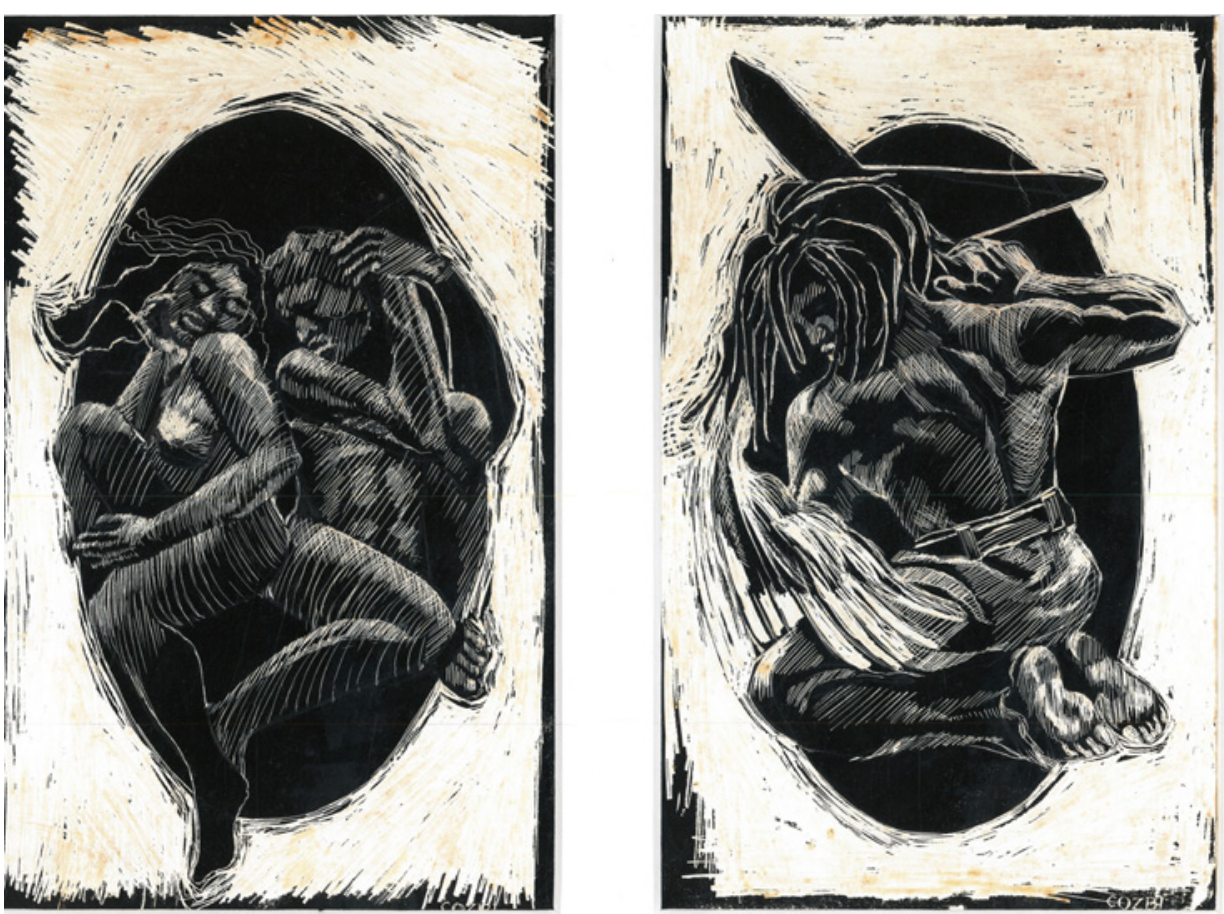

Figure 3. Cozbi Sanchez Cabrera, Man and Woman and Man and Technology, monochromatic scratchboard, circa 1990

found in their cooperative works. Creativity as protest through printed mediums of poetry and illustration is a correspondence between the author's and artist's works. The honouring and memorialising of the dead or the ancestors described and depicted in the poems and portraits is another. The reclamation by the creators of an identity and/or idiom(s) with which to convey artistic significance is yet another one.

What drew them to this project was the reanimation through poetic and visual arts of the nearly abandoned Venetian scole (schools) spaces of the Scola del Cristo and the Scola dei Mercanti, in the Cannaregio sestiere of Venice. The exhibit's curator, together with its Venetian collaborators, invited these Caribbean artists because of their experience with and knowledge of Venetian culture as well as for their themes, which resonated with the original activities of the adaptively reused spaces. For example, the original purpose of the Scola del Cristo, beginning in the Late Renaissance, was to provide burial for drowned persons; therefore, poetry by Matz and Sekou evoking this funerary theme was read. The original purpose of the Scola dei Mercanti, during the Medieval and Renaissance periods, was to procure 

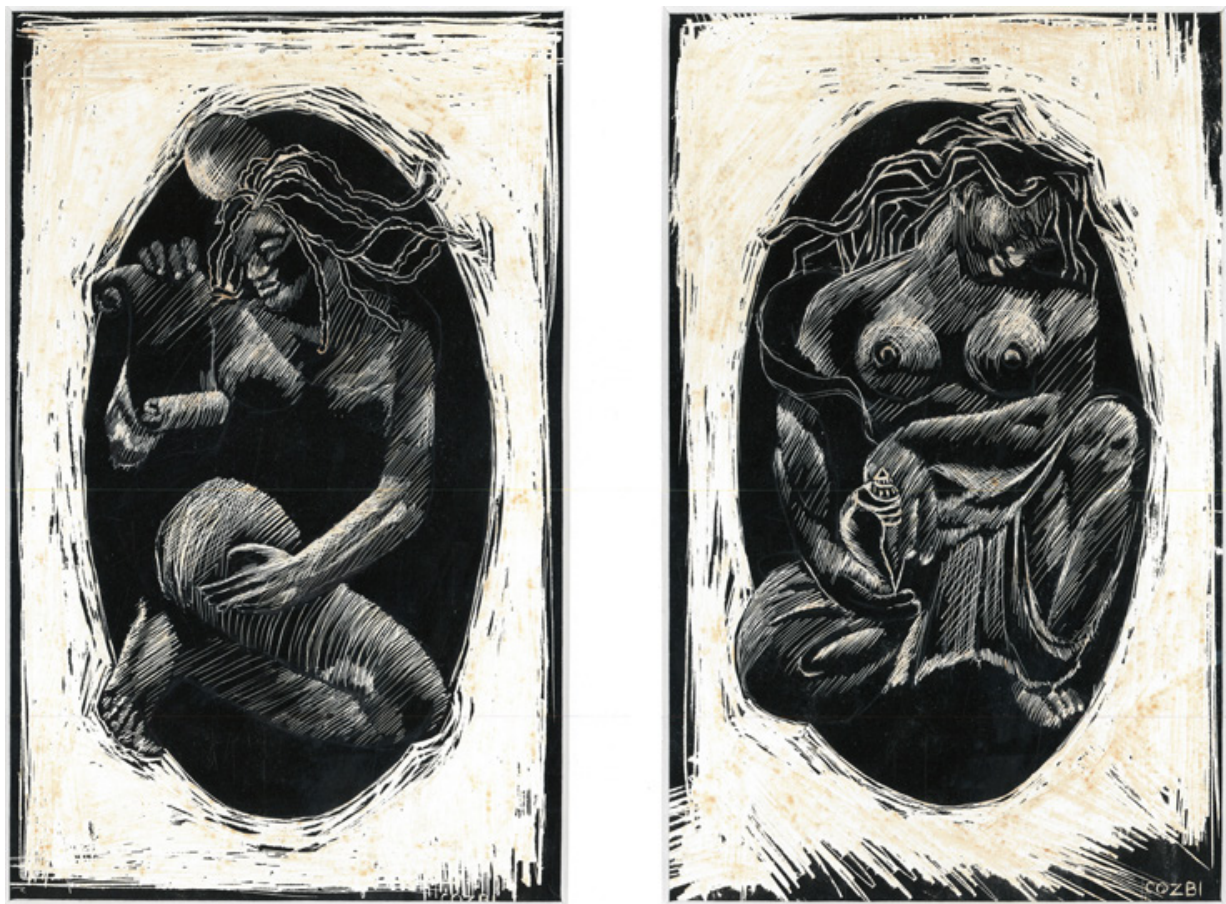

Figure 4. Cozbi Sanchez Cabrera, Woman with Knowledge and Woman with Marine Preservation, monochromatic scratchboard, circa 1990

and manufacture silk; thus, poetry aligned with its former use was explored there instead. The work of these artists as cultural corollary to the exhibit is distinctly identified as Caribbean, and their adoption of Venetian idioms, relative to the sea, travel, trade, and cultural reclamation in their works, makes them authentic interpreters, cultural explorers even, of Venetian culture.

Charles Matz II's past poetic performances included I Sonnambuli Giocosi (2013), published by Editrice Universitaria, in Venice, Italy, in 2013, as well as Columbus, the Moor, published by House of Nehesi Publishers in St. Martin, Caribbean, in 2015. I Sonnambuli Giocosi, a commedia, is based on Goldoni's brief stay in Feltre, Italy, in 1729 and his eventual exile in Paris, France. It was written primarily in Veneto dialect by Matz with occasionally interspersed words and sentences in French and German (though the author's original languages were English and German). The multi-genre performance narrative was presented at the Casa Carlo Goldoni in Venice, Italy, in March of 2014 and at a book salon in Belluno, Italy, in August 2014. Matz had taught and lived in Venice and Feltre from 1962 until 1984, a period during which critics note his writing had matured while being profoundly 
influenced by opera and by its Venetian precedent, Commedia dell'Arte.

Columbus, the Moor, on the other hand, is a tragic and redemptive piece, which, centred on the uncertain identity of Columbus and on his unexpected voyage to the Caribbean in 1429, had emerged from a lengthy inquiry by Matz. This research was sparked by the author's visit, together with a Contemporary Language muse from the Veneto, Mariagrazia Fia, to a place in Genoa, Italy where Columbus may have once lived:

The Saturnia, a ship of the Italia Lines, was where I met Columbus in person, or so I thought, in the 1960s... ......"Do you always sail on the Saturnia?" I asked... ... ..."No," he said quietly. "I flew back across the ocean once." The other passengers had been listening. We all looked suddenly at the waiter. "It was after the sinking of the Andrea Doria. I flew home. Just the once."

Mysteriously, secretly, I remembered Columbus, our waiter. For many years, this meeting with Columbus, as I thought it, made me want to celebrate him as a mysterious person, real, though elusive, courageous, despite stories blaming him for the travails of the Western Hemispheres where I was born. They were many stories; they were not history. These were mythical and heroic and, yet, they were real. Slowly, I passed through my own good and bad voyages...

... Once in lands believed to those of the beginning of a historic Columbus, I began to use my poetic performance experiences. Dramatic signs of him would explore the past by entering the past. Several languages with slightly different understandings of the legend, of the man, and of the heroics of all in the gales and currents of bravery created a memory of my own mystical encounter with Columbus. New World and Old, could be finally discovered in their grandness. Memories of earliest life recalled, as Montaigne rejoiced. Grand human spirits now recognized and praised when human cousins reunited after the sundering by mysteriously haunting events. ${ }^{1}$

The voices that the Columbus, the Moor text projects are heard by its audience in Arawak, the language of the native Taino islanders, in English, in Latin, and in Spanish. The script is primarily conveyed in English, and then translated into in Spanish, French, and Italian. Columbus, the Moor, was premiered in 1992 by an Italian and Spanish équipe at the Calderon de la Barca theatre in Valladolid, Spain. This city is where Columbus settled after his voyages, and where he died. Subsequently, Columbus, the Moor, was re-staged by the Historical Society and Museum of Art as well as broadcast via radio by a Spanish network, in Las Vegas, Nevada, in

1 Charles Matz II, email to Author, August 19, 2018. 
1992 - before ultimately being published by House of Nehesi Publishers as a four-language volume in St. Martin, in the Caribbean (both Matz and Sekou are known for the stylised use in their poetry of multiple languages as well as idioms and idiomatic expressions from various languages). The "Sepulchral Voices (Death Calling the Role)", part of Columbus, the Moor, was read by poet Lasana Sekou, who is described later in this essay, during the exhibit staging for Dimension: Two Venetian Scole.

A recent work by Jean-Ulrick Désert, titled The Waters of Kiskeya/Quisequeya, was displayed in the Dissolving into Consciousness: The Work of Caribbean Art exhibit at the Museum of Latin American Art (MoLAA) in Los Angeles, California, in 2017. It exemplifies an acquired idiom of cartography, which was inspired by Venetian maps studied by the artist during his residency at the Emily Harvey Foundation in Venice. A version of this map was also recently on view at the S 12 gallery in New York City from June 21 through July 12, 2018. An architectonic element in this drawing consists of a nine square grid that is overlaid upon the overall map. The grid alludes to and recalls the Venetian art of printing, a medium shared by both poetry and art, and its inherent role in disseminating knowledge. Tatiana Flores, curator of the MoLAA exhibit, described these maps:

Yachts and seaplanes replace the caravels of yore, and 'water-territorylines fracture the region,' akin to 'glass or ceramic shards,' articulating 'visual violence.' These lines are painted in red, and the sea they divide has been left colorless, signaling that the unnatural divisions created by colonialism were not only a humanitarian disaster but also an environmental one. Fish skeletons and dead coral reefs allude to the destruction and exploitation of the region, wrought not only by the early settlers but also by industries such as big oil and tourism... (Flores 2018, 1)

Two visual devices found in the maps focus on identity: the cartouche and the rainbow. The cartouche, shaped as the continent of Africa and outlined by aural frame, is a genial device which acts simultaneously on many different levels. Cartouches represented the artist's mark upon the artworks thus this specific one could be interpreted as a literal signature as well as an implied identity of its author. The rainbow, striated with bands of colour and inscribed with names of Caribbean literary figures (Edouard Glissant, Reinaldo Arenas, and Maryse Conde) is another device. Here, allegories of the forces of nature express godly elements so these names could be interpreted as implied influences as well as mystical presences.

Then there is Désert's "GLORIA", one part of a new media triptych consisting of videos, titled BLING-ECHO-GLORIA. BLING (Figure 5) critiques identity that is based on preconceived formulations whereas "GLORIA" (Figure 6) skirts identity favouring theatrical roles instead. The artist explained influences upon "GLORIA": 

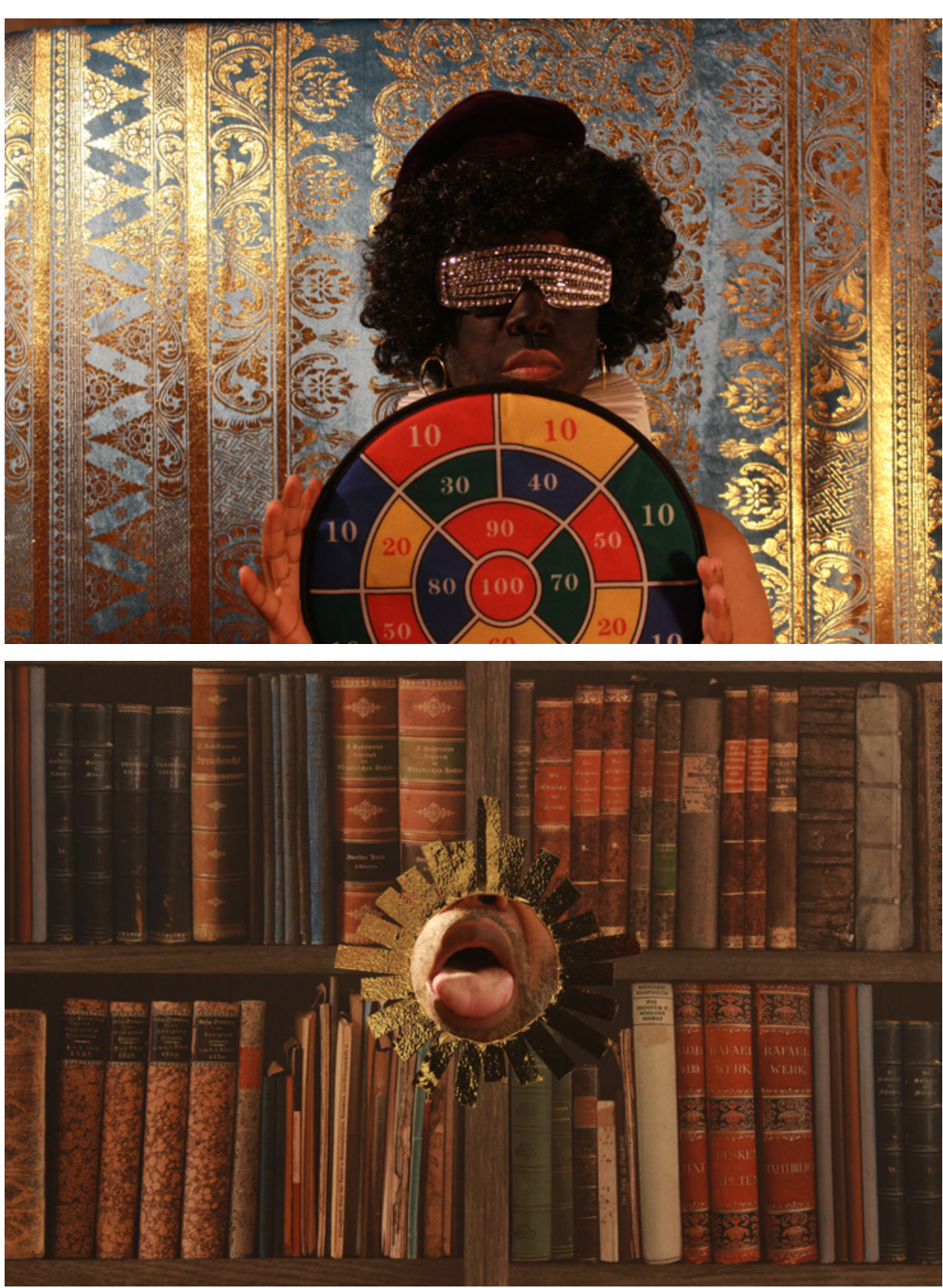

Figure 5 (up). Jean-Ulrick Désert, BLING, still from video, 2018

Figure 6 (bottom). Jean-Ulrick Désert, “GLORIA”, still from video, 2018 
inspired by the maxim 'see/hear/speak no evil,' attributed to the wise/ mystic apes... GLORIA begins and ends in the Venetian water, the Adriatic, which by extension becomes the Mediterranean Sea. The video ostensibly presents hunger, as embodied by the lips; it alludes to... ... a tactic where dignity is traded or relinquished as a strategy of survival... ......The theatre of want and poverty, where roles are sharply defined...... ...Hidden or exposed, the personal cost of refuge offers a certain capital. ${ }^{2}$

Désert's trio of allegories, also developed during his artist residency in Venice, are delivered through the video frame delineating the proscenium of a theatrical setting. These allegories and settings were likely influenced by actors and streetscapes of Commedia dell'Arte. "GLORIA" juxtaposes the artist's own identity to the stereotype of the moor. The refugee theme manifested in this video and, respectively, in Sekou's and Matz's poems from Book of The Dead and Columbus, the Moor, was suggested by the Venetians collaborating with the Dimension: Two Venetian Scole exhibit, owing to its currency that is directly impacting the Venetian community.

"GLORIA" was screened during the exhibit staging as part of a sequence, after a video of the reconstruction of the Scola del Cristo by various international architects, including Francisco Alba and Isaac Howland, and before the final video, named Atlantide, by Spoleto architect Fabio Fabiani, which is a composite of renderings that speak to the existential threat to and communal vulnerability of the City of Venice. Fabiani's Atlantide was also on view from July 7 through July 29, 2018, at the Carispo Foundation in Spoleto, Italy, on the occasion of the 61st Festival dei Due Mondi. The videos were projected adjacent to Holocube sculptures by Blake Carrington and Grayson Cox, devices that televised holograms that the participants could interactively recombine into new images.

The poet Lasana Sekou was first invited to read his recent poetry from Book of The Dead as well as poetry from an earlier critically acclaimed book, The Salt Reaper: Poems from the Flats (2005) as part of the Incroci di Civiltà (Crossings of Civilisations) and Poetry Vicenza. The literary events took place in Venice and Vicenza respectively in 2017. On those occasions, the poems that Sekou read had, significantly, also been translated into Italian by a team led by Caribbean Literature scholar Dr. Michela Calderaro. Sekou explained his acute perception of the essence of Caribbean and Venetian cultures:

What I did feel compelled to read in Venice, because of its provenance as a city of islands and water, were poems that somehow reflected aspects

2 Jean-Ulrick Désert, email to Author, June 17, 2018. 
of water or sea, and the aesthetics of salt linked to the Great Salt Pond, cradle of the St. Martin nation. ${ }^{3}$

Beyond the poems read and translated in Venice (Children of Man, Judgement, in Season, of Heritage and Fruit, and Sweet Lil' S'maatin), Book of The Dead also includes Saltern, a poem that is envisioned as an insular landscape, expressing its origins, and its salt monuments, addressing their oracles for divination of the island's future read through its past. This poem connects natural context to human existence demonstrating political activism as well as environmental consciousness. One also encounters Kiskeya in Book of The Dead, in this instance it is Kiskeya as the subject of a timely poem RECONOCIDO_en_QUISQUEYA, a poem formed as an immediate protest confronting resurgent racisms and genocidal tendencies in the Caribbean and throughout the world, including the Veneto region of Italy. These matters are the pressing issues of our contemporary times that concern Caribbean and Venetian audiences and communities alike thus Sekou's poems For/closure, Children of Man, The Angel of Death and LIBYA UNFINISHED were translated into Italian and read during Dimension.

"This is an important new work of world literature", wrote the Hong Kong/New York author Xu Xi in her foreword to Book of The Dead. Indeed, the contemporaneity of issues for Sekou, whether he is writing or performing from a metaphorical anchorage in St. Martin's Great Salt Pond or in the Laguna Veneta, is a constant. This is evidenced by the select poems from his forthcoming collection "Hurricane Protocol", which have recently appeared in the literary journal, Tripwire (2018, 171-6), reluctantly according to the poet himself. There is a raw urgency to these poems. All of the pieces within the 'protocol' set bear the same subtitle, "Hurricane Protocol", along with a different date as the name of each poem (9.8.17, 9.14.17, and 9.18.17), which echoes marine distress-calling, a plea to humanity as a whole. These poems recount the travails experienced by the St. Martin community during the devastating super-storms Irma and Maria. These disasters occurred in September of the Atlantic hurricane season in 2017, and garnered global news attention because of the unprecedented level of destruction on record that the storms brought on the poet's home island of St. Martin, and on neighbouring Caribbean countries and territories. As with previous collections, the message of hope of this poetry set is about prevailing over adversity and about resiliency to disaster. In his artist statement in Tripwire, Sekou briefly describes elements of the "Hurricane Protocol poems" that appear relative and intersectional to issues of mass-tourism impact on nature, vulnerability of inhabitation spaces, and marginalization of citizens - arguably a momentary juxtapo-

3 Lasana Sekou, email message to Author, July 9, 2018. 
sition of actuality to fiction, belying the Caribbean paradise as much as the Venetian utopia:

The images that tenant these poems are of a cyclonic provenance that has generated the loss of human life and ongoing displacement; destruction to homes and livelihood, to what was already a largely fragile infrastructure, an even more sensitive domestic food production and practically non-existent reserve, and to a highly stressed natural environment.

Sekou was born in Aruba in 1959, to St. Martin parents. He grew up in St. Martin before being taken by his mother to New York, USA, in 1972. After his studies in New York City and Washington, DC, Sekou returned to St. Martin in 1984.

The author/artist pairing for Dimension: Two Venetian Scole matched Sekou's poetry with the artwork of Cozbi Sanchez Cabrera. Cozbi Sanchez Cabrera's print-like scratchboard etchings for Sekou's Book of The Dead have a tradition of graphics that protested racism, which is presently the focus of an emergent history of art. The mono-chromatic technique was informed by bold prints that were designed and issued by early civil rights protests which initially started in New York City and later continued in other parts of the United States of America. This tradition of graphics, as a form of engraving, also stretches from its ancient roots and includes artwork of worker rights struggles, national liberation and anti-apartheid movements of the 20th century.

Although Sanchez Cabrera has yet to visit Venice, she was inspired to create these images that illustrate Book of The Dead while observing sculptural details of buildings throughout New York City that, much as the patere of the edifices of Venice, communicated symbols that were re-appropriated from other cultures imparting antiquity and deflecting misfortune. The statuary poise of the figures portrayed reinforces the epic narratives of the poems in Book of The Dead. Cozbi Sanchez Cabrera distilled the equivalence of printed literature and fabricated architecture through her illustrations that were projected as video accompanied by audio of Sekou's reading:

I chose to entomb the figures in ovals and shade them with the dimensionality one might find in an architectural frieze or low relief. A few of the figures or accoutrements seemed to 'leak' out of their prescribed places.

The reliefs, sparse in comparison to the text, are a nod to the permanence of edifice - expression common to all civilizations - neutral figures reflecting the builders, makers and stewards of the spaces we inherit, built to outlast an individual's lifetime. 
The dark figures ('black, blacker, blackest' as noted in the foreword by Xu Xi) are strong, earthy, earnest and African - at times kneeling, yet unapologetic. They represent a glimmer of a 'new' reflection, true to the insistence of survival and redress of misplaced identity. A reminder. ${ }^{4}$

Sanchez Cabrera's more recent work includes illustrations for the book, $M y$ Hair is a Garden (2018), which she authored. This children's book was reviewed in an article in The New York Times of June 14, 2018, headlined Why Do Kids' Books About Black Hair Draw So Much Attention - and Controversy? These illustrations as well as Sanchez Cabrera's handmade muñecas or dolls are intended to reshape preconceived visualisations of Caribbean and Central American women. This multimedia artist also makes quilts, which have a long narrative tradition especially among southern African-American women, such as the celebrated Gee's Bend quilters. Before her artist residency (2017-2018) at the prestigious Lincoln Center would be over, Cozbi Sanchez Cabrera's work was the subject of a solo exhibit at the Art Museum of Myrtle Beach, South Carolina in early 2017. The exhibit showcased her famous dolls, quilts, and over 20 of her illustrations from Patricia McKissack's children's book Stitchin' and Pullin' a Gee's Bend Quilt. By the year's end, the Lincoln Center Education Gallery in New York City exhibited a collection of muñecas entitled, "Cozbi A. Cabrera - Threads that Bind".

"The art of Cozbi Sanchez Cabrera has held an indelible fascination for me for over 30 years", said Sekou to New York University professor Jacqueline Bishop in a literary interview in the Jamaica Observer of May 6, 2018. While describing her own development as a Caribbean artist, Sanchez Cabrera recalled challenges in explaining the value of ephemeral designs, versus concrete fabrications, to her community. She recalled her acceptance into The Cooper Union Saturday Program, an art and architecture program which is preparatory to an exclusive merit-based scholarship at The Cooper Union itself. Attending the figure-drawing course at this academic institution is her bittersweet memory resulting in a controversy that ensued upon her community's awareness of the course's live modelling. The materials employed by this artist, as well as the processes followed by her, reflect a profound sensitivity towards resource sustainability. Cozbi Sanchez Cabrera, who left successful careers as an art and design director respectively at Atlantic Records and Sony Music to, in her own words, "discover, create and share pockets of grace and beauty", identifies herself as being of Caribbean descent, a native of Honduras, grew up and studied in New York City, and continues to live in the USA.

Concepts of identity and resilience that are manifested in the work of these Caribbean Contemporary artists resonate in Venice. A conceptual

4 Cozbi Sanchez Cabrera, email to Author, August 11, 2018. 
affinity between Caribbean authors and Venetian audiences was evident leading up to and present in events surrounding the Venice Biennale of Architecture 2018 and extending into the Biennale of Art 2019. The artists share with Venetians similar cultural concerns, like dialectical language, insular landscape, maritime economy and societal diversity. Concordant interests prompted Venetian citizens to invite the authors and artists to return again to Venice. This utopian community participated in lively discussions and multimedia performances during corollary happenings including those about effectively reanimated dead persons and spaces. These Caribbean authors' oeuvre is equally compelling to Venetians for the shared existential threats currently experienced by both Caribbean people and Venice citizenry.

\section{Bibliography}

Flores, Tatiana (2018). “Jean-Ulrick Désert's The Waters of Kiskeya/Quisqueya”. S 12, 21 June-12 July, 1.

Matz II, Charles (2013). I Sonnambuli Giocosi. Venezia: Editrice Universitaria, 1-77.

Matz II, Charles (2015). Columbus, the Moor. Philipsburg: House of Nehesi Publishers, 1-104.

Sekou, Lasana (2005). The Salt Reaper: Poems from the Flats. Philipsburg: House of Nehesi Publishers, 1-130.

Sekou, Lasana (2016). Book of The Dead. Philipsburg: House of Nehesi Publishers, 1-68.

Sekou, Lasana (2018). "Hurricane Protocol poems". Tripwire, 14, 171-6. URL https://tripwirejournal.com (2018-12-06).

Sanchez Cabrera, Cozbi (2018). My Hair is a Garden. New York: Albert Whitman \& Company, 1-32. 



\title{
The Sieve of Translation Reflections on the French Version of The Butcher Boy by Patrick McCabe
}

\author{
Silvia Boraso \\ (Università degli Studi del Piemonte Orientale «Amedeo Avogadro», Italia)
}

\begin{abstract}
This case study aims to analyse the difficulties a translator faces when dealing with a text that is built around the representation of oral language. In The Butcher Boy, the Irish author Patrick $\mathrm{McC}$ abe uses linguistic characterisation to confer social plausibility to his characters and builds the entire novel as a monologue the first-person narrator delivers in his own voice. The challenge such a variety of factors poses makes the translation of the book into any language an interesting subject of investigation. This contribution will draw a parallel between the original text and its French translation by focusing on the hermeneutic choices the translator has to make during the entire translating process.
\end{abstract}

Keywords Patrick McCabe. Translation as negotiation. Translation as hermeneutic process. Linguistic characterisation.

Born in 1955, Patrick McCabe is a renowned Irish novelist and playwright whose writings are mainly associated with the black comedy genre. ${ }^{1}$ The Butcher Boy (1992), the first of his novels to be acclaimed by both critique and public, ${ }^{2}$ is structured as a long first-person narration in which the protagonist and narrator, Francie Brady, tells the story of his life to an indefinite 'you' to whom he confides his darkest secrets. The oral quality of the récit legitimates the interpretation of Francie's monologue as an enunciation that he directly delivers to this unknown interlocutor with whom the reader may or may not choose to identify. ${ }^{3}$ Indeed, Francie

1 His main novels, all acclaimed by the critique, are The Butcher Boy (1992), Breakfast on Pluto (1998) - both shortlisted for the Booker Prize for Fiction - and Emerald Germs of Ireland (2001). McCabe also published a children's book, The Adventures of Shay Mouse (1985), and a collection of short stories, Mondo Desperado (1999). Moreover, he wrote radio plays for RTE, the national Irish channel, and for BBC Radio 4.

2 To the success of the novel contributes the appearance, in 1997, of the homonymous film directed by Neil Jordan.

3 The identity of Francie's interlocutor remains opaque. From the type of interaction created with the listener by the intimate quality of the narration one can infer that the 'you' Francie addresses is a sort of confessor, like a policeman, a priest, a worker from a charity institution 
seems to 'speak' to a public, as an actor wishing to break the fourth wall would by talking to the audience. The intimate atmosphere of complicity that is created in the novel by the confessional aspect of the narration (the same that arises from face-to-face conversation) allows the reader to experience a whole series of opposite feelings towards the narrated events - the revelation of the obscenities in which Francie indulges and the discovery of Mrs Nugent's brutal murder are viewed under a totally unexpected and original light deriving from the empathy that is gradually built up between the reader and the not-so-endearing narrator as a result of the confidential tone in which the story is told.

On the oral, mimetic nature of the novel depends the difficulty of translating Francie's narrative style into a different language - in the text resounds the unrefined voice of a deranged boy who belongs to that particular class of the Irish proletariat that in the aftermath of World War 2 could not afford to pay for their children's higher education. The narrator's socioeconomic situation requires a precise linguistic characterisation, whose social accuracy relies on the character's plausibility, all the more so since, as already mentioned, the entire narration is entrusted to Francie in the form of a long monologue. The translational challenges that stem from this variety of factors make interesting a comparison between the original novel and its translation into any language. Having read for personal interest the French translation of the book, Le garçon boucher, translated in 1996 by Énith Soonckindt-Bielok for the publishing house Plon, for this brief discussion I decided to draw a parallel between the English and the French version by recurring to close textual analysis.

At an aesthetic level, the peculiar characteristics of The Butcher Boy imply a remarkable interpretative effort on the translator's part during the process of translation, and this often entails, as Gadamer puts it, an 'emphasizing clarification' (1960, 345; Author's transl.) of the inferences deriving from the hermeneutic activity:

Limperativo della fedeltà, che vale per ogni traduzione, non può sopprimere le fondamentali differenze che sussistono tra le diverse lingue. Anche quando ci proponiamo di essere scrupolosamente fedeli ci troviamo a dover operare difficili scelte. Se nella traduzione vogliamo far risaltare un aspetto dell'originale che a noi appare importante, ciò può accadere solo, talvolta, a patto di lasciare in secondo piano o addirittura eliminare altri aspetti pure presenti. Ma questo è proprio ciò che noi chiamiamo interpretare. La traduzione, come ogni interpretazione, è una chiarificazione enfatizzante. (Gadamer 1960, 345)

(a sort of social worker), or even an imagined divine figure. This stratified indeterminacy undoubtedly facilitates the reader's identification with the recipient of the story. 
The imperative of fidelity that is valid for any translation cannot suppress the fundamental differences existing between the various languages. Even when one intends to be scrupulously faithful, one has to face the difficult situation of making tough choices. If one wants to stress in the target language a specific aspect of the original text that seems relevant to him, this can only occur if one pushes aside or even erases other aspects that are present in the text. However, this is precisely what I call interpreting. Translation, as any interpretation, is an emphasizing clarification. (Author's transl.)

By textual confrontation, this article endeavours to prove that the linguistic representation of the target version manages to highlight only some of the effects, intentions and contents which in McCabe's novel issue from the reproduction of the various idiolects. The nature of the linguistic peculiarities that are accentuated is determined by the interpretative choices the reader-translator made during the transposition of the text, choices that ineluctably presuppose a leveled/constricted rendition of the initial linguistic representation. Indeed, one of the translator's main tasks is, for each demanding passage, to sift through the set of possibilities and decide which translating technique between the widening and the narrowing of the lexical range he disposes of is the best option. This case study, then, aspires to examine the consequences of specific translational choices and, therefore, does not intend to be a reflection on translation in general. In the awareness of being only a partial overview of all the observable phenomena, its purpose is to identify the strategies adopted by SoonckindtBielok as far as the French translation of The Butcher Boy is concerned.

As already mentioned, the type of narration selected by McCabe for his novel needs a minute characterisation of the narrator's enunciative style in order to be effective. To grant Francie's idiolect plausibility, the language of the récit has to mimic the immediacy of talk and, consequently, must be both ungrammatical and apparently spontaneous. ${ }^{4}$ Actually, Francie's stream of words is not simply a mere imitation of orality, but is also functional to the construction of the empathic relationship with the reader/ spectator and to the characterisation of both Francie and the setting. ${ }^{5}$ Therefore, it seems interesting to determine, by drawing a close parallel between the two texts, what translational choices Soonckindt-Bielok made

4 This spontaneity is of course fictitious and the result of a meticulous planning on the author's part.

5 In fact, through Francie's words, McCabe renders both the historical and the geographical setting (the story takes place in Ireland during the '50s), establishes the social background not only of the narrator but also of the other characters whose utterances are reported - with different levels of manipulation - by Francie, and creates a subtle critique of the conversational norms that bourgeois conventions impose. 
in order to render in French the dialogical nature that characterises the protagonist's original narration.

In The Butcher Boy, McCabe reproduces what are the typical traits of verbal language within the boundaries of written narrative discourse. Despite the fact that the representation of speech is structured into multiple levels, ${ }^{6}$ this discussion is limited to the specific analysis of those aspects that constitute a translational obstacle for the French language. As far as the oral style of the narration is concerned, from the opening lines of the novel some differences can already be spotted between the source text and its translation. In particular, it is possible to discern a frequent recourse to pragmatic equivalence, ${ }^{7}$ which usually results in a rendering of the original grammatical incorrectness through an effect of colloquialism deriving from specific lexical choices:

When I was a young lad twenty or thirty or forty years ago I lived in a small town where they were all after me on account of what I done on Mrs Nugent. I was hiding out by the river in a hole under a tangle of briars. It was a hide me and Joe made. Death to all dogs who enter here, we said. Except us of course. ( $B B, 1 ;^{8}$ emphasis added)

Quand j'étais jeune il y a vingt trente ou quarante ans de ça j'habitais une petite ville où ils me couraient tous après à cause de ce que j'avais foutu sur Mme Nugent. Je m'étais caché dans un trou près de la rivière sous un enchevêtrement de bruyères. C'était une cachette que Joe et moi on avait faite. Mort à tous les chiens qui y entrent qu'on avait dit. Sauf nous bien sûr. (GB, 9; ${ }^{9}$ emphasis added)

These two passages show how Francie's ungrammaticality cannot be simply translated word by word, ${ }^{10}$ and the translator is forced to choose a lexical equivalence in her mother tongue in order to express the same perception of orality the English audience senses when reading McCabe's work. In the case of «I [had] done», where the omission of the auxiliary

6 For a taxonomy of orality, see Bazzanella 1994.

7 In this article I adopt the definition elaborated by Koller, who defines as «pragmatic» an equivalence that aims at maintaining in the target text the same effect produced in the source text. See Koller 1995.

8 Any bibliographical reference to McCabe's novel will be indicated by the initials $B B$ followed by the page number.

9 Any bibliographical reference to the French translation will be indicated by the initials $G B$ followed by the page number.

10 In his De optimo genere oratorum, Cicero already discouraged from translating verbum pro verbo. 
reminds one of demotic language, Soonckindt-Bielok employs the unorthodox verb foutre to recreate the same effect of colloquiality in the French version. The same kind of omission is present in the expression "me and Joe [had] made", where the use of the objective pronoun in frontal position is another 'mistake' made by the narrator (both because the first person pronoun should be a subjective one and because it should follow, not precede, the other subject of the sentence). In this case, the translator cannot but opt for the pleonastic employment of the subjects ("Joe et moi on"), which is typical of spoken French, but is obliged to conjugate the verb correctly so as to avoid a verbal construction that would sound excessively awkward and unnatural in French.

If one proceeds linearly and diachronically with the textual analysis, it soon becomes apparent that the translator's hermeneutic moves impose themselves on the original content and meaning of McCabe's novel, thus producing a 'new' - in the sense of "other" (see Paci 2008) - linguistic representation that differs at various degrees from the English version:

Then I stuck my nose out to see what was going on. Plink - rain if you don't mind!

But I wasn't complaining. I liked rain. The hiss of the water and the earth so soft bright green plants would nearly sprout beside you. This is the life I said. I sat there staring at a waterdrop on the end of a leaf. It couldn't make up its mind whether it wanted to fall or not. It didn't matter - I was in no hurry. Take your time drop, I said - we've got all the time we want. (BB, 1; emphasis added)

Puis j’ai glissé le nez dehors pour voir ce qui se passait. Ploc, et de la pluie en prime!

Mais je m'en suis pas plaint. J'aimais bien la pluie. Le sifflement de l'eau et la terre tellement molle que des plantes d'un vert éclatant auraient pu pousser d'un coup à deux pas de vous. Ça c'est la vraie vie j'ai dit. Je me suis assis là en contemplation devant une goutte d'eau au bout d'une feuille. Elle arrivait pas à décider si elle voulait tomber ou non. Ça avait pas d'importance. J'étais pas pressé. Prends ton temps goutte je lui ai dit on a tout le temps qu'il faut maintenant. (GB, 9; emphasis added)

The quotations above are indicative of the translating devices that the French translator adopts in an almost systematic way throughout the novel in order to render Francie's idiolect. Since it is not always possible to achieve a 'faithful' rendering of the original text, oral traits typical of colloquial French pervade the entire narration even when the English Francie 
speaks remains normative. ${ }^{\mathbf{1 1}}$ Paradigmatic in this sense are all the verbs in the negative form, expressed in French by omitting the ne the grammatical rule would require (ne... pas). Another example of the mechanisms regulating the transposition of orality is the use of $c ̧ a$, which is also distinctive of spoken, informal language.

Those translational choices - undeniably justifiable - originate from a series of Soonckindt-Bielok's interpretative moves that sometimes entail a sort of hypertranslation. This is due to the fact that the translator, who is first and foremost a reader of the text, presents the Francophone target public with a product that not only has to meet its demands, but that also carries and conveys a textual interpretation (the translator's). Emblematic in this regard is the last sentence of the passages above - McCabe recurs to a definite, precise use of punctuation (which, in his case, appears quite unusual); on the opposite, the same sentence in French is to be read in one breath. If the use of the dash, which in English symbolises the oral pause that denotes a change of topic, is not very common in French and, consequently, its omission is legitimate, the lack of a comma marking the prosody of the utterance is quite unjustified. The reason behind this deletion is probably the fact that, further on in the novel, Francie's narration gradually becomes an uninterrupted stream of words in which the use of punctuation is almost completely absent. However, at this stage of the story (this is the first page) deciding not to guide the reader in his or her textual inferences is more of an interpretative act than a mere translational choice. ${ }^{\mathbf{1 2}}$ This reading is further validated by the fact that in French, except for the last sentence that closes a dyadic dialogue, it is a comma, like in the source text, that marks the end of direct speech.

The hypertranslation just described is accompanied in Le garçon boucher by an opposite tendency, referred to as hypotranslation, which stems from the impossibility of finding solutions that maintain the polysemy and the metaphorical value of a term in the passage from a language to another. In the extracts above, for instance, one can notice that the sentences relating the narrator's perception of the natural world present subtle but meaningful differences. In fact, the English text is susceptible to possibilities of interpretation as far as Francie's weltanschauung is concerned, whereas in the target version the translator obliterates the nuances related to this

11 The concept of faithfulness has always been an object of debate in translation theory. However, it may be useful to cite here its definition by Umberto Eco: «il concetto di fedeltà ha a che fare con la persuasione che la traduzione sia una delle forme dell'interpretazione e che debba sempre mirare, sia pure partendo dalla sensibilità e dalla cultura del lettore, a ritrovare non dico l'intenzione dell'autore, ma l'intenzione del testo, quello che il testo dice o suggerisce in rapporto alla lingua in cui è espresso e al contesto culturale in cui è nato» (2003, 16; emphasis in the original).

12 Indeed, translation may well be seen as an interpretative process (see Gadamer 1960). 
potential construal by making narrowing translational choices - if the original lexicon conveys Francie's naïveté, gentleness and wonder in front of the rain, in French those connotations are completely absent. In this case, choosing equals not only selecting, but also sieving and excluding.

Another characteristic of Francie's récit is the use of lexical repetition. Since the immediacy of talk often implies the use of limited, generic lexicon that is redundantly re-employed within the same discourse, McCabe exploits this stylistic device to enhance the reader's sense of being in front of an oral enunciation rather than a written text. Although this locutionary strategy is valid for both languages, in French the systematic and extreme reiteration of the same word excessively disturbs the reading process. As the following passages illustrate, the translator is consequently forced to recur to a variation - even though not particularly effective in order to avoid excesses - of verba dicendi, which in English are usually limited to the generic to say:

One time we were standing in the lane behind the houses shading our eyes from the sun and Joe says: Did you see that plane Francie? I said I did. It was a tiny silver bird in the distance. What I want to know is, he said, how do they manage to get a man small enough to fit in it? I said I didn't know. I didn't know much about planes in them days. $(B B, 1-2$; emphasis added)

Une fois, on était debout sur le chemin derrière les maisons à protéger nos yeux du soleil et Joe a demandé: T’as vu cet avion, Francie? J'ai répondu que oui. C'était un minuscule oiseau argenté au loin. Ce que je veux savoir, il a dit, c'est comment ils arrivent à trouver un homme assez petit pour rentrer dedans? J'ai répondu que je savais pas. Je savais pas grand-chose sur les avions à cette époque. $(G B, 10$; emphasis added)

From these excerpts, it is apparent that, in the target version, the effects created by McCabe through the regular repetition of to say are somewhat dimmed. In Le garçon boucher, the only verb that is repeated is répondre, a generic lexical choice that only partially conveys the almost maniacal redundancy with which Francie uses words, and that it is hard to avoid interpreting as a sign, not even too veiled, of his obsessions. Furthermore, if one closely compares the two quotations above, it is evident that Francie's violation of the consecutio temporum is not reproduced in French - the translator limits herself to an alternation between the imparfait and the passé composé depending on whether the action is dynamic or punctual (in the sense that it does not extend in time). On the one hand, these expedients serve to meet the target readership's demands, which constitutes the ultimate goal of any translator; on the other hand, the changes that cannot be ascribed to the teleologic nature of translation and that consid- 
erably differ from the source text are to be attributed to the translator's interpretation of the language of the novel. That "T'as vu" uttered by Joe seems to support this hypothesis - the apocope that is introduced here probably derives from the need to underline and stress, in this instance even too much, the colloquial tone in which Francie's friend speaks as opposed to the oral, uncontrolled nature that already characterises the first level of narration. This necessity, which is completely absent from the dynamism of McCabe's novel, becomes inevitable when Soonckindt-Bielok opts for a systematization of talk that stems from her own interpretation of the English text..$^{13}$

As already mentioned, this homogenisation is functional and inevitable when reproducing as faithfully as possible an imitation of orality such as the one created in The Butcher Boy. However, at a communicative level faithfulness towards the source text is not always the most efficient choice ${ }^{14}$ when the translator detects a potential difficulty as far as the readers' comprehension is concerned, she adapts the language of the target version in order to facilitate to the French public the reception of the opaque meaning, which is intentionally blurred in English: ${ }^{15}$

Then I heard a clatter I thought I'd better get in the window to see what that was I thought maybe Grouse Armstrong or someone was in stealing the sausages again but when I got into the kitchen who's there only ma standing there and a chair sideways on the table. What's that doing up there ma I says it was fuse wire belonging to da just dangling but she didn't say what it was doing there she was just stood there picking at her nail and going to say something and then not saying it. I told her Mary's was shut could I still keep the sixpence she said I could Yee ha! I said and bombed off out to the border shop. ( $B B, 8$; emphasis added)

Puis j'ai entendu un cliquetis et j'ai pensé que je ferais mieux de passer par la fenêtre pour voir ce que c'était j'ai pensé que peut-être Grouse Armstrong ou quelqu'un était dedans en train de voler encore les saucisses mais quand je suis entré dans la cuisine voilà que je trouve m'man debout et une chaise en travers de la table. Qu'est-ce que ça fiche là m’man je lui demande c'était un fil électrique de ton père qui pendait

13 The translator's choice of putting at the centre of the translating process the reproduction of orality as the dominant effect of the text does not seem to take into consideration the implications that such a systematization of talk within the récit may have at a hermeneutic level. From my researches, it appears that Soonckindt-Bielok has not written critical contributions on The Butcher Boy, so my observations are derived from my personal reading and interpretation.

14 On the notion of faithfulness, see previous paragraphs.

15 More than translating, the translator seems to be explaining the text (see Paci 2017). 
mais elle me dit pas ce que ça fichait là elle restait plantée à se ronger un ongle sur le point de dire quelque chose pour finalement pas parler. Je lui ai expliqué que c'était fermé chez Mary mais est-ce que je pouvais garder quand même les six sous et elle m'a répondu que oui alors j'ai crié Youpi! et j'ai filé comme une flèche en direction de la boutique voisine. $(G B, 15$; emphasis added)

The juxtaposition of the two passages underlines the way in which in French, in order to make the reading process easier, inter-phrasal links are made explicit even though they correspond neither to the original text nor to its intention, that being that of showing the mental confusion bursting into Francie's language and modifying its internal cohesion and coherence. The episode described in the quotations above is emblematic of the frequent phenomena of denial and displacement characterizing Francie's psychological status. In fact, the narrator acritically describes the scene he witnessed as a child without being able to understand, or pretending not to understand, his mother's attempted suicide. In English, the mental derangement is reflected in the way Francie expresses himself - his style, in the way thoughts follow one another by association, hints at the different forms of the stream of consciousness; his use of sentence structuring mirrors his emotional state (Francie is so confused that he is unable to establish logical connections other than the coordinative conjunction and or the asyndeton). In French, by contrast, connective links are inserted in order to expedite the reader's understanding of what is happening. These links are basically an explanation of the narrated events that results from Soonckindt-Bielok's hermeneutic conclusions, which sometimes deviate, even though slightly, from the meaning of some enunciations. For instance, by opting for the possessive adjective "ton" she establishes that it is Mrs Brady the person that utters the sentence, whereas in English it seems that the source of the enunciation is Francie ("da" is the appellative he would normally use to refer to his father). Umberto Eco stresses the unavoidableness of the changes that occur during the translating process and underlines the lack of fair distribution within the exchanges generated in the passage of meaning from a language to another:

traducendo, non si dice mai la stessa cosa. L'interpretazione che precede ogni traduzione deve stabilire quante e quali delle possibili conseguenze illative che il termine suggerisce possano essere limate via. Senza mai essere del tutto certi di non aver perduto un riverbero ultravioletto, un'allusione infrarossa.

Ma la negoziazione non è sempre una trattativa che distribuisce equamente perdite e vantaggi tra le parti in gioco. Posso ritenere soddisfacente anche una negoziazione in cui ho concesso alla controparte più di quanto essa abbia concesso a me e tuttavia, considerando il mio 
proposito iniziale e sapendo che partivo in condizione di netto svantaggio, ritenermi egualmente soddisfatto. (Eco 2003, 16; emphasis in the original)

As already hinted, this contribution brings forward a practical example of what are the main translational criticalities McCabe's novel presents at both a narrative and hermeneutic level, in the awareness that other interpretative views may be applied to the translations of The Butcher Boy in other languages, as well as, obviously, to this very French version. Specifically, what is sketched here is a reflection, through a phenomenological analysis of the textual occurrences, on the fictional oral interaction that is created both in the original and the translated text. In the latter, Soonckindt-Bielok manages, even though within the limits of the 'negotiation' Eco writes of and through the necessary operations of equivalence, to reproduce the idea of an oral account, of a face-to-face conversation between the narrator and the reader. However, the implications of a narration that has as its intrinsic aesthetic that of being in mimetic relationship with talk are plural, and the effects of Francie's specific type of idiolect diversified. As mentioned above, McCabe achieves an accurate characterisation of the characters by exploiting the linguistic varieties they speak, which become at a further level a critique of verbal language and its conventions. ${ }^{16}$ Because of its complexity, this composite stratification characterising The Butcher Boy implies that in Le garçon boucher some aspects are inevitably left aside or just hinted at.

Since, consciously or not, the ultimate goal of translation must be that of meeting the demands of the target public, phenomena of domestication that make the understanding of the text easier to the Francophone readership are frequent in Le garçon boucher ${ }^{17}$ From an exegetical point of view, the series of operations to facilitate the readableness of the French text entails that some of the social, intellectual meanings that are conveyed through the representation of orality in English are inevitably lost in the target version. In fact, in French the traits that in written discourse conventionally portray speech are mainly denotative of the diaphasic variety of language and tend to overlook the diastratic and diatopic connotations McCabe employs in English. While the Anglophone reader of The Butcher Boy is aware of dealing with the typical idiolect of an Irish narrator belonging to the lower class and very poorly educated, the Francophone reader

16 Irish and Urban English connote the characters also at a social level and contribute to outline the postcolonial discourse of the novel (see following paragraphs).

17 To cite a few examples: "Newtown Road" $(B B, 7)$ becomes in French "Newton Road" (GB, $14)$; the use of a generic but more familiar "Londres" $(G B, 39)$ is preferred to the more specific but also more opaque "Camden Town" $(B B, 35)$. 
only perceives clearly the mimetic oral dimension of the narration because fewer and less evident are the instances in which the social dimension of speech is expressed. Those instances are easily acknowledgeable only when Francie reports entire sentences or fragments of dialogue he heard other people utter - by inserting the voice of the other characters within the boundaries of his own discourse, Francie provides the reader with a close juxtaposition of the linguistic varieties depicted in the novel, even though always filtered through his register and his subjectivity:

$\mathrm{H}^{\prime}$ ho when the priests get their hands on you there won't be so much guff outa you ye $h$ 'ho. I said I'm sorry Sergeant Sausage but he stubbed the fag exactly in the ashtray and said its too late for that me buck you shoulda thoughta that when you were in Nugent's up to your tricks! $H^{\prime}$ ho aye! (BB, 72; emphasis added)

Ho ho quand les curés t'auront mis la main dessus tu feras plus tellement le mariole ho ho. J'ai dit je suis désolé sergent Sausisse mais il a éteint sa cigarette dans le cendrier tout excité et il m'a répliqué c'est trop tard pour ça mon coco t'aurais dû y penser quand tu faisais l'andouille chez les Nugent! Hé hé oui. (GB, 72; emphasis added)

Through the semi-phonetic representation of the characters' voices, McCabe manages to reproduce the Irish accent and the demotic roots of their discourse. As the extracts show, when this diastratic connotation is more evident, a similar effect can be achieved in French by suggesting the characters' social background with terms deriving from the colloquial, argotic lexicon, though the diatopic characterisation portrayed in the source text gets inevitably lost. The neutrality to which translation is forced is a notion on which Françoise Grellet insists:

La plupart des traductions optent d'ailleurs pour une solution intermédiaire: le dialecte est rendu, mais il devient neutre. Il n'a presque aucune caractéristique régionale, ce qui est une façon de résoudre le problème du choix mentionné ci-dessus mais aboutit souvent à une perte certaine: une bonne partie de la saveur, de l'humour, de la richesse de certains dialectes anglais ou américains disparaissent en français. $(2014,199)$

This intermediate solution is determined by the interdependence that links the target text and the target readership; if the translator accepts what Grellet states above, the consequence is that the average reader provides the parameters for translation:

Translating as a teleological activity par excellence is to a large extent conditioned by the goals it is designed to serve, and these goals are set 
in, and by, the prospective receptor system(s). Consequently, translators operate first and foremost in the interest of the culture into which they are translating, and not in the interest of the source text, let alone the source culture. (Toury 1985, 19; emphasis in the original)

In French, the diastratic element is identifiable only as opposed to a more normative use of the language, mainly when characters engage in dialogical interactions. What follows is an excerpt in which it is possible to detect the stylistic difference between Francie's colloquial, trivial talking and Mrs Nugent's formal and grammatically correct speech:

J'ai croisé Joe sur le Diamond et il m'a dit fais attention Francie on est en guerre avec la Nugent. Elle est passée chez nous et elle va passer chez toi. Ça a pas loupé j'étais étendu en haut sur le lit et voilà qu'on frappe à la porte d'entrée. J'ai entendu m'man fredonner et ses pantoufles traîner sur le lino. Ah bonjour madame Nugent entrez donc mais la Nugent était pas d'humeur pour un ah bonjour entrez donc ou autre. Elle a attaqué m'man à propos des bandes dessinées et tout le bazar puis j'ai entendu m'man qui disait oui oui je sais bien sûr que je le ferai! Je m'attendais à ce qu'elle grimpe l'escalier à toute vitesse, m'attrape par l'oreille puis me jette en bas aux pieds de la Nugent et c'est ce qu'elle aurait fait si la Nugent avait pas commencé à parler des cochons. Elle lui a dit qu'elle connaissait les gens dans notre genre bien avant de partir en Angleterre et qu'elle aurait dû interdire à son fils de s'approcher de quelqu'un comme moi que peut-on espérer d'autre d'un foyer où le père traîne dans les cafés du matin jusqu'au soir, il ne vaut pas mieux qu'un cochon. Et ne croyez pas que nous ne savons pas ce qui se passe dans cette maison oh nous le savons que trop! Pas étonnant que le garçon soit comme il est quel avenir a-t-il à courir la ville à toutes les heures avec ses habits qui lui pendent dessus ça coûte quand même pas cher d'habiller un gamin que Dieu le garde ce n'est pas sa faute mais si on le voit encore avec notre Philip il y aura du raffut. Il y aura du raffut vous pouvez me croire!

Après ça m'man a pris mon parti et les derniers mots que j'ai entendus c'est la Nugent descendant la rue en nous lançant Des cochons pour sûr que toute la ville est au courant! (GB, 11-2; emphasis in the original)

Though long, the quotation is necessary for it shows that the options to which Soonckindt-Bielok can recur without preferring a specific regionalism to another - and, consequently, incurring in banal stereotypes and preconceived representations - are very limited. This limitation results in the fact that the most subtle linguistic peculiarities, identifiable at a first reading level in the original, need in French a closer juxtaposition 
between two or more varieties of language (in this case, Francie's and Mrs Nugent's) in order to be acknowledged.

The overall neutrality of the target text comes to be problematic when in McCabe's novel the language deliberately becomes metalanguage and reflects on Francie's social status. The previous extract, which reports the episode that triggers Francie's obsession with Mrs Nugent and internalization of his 'pigginess', is a good example of the way McCabe exploits linguistic representation to tackle socioeconomic issues. The transcription of other characters' sentences without quotation marks within Francie's stream of words is paradigmatic of his metamorphosis into what other people think he is - since he is called a "pig", he becomes one. ${ }^{18}$ The French version is unable to convey the specific critique, actualized in the original by the way speech is portrayed, of the complex and stratified Irish bourgeoisie (both Anglican and Catholic). The poor conditions and the sense of inferiority experienced by the 1950's proletariat, outclassed by the bourgeoisie of mainly English and Anglican origins, are symbolized by Francie's moral, mental and linguistic decadence; in the novel, it seems that Francie, condemned by historical and social determinism, cannot but become a clinic case. ${ }^{19}$ In French, this anthropological aspect, which is partly conveyed through linguistic representation in English, is necessarily transmitted by contents - bound to make choices that inevitably favour only some of the original features, the translator prefers enhancing the aesthetical and mimetic aspects of the text to portraying its social meaning, which is consequently put aside. The outcome of such an excluding selection is, perhaps unavoidably, an imperfect equivalence:

une bonne traduction ne peut viser qu'à une équivalence présumée, non fondée dans une identité de sens démontrable. Une équivalence sans identité. Cette équivalence ne peut être que cherchée, travaillée, présumée. (Ricœur 2004, 40; emphasis in the original)

What can be perceived as a partial loss of linguistic identity in the French translation may be considered as an inescapable factor of rendering such

18 By making Francie the emblem of the social inferiority of the colonised as opposed to the dominant status of the Anglican bourgeois ascendancy, McCabe tackles the complex question of Irish Postcolonialism. The possibility of considering Ireland as Great Britain's first, 'anomalous' colony has been an object of controversy for a long time, but only in the last decades scholars have started to centre their research around the issue. A renowned case is that of Edward Said, who sees in the subordinate position to the metropolitan culture in which the Palestinians live the same social inequality the Irish suffer under English hegemony (see Said 2003).

19 The question is much more complex. For this brief analysis, suffice it to say that the changes in human geography brought about by the different phases of evictions have contributed to forge Irish social stratification. 
a complex stratification as the one that characterises McCabe's novel. It is the result of Soonckindt-Bielok's hermeneutic, albeit legitimate, process (see Gadamer 1960) and of the inexorable linguistic 'insipidity' deriving from having to recur to aut-aut translating operations that opt, for lack of better options, for the 'lesser evil'. Moreover, in the case of works stemming from Postcolonial Literatures, the linguistic discourse poses a further challenge on account of the fact that the use of different registers implies, more than in other literary traditions, a reflection on identity values strongly linked to the socio-cultural context. The masterly elaborateness of The Butcher Boy, which derives from the intersection of anthropological themes and linguistic representation, forces the translator to put the original text through a sieve and to choose which aspects to privilege and which to leave aside.

\section{Bibliography}

Bazzanella, Carla (1994). Le facce del parlare. Un approccio pragmatico all'italiano parlato. Firenze: La Nuova Italia.

Benjamin, Walter (2006). "The Task of the Translator". Weissbort, Eysteinsson 2006, 297-309.

Carroll, Clare; King, Patricia (eds) (2003). Ireland and Postcolonial Theory. Cork: Cork University Press.

Eco, Umberto (2003). Dire quasi la stessa cosa. Esperienze di traduzione. Milano: Bompiani.

Gadamer, Georg (1960). “Dall'ermeneutica all'ontologia. Il filo conduttore del linguaggio". Salmon, Laura (ed.), Teoria della traduzione. Milano: Vallardi, 341-65.

Grellet, Françoise (2014). Initiation à la version anglaise. The Word Against the Word. Paris: Hachette.

Jakobson, Roman (2006). "On Linguistic Aspects of Translation". Weissbort, Eysteinsson 2006, 330-6.

Koller, Werner (1995). "The Concept of Equivalence and the Object of Translation Studies". Target, 7, 2: 191-222.

Levý, Jiří (2003). "La traduzione come processo decisionale". Salmon, Laura (ed.), Teoria della traduzione. Milano: Vallardi, 63-73.

McCabe, Patrick (1992). The Butcher Boy. London: Picador.

Nabokov, Vladimir (2006). "The Art of Translation". Weissbort, Eysteinsson 2006, 376-92.

Paci, Francesca Romana (2017). La Traduction: Défis et problématiques. URL https://bit.ly/2Evlb9M (2018-12-06).

Ricœur, Paul (2004). Sur la traduction. Paris: Bayard.

Said, Edward W. (2003). "Afterword: Reflection on Ireland and Postcolonialism". Carroll, King 2003, 177-85. 
Salmon, Laura (2003). Teoria della traduzione. Milan: Vallardi.

Soonckindt-Bielok, Énith (transl.) (1996). Le garçon boucher. Paris: Plon. Spivak, Gayatri Chakravorty (1998). "The Politics of Translation”. Weissbort, Eysteinsson 2006, 486-93.

Steiner, George (2013). After Babel. Aspects of Language and Translation [EPUB]. 3rd ed. New York: Open Road.

Toury, Gideon (1985). "A Rationale for Descriptive Translation Studies". Hermans, Theo (ed.), The Manipulation of Literature. Studies in Literary Translation. London; Sydney: Croom Helm, 16-41.

Venuti, Lawrence (2000). "Translation, Community, Utopia”. Venuti, Lawrence (ed.), The Translation Studies Reader. London-New York: Routledge, 468-89.

Venuti, Lawrence (2006). "Translation as Cultural Politics. Regimes of Domestication in English". Weissbort, Eysteinsson 2006, 546-57.

Weissbort, Daniel; Eysteinsson, Astradur (eds) (2006). Translation-Theory and Practice. Oxford: Oxford University Press. 

Interviste | Interviews | Interviews 



\section{"I Could Have Been Susanna From Thistlewood's Plantation Diary" \\ Jamaican Poet Ann-Margaret Lim Discusses Her Second Poetry Collection Kingston Buttercup}

Loretta Collins Klobah, Ari Hernández, Michelle Ramos Rodríguez (University of Puerto Rico, San Juan, Porto Rico)

On Reading Thistlewood's Diary

II

Susanna, dat's ma name

Don't confuse it wid de open-air African Savannah.

If yu look mi up In Miserable Slavery

you'll si mi listed under children,

wid Congo in bracket, page 29.

Ah was part o' de pickney gang in 1751

when him firs' tek mi

in de curing house.

Ah wet him bed him tek me in .

Each time ah wet de bed,

But him neva stop.

An'yu, who fin' him diary an' call mi favourite, Tell de worl' how dem whip me and Mazerine

For refusing backra an' him fren;

tell di worl' how Thistlewood

an' slavery ruin mi.

(Ann-Margaret Lim, excerpt from Kingston Buttercup)

In this interview, Ann-Margaret Lim discusses why in her two poetry collections, Festival of Wild Orchid (Peepal Tree Press, 2012) and Kingston Buttercup (Peepal Tree Press, 2016), she writes frankly about family relationships, her African, Chinese and Puerto Rican heritage, the rapes 
catalogued in Thomas Thistlewood's diary, and the domestic and societal violence of Jamaica's past that impacts the present, as well as art and popular music.

AUTHORS Your first poetry collection, The Festival of Wild Orchid (Peepal Tree Press, 2012), begins with poems about the arrival of your African and Chinese ancestors to Jamaica, the voyage of your father ("a brown boy in a yellow land" -"The Darkening"), who was taken as a youth to China to learn the culture, and hints about your mother, who left Jamaica for Venezuela, never to return, when you were young. In Kingston Buttercup, the complex family history is elaborated on as you describe your own recent journeys to China and Venezuela.

ANN-MARGARET LIM Yes, in the poem "At the Karaoke Bar, 21st Century Hotel", the speaker, in China, watches a karaoke singer, who flirts and orders her a whiskey, but she thinks of her father: "I remember you playing the harmonica, | the banjo, singing Chinese opera, | telling me stories of being brown in China, | being loved by a Chinese stepmother; | of girls intrigued by an oval face, | long brown limbs and massive waves of hair; | being the village basketball star". In "Yesterday", the speaker confides "Once I gulped a container of pills | wishing to never come back | to a room, a house without my mother". In "Venezuela Journal" the speaker asks about her mother "Did she think of me, as I of her | at the back of the plane | on the way to Venezuela?": "Today I sit at the window in the Hotel Alex, | sip Jamaican rum from the bottle $\mid \ldots$ and look down to see mi madre | in every brown-skinned woman | passing on the road..."

AUTHORS In Kingston Buttercup, some of the most powerful poems are in response to Thomas Thistlewood's 14,000-page diary, published in part as In Miserable Slavery (1750-1786), which documents his work and life as an overseer on the Egypt Plantation in Jamaica. When you were writing the poem "On Reading Thistlewood's Diary", which process was more difficult, reading his journal or writing your poems based on his diary? How did you decide on the personages to whom you would give a voice?

A.-M.L. Reading the journal was more difficult. I think all Jamaicans should read journals like this, though, so that we can appreciate where we are coming from and decide to treat each other better. The two women I referenced in the poems stayed with me, Phibba and Susanna. All the slaves were ill-treated, but these resonated most because I could have been either of them. Susanna could even be an ancestor of mine, and she, a girl, was raped repeatedly by a grown man. Susanna, for me, represents the voice from the grave that speaks out against paedophilia, carnal abuse. Susanna's psychological and 
physical reaction to the childhood rapes she suffered is documented by Thistlewood. Not only does he complain of her wetting the bed each time, but also of her fighting him off. Thistlewood ruined Susanna. What happened to her, and many others, still affects us now. It is one of the reasons why we should be owed reparations. I see my writing of Susanna's life in relation to the Tambourine Army Movement, which started in Jamaica in 2016, whereby victims of child sexual molestation have decided to publicly call the names of their abusers in the "Say their Names" and "Me, too" campaigns.

Phibba is the woman who was somehow able to 'stomach' criminal objectification of herself and women at large and benefit from her 'favouritism' as a means of economic empowerment. Susanna resisted and suffered irrevocable trauma, while Phibba somehow managed to 'earn her economic freedom'. When Thistlewood died, she was willed land and property and was a free woman. Both of these reactions represent choices that modern day women in Jamaica make. Too many women are raped in childhood. Some are destroyed, and visibly so. Others endure violence, viewing men as economic providers only, and seek to use them as such. It is an unhealthy cycle.

Living here and knowing our history, I become more convinced daily that the past and present in this country are constantly communicating, and that the miserable past of slavery keeps dictating the present day 'work plantations', inter-class relations and family and intimate relations.

AUTHORS Your poems critically engage with other source material related to slavery. The poem "Drawing" alludes to an illustration, "Full Freedom 1838", published in the book A Twelve Month's Residence in the West Indies, During the Transition from Slavery to Apprenticeship, by Richard Robert Madden. It depicts the moment of the Emancipation Proclamation in Jamaica. How do you see ekphrastic poetry as a means of revisiting history?

A.-M.L. I saw the drawing before reading Thistlewood's journal and was affected by it enough to write the poem. This is not my first attempt at ekphrastic poetry. In high school, I was drawn to West Indian history, and the fact that my high school was on a former plantation definitely helped to reinforce my awareness. I use illustrations to rewrite history from my perspective: "There's a ship | distant, unrigged, skeletal, | that squats, like memory, on the sea".

AUTHORS Could you tell us about your research process?

A.-M.L. The Internet represents an immediate source of information, so instead of waiting to go to a library to find pictures on slave auctions, or to find a West Indian History Book, I can just type 'slave auction 
pictures' in Google. The beauty of that is instead of just the specific West Indian reference I seek, I see more. I buck upon slaves like Philip in the poem "Philip, an Excellent Fisherman", a slave in the U.S. being auctioned: "Was there nowhere to sail to, | or were you only a fisherman | in bracketed waters, | the plantations your parenthesis?"

AUTHORS Poems make reference to other sorts of source materials, such as music and sculpture. In "On Reading Thistlewood's Diary", Ding Dong's song "Syvah" is referenced, and Christopher Gonzalez' tree-like sculpture of Bob Marley is admired in "The Artist". Do you follow a particular process when translating these source works into your writing?

A.-M.L. I may be so drawn to a piece of art, literature or music that poetry comes from that. I first saw the Gonzalez tribute statue for Marley as a 6th former in high school. We were touring the National Gallery of Jamaica, and I was told the story of the statue, why it was at the gallery and not in front of the National Stadium. The public wouldn't accept Gonzalez' vision of the reggae singer portrayed with tree roots and trunk instead of legs. I, however, loved the piece and thought it befitting of Marley. Then, meeting Gonzalez confirmed my love and preference for that sculpture over the one that was 'acceptable'. I later spent a day at the beach with Christopher Gonazalez. I saw how that rejection hurt him. For me, spending time with a 'rhatid', excellent artist, who met and loved Marley, was precious and exhilarating.

It is also the same process for my use of the song "Syvah" in my poem for Phibba. I live and breathe reggae and dance hall music. "Syvah" happened to be the 'hot' song at the time when I was reading Thistlewood's diary. The dance move, which involved squatting and flapping of the arms, reminded me of the act of release and flight. Below is a link to one of the videos doing the rounds. I interpret the dance moves like this: "And the takeoff | when the body comes fully into play | is the throwing off of shackles | ... And as the women release in | syvah, syvah, syvah | their hands like albatross' wings". ${ }^{1}$

AUTHORS In various poems, you pursue the theme of contemporary urban violence. Where do you find the material that inspired poems such as "Shaker Way", "Missing", and "Domestic: A Dyptych"?

A.-M.L. The thing about these poems is that they are personal. Shaker Way is where I live. Good Friday is celebrated in Jamaica, as the Friday on which Jesus was killed, and that was the day we woke and realised our neighbour's car was stolen. Some two years later, those same neighbours were held up in their homes, robbed and gun

1 Cf. https://www.youtube. com/watch?v=TZASt2iVsns\&list=RDTZASt2iVsns\&t=22 (2018-11-21). 
butted. "Missing" is also autobiographical, and, yes, "Domestic" is autobiographical: "She goes down, gets up, | goes down again. His hands | feet deliver blows. | A house looks up | intermittently from the paper". What those poems may remind people is that poets can be considered historians or journalists, who write down what's happening now, but with a different approach, different tools.

AUTHORS In both of your poetry collections, you include either a tribute or allusion to the confessional poet Sylvia Plath. Many of your poems about your African, Chinese and Puerto Rican heritage ("likkle Coromantine, quarter Chinese/Puerto Rican gal”, "Granmammy"), your family history, your abandonment at a young age by your mother, the early death of your brother and your estrangement from your father, as well as poems about love relationships, seem to be closely based on your own autobiography and sometimes traumatic experiences. Do you feel that you are contributing to confessional poetry in the Jamaican or larger Caribbean context?

A.-M.L. There was no deliberate attempt to be confessional or contribute to that genre. I know that I want to write the reality I live in, not what I think Caribbean poetry should sound and feel like, something I think we poets may have been trapped into thinking we should do. I don't come from the rural part of Jamaica, so I can't write much about growing up in the 'country' and have all the rustic, rural scenery some people come to expect from 'Caribbean poetry'. Plus, I will not fake it. So yes, I may be contributing to that body of Caribbean poetry considered confessional, but as I see it, it's not only that. There is that, and there is my preoccupation with history and how it colours the present. For me, that past and present conversation, at times, also joins with the confessional.

I think my poetry style is fluid. My poetry has been described as lyric. I aim for truth, and since my locale is mainly Jamaica, most of the truth I see and discover is set in Jamaica. Writing is therapy, an important part of the healing process, since to write the poem, you have to process the actions, thoughts, emotions, motives, etc. You have to be on the inside of what you're writing about. Writing is my guttural release.

Would you classify Olive Senior as confessional? Perhaps I would. I love her work. I am drawn to Shara McCallum, Safiya Sinclair - even Colin Channer's book Providential can be considered confessional. I love Shivanee Ramlocan's work. Some of it can be considered confessional, but there is also that 'trick'. There is so much intimacy, that you feel it is autobiographical, but it is not necessarily. 



\section{Doe Songs: Letting the Landscape Speak Through the Truest Language, Poetry}

Ann-Margaret Lim

(Head, Public Relations \& Media Affairs Unit at Ministry of Foreign Affairs \& Foreign Trade, Jamaica)

ANN-MARGARET LIM Your book Doe Songs communicates to the world where you are in life. You are now a mother, a daughter, a visual artist, a poet, a wife and a very good friend to Shivanee Ramlochan. Based on what the poems relate, friend would be an understatement, you two are sisters in poetry.

Your poem "Reader Beware" in its entirety below is just one example of how your friendship shines through.

"Reader, Beware"

For Shivanee Ramlochan

The poet is a flambeau.

The poet is a cutlass, a spear whittled

from the tallest cedar, sharpened to a fine point.

The poet cuts you without you even knowing it.

Yes, you are hurt but my God, it is beautiful.

You are tumbling outside yourself in wet handfuls now.

Nothing will hold. A field of cedar springs up

in the yawning where you once stood.

Why are you in tears?

The poems are hard to read and it is even harder to breathe. Something burns the back of your eyes, tickles your throat. Someone offers you food, offers you wine but you have no stomach for it.

The poem wants to find you hungry, hollow trembling inside your good shoes.

You might already be gone. 
You might have left the reading some time ago, but can't really be sure.

Nothing looks familiar except the poet's red dress.

It holds you steady in the dark room like high pitch oil flame.

You leave the reading walking behind yourself

alone in the streetlight, dissolving in your own shadow.

Reader, trust me when I tell you:

only the haunting remains.

A.-M.L. How does this friendship with Shivanee influence your poetry? Do you both attend workshop together or workshop each other's poems?

DANIELLE BOODOO-FORTUNÉ I met Shivanee in 2009 at the Cropper Foundation Caribbean Writers' Residential Workshop. A group of us spent three weeks in Toco, Trinidad writing and being workshopped by Funso Aiyejina and Merle Hodge. The workshop resulted in lasting, meaningful friendships with the other writers, and most of us still keep in touch. With Shivanee, what started as a mutual admiration for each other's work evolved into a kind of recognition of something in each other, a deep kinship that has grown over the years. We don't workshop or even read each other's work before publication, which I think comes from an understanding that the work needs to be what it will be. I respect her writing immensely and prefer to come to it with open hands when she is ready for it to be seen. She's my writing partner, and my poem-sister.

A.-M.L. Your poetry embraces the phenomenal in things. For want of a better word, it embraces the 'magical' in things. It keeps that childlike wonder, wherein things have that unexplainable element that is magic-making. The very first poem "To Enter My Mother's House" tells us that this poet lives simultaneously in reality and that spiritual realm associated with it, but it does not seem to be Christianity; it seems older. Will you be brave enough to finally name the phrase that this writing and awareness represents, if we are to say we are rejecting the Anglo Saxon term "Magic Realism"?

\section{Excerpt from “To Enter My Mother's House”}

The Heron god created daughter

on the last day. Not knowing

what to do with the longing

left over from creation, he poured it

into her open mouth, still warm

and echoing with earth. 
To enter my mother's house

I must walk backward with

smoke in my mouth.

To pass through the keyhole

I must become a spout

of water, a single hair

from an ocelot's back.

I must go back thirty years

to recreate myself, carve

my face on the unburnt tip

of a match, strike my teeth

thrice against her name.

D.B.-F. In the late stages of writing the poems in Doe Songs, I realised that all along I had been trying to build a kind of cosmology, a body of belief comprised of bits and pieces of things I had gathered growing up in rural northeast Trinidad, domestic rituals, submerged family histories, forest magic, things like that. I don't think I can give this a name without trying too hard to hold it too tight, but the poems do come from a place of belief that the natural world is a repository of memory and spirit, and that all this can be accessed by letting the landscape speak, which is what I try to do in Doe Songs, in my own way. To "Enter My Mother's House" is a poem about memory, walking backward, gathering the pieces. It is also a poem about letting the spirit in things speak, in people, places, objects, creatures. It is actually one of the later poems in the collection, in terms of the chronology of writing. There are the poems I agonised over, edited for months, and then there are the poems that just happened... "To Enter" was one that just happened.

A.-M.L. As "Coronory 11" and other poems illustrate, you are able to make objects of body parts and humans. For example, your heart becomes an object that can be put on the windowsill, like American pie, left to cool. Do you think this skill comes from the visual artist? Is this what happens when you are both poet and visual artist, or am I clutching at straws?

"Coronary II"

After a while, the words mean nothing. What is muscle, is vein, is needle 
is better than worse than

What can you bear

to live / not live

with?

What is this body

if not a map of punctures,

a glossary of bruises

What is this heart, dull, spongy and hot waiting too long now, left on the windowsill, left to dry, left empty

left with nothing to do but tick?

D.B.-F. For me, visual art and writing feed each other in very important ways. They do operate on different wavelengths, and I try to give each practice its separate space when the need is there, but I do think that because I do both, I am always thinking very visually. Making body parts into objects is something I've been doing in my art of late as well, hipbones, ribs and hearts stand out on their own, become both body parts and sacred objects.

A.-M.L. Are you the doe in Doe Songs? Following up on your answer to question 1, did you see yourself as doe and also dove while growing up, as in the poem "Sombra"? Is this part of letting the spirit in things and, in some cases, the spirit in you speak? You may be a doe now and a dove later?

D.B.-F. I don't think I ever really thought of myself as the doe in terms of a straightforward relationship, but the doe is a symbol that kept coming back to me during the process of writing these poems. The doe is both wild and vulnerable, a symbol of the feminine and the feral. I started drawing upon childhood memories of seeing the carcasses of red brocket deer brought home by hunters, and their own children attempting to raise the wild and terrified orphaned fawn. The fawn would almost always lose its will to live completely. The hunter and the doe, the hunter's child and the fawn, are all bound together, such as in the three part poem "Doe". This struck me even then as part of the very complicated relationship between people and place. We are more wild than not. By hurting, we are also hurt. Everything is in balance, and has its own cycle. Growing up on the periphery of the forest, it's 


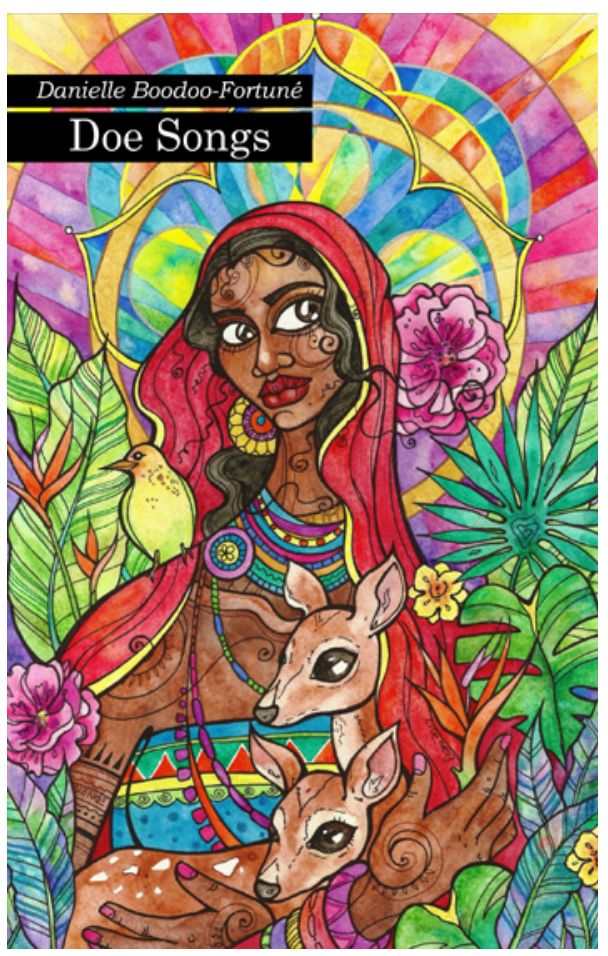

Figure 1. Cover of: Boodoo-Fortuné, Danielle. Does Songs. Leeds: Peepal Tree Press, 2018

something I've always known, and been truly fascinated by, especially now as I have become more aware of myself as mammal and mother. This is also true of the dove and all the other creatures in the book.

A.-M.L. In both "Sombra" and "To Enter My Mother's House" smoke is used effectively and to represent different things. Explain how it is used differently in both and what smoke represents for you. Also, is this part of the joy of poetry, where you explore different lives, strengths and characters of words?

D.B.-F. Smoke is a kind of veil between the 'real' world and the spiritual world. It's a marker of passage, or that a process is taking place, healing, birth, death or destruction. It is also ritual. Definitely this is part of the joy of poetry, the different lives of the word, and exploring the different dimensions and ways it affects the senses. "Sombra" explores that womb/shadow space between death and birth. I've actually never pointed this out before, but the title of the poem has its genesis in email correspondence with Loretta Collins Klobah, where, in a typo, she uses the Spanish word 'sombra' to refer to the womb, as 
I was pregnant at the time, and then adjusts it to English in a followup email. Upon first skimming the email, my first thought was that the word meant 'shadow'.

Excerpt from "Sombra"

If grief is bitter smoke,

the sting of ash

at the back of the throat,

then joy is a taste of honey,

warm rainwater, the quickening

of sugar on the tongue.

My mourning dove's heart

must find a way

to hold both.

Oh grandfather,

will you know each other

In the way that roots reach out

dreaming in the earth's deep sleep,

or meet in the middle of the bridge,

the endless, the sombra, resting space

between shadows?

A.-M.L. You actually seem to play with fire, smoke and ashes a lot. In the first poem "To Enter My Mother's House", you have to walk backward with smoke in your mouth. In "On Being Burnt", where it seems heartbreak is represented by fire and being burnt, nothing is left from your eaten self, but smoke. In "Praise Song in Carbon", there are arteries of fire under each taut surface, and all bodies burn. It seems, fire for you is really consuming. It harms; it cleanses, it seems to be in you. I could be wrong though. What exactly is fire for you in your world view? I share the third to last stanza from "A Poem on the World's Last Night" below.

It is late, and I am tired

of sweeping brimstone

beneath the bed,

pretending fires don't start

in quiet rooms.

D.B.-F. Fire is both ending and beginning, with smoke in the middle as a symbol of transition. Destruction, but newness, as in many religious 


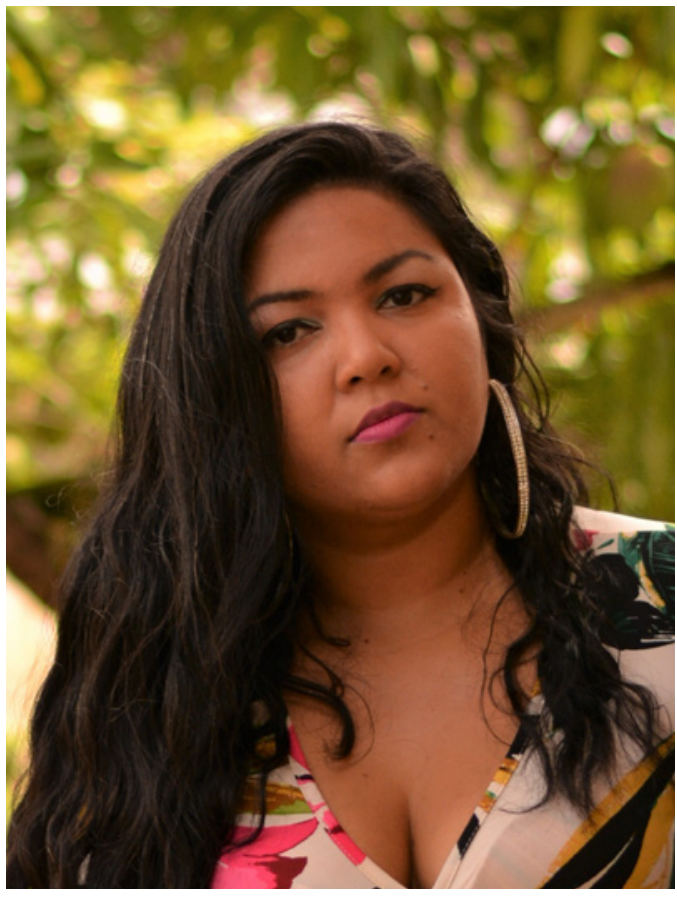

Figure 2. Danielle Boodoo-Fortuné, photo credit: Reynold Kevin Hacksaw, 2018

beliefs, and also in agricultural practice. It is also power, and connection to spirit.

A.-M.L. "Tangerines", set in Venezuela has special meaning for me. In that poem you are somewhat stranded in a strange place in Venezuela, and your mind travels to your mother. You see a tangerine vendor, and you think of your mother's crop of 'barren white citrus' the year before. You end the poem by asking your mother to gather her maps and run. We were both in Venezuela participating in the 12th World Poetry Festival that year, but I was not on that bus with you travelling to that location. It is also special for me, because I thought of my mother constantly in Venezuela, hoping I would find her there, the last place I was told she was. Like you, I wrote to my mother whilst in Venezuela.

What does traveling, particularly with fellow creatives, do for your poetry? Also how does that space away from your mother and other loved ones affect or influence your work?

D.B.-F. Yes, we were both in Venezuela that year! I know it was very special for you as well, though we were in different places. I travelled to Yaracuy where I was immediately attracted to the figure of Maria 
Lionza and the influence of the wild, fierce divine feminine that came through so strongly in the landscape and in the whole energy of the place. Returning to Caracas we actually missed the plane, and took a series of buses to get back, two of which broke down along the way. My guide's mother kept calling her while on the bus, and checking in on her, and checking in on me as well. While in Yaracuy, especially while around Sorte Mountain, I kept thinking of daughters and mothers and grandmothers, the powerful chain of birth and ancestry, how we were tucked away in our grandmother's hipbones from birth... the things we pass on, the things that go unhealed for generations. When I came back home from that short trip, I wrote intensely for months. It gave me a little breathing room to think, and infused my whole process with new life. We find the most familiar things in the strangest places, it seems.

A.-M.L. To say Emily Dickinson didn't travel much is an understatement, as she was indeed a homebody, yet she wrote copiously and excellent poems at that. Contrasting with her though, we have the diplomat Pablo Neruda, and others like him who travel a lot, including you, for example. Do we need to see the world to write?

D.B.-F. I don't think it affects whether we write, but perhaps how we write. The writing of women who didn't travel and had no choice in the matter is tremendously important, for instance. Motherhood has made it more difficult to commit to writing, but I've learned to write anywhere, if I need to. There are poems I wrote in my brief time in Venezuela or England, but there are also poems I wrote in the difficult final month of pregnancy, or housebound with my sleepless newborn. It's funny, the more intimate poems seem to be the ones written away from home. Some of the more ambitious ones were written in their first draft on my phone in the car, then refined afterwards.

A.-M.L. God, I am tired of being young

Upon this dead earth.

I am so tired of being

someone's daughter.

As you know the above is the last stanza from your poem "Letters from New Grace". There seems to be communication with your mother throughout the book. Her presence is a constant, and she is referenced often. For me she is a haunting. How would you say your mother's life and your life with her have influenced Danielle, the visual artist and poet?

D.B.-F. I am writing to my mother in many of my poems, perhaps my entire book. But I am also filling the gaps, raising different stories from 
the earth of my memory and hers, telling the stories my grandmother couldn't bear to tell, writing letters to the daughter I might have had, mining our collective memories for the whole of the things we cannot speak about. There is much a mother does not say to a daughter, yet still the daughter knows, in her very bones. There is a lot that women do not say. A good deal of my work is memory, and this starts with my mother and grandmother and their stories. It is also my memory.

A.-M.L. What for you, is the purpose of poetry?

D.B.-F. This is very hard! I tell people often that poetry saved my life, and it probably sounds quite ridiculous, but it's true. I found Lorna Goodison when I was younger, along with Jennifer Rahim, Olive Senior and many other phenomenal poets. It felt like a light had been switched on, and so much became illuminated. There was suddenly a way... many ways... to say things I'd always wanted to say. I still feel like this. Last week found me reading Ocean Vuong's Night Sky With Exit Wounds and crying my heart out in the corner while at an event with family members, not from sadness, but familiarity and recognition and the sheer power and beauty of it. Poetry is a language that feels truer than any other, for me.

A.-M.L. In addition to Shivanee Ramlochan's, whose poetry moves or inspires you to write?

D.B.-F. My first influences were Jennifer Rahim, Lorna Goodison, Olive Senior... so many phenomenal writers, but these remain my touchstones. Among my current favourites are Loretta Collins Klobah, Richard Georges, Andre Bagoo and Shara McCallum. Also currently in love with Warsan Shire, Ocean Vuong, Sarah Howe and quite a few others. I also adore the poetry of Louise Erdrich, who is primarily a novelist, but who writes about motherhood so beautifully and originally.

A.-M.L. The last question asks you to tell someone who has never read your work, what Doe Songs is about, giving an idea of the range of poems and techniques, styles and forms employed.

D.B.-F. Doe Songs is about memory, myth, magic and landscape. These poems are about women, about birth, motherhood, love and loss, but truly they are also about everything. I experiment with form, shape and music here, trying to feel out the inner sound of the poems, the rhythms, incantations and sense of them. It's a book that has been nine years in the making, and one I am deeply proud of. 



\title{
Ngũgĩ wa Thiong'o: an Interview
}

\author{
Giulia Barison, Beatrice Carmello, Asmaa El Hansali, Dalia Pratali Maffei
}

(International College, Università Ca' Foscari Venezia, Italia)

Waterlines (waterlinesproject.com) is a residency programme jointly organized by the International College of Ca' Foscari University of Venice, Venice Foundation and San Servolo Metropolitan Services of Venice. It combines writing with other artistic disciplines to reaffirm the role of Venice as a place of artistic and cultural production. In April 2018 the programme had the privilege of hosting one of the most prominent international intellectuals, Ngũgĩ wa Thiong'o, who lived in Venice for three weeks, participated in the literary festival "Incroci di civiltà" and two other public meetings with local artist Moulaye Niang 'Muranero'. On 11th April, Ngũgĩ was interviewed by students from the International College and Radio Ca' Foscari, the university web radio, on San Servolo island.

Ngũgĩ wa Thiong'o was born in Kenya in 1938. A many-sided intellectual, he is a novelist, essayist, playwright, journalist, editor, academic, and social activist. Imprisoned and exiled in the '70s because of his opposition to the regime, he has taught in many American universities, including Yale and New York University, and is currently Distinguished Professor of English and Comparative Literature at the University of California, Irvine. A UCI Medalist, Ngũgĩ is recipient of twelve Honorary Doctorates from universities all over the world and has been nominated for the Nobel prize many times. Following his well-known decision to abandon the English language and start writing his books in his mother tongue, Kikuyi, he has become an advocate of linguistic diversity in Africa and all over the world. His works, several of which have also been translated into Italian, include the essays Decolonising the Mind: The Politics of Language in African Literature (1986), Moving the Centre: The Struggle for Cultural Freedom (1993), and Secure the Base: Making Africa Visible in the Globe (2016), the memoir Dreams in a Time of War: A Childhood Memoir (2010), and the works of fiction A Grain of Wheat (1967), Petals of Blood (1977) and Wizard of the Crow (2006). The outcome of his Waterlines residency is the collection Venice Poems, written in Kikuyu and translated into English by the author, which were published in December 2018 by Damocle Edizioni with an Italian translation by Barbara Del Mercato and illustrations by Daniela Murgia. 
AUTHORS Thank you for meeting us. Our first question is: during your year in prison, you wrote your first novel in Kikuyu, your native Kenyan language. What did it mean to you then, to write in Kikuyu, and what does it mean now? Is abandoning English more of a sacrifice or a liberation to you?

NGŨGĨ WA THIONG It is definitely a liberation, because I think it is normal for people to always start from what's around them and then to add to the knowledge they have already acquired, so to where they come from. That's the normal cognitive process. You add what you know, you will be adding, and so on. And the more you know, the more you add, then you also get to know more, about the original space. So it is direct: you begin with the way you are, you're going to add and the further you go, the faster you see the connection between the way you are and wherever you are going. And then, the new experience makes you look at your own space, maybe with different eyes, and so on: it's mutual enrichment. That's the normal cognitive process.

Colonialism, instead, subverts that process: it seems you do not start with where you are, but with where you are not. And you can see how this works in relation to languages. It is the most normal thing, for all human communities, no matter where or who is, to start with the language - what you call mother tongue or the language of the culture in which you have grown up. And then you go and connecting that to other languages, and another language helps, a little bit more, you can even understand your own language better, by seeing it in relation to other languages. And your own knowledge of your own language can actually be put in a better position to understand other languages and other cultures. But colonialism subverts that, saying "no, if you are colonised, knowledge begins with this centre", the colony - in our case, metropolis or Europe and so on. This is obviously very negative to the colonised, because it is a sort of alienism from themselves. In the end, you identify more with "the language of glory", its history and the people who created that language. And by the same token, then, you establish a distance between your own language and the history and the culture carried by that language and by definition also from the people who created that language, so also your mother and your father and your grandparents and so on, so it is a terrible thing to be in that kind of position.

So for me writing in Kikuyu equals liberation: when I discovered my mother tongue, I reconnected with the language. But the difficulty I had, quite frankly, was to put it in a context. I was put in prison, because I was writing a play in Kikuyu language, in a place called Cameredo, Cameredo Community Educational Cultural Centre. It means to work with peasants, workers and so on. The play was held in November 1977 and the following month, December 1977 at 
midnight, I was put in a maximum-security prison. The importance of that was two things: one, at the end I asked myself more profoundly, why would an African government, postcolonial government, put me in prison for writing in an African language? Because I am aware that it was not just the language basis, but also what the language was saying and the fact that it could have been understood by everybody, or at least the community I came from. So part of that thinking was looking at the role of language in a colonial process no long as an intellectual or an academic exercise, but really as something which affected and impacted me. I remember that at the time I was put in prison I was not only the author of four novels in English (Weep Not, Child, The River Between, A Grain of Wheat and Petals of Blo$o d)$, I was the author of quite a few plays in English. Nothing much would have happened to me for writing those kind of novels, even though they were very critical of the postcolonial space and so on. So, that question of 'why' made me examine the role of language in a colonizational process and by the definition in the process of domination, in systems of domination. These thoughts I had when I was in prison in December 1978, would form, years to years later, in 1984, the basis of my work Decolonising the Mind. Those thoughts you find in Decolonising the Mind actually began systematically in prison.

The other thing which was very important for me was that, as a result of that thinking, making the decision now to write in Kikuyu, in prison. My argument was like this, if I can remember rightly: "I must write in the language which was the basis of my incarceration". It is kind of automatic: "you bring me here, so now I will do it, I'll do everything under your eyes". It is also kind of subversive, but this was very important for me in prison: to resist, often not in a physical sense, but in a mental sense, in a spiritual sense. Because by doing so I was affirming my right to my language. That's one thing, but something else also happened, which is psychological. Generally, the élites from formerly colonised places carry a big mountain and they don't always realise it. I realised I had it in prison. For many years I thought: "is it possible to write a novel in Kikuyu?" So you carry this body - I call it a mountain - which prevents you from seeing the obvious. "Is it logical? What's more smooth or more fluent in English or French or whatever it is? But my language, I can't even read it." So the sudden liberation of that mountain I carried made me think "Oh, my God: did I carry this burden all my life? Because, yeah, I can do it". I wrote the novel in Kikuyu in prison on the toilet paper, it is called Devil on a Cross. So for me that was a big act of liberation on a number of levels, first because I was in prison and I was resisting, I was asserting my right to freedom. But in another sense I was also liberating myself from the burden I had been carrying all my life. 
So, I use the religious tones: I saw the light in prison and I would never want to go back to the previous situation of darkness.

AUTHORS Don't you fear that writing in Kikuyu could mean that less people can read you and so you have a less positive impact in society? A second question: I read that you use a very interesting expression: "the most dangerous weapon that colonialists have is the cultural bomb"; can you elaborate on this expression and explain what you mean by "cultural bomb"?

N.W.T. About the second question: I use this image in Decolonising the Mind, because you can't really think of it, when people or anybody alienates you from your own language, it's a kind of alienation really from many things. First, from the knowledge carried by that language - so the knowledge of the area, the trees, the rivers, whatever, gone. Second, from the history of the community that made that language, gone. For a Language it takes many years, hundreds of years to be where it is. So, that external power completely whips out, like a hurricane, like a bomb that comes and clears everything that was there and it tries to plant something else on this terrain or contaminated ground, that's how I call it.

About the first question, the fact that if you write in an African language you would not be available to the others. If you meet an Icelandic writer, for instance, and they write in Icelandic, you don't ask them "why are you writing in Icelandic?". That's ridiculous, if you come to think of it. So if I am English and I write in English, people don't ask me "What do you mean? You write in English? Why? Why don't you write in Chinese? Or in Zulu?". So, the question itself arose and it arose also in Africa by the way, but it's the wrong question. Because you write in whatever language you can. So firstly, your native language, and then it can be translated or not. When you write a novel in English it doesn't mean that all the speakers in the world are English, right? And they are billionaires by now - I am teaching in English now, defending my salary in universities. In reality, and I can tell you for sure, even a Nigerian writer like Chinua Achebe, even when they write in English, they still sell more books in Nigeria than they do in the rest of the world. I sell more books in Kenya myself, because Kenya is still my base, even after all these years. And I sell more books in Africa than anywhere else. So, it does not mean that if you write in English all the English speakers will be running to buy your book. There is no correlation between writing in English and having your book. It's just something in the mind. The Yoruba people, for instance, there are about forty million in Nigeria alone. In Denmark, there are 4 million of them. Why is it that 4 million people can sustain a literature and 40 million people can not? Or in Iceland, always 
quoted by media, people are at least about 10 million and there are several Icelandic nations.

So, to answer your question: firstly, it's not important that you write in the world. I mean, that's fine and I am glad about what I hear and when I meet people around the world, but you don't have to write with the idea that "I am writing this, so an Italian reader can read me". It should be the other way around: "I am writing this so that the community around me will read me" and then, as the by-product, an Italian reader is able to access my work. That's a plus and a good thing, right? And then we have a historical parallel in Dante. What he says in Tuscan vernacular and this language became Italian. Some of his friends were telling him "Dante, if you write in Italian you're going to lose immortality, you will be gone. Write in Latin". The part I really like and I think is so beautiful is that Dante replies to him in perfect Latin to say "look, my vernacular is obvious to you, and it's full of muck and when you guys are very busy in Latin let me try and I write as much as I can from this 'island' full of muck". Now, those poets who were Christian men are not remembered today, but Dante is, everywhere. You deny it?

So that's what it means. The question of language dominance has nothing to do with language space. But it reflects an echo of economic, political and cultural inequalities of power. It has nothing to do with languages. Because when languages meet they can borrow from each other and they can relate. But when they meet in an unequal power relationship - which is what happens with my language, for instance - it has nothing to do with the languages at all. There's no language in the world that is more than another language, there are languages reflecting an unequal power in that society. That's what history is. English, inherently, is not more of a language than French, or French more of a language than Chinese, or Chinese more of a language than Swahili. Each language has its own unique musicality, even dialects of a language, like different musical instruments. So, in music we don't say that one instrument is better than another and to ban all of the other instruments except, for instance, the piano. But we say it with languages! They are telling us, "you get one instrument" and all its thoughts and feelings to express through that sound-system. The sound of violin, guitar, piano, these instruments of every community produce together this richness we call music. Wasn't that your question?

AUTHORS We are reminded that Dante wrote the Divina Commedia in Italian, but when he wanted to reach more people he actually wrote other works in Latin. If your final aim is to reach also Western people, isn't it better to write in English? 
N.W.T. Yeah but If I want to read Russian literature, I don't ask Russian writers to write in Kikuyu if I want to read them. There are two possibilities: I can learn Russian and therefore read the work in original, or I can still try to access the word and the tradition in translation. When you want to know more, to become an expert, then you learn Russian. In fact, if you want to reach more people then write in your language, because what people relate to in literature or arts generally is that element of Identification with whatever is been depicted. If someone speaks to you, even in translation, if the oral stories speak to me, the sense of speaking to me can only come from the self. So, the question of the self and peculiarity is very very important in all art. So, my own view that African people should not worry very much about reaching Chinese readers and so on - what language Chinese readers want to learn they can learn it, or they can wait for a book to be translated into Chinese.

What I'd like to say, what Dante says, however, is that sometimes it's a matter of necessity. All my novels and memoirs and drama and fiction now I write in Kikuyu, all my poetry. But I am also in a polemic war also, about language issues. What I am trying to argue and what Dante did, writing in Latin to his friend to show him "I can do it", is that I am making a conscious choice. And for me this is very important. In an ideal world, there's absolutely nothing wrong for an individual writer to write in a different language. You know Conrad right? Polish origins, Polish and French are his primary languages. And at 17 years old he learns English and then he writes this incredible novel in English and that's all right. But it's not that all Polish intellectuals write in English, it's an exception. However, if the whole Italian intellectual community brings their product in Chinese and they call it Italian literature, there's something a bit odd about that. Because Italian writers write in Italian. So, if there is an exception of one or two, that's fine and even interesting. But in Africa, a former colony, all of the intellectual production is in languages other than those of the countries people come from, that's the problem. If it's a question of one or two planning to write in whatever language they want to, that's very interesting. But there's something wrong when a whole intellectual community of inner group becomes captives of another language and culture.

AUTHORS There is a colonial heritage that tends to impose a tribal vision of the African people. In your opinion, can the term "tribe" be considered discriminatory, since the idea itself of tribe is produced by a colonialist vision of Africa?

N.W.T. Yes, it is. I'm fighting against this all the time in Africa: when someone says "I come from this tribe", I reply "Oh my God. What are 
you saying about your people?". Let's start with a few facts: Icelandic people are a quarter of a million. Yoruba people are forty million and you call them a tribe. Icelandic people, a quarter of a million, are a nation or a nationality of people, while forty million are an ethnic group or a tribe. It does not compute, even mentally, you can see the absurdity. When we talk generally about, let's say, the English, we call them the English or the English people, the Italian or the Italian people, Chinese or the Chinese people. But when we talk of Africa the word 'people' is thrown out of the window. Tribe. There is always a distinction between people. Everybody is 'people' except in Africa and some other places like Native American, those are tribes. But it becomes serious where people have internalised this and begin to call themselves like this and this is part of a negativity that will be used against them. This girl in front of me, that comes from Burkina Faso, she knows so many languages and I was so curious: she speaks Italian, English, French, Portuguese, she's learning Chinese, but especially she knows a Burkina Faso language, Moré. I was so impressed. That's very good. Because in reality, that's the way I put it everywhere, if you know all the languages of the world and you don't know your mother tongue or the language of your culture, this is actually enslavement. If you know your mother tongue or the language of your culture and all the other languages in the world there's empowerment, as opposed to enslavement.

So, we were speaking about tribe. Tribe is a negative connotation. And even when I was in India I was talking with a community there and they called themselves a tribe, I asked "why do you have to call yourself a tribe, so they call you like this? You are people, like other people are, they are names you call yourself, I am Italian, that's fine but they don't do it in Africa, they don't say "I am a tribesman" all the time.

AUTHORS Another question concerning language: you said that if you don't know your mother tongue or the language of your ancestors, this is a kind of enslavement. What about the African countries, like the North African countries or like Egypt, Sudan, Tunisia, Lybia, Algeria that are still undergoing waves of arabisation that have led to the loss of some of their native languages. Do you consider it as also a kind of enslavement?

N.W.T. Wait, this is a bit complex. Because from what I understand, now Arabic is spoken by almost everybody.

AUTHORS Yes, Tunisia and Egypt are almost fully Arabophone right now. In Algeria and Morocco there are still significant Amazigh communities. But officially speaking, Arabic is used. 
N.W.T. So, let me turn to the case of Arabic and Amazigh. These are colonial troubles, by the way, especially in Algeria, we all followed that war and I remember that the question of the Arabic language was very important in the struggle against the French, because it was a symbol of fighting, even in a linguistic and cultural way. But now, within Algerians and Moroccan, the Amazigh language is marginalised by Arabic: it's as if we reproduce the whole hierarchical relationship back into our own communities. So, Arabic is spoken a lot by people in Egypt, while Arabic marginalises the native African or the languages which are native to that area, like Amazigh - that's a good example - and others. In the end, for Amazigh people it's enslavement: they abandoned their languages and they speak only Arabic. Liberation would be like this: speaking Amazigh, then Arabic, as the language of power, then maybe French, English or whatever. That's empowerment.

AUTHORS What about the generations that do not speak Amazigh?

N.W.T. They have to start with whatever they have: in Africa, in Kenya, there are children who were brought up by their parents without any knowledge of their mother tongue. So, their parents have consciously put them in a little prison. But when you are put on a prison, you break out, not physically. How do you break out? Not by saying I got this language, Arabic or English, for instance. But if I have to make one more choice, what language do I add? I would add the language they led me to abandon. So that way I am using the power of the language that I have, that is now my mother tongue, Arabic or French, it doesn't matter, and I connect the language of the community. That's power.

AUTHORS Another question concerns the psychological factor attached to the mother tongue. What is the importance of using one's native language with its four dimensions: the social one, the psychological one, the issue of preservation of the cultural heritage and that of rebellion against colonisation?

N.W.T. The problem we have, and it's a good problem to have, is this: all languages have their own unique musicality. So Arabic has its musicality and French as well. And in themselves they produce great literatures and so on which I want to read, you want to read, everyone wants to read and so on. The problem, as I said earlier, is when we say that the musicality of a specific language is the only musicality possible or the ideal musicality. The musicality is something like a loan, you know. To make it very clear: if you are born a speaker of a given language, even though it is an acquired language as English or Arabic, you can not say "ah I abandon it". As an individual this time, if you have to add another language, that would be the language of the community. 
Now we are talking about communities, not about individuals: I think there will be a gorgeous struggle within Africa and outside Africa for a recognition of multilingualism as an equally valid basis for nation, development and so on, so that we fight to change the policies. You see, in Morocco or Algeria there is nothing wrong with Arabic: what's wrong is not 'Arabicness', it is its relation to Amazigh. Amazigh can have a very mutually and enriching relation with Arabic and even French, to be a productive and creative relationship. Even when they say Amazigh must be down there and Arabic or French or another language becomes the 'graveyard' of Amazigh. This is what we really have to change. So, there is a distinction with individual choice: if you are born here in Italy, for instance, Italian is your mother tongue, but if you want to connect to another language and you learn it, this is a conscious choice and, to me, a path of empowerment.

AUTHORS With all the scars left by colonialism and the problems of the tribal visions, would it be possible to envision a united Africa, like a federal community, along the lines of Europe or the United States?

N.W.T. I believe in Africa's unity, without making a distinction between Africa and North Africa. I do not accept Sahara as a division. I do not accept the idea that there is somehow an Africa that is not a proper Africa; a European Africa, and that there is an Africa that is subSaharan, and a proper Africa. This is a fiction that was passed in the 19th century and was highly articulated by the German philosopher Hegel. They saw a given civilisation that lasted over than three thousand years with an incredible work in architecture, art, astronomy, mathematics and more. The system of government in the States, although embodied in the Pharaonic society, they cannot credit it to Africa. They say there is a 'proper Africa', the South of the Sahara, and a Mediterranean or a European Africa which is North of the Sahara. They say that the northern part is good because it is European, whereas the 'proper Africa' is inhabited by savages. Those stereotypes are internalised by Africans themselves; they internalised negativity about themselves, their countries, history, people and continent, to the point that they come to see themselves and the related communities through the eyes of the colonizers. What is the responsibility of Africa on Africa? This is exactly what I talk about in Decolonising the Mind.

AUTHORS A question about gender and colonialism: could we have, potentially, a book titled The History of Kenya Written by its Women? Are there language varieties spoken by women?

N.W.T. The question of the culture of language differentiation within African languages is very important. Those languages per se have 
nothing inherently more democratic in them. Languages reflect other conditions, economic and political. So, if there is any kind of gender inequality or any other inequality, the author will reflect that through the language. And the dominant group - be it males or social wealth groups - will always try to use the language in such a way that the only usage of that language in the world will be through their eyes. I address this issue in my novel The Wizard of the Crow. One of the characters in the novel, she is a leader of a revolutionary movement. Nawira is a name of a woman, and it also means 'walker', and she is the leader of this movement. Nawira talks about all of the issues of women, be it class, gender or colour, which are all connected issues that have to be addressed together. What Nawira says is that the liberation of the woman walker would actually mean the liberation of all women. So, we say that all women can identify in a way or another with that woman walker, even a queen of a country as a woman whose economic situation is better can find a connection with that woman walker. The importance of all of these factors lies in the way they relate to one another. In fact, I can go further and give an example that I mentioned in my fable The Upright Revolution in which the body and the way it is structured. You look at the head, the top, a mixture of reason and coolness. And the body, arms and heart, the productive system are a mixture emotion and production. Production is very important, and one of the most primary productions is the one made by women who carry a human being for nine months. What I find interesting in all societies is that people who produce are always marginalised by people in power. The walking people, the ones who make life possible, and make all things be. So, the woman walker's liberation is a liberation of all women.

AUTHORS Do you think that the word 'ethnicity' is a colonial heritage like the word 'tribe'?

N.W.T. They of course do not carry the same connotation, although ethnicity has a colour connotation. The idea of tribe with all of the colonial connotations it carries, infers a kind of fear in people. That is why you can fear an Afghan leader described as Yoruba tribesman. When it comes to the analysis of African politics, instead of looking at the situation's complexity, in the West, particularly the press, they just check which community a person comes from and journalists do not try to find the cultural, political, philosophical and ideological differences between the so-called 'tribes', they instead say, for instance, "this comes from tribe Morocco and the other from tribe Italy". Therefore, the problem is reduced to the fact that one is Moroccan and the other is Italian. It is a very simplistic way of looking at African problems. The tribal concept has also the notion of not having arrived yet at the 
model. The word tribe for me has a very negative connotation. My own solution is simple: we can call the people by the name they call themselves. If a person calls him/herself Amazigh, then we can call him/her Amazigh, just like we would call a person English or Italian.

AUTHORS How can young diasporic people who live in Italy, who came here when they were kids and are no longer connected to their root cultures, reconnect to them?

N.W.T. It always starts with who you are, even if you are born here in Rome. It is always about the choices you make. You can choose to reconnect with your language or forget it. If I am here in Italy I will try to learn as much Italian as possible. But then, it is about the other choices you make. You can always learn your own language and add it to Italian, and it is the most logically organic thing to do. You do not replace Italian, you add a language to Italian. This will help you to contribute to your African language from the very fact that you have an experience from the outside. The problem I see with diaspora is that the way the culture in which we grow, the dominant culture, looks at Africa is very negative and that one actually can internalise it. To me, using a religious terminology, diaspora youth can add something positive to their native cultures by looking at the story of Moses, who is brought up in Egypt: he later uses that knowledge and upbringing to help his relatives to find their way to Palestine. So, everybody has their 'Palestine', so to speak. So, the knowledge we have here, we should not see it in a negative sense unless we internalise the negativity that one culture carries about the other. But if we can use that knowledge, it should be extremely liberating. 

Recensioni | Reviews | Comptes rendus 



\title{
Parisot, Yolaine (2018). Regards littéraires haïtiens. Cristallisations de la fiction-monde. Paris : Classiques Garnier, 385 pp. Coll. Bibliothèques francophones
}

\author{
Alessia Vignoli \\ (Uniwersytet Warszawski, Polska)
}

Relire la fiction haïtienne contemporaine dans une perspective qui prend en considération les arts visuels et les autres systèmes linguistiques : tel est l'objectif de Yolaine Parisot, professeure en littératures francophones et comparées à l'université Paris-Est Creteil, dans son dernier ouvrage, Regards littéraires haïtiens. Cristallisations de la fiction-monde. Dans son étude de cette littérature née d'une révolution d'esclaves déclenchée - croiton - par une cérémonie vodou, universellement reconnue pour son « inépuisable vitalité »(15), Parisot s'arrête sur un corpus sélectionné d'ouvrages dans le but de «fonder historiquement la lecture de la fiction haïtienne contemporaine comme phénoménologie de l'art, du politique, de soi » (27), comme elle le souligne dans l'introduction. À l'aide d'un concept d'inspiration glissantienne comme celui de « fiction-monde », l'auteure se propose de tracer le parcours de la littérature haïtienne en se focalisant sur ses relations avec les autres arts, en particulier la peinture et le cinéma.

Le volume est divisé en trois parties. La première partie, "Lécole haïtienne du regard », s'ouvre par une première section, "Constellations artistiques », où sont d'abord mentionnées certaines parmi les nombreuses revues "qui ont contribué à écrire l'histoire de la littérature haïtienne » (37) : La Revue indigène, Conjonction, Chemins critiques et Boutures. À partir de l'évocation des mouvements nés dans l'entre-deux guerres « que produisirent l'essor de l'ethnologie et la redéfinition subséquente du concept de culture » (38) - la Harlem Renaissance, l'Indigénisme haïtien, les indigénismes de l'Amérique hispanique et la Négritude - Parisot s'arrête sur une figure centrale qui a ouvert Haïti au monde : Jacques Roumain. Dans sa présentation de la «constellation Jacques Roumain » (38), l'auteure prend en considération le roman paysan Gouverneurs de la rosée (1944) et la mise en scène du regard dans le récit, où s'exprime la « poétique oculaire » (57) élaborée par le poète et romancier indigéniste. 
Sont ensuite évoquées les conférences données à Port-au-Prince en 1943 par Alain Locke, théoricien de la Harlem Renaissance, et leurs résonances dans le roman de Yanick Lahens Dans la maison du père (2000).

Dans cet ouvrage la romancière, qui situe l'action dans les années ' 40 , réalise une « mise en fiction des débats ouverts, en Haïti, par les conférences de Locke » (61), visible en particulier dans la vocation de la protagoniste pour les danses vodou. En ce qui concerne la représentation de la peinture dite 'naïve', présente aussi dans le roman de Lahens, Parisot mentionne Dany Laferrière et son ouvrage Je suis un écrivain japonais, où l'auteur évoque les peintres primitifs, Compère Général Soleil (1955) de Jacques-Stephen Alexis et l'œuvre romanesque de Jean Métellus - Jacmel au crépuscule (1981), Une eau-forte (1983) et Louis Vortex (1992) - où l'écrivain intègre la peinture naïve haïtienne et transpose le pittoresque dans ses descriptions.

Dans la section suivante de la première partie, " Propositions historiques et philosophiques », l'auteure s'interroge sur la place d'Haïti et de sa littérature dans le domaine des études postcoloniales, en précisant que la diffusion d'ouvrages d'origine haïtienne dans d'autres champs littéraires, en particulier le champ littéraire français et celui d'Amérique du Nord, à partir du XXIe siècle, a contribué de manière décisive à l'augmentation d'intérêt à l'égard d'Haïti, dont la littérature est de plus en plus étudiée par la communauté scientifique internationale. Parisot prend ensuite en considération l'œuvre de René Depestre et de Dany Laferrière pour montrer que les érotismes des deux auteurs sont liés à la « réactualisation de la dialectique hégélienne dans les contextes de la décolonisation, puis de la mondialisation » (92). Après un survol de la dynamique du regard et de la dichotomie des genres dans Amour, Colère et Folie (1968) de Marie VieuxChauvet et Les Chemins de Loco-Miroir (1990) de Lilas Desquiron, l'auteure aborde l'œuvre de Jacques-Stephen Alexis pour étudier les relations entre connaissance et perception dans Les arbres musiciens (1957).

La première partie se termine par une étude croisée du réalisme magique et du réalisme merveilleux haïtien, qui se prolonge au début de la deuxième partie, « Pour une fiction-monde ». Dans la section appelée « Le répertoire et le laboratoire ", l'auteure analyse L'Espace d'un cillement (1959) de Jacques-Stephen Alexis et l'« autobiographie américaine » de Dany Laferrière, afin de montrer l'héritage du réalisme merveilleux dans l'œuvre de Laferrière, " écrivain primitif » (155). Parisot présente ensuite le Spiralisme, né dans la moitié des années '60 des réflexions de Frankétienne, René Philoctète et Jean-Claude Fignolé, et sa théorie de la représentation. Ce mouvement symbolise un nœud central dans l'histoire de la littérature haïtienne car il « développe une poétique oculaire du doute, du soupçon » (162) et définit une « littérature de la perception » (176) où « l'allégorie, le fantastique, le grotesque, la parodie, les potentialités du langage et l'humour s'intègrent à une distanciation visant à redonner au sujet la place qu'il a perdue dans le monde » (175). 
Pour ce qui est des relations entre l'école haïtienne du regard et le cinéma, l'œuvre de Dany Laferrière est présentée comme celle qui exploite au mieux le potentiel médiatique de la «cinématique du regard » (176) qui se manifeste dans le roman haïtien à partir de Gouverneurs de la rosée de Jacques Roumain. De Laferrière, qui intègre dans ses réflexions la culture pop et celle médiatique, à la lodyans, genre populaire « qui relève à la fois du théâtre et du récit » (180) et définie comme « art de la miniature » (183) par Georges Anglade qui l'a théorisée, les références intermédiales dans la littérature haïtienne continuent d'être exploitées.

La section suivante, « La chair du monde au miroir du roman », prend en examen trois modèles du « roman de la dictature » : Amour, Colère et Folie (1968) de Marie Vieux-Chauvet, Le Mât de cocagne (1979) de René Depestre et l'œuvre d'Émile Ollivier (Mère solitude, 1983 ; Passages, 1991 ; Mille Eaux, 1999). Appartenant à la même génération d'Ollivier, le romancier Gérard Étienne avec Vous n'êtes pas seul (2001) revient sur la dictature duvalieriste dans une époque tardive et se rapproche ainsi d'un corpus féminin dont font partie entre autres The Dew Breaker (2004) d'Edwidge Danticat, Saisons sauvages (2010) de Kettly Mars et La Mémoire aux abois (2010) d'Évelyne Trouillot. Parisot mène ensuite une réflexion sur le carnaval et la zombification dans la littérature haïtienne en repérant un «tournant dans la mimesis haïtienne » (213) dans Les Urnes scellées (1995) d'Émile Ollivier, pour l'exploration à l'intérieur du récit du topos descriptif du cimetière, que l'on retrouve dans des romans récents de Lyonel Trouillot et Louis-Philippe Dalembert, parmi d'autres.

La troisième partie, «Archéologies de soi », évoque d'abord, dans la section «Les cristallisations de l'histoire immédiate », l'interrogation sur le pouvoir des arts élaborée par Lyonel Trouillot dans deux de ses romans : L'Amour avant que j'oublie (2007) et La Belle amour humaine (2011). Parisot propose ensuite une réflexion sur la transposition de l'histoire immédiate en littérature en utilisant le concept de « littératures de l'urgence » (236), emprunté aux littératures de l'Amérique du Sud. Lauteure distingue deux nouveaux corpus qui ont émergé récemment : « un corpus qui revisite la période dictatoriale au prisme de l'histoire immédiate et un corpus né du tremblement de terre de janvier 2010 » (237). À l'intérieur de ce vaste corpus, la critique prend en considération quelques fictions politiques comme Bicentenaire (2004) de Lyonel Trouillot, La Couleur de l'aube (2008) de Yanick Lahens, La Mémoire aux abois (2010) d’Évelyne Trouillot et Un alligator nommé Rosa (2007) de Marie-Célie Agnant. Après une analyse de la lodyans comme forme qui « offre un modèle de représentation de l'urgence prêt à l'emploi » (252), l'auteure dresse un panorama de la littérature de l'après-séisme en choisissant quelques romans pour illustrer la variété de ce corpus, où l'éthos de l'écrivain est confronté au « choc de l'instant » (257).

La section «Fictions-monde, mises en scène de l'écrivain » porte sur l'étude de quelques auteurs et de leurs « mises en scène singulières » 
(269) : René Depestre et son « érotisme solaire » (281), Émile Ollivier et son identité " rhizomatique et toujours schizophrène » (294), Marie Vieux-Chauvet, dont l'écriture se situe « au-delà du trauma » (310) et Dany Laferrière et sa bio-fiction ouverte au monde.

À la fin du parcours tracé par Yolaine Parisot, où l'étude de la fiction-monde haïtienne est toujours accompagnée de renvois aux arts visuels, l'auteure avoue que «le risque est grand de se perdre dans la vertigineuse mutation qu'opère la fiction littéraire haïtienne, passant, en l'espace d'un peu plus de soixante-dix ans, de la spécularité du roman [...] aux ramifications innombrables des formes réflexives » (333). Parisot dans son analyse a abordé le contemporain littéraire haïtien en soulignant son unicité, en tant que " littérature nationale dont la naissance fut une réponse à la dialectique hégélienne du maître et de l'esclave » (333). Elle a ainsi montré que la création artistique de la Première République noire, née d'une révolution d'esclaves bien avant les indépendances des autres pays de la Caraïbe, de l'Amérique Latine et les luttes des Africains Américains, échappe à la « critique postcoloniale de l'universalisme » (334) et ne peut qu'être étudiée à partir d'une approche plurielle et ouverte sur le monde. 


\title{
Marty, Anne (2017). La littérature haïtienne dans la modernité. Paris : Éditions Kathala, 273 pp.
}

\author{
Fabiana Fianco
}

(Università Ca’ Foscari Venezia, Italia)

Digne du plus grand intérêt, La littérature haïtienne dans la modernité est une contribution concise et essentielle à la mise en valeur d'une riche production haïtienne moderne. La particularité de l'ouvrage tient au parcours singulier de son auteur : professeure pendant plus de trente ans dans l'Éducation nationale, critique littéraire, chercheuse universitaire et femme très active dans les milieux associatifs d'Haïti, Anne Marty est aujourd'hui parmi les spécialistes les plus compétentes de littérature francophone. Son œuvre est présentée comme le résultat de plus de quarante années d'études et de recherches en littérature haïtienne, qui sont d'ailleurs témoignées par deux autres textes signés par l'auteure. ${ }^{1}$ Son dernier ouvrage s'inscrit harmonieusement dans le sillage de ces publications et se distingue notamment pour sa potentialité pédagogique et didactique. Marty propose des articles parus entre 2000 et 2013 dans des revues comme Pour Haïti, Il Tolomeo, Interculturel Francophonies, Notre Librairie et le magazine Amina. Il s'agit là d'une sélection de textes dont certains ont été écrits en vue d'une conférence, alors que d'autres ont été publiés avant toute prise de parole publique.

Dans la préface à l'œuvre, Yves Chemla nous prévient quant à la nature générique du livre : loin d'être une collection d'essais, il est un «projet assumé et qui fait sens progressivement »(6). Sa dynamique repose en effet sur trois axes thématiques - littérature haïtienne, expression féminine et réception étrangère - qui régissent les trois parties qui le constituent : " Particularités de la littérature haïtienne d'expression française »; "Quelques spécificités de l'écriture féminine ; « Euvres et auteurs bien reçus par les éditeurs étrangers ». Dans la première partie, Marty s'interroge sur les spécificités propres à la littérature haïtienne et sur les relations tissues au cours des siècles avec la littérature française. Si aujourd'hui il est indéniable que la littérature haïtienne est la plus féconde des Caraïbes avec tout ce qui la caractérise, il est aussi vrai que c'est

1 Marty, Anne (1995). Le personnage féminin dans les romans haïtiens et québécois de 1938 à 1980. Lille : Presses Universitaires du Septentrion; Marty, Anne (2000). Haïti en littérature. Paris : Éditions Maisonneuve et Larose ; La Flèche du Temps. 
par l'éloignement progressif des modèles provenant de la Métropole que cette unicité a pu voir le jour. Bien que le sujet puisse paraître épuisé à la lumière de nombreuses études qui l'ont précédemment abordé, Marty décortique ce rapport sous un angle assez spécifique qui rend compte de la recherche problématique d'une langue d'expression littéraire chez les auteurs haïtiens. Elle s'interroge ainsi sur la nature du français écrit tel qu'imposé en Haïti depuis les débuts de la colonisation, pour ensuite se pencher sur les conflits esthétiques et linguistiques qui ont amené les écrivains à se partager entre fidèles imitateurs d'un côté et audacieux novateurs de l'autre. En parcourant les moments clés de l'évolution du système éducatif haïtien, l'auteure se focalise sur les potentialités littéraires offertes par la dichotomie linguistique français/créole, qui amène au surgissement de phénomènes de créolisation, d'intertextualité et de transtextualité analysés par des références directes aux textes de la tradition.

Aussi, la langue de l'écrivain haïtien est considérée dans un contexte mondial où il est de plus en plus difficile de définir la provenance d'un auteur par les seuls traits linguistiques. De cette manière, la prise en compte de la question linguistique n'empêche pas un élargissement de la thématique et devient l'occasion pour décrire les particularités culturelles de l'écriture haïtienne. En passant des thématiques proprement haïtiennes finalisées à l'émancipation du modèle étranger aux valeurs populaires inscrites en littérature, Marty en profite aussi pour parler du délicat processus de métissage linguistique qui a déclenché les premiers grands débats sur la langue littéraire de l'île caribéenne. Pour le lecteur moins avisé c'est donc l'occasion pour parcourir les œuvres majeures du répertoire haïtien et pour découvrir les innovations linguistiques qui les ont rendues telles, comme l'écriture en créole ; la graduelle naturalisation du français ; l'insertion dans les textes de traductions en créole ou la persistance d'un français très classique et travaillé.

Après ce survol général sur les particularismes littéraires haïtiens, la deuxième partie du livre a le mérite de focaliser l'attention sur un type d'écriture que Léon-François Hoffmann affirmait ne pas avoir fait l'objet d'une étude distinctive il y a une trentaine d'années $(1982,127)$. C'est l'écriture féminine que Anne Marty étudie et approfondit depuis ses premières publications et qu'elle a magistralement analysée en perspective comparative dans sa thèse de doctorat soutenue en 1995. En lui reconnaissant la juste place à côté de la production masculine, elle parcourt l'émergence des premières voix féminines et en suit l'évolution dans plusieurs genres littéraires. L'intérêt porte notamment sur l'être-femme littéraire et sur la volonté des écrivaines de se démarquer d'une narration masculine qui a longtemps perpétué des faux stéréotypes. L'ordre établi est contesté de différentes manières et Marty nous fait part de cet esprit de révolte en se focalisant sur les personnages féminins qui luttent contre l'exclusion et qui connaissent la rivalité mère/fille. Parmi plus d'une trentaine d'auteures 
citées - à commencer par Ida Faubert et Virginie Sampeur jusqu'à Jacqueline Scott-Lemoine - c'est à Yanick Lahens qu'est reconnue une place privilégiée. En s'appuyant sur le roman La Couleur de l'aube et sur le recueil de nouvelles La petite corruption, Marty aborde la représentation du sexe et du corps féminin, un thème très récurrent chez les romancières de la modernité. La mémoire de la violence corporelle est également prise en compte comme spécifique d'une écriture féminine où le moi-narrateur fait face au pire avec une lucidité et une sincérité audacieuses. L'attention pour Lahens n'empêche pourtant pas la prise en compte des manifestations du corps chez d'autres romancières dont l'écriture est façonnée par la danse, la maternité, le sexe, la couleur de peau et le « moi » psychologique du « je » littéraire (101). En clôture, une partie importante est consacrée à la poésie féminine haïtienne, une production d'habitude étouffée par celle masculine mais qu'à partir des années '60 voit la poésie devenir de plus en plus mixte. Par les textes de la poétesse haïtienne Michaëlle Lafontant, Marty nous introduit à la poésie à cheval entre XXe et XXIe siècle, tandis qu'avec Pascale Blanchard-Glass, Jacqueline Scott-Lemoine et Maggy De Coster elle donne la parole aux femmes de l'exil.

La troisième et dernière partie de l'ouvrage n'en est pas moins intéressante. Le lecteur est invité à connaître les auteurs qui ont marqué et qui marquent encore aujourd'hui la littérature haïtienne moderne. Et cela de manière très originale, c'est-à-dire à travers l'œil de la réception de la critique littéraire. Marty publie dans cette section toute une série de comptes rendus d'auteurs en ordre alphabétique. Cette partie a une utilité extrême si on la considère comme la possibilité d'obtenir un panoramique global de la littérature haïtienne d'une grande partie du XXe siècle. Néanmoins, une petite faiblesse est à reconnaître : la minorité d'écrivains femmes (Marie Vieux-Chauvet et Yanick Lahens) contre treize écrivains hommes. Serait-ce dire que l'écriture féminine n'est pas bien reçue à l'étranger ?

Dans l'ensemble, La littérature haïtienne dans la modernité a tous les atouts pour devenir une excellente introduction à la littérature haïtienne. L'ouvrage donne la possibilité de parcourir les évènements clé de l'histoire coloniale et postcoloniale haïtienne à travers les textes littéraires, en offrant aux lecteurs moins équipés les instruments nécessaires pour la contextualisation des propos avancés. À l'enseigne de la diffusion d'un savoir parfois difficile à repérer dans une seule publication, le livre d'Anne Marty interroge la modernité haïtienne dans sa variété et sa multiplicité, sans pourtant oublier le rapport indissoluble que celle-ci entretient avec les commencements de cette même littérature. Aussi, par le traitement de sujets qui ont d'habitude peu de place dans les anthologies francophones et par un regard inclusif, cet ouvrage s'avère un point de départ à valoriser pour saisir les enjeux essentiels au cœur du contemporain. 


\section{Bibliographie}

Hoffmann, Léon-François (1982). Le roman haïtien : idéologie et structure. Sherbrooke : Les Éditions Naaman.

Marty, Anne (1995). Le personnage féminin dans les romans haïtiens et québécois (1938-1980) [thèse de doctorat]. Lille : Presses Universitaires du Septentrion.

Marty, Anne (2000). Haïti en littérature. Paris : Éditions Maisonneuve et Larose ; La Flèche du Temps. 


\title{
Martelly, Stéphane (2016). Les jeux du dissemblable : folie, marge et féminin en littérature haïtienne contemporaine. Montmagny : Éditions Nota bene, 379 pp.
}

\author{
Fabiana Fianco \\ (Università Ca’ Foscari Venezia, Italia)
}

Dans un panorama littéraire haïtien où le contemporain occupe une place de plus en plus privilégiée par la critique, l'essai de Stéphane Martelly se révèle d'une nouveauté frappante. Issu de sa thèse de doctorat soutenue en 2014 à l'Université de Montréal, cet ouvrage offre une lecture transdisciplinaire d'une écriture désignée depuis les années '90 comme " poétique de la dégradation » ou du « délabrement ». Il s'agit d'une écriture qui dégage la « consistance indéterminée » de l'inconnu, une forme "imprécise et violente » (9) qui tente d'émerger dans le littéraire haïtien contemporain et que Martelly identifie comme une dissemblance tant évidente que difficile à cerner. Cette dissemblance elle la retrouve dans les œuvres majeures d'une littérature contemporaine située entre les années '60 et le séisme de 2010 : on trouve ainsi Marie Vieux Chauvet, Frankétienne, Davertige, Jan J. Dominique et Lyonel Trouillot. Un corpus restreint mais symbolique qui reflète les tendances haïtiennes contemporaines telles que la perte de points de repère et de sens ; la recherche de définition identitaire ; un sentiment général de défaite et d'inquiétude. C'est dans ce contexte que s'inscrit l'étude de Martelly, là où le littéraire est envahi par la folie et par l'émergence du féminin.

Ce sont là les axes centraux autour desquels l'argumentation évolue, en reconnaissant une place légitime à deux sujets peu abordés par les études littéraires haïtiennes. Martelly envisage la folie et le féminin en tant qu' « expressions concourantes de la dissemblance » (11) et les analyse à partir d'un questionnement clé qui sous-tend toute sa démarche analytique :

Après plusieurs siècles de littérature haïtienne, un sujet féminin a-t-il réussi à émerger et à s'accomplir en tant que sujet ? Et si un tel enjeu d'écriture a réussi à se réaliser, quel rapport entretient ce sujet avec la folie, figure emblématique d'étrangeté, de radicale subversion et de silence, figure banalisée et pourtant insaisissable d'altérité absolue qui 
se dresse, menaçante, sur toute identité, toute parole, toute signification? (11)

Dans cette optique, les œuvres sélectionnées permettent à Martelly d'explorer les points de convergence entre la folie et le féminin, en montrant les enjeux au cœur d'un « je » de dissemblance qui n'est pas encore nommé (12). Le parcours qui suit les mouvements du dissemblable s'articule en trois chapitres où l'auteure joue entre écriture académique et création personnelle. Poète et plasticienne ainsi qu'écrivaine, elle en profite pour prendre du recul critique et artistique sur son objet de recherche. Sans se limiter à commenter les œuvres du corpus, Martelly les assimile et les restitue aux lecteurs à travers un regard interdisciplinaire. Le résultat consiste en une série de peintures annexes qui symbolisent ses premiers tâtonnements au sujet de la folie. En outre, à partir du deuxième chapitre elle insère également des textes où elle remanie son objet d'étude sous forme poétique et qu'elle intitule respectivement Folie I, Folie II et Folie III. Tant dans la lecture critique et académique que dans la création personnelle les objectifs de sa recherche sont clairs et novateurs. Ils portent « davantage sur le fonctionnement du texte et les théories qu'elle peut convoquer que sur des hypothétiques exhaustivités contextuelles, historiques ou interprétatives » (13).

Ainsi, Martelly adopte une approche critique qui n'est ni culturaliste ni thématique, mais plutôt rhétorique, herméneutique et énonciative. Elle se penche sur l'identification du sujet féminin reconfiguré comme posture énonciative et figure littéraire au-delà du genre, du sexe et du personnage féminin (14). De là, elle s'interroge sur la mise en rapport entre ce sujet féminin et la folie, les deux étant des visages de l'altérité qui laissent des traces dans le langage. Aussi, l'essai est une tentative d'analyser les textes choisis selon la lecture d'un défaut, d'un manque textuel provoqué précisément par la rencontre entre folie et féminin. Martelly se propose de faire émerger la langue de la folie conçue comme « l'absence essentielle où se risque et se joue la création » (337). Elle ponctue notamment la difficulté énonciative de la femme, dont la subjectivité ébranlée par la folie peine à atteindre une expression complète et définie. L'auteure envisage de cette manière le dissemblable et sa folie par le biais des modalités énonciatives exploitées pour surgir et s'imposer dans le texte.

Lanalyse textuelle est progressive et se déploie en trois étapes qui coïncident avec la division des chapitres. Le premier - « Parcours critiques et déchiffrements heuristiques » - est l'occasion pour Martelly de parcourir l'évolution historique du thème de la folie et de mieux préciser les assises théoriques de son approche rhétorique. Elle procède à cet éclaircissement à l'aide du roman Folie de Marie Vieux Chauvet, le texte qui mieux marque la rupture dans la représentation du féminin en littérature haïtienne après Amour et Colère. Contrairement aux nombreuses études conduites sur la 
trilogie de Chauvet, Martelly veut montrer que la folie est plus un effet de lecture qu'un thème ou un objet (34). La sensation provoquée par Folie est ainsi celle d'un trou creusé dans le texte qui construit et défait en même temps toute signification (86). L'intérêt de Martelly devient alors celui d'interpréter les interactions entre folie et littérature dans l'œuvre de Chauvet, où la première évoque l'absence de sens alors que la deuxième renvoie paradoxalement à une expérience de langage qui finit toujours par dire quelque chose, malgré la limite que le silence impose à l'œuvre. Linterrogation sur la folie du roman signifie ainsi comprendre le projet rhétorique qui fait exister l'œuvre même. Cela implique également lire les ruptures provoquées par les jeux énonciatifs du texte dans la perspective 'heuristique' d'une écriture qui procède en cercles concentriques.

Ensuite, dans le deuxième chapitre - «Détours/traces : marques et mémoires du contemporain haïtien »-l'auteure dresse un portrait du scénario politique haïtien en analysant ses traces et ses effets dans Anthologie secrète de Davertige et dans l'œuvre homonyme de Frankétienne. La focalisation se déplace maintenant sur la folie et sur le sujet poétique qui en est affecté en prenant appui sur « les notions de la marge et de la mémoire » (126). Martelly identifie deux mouvements qui travaillent le contemporain littéraire : d'un côté, elle observe un effort collectif de conservation et de préservation de la mémoire dans un contexte fortement répressif et dictatorial ; de l'autre, elle constate l'incorporation subjective du vécu historique qui amène à la contestation individuelle nourrie par un sens de décentrement. C'est précisément dans ce « lieu conflictuel » (129) entre marge individuelle et mémoire collective que Martelly inscrit sa réflexion, afin de restituer les réactions du sujet dans la multiplicité de son énonciation. De même que la folie et le féminin, la mémoire et la marge sont en effet conçus comme des processus rhétoriques. Étant considérés comme les résultats d'une opération d'écriture, la mémoire traumatique et la marge sont analysées comme les témoignages énonciatifs d'un mécanisme de différenciation ainsi que d'affirmation de liberté. Aussi, en faisant dialoguer folie et mémoire, Martelly se place du côté des sujets d'écriture qui ont de la peine à trouver un espace autonome d'énonciation. En creusant dans le silence et dans le manque de parole, l'auteure pousse donc sa recherche vers une périphérie littéraire souvent ignorée.

Dans cette perspective, le chapitre conclusif - « Lire/Créer : Féminin et art dangereux de la dissemblance »- renoue les propos des deux premiers en une parfaite symbiose. Il s'attache à des "œuvres de filiation larvée » (337), Les enfants des héros par Lyonel Trouillot et Mémoire d'une amnésique par Jan J. Dominique, deux textes qui permettent de saisir les modalités d'expression des sujets féminins marginalisés dans la folie. La femme est dessinée comme l'archétype de l'altérité et de la différence, une " figure satellite » occupée à négocier son existence dans la marge (324) et dans la périphérie du fou (262) par une langue qui détermine le 
sujet comme 'autre'. Entre marge et folie, Martelly suit la construction du féminin comme un dissemblable qui essaie de pallier " un défaut de représentance » (224). Sa recomposition et son affirmation deviennent possibles grâce à la parole et aux silences féminins, nécessaires à montrer « la rhétorique et le visage de la folie » (294), là où cette dernière peut même se manifester par un refus d'expression ou un échec du langage. L'intérêt du chapitre est d'étudier comment l'ombre du féminin bouscule non seulement l'énonciation créative et subjective d'une dissemblance supposée, mais aussi sa lecture critique.

Par ce parcours triparti, Les Jeux du dissemblable donne magistralement voix à l'absence et à l'oubli dans la textualité haïtienne contemporaine selon une 'lecture par le trou'. Le féminin, la folie et la marge sont reconsidérés comme manifestations complexes d'un dissemblable qui peut évoluer en tant que projet énonciatif et rhétorique. Néanmoins, Martelly avoue les limites de tels objets, en les définissant comme une matière qui reste toujours quelque peu insaisissable dans l'écriture et parfois difficile à y être enfermée. Ce manque - qu'il soit envisagé sous la forme d'un silence, d'un défaut ou d'un vide - est pourtant positivement envisagé comme une potentialité de création. Défini par Robert Berrouët-Oriol comme « un livre majeur et de grande érudition ${ }^{1}{ }^{1} l^{1}$ 'œuvre de Martelly est en dernière analyse extrêmement claire tant dans son argumentation que dans ses finalités de recherche. En contact avec des sujets que d'après Martelly aucune approche critique ne saurait entièrement recouvrir, l'auteure se risque dans une analyse dépourvue d'outils théoriques de référence. L'œuvre a ainsi le mérite de ramener le lecteur à la forme la plus pure et la plus simple de l'investigation littéraire, c'est-à-dire à l'essentialité du texte.

1 Berrouët-Oriol, Robert (2016). Aménagement linguistique et littérature. URL http:// berrouet-oriol.com/uncategorized/les-jeux-du-dissemblable/ (2018-11-20) 


\title{
Parisot, Yolaine (éd.) (2016). « Dany Laferrière : mythologies de l'écrivain, énergie du roman » (2016). Interculturel Francophonies, 30, novembre- décembre, 321 pp.
}

\author{
Alba Pessini \\ (Università di Parma, Italia)
}

Les contributions de ce numéro d'Interculturel Francophonies, dirigé par Yolaine Parisot et consacré au premier écrivain haïtien devenu immortel sous la Coupole de l'Académie française, se partagent en trois volets dont le premier intitulé « La littérature, les arts, le cinéma et moi » se penche sur l'élément autobiographique de la production laferrienne. L'œuvre de Dany Laferrière a souvent interrogé les critiques qui ont sondé sa démarche autobiographique et tenté de démêler le vrai du vraisemblable, l'effet de réel de la pure fiction. A travers trois romans de Laferrière considérés comme les plus autobiographiques - Le Cri des oiseaux fous, Pays sans chapeau et l'Enigme du retour -, Christiane Ndiaye s'attache à mettre en lumière comment les aspects qu'elle considère autobiographiques glissent vers la fiction mais aussi comment tout roman est le résultat de l'invention d'un monde qui puise dans le quotidien. Deux dimensions mémorielles se mêlent ainsi dans l'univers romanesque de Laferrière, la mémoire du vécu et la mémoire littéraire, cette dernière interpellant, au gré des œuvres choisies, des textes différents en fonction des messages et des enjeux qu'elles entendent véhiculer. Toutefois, selon Ndiaye, Laferrière procède " constamment à une sorte de reconnaissance des textes marquants de la bibliothèque mondiale, comme pour signifier que tout ce déjà sémiotisé n'est plus de notre temps, que l'écrivain d'aujourd'hui ne peut pas les prendre pour modèles »(36), que ce dernier est pressé par l'urgence de trouver sa propre voie/voix. Dans «Autoreprésentation et invention d'identités chez Dany Laferrière », Cheikh M.S. Diop met en garde le lecteur qui aurait facilement tendance à embrigader les textes laferriens dans un genre d'emblée trop évident, celui des récits autobiographiques. Il interroge le « je » de trois œuvres laferriennes, Comment faire l'amour avec un Nègre sans se fatiguer, Je suis un écrivain japonais et L'énigme du retour et traque les ruses de l'écrivain dans l'image textuelle de lui-même qu'il diffuse. La contribution de Mylène F. Dorcé, « Dany Laferrière : de l'écrivain intarissable au cinéaste insaisissable », propose une comparaison 
entre les textes Comment faire l'amour avec un Nègre sans se fatiguer, Le Goût des jeunes filles, Comment conquérir l'Amérique en une nuit et Vers le Sud et leurs respectives adaptations cinématographiques. Dorcé focalise son attention sur des types de personnages (Le Nègre, la Blonde/Blanche, Le Blanc, La Négresse) qu'elle dissèque pour saisir leurs rôles et leurs fonctions au gré des romans et des représentations filmiques. Le dernier article de Hidehiro Tachibana qui clôt ce premier volet interroge la notion de masque que le critique, au fil de son analyse, entend bien faire tomber et dévoiler ainsi la part fictive et autobiographique de l'œuvre laferrienne.

La deuxième partie, "L'énergie du roman », s'intéresse de plus près aux rouages de l'écriture romanesque et s'ouvre avec l'étude d'Yves Chemla, « La Confidence de l'arrivée ». Il s'agit pour ce dernier d'interroger les ambiguïtés de l'énigme de l'arrivée', un moment qui intervient à plusieurs reprises au sein de l'Autobiographie américaine, notamment dans J'écris comme je vis et Je suis fatigué, et sur laquelle le critique se penche de façon plus systématique dans Chronique de la dérive douce en considérant les techniques narratives auxquelles Laferrière recourt. Selon Chemla, cet épisode pourrait se présenter comme un autre « topos du roman de la décolonisation, tout comme l'était la transformation de soi par l'école » (127); cependant il insiste aussi sur le fait que Laferrière est à même de construire un récit qui fait fi des modèles. Il nous présente donc Chronique de la dérive douce " comme un récit subversif à plusieurs titres : il transforme l'énigme de l'arrivée en un bref récit de formation. Et subvertit le récit de cette formation en prenant en charge la complexité et les contradictions qui traversent l'être lui-même comme la société qui l'accueille » (142). Alessia Vignoli dans «Je bouge : donc je suis ? (Du mouvement et de l'immobilité chez Laferrière) » cherche à dépasser l'insertion de l'œuvre de Laferrière dans une littérature de l'exil stricto sensu et préfère se concentrer sur la dialectique du mouvement et de l'immobilité dont l'antithèse, selon Vignoli, n'est qu'apparente puisque « l'évolution de la fonction du déplacement aboutit à une superposition entre mouvement et immobilité aux contours flous et instables » (162). La contribution de Ching Selao intitulée " 'Si le sexe est sain, il n'est pas innocent'. Sexe et pouvoir dans l'œuvre de Dany Laferrière » analyse la mise en scène des rapports de force entre les sexes et plus particulièrement entre les noirs et les blanches et interroge le sexe dans ses différentes fonctions, comme monnaie d'échange ou encore comme acte subversif.

Dans le troisième et dernier volet de ce recueil il est question de la relation que l'œuvre de Laferrière entretient entre « La littérature, le monde et nous ». Oana Panaïté nous introduit à la « Mondialisation culturelle et mondialité poétique chez Dany Laferrière » et montre comment l'écrivain originaire de Petit-Goâve " conçoit des mondes fictionnels où la mémoire du pays natal n'est guère effacée ou rejetée mais intégrée à d'autres thématiques englobantes telles que l'identité en mouvement, le vécu 
de la création artistique, et la dynamique et les pathologies du monde contemporain » (198). Païnaté approfondit les réflexions métacritiques que Laferrière élabore dans plusieurs ouvrages depuis 2001 pour tenter de mettre en évidence ce qu'elle appelle « une écriture du pays [qui] se nourrit aux sources multiples de la mémoire et de la diction littéraire classique et contemporaine » (198). Selon Françoise Naudillon dans "Ombres et lumières : Windsor Klébert et ses leçons d'immortalité », il manquerait un onzième tome à l'Autobiographie américaine de l'écrivain, celui qui prendrait en considération les différentes stratégies mises en acte pour se construire une célébrité. Qu'à cela ne tienne, Naudillon s'en charge et parcourt les étapes de ce succès en dégageant les mécanismes qui le soustendent en une série de 5 leçons (Leçon $\mathrm{nr}$. 1 : se faire connaître ; Leçon nr. 2 : se faire reconnaître, etc...). Florian Alix s'intéresse, lui, à la posture « académicienne » de Laferrière qu'il illustre à travers L'Art presque perdu de ne rien faire et compare l'écriture de Laferrière essayiste avec celle d'autres écrivains (Léopol Sedar Senghor, François Cheng, Amin Maalouf), eux aussi élus à l'Académie, et leurs essais respectifs. Les articles de cette dernière partie s'articulent tous autour d'un même dénominateur commun qui est représenté par l'essai de Jérome Meizoz sur la posture littéraire ( $\mathrm{Po}$ stures littéraires. Mises en scène modernes de l'auteur. Genève : Slatkine Erudition, 2007). La dernière contribution de Véronique Bonnet, « Dany Laferrière : le lecteur dans sa baignoire ou l'art de bien lire », comme son titre l'indique, s'attache à la posture du lecteur et enquête sur cette figure qu'il s'agisse du narrateur des textes ou encore de l'écrivain lui-même. Bonnet insiste sur le fait que « la pratique de la lecture littéraire [...] et la mise en scène de l'acte de la lecture, apparaissent chez lui avec une forte régularité, se renforcent au fil du temps » (255). Elle nous convie à découvrir la bibliothèque-monde de l'écrivain au fil de ses œuvres mais ne passe pas non plus sous silence la place que tient le lecteur de Laferrière « au cœur du processus de création et de fabrique littéraire ».

L'entretien avec l'écrivain conduit par Yolaine Parisot ne s'affiche pas comme une simple annexe pour clore un ouvrage déjà bien riche, il s'insère plutôt comme un dialogue renouvelé avec les contributions qui se sont succédés et qui tentent de lever le voile sur une œuvre toujours prête à nous offrir de multiples perspectives et des approches stimulantes. 



\title{
Charles, Jean-Claude (2018). Baskets. Coordonné par Alba Pessini. Montréal : Mémoire d'Encrier, 336 pp. Chronique
}

\author{
Emanuela Cacchioli \\ (Università di Parma, Italia)
}

Un voyage dans l'espace, ainsi que dans le temps, dans la littérature, le cinéma, les différentes manifestations culturelles et anthropologiques que le lecteur peut détecter dans le monde entier. Pour marcher et suivre ce périple, il faut des chaussures confortables, des Baskets, qui nous permettent de « fouler le sol, qu'il soit de bitume, dans les grandes métropoles, ou de sable, celui des déserts américains ou africains » (7-8). Grâce à ces derniers mots - que nous lisons dans la Préface rédigée par Alba Pessini - nous comprenons la signification du titre du roman et nous abordons un déplacement spatial aux côtés de Jean-Claude Charles, écrivain haïtien mort à Paris en 2008. Ce volume - inédit jusqu'à présent - a été publié par la maison d'édition Mémoire d'encrier qui, depuis quelques années, a entrepris un vaste projet de réédition des ouvrages de l'auteur. Il est difficile de trouver une définition univoque pour ce volume : nous adoptons le soustitre " récits de voyage » pour résumer en une expression cette collection d'instantanés suggestifs, de bribes de dialogues, d'impressions, détails, rencontres intéressantes, réflexions, au langage vif, immédiat mais également poétique qui saisit la spécificité d'un lieu, d'une ville, d'un paysage. Ce dernier mot comprend l'acception géographique, mais aussi humaine avec ses implications culturelles, sociales, historiques et littéraires. Un texte étonnant qui transporte le lecteur dans un voyage subjectif et réel. Subjectif car ce sont les impressions d'un voyageur qui observe la réalité et en donne au lecteur une image personnelle et transfigurée par ses lectures (concernant les faits divers et la littérature), ses connaissances cinématographiques, historiques, politiques, linguistiques. Il s'agit d'un voyage réel aussi puisque les constats sur la société, la topographie, les traditions culturelles, religieuses, gastronomiques et les descriptions des musées, des librairies, des cafés, des monuments sont fournies au lecteur comme s'il s'agissait d'un guide touristique concret et en même temps original, hors du commun. Comme nous l'explique Alba Pessini, dans le commentaire qui clôt le volume, Baskets naît comme un recueil des articles que Charles a publiés dans le supplément du Monde intitulé « Le Monde 
sans visa » entre 1986 et 1996. L'écrivain avait l'intention de les publier en volume et avait déjà conçu la structure de l'ouvrage, mais les carnets sont restés dans un tiroir jusqu'à aujourd'hui quand la chercheuse les a ramenés au jour, en a établi l'édition et coordonné la publication avec une préface et une postface nous fournissant le contexte d'écriture et une analyse très intéressante de quelques aspects du volume comme le recours à la dimension littéraire du texte, l'ancrage de l'écrivain aux espaces et le rituel de la lecture des quotidiens locaux.

Un volume qui mérite d'être connu et lu parce que l'univers de JeanClaude Charles est dans ces récits : ses fuites dans l'espace nous livrent sa vision du monde, sa volonté d'être nomade, de se déraciner et de se nourrir de tout ce qu'un lieu et ses habitants peuvent lui donner. C'est la raison pour laquelle chaque paysage (peu importe qu'il soit urbain ou naturel comme le désert ou l'océan) se lie à des réflexions profondes sur l'histoire (qu'il s'agisse de la dictature de Duvalier, de la déportation des Africains en Amérique, de la lutte d'émancipation des Noirs ou du mur de Berlin), la société (et la transformation des villes au niveau topographique qui suit une période de surpeuplement ou de migration) et l'économie (quand il faut choisir entre un tourisme aliénant ou pas). Charles n'oublie jamais, pour chaque lieu, de laisser une large place aux êtres humains qui y habitent, ces derniers confèrent à l'espace son identité et sa véritable valeur : à partir d'un détail ou d'une réplique l'écrivain nous met en contact avec la richesse humaine constitutive de la beauté du monde.

Dans ce périple qui s'étale sur trois continents (l'Europe, l’Amérique et l'Afrique), la France et les États-Unis gardent une place importante au niveau de la consistance des références géographiques ainsi que culturelles. Cependant c'est Haïti qui demeure un repère incontournable et la véritable toile de fond. On dirait que le pays natal est la destination ultime de la première partie de son voyage quand Charles rentre à Port-au-Prince après la chute de la dictature de Duvalier. Il s'arrête à New York et à Miami avant d'atterrir dans la capitale haïtienne pour rencontrer les plus grandes communautés d'émigrés. Baskets se termine sur cette image : après un nouveau retour dans son pays, l'écrivain se dirige vers la Floride et il retrouve à nouveau quelques membres de Little Haïti. Cependant sa terre natale n'est pas l'objectif de ce périple : le mouvement, les rencontres, bref le déplacement spatio-temporel et ses implications culturelles et humaines représentent le but de l'auteur afin de s'ancrer encore une fois dans l' « enracinerrance » qu'il proclame.

La publication de Basket est un vrai cadeau que Mémoire d'encrier et Alba Pessini ont fait aux lecteurs de Jean-Claude Charles et aux chercheurs qui veulent approfondir la connaissance de cet écrivain, disparu trop tôt, mais qui a laissé un patrimoine littéraire immense bien qu'encore peu connu. 


\section{Sénac, Jean (2018). L'Enfant fruitier. Série Petits inédits maghrébins 1, 91 pp. Alger : El Kalima. Collection Djib}

\section{Hadj Hamou, Abdelkader (2018). L'Offense. Série Petits inédits maghrébins 2, 141 pp. Alger : El Kalima. Collection Djib}

Alice Petruzzellis

(Università degli Studi di Trieste, Italia)

Le edizioni El Kalima hanno lanciato nel 2018, sotto la direzione di Guy Dugas, la collana «Djib» in cui ad oggi sono previsti sei inediti magrebini in lingua francese. Le opere in corso di pubblicazione, in formato tascabile, nel 2018, toccano differenti temi e generi letterari: sono previsti tre drammi teatrali, una raccolta di poesie e due saggi. Gli autori sono per la maggior parte algerini, ad eccezione di Driss Chraïbi, marocchino. Ogni testo è preceduto da una breve introduzione scritta da studiosi che presentano l'autore, il testo e la collocazione del manoscritto prima della sua pubblicazione. Le prime due opere della collana sono L'Enfant fruitier, raccolta poetica di Jean Sénac e L'Offense, dramma di Abdelkader Hadj Hamou.

L'Enfant fruitier raccoglie 20 poesie, scritte da Sénac tra il 1951 e il 1952, durante il soggiorno in Francia. Come illustra Dugas nella presentazione dell'opera, vi si possono ritrovare tre nuclei tematici principali: la contrapposizione tra Francia e Algeria, l'influenza esercitata sull'autore dai poeti dell'Hexagone, tra cui soprattutto Rimbaud e Verlaine, e il tema dell'amore omosessuale. Risulta molto interessante la scelta di mantenere nella stampa le correzioni effettuate da Sénac nel manoscritto; alcune poesie sono accompagnate dalla copia dell'originale manoscritto, mostrando quindi la grafia, le incertezze e le diverse fasi che hanno portato l'autore a raggiungere il risultato finale. La raccolta non aveva fine editoriale: l'autore, suggerisce Dugas nell'introduzione, aveva optato per l'autocensura, probabilmente a causa dei temi che non si conciliavano con l'immagine che la famiglia e la cerchia letteraria algerina, in pieno sviluppo negli anni '50, avevano di lui.

Riportare alla luce questi componimenti è importante per la conoscenza approfondita dell'opera di Sénac, autore di temi universali e al contempo radicati nella cultura di appartenenza; il testo è il primo componimen- 
to scritto dall'autore fuori dall'Algeria e dimostra come la sua crescita personale e intellettuale sia stata certamente influenzata dal soggiorno francese. Il rapporto con la Francia è veicolo di un duplice messaggio: legame con la Francia e rievocazione nostalgica dell'Algeria e della madre costituiscono i due poli del dettato poetico di Sénac. Si aggiunga che Sénac, ne L'Enfant fruitier, esprime magistralmente l'ambivalenza dell'io, tra censura e libertà, tra curiosità e nostalgia, con toni che permettono ancor oggi l'identificazione da parte del lettore.

Completamente diverso è il secondo inedito della collana, il dramma in tre atti di Abdelkader Hadj Hamou intitolato L'Offense. La pubblicazione è curata da Hadj Miliani, professore di letteratura all'università di Mostanghanem e intellettuale attivo nella promozione della cultura algerina. L'opera, composta nel 1910, rappresenta uno scritto giovanile e, come sostiene Miliani, acerbo dell'autore. Risulta inoltre essere una delle prime testimonianze letterarie in lingua francese scritte da un autore musulmano algerino. Il dramma, mai pubblicato né messo in scena, racconta le vicissitudini di un giovane insolente e libertino e del padre, un uomo buono legato al figlio da un amore incondizionato ma sfortunatamente unilaterale. L'opera si conclude con la redenzione del ragazzo attraverso il suicidio purificatore, del protagonista e della sua innamorata, anch'essa dissoluta e priva di cuore.

Un aspetto interessante de L'Offense è la totale assenza di riferimenti geografici e culturali che possano permettere di identificare i personaggi e l'autore. Il dramma rappresenta una situazione universale, quella di un padre pronto ad accettare i capricci del figlio. Ancora una volta l'universalità dei temi trattati rappresenta il fulcro di questa collana di «Petits inédits maghrébins», nome della serie tascabile.

L'intento moralistico del dramma volge tutta la trama verso la contrapposizione tra bene e male: i personaggi stessi sono archetipi di questa coppia complementare e risultano privi di una reale introspezione psicologica. Hamou, tuttavia, è abile nel guidare le preferenze del lettore (e dell'eventuale spettatore) durante i tre atti; i due giovani nel primo e secondo atto vengono raffigurati in maniera assolutamente negativa, poiché affiancati a personaggi che rappresentano simbolicamente il loro alter ego positivo; nel terzo atto, al contrario, il lettore è portato a provare una forte simpatia per i due, caduti nelle mire di feroci banditi, personaggi negativi per eccellenza. Nonostante l'opera possa sembrare piatta e poco sviluppata agli occhi del lettore contemporaneo, risulta comunque interessante, poiché presenta, come già detto, uno dei primi testi di una neonata cerchia letteraria di intellettuali autoctoni che utilizzano la lingua francese per esprimere le proprie opinioni e passioni.

Qui risiedono il valore e l'importanza del progetto editoriale che mostra un lato particolare della letteratura magrebina in lingua francese, caratterizzata da temi radicati nel territorio e al contempo universali, dove l'io 
e la personalità degli autori spiccano in un concatenarsi di similitudini tra opere, così lontane tra loro per genere letterario, ma così vicine per origini e finalità estetiche. Si potrebbe suggerire che l'obiettivo ultimo di questa collana sia portare alla luce perle inedite che potrebbero essere fondamentali per approfondire gli studi sulla letteratura magrebina di lingua francese e, più in generale, sulla letteratura francofona. 



\section{Puccini, Paola ; Kirouac Massicotte, Isabelle (éds.) (2017). Bien-être en ville : espaces urbains, langues, culture et société. Bologna : I libri di Emil, 160 pp.}

Sara Del Rossi

(Uniwersytet Warszawski, Polska)

Les actes du colloque Bien-être en ville : espaces urbains, langue, culture et société, organisé par le Centre interuniversitaire d'études québécoises (CISQ), confirment le caractère pluri- et interdisciplinaire du Centre, qui se veut l'espace de rencontre d'un « dialogue entre le Québec et l'Italie » (7) autour du thème choisi. En effet, même si les collaborateurs appartiennent à des domaines d'étude différents, leurs contributions s'enchaînent dans un rayonnement d'échanges intellectuels dépassant toutes frontières. Le but général est de faire comprendre que le bien-être n'est pas seulement une question d'infrastructures, mais qu'il concerne aussi et surtout l'individu et sa nécessité de regagner sa place à l'intérieur de la ville.

La contribution de Dino Gavinelli, « La ville entre dynamisme économique et cohésion socio-territoriale », peut être considérée comme un cours introductif au thème choisi, vu qu'on y retrouve l'explication de plusieurs concepts-clés de la géographie sociale (gentrification, ségrégation, etc.) employés dans la totalité de l'ouvrage. L'idée principale est la mise en valeur du rétablissement de l'égalité des conditions sociales et économiques, estimée plus importante que le renforcement des infrastructures. L'exemple donné est celui de la ville de Montréal qui, à partir des années '90, lutte contre l'inégalité socio-économique parmi les quartiers anglophones et francophones à travers la promotion de " réformes sociales et revitalisation urbaine » (24). Cette thèse est supportée par un historique des réformes adoptées pour combattre « la ségrégation sociale » (22), qui démontre que la justice sociale est fondamentale pour le bien-être des citoyens, beaucoup plus que les richesses matérielles.

L'une des reformes sociales réalisées est celle concernant l'insertion professionnelle des jeunes provenant des quartiers défavorisés de Montréal, qui constitue le sujet de l'analyse sociologique de Marco Alberio et de DianeGabrielle Tremblay dans « L'intégration socioprofessionnelle des jeunes en difficulté socioéconomique et l'importance des initiatives locales. L'exemple des entreprises d'insertion à Montréal ». Il s'agit de la publication des 
résultats d'une enquête auprès de jeunes travailleurs et gestionnaires de dix entreprises qui s'occupent de leurs parcours d'insertion professionnelle. Le but est de comprendre la dynamique de (ré)adaptation du point de vue des jeunes et aussi des entreprises, pour avoir une vision globale des entraves trouvées et penser à un moyen pour améliorer les conditions d'insertion. Les entretiens se concentrent sur des aspects plurielp les conditions familiales et sociales des jeunes, les instruments utilisés par les gestionnaires pour la formation et les côtés économique et psychosocial. Les résultats des entretiens mettent en exergue le besoin d'un suivi de deux ans afin d'assurer la continuité et la bonne réussite du processus d'intégration, sans oublier sa portée au niveau économique et social.

L'étude comparative "Bien-être : avoir un certain contrôle sur sa vie. Les pratiques des pairs-aidants à Bologne et à Montréal » de Maurizio Bergamaschi clôt la partie sociologique de l'ouvrage. Le pair-aidant, nouvelle figure professionnelle du travail social, constitue le sujet de la recherche de Bergamaschi qui s'interroge sur la manière dont ce rôle professionnel, désormais fondamental pour l'aide aux jeunes de la rue et aux sans-abri, est influencé par les contextes sociaux, économiques et politiques. L'étude prend en considération deux contextes éloignés : la ville de Bologne en Italie et celle de Montréal au Canada. Après avoir présenté les résultats de ses recherches, l'auteur dresse une liste des différences entre les deux pôles, en soulignant le fait qu'en Italie le pair-aidant est bloqué dans son rôle, sans possibilité d'avancement ; par contre, au Québec l'escalade sociale est possible, mais sa condition est plus vulnérable qu'en Italie, car les gens désignés habitent encore la rue, en proie à toute sorte de tentation.

La dégradation des jeunes de la rue et des sans-abri est l'un des facteurs du mal-être en ville, qui s'ajoute à la corruption, la mauvaise administration et le manque de services et infrastructures. La suburbanisation est la réponse pratique de la part des classes aisées au malaise urbain, auquel elles substituent une autarcie du quartier, qui supplée l'incapacité du gouvernement central. Harold Bérubé, dans son article « La suburbanisation comme pratique antiurbaine à Montréal (XIXe-XXe siècles) », examine la situation des suburbs de Montréal et leur organisation. La classe bourgeoise montréalaise, en particulier celle anglo-protestante, a institué des normes urbanistiques sévères qui rendent plus difficile l'accès à la communauté, jusqu'à former des formes de «zonage » (63) et de « ségrégation » (63). L'historien, à travers un parcours qui prend en analyse cette pratique dès ses origines, démontre comment le manque de confort au centre-ville est toujours en train de créer des univers qui se veulent séparés du marasme urbain, mais qui en même temps ne s'en éloignent pas car ils en dépendent économiquement et culturellement.

En effet, Montréal est une ville riche en culture, qui offre un large éventail d'activités socioculturelles, mais au-delà des zones célèbres pour leur vitalité sociale, la ville cache des lieux à (re)conquérir. La contribution 
d'Anna Giaufret, «Les interstices de Montréal : exploration, représentation, pratiques dans l'espace public urbain », se focalise sur l'observation de diverses pratiques des Montréalais de vivre la ville, en particulier sur la redécouverte et l'appropriation des espaces interstitiels (Fischer). L'étude s'appuie surtout sur les théories de la géographie sociale d'Henri Lefebvre en ce qui concerne « les relations entre espace vécu, perçu et conçu » (69) et prend en analyse deux ouvrages graphiques - La guerre des rues et des maisons (Sophie Yanow) et Cartographie éphémère (Dominique Ferraton) - et la pratique sociale de la guerrilla potagère. Giaufret souligne l'importance de la réappropriation des espaces-résidus, « voués à la disparition » (78), comme pratique de vivre à fond la ville, mais aussi un moyen de « laisser une trace » (86) dans un espace en évolution perpétuelle.

La façon de vivre la ville est aussi au centre de la contribution « Habiter l'ellipse » de Jean-François Plamondon. L'auteur analyse les relations entre le personnage et la ville dans trois ouvrages de l'extrême contemporain québécois : Autobiographie de l'esprit (Élise Turcotte, 2013), L'album multicolore (Louise Dupré, 2014) et Recommencements (Hélène Dorion, 2014). En s'appuyant sur des méthodologies et concepts multiples comme la « seconde modernité » (Anthony Gidden à la page 92), la narratologie, l'autobiographie et le concept d'habiter dans le sens d'expérimenter (Thierry Parquot et Augustin Berque), Plamondon démontre que le parcours de la quête identitaire des protagonistes suit une trajectoire elliptique. En effet, dans les trois ouvrages autobiographiques, à l'action d'habiter un nouvel espace correspond une phase d'expérience qui enrichit le sujet. Cet enrichissement de l'être apporte une variation à la ligne plate du vécu en la courbant jusqu'à la successive phase de retirement, c'est-à-dire quand l'habiter se fait habitude. La forme elliptique, due à la correspondance spéculaire entre intériorité et extériorité, résume ainsi toute existence, de la découverte à la dernière demeure.

Le thème du rapport entre personnage et ville se retrouve aussi dans l'intervention «La ville, du récit à l'écran : le cas de Et au pire, on se mariera » de l'écrivaine Sophie Bienvenue. La romancière témoigne des obstacles rencontrés au cours de l'adaptation filmique de son roman Et au pire, on se mariera (2011), qu'elle a confiée à la cinéaste Léa Pool, après avoir échoué en essayant elle-même. Ce qui a touché l'auteure a été la difficulté de la transposition des espaces et des rapports de la protagoniste avec la ville de Montréal, en particulier avec son quartier, Centre-Sud. En effet, non seulement il faut rendre la ville à partir de la vision du personnage, mais aussi ses habitants et les relations entre et avec eux. De plus, à la « symbolique du lieu » (112) s'ajoute la « pertinence du lieu » (110), c'est-àdire réussir à trouver une location qui correspond à l'environnement décrit trois ans avant, avant la gentrification du quartier et sa transformation.

L'adaptation n'est pas seulement filmique, mais aussi linguistique et culturelle, comme dans le cas de la traduction littéraire. Tout comme Sophie 
Bienvenue témoigne des joies et des souffrances de l'adaptation filmique, Cristiano Felice témoigne dans son article « La vision de la ville chez Nadine Bismuth, Ying Chen et Marie Hélène Poitras », des mésaventures et des difficultés du traducteur. Felice examine «l'impact linguistique sur la vision de la ville » (115) et ses effets sur la traduction des ouvrages de fiction de trois auteures : Nadine Bismuth (Les gens fidèles ne font pas les nouvelles, Scrapbook et Êtes-vous mariée à un psychopathe ?), Ying Chen (Les lettres chinoises) et Marie Hélène Poitras (Soudaine le Minotaure). Le passage de la «forte participation émotive » (118) de la langue de Bismuth à la clarté descriptive de l'immigrée chinoise chez Chen, jusqu'à aboutir aux changements des registres du style labyrinthique de Poitras, démontrent la complexité et la variété que la langue peut assumer de quartier en quartier. Les traductions en bas de page constituent la mise en pratique des analyses stylistiques de l'auteur-traducteur et permettent la compréhension de son travail d'adaptation linguistique et stylistique.

Si Felice propose d'écouter le style et les registres pour les transposer sur page, Sherry Simon incite à écouter la ville et sa multitude de langues et registres afin de la vivre pleinement. Dans son article « Redessiner la carte des diversités à Montréal » l'auteure de Le Trafic des langues (1994) encourage la considération des quartiers absents, ceux qui n'ont pas encore le poids historique et social, la personnalité et le caractère des «lieux mythologiques » (126). Le projet de redessiner la carte des diversités montréalaises a comme but de faire ressortir la "prolifération » (127) de cette ville et sa richesse linguistique, culturelle et sociale. Le Boulevard Saint-Laurent est désigné en tant que lieu d'union entre absence et présence, un espace où se retrouvent « l'ici et l'ailleurs, le passé et le présent, l'actualisation des différents niveaux de relations sociales et des différentes temporalités » (129). Cet espace de démarcation et de frontière, où débouchent divers quartiers de la ville, résume le caractère de Montréal, son être un lieu d'échange et de circulation, non simplement une ville multilingue, mais une ville « traductionnelle » (132) où « l'entrecroisement des imaginaires » (132) est possible.

Le concept de ville traductionnelle de Sherry Simon est repris par Gerardo Acerenza dans sa contribution « Affichage bilingue et traduction à Montréal : un état des lieux ». Dans son analyse de l'affichage public et commercial pour évaluer le niveau du bilinguisme dans la ville de Montréal, Acerenza nuance l'exaltation de Simon et ses considérations sur le rôle de Montréal en tant que ville à l'avant-garde en ce qui concerne les relations entre les nombreuses langues qui la peuplent. Les exemples de fausses traductions, des traductions littéraires et des ambiguïtés linguistiques trouvées dans les panneaux bilingues soulignent la situation d'« inconfort linguistique » (141) dans laquelle vit la communauté francophone montréalaise. Cet état des lieux n'est qu'un indice de la tension linguistique présente au Québec entre l'anglais et le français, une tension 
qui est source d'un malaise qui ne semble pas s'apaiser malgré les mesures législatives adoptées.

"Linguistique, traductologie, analyse du discours, littérature, sociologie, urbanisme, étude du paysage, géographie, histoire, etc. » (7), le nombre des disciplines qui s'adonnent à l'étude du bien-être en ville est étonnant et la multitude d'approches utilisée confirme l'importance universelle de cette thématique. L'ouvrage exalte le rôle de la solidarité sociale et culturelle comme facteur fondamental du bien-être, qui se réalise uniquement à travers l'inclusion sociale, économique et linguistique. L'esprit de collaboration non seulement est encouragé par tous les intervenants, mais est mis en pratique à chaque page, à chaque passage d'une section à l'autre, d'un article à l'autre, dans un dialogue continu. Le CISQ confirme encore une fois son caractère d'ouverture et d'échange, propre d'un Centre qui se base sur la collaboration parmi dix universités italiennes et le Québec. 



\title{
Puccini, Paola ; Kirouac Massicotte, Isabelle (éds.) (2016). Langue et pouvoir. Bologna : I libri di Emil, 128 pp.
}

\author{
Sara Del Rossi \\ (Uniwersytet Warszawski, Polska)
}

Le binôme thématique langue/pouvoir est central dans le contexte de la société québécoise, d'autant plus que, depuis la Charte de la langue française (1977), l'État du Québec se propose de « légiférer » (9) sur la question de la langue, comme l'affirment Paola Puccini et Isabelle Kirouac Massicotte dans l'introduction aux actes du colloque Langue et pouvoir, organisé par le Centre interuniversitaire d'études québécoises (CISQ). L'ouvrage se propose de réfléchir autour de ce thème double, du XIXe siècle à nos jours, à partir de plusieurs points de vue et à travers une approche multidisciplinaire qui a sa base dans la linguistique et la sociologie.

La contribution de Nadine Vincent, «L'élite du Québec à l'assaut du français québécois : 150 ans de dénigrement dans la presse écrite », peut être considérée comme une traversée historique de l'évolution des rapports entre la langue et le pouvoir au Québec. Lauteure compare les discours sur la langue française dans la presse québécoise du XIXe au XXIe siècle, en soulignant que le contexte sociopolitique est indissociable de la question linguistique. Avant de procéder à l'analyse du corpus, elle donne un court mais essentiel survol de l'histoire de la construction identitaire au Québec par rapport à la langue française. Cet excursus historique et sociolinguistique permet de comprendre le choix de la moitié du XIXe siècle comme point de départ de l'analyse, c'est-à-dire la période successive à l'Union Act (1840), la constitution qui sanctionne l'assimilation politique et linguistique des francophones. À l'insécurité identitaire correspond une insécurité linguistique, à laquelle les lettrés de l'époque s'opposent avec une « chasse aux particularismes » (57) du français canadien, afin de faire primer la pureté linguistique et la standardisation, censées être les seuls outils pour combattre l'annihilation identitaire. Vincent remarque aussi que le même processus se retrouve chez les intellectuels québécois de nos jours qui, dans une période d'inquiétude politique, cherchent à endiguer l'anglicisation massive du français québécois à travers un retour au purisme et à la standardisation linguistique. 
Le thème de la correction de la langue et de sa standardisation est au centre de l'article de Wim Remysen " 'La science des mots n'est pas forte à la Minerve et au Canada': l'idéologie du standard et le pouvoir de la norme dans le journal montréalais Le Pays (1852-1871) ». À partir des études sociolinguistiques de Norman Fairclough et Lesley et James Milroy sur la standardisation linguistique et le lien avec le pouvoir, Remysen s'interroge sur les modalités de discréditation de l'adversaire politique à travers la dépréciation linguistique qui a eu lieu au XIXe siècle dans les pages du journal montréalais Le Pays. L'analyse souligne le rôle essentiel des médias dans la divulgation de l'idéologie dominante, qu'elle soit politique ou linguistique, mais surtout l'importance capitale de la maîtrise de la langue pour l'inclusion dans les débats politiques et sociaux, en particulier dans un contexte d'insécurité identitaire et politique comme celui du Québec au XIXe siècle.

Le rapport entre maîtrise de la langue et crédibilité se retrouve aussi dans l'article d'Annette Boudreau « Idéologie du standard et construction de la différence au Canada : le bilinguisme de Claudette Bradshaw et Justin Trudeau ». Après avoir illustré les caractéristiques qui définissent le concept d'idéologie linguistique à l'aide des théories de Paul Kroskrity, sans toutefois oublier d'autres noms importants de l'anthropologie linguistique, l'auteure observe les diverses réactions de la presse aux pratiques linguistiques de deux politiciens : Claudette Bradshaw et Justin Trudeau. Le but est de montrer que la façon de parler est un facteur de discrimination sociale réel, mais socialement et universellement accepté. Lauteure se concentre en particulier sur la question de l'accent chez les deux sujets bilingues, dont elle présente l'habitus (Bourdieu), et sur les réactions du public, en particulier les journalistes, face au "savoureux accent acadien » (82) de Bradshaw et à celui de Trudeau, considéré par ses adversaires comme un « cégépien anglophone » (83). Les résultats des considérations publiques montrent que les médias et leurs agents sont des vecteurs portant des idéologies linguistiques, tout comme les linguistes et d'autres spécialistes de la langue. Boudreau confirme le lien indissociable entre usage fautif de la langue et crédibilité, lien qui dépasse le niveau linguistique et normatif, pour aboutir aux sphères politiques et sociales.

Le rapport langue-politique-société est repris aussi par la contribution d'Isabelle Kirouac Massicotte, "Questions de féminisation linguistique : son application dans les discours féministes contemporains au Québec ». L'auteure se propose de vérifier les théories de Pierre Larrivée, selon qui la gauche serait plus favorable à la féminisation linguistique, à travers l'étude comparée des techniques de féminisation dans trois discours politiques de trois factions différentes de la gauche au Québec, de celle plus modérée à celle plus extrémiste. Après une première partie consacrée à la présentation de diverses techniques possibles, l'auteure se penche sur l'analyse du corpus choisi : Le mal du pays (2012) de Lise Payette, De co- 
lère et d'espoir (2011) de Françoise David et le manifeste Nous sommes avenir (2012) de l'association étudiante CLASSE. Les trois textes ont été rédigés par des femmes, exception faite pour le manifeste qui est un fruit de la collectivité. L'analyse prend en considération les extraits où la féminisation a été opérée, en observant quelles techniques ont été choisies et leur valeur d'un point de vue sociolinguistique. Cette stratégie d'analyse a permis à Kirouac Massicotte de confirmer les théories de Larrivée sur la correspondance entre progressivité linguistique et progressivité idéologique, mais aussi de donner une vue d'ensemble utile des stratégies de féminisation, qui permet sans doute la familiarisation avec ces pratiques, surtout pour un public peu habitué.

Létude de Cristina Brancaglion, « 'Notre vocabulaire et le Dictionnaire de l'Académie française' : l'attraction de l'autorité normative française au Québec au début du XXe siècle », explore les relations entre l'Académie française et la Société du parler français au Canada (SPFC). L'auteure prend en considération l'évolution des rapports entre ces deux institutions en analysant un corpus d'articles parus sur le Bulletin du parler français au Canada, l'organe de diffusion du SPFC. Dans un premier temps, les contacts entre les deux organes sont « respectueux et complaisants » (40) : d'un côté les jeunes Canadiens voient dans ce rapport une possibilité unique pour s'affirmer et cèdent souvent à l'obséquiosité ; de l'autre côté, le monstre sacré français se plaît de son jeune admirateur et le flatte et l'encourage dans sa « lutte contre l'anglicisme » (38), jusqu'à justifier les canadianismes abondants en soulignant leur origine bretonne et normande. Pourtant, le rôle de «mère » (39) et «sœur » (39) protectrice revêtu par l'Académie s'affaiblit de plus en plus; en effet, les quarante immortels perdent leur aura de sacré au fur et à mesure que les Canadiens bâtissent leur identité linguistique, incapables de comprendre et accepter les évolutions et les créations lexicales du nouveau continent. Brancaglion souligne, enfin, comment la SPFC a joué un rôle essentiel pour l'autonomisation et la légitimation du français québécois dans la lutte pour se libérer de sa « double insécurité » (47) : le fait d'être une minorité au sein du Pays, mais aussi sa condition de français dit vernaculaire.

L'ouvrage se termine par la contribution littéraire de Marco Modenesi " 'Le passé ne s'arrête jamais'. Langue et pouvoir chez Jacques Poulin ». L'écrivain québécois est notamment très attentif à la question de la langue, qui est omniprésente dans toute son œuvre, que ce soit sous forme de personnages strictement liés à la langue (écrivain, traducteur, bibliothécaires, etc.) ou tout simplement à travers son évocation dans les discours et dialogues. Toutefois, comme l'affirme Modenesi, Jacques Poulin n'a jamais brillé pour son militantisme, au contraire de ses collègues protagonistes des débats linguistiques depuis les années '70, il suffit de citer Michèle Lalonde et son poème Speak White pour l'affranchissement de la domination de la culture anglaise et de sa langue. Grâce à l'analyse attentive 
de trois romans de Poulin - Chat sauvage (1998), L'anglais n'est pas une langue magique (2009) et L'Homme de la Saskatchewan (2011) - Modenesi réussit à mettre en exergue la subtile dénonciation du romancier québécois à propos du « binôme dominant/dominé » (114) et des préjugés sur la langue française.

Le colloque organisé par le CISQ est l'épreuve que la thématique langue/ pouvoir est toujours actuelle et qu'elle intéresse plusieurs champs d'étude. Les contributions présentées offrent un tableau de la société et de la culture québécoises du XIXe siècle à nos jours et analysent et approfondissent la question langue/pouvoir sous diverses perspectives. L'ouvrage est sans doute adressé à un public spécialisé, toutefois les survols historiques et méthodologiques permettent aux néophytes aussi de s'en approcher. De plus, les cas hétérogènes sont quand même liés par une forte cohérence, qui empêche de tomber dans « le piège d'un militantisme aveugle » (127), mais qui donne, au contraire, des pistes de réflexions et des méthodologies qui peuvent être appliquées et approfondies aussi dans d'autres contextes de minorité linguistique. 


\section{Pfersmann, Andréas ; Porcher-Wiart, Titaua (éds.) (2017). « Francophonies océaniennes ». Interculturel Francophonies, 31, juin-juillet, 417 pp.}

Anna Michieletto

(Università Ca’ Foscari Venezia, Italia)

Ce volume d'Interculturel Francophonies, consacré à la littérature francophone des Îles de l'Océan Pacifique, commence par une " Ouverture » qui contient une « Présentation » et un « Court panorama de la littérature de Nouvelle-Calédonie ». Dans la présentation, Pfersmann et Porcher-Wiart font d'abord référence au concept de " littérature-monde » (11) d'Edouard Glissant, qui reviendra tout le long du livre, pour contextualiser ce monde insulaire francophone de "sociétés de l'oralité » (13) peu connu, qui englobe la Nouvelle-Calédonie, Wallis et Futuna, le Vanuatu et la Polynésie française. Dominique Jouve, après un aperçu historique de la NouvelleCalédonie, s'interroge sur l'existence d'une " matière littéraire spécifique » (27) de cet archipel, sur les possibles liens avec les littératures anglophones de son aire géographique et sur son positionnement en tant que « littérature en émergence » (27) au niveau de la création et de la reproduction. La diglossie marquée par l'usage du français, langue liée à la colonisation, et par l'identité kanak, fondée sur le paysage et la tradition des ancêtres, caractérise la réflexion des écrivains au XXe siècle et sera soulignée dans tout le volume. L'article retrace les étapes de la littérature écrite concernant la Nouvelle-Calédonie à partir des récits de Cook et de La Billardière, en passant ensuite par les relations de voyage et l' «écriture de l'exil » (30) des déportés pendant la période coloniale. Dans la poésie des années 1860 et 1870, les mythes européens de l'exotisme et du bon sauvage qui habite une sorte de paradis terrestre côtoient la vision noire des bagnards, parmi lesquels Louise Michel, déportée de la Commune, qui reconnaît l'Autre kanak avec une sensibilité fraternelle et romantique. À la même époque remonte la dure et malheureuse réalité peinte par les colons exploiteurs des mines de nickel. Àprès la Première Guerre mondiale, avec Baudoux, Laubreaux et Mariotti, l'Autre est encore considéré un sauvage, inhumain, refoulé dans les réserves, tandis que pour en arriver enfin à un dialogue entre les cultures il faut attendre les années '70. Nicolas Kurtovitch préfère à la notion de « métissage » celle d' « interface » culturelle (46) et évite tout 
particularisme linguistique pour atteindre « un effet d'universalité » (46). Pierre Gope et Ismet Kurtovitch, au contraire, développent « un langage théatral directement lié aux parlures quotidiennes, afin de « tisser un lien identitaire fort avec le public » (47). En parallèle au développement des mouvements politiques indépendantistes, on assiste à un renouveau littéraire et thématique dans les chansons, les $\mathrm{BD}$, les nouvelles, les concours de slam et la recherche linguistique : Déwé Gorodé a bouleversé la poésie, Wamo s'est lancé dans un nouveau genre entre poésie et oralité, Purawa écrit sur Facebook, Hnacipan transmet aux jeunes son savoir, revues et maisons d'éditions contribuent à la diffusion de cette " écriture du Pacifique » (58) qui a atteint des « accents trans-océaniens » (58).

Nous retrouvons Pierre Gope, auteur issu d'une famille de chefs et médiateurs, dans la section «Questions identitaires et politiques ». Eddy Banaré analyse la pièce Okorenetit ? Où est le droit ?, où Gope met en scène "un espace travaillé par son héritage colonial" (65), dans lequel on essaye de dialoguer avec la France pour « la reconnaissance du trauma colonial et l'engagement d'un processus de décolonisation » (66). Il fait référence aux « Evénements » de 1984-1988 et aux accords de Matignon-Oudinot et de Nouméa (1998). La pièce, éditée en 1997, illustre une démarche de reconfiguration identitaire liée à la politique et à l'Histoire. Il s'agit d'un véritable théâtre kanak à partir de la troupe et des espaces scéniques, que Gope a créé après sa découverte de la dimension thérapeutique du théâtre francophone africain. La pièce, qui a un fort aspect éthique, aborde une thématique compliquée : la résolution juridique d'un cas de viol collectif, pratique courante en milieu kanak. La victime demande justice au tribunal traditionnel, puis au tribunal français. Le théâtre est ainsi appelé à un rôle de dénonciation de la « quotidienneté tragique » (72) et de «prévention sur le terrain » (75). Gope insère des chants en nengone, sa langue maternelle, « moment d'exclusion-inclusion » (76) pour le public européen. Gope situe sa mise en scène contemporaine dans un espace difficile, de seuil : la tribu est accusée comme « lieu de non-dit » (79), la recherche de justice de la jeune fille passe par la rupture générationnelle et « le franchissement symbolique d'espaces structurants la Nouvelle-Calédonie » (79).

On passe ensuite à la poésie polynésienne avec l'article de Mounira Chatti, «Un mot qui se perd, c'est un trésor social qui disparaît ». L'auteure présente le poète Henri Hiro, qui vise dans ses ouvrages à s'approprier le français, mais en gardant l'importance de sa culture et de son identité polynésiennes face au risque de voir leur assimilation ou leur dissolution. À ce propos, elle rappelle le « devoir d'originalité » (90) prôné par Aimé Césaire et Kateb Yacine, qui dans son roman Nedjma veut « montrer en français que l'Algérie n'était pas française » (91). Appartenant à une famille d'écrivains, Déwé Gorodé suit elle aussi ce chemin, afin d'exprimer en français la « conception kanak des choses » (92). Chatti expose donc à titre d'exemple cette démarche de Gorodé dans la nouvelle «Benjie, mon 
frère... », qui appartient au recueil L'Agenda (1996). L'histoire raconte d'un enfant kanak qui aide un jeune de la ville à redécouvrir ses racines culturelles et religieuses et à devenir militant. Le traumatisme colonial est pourtant trop fort et la nouvelle se termine par la mort du narrateur : Gorodé condamne la colonisation, mais également « l'institutionnalisation du mouvement indépendantiste » (95).

Un point de vue très original nous est offert par Fritz Peter Kirsch, qui procède à la comparaison de quatre romans contemporains : deux tahitiens en français et deux occitans en langue d'oc, appartenant à « deux littératures dominées » (99) . Kirsch se situe, lui aussi, parmi les défenseurs d'une « littérature-monde en français » (99), caractérisée par la diversité. Voilà pourquoi il refuse l'idée "d'une Francophonie avec majuscule, unie et fraternelle » (99) et suggère « une nouvelle approche de la littérature française parisienne-hexagonale » (100). Il note comment la littérature tahitienne se rapproche de celles d'Amérique Latine et des États-Unis : elle se lance dans l'engagement sans passer préalablement par la phase du « régionalisme folklorisant » (112) et elle fait sienne la langue de l'ancien colonisateur. Au contraire, dans les romans en langue d'oc analysés, la lutte contre la domination passe par « la conquête d'un univers alternatif » (112) qui déconstruit les normes et les conventions culturelles.

Andréas Pfersmann illustre au début de son article un sonnet de JeanMarc Tera'ituatini Pambrun, Le regard des vaincus. Il veut nous faire entrer d'emblée dans la critique du colonialisme chez Spitz, Peu et, évidemment, Pambrun. La domination française dans les îles du Pacifique est caractérisée par les essais nucléaires. Cette politique a été appuyée par « l'élite 'demie', la minorité privilégiée [...] complice du colonisateur » (119). Le peuple, bien que pauvre et aliéné, n'est pas pourtant exempt de caricaturer le modèle et l'idéologie dominants. L'école joue à ce propos un rôle déstructurant par rapport à l'identité sociale et culturelle : elle produit des adultes « souséduqués, [...] futurs travailleurs sous-formés que l'on pourra sous-payer » (124) dans un monde modernisé et consumériste. Lécole du dimanche, tenue en tahitien par les missionnaires protestants, aide à préserver le patrimoine culturel autochtone, mais malheureusement n'arrive pas à éviter l'échec social du protagoniste de Hombo. Transcription d'une biographie (2002) de Spitz. Le mot « hombo » définit les jeunes marginalisés. L'auteure considère le christianisme «le traumatisme fondateur » (129). Pambrun préférerait retourner au paganisme originaire, ce qui ne convainc pourtant pas Pfersmann, qui indique comme stratégies de résistance « le militantisme politique et/ou syndical ; la reconquête de pratiques culturelles ancestrales ; la réappropriation de l'histoire des vaincus et de la mémoire collective » (130-1) et enfin une écriture où tout se révèle être politique.

L'article d'Odile Gannier, qui ouvre la section "L'enjeu des langes », propose un tableau du Pacifique du Sud caractérisé par la présence de littératures « excentrées » (145), anglophones et francophones, qui rejettent 
les concepts de littératures du Commonwealth ou de la Francophonie. À partir du bouleversement qui a suivi la publication du manifeste de 2007 Pour une littérature-monde en français (146), auquel d'autres auteurs de ce volume ont fait référence, on invoque donc l'usage de la langue comme « bien commun » (153) dans ses variantes les plus créatives, selon les contextes culturel, géographique et chronologique. Par exemple, dans Tergiversations et rêveries de l'écriture orale, Flora Devatine s'interroge sur le processus scriptural d'une manière exhibée et très explicite. En Polynésie le "reo ma'ohi », qui indique «l'ensemble des langues polynésiennes du territoire » (150), est langue officielle depuis 1980, il est enseigné à l'école et peut enfin instaurer un dialogue avec la langue du colonisateur à partir d'une "reconnaissance mutuelle » (151). Les limites des « littératures de l'exiguité » (151) concernent cependant la diffusion et la maîtrise de la langue de la part du lecteur aussi bien que de l'écrivain. L'utilisation d'une langue locale dans une publication en français, que ce soit à travers l'introduction de termes vernaculaires, de dialogues ensuite traduits ou de tournures syntaxiques, dépayse le lecteur européen et l'invite dans un univers autre. Dernièrement les glossaires finaux ne fournissent plus toutes les traductions : ce « libre usage de la langue [...] est un acquis littéraire et culturel » (163). Les écrivains apprivoisent ainsi le français et délimitent un espace d'autonomie dans les frontières de la langue.

Titaua Porcher-Wiart se penche, elle aussi, sur le recueil Tergiversations et rêveries de l'écriture orale (1998) de Flora Devatine, caractérisé par une « oralité savamment recostruite » (306), qui renvoie au slam et à des performances traditionnelles telles que l'orero (300) ou le paripari fenua (303), et par l'omniprésence des corps de l'écrivaine et du lecteur. Ecrire constitue en effet pour Devatine « un parcours de la corporéité par une exploration des formes du sentir corporel » (296), qui s'exprime à travers un remaniement de la langue et qui manifeste une contre-culture.

À l'intérieur de ce contexte multilinguistique, Stéphanie Geneix-Rabault se focalise sur le slam des auteurs kanak en Nouvelle-Calédonie. Il s'agit d'un espace créatif «basé sur des relations complexes, mais néanmoins inclusives et dynamiques » (174), où les cultures et les langues (le français et les langues locales) témoignent de la diversité du territoire et se mettent à l'écoute les unes des autres d'une nouvelle manière. Ces textes, que l'on a la possibilité de savourer tout au long de l'article, sont aussi susceptibles d'être exportés grâce à la création du Poemart (Pole d'Export de la Musique et des Arts de Nouvelle-Calédonie). L'auteure termine en se demandant si le slam pourrait contribuer à la construction d'un nouveau type de lien socio-linguistique, car il a réussi à « dépasser les rapports de domination du français » (188) : il introduit en effet des passages en différentes langues et promeut l'usage de ces dernières en tant qu'outils d'une « écriture hybride et métissée » (187), qui exprime l'oralité et l'identité, étroitement liées à la musique et à la danse. 
Dans le même contexte de la Nouvelle-Calédonie, Hamid Mokaddem analyse le «premier essai en langue française d'un auteur kanak » (197) : l'épopée de la guerre d'Ataï. Le texte, qui relate des événements de 1878, date de 1965. Mokaddem passe ensuite à Sous les cendres des conques de Déwé Gorodé, auteure militante qui a été aussi incarcérée et qui propose dans son ouvrage une scansion temporelle basée sur le calendrier cyclique kanak de la culture de l'igname. Nous retrouvons encore une fois le dramaturge Pierre Gope et son attachement à sa langue maternelle, le nengone, qui lui permet de visualiser ce qu'il traduit sur la scène d'Okorenetit ? Où est le droit ? et de montrer une " société en devenir » (213) dans le développement de conflits sociaux très complexes. Mokaddem reconnaît à ces textes « des richesses langagières insoupçonnées » (214) et propose l'étude de ces « intermondes »(214) pour en dégager les « sens pluriels » (214) et leur façon de «faire devenir autre une même langue » (214).

Audrey Ogès illustre la notion $\mathrm{d}^{\prime}$ " interlangue » : une nouvelle langue conçue à partir du français et de la vision du monde kanak. Flora Devatine, Déwé Gorodé et Chantal T. Spitz travaillent sur la langue dans le but de dépasser le traumatisme colonial et affirmer leur identité. La référence à Edouard Glissant et à d'autres auteurs caribéens pour leur volonté de « créolisation du monde » (223) est incontournable. Pour les trois auteures océaniennes, écriture est synonyme de lutte : leur chaos intérieur sort violemment et provoque une souffrance thérapeutique, qui leur permet de survivre et d'exprimer leur identité et leur « interlangue subjective » (240). Des récits hybrides, une tension et un désordre linguistique qui refusent ou plient la norme reflètent la résistance centrifuge à l'homogénéité, vers une « symphonie des langues » (226). De la simple traduction à une fusion entre les idiomes, on fait jaillir une nouvelle réalité littéraire : " pour créer un sens nouveau, il faut une langue nouvelle » (236) qui passe par un « corps à corps » (236) avec le français.

Le troisième volet du volume, consacré à « Corps/parole/espace : regards anthropologiques et écocritiques », décrit des perspectives interdisciplinaires suivant « l'aspect écologique de l'exégèse » (249), qui amène à tisser un « discours éthique » (250). Anne-Sophie Close analyse le corpus littéraire polynésien dans son lien avec l'environnement (le « corps du monde », 249) et le corps humain. "Lautochtonie se manifestant par un rapport symbiotique et sensoriel à l'environnement » (251), il est possible d'effectuer une « lecture tidalectique » (251), à savoir une lecture qui reflète le mouvement de la marée, son flux cyclique, circulaire et fluide et son appartenance aux îles. Cette lecture a été proposée notamment pour Tergiversations et rêveries de l'écriture orale de Devatine. Cet ancrage, au territoire d'un côté et aux formes traditionnelles orales de l'autre, pousse à la déconstruction dans des romans polyphoniques (253-4) où « la violence corporelle répond [...] à la violence sensorielle et environnementale » (260). Les personnages sont des « ex-ilés » (257), aliénés et schizo- 
phréniques à cause du détachement de leur identité culturelle. Ils doivent donc reconnaître leur appartenance au territoire et à son peuple, comme il arrive dans Le Roi absent de Moetai Brotherson ou dans L'île des rêves écrasés de Chantal T. Spitz. Le corps, "vaisseau de reterritorialisation » (262), permet à l'individu de se recentrer dans son « paysage textuel et sensoriel/environnemental » (264) et de reconquérir son identité. Le corps textuel aide à s'émanciper et à passer à l'action.

Julia L. Frengs souligne elle aussi la relation entre la terre et le corps qui est au centre de la pensée kanak. Elle propose « une approche écocritique post (ou anti) coloniale » (274), suivie d'exemples textuels de Peu, Spitz, Gorodé, Pourawa et Gope, focalisés sur les «violations des droits humains environnementaux » (274). Ces auteurs s'occupent notamment des essais nucléaires en Polynésie française, de l'exploitation du nickel en Nouvelle-Calédonie et de la promotion du modèle consumériste occidental dans tout le Pacifique océanien. La littérature de cette région s'avère donc un outil de dénonciation des injustices contre les sociétés humaines et les milieux naturels locaux et un lieu de contestation éco-éthique qui se rattache aux discussions transnationales sur l'avenir économique et les choix complexes du monde globalisé.

Le dernier article de cette section se détache des autres en nous présentant « L'écharde, roman 'australien' de Paul Wenz », " nomade [...] en quête d'aventure » (324), exception francophone dans la littérature anglophone de l'île. Le livre, publié en 1931, est centré sur la rencontre de la protagoniste, anglaise, avec la vastité et les spécificités du paysage australien.

«Francophonies océaniennes » présente donc différentes perspectives de lecture d'une (ou plusieurs ?) littérature peu connue et qui a difficilement accès aux éditeurs européens. Ses auteurs se caractérisent par leur « opposition, assimilation, détournement, appropriation, émancipation » (13) vis-à-vis de la culture dominante. Cette plongée dans le temps et dans l'espace océanien se termine par une conclusion et une orientation bibliographique. L'« Anthologie portative de la littérature francophone du Pacifique » qui la précède permet au lecteur de savourer plusieurs typologies des textes, en poésie et en prose, analysés dans les articles tout au long du volume et qui bien représentent la littérature de Tahiti et de la Nouvelle-Calédonie. 


\section{Bernovsky, Victor (2017). « Francité, américanité et indianité dans le roman québécois contemporain ". Interculturel Francophonies, 32, novembre-décembre, $326 \mathrm{pp}$.}

Veronica Brunetto

(Università Ca'Foscari Venezia, Italia)

Le numéro 32 d'Interculturel Francophonies traite de l'identité québécoise dans le roman contemporain québécois. Aux trois sphères d'influence affectant le sentiment d'appartenance québécois, à savoir la francité, l'américanité et l'indianité, une variable est ajoutée : l'immigration.

\section{Première partie : «Survol de l'évolution de la société québécoise »}

La première partie de ce numéro de la revue offre un point d'approche socio-historique, en retraçant un aperçu de l'évolution de la société québécoise, rapide, mais exhaustif, qui donne un outil pour comprendre les enjeux littéraires et identitaires des articles suivants.

Dans l'article «S'intégrer progressivement dans les dynamiques des Amériques : rencontrer l'autre » (27-40), Patrick Imbert rappelle le rôle joué par les différentes nationalités dans la constitution du Québec. Néanmoins, l'auteur remarque que les facteurs d'une première conception identitaire québécoise provenaient des codes historico-culturels liés aux dynamiques commerciales monopolisées par les élites anglophones. À partir de cela, la littérature a été consacrée aux valeurs traditionnelles jusqu'aux années '50. Par contre, la génération d'après la Deuxième Guerre mondiale change la perception des cultures minoritaires par rapport à la culture majoritaire. Celle-ci, aujourd'hui, embrasse la diversité ethnoculturelle du Pays dans une perspective interculturelle, "dans l'esprit du pluralisme, un modèle [...] axé sur la recherche d'équilibres entre des impératifs souvent divergents » (32). D'ailleurs, la question linguistique et la discrimination anglophone relèguent le Québec francophone à une position de minorité, une position caractérisant la littérature d'émigration également. L'identification entre les Québécois et les émigrés offre un nouveau point de vue, dilatant une identité plurielle à l'échelle d'une géographie mondialisée et relationnelle. 
La contribution de Jean-François Létourneau, intitulée « La durée réelle du continent » (41-52), renseigne le lecteur sur la véritable permanence du continent américain, qui n'est pas tout à fait « Nouveau », car son histoire ne commence pas en 1492, comme une vision européocentriste l'a fait longtemps croire. L'auteur illustre plusieurs aspects de ce malentendu créé par la quête identitaire des descendants européens s'estimant enracinés sur le territoire d'un autre continent. La seule voie de guérison semble être le voyage aller-retour sur le continent même, pour découvrir l'Amérique en renouant avec ses véritables racines, " en s'inspirant [...] des cultures autochtones » (46). Ainsi, Létourneau propose aux lecteurs les clefs pour comprendre l'influence grandissante de la culture des Autochtones à l'intérieur de la littérature québécoise contemporaine.

\section{Deuxième partie : « Transformations des littératures autochtones«}

À la suite de cette esquisse socio-historique, François Paré et Jeanette Den Toonder abordent la question littéraire de la société québécoise. Tout d'abord, Paré donne un cadre général du panorama littéraire québécois, tandis que Den Toonder entre dans le vif de deux œuvres contemporaines : Ourse bleue de Virginia Pésémapéo Bordeleau et Ru de Kim Thúy.

Dans « La littérature québécoise du 21ème siècle. Cœur et marges de l'Amérique » (55-67), François Paré prône la richesse et la complexité de la littérature québécoise, en présentant les « grands champs thématiques et institutionnels qui [...] semblent rendre compte » (56) de ses tendances atypiques. Il s'agit du renouvellement du milieu de l'édition littéraire relevée par une multiplication d'initiatives indépendantes. Ce renouvellement a ouvert le champ à des orientations littéraires inédites, comme la redécouverte du lyrisme intimiste, où la quête existentielle du sujet est apaisée par la réappropriation du paysage extérieur, source de l'identité. Cette révolution éditoriale a porté également à la légitimation des littératures autochtones d'expression française, dont « la dimension pédagogique [...] reste centrale à un processus de guérison qui implique l'ensemble de la société québécoise » (61). L'auteur termine sa contribution en citant quatre écrivains québécois contemporains, Hélène Dorion, Pierre Nepveu, Catherine Mavrikakis et Louis Hamelin. La reconnaissance que la critique a réservée à ces auteurs est emblématique de l'envergure de la littérature québécoise, bien qu'elle soit encore méconnue au grand public.

De fait, l'article de Jeanette Den Toonder, « Nouvelles perspectives sur l'écriture féminine au Québec. Liens familiaux, voyages et souvenirs dans Ourse bleue (2007) et $R u$ (2009) » (69-88) étudie les développements de l'écriture féminine au Québec, en considérant à la fois « l'éveil du féminisme autochtone au Canada et l'ancrage de l'écriture des femmes mi- 
grantes au sein de la littérature québécoise » (71). Dans $R u$ de Kim Thúy et Ourse bleue de Virginia Pésémapéo Bordeleau la réflexion identitaire est inscrite dans les rapports intergénérationnels entre mère et fille, obligées à l'exil, ou au voyage physique. Le rôle de passeuses de la mémoire rend les femmes des intermédiaires de la communauté, dont on entend la voix à travers les expériences individuelles. Les questions liées à l'appartenance géographique, aux relations avec les proches et au passé de ces femmes « vivant entre deux cultures » (83) trouvent une réponse dans le métissage, clef pour un nouvel équilibre individuel et social.

\section{Troisième partie : « La problématique de la francité et de l'indianité 》}

Dans la troisième partie de ce numéro de la revue, Nicolas Davignon et Sylvie Vignes traitent de la quête identitaire québécoise lorsque la question de l'indianité s'entrecroise avec celle de la francité. Cela, en examinant les romans Petit Homme Tornade (1996) de Roch Carrier et Ce qu'il reste de moi (2015) de Monique Proulx.

Au cours de son article « De la dualité à l'unité dans Petit Homme Tornade de Roch Carrier. Le voyage identitaire : le Québécois face à l'Amérique » (91-108), Davignon illustre la représentation du conflit créateur congénital à l'identité québécoise dans le roman de Carrier. Ici la dualité originaire est incarnée par Robert Martin, un homme blanc et Charlie Longsong, un vieil indien. Les deux protagonistes matérialisent, par leur rencontre, le choc entre « deux systèmes de valeurs : celles américaines (nature, nomadisme, liberté, etc.) face à celles européennes (culture, sédentarité, stabilité, etc.) » (92). Une opposition immobilisant dans un passé de souffrance, qui sera finalement surmontée au niveau du présent, où le métissage des cultures indienne, française et canadienne est la source pour une identité unique.

$\mathrm{Au}$ cours de l'article «Utopie et identité montréalaise dans Ce qu'il reste de moi de Monique Proulx »(109-26), Sylvie Vignes observe les entrelacements des voix dans le roman polyphonique de Proulx, afin de montrer la constitution complexe de l'identité montréalaise. La narration symbolise le passé utopique de Montréal, par le récit de l'histoire de Jeanne Mance et par son avenir multiculturel. Ce texte montre que tous les personnages du roman agissent pour un but transcendant la trivialité de la vie contemporaine, tout comme le but de la «Folle entreprise » de Jeanne Mance. Ainsi, Proulx même affirme que la « motivation profonde d'atteindre le meilleur de nous est le vrai sujet $»^{\mathbf{1}}$ de l'œuvre.

1 «Rencontre avec Monique Proulx : le cœur battant de Montréal, Ce qu'il reste de moi, ou Montréal comme microcosme de l’humanité ». Le Devoir, 25 avril 2015. 


\section{Quatrième partie : « Francité et/ou américanité 》}

À l'intérieur de la quatrième partie, les questions soulevées par l'ascendant de la culture française et de l'appartenance à l'Amérique entrent en jeu dans le discours sur l'identité québécoise.

Adrien Guyot développe au fil de l'article intitulé " Une Amérique en filigrane » (129-46), l'évolution du concept d'américanité dans l'identité culturelle et littéraire québécoises. À partir de la fondation de la Nouvelle-France, Guyot relate les passages qui font surgir un imaginaire typiquement américain, pour aboutir au cadre critique multidisciplinaire actuel. En raison d'un « contexte mondial caractérisé par de nombreuses mutations sociodémographiques » (129) et des événements qui ont marqué la politique étatsunienne, il y a eu une « réactualisation mythique continentale » (142) en ce qui concerne la définition de l'identité québécoise. De fait, c'est l'appartenance au continent américain qui atteste l'identité du sujet, qui est libre de se déterminer, aujourd'hui, par rapport au « métissage biologique et culturel » (143).

Après la reconstruction de Guyot, Jean Morency montre dans son article la représentation littéraire de la francité, de l'américanité et de la franco-américanité : "Entre américanité et francité : Les yeux bleus de Mistassini de Jacques Poulin » ( 147-59). Morency explique que la francité dérive de l'influence « livresque, artistique, culturel[le] » (150) exercée par la France jusqu'aux années ' 50 . En revanche, l'américanité est le fruit de l'adaptation des collectivités québécoises à un milieu nouveau et de leur perception des paradigmes socioculturels étatsuniens. Enfin, la franco-américanité est issue de la mémoire collective provenant de « l'expansion territoriale [...] qui remonte à la Nouvelle-France » (149). Pendant les vicissitudes du roman, ces trois tendances sont incarnées par trois figures d'écrivains emblématiques : Ernest Hemingway (américanité), Gabrielle Roy (franco-américanité) et Philippe Rollers (francité).

L'article "L'Amérique est aussi un roman québécois » (161-71) de l'écrivaine Madeleine Monette, présente un point de vue interne sur la question identitaire, celui de Monette même. En tant que migrante, l'auteure expose sa double vie au sein des États-Unis : montréalaise, elle vit à New York depuis 30 ans. Entre deux langues et deux cultures, Monette perçoit « l'hybridité de [s]on devenir sur le continent américain, [s]a façon particulière de [s]'inventer à la croisée d'autres cultures » (162). Une identité en devenir où l'écrivaine est décentrée pour mieux se réorienter. Cette réorientation caractérise également la littérature québécoise, qui a décentralisé la nation, l’histoire et la mère patrie. Tout comme les auteurs qui l'ont précédée, Monette déclare qu'il y a une « multiplicité des centres » (170) marquant l'identité et la littérature québécoises positivement.

Jimmy Thibeault met l'accent sur les causes de cette identité multiple dans sa contribution intitulée « L'année la plus longue de Daniel Grenier : 
remonter la trace de la franco américanité »(173-98). Selon Thibeault, le revirement de la conscience identitaire au Québec procède de l'échec du referendum des années '80. Depuis cela, il y a eu un " éclatement des repères spatiaux » $(173)^{2}$ et temporels. D'une « société pensée » (174), où le mythe collectif s'inscrit dans l'Histoire nationale (de longue durée), la littérature converge vers la représentation d'une "société vécue 》 (174), où l'expérience subjective (de courte durée) s'ouvre à l'ailleurs. La quête de l'identité individuelle coïncide avec celle de l'identité culturelle. L'histoire de la présence française sur le sol américain mise en scène par le roman L'année la plus longue de Daniel Grenier le décrit admirablement. Les récits de la vie extraordinaire d'Aimé Bolduc et de sa progéniture offrent une relecture humanisée de l'histoire, dès la Conquête de 1759 jusqu'au futur (2047), en passant par le 11 septembre 2001. Une relecture qui « permet [au sujet] de créer, dans son présent, sa propre histoire et de l'inscrire dans la longue durée » (195).

Dans le sillage du voyage, Victor Bernovsky analyse « Les héros de Jacques Poulin sur la route américaine » (199-219). Bernovsky lie la francité et l'américanité des personnages - en distinguant l'apport des cultures anglophone et amérindienne - pour aboutir à définir l'identité québécoise, résultat du métissage. Celui-ci est le point d'arrivée de la quête symbolisée par le voyage de Volkswagen blues; une quête "à trois dimensions : historique, géographique et spirituelle » (201). En effet la recherche des origines ancestrales se déroule parallèlement à l'exploration territoriale : Jack et Pitsémine reviennent sur les faits historiques répandus sur la route, grâce aux ouvrages historiques et aux musées commémoratifs qu'ils visitent. De plus, tout au long de la narration, l'intertextualité rapporte au lecteur le discours sur l'Amérique, tandis que le langage est utilisé comme un outil critique. Effectivement, les expressions en anglais utilisées par les protagonistes semblent renvoyer au danger de l'assimilation et à la violence indissociable de l'expansion coloniale anglophone.

La question de l'intertextualité est en outre touchée par Voichita-Maria Sasu dans l'article « Extraterritorialité et intertextualité comme manifestations de l'américanité dans La Pêche blanche de Lise Tremblay » (221-31). Sasu souligne l'influence réciproque du Québec et des États-Unis, à partir des mythes fondateurs affins et des thèmes intertextuels, comme " l'image du vagabond et de l'errance, la marginalité, la solitude » (221). Ces thèmes se retrouvent dans La Pêche blanche, roman où deux frères incarnent les pôles opposés caractérisant leurs vies et l'imaginaire Nord/Sud (froid/

2 Citation tirée de Kwaterko, Józef (2001). « Clivages, ex-centricité, nomadisme : l'identité culturelle et l'imaginaire de l'espace dans le roman québécois ». Lintvelt, Jaap ;Paré, François (s.d.), Frontières flottantes. Lieu et espace dans les cultures francophones du Canada / Shifting Boundaries. Place and Space in the Francophone Culture of Canada. Amsterdam ; New York : Éditions Rodopi, 147-59. 
chaleur, bruit/silence, solitude/foule, contrainte/liberté). À Saguenay vit Robert, à San Diego vit, au cours de l'hiver, Simon. Tous les deux sont écrasés par un sentiment d'inadéquation, qu'ils parviendront à surmonter grâce à une "révolte tranquille » (228), qui changera leur relation à la nature : une force « puissante puisqu'elle peut vaincre jusqu'au temps [et] doit être un point d'appui pour les cœurs en dérive » (229).

L'article « Le français de France dans Folle de Nelly Arcan et Fleurs de crachat de Catherine Mavrikakis » (233-47) d'Élisabeth Nardout-Lafarge expose un aspect autrefois prééminent dans la littérature québécoise : l'usage du français de France. Dans Folle d'Arcan s'expriment « le conflit des genres [...] et l'attitude coloniale de l'ex-amant, jusqu'à en faire l'archétype négatif du Français au Québec » (236). Par contre, Dans Fleurs de crachat, Mavrikakis peint la différence entre le français hésitant du Québec et celui triomphant de France, en relevant le passéisme ridicule de celui-ci, réduit à une langue vaniteuse et inoffensive. Pourtant, Nardout-Lafarge remarque que « les deux textes [...] attachent à la parole française les mêmes traits de maîtrise, de force, de domination » (243), signe d'un trait originaire encore dominant.

Dans «Quatre versions américano-québécoises du mythe d'Icare ou les paradoxes de la réussite » (249-70), Eva Voldřichová Beránková présente la trilogie d'Éric Plamondon 1984. Un modèle d'écriture « polyphonique et nerveuse » (251), qui communique les informations par bribes (modalité typique de la post modernité, en opposition aux genres du passé). L'auteure expose les trames des trois tomes, à savoir Hongrie-Hollywood Express (2011), Maïonnaise (2012) et Pomme S (2013), dont les trois protagonistes - Johnny Weissmuller, Richard Brautigan et Steve Jobs - incarnent trois «Icare » du rêve américain. La perspective d'un quatrième héros, Gabriel Rivages, éclaircit le lecteur sur le succès des trois figures. C'est la progéniture qui en détient la clef, car la seule façon de survivre à sa mort/déchéance dépend de l'interprétation et du récit qu'en fera la postérité. Par conséquent, « le mot définit le monde, le transmet et le 'sauvegarde' » (267).

Dans « La transparence du réel. Le cycle du voyage de Louis Gauthier et la contre-culture » (271-81), Frédéric Rondeau propose un survol sur la série de quatre récits de voyage de Louis Gauthier, expression de l'héritage de la contre-culture. Au Cours de Voyage en Irlande avec un parapluie (1984), Le pont de Londres (1988), Voyage au Portugal avec un Allemand (2002) et Voyage au Maghreb en l'an mil quatre cent de l'Hégire (2011), il y a une désillusion progressive - concrète et symbolique - des idéaux animant la contre-culture. Toutefois, l'écriture témoigne d'un lien avec ces idéaux déçus, car elle incarne la transposition la plus fidèle possible de l'expérience. Arsenault, écrivain en quête identitaire sur la route, trouve un réconfort dans l'écriture, qu'il abandonne pour une période par manque de transparence. Après son retour au Québec et sa réconciliation avec son identité profonde, il se consacre à un projet envisageant « la transcription 
la plus efficace de ce à quoi j'assist[e], non la plus lyrique » (278), véritable legs de la contre-culture.

Cet excursus sur la question de l'identité québécoise se termine par l'article de Jérôme Melançon traitant du voyage au Canada comme espace de définition du soi. Dans « L'Ouest canadien comme terre de transformation : du fédéralisme comme arrière-plan de quelques romans québécois » (283-310), l'auteur analyse quatre romans : Edmonton (2013) de Guillaume Berwald, Go West, Gloria (2014) de Sarah Rocheville, Le mur mitoyen (2014) de Catherine Leroux et Okanagan (2016) de Sara Lazzaroni. La fuite, thème commun aux romans, assume des significations différentes, cependant, elle rend possible un éloignement qui ouvre à l'exploration et à la transformation du soi. De fait, cette transformation " requiert l'épreuve. Le sentiment de l'absence, le séjour dans un élément étranger [...] mais aussi un retour » (298). Ainsi, en facilitant les transmigrations interprovinciales, le fédéralisme est à l'origine d'une dialectique des lieux lointains et prochains par rapport au Québec. Une situation qui aboutit à la constitution d'un arrière-plan physique et psychologique pour l'écartement, l'isolement et ensuite la réinvention de soi. Pour conclure, la question identitaire demeure indissociable non seulement du pays natal, mais aussi d'un contexte qui représente un ailleurs méconnu, déboussolant, une sorte d"autre' géographique. 



\title{
Biondi, Carminella (éd.) (2016). 1789: les colonies ont la parole. Anthologie, tt. 1-2. Paris : L'Harmattan, 498 pp. Autrement mêmes
}

\author{
Elena Fermi \\ (Università degli Studi di Torino, Italia ; Paul Valéry-Montpellier III, France)
}

L'ouvrage qui va être présenté par la suite est une anthologie dans laquelle Carminella Biondi, professeur de littérature française à l'Université de Bologne, a regroupé tous les écrits rédigés et tous les discours tenus au sujet des colonies par les membres de l'Assemblée nationale française au cours de l'année 1789. L'anthologie fait partie de la collection « Autrement mêmes » dirigée par Roger Little, professeur émérite de Trinity College de Dublin, qui est consacrée à la réédition de textes introuvables en dehors des bibliothèques spécialisées, normalement rédigés par un écrivain blanc et traitant des Noirs ou, plus généralement, de l'Autre. L'ouvrage se divise en deux tomes, précédés par une introduction de Carminella Biondi qui présente et analyse les écrits et les discours que le lecteur pourra trouver et lire ensuite dans l'anthologie. L'auteur se concentre notamment sur les questions fondamentales de la relation, toujours problématique, entre les colonies et la métropole, sur le problème des gens de couleur et sur ceux de la traite négrière et de l'esclavage, qu'elle identifie comme étant au centre des différents écrits et discours proposés. Afin de rendre compte de ces différentes questions et de permettre au lecteur un repérage immédiat, les deux tomes sont divisés chacun en deux sections, qui présentent les quatre thèmes cités ci-dessus. Dans le premier tome on trouve, d'un côté, les textes concernant les relations entre les colonies et la métropole et, de l'autre, ceux qui concernent le statut des gens de couleur; le deuxième tome rassemble, en revanche, les documents qui se rapportent à la traite négrière et au phénomène de l'esclavage.

La première section intitulée « Nous aussi nous sommes Français. Vivent les colonies! », présente des réclamations, des doléances, des remontrances et des requêtes faites à l'Assemblée des États Généraux par les colons des Antilles, de Saint-Domingue et du Sénégal, ainsi que des motions et des discours des députés siégeant à l'Assemblée qui rendent compte des questions relatives aux colonies. Même si les problèmes concernant les possessions françaises d'outre-mer sont très nombreux, Biondi affirme qu'ils peuvent être regroupés, du point de vue des colons, en deux grandes 
catégories. La première met l'accent sur la liberté de commerce et d'approvisionnement, c'est-à-dire le fait de ne plus être lié du point de vue commercial uniquement à la métropole, tandis que la deuxième demande l'opportunité d'affaiblir le pouvoir absolu des administrateurs coloniaux qui rend impossible aux colons toute intervention dans la gestion du territoire qu'ils habitent et dont ils connaissent donc bien mieux les caractéristiques et les besoins. On relève ici l'exception constituée par Saint-Domingue, la seule colonie à avoir élu ses députés qui la représentent, même si de manière irrégulière, à l'Assemblée, car les directives royales et ministérielles n'en prévoyaient pas la convocation.

La deuxième section s'intitule «LAristocratie de la peau » et regroupe les réclamations présentées à l’Assemblée par les « Gens de couleur », expression avec laquelle on identifiait généralement les habitants des colonies qui dérivaient du croisement entre un homme blanc et une femme noire et de tous les croisements successifs. Ces réclamations dérivent du fait que le nombre et les richesses de ces colons, que la métropole considère comme des personnes de deuxième catégorie, ont augmenté rapidement et qu'ils sont désormais devenus une force que les Blancs considèrent - à tort ou à raison - comme une menace à leur pouvoir.

La troisième section, «Un trafic aussi funeste que déshonorant. Pour et contre la traite des Noirs », regroupe une série de textes dans lesquels les députés s'interrogent sur ce phénomène, évidemment intolérable par une Assemblée qui proclame les principes de la liberté et de l'égalité de tous les hommes, à moins que l'on n'accepte l'idée que les Noirs n'appartiennent pas à l'espèce humaine. Les discours et les textes présentés mettent en évidence - suivant Biondi - l'impasse où l'Assemblée est destinée à aboutir : l'abolition de la traite entraîne avec elle la remise en question des bases sur lesquelles se fonde l'économie coloniale et donc, si les discours sont très sévères dans la condamnation sur le plan des principes, ils sont bien conscients que ce système ne peut pas être effacé d'un trait de plume.

La quatrième et dernière section, « Mangez plutôt de l'herbe et soyez juste. Pour et contre l'esclavage », présente enfin les discours et les textes qui posent la question de l'abolition tout court de l'esclavage, de l'affranchissement donc des esclaves qui travaillent dans les colonies sur lesquels le maître a un pouvoir absolu de vie et de mort. Biondi fait remarquer, à ce propos, la prudence que tous les députés ont dans leurs déclarations, suivant lesquelles l'abolition devra se faire, un jour, mais les temps ne sont pas encore mûrs. Le problème est toujours le même : la prospérité des possessions coloniales se base, en grande partie, sur l'exploitation de cette main d'œuvre gratuite dont la métropole profite largement et les bases de ce système seraient ébranlées par une décision de ce type.

La lecture de cette anthologie donne donc un panorama des discussions et des débats autour des colonies qui ont eu lieu, au cours de 1789, à l'inté- 
rieur de l'Assemblée constituante. L'auteur veut mettre en relief que, si une certaine attention a été portée sur les problèmes concernant les possessions d'outre-mer par les représentants de la métropole, aucune décision importante n'a cependant été prise, car aucun modèle alternatif d'organisation de ces territoires n'avait été prévu. Biondi remarque également que ces discussions ne sont qu'un début et que, même si elles n'aboutissent à rien en 1789, elles permettront d'arriver, le 4 février 1794, à l'abolition complète de l'esclavage proclamée par la Convention nationale.

L'anthologie est enrichie par une série d'illustrations provenant des collections des auteurs et présente une documentation très riche qui permet au lecteur d'approfondir le sujet : on y trouve une bibliographie raisonnée des textes présentés (discours, lettres, ordonnances, précis, réflexions, requêtes) qui, tout en ne prétendant pas à l'exhaustivité, constitue cependant un instrument fondamental pour démarrer d'ultérieures études, et une bibliographie critique. Toutes les deux suivent l'introduction à l'ouvrage et précèdent la partie consacrée aux textes. En annexe au deuxième volume, le lecteur peut aussi trouver une chronologie des principaux événements concernant le sujet traité qui ont eu lieu entre le 18 février 1788 et le 30 décembre 1789, les Cahiers des États Généraux, avec des déclarations des députés des trois ordres concernant les colonies, l'esclavage et la traite des Noirs, les biographies des auteurs et encore les déclarations concernant les mots à utiliser afin d'identifier les lieux et les gens qui faisaient l'objet des discussions. 



\section{Modenesi, Marco (éd.) (2017). « Jouer avec les mots ". Ponts, Langues littératures civilisations des Pays francophones, 17, 245 pp.}

Veronica Brunetto

(Università Ca' Foscari Venezia, Italia)

Lenuméro 17 de Ponts estconsacréauxjeux de mots, au sens des « manipulations de la langue française que la dextérité et les stratégies de chaque francophone peuvent assurer » (7), comme l'explique Marco Modenesi dans l'éditorial de la revue. Ainsi, les jeux de mots sont valorisés dans une perspective de longue haleine, qui englobe figures rhétoriques, transformations des mots, néologismes, voire combinaisons syntagmatiques issues de l'intersection entre français et créole. Les quatre premiers articles montrent à la fois la variété des jeux de mots francophones et la fonction de leur côté humoristique : de la critique socio-politique, à la défense de la langue, en passant par celle de la culture et de l'identité. Suit une étude libre sur le dictionnaire bilingue Nuovo Garzanti di francese (1992), où le français non hexagonal est enregistré dans un esprit avant-gardiste. En conclusion, la revue propose des notes de lecture concernant la francophonie de différentes aires géographiques, occasions pour un développement ultérieur de la question.

\section{1 « Jeux de mots qui percutent, jeux de mots qui enquêtent : la rhétorique engagée d'Abdelhak Serhane»}

Dans son article sur Abdelhak Serhane, "Jeux de mots qui percutent, jeux de mots qui enquêtent : la rhétorique engagée d'Abdelhak Serhane », Francesca Todesco met en évidence l'ironie virulente qui caractérise l'œuvre littéraire de l'écrivain et poète marocain. Une ironie d'antiphrase et de mention, où les éléments du « vocabulaire adversaire » (15) propres aux valeurs et aux comportements du système traditionnel, sont réorganisés, afin de souligner l'hypocrisie du système lui-même. L'analyse de Todesco illustre la présence d'une hypertextualité de dérivation, soit d'un procédé de transformation et d'imitation de textes préexistants, ${ }^{1}$ dont l'uti-

1 En guise d'exemple Todesco rapporte la transformation des prières du Coran.

DOI 10.30687/Tol/2499-5975/2018/20/032

Submitted: 2018-09-08

(c) 2018 | (c) Creative Commons Attribution 4.0 International Public License 
lisation détermine des catégories différentes. ${ }^{2}$ Todesco nous en donne plusieurs exemples saillants à travers la transcription de quelques passages littéraires. L'auteure examine aussi une partie représentative de la production lyrique serhanienne, où sa rhétorique change profondément de forme, en procédant « d'une recherche consciente des effets de soulignement [où] la forme fait toujours chatoyer le sens » (28).

Enfin, Todesco relève comment les jeux de mots de Serhane constituent des «lieu[x] d'un métalangage critique » (31) qui nous parle des idées, des valeurs, voire de la quête intime de l'écrivain.

\section{2 «Visées stratégiques de l'humour linguistique dans le satirique Le Messager Popoli »}

Dans son article Cécile Madiga retrace les objectifs du journal satirique camerounais Le Messager Popoli, en considérant un échantillon d'une centaine de numéros, de 1993 jusqu'à 2015. L'examen de Madiga relève que la méthodologie des procédés rhétoriques utilisés tient « aussi bien du français classique que du substrat linguistique de l'oralité des langues en présence, susceptibles de produire le rire par la distance par rapport à la norme du français » (36; emphase dans l'original). Les visées stratégiques de ces procédés concernent le plaisir (visée ludique), la vente (visée marketing), la critique socio-politique (visée critique) et la légitimation du français endogène (visée identitaire).

Comme nous l'avons anticipé, pour la visée ludique les journalistes exploitent la distance entre le français standard et une forme issue de la manipulation du plurilinguisme. Par contre, le message caché par la devise du Messager Popoli, « Rira bien qui lira le premier », mire à captiver l'attention du lecteur-acheteur potentiel, obligé de se procurer le journal « le premier » sous menace d'en perdre le contenu ludique (visée marketing). Quant à la visée critique, elle apparaît dans le titre Le Messager Popoli, interprétable sur trois niveaux, dont la signification finale résulte : « Moi - Nyemb Popoli [fondateur du journal] - je suis le messager du peuple, contre son oppresseur Paul Biya [Président du Cameroun depuis 1982] » (42). Pour conclure, la variation du français endogène est revalorisée, donc légitimée, par la transgression de la norme syntaxique, lexicale et sémantique, en connivence linguistique avec le locuteur local (visée identitaire).

2 En particulier, il s'agit des catégories individuées par Gérard Genette dans son œuvre $\mathrm{Pa}$ limpsestes (Paris : Le Seuil, 1982), selon les modes de la dérivation de la transformation et de l'imitation en fonction des régimes ludique, satirique, ou sérieux. Le rapport de transformation entre le texte source et le texte cible affecte les catégories de la parodie, du travestissement et de la transposition, tandis que l'imitation du texte source peut donner origine aux catégories du pastiche, de la charge et de la forgerie. 


\section{3 «Ironie et jeux de mots au Québec : enjeux socio-culturels »}

L'article de Chiara Molinari touche les aspects socioculturels relatifs au débat linguistique au Québec, dans ses traits humoristiques. Pour donner un aperçu sur la spectacularisation qui peut découler du sentiment linguistique québécois, Molinari décrit les cas spécifiques de l’humoriste sénégalais Boucar Diouf et du journaliste Antoine Robitaille. Installé au Quebéc depuis les années '90, Boucar Diouf maîtrise parfaitement à la fois le français standard et le français québécois. Les jeux de mots et les figures rhétoriques au sein de ses sketchs reposent sur des agencements sémantiques et phonétiques dont les caractères culturels et identitaires soulèvent souvent des questions épineuses, comme, par exemple, les dangers de l'anglicisation et le métissage.

Quant au journaliste Antoine Robitaille, son blogue Mots et maux de la politique, ${ }^{3}$ annonce son approche critique et ludique à partir du titre, d'où se révèle le double but d'amuser et d'observer la langue et la scène politique québécoises. En particulier, les jeux de mots des catégories et des billets du blogue relèvent « des procédés ludolinguistiques [...] complexes (créations néologiques, aptonymes, noms de famille) »(68-69).

Finalement, il est évident que tant les sketchs de Diouf que les jeux de mots de Robitaille présupposent un arrière-plan socioculturel et sociolinguistique, afin d'en comprendre les questions sociales, identitaires et politiques sous-jacentes.

\section{4 « La rigoladerie héroïque de Raphaël Confiant »}

Dans cet article Francesca Paraboschi analyse l'attitude de l'écrivain martiniquais Raphaël Confiant par rapport au patrimoine oral traditionnel, « qui exploite les jeux des sonorités et les enlacements de sens [...] dans des buts à tour mnémoniques, ludiques ou satiriques » (73). D’abord, les romans intéressés ${ }^{4}$ par cette étude manifestent la volonté de sauvegarder l'héritage culturel et linguistique créole par le biais de l'écriture. De fait, la langue littéraire de Confiant provient d'une manipulation ingénieuse et déroutante du français, créolisé en vertu de la condition de diglossie et de la variété lexicale créole en Martinique. En deuxième lieu, nous assistons au déploiement d'une rhétorique créole, impliquant des figures de style, voire

3 Le blogue a été hébergé sur le site du quotidien Le Devoir, à partir de 2009 jusqu'à 2016.

4 Notamment les romans qui figurent dans la bibliographie de l'article : Bitako-a (Fort de France : Gerec, 1985) ; Chimères d'En-ville (Paris : [Ramsay, 1997] Librio, 1998) ; Le Nègre et l'Amiral (Paris : [Grasset, 1988] Le Livre de Poche, 2004) ; L'Allée des Soupirs (Paris : [Grasset, 1994] Gallimard, 2010) ; La Vierge du Grand Retour (Paris : [Grasset, 1996] Gallimard, 2009) ; Le meurtre du Samedi-Gloria (Paris : [Mercure de France, 1997] 
des « formes propres à l'oral où l'on devine des calques du créole » (79). Pour comble de créolisation, l'auteur parvient à plonger le lecteur au cœur de la mentalité martiniquaise, en adoptant une structure du roman non conventionnelle, fortement déstabilisante. Même la narration décrit des situations grotesques, qui sont censées accentuer les traits d'une société visionnaire et extravagante. Néanmoins, ce tableau palpitant de la société martiniquaise, peuplée des personnages caricaturaux, cache derrière le rire des situations comiques l'héritage d'une condition identitaire difficile, issue de l'oppression esclavagiste. Ainsi, au fond, le rire rappelle l'ancien rire amené par le conteur traditionnel, autrefois symbole de résistance. Ce rire continue à s'offrir comme interprète de la société créole à travers la création linguistique et littéraire de Raphaël Confiant, une création ludique et engagée au même temps.

\section{5 «La française hors de France à l'épreuve de l'italien dans le Nuovo Garzanti di Francese de 1992 »}

À l'intérieur de la section «Études libres », Monica Barsi attire notre attention sur un thème proche aux jeux de mots: "l'histoire de la lexicographie bilingue où la traduction est la clé interprétative des deux cultures mises en contact »(105). De fait, l'intercompréhension joue un rôle anticipateur quant à l'intelligibilité des jeux de mots entre deux langues étrangères ; voilà pourquoi il est intéressant de découvrir la façon de répertorier les variantes francophones dans un ouvrage bilingue, qui inclut, de façon avant-gardiste, les variantes des Pays comme la Suisse, la Belgique, le Canada et l'Afrique francophone. De plus, l'esprit novateur du dictionnaire Nuovo Garzanti di Francese relève aussi de sa composition, élaborée à une époque dépourvue de technologies informatiques utilisables. En s'appuyant sur un corpus constitué par les entrées francophones des lettres A, M, T, Barsi établit les différentes manières de les traduire (par un/deux équivalent/s, par une explication, à l'aide des mots vedettes, etc.). Par la suite, l'auteure a rapporte en détail l'organisation de l'ouvrage, indispensable pour amplifier notre connaissance de la « doxa linguistique de la génération qui s'en servait et de mesurer les dimensions d'un savoir collectif et partagé sur la francophonie » (114; emphase dans l'original).

Gallimard, 2010) ; La panse du Chacal (Paris : [Mercure de France, 2004] Gallimard, 2006) ; L'Hôtel du bon plaisir (Paris : [Mercure de France, 2009] Gallimard, 2010) ; Case à Chine (Paris : Mercure de France, 2007) 


\section{Organisation internationale de la Francophonie [2006] (2013). Le Mouvement panafricaniste au vingtième siècle. Paris, 640 pp.}

Giacomo Bottosso

(Università degli Studi di Trieste, Italia)

Le Mouvement panafricaniste au vingtième siècle, pubblicato dall'Organisation internationale de la Francophonie (OIF) e disponibile gratuitamente in versione digitale nel sito dell'organizzazione, ${ }^{1}$ non è una semplice raccolta di conferenze: è un manifesto. L'OIF, sotto la guida del segretario Abdou Diouf, ha racchiuso in un unico testo i principali incontri di carattere panafricanista a partire dalla Prima conferenza panafricana, svoltasi a Londra dal 23 al 25 luglio del 1900 (data con la quale si fa coincidere la nascita del termine panafricanismo) fino alla Seconda conferenza degli intellettuali dell'Africa e della diaspora, tenutasi a Salvador di Bahia, Brasile, dal 12 al 14 luglio del 2006; durante le riunioni, che hanno avuto luogo in Europa, Africa e America lungo tutto il ventesimo secolo, i principali leader ed esponenti di varie associazioni e organizzazioni hanno posto l'attenzione sulle più importanti battaglie politiche per il riconoscimento dei diritti negati ai paesi e ai popoli colonizzati e hanno sottolineato l'importanza e l'influenza della cultura nello sviluppo delle popolazioni dell'Africa e della diaspora. L'Africa si pone come esempio per nuove soluzioni a problemi ecologici ed economici mondiali e come fonte di forme alternative di governo su basi storiche tipiche di antichi regni africani: leggere Le Mouvement panafricaniste au vingtième siècle significa ripercorrere il cammino dei figli di quegli uomini sul cui sangue è stato edificato un mondo che gli eredi degli schiavi stessi vogliono, ora, salvare dai danni prodotti dai loro vecchi padroni.

La prima parte del testo illustra i temi alla base della nascita e dallo sviluppo del movimento panafricanista nel diciannovesimo secolo, ovvero il rifiuto dello schiavismo, la sensibilizzazione nei confronti delle condizioni di arretratezza delle colonie e nei confronti dell'inferiorità sul piano umano e civile cui erano relegati gli individui di colore. Caratterizzato dall'affer-

1 Cf. http://mediatheque.francophonie.org/IMG/pdf/0IF-Le-mouvement-panafricanisteau-XXe-s.pdf (2018-11-20). 
mazione del modello colonialista nel Terzo mondo, il Novecento è il secolo in cui si intensificano le iniziative del movimento volte al raggiungimento della pari dignità per i neri: prendendo vita in tre continenti distinti e nelle rispettive realtà sociali, culturali e politiche, le varie organizzazioni attive per la causa panafricana agiscono in maniera indipendente: è per questo che le conferenze e i congressi rappresentano un momento fondamentale per la presa di coscienza e per l'unità di un movimento che intende legare il destino di individui con origini comuni, ma con storie e identità diverse. Le città scelte per i primi incontri rappresentano una sfida all'Occidente: Londra e Parigi, a cui si aggiungono, in seguito, Bruxelles e Lisbona, le capitali dei maggiori imperi coloniali europei. Gli albori del movimento panafricanista sono contraddistinti da un fermento proveniente soprattutto dagli Stati Uniti, dalle isole dei Caraibi e dalle colonie inglesi in Africa. Tra le personalità più carismatiche della prima metà del ventesimo secolo, infatti, si distinguono il trinidadiano H.S. Williams (1869-1911) e gli haitiani Anténor Firmin (1850-1911) e Benito Sylvain (1868-1915), pionieri del movimento; il liberiano E.W. Blyden (1832-1917), l'americano di nascita ma ghanese di adozione W.E.B. Du Bois (1868-1963) e il giamaicano Marcus Garvey (1887-1940), le cui divergenze politiche sono state fonte di contrasti all'interno del movimento. Du Bois, in particolare, si è affermato come leader principale a partire dal 1919, anno del Congresso panafricano di Parigi, fino alla propria morte, avvenuta ad Accra, poco tempo dopo aver ottenuto la cittadinanza ghanese: la sua linea radicale di pensiero e di azione ha influenzato in maniera decisiva l'orientamento del movimento panafricanista, causando un frequente accostamento a quello comunista.

Il quindicennio che segue il 1945, anno del passaggio di testimone tra la prima e la seconda generazione di intellettuali del movimento, è il periodo in cui si consolida la posizione africanista del movimento: l'era della negritudine e del faraonismo. Ponendosi in netta opposizione al razzismo scientifico sostenuto dalle teorie colonizzatrici, secondo le quali l'Europa avrebbe contribuito alla civilizzazione dell'arretrata Africa con la sua conquista, Senghor e il poeta e politico martinicano Aimé Césaire (1913-2008) guidano una resistenza composta da storici e poeti. L'indagine che mira all'esaltazione di grandi civiltà e degli imperi africani e, quindi, dell'identità nera si colloca al centro del progetto artistico del movimento panafricanista, grazie al contributo di altri due poeti francofoni, il guianese Léon-Gontran Gamas (1912-1978) e il senegalese Alioune Diop (19101980), fondatore della rivista di riferimento per gli intellettuali di colore Présence Africaine; oltre agli uomini dell'Africa e della diaspora, partecipano alla riabilitazione della comunità nera anche storici e intellettuali europei e arabi. Altri, però, come il fisico e antropologo senegalese Cheikh Anta Diop (1923-986) e lo psichiatra e filosofo Frantz Fanon (1925-1961) ritengono limitato il lavoro dei poeti della negritudine: il primo critica le insufficienti prove scientifiche a supporto della negritudine e pone l'Egitto 
come epicentro della scienza occidentale, mentre il secondo, connazionale e allievo di Césaire, accusa il movimento della negritudine di essere un tentativo di compiacere gli occidentali ribaltando i loro stessi argomenti, piuttosto che recuperare la propria identità e prendere le distanze da chi li ha sempre considerati inferiori.

Nella seconda metà del Novecento, il processo di decolonizzazione trasforma l'Africa: sulla scia di Egitto, Marocco e Tunisia, prime colonie a conquistare l'indipendenza, e soprattutto del Ghana, primo tra i paesi sub-sahariani a riacquisire la propria sovranità nel 1957 sotto la guida di Kwame Nkrumah (1909-1972), gli stati africani si pongono l'obiettivo di fondare una federazione di nazioni indipendenti, spinti dal desiderio di autonomia dalle logiche imperiali e dalla riscoperta dell'identità e della cultura nera. Léopold Sédar Senghor (1906-2001), politico e scrittore senegalese, presidente del proprio paese, conia il termine Africanité, per mezzo del quale identifica «l'ensemble des valeurs africaines de civilisation» (359) che caratterizza ogni uomo e donna d'Africa e della diaspora. E a partire di questo nuovo concetto che, quindi, Senghor preme per l'unificazione degli stati africani in una confederazione, in quanto l'africanità si manifesta nella stessa misura sia tra i popoli berbero-arabi che in quelli 'negro-africani'. L'appartenenza culturale, insieme alla condivisione delle stesse sorti di ex colonie sottosviluppate prede del capitalismo occidentale, deve essere il motore per l'unità delle nazioni africane, allo scopo di favorire lo sviluppo dell'economia del continente sulla base della valorizzazione degli Africani. La lotta per l'indipendenza dall'Occidente attira l'attenzione di intellettuali da tutto il mondo, che ne riconoscono e difendono la causa. Da questo momento, l'Africa diventa l'unica sede delle conferenze del movimento panafricanista.

Ma il punto più significativo all'interno del percorso del movimento, sul quale è doveroso soffermarsi, riguarda il cambiamento degli interlocutori del movimento stesso: se durante i primi decenni i leader panafricanisti coinvolgono indirettamente nei propri discorsi i governanti europei e, in generale, i bianchi, per richiedere ciò che spetta a ogni essere umano, ora è quasi esclusivamente con se stessi che dialogano. I capi di stato e gli intellettuali africani e della diaspora pongono in primo piano l'importanza della storia del continente, dell'originalità culturale e della produzione artistica africana, per favorire lo sviluppo delle ormai ex colonie e l'allontanamento dalla mentalità capitalista europea: le ultime conferenze, infatti, si concentrano sul contributo culturale e scientifico dell'Africa in un mondo multiculturale come quello del terzo millennio e su temi di rilevanza centrale per lo sviluppo dei popoli africani quali l'istruzione, la sanità e il progresso scientifico ed economico. I presidenti dei paesi africani e della diaspora, i rappresentanti delle organizzazioni politiche e gli intellettuali neri si impegnano a contribuire alla rinascita del continente attraverso soluzioni che coinvolgano l'Africa in maniera diretta, sottraendosi al ruolo di 
subalterni. La parola chiave che costituisce il tema centrale dei congressi degli ultimi decenni è, infatti, Renaissance, un concetto preso in prestito dalla cultura occidentale, ma che serve come riferimento per la fondazione di un'umanità nuova, rinnovata, simile a quella teorizzata da Fanon in Les Damnés de la terre:

La renaissance africaine est essentielle pour que le XXIe siècle marque le début d'une ère dans laquelle tous les peuples et tous les pays partagent les bienfaits de la richesse et de la culture, dans le plein respect de la dignité des droits et des valeurs de tous les êtres humains, et notamment des enfants, des femmes et des personnes âgées, quelles que soient leur appartenance ethnique ou leurs croyances. (611-12) 


\title{
Chambers, lain; Curti, Lidia; Quadraro, Michaela (a cura di) (2014). Ritorni critici. La sfida degli studi culturali e postcoloniali. Milano: Meltemi, 245 pp. La politica del ritorno: per una spettrologia dell'accademia
}

\author{
Eleonora Meo \\ (Università degli Studi di Napoli “L'Orientale”, Italia)
}

\begin{abstract}
Haunting raises specters, and it alters the experience of being in time, the way we separate the past, the present, and the future. These specters or ghosts appear when the trouble they represent and symptomize is no longer being contained or repressed or blocked from view. The ghost, as I understand it, is not the invisible or some ineffable excess. The whole essence, if you can use that word, of a ghost is that it has a real presence and demands its due, your attention.
\end{abstract}

(Gordon 2008, xvi)

La Sala conferenze di Palazzo Du Mesnil, un palazzo storico del XIX secolo acquistato nel 2000 dall'Università degli Studi di Napoli "L'Orientale" come sede del Rettorato, è un luogo infestato da un ritorno, dove gli spettri del passato e quelli di possibili futuri perduti si sono dati appuntamento per perseguitare l'incerto e inquietante presente accademico di cui quella sala è simbolo. Quando le conseguenze di cieche politiche di razionalizzazione economica dell'università pubblica italiana - fatte di tagli, chiusure di corsi di laurea e disinvestimenti nella ricerca - vengono ignorate, ecco che le presenze (the haunting) si manifestano. Nei suoi interni sfarzosi in stile neoclassico e barocco arricchiti da intarsi in legno, affreschi a carattere mitologico, stucchi, marmi e da riquadri intagliati nelle porte, il 18 e 19 novembre 2016 si è tenuto il seminario Ritorni Critici, organizzato dall'ormai estinto Dottorato in Studi Culturali e Postcoloniali del Mondo Anglofono. Questo ritorno ha voluto rievocare un lungo percorso, iniziato negli anni '70 da un gruppo di ricercatrici e ricercatori di quella che si è successivamente definita come 'scuola napoletana degli studi 


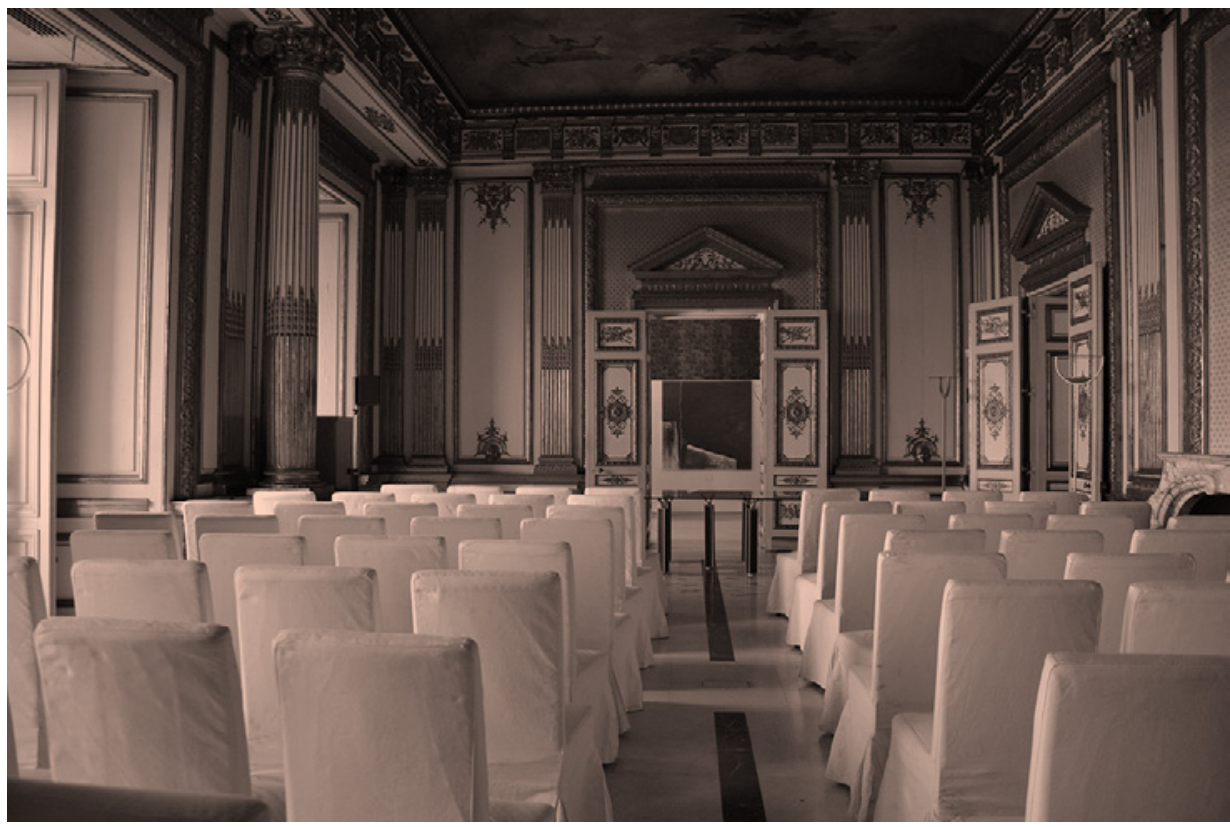

Università degli Studi di Napoli “L’Orientale”, Sala conferenze, Palazzo Du Mesnil. @ 2013 Marilena Galella. Fonte: http: //www . marilenagalella. net/esposizioni/esposizionepalazzo-du-mesnil-universita-degli-studi-di-napoli-lorientale-2011/.

culturali'1 $^{\mathbf{1}}$ - il primo incubatore italiano degli studi culturali britannici - e continuato con la nascita, nel 2000 (stesso anno in cui Palazzo Du Mesnil fu acquistato), del Dottorato in Studi Culturali e Postcoloniali del Mondo Anglofono. Il Dottorato, a seguito di una ristrutturazione dei corsi dottorali (e a differenza, almeno per ora, di Palazzo Du Mesnil), è stato chiuso definitivamente nel 2015, con l'addottoramento dei suoi ultimi superstiti.

Il seminario Ritorni Critici - di cui il volume Ritorni critici. La sfida degli studi culturali e postcoloniali a cura di Iain Chambers, Lidia Curti e Mi-

1 Come ricorda Marta Cariello, tra le autrici del volume, in un articolo apparso nel $2015 \mathrm{su}$ postcolonialitalia: "A partire dalla metà degli anni '60 iniziò infatti la liaison tra il Centro di Birmingham e l'Università Orientale di Napoli (ed è utile chiarire, a tal proposito, che a Napoli gli studi culturali prima, e postcoloniali poi, trovano sede e si sviluppano all'Orientale in modo pressoché esclusivo). Prima Lidia Curti, nel 1964-65, poi altre studiose, tra cui Marina Vitale e Adi Mineo, trascorsero in quegli anni periodi di studio e ricerca a Birmingham. A sua volta Stuart Hall fu ospitato per la prima volta all'Orientale poco prima del ‘68 e poi ancora diverse volte nei fertili e turbolenti anni ' 70 . Ulteriore momento di svolta è costituito, poi, dall'approdo di Iain Chambers a Napoli prima, e poi all'Orientale, ormai più di 30 anni fa» (http://www.postcolonialitalia.it/index.php?option=com content\&view=arti cle\&id=105: cariello-intervento\&catid=27: interventi\&Itemid=101\&lang=it (2018-11-27). 
chaela Quadraro è la restituzione in forma cartacea - è stato dunque ciò che la sociologa americana Avery Gordon ha definito, seppur in un altro contesto, un «haunting remainder» (Gordon 2008, 25): il resto di ciò che è stato represso o che è rimasto irrisolto, ciò che ricompare quando «what's been in your blind spot comes into view» (Gordon 2008, xvi), rendendo percepibili gli effetti passati e presenti dei complessi rapporti sociali in cui siamo immersi. Gordon, infatti, considera gli spettri come 'figure sociali' che ritornano poiché la violenza sociale che hanno subìto è rimasta irrisolta o è stata rimossa. Un ritorno, dunque, che è politico in quanto portatore di un'istanza precisa, il cui fine è provocare qualcosa che 'deve essere fatto' o che deve essere continuato.

In questo senso, il volume Ritorni critici. La sfida degli studi culturali $e$ postcoloniali, dunque, non è solo il risultato di «un laboratorio di lunga durata» - come scrivono i curatori nella loro nota introduttiva - «nato dal proposito di rinnovare i linguaggi delle analisi critiche dei testi e delle pratiche culturali nell'epoca contemporanea», ma diviene sfida e sintomo dello smantellamento neoliberale dell'università come laboratorio di saperi critici, l'alternativa storica che rifiuta di essere silenziata dal processo di 'spettralizzazione' (Mbembe 2002, 25) dell'accademia italiana.

Per 'spettralizzazione' qui s'intende un processo neoliberista di progressiva mercificazione del sapere e di svuotamento del mondo accademico italiano, fatto di: tagli alle università pubbliche e delle risorse destinate alla ricerca, blocco del turn over, aumento dei costi - in termini di tasse, precarizzazione e lavoro gratuito - a carico di studenti/ricercatori/assegnisti/dottorandi, incessante ridimensionamento ed eliminazione di corsi di laurea in scienze umane e sociali a favore delle discipline utili all'economia e conseguente inesorabile deflusso di cervelli dalle università e dai centri di ricerca. Da questo punto di vista, allora, la 'spettralizzazione' dell'accademia altro non è che un elemento della maledizione capitalista.

I saperi critici, e chi li pratica, stanno letteralmente scomparendo all'interno di un'accademia settata sulle coordinate della «società del controllo» (Deleuze 1990), prodotto di uno «Stalinismo di mercato» (Fisher 2009) che si basa sulla burocratizzazione tardo capitalista e sul puntare tutto, tramite sistemi di valutazione, sulla rappresentazione di un sistema (universitario) che produce, che è efficiente, nonostante al suo interno nulla si muove o migliora. Un sistema, dunque, dove il simbolo del raggiungimento di un obiettivo ha più valore della qualità o del raggiungimento vero di quell'obiettivo. Un sistema che opera attraverso il 'rinvio indefinito' della fine della formazione (secondo l'ideologia del Lifelong Learning), nei cui labirinti il soggetto non smette mai di essere o sentirsi studente/candidato/ tirocinante/junior di qualcuno, anche molto dopo che ha terminato il suo percorso di studi universitari o il Dottorato di Ricerca. Come ricordava Gilles Deleuze in «Post-scriptum sur les sociétés de contrôle» (1990): 
Il principio regolatore del 'salario secondo il merito' non manca di sedurre la stessa Pubblica istruzione: in effetti, così come l'impresa sostituisce la fabbrica, la formazione permanente tende a sostituire la scuola, e il continuo controllo a sostituire l'esame. È il mezzo più sicuro per consegnare la scuola all'impresa. Nelle società disciplinari non si faceva che ricominciare (dalla scuola alla caserma, dalla caserma alla fabbrica), mentre nelle società di controllo non si finisce mai con nulla, in quanto l'impresa, la formazione, il servizio sono gli stati metastabili e coesistenti di una stessa modulazione, di uno stesso deformatore universale. [...] Molti giovani pretendono stranamente di essere 'motivati', richiedono stage e formazione permanente; sta a loro scoprire di che cosa diverranno servi, così come i loro antenati hanno scoperto, non senza dolore, la finalità delle discipline. («Post-scriptum sur les sociétés de contrôle» in Deleuze 2000, 236-41; corsivi nell'originale)

Al centro del dispositivo accademico (Chambers, Guarracino 2017) opera, invisibile, il cosiddetto «grande Altro» (Fisher 2009), ovvero quella struttura simbolica impersonale che si manifesta solo tramite il diligente operato di ciascuno dei suoi luogotenenti (addetti, burocrati, impiegati, segretari, collaboratori, direttori, supervisori, ecc.), un meccanismo capillare senza conducente che funziona esclusivamente in base al fatto che la società vi crede. Si può certo sostenere che il grande Altro riesca a mettere al lavoro una mole di singolarità ai fini di stimolare la nostra personale «libido per la burocrazia» (Fisher 2009, 55), e tuttavia, malgrado i tentativi di identificare le motivazioni di un simile declino, resta difficile fornire una spiegazione tanto sulla cronica mancanza di fondi di ricerca o di sbocchi all'interno delle nostre università quanto sulla costante sottovalutazione delle Scienze umane e sociali italiane come settore degno di investimento. Questi interrogativi, è chiaro, resteranno senza risposta. Come rileva Mark Fisher in Capitalist Realism. Is There No Alternative, l'eventuale risoluzione dell'enigma abita all'interno stesso di questa maledizione. Ricordando lo scrittore che ci ha consegnato uno dei ritratti più 'realisti' della burocrazia, Franz Kafka, egli sottolinea:

The quest to reach the ultimate authority who will finally resolve K's official status can never end, because the big Other cannot be encountered in itself: there are only officials, more or less hostile, engaged in acts of interpretation about what the big Other's intentions. And these acts of interpretation, these deferrals of responsibility, are all that the big Other is. (Fisher 2009, 55)

La 'spettralizzazione' dell'accademia, ovviamente, è tutto questo e molto altro. Operare una spettrologia dell'accademia significa, pertanto, non soltanto fare i conti con una condizione macabra dove l'essenza vitale delle 
soggettività viene inesorabilmente drenata ma, soprattutto, significa riuscire a percepire gli spettri quando essi ritornano, riconoscere le loro istanze politiche e le storie sospese del passato e dei presenti futuri che portano con sé. Si tratta, in sostanza, di diventare sensibili ai fantasmi dell'accademia.

Questa riflessione vuole semplicemente cercare di mettere in luce il contesto in cui il volume Ritorni critici ha preso forma, e sottolineare perché può essere un importante punto di partenza. Probabilmente, infatti, a un lettore italiano alle prime armi con gli studi culturali e postcoloniali il volume potrebbe sembrare il semplice lancio di una sfida ai tradizionali canoni disciplinari - d'altronde è anche questo che segnò la nascita di tali studi in ambiente anglosassone. Un lettore più esperto, d'altronde, potrebbe probabilmente essere portato a pensare che Ritorni critici sia invece l'ennesimo volume di teoria culturale e postcoloniale, un campo che negli ultimi decenni, da un certo punto di vista, ha guadagnato sempre più terreno in Italia, facendosi largo negli interstizi di diverse discipline. In realtà Ritorni critici, come si è detto, non lancia nessuna 'nuova' sfida, bensì il ritorno - e qui si trova il significato politico di questa rinnovata interruzione accademica - della sfida italiana degli studi culturali e postcoloniali.

Ad aprire la prima parte del volume «Poetiche, genere e linguaggi» è proprio un'interrogazione di Iain Chambers: «Com' è possibile pensare a un approccio interdisciplinare degli studi culturali nelle rovine dell'università attuale, quella ottocentesca e illuministica [...]?» (Chambers, «L'(im)possibilità degli studi culturali», 13). L'autore ricorda come gli studi culturali, nascendo da un dissidio interno alla propria formazione culturale, si fondino sulla sfida politica della necessità di esporre costantemente ciò che definiamo 'cultura' e ciò che definiamo 'critica' a «domande non autorizzate», attraverso percorsi di spaesamento. I venti contributi della raccolta, seppur brevi, riescono a rendere le molteplici traiettorie che gli studi culturali e postcoloniali possono seguire in modi del tutto imprevisti, a partire da angolazioni e punti di partenza anche molto diversi. Attraverso l'arte, la danza, la letteratura, il visuale, la street art, l'estetica, il femminismo, la poetica, la sign-ature (la letteratura in lingua dei segni), le cartografie del suono, il pensiero queer, il digitale, la politica dell'eco, le ecologie sociali, il volume si fa «mappa non autorizzata» (Chambers 2017, 49) - e non compiuta - a partire dalla quale, attraverso lenti caleidoscopiche, è possibile analizzare la complessità culturale dell'attuale congiuntura globale.

Celeste Ianniciello, nel suo saggio, indaga in tal senso le risposte dell'arte alle attuali cartografie della Fortezza Europa attraverso le mappe delle artiste Mona Hatoum e Bouchra Khalili. Mappe che, proponendo una cartografia alternativa, evocano lo sconfinamento delle geografie e dei confini, trasportandoci in uno spazio critico eterotopico e interrogando le nostre posizioni culturali e identitarie. L'arte come forma di pensiero e di produzione teorica è rievocata anche da Marina Vitale nel suo saggio, ricordando come la ricerca teorica non debba mai essere fine a se stessa 
o sovrimposta ai fenomeni culturali poiché questa deve esservi «sempre posta accanto, pensando con essi» (Vitale 2018, 141; corsivo nell'originale). Lidia Curti, nel suo contributo, ricostruisce la genealogia erratica dei percorsi femministi e del pensiero intersezionale nel suo rapporto con la soggettività. Lautrice femminista, facendo appello proprio alle risposte che possono venire dagli spazi creativi, poetici e narrativi, ci mette in guardia dai pericoli di ricadere in una visione trasparente e univoca della trasversalità.

Focalizzandosi sugli aspetti epistemologici del problema della modernità, le autrici e gli autori del volume sfidano i regimi di verità accademici che vorrebbero la sclerotizzazione, e l'addomesticamento, del sapere in campi disciplinari ben definiti e trasparenti alla logica neopositivista della razionalità accademica.

Il volume, e il ritorno di cui è traccia, lancia dunque un'eco a tutti coloro che in Italia si occupano di saperi critici e che con grosse fatiche (e rinunce) portano avanti le proprie ricerche all'interno (e fuori) dei dipartimenti e dei programmi dottorali. Come rievoca l'ultimo contributo:

L'eco è in effetti il suono di qualcosa che non esiste, o almeno non più: un suono fantasma, il suono di un fantasma. Nondimeno esiste, nella misura in cui i nostri sensi ne fanno esperienza. (D’Aquino 2018, 240)

Si tratta, allora, di iniziare a imparare a vivere con (tra) i fantasmi (Derrida 1993) e ad ascoltare una richiesta di attenzione, provando a immaginare cosa accadrebbe al mondo universitario italiano se riconoscessimo le storie che questi spettri portano con sé.

\section{Bibliografia}

Chambers, Iain; Curti, Lidia; Quadraro, Michaela (a cura di) (2018). Ritorni critici. La sfida degli studi culturali e postcoloniali. Milano: Meltemi. Chambers, Iain; Guarracino, Serena (2017). «Derive critiche e modernità non-autorizzate. Una conversazione con Iain Chambers». Altre Modernità. Gli Studi Culturali e l'università italiana 9. DOI doi. org/10.13130/2035-7680/9003.

Curti, Lidia (2018). «Percorsi femministi: etica e estetica della diversità». Chambers, Curti, Quadraro 2018, 25-37.

D'Aquino, Brian (2018). «Riavvolgendo il nastro della storia: appunti per una politica dell'eco». Chambers, Curti, Quadraro 2018, 235-45.

Deleuze, Gilles (2000). Pourparler 1972-1990. Macerata: Quodlibet.

Demos, T.J. (2013). Return to the Postcolony. Berlin: Sternberg Press.

Derrida, Jacques (1993). Spectres de Marx. L'État de la dette, le travail du deuil et la nouvelle Internationale. Paris: Galilée. 
Fisher, Mark (2009). Capitalist Realism. Is There No Alternative?. Winchester (UK): Zero Books.

Fisher, Mark (2013). Ghosts of My Life: Writings on Depression, Hauntology and Lost Futures. Winchester (UK): Zero Books.

Gordon, F. Avery (2008). Ghostly Matters. Haunting and the Sociological Imagination. Minneapolis (MN): New University of Minnesota Press.

Höller, Christian (2002). «Africa in Motion: An Interview with the PostColonialism Theoretician Achille Mbembe». Springerin, 3(2), n.p.

Ianniciello, Celeste (2018). «Oltre la fortezza: i confini, i transiti e le risposte dell'arte». Chambers, Curti, Quadraro 2018, 77-86.

Mbembe, Achille (2002). «The Power of the Archive and its Limits.» Hamilton, Carolyn; Pickover, Michelè (eds), Refiguring the Archive. Dordrecht: Springer, 19-26.

Vitale, Marina (2018). «L'interruzione degli studi culturali». Chambers, Curti, Quadraro 2018, 135-46. 



\title{
Kirsch, Adam (2016). The Global Novel: Writing the World in the 21st Century. New York: Columbia Global Reports, 105 pp.
}

\author{
Yuqian Cai \\ (Università Ca’ Foscari Venezia, Italia)
}

The global novel is the mirror and the lamp of the global age. As 'globalisation' entered common parlance in the '80s, a notion of 'global novel' also appeared, and writers like Ishiguro could confidently proclaim in 1987 that "I am working myself up to writing a kind of epic global novel. I suppose a lot of people are always working themselves up to writing that kind of novel". ${ }^{1}$ But the 'global novel' was not and is not always desired, and the phrase did not gain currency in literary studies until the past decade. Adam Kirsch's 2016 book, ${ }^{2}$ published by Columbia Global Reports, is the first introductory attempt to establish the global novel as a legitimate category, paradigmatic for "writing the world in the 21st century".

The legitimacy of the global novel has been contested across the Atlantic, and Kirsch writes "World Literature and Its Discontents" as the first chapter of his defence. Dissenters criticise world literature on aesthetic and political fronts, disparaging the global novel as "diluted and deracinated", plagued by semiotic problems of "the Untranslatable"3 and stylistic deficiencies coupled with political and economic complicities. A common charge is that this genre avoids difficult particularities to maximise readability through simplified language and representation, making foreignness a homogenising commodity in a capitalist world, and hence mediocrity prevails with dumbing-down effects, preventing genuine encounter with differences and challenges. Tim Parks, for instance, deplores the rise of the global novel practiced by Ishiguro and others, and feels nostalgic for writers like Jane Austen who exemplify "culture-specific clutter and linguistic virtuosity", not streamlined tropes or "overstated fantasy

1 Bigsby, Christopher (2008). "In Conversation with Kazuo Ishiguro". Conversations with Kazuo Ishiguro. Jackson: University Press of Mississippi.

2 Quotations retrieved from Kindle edition of the book.

3 Apter, Emily (2013). Against World Literature. New York: Verso.

DOI 10.30687/Tol/2499-5975/2018/20/035 
devices of a Rushdie or a Pamuk". ${ }^{4}$ Moreover, whereas some editors bemoan a collapse of postcolonial radicalism and anti-imperialism into such axioms as "freedom of speech" following Rushdie's (1988) and the end of the Cold War, ${ }^{\mathbf{5}}$ critics and writers like Minae Mizumura worry that world literature triumphs at the expense of linguistic and mental diversities, advancing rather than checking the imperialism of the English language backed by the hegemony of the United States, and making literatures in other languages provincial or peripheral to what Pascale Casanova calls "the world republic of letters". ${ }^{6}$ But even if aesthetic-political ideals render contemporary world literature "compromised and complaisant", Kirsch insists that the global novel can be more stimulating and enriching than impoverishing.

In theory, Kirsch is largely correct to affirm the possibility and desirability of the global novel. Detractors have every right to remain sceptical, but writing the global novel, as Kirsch says, means "a basic affirmation of the power of literature to represent the world". A new development of "the preeminent modern genre of exploration and explanation", the global novel arises not from writers' desire to gain critical or commercial rewards, but from the condition of life in a global age, and from the potential of fiction to reckon with life and "reveal humanity to itself". Unlike the 18th century when Austen could blithely say "it is a truth universally acknowledged," the 21st-century novelist, Kirsch argues, "must dramatize that unity [of human nature], by plotting local experience against a background that is international and even cosmic". Thus, he suggests:

A global novel can be one that sees humanity on the level of the species, so that its problems and prospects can only be dealt with on the scale of the whole planet; or it can start from the scale of a single neighborhood, showing how even the most constrained of lives are affected by worldwide movements. It can describe a way of life common to people in many places, emphasizing the interchangeability of urban life in the twenty-first century; or it can be one that emphasizes the importance of differences, and the difficulty of communicating across borders. It can deal with traditional cultural markers like appearance and behavior or with elusive cosmic intuitions that seem to transcend place.

4 Parks, Tim (2010). "The Dull New Global Novel”. The New York Review of Books, February 9, 2010. URL https://wWw.nybooks.com/daily/2010/02/09/the-dull-new-global-novel/ (2018-12-13).

5 N+1 editors (2013). "World Lite", in "The Evil Issue". Issue 17, N+1. URL https://nplusonemag.com/issue-17/the-intellectual-situation/world-lite/ (2018-12-13).

6 Casanova, Pascale (1999). Republique mondiale des lettres. Paris: Editions du Seuil. 
Given such a variety of approaches already perceivably taken by writers, the global novel seems to be "not a unitary genre", but rather "a medium" for all sorts of stories, sharing experience and imagination of coming to grips with cosmopolitanism, and making world literature tantamount to the literary representation and construction of "a meaningfully global consciousness".

Kirsch's argument by example, however, is only half-convincing, since the calibres of his chosen authors vary so much that some of them undermine rather than underline his defence. In the next five chapters, Kirsch turns to empirical evidence provided by supposedly 'representative' novels from the "pantheon of world literature": Pamuk's (2002), Murakami's (2009), Bolaño's (2004), Adichie's (2013), Hamid's (2007), Atwood's (2003), Houellebecq's (2005), and Ferrante's Neapolitan quartet (20112014). Writing in various languages and forms, these eight novelists have merely one thing in common, that they have "reached worldwide audiences" and achieved their status as "leading figures" in a globalised literary market. Their novels are more or less best-sellers, but there is no critical consensus on their literary quality. Overlooking the latter and asserting that "other studies of world literature" would be incomplete without considering all these writers, Kirsch seems to equate his 'pantheon' with the marketplace, and he overstates the importance and correctness of his list. He does address aesthetic and political questions about the novels, as he is aware of the disparities between the authors he discusses in pairs, i.e. Murakami and Bolaño, Adichie and Hamid, Atwood and Houellebecq. However, Kirsch's indiscriminate inclusion of all them into his pantheon creates a fundamental weakness of his book, vulnerable to the criticisms it seeks to defend against, such as the judgment that world literature is a commodity lacking style or taste. To give a more adequate defence, Kirsch had better focus on the literary value of the global novel, instead of relying on some other criterion.

If Kirsch's second chapter on Pamuk and final one on Ferrante are relatively unproblematic, then troubles lay in the three chapters in between, which put thematically linked novels in pairs. Kirsch juxtaposes with as two novels about alternate realities, but he merely alludes to what becomes explicit when the same chapter is republished online under the title "Murakami vs. Bolaño: Competing Visions of the Global Novel" (2017): treating Murakami as "a test case for the aesthetic and even moral validity of global literature", Kirsch does not draw a clear-cut conclusion, until the new title pits Murakami against Bolaño, implying that Murakami is perhaps more of a negative example that attracts criticisms like Tim Parks'. Murakami's prose style is simplistic, his cultural references quintessentially Western, his characters mostly urban isolates devoid of society, politics, or history, and hence his story is readily translatable and "stripped for export". By contrast, Bolaño's novel reflects his sensibility that "the world is divided 
into zones of immunity and vulnerability". On the one hand, Europe seems to be "a zone of peace, culture, and self-absorption", where people occupy themselves "with study and with love"; but, the European "hypercivilization" can also be violently intolerant of "foreigners and immigrants who do not share it". On the other hand, Bolaño takes readers to "the sick heart of contemporary reality", like the fictional city of Santa Teresa on the Mexican-American border; by compelling readers to recognize the injustices and crimes propagated by the global economy and its moral contradictions, represents arguably a better species of world literature that undoes "the complacency of global citizenship". In light of Kirsch's essay "In Defence of the Global Novel" (2017), one may infer that while Murakami's writing is stylistically "more culture-industry product than work of art", Bolaño is intellectually "cannier, more self-reflexive and creatively resourceful", writing the true kind of global novel "defined as a novel for which being global is itself a problem", which stimulates "the empathetic imagination of difference a globalised world so direly needs."

If Murakami's position in the "pantheon of world literature" ought to be shaky, the same can be said about Hamid and Houellebecq because of their stylistic or ethical deficiency. Kirsch pairs Adichie with Hamid as two migrant writers "to America and back"; however, is a lightweight novella, a dramatic monologue in which only one character (the narrator) appears real, and hence it is aesthetically substandard as a candidate for the global novel, no matter how cleverly constructed and politically provocative it is. Kirsch shrewdly points out that Hamid's narrator is infatuated with America despite his anti-Americanism, problematizing the global order while fostering an internationalist consciousness. Still, it is unnecessary to use two novelists for making one point, that migrant literature provides a significant portrait of an age in which millions of people cross borders in both directions. Similarly, Atwood and Houellebecq are strange bedfellows whom Kirsch puts together for their fiction of global apocalypse; their novels share many features, but "the Canadian feminist and the French misogynist" should not be equal contenders for the authority or "the right to represent the world in fiction", not to mention that their dystopias may well be "an imperialism of imagination" and "a kind of colonialism" that conflate the experience of modern Western societies with the essence and fate of all humanity. Kirsch cautions about both writers' outlooks, but again, he may easily do without Houellebecq, or Hamid.

By contrast, the choice of Pamuk is expected and that of Ferrante reasonable, even though none would be universally or unquestionably accepted as a representative global novelist. Despite objections from Turkey, Kirsch describes Pamuk as an ambassador to the United Nations of "the world's literary consciousness". Take for example, while its framework is primarily Western rather than Eastern as manifested by the literary conventions and allusions it draws on, the novel represents often repressed voices, which 
protest the hegemony of Western narratives. Furthermore, quoting from a character - "I'd like to tell your readers not to believe anything you say about me, anything you say about any of us. No one could understand us from so far away" - Kirsch contends that the novelist acknowledges as well as negates the impossibility of "cultural translation," which is foundational to world literature, by allowing the reader to understand and feel the character as a real human being. Although Pamuk's position may be overdetermined when he writes about the East vis-à-vis the West, as Kirsch argues, "there remains the hope that the novel itself might be a genre that encompasses these divisions, not by transcending them in the name of a universal art, but by allowing all points of view to express themselves". Such diversification of perspectives and voices is evident in Ferrante's Neapolitan novels as well, which interweave the dialectic of the local and the global with other themes like "violence against women," an issue commonly binding the global novelists. As mentioned above, there are many ways to write a global novel; by analysing the work of eight novelists, Kirsch offers almost a typology of what the global novel can be. It is not thorough or impeccable, and it exaggerates the representativeness of some writers, but overall, Kirsch's book is a valuable albeit flawed effort to legitimize the global novel.

Additionally, while Kirsch's fundamental flaw comes from a neglect of literary value, to better meet the aesthetic and ethical challenges faced by writers and questioned by critics, one may think twice about "global classicism," proposed by Michael Lind in "World Books" (2015). ${ }^{7}$ Lind may be "elitist" and "conservative", and Kirsch is right that modernism is not necessarily the foe of classicism, since the modernism of Pound and Joyce is also "radically innovative classicism". But Lind's suggestion, as aptly summarised by Kirsch, is sound, that "[t]he global quality of such writing consists not in popularity across cultures, but a cosmopolitan appropriation of the best models of the past, regardless of their linguistic or national origin". Such cosmopolitan classicism, if treated properly, can be rooted and balanced, and compatible with global democratization.

7 Lind, Michael (2015). "World Books". The Smart Set, Oct. 6. URL https://thesmartset. com/world-books/ (2018-12-13). 



\section{Concilio, Carmen; Festa, Maria (eds) (2016). Word and Image in Literature and the Visual Arts. Milano: Mimesis International, 397 pp.}

Isabella Bruschi

(Università degli Studi di Torino, Italia)

The picture on the book cover reproduces a poster advertising an engraving company. It shows a man, a brush in his right hand, concentrated on his painting; the slogan above goes: "ILLUSTRATION BEATS EXPLANATION". The poster evocatively ushers the reader into the recurrent theme of the essays in the volume. It brings the suggestive power of images to the foreground, but also reminds us of the long-standing competition for primacy between word and image, while being evidence, at the same time, of how words and images can throw light on each other.

The essays collected in the volume all address the intersection between visual and verbal codes in the most varied contexts. Besides providing a theoretical background to the implications of such an intersection, the essays deal with the relationship between literary classics and the cinema or the graphic novel, they concentrate on the writers' capacity of creating images out of words, they discuss the hybrid nature of works that combine different languages: images that range from photographs and drawings to films intertwined with the most diverse written texts - fiction, poetry, articles, reportages. The multidimensionality of the works taken into account by the contributors to the volume appears to be a fundamental constituent of counter-canonical aesthetics that would allow to render more appropriately the complexities of our world and, more specifically, to lend a more open eye to postcolonial realities. Several essays in fact deal with postcolonial issues and the connected phenomenon of contemporary migrations.

Interesting parallels are also drawn between pieces of writing and images which did not originate within the same project, but connect with the same discourse. For instance, Carmen Concilio, in her essay "South-Africa's New Archive. Literature, Photography and the Digital Humanities", associates a well-known photograph by David Goldblatt with a passage from Nadine Gordimer's No Time like the Present, an excerpt from NoViolet Bulawayo's We Need New Names and a chronicle published in the online journal Johannesburg the Salon, to show how they converge to convey the same message about the Zimbabwean refugees' aspirations frustrated by 
political failures in South-Africa. Similarly Chris Ledochowski's shots of life in South-African townships relate to the description of the same places in J.M. Coetzee's Age of Iron or in Sindiwe Magona's Mother to Mother. Further on Concilio observes how all David Golblatt's "photographic errands" are complementary to Ivan Valdislavić's "latest literary urban errands, both fictions and non-fictions" (71). This similarity has produced a deliberate cooperation between Golblatt and Valdislavić, whose novel Double Negative was written to accompany the former's photographic project of $T$. J.; besides working as a metaphor of the protagonist's diasporic conscience divided between Johannesburg and London, the title Double Negative is interpreted by Concilio as an allegory of the relationship between novel and photo album. One of Valdislavić's short stories, Journal of a Wall and some fragments from his Portrait with Key find their 'material' equivalent in the walls decorated by William Kentridge, as Paola Quazzo points out in her essay. Both the writer and the visual artist - each through their own language - attach manifold meanings to the idea of wall: a symbol of prevarication and seclusion that can be turned into a manifesto of freedom and creativity, a meeting point, the metaphor of an urban space - be it South-African or worldwide - that keeps the multilayered memories of the people inhabiting it.

A further interplay between visual and verbal codes is highlighted by Ilaria Oddenino in "Portraying Migration in Contemporary Europe: Two Comparative Approaches", where she compares the reportage Lettre à une Calesienne (first published in the French journal Revue XXI) by Emmanuel Carrère and the film Fuocoammare by Italian director Gianfranco Rosi. What the two works have in common is not just the topic they treat (namely the plight of migrants who reach Europe from Africa crossing the Mediterranean and the attitude of the local people and authorities, no matter whether Italian or French) but also the authors' approach to the issue: both journalist and director tell stories of migration adopting the partial - if not stereotyped - vision of the residents embodied by two imaginary characters (a woman from Calais and a boy from Lampedusa) as the starting point of their narrations, blending thus documentary and fictional elements.

Postcolonial Italy is the focus of Pietro De Andrea's essay too. It deals in fact with three Italian films, Ermanno Olmi's Il Villaggio di Cartone, Gabriele Del Grande, Khalid Soliman Al Massiry and Antonio Agugliaro's Io Sto con la Sposa, Daniele Gaglianone's La Mia Classe and Wu Ming's short story Momodu. They all tell migrants' stories and all, though in different ways and degrees, adopt counter-canonical aesthetics, either disrupting spaces, or mixing real and fictional elements, or blending genres and languages, or reversing the chronological order of events. This last is the case of Momodu that is narrated backward, from outcome to origin (which is materially reflected by the order of the chapters going from 13 
to 0 ); thus the truth about a crime supposedly committed by a Gambian immigrant is progressively uncovered to reveal that the accusation was built up to cover the misdeed of a carabiniere, whose hate for migrants is rooted in the Italian colonial past. The upside-down structure of Wu Ming's story, as well as the hybridity and fluidity of the films taken into account represent a kind of aesthetics that takes on a political value. This is exactly the point De Andrea makes: the non-canonical modes of narration analysed contribute to the dismantling of a hegemonic reading of the phenomenon of migration and work as a denunciation of the absurdities and cruelties of European policies relating to it.

In her essay Luisa Pellegrini presents two further works that join words and images to address once again the issue of migration: Asylum Seekers, produced by the cooperation of three professional photographers and two anthropologists, is a photo book that deals with the issue from an ethnographic/sociological point of view, and Mare Nostrum, a graphic novel by Fabio Visintin, where the bodies of drown migrants, drifting in the salt water that envelops their tragedies in a sort of surreal atmosphere, tell their stories to the animals of the sea, the only ones capable of listening to them sympathetically.

Another interesting example of connection between verbal and visual elements is Lives of Shadows by the Canadian writer Barbara Hodgson. In her original novel the images interact with the narrative threads and temporal leaps at different stages and with different functions, as Maja Duranovic observes: the intersection of photographs, postcards, maps, drawings, old newspaper articles, Arabic inscriptions gives a visual perspective to the words, complements the readers' mental images and provides them with clues to identify the characters, or, as in the case of newspaper clippings, it serves to challenge the official narration of the bombings of Damascus by juxtaposing it to the fictional characters' reports of their daily experiences of war.

Teju Cole's deliberate effort to interweave literature and photography is the subject of Maria Festa's essay. As Festa indicates Cole's blending of words and images shows his attempt to produce a narrative where both have equal value. However Cole's essays also provide us with reflections about photography in terms of what it reveals or hides, of its relationship with memory and history. In Cole's fictional work, whether complemented by images or not, photography is central: unlike his novella, Everyday is for the Thief, where photographs are meant to give a more substantial view of Lagos and Nigeria, or his journal Punto d'Ombra, where they preserve the memory of his wanderings around the world, the novel Open City does not displays images, yet, as Festa writes, the reader is led to look at words using eyes as a camera.

The end of Apartheid in South Africa gave international visibility to photographic reportages unknown till then; this opened a previously un- 
explored perspective on the nature of photography and its interpolation with literary and narrative discourse, while tackling the new realities of the Rainbow Nation. In this context Concilio gives account of a seminal work notable for its hybridity: blank_Architecture, Apartheid and After by Hilton Judin and Ivan Valdislavić. The compedium combines photos, drawings, maps with essays covering disparate fields ranging from sociology to urban planning, advertising and short narrative fictions. Concilio also touches on post-Apartheid Digital Humanities and Web Archives. The flourishing of these currents is to be found in such initiatives as the online journal, The Johannesburg Salon or the VIADUCT 2015 Platform launched by the University of Johannesburg, or the Web Project Twenty Journey initiated by the three photographers, Sean Metelerkamp, Sipho Mpongo and Wikus de Wet. All these works concur to discuss the question of the responsibility of the artists who represent contemporary South-Africa and, more in in general, our present world, and they do so by bringing together the most diverse codes: still and moving images, poems, travel impressions, narratives. All this shows how photo essaysm is establishing itself as a powerful and effective new genre, as a new cultural phenomenon and, because of its hybridisation of words and images, it turns out to be particularly versatile and apt to read our postcolonial era.

In our 'network civilisation' images have acquired a tremendous power: to paraphrase Paul Virilio, they can be an instrument of freedom revealing the obscure side of reality, yet they could also obliterate reality, substituting it with an illusory virtual one. The centrality of images in shaping public opinion poses an urgent demand for tools to interpret and discriminate. The volume edited by Concilio and Festa provides us with critical keys towards a healthy understanding of the impact images have on our comprehension of the world, but it also shows us how they can usefully interact with words. 


\title{
Collins Klobah, Loretta (2018). Ricantations. Leeds: Peepal Tree Press Ltd, 138 pp.
}

\author{
Michela A. Calderaro \\ (Università degli Studi di Trieste, Italia)
}

Loretta Collins Klobah, whose new poetry collection, Ricantations, was named a "summer recommendation" by the British Poetry Book Society, is recognised as one of the main poetical voices within the Puerto Rican literary scene, and one of the most vocal supporter of women's plights both in Puerto Rico and in all other Caribbean islands.

She teaches Caribbean literature, creative writing, and medical humanities courses at the University of Puerto Rico. She has published both poetry and academic articles in such journals as Small Axe, Anthurium: A Caribbean Studies Journal, Image and Narrative, Jamaica Journal, Journal of Commonwealth and Postcolonial Studies, South Asian Popular Culture, and Literature and Medicine. Most noteworthy among the many awards she has received are the Pam Wallace Award for an Aspiring Woman Writer, the Award of The Academy of American Poets, and the Pushcart Prize. She has also won grants from the National Endowment for the Humanities, a Fulbright Award, an International Council for Canadian Studies Fellowship, and a Rockefeller Grant. She has been an invited poet at the Bim Literary Festival in Barbados, Calabash International Literary Festival in Jamaica, NCG Bocas Literary Festival in Trinidad \& Tobago, and the St. Martin Book Fair. She was also nominated as one of the poetry judges for the 2018 OCM Bocas Award in Caribbean Literature.

It is always interesting, before opening a book, be it poetry or prose, to take a close look at the front cover: it tells you more about what is inside than the blurbs on the back cover.

The cover art of The Twelve-Foot Neon Woman, Collins Klobah's debut collection for which she won the 2012 OCM Bocas Prize for Caribbean Literature, is a reproduction of a mural by a Puerto Rican graffiti artist named Ske Pagan and the TNX crew. A poem in the collection, entitled "La Madonna Urbana" (who is that "twelve-foot woman painted onto the wall" who gives the collection its title on page 12) is actually about another mural by Ske. The poem offers us a glimpse of humanity protected by "Our Lady of Barrio Obrero, la señora profana, la madonna boricua, | la cabrona, la Gata". We witness the lives of real, actual people whom the poet has encountered: we meet Jorge, "in fishing galoshes and sweat-soused shirt"; 
Alejandra; and Juanito, who has AIDS and "limps barefooted on sun-melted asphalt". And while the barrio gets slowly and quietly ready to sleep, Juanito sings together with Our Lady of Providence a song that opens the door to hope, notwithstanding the harsh lives of the barrio's inhabitants.

Hope, indeed, is one of the emotions you're likely to experience reading Collins Klobah's visionary new collection of poetry. Hope and music are elements that run through all of Collins Klobah's poems, and in Ricantations she makes the reader dance to the sound of boleros, salsa, calypso ("Man Haffi Try", or "Night Watch", to name just a couple of poems).

Music and hope are also present on the front cover of Ricantations, with a reproduction of artist Samuel Lind's oil painting Ángel Plenero. This painting, which in Collins Klobah's own words is one of resurrection, is closely related to what the poet experienced and saw during Hurricane Maria and the devastation that ensued in its wake on the island, all described in the collection's title poem. However, the traumatic experience did not weaken the resolve of most determined citizens, the poet included, to stay on the island.

The painting may also be viewed as a sort of introduction to the entire collection, with details of it found scattered throughout all poems. In an interview conducted by artist Jacqueline Bishop and published on The Sunday Observer (June 3, 2018), Collins Klobah's herself describes how the painting helped the book to take shape:

Every element in the painting relates to the poems in Ricantations, from the symbolism of death and resurrection, to the winged angel dressed in two-toned shoes and fedora, the breaking of the chains on the Plena drum, the mysterious plant life, and the mythic sky and stars. "Plenero" means "a musician of Plena", one of our traditional singing, dancing and drumming forms in Puerto Rico, which involves the playing of a handheld drum that looks like a tambourine without the metal discs.

Some of Samuel Lind's works, his bronze statue and paintings of Osain, are the inspiration for Collins Klobah's poem "Osain". Osain is one of the Orishas, who are the primary gods and goddesses of the Yoruba religion; he is the god, or spirit, of the forest, the guardian and keeper of plants and nature. Lindt's painting depicts him running through a mangrove swamp, and turning into a mangrove tree.

In Collins Klobah's poetry nature has always played an important role. This collection brings to the fore her fascination with the way nature intertwines with both female and male figures, and shows how nature can actually sooth and change the lives of people who grow up amid violence and death.

There is indeed a very high level of violence in Puerto Rico, but often, in Collins Klobah's collection, this violence can be transformed into something good. 
In the poem "He talks to a Butterfly" the protagonist is Joe, an actual person, an ex-convict, who works as a caretaker of butterflies at 'Aula Verde', a butterfly farm. The Puerto Rico administration had set up a program aimed at rehabilitating convicts through work at the butterfly farm, so they could then rejoin society. In a delicate and elegant voice, Collins Klobath tells us about Joe's tender harvesting of eggs in "the copulario | here monarchs and orange fritillaries | achieve butterfly positions". His love and dedication to butterflies is cathartic and transforms his life.

Loretta Collins Klobah's poetic texts intersect with existing, mainly historical, texts, leading to completely new and surprising formal effects. Myths and monsters of the past, grotesque beings, human and supernatural, historical figures belonging not only to Puerto Rico but also to other Caribbean islands, are thus fused into daily life. The stories of these creatures become canvasses where, through a process of substitution and superimposition, a new vibrant reality is brought to life.

Collins Klobah weaves a web that connects all the Caribbean islands and then reaches outside them, towards other shores and other lands. She uses geography, culture, language, time and space as tools, as if she were in front of a loom, interlacing diverse strands to build an intricate pattern.

Making use of Bachtin's chronotope, a theory regarding how space and time intersect each other, Collins Klobah manipulates time and space, fusing different realities and leading to the creation of manifold portals. Often the portals open to a new space, where the space and the time of the dead merge with those of the living - as in "Come, Shadow" for instance, where the poet can clearly perceive the presence of her dead mother, or in "Night of Charcoal Sky and Sea" where time lived and time dreamed blur and become indistinguishable.

Through these portals Collins Klobah brings together mythical elements of the past into our present - as, for example, the "gargoyle" which flies over us, today. The gargoyle embodies different times and different spaces, observing ghosts alongside the living, and making us feel how hundreds of years of suffering, deprivation, excruciating work in the cane fields affect life today.

Just at the moment

when the guard's

head drops sideways,

he hears the beating

windstorm of heavy,

feathered wings flogging

him to the ground,

then lifting off into the dark sky,

wings taller than a man,

smelling of seaweed and rot. 
[...]

Perching on a roof of a company village -

[...] the gargoyle glimpses ghost girls playing

a ghost son $[\ldots]$

as he carries a warm lunch in a fiambrera to his father

in the cane fields

Spanish and English, but also dialects, embark us on a whirlwind voyage. Loretta Collins Klobah grew up in a plurilingual and multicultural family, and thus the issues of identity and belonging, geography, language and culture play an important role in her work.

She was born in Merced, California; her mother was of Spanish and Scottish heritage; her father Cherokee and Irish; other close relatives came from Mexico. Though Spanish is the main language spoken in Puerto Rico, English is the language used in schools, so one is bound to continuously switch between languages, both at home and outside it. Language is also greatly affected by the waves of migration of islanders to and from the 'mainland' - the United States, of which Puerto Rico (Spanish for «Rich Port») is an unincorporated territory.

Puerto Rico, with its mountains, its pueblos, its inhabitants and their resilience, and especially its history, is set at the centre of Collins Klobah's poetic web. But then her poems carry us also to London, to Mexico, to Trinidad, all while switching from one language to another, from one historical or personal event to another.

Ricantations is also a moving autobiographical portrait of Collins Klobah's life. In "Come, Shadow", for example, she describes visiting her hospitalized schizophrenic mother, and how she still feels her presence:

After two years on the other side, she gave up on visiting my dreams then a decade of non-intervention, quiet ashes in a buried tin box.

Today she is detectable in my peripheral vision, everywhere in my head - annunciation.

Other poems describe real people whom she has met, or events she has taken part in. Every poem gives us a glimpse of life in the Caribbean, or helps us see how events, or injustices, related to the islands have a bearing on life outside the Caribbean and should be addressed by all of us. 
Necrologi | Obituaries | Nécrologies 



\title{
In Memoriam : Anne Marty (1947-2018)
}

\author{
Alessandro Costantini \\ (Università Ca' Foscari Venezia, Italia)
}

Anne Marty n'est plus avec nous.

J'ai reçu avec une tristesse infinie la nouvelle du décès d'Anne, le matin du 4 juin dernier, des suites d'un cancer fulgurant : elle laisse ses enfants Christophe et Vincent et toute sa famille, à laquelle elle avait décidé, depuis quelque temps, de se consacrer entièrement.

Femme de terrain très active dans les milieux associatifs haïtiens, journaliste et critique militante dès ses débuts, elle a fondé une revue (Pour Haïti), où elle a travaillé incessamment, directement et indirectement, pour la cause du progrès et de la liberté culturels haïtiens.

Son activité de critique littéraire est marquée de l'originalité de son parcours et de la multiplicité de ses expériences, versées dans le moule d'une formation scientifique rigoureuse de chercheur universitaire, qui l'a menée jusqu'au Doctorat d'État ès lettres et sciences humaines à la Sorbonne.

Nous avons collaboré plusieurs fois, Anne et moi, depuis ce jour lointain, en 1987, lorsque nous nous sommes rencontrés pour la première fois, chez elle, à Maisons-Alfort.

J'étais un jeune chercheur qui débutait dans la critique de la littérature haïtienne et - mouvant ses premiers pas dans un territoire, mieux, dans un monde qui lui était presque inconnu - cherchait partout de l'aide, des renseignements, des suggestions. C'est ainsi, m'étant rendu au Consulat d'Haïti à Paris, que j'y ai reçu l'excellent conseil de contacter Anne Marty : vous verrez, m'avait-on dit, elle vous aidera beaucoup ; elle connaît très bien toute la littérature haïtienne et pourra vous donner tous les renseignements et l'aide dont vous avez besoin. Et ce fut ainsi, car son amour pour Haïti et pour la littérature et culture haïtiennes était infini.

A commencé alors entre nous, bien que de loin la plupart du temps, une amitié personnelle et scientifique qui a duré trente ans ; on se tenait au courant de nos recherches réciproques, on collaborait aussi : j'ai eu le plaisir de publier moi-même ou de faire publier en Italie plusieurs de ses articles, interviews, comptes rendus... les fruits de la passion critique qui l'animait et qui a vivifié non seulement son activité critique, mais aussi son enseignement universitaire à l'université de Créteil, qui est venu se joindre à son plus ancien enseignement dans le secondaire. 
Elle est l'auteure de trois livres. Le premier, sa thèse de doctorat, $L e$ personnage féminin dans les romans haïtiens et québécois, a paru en 1997, suivi par deux autres, Haïti en littérature en 2000, et La littérature haïtienne dans la modernité en 2017. Aussi, elle a participé au Dictionnaire universel des littératures (1994) et a écrit de nombreux articles sur la littérature haïtienne dans différentes revues, entre autres : Notre Librairie, Afrique littéraire et artistique, Collectif Parole, Conjonction, Pour Haïti, Il Tolomeo, Interculturel, Interculturel/Francophonies.

J'avais reçu d'elle, il y a quelques mois seulement, justement son dernier livre, pour en faire faire un compte rendu dans la revue que je dirige et avec laquelle elle a souvent collaboré avec plaisir pour le plus grand profit de la littérature haïtienne, à laquelle nous avons tous les deux consacré tant d'années et d'énergies, mais Anne bien avant, bien plus et bien mieux que moi.

Ce numéro du Tolomeo présente donc un beau compte rendu de $L a$ littérature haïtienne dans la modernité. Ce legs critique sera, je crois et j'espère, la meilleure façon de prendre congés d'Anne, un congés dont la soudaineté et la dureté et l'injustice nous laissent muets, car il est difficile d'accepter la perte d'une personne si chaleureuse, affectueuse, sérieuse, compétente, généreuse.

Elle est maintenant, je crois, nan Ginen, en paix, à veiller sur ceux et sur ce qu'elle aimait.

Adieu Anne. Et merci. 


\title{
In memoriam: Meena Alexander (1951-2018)
}

\author{
Shaul Bassi \\ (Università Ca’ Foscari Venezia, Italia)
}

Meena Alexander è mancata il 21 novembre 2018, pochi giorni dopo la sua ultima apparizione pubblica, immersa nell'affetto della famiglia e devota alla poesia fino alla fine. La sua vita e la sua opera hanno incarnato alla perfezione la condizione postcoloniale. Nata nel 1951 ad Allahabad, in India, da una famiglia cristiana siriaca del Kerala, era cresciuta in Sudan e aveva poi studiato in Inghilterra, dove si era specializzata nella letteratura inglese del romanticismo; si era trasferita poi negli Stati Uniti nel 1979, dove ha passato gran parte della sua vita, insieme al marito David Lelyveld, storico dell'India, e ai due figli. Questa esistenza cosmopolita, continuamente dislocata e riterritorializzata è sempre stata fonte di smarrimento, di strappi mai ricuciti del tutto ma anche fonte inesauribile di ispirazione poetica e critica. Si pensi solo alle lingue con cui era cresciuta: il malayalam dei genitori, la hindi della comunità nazionale, l'arabo appreso in Sudan, l'inglese dell'istruzione postcoloniale, e il francese degli studi universitari cominciati a Khartoum e proseguiti a Nottingham. In un'intervista rilasciata a Marco Fazzini confessava:

I started writing poetry young, when I was eleven of twelve. The reason why I keep writing is still the same. For me, it is the music of survival. There is an inner voice that speaks to me, makes music out of words, makes notes out of syllables, makes rhythms out of what words cannot reach... Poetry and place are always bound up together. If poetry is the music of survival, place is the instrument on which that music is played, the gourd, the strings, the fret. I have sensed the truth of this, but certain difficulties befell me... Lacking just one single place to call my home and shorn of a single language I could take to be mine, and mine alone I felt stranded in the multiplicity that marked my life. Its rich coruscating depth drew me, or so I felt, into grave danger. It took me quite awhile to realize that I did not have to feel strung out and lost in the swarm of syllables. Rather, the hive of language could allow me to make a strange and sweet honey, the pickings of dislocation. (Fazzini 2017, 180-2)

Larrivo negli Stati Uniti è tutt'altro che una facile ricomposizione di questi frammenti, e nemmeno la New York crocevia di genti può accomodare 
armoniosamente tutte queste parti. Nel suo volume autobiografico del 1993, dall'emblematico titolo Fault Lines, si trova un momento rivelatore, in cui la dolorosa frammentazione viene insieme accettata e trasfigurata in una nuova concezione estetica:

My life was so torn up into bits and pieces of the actual that I depended on the poems, irruptions of the imaginary to make an internal history for me. I focused in on the silk banner outside the museum entrance. It had a sign advertising "The Golem in Jewish Art." The mud monster. Wasn't that what the golem was? Mary Shelley's monster came to mind, the creature made with bits and pieces of flesh and stapled together with Frankenstein's miserable magic. I shut my eyes to cut out the ugliness of it, the grotesque joints, the stitches that showed, the split seams. (Alexander 2003, 125)

È difficile oggi, con un multiculturalismo spesso superficiale ed esotizzante diventato fenomeno di consumo, ricordare quanto autori come Alexander siano stati pionieristici nel decolonizzare e denazionalizzare la letteratura e la poesia. Hanno saputo renderla compiutamente cosmopolita in un senso radicalmente diverso da quello, squisitamente eurocentrico, dei grandi maestri del modernismo, che sceglievano il mondo come casa ma erano comunque saldamente radicati in antiche culture nazionali egemoni. Meena Alexander non ha mai celebrato il dislocamento come una condizione felice e privilegiata, sottolineando spesso lo smarrimento e l'alienazione che possono derivare da un continuo spostarsi di luogo in luogo. Nelle sue poesie incontriamo spesso donne esuli e rifugiate, in fuga dalla guerra e dalla violenza, così come incontriamo personaggi provenienti da mondi radicalmente diversi che come per magia si sovrappongono.

Sebbene alcune poesie fossero già apparse in traduzione araba ai tempi del Sudan, Alexander comincia a pubblicare poesie in India con The Bird's Bright Ring (1976), I Root My Name (1977), and Without Place (1978), rappresentando una seconda generazione di poeti anglofoni seguita ai pionieri nati negli anni '30, Nissim Ezekiel, Kamala Das e A.K. Ramanujan. A segnare una svolta decisiva è significativamente la sua prima raccolta 'americana', House of a Thousand Doors (1988), che sarà seguita da The Storm (1989), Night-Scene, the Garden (1989), River and Bridge (1995), Illiterate Heart (2002), Raw Silk (2004), Quickly Changing River (2008), Birthplace with Buried Stones (2013), e la recentissima Atmospheric Embroidery (2018).

'Distinguished Professor' di letteratura inglese allo Hunter College e al CUNY Graduate Center, Alexander aveva sempre accompagnato la sua vocazione poetica al lavoro critico, con opere in cui la felicità della scrittura creativa si faceva sempre sentire nel rigoroso lavoro di analisi culturale. Dopo due studi critici sul romanticismo inglese (uno dedicato a Mary Wollstonecraft, Dorothy Wordsworth e Mary Shelley, e uno sulla fenomenologia 
del Romanticismo) scritti all'inizio della carriera, i temi della sua poesia e della sua vita si allineano nei saggi di The Shock of Arrival: Reflections on Postcolonial Experience (1996), che mettono la migrazione al centro della condizione postcoloniale, e rappresentano anche un particolare momento storico in cui l'asse della riflessione teorica si sposta sempre di più dalla 'madre patria' inglese verso gli Stati Uniti, e New York in particolare. Anche il successivo Poetics of Dislocation (2009) ne è conferma, con Alexander che si rivela anche generosa e attenta critica di altri scrittori contemporanei. Oltre alla già citata autobiografia, nella sua versatile produzione si ricordano anche i due romanzi Nampally Road (1991) e Manhattan Music (1997), ricchi di spunti autobiografici e di elementi lirici. Numerosissimi i saggi e le introduzioni ad antologie: il suo ultimo lavoro critico è stata la curatela di una preziosa antologia di scrittori indiani che meditano sulla propria arte (Name Me a Word. Indian Writers Reflect on Writing, 2018) dove la vena metaletteraria dell'autrice, in cui la scrittura si manifesta sempre come atto anche intrinsecamente fisico e corporeo, dà voce a un'ampia rosa di autori in varie lingue dell'India.

E impossibile riassumere in poche righe il senso e lo stile di una poetessa prolifica e sempre attenta a interpretare il presente, ma può essere utile ricordare alcuni dei temi che costituiscono l'asse portante dei suoi versi, sempre concreti nel partire da folgoranti e vivide immagini di cose e persone. Il corpo, dolente, ferito, martoriato, ma anche desiderante, sensuale, espressivo è una presenza costante, spesso messo a dura prova dalla violenza, soprattutto quella contro le donne.

Coraggiosa e diretta nell'incorporare dolorosi elementi autobiografici (dagli abusi sessuali subiti da parte di un parente, alla complessa relazione con l'anziana madre, alla sorella disabile, al trauma dell'11 settembre), nelle sue liriche Meena Alexander fonde spesso diversi piani temporali e diverse parti del mondo, in un complesso gioco tra memoria e luogo. Tipico in questo senso la fusione delle acque del Kerala ancestrale con quelle di Venezia, città a lei molto cara e che aveva visitato più volte, risiedendovi anche per lunghi periodi (e perdendovisi con grande disinvoltura, non nascondendo la sua mancanza di senso dell'orientamento). In Italia aveva trovato molti estimatori e amici. Le sue poesie sono apparse in traduzione italiana nelle antologie Poeti indiani del Novecento di lingua inglese (a cura di Shaul Bassi, 1998), L'india dell'anima. Antologia di poesia femminile indiana contemporanea in lingua inglese (a cura di Andrea Sirotti, 2006), Otto Poesie (a cura di Marco Fazzini, 2011). Aveva partecipato nel 2008 alla primissima edizione del festival di letteratura Incroci di civiltà e nel 2011 al convegno Europe and the Americas. Intra and Intercontinental Migrations, organizzato da Daniela Ciani, che a lei ha dedicato il saggio «Voci migranti dall'Asia agli USA: Meena Alexander, una poetessa indiana a New York» (Ciani 2009). Una prima residenza nel 2011 aveva ispirato varie poesie veneziane, tra cui Acqua alta, che era 
stata musicata dal compositore svedese Jan Sandström per un concerto dedicato al tema dei cambiamenti climatici. Nell'estate del 2016 era stata invitata da Beit Venezia. Casa della cultura ebraica a un'altra residenza dedicata al cinquecentenario del Ghetto di Venezia, e da lì nascono le poesie su Sara Copio Sullam pubblicate nel volume Poems for Sarra/Poesie per Sara (2018), con opere di Meena Alexander tradotte da Anita Pinzi, Rita Dove, e Esther Schor, oltre a uno spettacolo andato in scena a New York curato da Ted Hardin ed Elizabeth Coffman. In un commovente omaggio del figlio Adam Kuruvilla, scritto per tenere aggiornate le tante persone che le si erano strette intorno quando la grave malattia che l'aveva colpita si era ripresentata una seconda volta, leggiamo che Meena Alexander ha lasciato questo mondo ascoltando Bach e le poesie di Tagore, accettando serenamente e coraggiosamente che il suo cammino era arrivato alla fine, e condividendo un messaggio che riassume perfettamente la profondità e intensità di una instancabile viaggiatrice dell'anima: "She spoke of birth and death and the veil between from which she could see so many things she had not seen before and at whose edge she hovered in grace. Love is the thing she said, love is what matters".

\section{Attar}

Soon after we met you set a tiny bottle in my palm. It had blue whorls and a gold stopper,

Glass blown in the furnaces of Hyderabad. Open it you whispered,

They call it Attar of the First Rains on Dry Earth:

Pick a piece of wool cotton and pour a drop on it,

Then set it in the broken window frame:

Remember this is the odor of earth and air

This perfume summons souls.

(Meena Alexander. "Attar”. Ciani Forza, Daniela; Francescato, Simone (a cura di), Il profumo della letteratura. Milano: Skira, 2014, 359).

\section{Attar}

Appena incontrati mi posasti nel palmo una boccetta. Aveva spirali blu e un tappo d'oro,

Vetro soffiato nelle fornaci di Hyderabad.

Aprilo, sussurrasti,

Lo chiamano l'Attar delle Prime Piogge sulla Terra Riarsa:

Prendi un batuffolo d'ovatta e versaci una goccia,

Mettilo nella cornice d'una finestra infranta:

Ricorda che questo è l'odore della terra e dell'aria

Questo è il profumo ch'evoca anime.

(traduzione di Marco Fazzini) 


\section{White Nile, Love Song}

Coarse sunlight, cotton shirt, fistful of dates, bitten orange, your sandals, my scarf.

Behind us

a room with a polished floor, roses in extremis.

Will you love me after the end, before the beginning, by a stream of Nile water, bloodied by roses?

(Meena Alexander. "White Nile, Love Song". Otto poesie. Venezia: Sinopia Libri, 2011, 29)

\section{By the Zattere}

A swallow perched on a black stone At the edge of our courtyard.

A bird of parting if there ever was one. Who knew it could sing like that?

Alphabets rose from damp soil, They glimmered in the light of the cosmos Then burst into particles, Turquoise, ruby, jade, shadowy onyx. Where was the ink to etch our syllables? Bits of light tore free in the wind. I tried to play my violetta d'amore On rocky ground

By the rim of the Zattere, In intemperate sunlight, trailing my skirts.

Who will remember me?

(Meena Alexander. "By the Zattere". Alexander, Meena; Rita Dove; Esther Schor, Poems for Sarra/ Poesie per Sara. Venezia: Damocle, 2018, 44-5.

\section{Nilo bianco, canzone d'amore}

Luce garbata, camicia in cotone, una manciata d'appuntamenti, un'arancia morsicata, i tuoi sandali, la mia sciarpa.

Dietro di noi una stanza dal pavimento lucidato, rose in extremis.

Mi amerai dopo la fine, prima dell'inizio, accanto a un rivolo del Nilo, insanguinato dalle rose?

(traduzione di Marco Fazzini)

\section{Alle Zattere}

Una rondine si posò su una pietra nera Al margine del nostro cortile.

Uccello di commiato se mai ce ne fu uno.

Chi immaginava potesse cantare così?

Alfabeti si levarono dal suolo umido, Brillarono nella luce del cosmo

Scoppiarono in particelle, Turchese, rubino, giada, ombrosa onice. Dov'era l'inchiostro per incidere le nostre sillabe? Frammenti di luce si liberarono, strappati dal vento. Provai a interpretare la mia violetta d'amore Su terra rocciosa

Al margine delle Zattere

Sotto la luce inclemente del sole, trascinando le mie gonne.

Chi si ricorderà di me?

(Traduzione di Anita Pinziw) 


\section{Bibliography}

Alexander, Meena (2003). Fault Lines. New York: The Feminist Press at CUNY.

Alexander, Meena; Rita Dove; Esther Schor (2018). Poems for Sarra/Poesie per Sara. Venezia: Damocle.

Ciani, Daniela (2009). «Voci migranti dall'Asia agli USA: Meena Alexander, una poetessa indiana a New York». Oltreoceano, 3, 97-107.

Fazzini, Marco (2017). The Saying of It. Conversations on Literature and Ideas with 13 Contemporary English-language Poets. Pisa: Edizioni ETS. 
Bio-bibliografie | Bio-bibliographies | Bio-bibliographies 



\title{
Bio-bibliographies
}

\begin{abstract}
ALVAREZ DOMENECH, Irene | ialvarezdomenech@gmail.com Irene Alvarez Domenech recently received her master's degree in French and Francophone Studies, a double degree from Sorbonne University in France and Ca' Foscari University of Venice in Italy. Her research is mainly focused on European fin-de-siècle literature but she is also interested in twentieth-century modernist and postcolonial authors. She is currently preparing a PhD on the mutual influences between the Decadent literary movement and the medical theories of the second half of the nineteenth century.
\end{abstract}

AUTIERI, Arianna | arianna.autieri@gmail.com In October 2018 Arianna Autieri started her PhD studies at Warwick University (UK), where she has been awarded a CADRE (Centre for Arts Doctoral Research Excellence) Scholarship. In her research, she is investigating the linguistic musicality of James Joyce's Ulysses and its relevance in translation. She previously graduated in Foreign Languages and Literatures at University of Milan in 2017, with a thesis on the translation of the musical aspects of James Joyce's prose in The Dead, A Portrait of the Artist as a Young Man, and "Sirens". She also graduated in Classical Music at Milan Conservatory in 2014. She taught music since right after her graduation in 2014, and in the school year 2017-2018 she was a teacher of English Language and Literature at Liceo Classico Beccaria in Milan. Her research interests are Joyce, music and literature studies, and translation.

BADR Maha | maha_badr@hotmail.it Maha Badr is an associate professor at The Lebanese University since 2008. She received her PhD in Language, Civilisation and French Literature from the University of Poitiers-France (2007), and the Master's degree from the University of Lyon II - France (2002). She is an author of multiple articles and of a book titled Georges Schehadé ou la poésie du réel edited by L'Harmattan, Paris, in September 2010. She participated in regional and international conferences. Research interests : poetry, modern and contemporary literature, francophone literature.

BORASO Silvia | silviaboras093@gmail.com Silvia Boraso graduated in 2017 from University of East Piedmont. Her thesis Writing Orality. Representations of Speech in Five Postcolonial Novels is a comparative study that analyses, from both a linguistic and hermeneutic perspective, the ways in which orality is reproduced within the boundaries of narrative discourse. Her research mainly focuses on the representation of language in the Postcolonial novel and on the socio-linguistic characterisation of narrators.

BOTTOSSO Giacomo | botossogiacomo@gmail.com Giacomo Bottosso completed his Master's Degree in Foreign Languages and Literatures at the University of Trieste, discussing a dissertation entitled Razza, genere e biopolitica: una lettura necropolitica di Sony Labou Tansi. Since February 2018 he has been teaching French Literature as adjunct professor at the Department of Humanities, University of Trieste.

BRUNETTO Veronica | brunetto.veronica@gmail.com In July 2016 Veronica Brunetto graduated in European, American and Postcolonial Languages and Literatures at Ca' Foscari University of Venice, with a dissertation called La metamorphose dans le conte folklorique haitien: Deux perspec-

DOI 10.30687/Tol/2499-5975/2018/20/040

Submission 2018-11-28

(c) 2018 | () Creative Commons Attribution 4.0 International Public License 
tives d'analyse. In December 2016 she published a review of the new edition of Louisiana Folktales, Lapin, Bouki, and Other Creole Stories in French Dialect and English Translation, by Alcee Fortier, in Il Tolomeo journal (no. 18). She is continuing her research on Francophone Literatures, especially in what concerns folk tales' presence in written literature.

BRUSCHI Isabella | isabella.bruschi@gmail.com Isabella Bruschi is a teacher of English Language and Literature at High School (Liceo Classico "V. Alfieri", Turin) and has been a teacher-trainer at the post-graduation School for Foreign Languages Teachers (University of Turin and East Piedmont). She has researched in the field of Indo-Anglian Literature, in particular she has dealt with Partition Literature, the subject of her PhD dissertation. Her publications relate both to her teaching and academic activities.

CACCHIOLI Emanuela | emanuela.cacchioli@gmail.com Emanuela Cacchioli obtained her PhD in Comparative Literatures at the University of Genova in 2015. Her thesis, Relectures du mythe d'Antigone dans les littératures francophones extra-européennes, has been published by L'Harmattan. Her main fields of interest are Francophone Literature, in particular the Haitian one, intertextuality, rewritings and contemporary French Literature. She has published several articles in Italian, French, Canadian specialised reviews and in books. She is member of CRLC at the University of Paris IVSorbonne where she also took part in OBVIL project that promoted research in Digital Humanities.

CAI Yuqian | 870192@stud.unive.it Yuqian Cai is an MA student in the Joint Degree in English and American Studies at Ca' Foscari University of Venice and the University of Graz; he also holds an MA in East Asian Studies from Yale University.

CALDERARO Michela | michela@rialto.com Michela A. Calderaro has been an Associate Editor of Calabash. A Journal of Caribbean Arts and Letters for the entire decade of its publication and head of its book reviews section; she has also been the Editor of the Ford Madox Ford Newsletter (1999-2006). Her critical works include a book on Ford Madox Ford, numerous articles on British, American and Anglophone Caribbean writers, and a series of interviews with major Caribbean poets. In both her critical works and interviews she focuses on issues of identity and memory, and the way (personal and collective) memory is processed by artists. She is currently working on a biography of Creole writer Eliot Bliss, and has edited a collection of Bliss' unpublished poems, Spring Evenings in Sterling Street.

COLLINS KLOBAH Loretta | collinsklobah.loretta@gmail.com Loretta Collins Klobah is Full Professor of Caribbean Literature, Creative Writing, and Medical Humanities courses at the University of Puerto Rico, Río Piedras Campus, in San Juan, Puerto Rico. She received an MA in English-Creative Writing from California State University, Fresno, an MFA in poetry writing from the University of lowa Writer's Workshop, and a PhD in English, with an emphasis on Anglophone Caribbean Literature, from the University of lowa. Her poetry collection The Twelve-Foot Neon Woman (Leeds: Peepal Tree Press, 2011) received the 2012 OCM Bocas Prize in Caribbean Literature in the category of poetry and was short listed for the 2012 Felix Dennis Prize for Best First Collection in the Forward Poetry Prizes. Her poetry collection Ricantations is forthcoming from Peepal Tree Press in June, 2018. It has been named a Summer Recommendation by the British Poetry Book Society. She has been the recipient of numerous awards for poetry writing, including those of the Academy of American Poets, Pushcart Prize, and the Pam Wallace Award for an Aspiring Woman Writer. She has served as a judge for the poetry category of the 2018 OCM Bocas Lit Fest in Trinidad and Tobago. She has also been a recipient of a faculty development grant from the National Endowment for the Humanities, a Fulbright Award, an International Council for Canadian Studies Fellowship, and a Rockefeller Grant, at the University of Georgia, Athens. Her academic articles have been anthologized, as well as published in such journals 
as Small Axe, Anthurium: A Caribbean Studies Journal, Image and Narrative, Jamaica Journal, Journal of Commonwealth and Postcolonial Studies, South Asian Popular Culture, and Literature and Medicine.

\section{Co-authors:}

- Ari HERNÁNDEZ is taking graduate coursework related to Caribbean literature in the Department of English, University of Puerto Rico, Río Piedras Campus.

- Charlotte LÓPEZ is an MA student in the Cultural Administration Program at the University of Puerto Rico, Río Piedras Campus.

- Michelle RAMOS is an MA student in English, with an emphasis in literature, in the Department of English, University of Puerto Rico, Río Piedras Campus.

- Nilo Mahatma CAITUIRO MONGE recently completed a Master's Degree in Cultural Administration at the University of Puerto Rico, Río Piedras Campus.

COSTANTINI Alessandro | costalex@unive.it MA in Foreign Languages and Literatures at Venice University. Scholarship fellow (MAE, France; CNR., Italy) at École des Hautes Études en Sciences Sociales, Paris. "Diplôme de l'École" and "DEA" in Semiotics at É.H.É.S.S. (Paris). PhD in Literary Sciences. Since 1992, researcher and assistant professor of French and Francophone literatures at Ca' Foscari University of Venice; at present associate professor of French Literature and Francophone Studies. His research and teaching work focuses on Francophone literatures (mainly Haitian and Caribbean), French Algeria literature, History of French culture (French colonisation, Franco-belgian comics), literary diglossia, Francophone linguistics. Editor-in-chief of the journal /l Tolomeo. Member of the Editorial Board of Interfrancophonies and member of the Scientific Committees of the journals Interculturel/Francophonies, Lingue e linguaggi and Regards croisés (Linguistic and Literary Studies Collection, Macerata and Clermont-Ferrand Universities). Books: Roumain, Jacques. Signori della rugiada. A cura di Alessandro Costantini. Roma, Edizioni Lavoro, 1995; Fantasmi narrativi e sovversione linguistica nel romanzo haitiano moderno e contemporaneo. Milano: Cisalpino-Ist. Ed. Universitario, 2002; "La langue polyphonique de Jacques Roumain". Roumain, Jacques, Euvres complètes. Paris, ALLCA XX, 2003, 1429-67; Le roman haïtien: intertextualité, parentés, affinités. Sous la direction de Yves Chemla et Alessandro Costantini. Lecce: Alliance Française, 2007; "Nouvelles formes de l'engagement dans les littératures francophones”. Intefrancophonies, 7, 2016, 1-180.

COZzıO Federica | federica.cozzio@gmail.com Federica Cozzio has a Bachelor Degree in Cultural Heritage Studies (curriculum History and Preservation of Theatrical and Film Heritage) from the University of Milan and a Master in Cultural Anthropology, Ethnology and Ethnolinguistic from Ca' Foscari University of Venice. Her main fields of research focus on Anthropology of Space, Development and Tourism. She is also interested in the interdisciplinary dialogue between Anthropology and Literature, and between Anthropology and Cinema. She directs documentary films and has made field research in Haiti and New Orleans.

DEL ROSSI Sara | saradelrossi88@gmail.com Sara Del Rossi is a PhD candidate in Romance Languages and Literatures at the University of Warsaw, where she is continuing her research in the field of the Caribbean, and most particularly Haitian, oral literature. Her main interests are the different ways in which the diaspora (France, Québec and USA) memory influences the contemporary oral genres (folktales, proverbs, plays, slams...). Last publications: "Mèt kontè se manti ! Maitre conteur, tu mens 
ou tu (te) trompes ! Le passage de la simulation narrative à la confession autobiographique dans le conte haïtien en diaspora" (Echo des études romanes, 2017) and "Rites de passage et performativité. Le conte orale haitien en diaspora québécoise” (Rites et ritualisation, 2018).

DORGELÈS Houessou | dorgeleshouessou@yahoo.fr Dorgelès Houessou is a doctor in stylistics and textual linguistics fields, graduated from the University Felix Houphouet Boigny of Abidjan-Cocody in 2013 after a doctoral thesis on the decompartmentalization and generic categorization of the inauguration speech through the Ivorian case. He is currently teaching French stylistics at the Department of Modern Letters of the UFR Communication, Environment and Society at the Alassane Ouattara University of Bouaké (Ivory Coast). His research is part of a multidisciplinary perspective and combines the fields of stylistics, pragmatics, semiology and discourse analysis. He participated in about fifteen symposiums and international study days and was part of the coordinators of the Abidjan symposium on urban languages in Africa (March 13-15, 2019). He also has about twenty publications in collective works and international journals including EID \& A, Textimage, and Cahiers de Narratologie.

FERMI Elena | elena.fermi50@gmail.com During June 2000 she graduated in French Language and Literature at the University of Parma and in 2001 she got a Master's Degree in French and Comparative Literature at the University of Paris X - Nanterre La Défense and in 2006 a PhD in French Studies at the University of Turin and Paul Valéry-Montpellier III. Her main areas of interest are historic avant-guard, the relationships between art and literature in early 20th century and francophone literature. Her publications include essays about Jean Cocteau and his relationships with Italian painters and artists. She deals with the organisation of cultural events (in particular exhibitions of contemporary art). Since 2014, she contributes with some reviews at the section "Letterature francofone extraeuropee" of the periodical Studi francesi.

FIANCO Fabiana | fabiana.fianco@gmail.com Fabiana Fianco graduated in Modern Languages and Literatures at University of Trento in 2016 with a dissertation called De la Négritude à la Créolité, which analyses the evolution of the francophone racial perspective through the most relevant literary theories conceived between the 1930s and the 1990s. In 2018, she completed a double degree program between the University of Lausanne and Cà Foscari University of Venice, where she obtained her Master's degree in European, American and Postcolonial Language and Literature. Her thesis explored the themes of sexuality and corporeality in the work of African and Haïtian female novelists in contemporary times (1970-2013). She currently teaches French language in high school.

GUADELOUPE Francio | f.e.guadeloupe@uva.nl Francio Guadeloupe, the Aruban born Social \& Cultural Anthropologist, served for four years as the President of the University of St. Martin (USM), until hurricane Irma led to the closure of the institution in the Dutch West indies. He is currently employed at the University of Amsterdam, the Netherlands. He is the author of the monograph, Chanting Down the New Jerusalem: Calypso, Christianity, and Capitalism in the Caribbean (University of California Press, 2009). Essays of Guadeloupe have appeared in Transforming Anthropology, Latin American and Caribbean Ethnic Studies, Social Analysis, Etnofoor, Women Studies International Forum, and the Journal for the Study of Religion

LIM Ann-Margaret | annyinpin@hotmail.com Ann-Margaret Lim is the author of the debut poetry collection The Festival of Wild Orchid (Peepal Tree Press, 2012), which was nominated for the UK Guardian First Book Prize and received an Honorable Mention for the 2013 OCM Bocas Prize for Caribbean Literature. Her second collection, Kingston Buttercup (Peepal Tree Press, 2016) was long-listed for the 2017 OCM Bocas Prize, in the poetry category. Her 2014 Calabash Literary Festival reading earned her a feature alongside former Jamaican Poet Laureate, Prof. Emeritus Mervyn Morris, in the Ebony Magazine article: "Six Caribbean Writers to Discover this Summer" (2014). She has represented Jamaica in festivals in Venezuela and Colombia, and at the St. Martin Book Fair. She 
is published in anthologies and journals in the Caribbean, the United Kingdom, the US and South America. She lives in Red Hills, Jamaica.

SPENCER MATZ Margaret | matzmargaret@gmail.com Margaret Matz is an AIA-registered architect and president of Milestone Architecture, PLLC, in New York City. The former assistant professor at New York Institute of Technology and Pratt Institute School of Architecture, has lectured on art and architecture in the USA, St. Martin, and Venice, Italy. Matz has served as project architect, project manager, senior designer, and executive principal with major architectural firms. For 20 years, the award-winning architect has been designing new, restoration and sustainable renovation projects for academic and health care institutions and corporate and retail organizations in the USA, Ireland, and Italy.

MEO Eleonora | eleonorameo@hotmail.it Eleonora Meo completed her PhD in International Studies at the University of Naples "L'Orientale". Her thesis, Visual Culture: a Postcolonial Critique of European Citizenship, explored the concept of "visual citizenships" and linkages between colonialism, European citizenship and the emerging of counter-narratives in contemporary visual art. Her principal research interests stem from cultural studies, feminist theory and postcolonial studies, focusing on visuality, racism, cultural representation, citizenship and migration. She has recently co-edited a book on modernity and postcolonial critique, Genealogie della modernità: teoria radicale e critica postcoloniale (Meltemi, 2017). She is currently preparing her thesis for publication.

MESSULAM Federica | federica_messulam@yahoo.it Federica Messulam graduated from the School of Modern Languages for Interpreters and Translators of Trieste. She is mainly interested in authors coming from the Caribbean area. Among them, she translated a collection of short stories by Lorna Goodison, the first Jamaican graduate poet. She has collaborated with the cultural events Incroci di Civiltà and Poetry in Vicenza, presenting in 2015 a selection of poems by the poet Shara Mc Callum, and in 2017 a selection of poems by Lasana Sekou. She is currently working with a team of translators at the first collection of works by Lasana Sekou in Italian, for the House of Nehesi Publishers publishing house.

MICHIELETTO Anna | leilaflower@yahoo.it After following her studies in French and English languages and literatures with a particular focus on post-colonial studies, Anna Michieletto obtained her MA at Ca' Foscari University of Venice with highest honours in 2006. She has taught English and French language and literature in a secondary school in Italy and Italian language for foreigners at "Laboratorio Internazionale della Comunicazione" in Gemona del Friuli. She worked as a teacher of French language in Spain (EU project "Comenius") and she taught Italian at Hacettepe University in Ankara (Turkey). She has collaborated with II Tolomeo, literary review. She obtained another MA in anthropology at Ca' Foscari University of Venice in 2012 and is very interested in travelling and studying other cultures.

PESSINI Alba | alba.pessini@unipr.it Alba Pessini teaches French literature and language at the University of Parma. She obtained her PhD in Comparative Literature at University Sorbonne-Paris IV and the University Alma Mater Studiorum in Bologna. Her research mainly focuses on contemporary French and Francophone literatures, particularly on the works written in French by authors from the Caribbean (The Antilles, Haiti, Guiana). She wrote her thesis on the Haitian writers from the Diaspora, investigating issues such as exile, migration and relocation. Her interests also include women's literature (Nathalie Sarraute, Irène Némirovsky, Yanick Lahens, Evelyne Trouillot) and the issue of food in postcolonial literature.

PETRUZZELLIS Alice | alice.petruzzellis@hotmail.it Alice Petruzzellis is a former student of foreign languages and literatures in Trieste. She graduated with a bachelor's degree in Oriental languages in Bergamo, where she approached the Arabic language and culture. She got a master's 
degree in foreign languages and literatures at the University of Trieste in 2018. There, she started to take an interest in French feminism and in the colonial issues of the Maghreb, studying the relationship between local and French writers.

PUCCIARELLI Edvige | edvige.pucciarelli@gmail.com PhD, she is an independent scholar. As a contract lecturer, she has taught English literature at the Università Cattolica del Sacro Cuore of Milan and at the Università of Bergamo and English language at the University of East-Piedmont. Her main fields of interest are those of English Renaissance Literature and Culture and Canadian Literature. Her recent publications include: A Reading of the Imperial Theme in Shakespeare's "Antony and Cleopatra" (Simplegadi); "Paesaggio morale in Free Radicals di Alice Munro" (II Tolomeo); Il Mito di Albione, insularità e identità nell'Inghilterra giacomiana (Anicia); Gli angeli caduti di Morley Callaghan (Universitas Studiorum). She has also worked on Translation Studies and her translations have been published in Italy and abroad.

ROMERO Ivette | Ivette.Romero@Marist.edu Ivette Romero, born in New York and raised in Puerto Rico, is a Professor of Spanish in the Department of Modern Languages and Cultures at Marist College, in Poughkeepsie, New York. At Marist, she is faculty coordinator of Marist Abroad Programs for Italian and Spanish, and founder/coordinator of the Latin American and Caribbean Studies Program. Besides co-edited volumes, Romero's work has been published as chapters in various essay collections and journals such as Anales del Caribe, Callaloo, Caribbean Quarterly, Emisférica, Mango Season, Nineteenth-Century Literature Criticism, Sx salon, and Sargasso.

VIGNOLI Alessia | alessia.vignoli89@gmail.com Alessia Vignoli is a PhD candidate in French and Francophone Studies at the University of Warsaw (Poland). Her main research topic is the Caribbean literature of French expression, especially Haitian post-earthquake literature. Her PhD dissertation focuses on the representation of natural disasters in Haitian and French Caribbean literature. Last published works: "Le roman post-sismique en Haïti: une écriture (post)contemporaine de l'extrême" (Kwartalnik Neofilologiczny, 3, 2018) ; "De l'éruption rhétorique: étude de l'hypotypose dans la littérature catastrophique franco-antillaise" (Folia Litteraria Romanica, 11, 2017) ; "Des secrets sous les décombres: la (dis)simulation dans le roman post-sismique haïtien" (Écho des études romanes. Revue semestrielle de linguistique et littératures romanes, 2, 2017).

ZOPPELLARI Anna|zoppelan@units.it Anna Zoppellari is Associate Professor of French Literature at the University of Trieste. Her main areas of interest are contemporary Francophone literatures and the relationships between literature and visual arts. She published several articles on French and Maghribi authors.

The authors of the interview with Ngũgĩ wa Thiong'o are alumni of the International College of Ca' Foscari University of Venice.

Giulia BARISON (Treviso, 1994-12-18). MA degree in Italian Philology at Ca' Foscari University of Venice. Now PhD in Romance Philology at University of Siena.

Beatrice CARMELLO (Treviso, 1994-05-24). MA in Ancient Civilisations at Ca' Foscari University of Venice.

Asmaa EL HANSALI (El Jadida, 1992-08-08). MA joint in English and American studies.

Dalia PRATALI MAFFE (Treviso, 1994-12-17). MA degree in Ancient Civilisations at Ca' Foscari University of Venice. 



\section{Rivista annuale \\ Annual journal \\ Revue annuelle}

Università Ca' Foscari Venezia

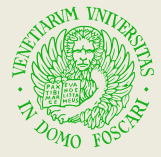

Università

Ca'Foscari

Venezia

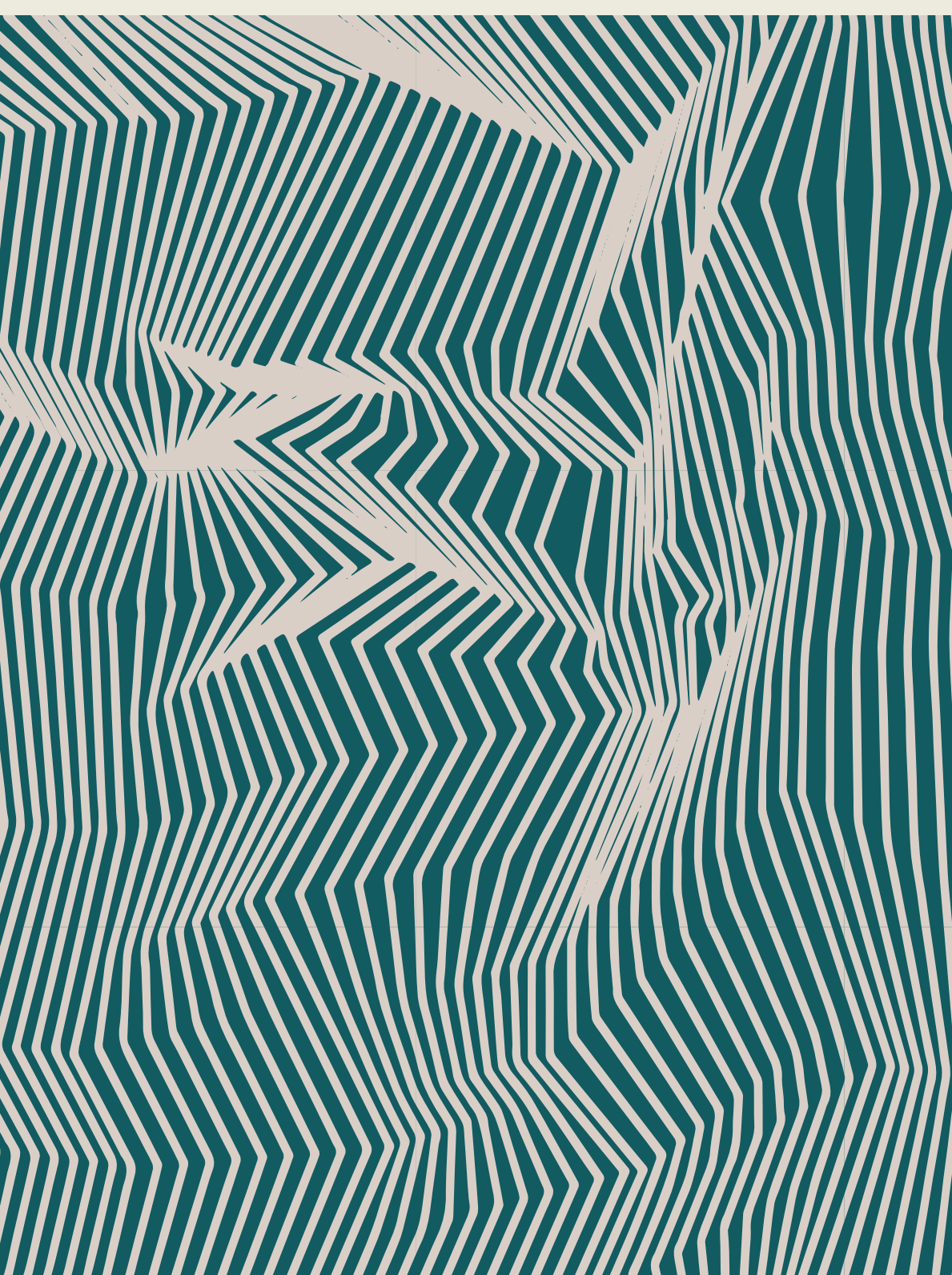

